LBL --31139

DE92 000963

\title{
The Electrical Resistivity Method in Cased Boreholes
}

\author{
Clifford J. Schenkel \\ (Ph.D. Thesis) \\ Engineering Geoscience \\ University of California \\ and \\ Earth Sciences Division \\ Lawrence Berkeley Laboratory \\ University of California \\ Berkeley, California 94720
}

May 1991

This work was supported in part by the U.S. Environmental Protection Agency under Interagency Agreements DW89932611 and DW89933758, through U.S. Department of Energy Contract DE-AC0376SF00098, and in part by Paramegnetic Logging, Inc.

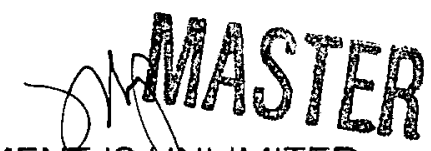




\section{DISCLAIMER}

This report was prepared as an account of work sponsored by an agency of the United States Government. Neither the United States Government nor any agency Thereof, nor any of their employees, makes any warranty, express or implied, or assumes any legal liability or responsibility for the accuracy, completeness, or usefulness of any information, apparatus, product, or process disclosed, or represents that its use would not infringe privately owned rights. Reference herein to any specific commercial product, process, or service by trade name, trademark, manufacturer, or otherwise does not necessarily constitute or imply its endorsement, recommendation, or favoring by the United States Government or any agency thereof. The views and opinions of authors expressed herein do not necessarily state or reflect those of the United States Government or any agency thereof. 


\section{DISCLAIMER}

Portions of this document may be illegible in electronic image products. Images are produced from the best available original document. 
Copyright (C) 1991 by Clifford J. Schenkel

The Goverment reserves for itself and others acting on its behalf a royalty free, nonexclusive, irrevocable, world-wide license for governmental purposes to publish, distribute, translate, duplicate, exhibit, and perform any such data copyrighted by the contractor. 


\title{
The Electrical Resistivity Method in Cased Boreholes
}

by

\section{ClifFord J. SCHENKEL}

\begin{abstract}
The resistivity method in cased boreholes with downhole current sources is investigated using the integral equation (IE) technique. The casing and other bodies are characterized as conductivity inhomogeneities in a half-space. For sources located along the casing axis, an axially symmetric Green's function is used to formulate the surface potential and electric field (E-field) volume integral equations. The situations involving off-axis current sources and three-dimensional (3-D) bodies is formulated using the surface potential IE method. The solution of the 3-D Green's function is presented in cylindrical and Cartesian coordinate systems. The methods of moments is used to solve the Fredholm integral equation of the second kind for the response due to the casing and other bodies.

The numerical analysis revealed that the current in the casing can be approximated by its vertical component except near the source and the axial symmetric approximation of the casing is valid even for the 3-D problem. The E-field volume IE method is an effective and efficient technique to simulate the response of the casing in a half-space, whereas the surface potential approach is computationally better when multiple bodies are involved.

Analyzing several configurations of the current source indicated that the casing response is influenced by four characteristic factors: conduction length, current source depth, casing depth, and casing length. The conduction length, the most important factor, relates the casing conductance with the conductivity of the host medium and is an indicator of the ability of the pipe to carry the current along its length. When the source is located within the casing, the characteristic parameters can be reduced to three ratios: the conduction length to casing length
\end{abstract}


(conduction ratio), the source position to casing length, and the casing depth to casing length. For a conduction ratio that is approximately greater than two, the fields from the casing are similar to those produced by a line source. When the source is located beneath the casing, the distortion of the fields is also dependent on the casing-source separation distance. For a current source near the casing ( $\ll 100$ casing diameters), the casing greatly distorts the fields when compared to those produced by a pole source. When the source is greater than 100 casing diameters from the pipe, only the region near the casing is affected. The numerical simulations indicate that cross-hole and downhole to surface time monitoring studies may be conducted with very little casing effect. An energized casing or a dipole source at the end of the casing can enhance the anomalies produced by a conductive zone. The use of casing in experiments that monitor injection or extraction processes may be advantageous.

Numerical analyses indicate that resistivity measurements through metal casing (MTC) is feasible provided one can distinguish voltage differences in the order of 10 's $\mu \mathrm{V} / \mathrm{m}$ per Ampere. The discontinuity of the E-fields correspond to the layer boundary and the rate of change of the E-field is inversely proportional to the resistivity of the adjacent formation. For a simple layer model, the formation resistivity can be estimated by the ratio of the potential and its second derivative provided that the casing conductance can be obtained. For an unknown casing conductance, two set of measurements are needed to calculate the formation resistivity. At the places where the contact resistance is low, the current tends to leak into the formation more readily and the formation resistivity will be underestimated. A transmission network, to incorporate the current channeling effects, can be used to simulate the MTC method. The borehole fluid resistivity has negligible effects on the resistivity measurements in the casing. Variations of the casing conductance only affect the measurements when the discontinuity lies within the span of the array, while variations of the cement annulus have great effects on the estimate of the formation resistivity. Geometric factors, such as length and radius of the casing, as well as the array location within the casing affect the resistivity measurements. The resolution of the layer boundary is limited to the electrode spacing needed 
to estimate the second derivative. The radius of investigation is independent of the electrode spacing: using different electrode spacings will not provide any additional information about the conductance of the cement or the formation. To determine the true formation resistivity in the presence of a cement annulus is not possible without prior knowledge of the thickness or resistivity of the cement.

The downhole to surface field experiment, conduced at a Dupont test well near Waverly, Tennessee, showed that the signals can be measured in a resistive environment. Not only are casing effects large for measurements near the well, but they can be seen at the far electrodes even for sources located far below the casing. The modeling indicated that strong interactions between the casing and near surface formations exist.

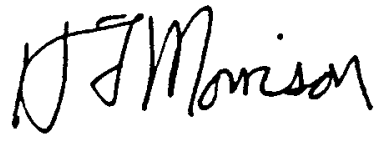




\section{TO THE MEMORY OF MY BROTHER, SAM.}

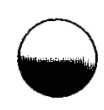




\section{ACKNOWLEDGEMENTS}

I would like to express my sincere gratitude to my research advisor, Frank Morrison for his patience and guidance during my "brief" stay at Berkeley. His knowledge and wisdom are only exceeded by his friendly personality and enthusiasm for life. I am lucky to have worked with him. I am also grateful to my other committee members, Alex Becker and David Donoho, whose insights and observations have always made my discussions with them enlightening. A special thanks to Ki Ha Lee. Without his help in the initial stages, this thesis would have never gotten off the ground. To Dave Kennedy, for many fruitful chats about integral equations, Green's functions, and singularities, although at times these conversations were like the blind leading the blind.

I am appreciative to my long time friends who kept in touch to share my experiences. To my friends in EG, MSME, and LBL with whom I have been able to share many good times. I would also like to mention the "Mining Association" basketball group for guaranteeing me at least one day a week, if not more, of soreness, aches, and pains.

I would like to thank my parents for supporting me in all my endeavors and my sister, Marty, for reminding me of what real life is like. I am very thankful to have such a devoted companion in Calie. The wag of her tail has always made the daily grind much easier to bear. Finally, my heart goes out to Maria Toyofuku. I am very fortunate to have someone like you to share my life. Your love and support has meant, and always will be, much more to me than I can put into words.

Without the financial support that I received while attending Berkeley, I would not have been able to start, yet alone complete, this thesis. I am indebted to Chevron and the department of MSME for awarding me fellowships. To Aldo Mazzella and the EPA for supporting the majority of this research, and to Banning Vail and Paramagnetic Logging, Inc. for funding me during the final phase of this thesis.

This work was partially supported by the United States Environmental Protection Agency through Interagency Agreement Number DW89932611 and DW89933758 to Lawrence Berkeley Laboratory. 
I have made this letter longer than usual because I lacked the time to make it short.

BLAISE PASCAL (1623-1662) 


\section{TABLE OF CONTENTS}

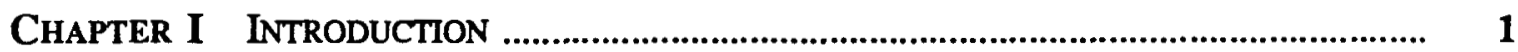

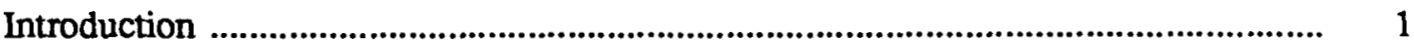

Previous Research ........................................................................................ 2

Scope of Research ............................................................................................... 5

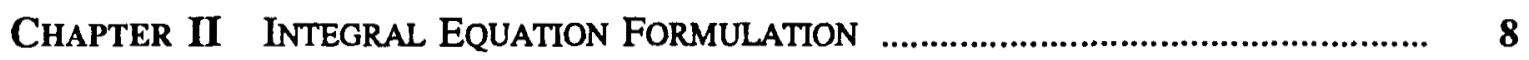

Introduction .................................................................................................. 8

2.1 Surface Potential Integral Equation ......................................................... 10

2.1.1 Potential Field Equation ................................................................. 10

2.1.2 Surface Integral Equation Formulation ......................................... 11

2.1.3 Singularity ...................................................................................... 17

2.1.4 Half-Space Problem ..................................................................... 18

2.1.5 Multiple Bodies .......................................................................... 20

2.2 Electric Field Volume Integral Equation .................................................... 22

2.2.1 Electric Field Equation ................................................................. 23

2.2.2 Solution of the Field Equation ........................................................ 25

2.2.3 Electric Field Integral Equation Formulation .................................... 26

2.3 Numerical Solution of the Integral Equations .............................................. 29

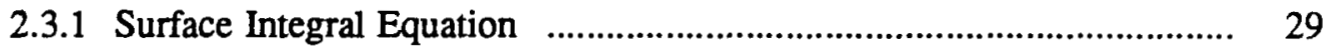

2.3.2 Volume Integral Equation .......................................................... 30

2.3.3 Matrix Equation ...................................................................... 31

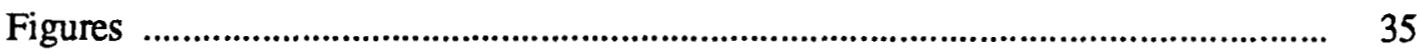

Chapter III Green's Function Solutions ................................................... 40

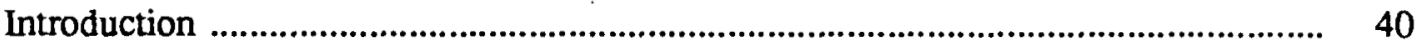

3.1 Axisymmetric Problem ........................................................................... 42

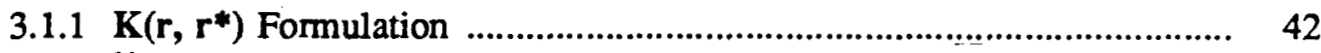

3.1.2 $\boldsymbol{\Gamma}\left(r, r^{*}\right)$ Formulation ................................................................. 46

3.2 Three Dimensional Problem ........................................................................ 50

3.2.1 Cartesian System $\mathbf{K}\left(\mathbf{r}, \mathbf{r}^{*}\right)$ Formulation ........................................ 50

3.2.2 Cylindrical System $\vec{\Gamma}\left(r, r^{*}\right)$ Formulation ........................................ 53

3.3 Off-axis Geometry and Axisymmetric Approximation .................................. 56

Figures ............................................................................................... 59 
ChaPTER IV CONSISTENCY, ACCURACY, AND VALIDITY .......................................... $\quad 70$

Introduction ................................................................................................ $\quad 70$

4.1 Self Consistency \& Accuracy ........................................................................ 71

Convergences ..................................................................................... 71

Reciprocity ...................................................................................... 72

4.2 Validity by Comparison ............................................................................... 74

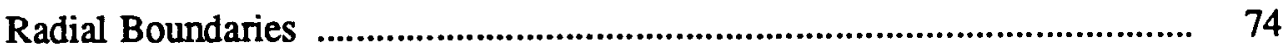

Horizontal Boundaries .......................................................................... 75

Dual Boundaries ................................................................................. 75

Axisymmetric Approximation .................................................................. 76

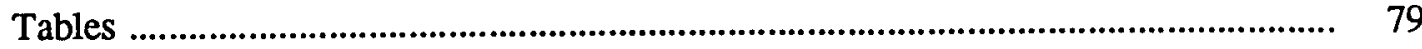

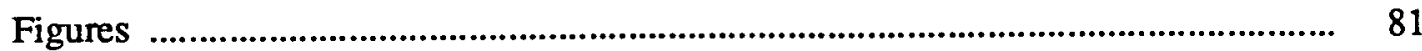

Chapter V Numerical ANALYSIS of CASING EfFects ......................................... 90

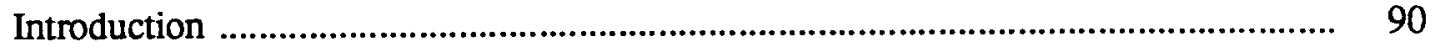

5.1 Source within Casing ………….................................................................... 92

5.1.1 Semi-Infinite Length Pipe ................................................................. 92

5.1.2 Finite-Length Pipe .......................................................................... 95

5.1.3 Potentials in the Medium .................................................................... 97

5.1.4 Line Source Approximation …………………….............................. 100

5.1.5 Summary ...................................................................................... 101

5.2 Source Beyond Casing End ………………………….................................. 102

5.2.1 E-fields in the Casing ...................................................................... 102

5.2.2 Potentials in the Medium ................................................................... 103

5.2.3 Summary .................................................................................... 105

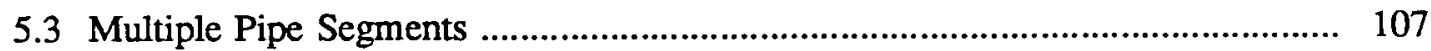

5.3.1 E-fields in the Casing ....................................................................... 108

5.3.2 Potentials in the Medium ................................................................ 108

5.3.3 Additional Segments ...................................................................... 109

5.3.4 Summary ................................................................................. 110

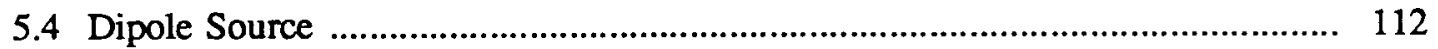

5.4.1 Conduction Length ........................................................................ 112

5.4.2 Dipole Gap Length ......................................................................... 113

5.4.3 Lower Segment Length .................................................................. 114

5.4.4 Surface Response ......................................................................... 115

5.4.5 Summary ................................................................................... 115

5.5 Numerical Examples .............................................................................. 117

5.5.1 Injection Monitoring Simulations .................................................. 117

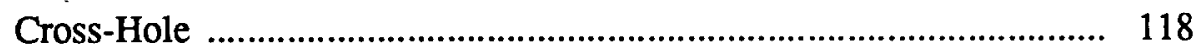

Downhole to Surface …............................................................ 119

Conclusions .............................................................................. 122

5.5.2 Measurement While Drilling Telemetry ............................................... 122

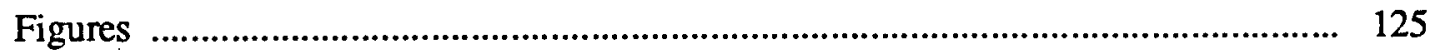


Chapter VI Resistivity Measurement Through Casing

Introduction

6.1 Resistor Circuit Relation

6.2 Electrode Arrays for Unknown Conductance ................................................ 232

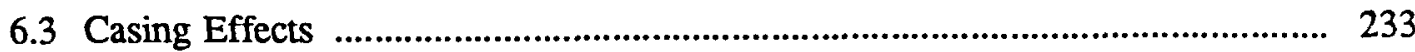

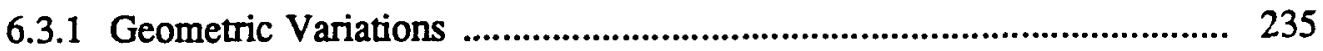

6.3.2 Conductance Variations ............................................................... 237

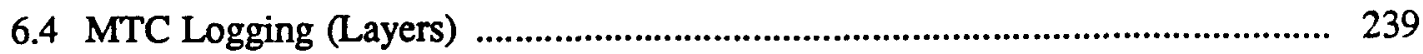

6.5 Borehole Fluid Effects ................................................................................. 241

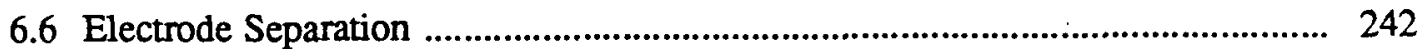

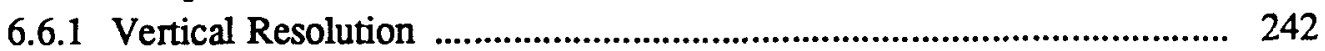

6.6.2 Radius of Investigation ............................................................. 243

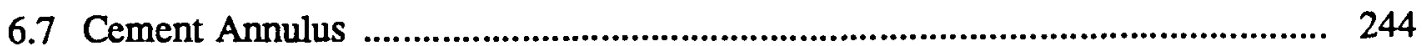

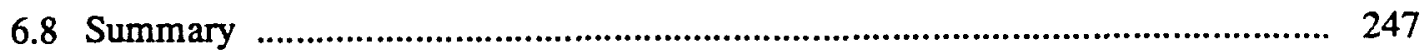

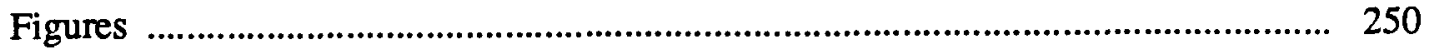

Chapter VII FIEld EXAMPLE - Downhole to SuRface Resistivity SurVey ...... 275

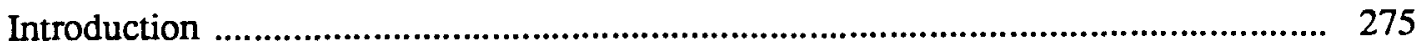

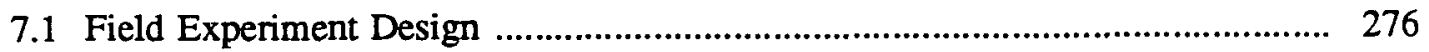

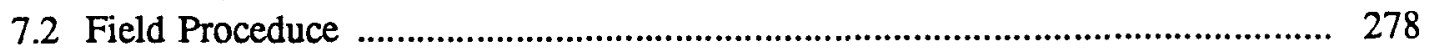

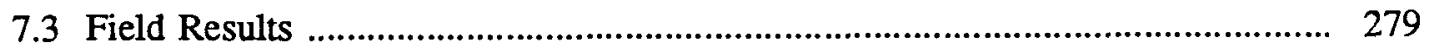

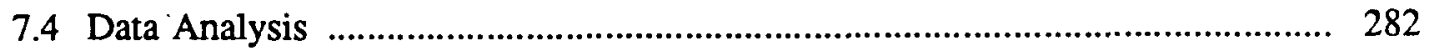

7.4.1 Apparent Resistivities ............................................................... 282

7.4.2 Numerical Models ...................................................................... 284

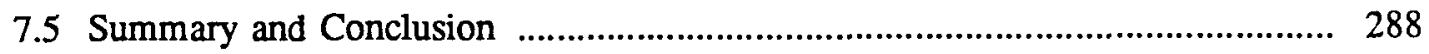

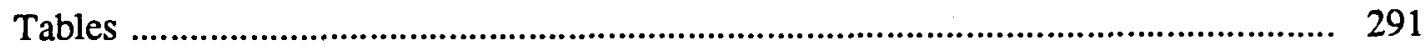

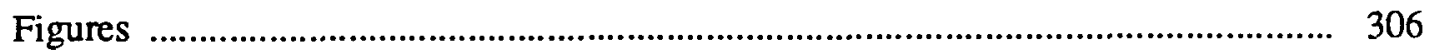

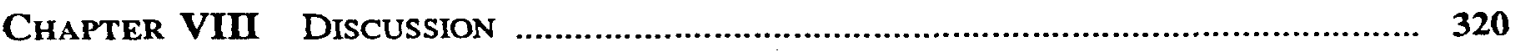

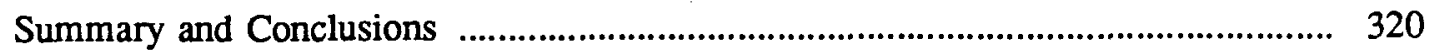

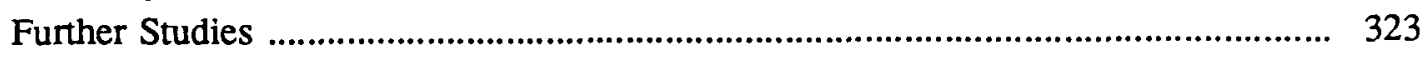

REFERENCES

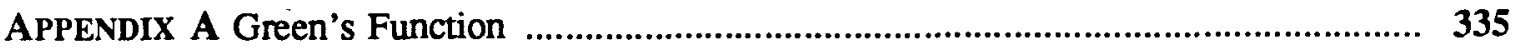

APPENDIX B Numerical Approximation: Method of Moments ..................................... 340

APPENDIX C Axisymmetric Green's Function ............................................................. 344

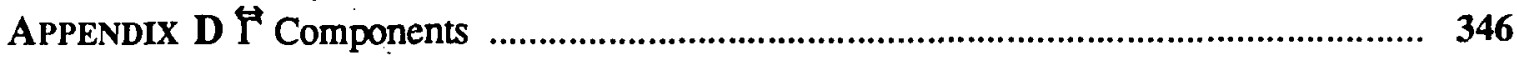

APPENDIX E Surface Gradient Integral ............................................................ 348 


\section{Chapter I}

\section{INTRODUCTION}

The use of downhole current sources in resistivity mapping can greatly enhance the detection and delineation of subsurface features (Merkel and Alexander 1971, Daniels 1978, and Daniels and Dyck 1984). Theoretical studies of a spheroidal target (Snyder and Merkel 1973, Dobecki 1980, and Lytle 1982), as well as numerical integral equation results for arbitrary objects (Daniels 1977, Yang and Ward 1985, and Beasley and Ward 1986) have shown an enhanced response of the body when downhole sources are used. Wilt and Tsang (1985), using a three-dimensional finite difference modeling algorithm developed by Dey and Morrison (1979) to simulate the migration of contaminants, indicated that nearly an order of magnitude sensitivity can be obtained with the mise-à-la-masse configuration. Asch (1990) presented a differencing scheme to minimize near-surface variations which further enhances the sensitivity to changes in resistivities at depth. Sensitivity to variations in resistivities makes the downhole methods ideally suited for monitoring subsurface processes such as injection in enhanced oil recovery, leakage or injection of contaminants at a waste site, or extraction and reinjection processes in geothermal production.

For many of the above applications, the wells are cased with steel, which distorts the fields in the medium and leads to an erroneous interpretation of the field data (Holladay and West 1984 and Williams and Wait 1985). If the casing is used as an electrode, the results may be unpredictable because the current leaves the pipe irregularly due to the variability of the contact resistance between the casing and formation (Kauahikaua et al., 1980).

The purpose of this work is to examine the resistivity method for current sources in wells cased with steel. This research begins with an analysis of the distortion of the fields in 
the half-space when current sources are placed on the axis and beyond the end of the casing. Since many holes are left uncased for some distance at the bottom, the first objective was to determine how interpretable data would be for downhole to surface and cross-hole configurations of electrodes. Through an understanding of how the fields are distorted by the interaction of the casing, source, and host medium, one may develop methods which compensate or minimize the deviations. With this knowledge, field tests can be designed to reduce the distortion caused by the casing or even to use the casing effect in the experiment. Since the formulation of the numerical solution for this problem was perfectly general, a second objective of the research became the analysis of the fields when the current source was inside the casing.

\section{Previous Research}

The effect of casing on electrical survey data has been the subject of numerous studies in the geophysical literature. In this section, the publications on resistivity measurements in the presence of the casing are reviewed.

Several authors have investigated the distortion effects of nearby casing on surface resistivity configurations. Wait (1983) analyized the effect of a vertical semi-infinite conductor on the apparent resistivity for a surface pole-pole array. His numerical results indicate that the apparent resistivity can be seriously affected by the conductor even when the electrodes are located at a distance of more than ten times the pipe radius. In his formulation, a surface impedance on the conductor was incorporated to allow for an oxidized layer or coating. This impedance layer appears to reduce the effects of the conductor on the apparent resistivity.

Holladay and West (1984) studied the effects of well casing on surface dipole-dipole resistivity and induced polarization (IP) surveys. To examine the factors which affect the measurements, they used the semi-infinite pipe solution and an integral solution approach using the Galerkin weighted residuals technique to determine the currents in the pipe. They studied the effects of the casing length on the apparent resistivities. In the evaluation of the 
field data, multiple pipes were used. In obtaining the response of the multiple pipes, they ignored the casing interactions and determined the response by the superposition of the fields due to a single pipe at several casing locations. They indicated that the metal casing may strongly distort the response of the desired signal on surface surveys. Important factors included the pipe conductance, the distance of the pipe from the survey stations, and the pipe length and radius.

Johnson et al. (1987) also investigated the effects of a finite length pipe for surface surveys. They assumed that the pipe can be approximated by a set of finite length current line sources which resulted in a set of linear equations. The potentials are obtained by matrix inversion of the linear equations. In their study, a thin surface layer was introduced to account for the contact resistance between the pipe and host medium. They calculated the apparent resistivities for pole-pole, Schlumberger, and dipole-dipole arrays. In their pole-pole analysis, they showed that the apparent resistivity near the casing decreases as the pipe lengthens.

In most of the studies of subsurface electrode configurations, the currents are injected in the borehole and the potentials are measured at various points in the medium. If a well is cased in steel, point source approximations cannot are invalid. Sill and Ward (1978) used the well casing as a buried electrode for their mise-à-la-masse experiment at the Roosevelt Hot Springs geothermal field in Utah, as did Kauahikaua et al. (1980) for their mise-â-la-masse mapping of a high temperature geothermal reservoir in Hawaii. Sill (1983) used the well as a source to monitor an injection test at Raft River, Idaho to determine if measurable changes that might indicate the direction of fluid flow could be observed. Rocroi and Koulikov (1985) delineated a known resistive hydrocarbon deposit in the USSR by injecting current into two cased wells.

All of these studies were based on the common assumption that a constant line source can be used to represent a pipe excited by a source in or on it. The highly conductive grounded casing is supposed to act like a transmission line which distributes the current along 
its length. If the casing is very long or the surrounding formations are conductive, the current will not extend uniformly along the entire length of casing. This may have occurred in the experiment of Kauahikaua et al. where they indicated that the current appeared not to flow in the lower section of the casing.

Other studies, which do not use the line source approximation, attempt to solve the problem analytically. In early works, the solutions for the pipe response were obtained by making a simplifying assumption that it can be represented by a solid cylinder extented to infinity (Wait 1952 and Smythe 1968). Later, the cylindrical multi-layered infinite or semi-infinite pipe response was investigated for both direct current (DC) and electromagnetic (EM) sources located inside or outside the pipe (Otto 1968, Gianzero and Rau 1977, and Wait 1982, and Wait and Williams 1985).

LaBrecque and Ward (1988) investigated the effects of well casing on surveys for fracture location using a downhole source. They used the pipe model of Holladay and West (1984) and combined it with Beasley and Ward's (1986) rectangular body model to produce an integral equation for the electric fields. Like Holladay and West, they used the twodimensional approximation where the electric fields in the pipe are radially symmetric. The fracture was modeled with a thin vertical prism so that only a single row of elements was needed to define the target. Their study showed that large distortions of the apparent resistivities may occur when the source is near the end of the casing. They indicated that for borehole to surface surveys the effects of the casing are small for a source completely below the casing and for receivers at least one casing-length away from the pipe.

Two non-conventional techniques, measurements while drilling (MWD) and measurements through casing (MTC), measure signals in the presence of a steel drill string or casing. These methods have been developed due to technological advances in electronics so that minute signals may be measured in hostile environments. The MWD method collects realtime information during drilling operations. Holbrook (1985) determines properties of the invasion zone with resistivity measurements during drilling. Sorensen (1989) has measured formation 
resistivity while auger drilling using the "Ellog" method. The MTC logging method measures the electrical resistivity of the formation through metal casing. Several patents, Kaufman (1989), Vail (1989a,b), and Gard et al. (1989), describe devices that are capable of measuring through casing, but only Vail is known to have developed and tested such a tool. These nonconventional resistivity applications are investigated in greater detail in the thesis.

\section{Scope of Research}

Four tasks constitute the objectives of this thesis. The first is the development of the theory and computer algorithms based on the integral equation method to determine the potentials due to a cased well and other three-dimensional objects in the half-space (Chapters IIIV). The second is the evaluation of the casing effect and the parameters that influence the behavior of the fields (Chapter V). The third is the numerical analysis of several field situations involving steel cased wells: downhole current electrode surveys, measurement-whiledrilling, and measurements through casing logging (Chapters V-VI). The last is the application of the algorithm to evaluate the field data from a survey conducted in a cased well (Chapter VII).

Chapters II and III present two forms for the theoretical formulation of the integral equation method. The first makes use of the surface integration of the potential fields. The second uses a the volume integral of the electric fields. In Chapter II, the integral equations are derived from fundamental expressions. Chapter III evaluates the Green's function expressions for both types of integral equations.

The application of Green's theorem to the field equations results in a Fredholm integral equation of the second kind for the unknown fields. The integral equation expresses the unknown (field) function as superposition of the primary field caused by the applied source and the scattered field due to the inhomogeneities. For this problem, the inhomogeneities are caused by a conductivity contrast between the disturbing bodies (pipes and other objects) and the background medium. In deriving the expression for the integral equations, a ring Green's 
function is required for the axial symmetric problem and a point Green's function is used for the three-dimensional problem.

The integral equation is solved by an approximate method of point matching over subsectional bases (Harrington, 1968). This method involves the expansion of the unknown function into a series of weighting and basis functions at $N$ discrete points in or over the region of interest. Each basis function exists only over a subsection of the region and the corresponding piecewise constant weighting function will only affect the approximation of the unknown function over that subsection. This results in reducing the integral equation into a set of linear equations which must be satisfied at the discrete points. The linear equations are simultaneously solved to determine the unknown basis functions. Once the basis functions are found, the linear form of the integral equation is used to calculate the fields in the medium.

The accuracy and validity of the theoretical formulation and the computer algorithm is tested in Chapter IV. Convergence and reciprocity properties of the numerical results are used to check the accuracy or self-consistency of the method. The validity of the formulation and algorithms is checked by comparison to known analytic solutions and published results. The analytic solutions for a semi-infinite vertical annulus and horizontally layered media are used to check the numerical results for models with radial and horizontal boundaries, respectively. The situation involving both boundaries is checked by comparison to the numerical results of Gianzero and Anderson (1982).

In Chapter V, the casing effects are examined for several situations involving steel cased wells and downhole sources. The semi-infinite vertical annulus and the electric field volume integral equation method are used to determine the important parameters for the fields within the pipe. In the half-space, the electric field volume integral equation method is used to illustrate the pipe effects on the potential fields. Position, thickness, diameter (radius), and length of the pipe, as well as the conductivities of the casing and background medium are the important parameters which influence the casing response.

Several current electrode configurations are used to determine the casing influence on 
downhole to surface and cross-hole measurements. Additionally, these effects are examined for a configuration in which the casing segments are separated by insulators of various length and are used as downhole current and potential electrodes. By separating several segments in a well, multiple downhole sources or receivers can be used to image a target The response of the medium to a dipole source at the end of the pipe is also examined.

Several numerical examples are used to simulate practical situations. The first simulation involves the monitoring of an injection plume with cross-hole and downhole-surface measurements. The second is the transmission of a signal through the earth during MWD operations.

Chapter VI presents the numerical analysis of the MTC method. Kaufman (1990) investigated the behavior of the potential and its derivative for a borehole with casing based on models of an infinite-length vertical annulus in a homogeneous medium. This chapter examines the resistivity measurements through a finite-length casing in a homogeneous or layered medium. The algorithm based on the IE approach is used to investigate the effect in variations of the casing conductance, borehole fluid resistivity, and cement annulus. Geometric factors, such as casing dimensions and location within the casing, may affect the resistivity measurements and must be studied. In addition to these topics, the relations of layer boundary resolution and radius of investigation to the electrode spacing are examined.

The evaluation of a downhole-surface resistivity survey conducted near Waverly, Tennessee is presented in Chapter VII. Three radial dipole surface arrays measure the voltages for a succession of downhole current sources. The primary objective of the survey was to determine if borehole to surface measurements were feasible in a resistive environment. Data of acceptable quality was analyized by numerically simulating the apparent resistivities. The surface IE method was used to produce the surface response of a partially cased well in a high resistive (> $1000 \Omega \cdot \mathrm{m}$ ) layered medium. Finally, in Chapter VIII, the results of this study are summarized and discussed. 


\section{Chapter II}

\section{INTEGRAL EQUATION FORMULATION}

Physical phenomena which can be represented by fields are usually expressed by partial differential equations. A solution is found when the field, govemed by the partial differential equation (PDE), satisfies the particular set of boundary conditions which are appropriate to the given physical situation.

For geophysical situations, the geometry of the boundary value problem is so complex that solutions are usually obtained numerically. The finite difference and finite elements method, the most commonly used techniques, require a complete gridding of the solution domain which must be fine enough to resolve the features of interest, usually the inhomogeneities. However, strong constraints are placed on the resolution of the field for a large domain due to the limited memory of the computer. Another drawback to these methods is that the solution of the field is calculated for the entire domain although one may only need a solution for a single point.

For situations where finite-size inhomogeneities are located within a domain, the integral equation (IE) may be a more convenient technique. The boundary value problem is recast into an IE which incorporates the boundary conditions directly into the formulation. This method involves only the inhomogeneities or their boundaries, and any external sources which are usually specified. Thus, the solution within the entire domain need not be calculated, although it may be obtained from the IE.

The use of the IE method to model geophysical problems is well documented in the literature. Typically in electrical problems, the Fredholm integral equation of the second kind is obtained by applying Green's theorem to the field equation and employing the boundary 
conditions. In terms of scattering theory, the IE is the representation of the total field which is the superposition of a primary field and the scattered field. The primary or incident field arising from a known source radiates in an idealized medium, usually homogeneous or layered. The scattered field, also known as the secondary field, is due to the inhomogeneities with contrasting properties located in the idealized medium.

The scattered field can be described by a surface charge distribution that accumulates on the surfaces of the homogeneities. This method is typically used for the DC resistivity problem. Several examples of the IE involving the surface charge distribution can be found in Dieter et al. (1969), Snyder (1976), or Eskola (1979). The other method, commonly used in electromagnetic (EM) modeling, is to describe the scattered field by a volume distribution of current density or current dipole moment within the inhomogeneity (Hohmann, 1971, Ting and Hohmann, 1981, SanFilipo and Hohmann, 1985, and Robertson, 1987).

This chapter will present the derivation of surface and volume IE methods as applied to the DC resistivity problem. The first approach uses the surface integral of the potential on the inhomogeneity to calculate the potentials in the medium. This "surface potential method" is a description of the physical phenomenon of the surface charges accumulating on the discontinuities in the medium. Commonly, the surface IE solves for the surface charges on the inhomogeneity, but the method using surface potentials is much simplier to use. Only one IE is required to solve the problem, whereas the surface charge method needs two equations: one to calculate the surface charges and another to find the potential function at the field point. The formulation of the surface potential boundary IE approach follows that of Hvozdara (1982 and 1983) and Eloranta (1986). The second method determines the potential fields in the medium by using the volume distribution of current density. This method is not commonly used for DC resistivity modeling, although Holladay and West (1984) and Beasley and Ward (1986) have applied this technique. For this chapter, the familiarity with Green's functions is helpful and can be found in Kellogg (1953), Morse and Feshbach (1953) or Tai (1971). 


\subsection{Surface Potential Integral Equation}

The boundary or surface IE method incorporates the surface boundaries of the inhomogeneities in its formulation. The surface charges on the boundaries can be described as a source density distribution. The IE technique involving a source density distribution is referred as the "source density" method. This technique requires that the source density distribution must first be solved and the field solution is then calculated from an integral expression that relates the field to the source density distribution. This method requires two integral equations, one for the source density distribution and another for the field solution. If the boundary values of the field play the role of the source density distribution, the formulated expression is a "direct" boundary IE. This method makes use of Green's theorem which results in the expression of the harmonic function ${ }^{1}$ as the superposition of a (surface charge) single-layer and a (dipole) double-layer potential function.

This chapter will present the derivation of the direct boundary IE formulated for the DC resistivity problem in an infinite medium. The harmonic function is the scalar potential field which is derived from the continuity equation, Faraday's and Ohm's laws. For this problem, the single-layer and double-layer functions will contain the infinite medium Green's function and its normal derivative, respectivity. By applying the boundary conditions, the IE is reduced to the double-layer function containing the harmonic function and the normal derivative of the Green's function.

\subsubsection{Potential Field Equation}

The partial differential equation which govems the potentials can be derived from fundamental relations of electromagnetic (EM) theory. For a point source of current located at $\mathbf{r}_{\mathbf{o}}$, the continuity equation at a field point $\mathbf{r}$ is (Panofsky and Phillips, 1964):

1 A function is harmonic within a closed region if its second derivatives exist and are continuous, and it satisfies Laplace's equation at all points of that domain inducing its boundary (Kellogg, 1953). 


$$
\nabla \cdot \mathbf{J}(\mathbf{r})=I \delta\left(\mathbf{r}-\mathbf{r}_{\mathrm{a}}\right)
$$

where $\mathbf{J}$ is the current density, $I$ is the current of the current source, and $\delta\left(\mathbf{r}-\mathbf{r}_{\mathrm{o}}\right)$ is the Dirac delta function defined in Appendix A. Using Faraday's law and noting that the electric field, $\mathbf{E}$ is irrotational:

$$
\nabla \times \mathbf{E}=-\frac{\partial \mathbf{B}}{\partial t}=\mathbf{O}
$$

The electric field can be written as a gradient of a scalar potential, $\phi:$

$$
\mathbf{E}=-\nabla \phi
$$

Using Ohm's law:

$$
\mathbf{J}=\sigma \mathrm{E}
$$

and equation (2.1.1-3), substituting into equation (2.1.1-1) yields the differential equation:

$$
\nabla \cdot-\sigma \nabla \phi=I \delta\left(\mathbf{r}-\mathbf{r}_{\mathrm{o}}\right) \quad \rightarrow \quad \nabla^{2} \phi=-\frac{1}{\sigma}\left[\nabla \sigma \cdot \nabla \phi+I \delta\left(\mathbf{r}-\mathbf{r}_{\mathrm{o}}\right)\right]
$$

For a homogeneous region, there is no conductivity gradient and equation (2.1.1-5) becomes the goveming field equation, the familiar Poisson's equation:

$$
\nabla^{2} \phi=-\frac{I}{\sigma} \delta\left(\mathbf{r}-\mathbf{r}_{0}\right)
$$

As can be seen from equation (2.1.1-6), the scalar potential is the harmonic function which is being sought.

\subsubsection{Surface Integral Equation Formulation}

From the governing field equation (2.1.1-6) and with the use of the Green's function, Appendix A, the IE will be formulated. As stated previously, the harmonic function can be represented as the sum of the single-layer and double-layer potential functions. However, by applying the boundary conditions on the inhomogeneity, the expression of the harmonic function in the IE can be reduced to a double-layer potential and the applied extemal fields.

Consider an inhomogeneity of conductivity $\sigma_{1}$ and volume $V_{1}$ in an infinite homogeneous medium of conductivity $\sigma_{0}$ with volume $V_{0}$ (Figure $2-1$ ). The boundary of region 0 is the sur- 
face $\Pi$ of the body (normal unit vector $n_{\Pi}$ point towards medium 0 ) and surface $\Gamma$ of the sphere at infinity (outward normal unit vector $n_{\Gamma}$ ). Region 1 has surface $\Pi$ as its boundary.

\section{Source in the Host Medium}

Assume there is a point source of current $I$ located at $r_{0}$ in region 0 . The differential expression which describes the potentials in the homogeneous region is:

$$
\nabla^{2} \phi_{0}(\mathbf{r})=-\frac{J}{\sigma_{0}} \delta\left(\mathbf{r}-\mathbf{r}_{\mathrm{\alpha}}\right)
$$

In region 1, there are no sources and Laplace's equation is valid:

$$
\nabla^{2} \phi_{1}(\mathbf{r})=0
$$

The boundary conditions are:

$$
\begin{aligned}
\phi_{0}(r) & =\phi_{1}(r) & & (r \text { on } \Pi) \\
\sigma_{0} \frac{\partial \phi_{0}(r)}{\partial n_{\Pi}} & =\sigma_{1} \frac{\partial \phi_{1}(r)}{\partial n_{\Pi}} & & (r \text { on } \Pi) \\
\lim _{|r| \rightarrow-} \phi_{0}(r) & =0 & & (r \text { on } \Gamma)
\end{aligned}
$$

If the field point of interest, $\mathbf{r}$ is located in region 0 , the Green's function (Appendix A) satisfies the same differential equation as the potential function, $\phi_{0}$ :

$$
\nabla^{2} g\left(\mathbf{r}, \mathbf{r}^{*}\right)=-\delta\left(\mathbf{r}-\mathbf{r}^{*}\right)
$$

with the boundary condition:

$$
\lim _{r \rightarrow r^{*} \rightarrow-g} g\left(r, r^{*}\right)=0
$$

The solution satisfying equations (2.1.2-4) and (2.1.2-5) is:

$$
g\left(\mathbf{r}, \mathbf{r}^{*}\right)=\frac{1}{4 \pi} \frac{1}{\left|\mathbf{r}-\mathbf{r}^{*}\right|}
$$

Multiply equation (2.1.2-1) by $g$ and equation $(2.1 .2-4)$ by $-\phi_{0}$, add the two expressions, interchange $\mathbf{r}$ and $\mathbf{r}^{*}$, and integrate over the volume $V_{0}$, resulting in:

$$
\begin{aligned}
\int_{V_{0}}\left[g\left(\mathbf{r}, \mathbf{r}^{*}\right) \nabla^{*}{ }^{2} \phi_{0}\left(\mathbf{r}^{*}\right)\right. & \left.-\phi_{0}\left(\mathbf{r}^{*}\right) \nabla^{* 2} g\left(\mathbf{r}, \mathbf{r}^{*}\right)\right] d v^{*} \\
& =\int_{V_{0}}\left[-\frac{I}{\sigma_{0}} g\left(\mathbf{r}, \mathbf{r}^{*}\right) \delta\left(\mathbf{r}^{*}-\mathbf{r}_{0}\right)+\phi_{0}\left(\mathbf{r}^{*}\right) \delta\left(\mathbf{r}^{*}-\mathbf{r}\right)\right] d v^{*}
\end{aligned}
$$


The right side of equation (2.1.2-7) is:

$$
-\int_{V_{0}}\left[\frac{I}{\sigma_{0}} \delta\left(\mathbf{r}, \mathbf{r}^{*}\right) \delta\left(\mathbf{r}^{*}-\mathbf{r}_{0}\right)\right] d v^{*}+\int_{V_{0}}\left[\phi_{0}\left(\mathbf{r}^{*}\right) \delta\left(\mathbf{r}^{*}-\mathbf{r}\right)\right] d v^{*}=-\phi_{\mathrm{P}}(\mathbf{r})+\phi_{0}(\mathbf{r})
$$

where the first term of the right side of equation $(2.1 .2-8)$ is the equation for the incident (primary) field of a point source due to current, 1 :

$$
\phi_{\mathrm{p}}(r)=\frac{I}{\sigma_{0}} g\left(r, r_{0}\right)=\frac{I}{4 \pi \sigma_{0}} \frac{1}{\left|r-r_{0}\right|}
$$

The left side of (2.1.2-7) is converted to surface integrals by Green's theorem: ${ }^{2}$

$$
\begin{aligned}
\int_{S_{0}}\left[g\left(\mathbf{r}, \mathbf{r}^{*}\right) \nabla^{*} \phi_{0}\left(\mathbf{r}^{*}\right)\right. & \left.-\phi_{0}\left(\mathbf{r}^{*}\right) \nabla^{*} g\left(\mathbf{r}, \mathbf{r}^{*}\right)\right] \cdot \mathbf{n}\left(\mathbf{r}^{*}\right) d s^{*} \\
= & \int_{\Gamma^{\prime}}\left[g\left(\mathbf{r}, \mathbf{r}^{*}\right) \nabla^{*} \phi_{0}\left(\mathbf{r}^{*}\right)-\phi_{0}\left(\mathbf{r}^{*}\right) \nabla^{*} g\left(\mathbf{r}, \mathbf{r}^{*}\right)\right] \cdot \mathbf{n}_{\Gamma}\left(\mathbf{r}^{*}\right) d s^{*} \\
& \quad+\int_{\Pi}\left[g\left(\mathbf{r}, \mathbf{r}^{*}\right) \nabla^{*} \phi_{0}\left(\mathbf{r}^{*}\right)-\phi_{0}\left(\mathbf{r}^{*}\right) \nabla^{*} g\left(\mathbf{r}, \mathbf{r}^{*}\right)\right] \cdot\left[-\mathbf{n}_{\Pi}\left(\mathbf{r}^{*}\right)\right] d s^{*}
\end{aligned}
$$

As $\left|\mathbf{r}^{*}\right| \rightarrow \infty$, the first surface integral of equation (2.1.2-10) vanishes as the result of the properties of the Green's function and potential at infinity. Therefore, the integral equation in region 0 is:

$$
\phi_{0}(\mathbf{r})=\phi_{\mathrm{p}}(\mathbf{r})-\int_{\Pi} g\left(\mathbf{r}, \mathbf{r}^{*}\right) \frac{\partial}{\partial n^{*}} \phi_{0}\left(\mathbf{r}^{*}\right) d s^{*}+\int_{\Pi} \phi_{0}\left(\mathbf{r}^{*}\right) \frac{\partial}{\partial n^{*}} g\left(\mathbf{r}, \mathbf{r}^{*}\right) d s^{*}
$$

Equation (2.1.2-12) is the general form found in Stratton (1941) where the harmonic function may be expressed as the superposition of the fields due to the applied sources within region 0 and to sources or charges outside the region. These charges outside the region can be replaced by an equivalent surface distribution on $\Pi$ which will produce the proper value of the harmonic function within the region. The equivalent surface layers are the single-layer (left integral) and double-layer (right integral) potential functions. The single-layer is

2 Green's theorem is:

$$
\int_{v^{*}}\left[\psi \nabla^{*} \theta-\theta \nabla^{*} \psi\right] d v^{*}=\int_{s^{*}}\left[\psi \nabla^{*} \theta-\theta \nabla^{*} \psi\right] \cdot n^{*} d s^{*}=\int_{s^{*}}\left[\psi \frac{\partial \theta}{\partial n^{*}}-\theta \frac{\partial \psi}{\partial n^{*}}\right] d s^{*}
$$

where $V^{*}$ is the region of interest $S^{*}$ is the surface enclosing $V^{*}, \mathrm{n}^{*}$ is the outward unit normal vector on $S^{*}$, and $\partial \psi / \partial n^{*}$ is the directional derivative of the function $\psi$ in the normal direction to the surface. 
equivalent to the potential of a single layer of charge distributed over $\Pi$. The double-layer represents the potential produced a layer of dipoles on the surface $\Pi$.

In region 1, Laplace's equation is valid for the potential function, $\phi_{1}(r)$ and the Green's function since the point of interest is outside the body. Hence, the governing equations for the potential and Green's function are:

$$
\begin{aligned}
\nabla^{2} \phi_{1}(r) & =0 \\
\nabla^{2} g\left(r, r^{*}\right) & =0
\end{aligned}
$$

Using the same procedure employed to obtain equation (2.1.2-7) yields:

$$
\int_{V_{1}}\left[g\left(\mathbf{r}, \mathbf{r}^{*}\right) \nabla^{* 2} \phi_{1}\left(\mathbf{r}^{*}\right)-\phi_{1}\left(\mathbf{r}^{*}\right) \nabla^{* 2} g\left(\mathbf{r}, \mathbf{r}^{*}\right)\right] d v^{*}=0
$$

Applying Green's theorem, results in the following relation:

$$
\int_{\Pi} g\left(\mathbf{r}, \mathbf{r}^{*}\right) \frac{\partial}{\partial n^{*}} \phi_{1}\left(\mathbf{r}^{*}\right) d s^{*}=\int_{\Pi} \phi_{1}\left(\mathbf{r}^{*}\right) \frac{\partial}{\partial n^{*}} g\left(\mathbf{r}, \mathbf{r}^{*}\right) d s^{*}
$$

Using the continuity of normal current density boundary condition, equation (2.1.2-3b), and substituting for the left side of equation (2.1.2-15), gives a relation of normal surface derivative of the potential in terms of the normal surface derivative of the Green's function:

$$
\int_{\Pi} g\left(\mathbf{r}, \mathbf{r}^{*}\right) \frac{\partial}{\partial n^{*}} \phi_{0}\left(\mathbf{r}^{*}\right) d s^{*}=\frac{\sigma_{1}}{\sigma_{0}} \int_{\Pi} \phi_{1}\left(\mathbf{r}^{*}\right) \frac{\partial}{\partial n^{*}} g\left(\mathbf{r}, \mathbf{r}^{*}\right) d s^{*}
$$

By using the continuity of the potential boundary condition, equation (2.1.2-3a), letting:

$$
\phi\left(\mathbf{r}^{*}\right)=\phi_{0}\left(\mathbf{r}^{*}\right)=\phi_{1}\left(\mathbf{r}^{*}\right) \quad\left(\mathbf{r}^{*} \text { on } \Pi\right),
$$

substituting equation (2.1.2-10) into the right side of equation (2.1.2-17), and inserting this into the integral of equation (2.1.2-12) will give (after some minor algebra):

$$
\phi_{0}(\mathbf{r})=\phi_{\mathrm{P}}(\mathbf{r})+\left[1-\frac{\sigma_{1}}{\sigma_{0}}\right] \int_{\Pi} \phi\left(\mathbf{r}^{*}\right) \frac{\partial}{\partial n^{*}} g\left(\mathbf{r}, \mathbf{r}^{*}\right) d s^{*}
$$

Equation (2.1.2-18) is the desired IE for the potential at a point in the region 0 . This IE has eliminated the single-layer function such that the harmonic function $\phi_{0}$ is expressed as the sum of the extemal sources and the double-layer potential function distributed over the surface 
of the inhomogeneity. The double-layer function is the surface integral that contains the normal derivative of the Green's function and the unknown boundary values of the function $\phi$.

\section{Source in the Inhomogeneity}

Now, consider the field point $r$ to be in region 1 . The goveming equations for the potential and the Green's function are:

$$
\begin{aligned}
\nabla^{2} \phi_{1}(r) & =0 \\
\nabla^{2} g\left(r, r^{*}\right) & =-\delta\left(r-r^{*}\right)
\end{aligned}
$$

with the boundary conditions of equations (2.1.2-3) and (2.1.2-5) for the potential and Green's function. Using Green's theorem for $\phi_{1}$ and $g$ results in :

$$
\begin{aligned}
\int_{V_{1}}\left[g\left(\mathbf{r}, \mathbf{r}^{*}\right) \nabla^{*} \phi_{1}\left(\mathbf{r}^{*}\right)\right. & \left.-\phi_{1}\left(\mathbf{r}^{*}\right) \nabla^{*} g\left(\mathbf{r}, \mathbf{r}^{*}\right)\right] d \nu^{*}=\int_{V_{1}} \phi_{1}\left(\mathbf{r}^{*}\right) \delta\left(\mathbf{r}^{*}-\mathbf{r}\right) d v^{*}=\phi_{1}(\mathbf{r}) \\
& =\int_{\Pi}\left[g\left(\mathbf{r}, \mathbf{r}^{*}\right) \nabla^{*} \phi_{1}\left(\mathbf{r}^{*}\right)-\phi_{1}\left(\mathbf{r}^{*}\right) \nabla^{*} g\left(\mathbf{r}, \mathbf{r}^{*}\right)\right] \cdot \mathbf{n}_{\Pi}\left(\mathbf{r}^{*}\right) d s^{*} \\
\rightarrow & \phi_{1}(\mathbf{r})=\int_{\Pi} g\left(\mathbf{r}, \mathbf{r}^{*}\right) \frac{\partial}{\partial n^{*}} \phi_{1}\left(\mathbf{r}^{*}\right) d s^{*}-\int_{\Pi} \phi_{1} \frac{\partial}{\partial n^{*}} g\left(\mathbf{r}, \mathbf{r}^{*}\right) d s^{*}
\end{aligned}
$$

With the current source in region 0 , the goveming equations in region 0 for the potential and Green's function are:

$$
\begin{aligned}
\nabla^{2} \phi_{0}(\mathbf{r}) & =-\frac{I}{\sigma_{1}} \delta\left(\mathbf{r}-\mathbf{r}_{0}\right) \\
\nabla^{2} g\left(\mathbf{r}, \mathbf{r}^{*}\right) & =0
\end{aligned}
$$

Again using Green's theorem and the boundary condition of $\phi_{0}$ and $g$ at infinity:

$$
\begin{array}{r}
\int_{V_{0}}\left[g\left(\mathbf{r}, \mathbf{r}^{*}\right) \nabla^{* 2} \phi_{0}-\phi_{0}\left(\mathbf{r}^{*}\right) \nabla^{* 2} g\left(\mathbf{r}, \mathbf{r}^{*}\right)\right] d v^{*}=-\int_{v_{0}} \frac{I}{\sigma_{1}} g\left(\mathbf{r}, \mathbf{r}^{*}\right) \delta\left(\mathbf{r}^{*}-\mathbf{r}_{0}\right) d v^{*}=-\phi_{\mathrm{P}}(\mathbf{r}) \\
\rightarrow \phi_{\mathrm{P}}(\mathbf{r})-\int_{\Pi}\left[g\left(\mathbf{r}, \mathbf{r}^{*}\right) \frac{\partial}{\partial n^{*}} \phi_{0}\left(\mathbf{r}^{*}\right)-\phi_{0}\left(\mathbf{r}^{*}\right) \frac{\partial}{\partial n^{*}} g\left(\mathbf{r}, \mathbf{r}^{*}\right)\right] d s^{*}=0
\end{array}
$$

Using the boundary conditions, equations (2.1.2-3a) and (2.1.2-3b), on equation (2.1.2-20), results in the following expression:

$$
\int_{\Pi} g\left(\mathbf{r}, \mathbf{r}^{*}\right) \frac{\partial}{\partial n^{*}} \phi_{1}\left(\mathbf{r}^{*}\right) d s^{*}=\frac{\sigma_{0}}{\sigma_{1}}\left[\phi_{P}(\mathbf{r})+\int_{\Pi} \phi \frac{\partial}{\partial n^{*}} g\left(\mathbf{r}, \mathbf{r}^{*}\right) d s^{*}\right]
$$


Substituting into equation (2.1.2-20), yields:

$$
\begin{aligned}
\phi_{1}(\mathbf{r}) & =\frac{\sigma_{0}}{\sigma_{1}}\left[\phi p(r)+\int_{\Pi} \phi\left(\mathbf{r}^{*}\right) \frac{\partial}{\partial n^{*}} g\left(\mathbf{r}, \mathbf{r}^{*}\right) d s^{*}\right]-\int_{\Pi} \phi\left(\mathbf{r}^{*}\right) \frac{\partial}{\partial n^{*}} g\left(\mathbf{r}, \mathbf{r}^{*}\right) d s^{*} \\
& =\frac{\sigma_{0}}{\sigma_{1}} \phi_{\mathrm{P}}(\mathbf{r})+\left[\frac{\sigma_{0}}{\sigma_{1}}-1\right] \int_{\Pi} \phi\left(\mathbf{r}^{*}\right) \frac{\partial}{\partial n^{*}} g\left(\mathbf{r}, \mathbf{r}^{*}\right) d s^{*}
\end{aligned}
$$

Factoring out $\sigma_{0} / \sigma_{1}$, equation (2.1.2-24) is:

$$
\phi_{1}=\frac{\sigma_{0}}{\sigma_{1}}\left[\phi_{\mathrm{P}}(\mathbf{r})+\left[1-\frac{\sigma_{1}}{\sigma_{0}}\right] \int_{\Pi} \phi\left(\mathbf{r}^{*}\right) \frac{\partial}{\partial n^{*}} g\left(\mathbf{r}, \mathbf{r}^{*}\right) d s^{*}\right]=\frac{\sigma_{0}}{\sigma_{1}} \phi_{0}(\mathbf{r})
$$

Equation (2.1.2-25) shows that the formula for the potential in region 1 can be expressed in a form that involves the IE of region 0 , equation (2.1.2-10), multiplied by the conductivity ratio of the host and inhomogeneity. This relation is important when developing an algorithm to solve for the potentials in both regions. Equation (2.1.2-25) allows a single routine to calculate the potentials in both regions by merely incorporating a condition statement within the routine.

For the mise-a-la-masse situation (sources within the inhomogeneity), the potential must now satisfy Poisson's equation for field points in region 1 and Laplace's equation in region 0. The Green's function will satisfy its respective governing equation for its domain. By following the same procedures as before, equations (2.1.2-18) and (2.1.2-24) are also the expressions for the mise-à-la-masse case.

An advantage of the surface potential IE, equation (2.1.2-18), over the IE involving the surface charge distribution is the relaxation of the restriction that the boundary be a Liapunov (smooth) surface. It can be applied to the more general Kellogg regular surfaces, allowing inclusions of corners or edges (Brebbia et al., 1984). Another advantage is only one IE is required to determine the potentials on the boundary and in the medium. Whereas, the other method needs two expressions: one to find the surface charges and the other to calculate the potential in the medium. 


\subsubsection{Singularity}

The evaluation of the singularity of the double-layer potential function follows the discussion of Okabe (1976). The surface integrals of equations (2.1.2-18) and (2.1.2-24) are improper since the integrand goes to infinity as $\mathbf{r}$ approaches $\mathbf{r}^{*}$. To investigate the behavior of the surface potential near the singularity, the surface boundary, $\Pi$ is broken into two surfaces: one is an arbitrarily small disc of radius $\varepsilon \ll 1$ containing the point $r^{*}$ with surface $\Pi_{\varepsilon}$; the other involves the remaining surface of $\Pi-\Pi_{\varepsilon}$ which excludes $r^{*}$ (Figure 2-2). Now the integral of equation (2.1.2-18) can be written as:

$$
\int_{\Pi} \phi\left(\mathbf{r}^{*}\right) \frac{\partial}{\partial n^{*}} g\left(\mathbf{r}, \mathbf{r}^{*}\right) d s^{*}=\int_{\Pi-\Pi_{\varepsilon}} \phi\left(\mathbf{r}^{*}\right) \frac{\partial}{\partial n^{*}} g\left(\mathbf{r}, \mathbf{r}^{*}\right) d s^{*}+\int_{\Pi_{\varepsilon}} \phi\left(\mathbf{r}^{*}\right) \frac{\partial}{\partial n^{*}} g\left(\mathbf{r}, \mathbf{r}^{*}\right) d s^{*}
$$

The first integral over $\Pi-\Pi_{\varepsilon}$ is proper, since the singular point has been removed from the integrand, and can be evaluated. Since $\varepsilon$ can be arbitrarily small, the potential function is essentially constant over the area $\Pi_{\varepsilon}$ with the value of $\phi\left(r^{*}\right)$ which can be taken out of the integral:

$$
\int_{\Pi_{\mathcal{E}}} \phi\left(\mathbf{r}^{*}\right) \frac{\partial}{\partial n^{*}} g\left(\mathbf{r}, \mathbf{r}^{*}\right) d s^{*}=\phi\left(\mathbf{r}^{*}\right) \int_{\Pi_{\mathcal{E}}} \frac{\partial}{\partial n^{*}} g\left(\mathbf{r}, \mathbf{r}^{*}\right) d s^{*}=-\phi\left(\mathbf{r}^{*}\right) \frac{\partial}{\partial n} \int_{\Pi_{\mathfrak{e}}} g\left(\mathbf{r}, \mathbf{r}^{*}\right) d s^{*}
$$

where the relation between the normal derivatives of the Green's function (2.2.1.3) was used.

To evaluate the integral, a cylindrical coordinate system was used where the origin is located at the center of the disk. The field point $\mathbf{r}$ is placed a distance $\beta$ from the surface on the vertical axis, which coincides with the normal unit vector of the surface. The surface position $\mathbf{r}^{*}$ is located a distance $\alpha$ from the origin on the tangential axis (Figure 2-2). From Figure (2.1.3-2), the following relations hold:

$$
\begin{gathered}
\left|\mathbf{r}-\mathbf{r}^{*}\right|=\left[(\beta-0)^{2}+(0-\alpha)^{2}\right]^{1 / 2}=\left(\beta^{2}+\alpha^{2}\right)^{1 / 2}, \\
\frac{\partial}{\partial n}=\frac{\partial}{\partial \beta} \text { and } d s^{*}=\alpha d \alpha d \theta
\end{gathered}
$$

Substituting these into the integral of equation (2.1.3-2) will give:

$$
-\frac{\partial}{\partial n} \int_{\Pi_{\varepsilon}} g\left(\mathbf{r}, \mathbf{r}^{*}\right) d s^{*}=-\frac{1}{4 \pi} \frac{\partial}{\partial n} \int_{\Pi_{\varepsilon}} \frac{1}{\left|\mathbf{r}-\mathbf{r}^{*}\right|} d s^{*}=-\frac{1}{4 \pi} \frac{\partial}{\partial \beta} \int_{0}^{\varepsilon} \int_{0}^{2 \pi} \frac{\alpha \dot{d} \alpha d \theta}{\left(\beta^{2}+\alpha^{2}\right)^{1 / 2}}
$$




$$
=-\frac{1}{2} \frac{\partial}{\partial \beta} \int_{0}^{\varepsilon} \frac{\alpha d \alpha}{\left(\beta^{2}+\alpha^{2}\right)^{1 / 2}}=-\frac{1}{2} \frac{\partial}{\partial \beta}\left[\left(\beta^{2}+\varepsilon^{2}\right)^{1 / 2}-\beta\right]
$$

By letting $\mathbf{r} \rightarrow \mathbf{r}^{*}$, which is equivalent to $\beta \rightarrow 0$, the evaluation of the integral is:

$$
\begin{aligned}
-\frac{\partial}{\partial n} \int_{\pi_{e}} g\left(\mathbf{r}, \mathbf{r}^{*}\right) d s^{*} & =-\frac{1}{2} \lim _{\beta \rightarrow 0} \frac{\partial}{\partial \beta}\left[\left(\beta^{2}+\varepsilon^{2}\right)^{1 / 2}-\beta\right] \\
& =-\frac{1}{2} \lim _{\beta \rightarrow 0}\left[\frac{\beta}{\left(\beta^{2}+\varepsilon^{2}\right)^{1 / 2}}-1\right]=\frac{1}{2}
\end{aligned}
$$

The use of relations (2.1.3-5), (2.1.3-2), and (2.1.3-1) and substitution into equation (2.3-20) will yield the IE which is now proper everywhere on $\Gamma$ :

$$
\phi_{0}(\mathbf{r})=\phi_{\mathrm{p}}(\mathbf{r})+\left[1-\frac{\sigma_{1}}{\sigma_{0}}\right]\left[\int_{\Pi} \phi\left(\mathbf{r}^{*}\right) \frac{\partial}{\partial n^{*}} g\left(\mathbf{r}, \mathbf{r}^{*}\right) d s^{*}+\frac{1}{2} \phi\left(\mathbf{r}^{*}\right) \delta\left(\mathbf{r}, \mathbf{r}^{*}\right)\right]
$$

where $\delta\left(r, \mathbf{r}^{*}\right)$ is the Kronecker delta function defined by:

$$
\delta\left(\mathbf{r}, \mathbf{r}^{*}\right)= \begin{cases}1, & \mathbf{r}=\mathbf{r}^{*} \\ 0, & \mathbf{r} \neq \mathbf{r}^{*}\end{cases}
$$

The numerically integration of equation (2.13-6) can be performed as though the singularity did not exist in the integrand since the singularity has been excluded (theoretically) in the formulation. After the numerical integration, the contribution of the singularity (if applicable) can then be added to the numerical solution.

\subsubsection{Half Space Problem}

The derivation of the half-space problem is similar to that of the whole-space. The initial configuration is the same as Figure (2-1) except that region 0 which was surrounded by an infinite radius sphere $\Gamma$ is now enclosed by two surfaces. The first is a hemisphere, again called $\Gamma$, located at infinity with unit normal $n_{\Gamma}$. The second is the planar half-space surface $\Psi$ with unit normal $\mathbf{n}_{\Psi}$. Figure (2-3) illustrates the half-space situation.

The same PDE govem the behavior of the potentials: Poisson's equation for the medium containing an external source and Laplace's equation for the source-free region. In addition to the boundary conditions of the potentials and the normal current densities of equation (2.1.2- 
2 ), there is a boundary condition for the planar half-space surface, $\Psi$ :

$$
\sigma_{0} \frac{\partial \phi_{0}}{\partial n_{\Psi}}=0 \quad(r \text { on } \Psi)
$$

The Green's function satisfies the same equations as in Section 2.1.2. In addition to the Green's function behavior at infinity, a boundary condition is needed for the surface $\Psi$, equation (2.2.1.4),

$$
\frac{\partial g\left(\mathbf{r}, \mathbf{r}^{*}\right)}{\partial n_{\Psi}}=0 \quad\left(\left|\mathbf{r}-\mathbf{r}^{*}\right| \text { on } \Psi\right)
$$

The solution satisfying the goveming equation and the boundary conditions will result in the half-space Green's function, equation (2.2.1):

$$
g\left(r, r^{*}\right)=\frac{1}{4 \pi}\left[\frac{1}{\left|r-r^{*}\right|}+\frac{1}{\left|r-r_{i}^{*}\right|}\right]
$$

where $\mathbf{r}_{l}^{*}$ is the image location of the source position $\mathbf{r}^{*}$.

Applying Green's theorem to the field equation for the Green's function and potentials will result in the same expression as equation (2.1.2-10) plus another surface integral to account for the planar surface $\Psi$ which is:

$$
+\int_{\Psi}\left[g\left(\mathbf{r}, \mathbf{r}^{*}\right) \frac{\partial \phi_{0}\left(\mathbf{r}^{*}\right)}{\partial n_{\Psi}^{*}}-\phi_{0}\left(\mathbf{r}^{*}\right) \frac{\partial g\left(\mathbf{r}, \mathbf{r}^{*}\right)}{\partial n_{\Psi}^{*}}\right] d s^{*}
$$

The integrand will vanish when the boundary conditions (2.1.4-1) and (2.1.4-2) are applied to this term. The same procedure that was applied in Section 2.1.2 can be employed to obtain the IE for all the half-space cases:

$$
\phi_{0}(\mathbf{r})=\phi_{\mathrm{P}}(\mathbf{r})+\left[1-\frac{\sigma_{1}}{\sigma_{0}}\right] \int_{\mathrm{n}} \phi\left(\mathbf{r}^{*}\right) \frac{\partial}{\partial n^{*}} g\left(\mathbf{r}, \mathbf{r}^{*}\right) d s^{*}
$$

where the primary potential term of equation (2.1.4-5) also involves an image term:

$$
\phi_{\mathrm{P}}(\mathrm{r})=\frac{I}{\sigma_{0}} g\left(\mathrm{r}, \mathrm{r}_{0}\right)=\frac{I}{4 \pi \sigma_{0}}\left[\frac{1}{\left|\mathrm{r}-\mathrm{r}_{0}\right|}+\frac{1}{\left|\mathrm{r}-\mathrm{r}_{0,1}\right|}\right]
$$

and $r_{0, I}$ is the image location of the source location $r_{o}$. Figure $(2.1-4)$ shows the inhomogeneity and external source with their images. 


\subsubsection{Multiple Bodies}

The expressions for a single body in a homogeneous medium that have been derived in the previous sections can be extended to incorporate multiple bodies. The derivation in this section will deal with two bodies in a homogeneous space. The extension to the multiple bodies case will be apparent from the derivation.

Consider two separated bodies of conductivities, volumes, and boundaries of $\sigma_{1}, V_{1}, \Pi_{1}$ and $\sigma_{2}, V_{2}, \Pi_{2}$, respectively, set in a homogeneous medium with a conductivity of $\sigma_{0}$, volume $V_{0}$, and outer boundary $\Pi_{0}$. The directions of the normal unit vector of the surfaces are shown in Figure (2-5). For the field point and current source located in region 0 , the following field equations for the potentials and Green's function are:

$$
\begin{aligned}
& \left.\nabla^{2} \phi_{0}(r)=-\frac{I}{\sigma_{0}} \delta\left(r-r_{0}\right) \text { and } \nabla^{2} g\left(r, r^{*}\right)=-\delta\left(r-r^{*}\right) \quad \text { (in region } 0\right)(2.1 .5-1) \\
& \nabla^{2} \phi_{1}(r)=0 \quad \text { and } \nabla^{2} g\left(r, r^{*}\right)=0 \quad \text { (in region 1) }(2.1 .5-2)
\end{aligned}
$$

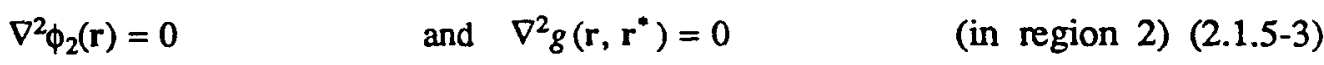

The boundary conditions for the potentials are:

$$
\begin{gathered}
\lim _{|r| \rightarrow \infty} \phi_{0}(\mathbf{r})=0 \\
\phi_{0}(\mathbf{r})=\phi_{1}(\mathbf{r}) \quad \text { and } \quad \sigma_{0} \frac{\partial \phi_{0}(\mathbf{r})}{\partial n_{\Pi_{1}}}=\sigma_{1} \frac{\partial \phi_{1}(\mathbf{r})}{\partial n_{\Pi_{1}}} \\
\phi_{0}(\mathbf{r})=\phi_{2}(\mathbf{r}) \quad \text { and } \quad \sigma_{0} \frac{\partial \phi_{0}(r)}{\partial n_{\Pi_{2}}}=\sigma_{2} \frac{\partial \phi_{2}(\mathbf{r})}{\partial n_{\Pi_{2}}}
\end{gathered}
$$

Applying Green's theorem to equation (2.1.5-1) and using equations (2.1.5-4) and (2.3-5) boundary conditions, gives:

$$
\begin{aligned}
\int_{V_{0}}\left[g\left(\mathbf{r}, \mathbf{r}^{*}\right) \nabla^{*} \phi_{0}\left(\mathbf{r}^{*}\right)\right. & \left.-\phi_{0}\left(\mathbf{r}^{*}\right) \nabla^{*} g\left(\mathbf{r}, \mathbf{r}^{*}\right)\right] d v^{*}=-\phi_{\mathrm{P}}(\mathbf{r})+\phi_{0}(\mathbf{r}) \\
=--\int_{\Pi_{1}}\left[g\left(\mathbf{r}, \mathbf{r}^{*}\right) \frac{\partial}{\partial n_{1}^{*}} \phi_{0}\left(\mathbf{r}^{*}\right)-\phi_{0}\left(\mathbf{r}^{*}\right) \frac{\partial}{\partial n_{1}^{*}} g\left(\mathbf{r}, \mathbf{r}^{*}\right)\right] d s^{*} & \\
& -\int_{\Pi_{2}}\left[g\left(\mathbf{r}, \mathbf{r}^{*}\right) \frac{\partial}{\partial n_{1}^{*}} \phi_{0}\left(\mathbf{r}^{*}\right)-\phi_{0}\left(\mathbf{r}^{*}\right) \frac{\partial}{\partial n_{1}^{*}} g\left(\mathbf{r}, \mathbf{r}^{*}\right)\right] d s^{*}
\end{aligned}
$$

where the notation for the partial derivatives, $\frac{\partial}{\partial n_{\Pi_{i}}}=\frac{\partial}{\partial n_{i}}$, is used for convenience. 
For region 1, Green's second identity yields:

$$
\begin{aligned}
\int_{V_{1}}\left[g\left(\mathrm{r}, \mathrm{r}^{*}\right) \nabla^{*} \phi_{1}\left(\mathrm{r}^{*}\right)\right. & \left.-\phi_{1}\left(\mathrm{r}^{*}\right) \nabla^{* 2} g\left(\mathrm{r}, \mathrm{r}^{*}\right)\right] d v^{*}=0 \\
& =\int_{\Pi_{1}}\left[g\left(\mathrm{r}, \mathrm{r}^{*}\right) \frac{\partial}{\partial n_{1}^{*}} \phi_{1}\left(\mathrm{r}^{*}\right)-\phi_{1}\left(\mathrm{r}^{*}\right) \frac{\partial}{\partial n_{1}^{*}} g\left(\mathrm{r}, \mathrm{r}^{*}\right)\right] d s^{*}
\end{aligned}
$$

which leads to:

$$
\int_{\Pi_{1}} g\left(\mathbf{r}, \mathbf{r}^{*}\right) \frac{\partial}{\partial n_{1}^{*}} \phi_{1}\left(\mathbf{r}^{*}\right) d s^{*}=\int_{\Pi_{1}} \phi_{1}\left(\mathbf{r}^{*}\right) \frac{\partial}{\partial n_{1}^{*}} g\left(\mathbf{r}, \mathbf{r}^{*}\right) d s^{*}
$$

Using boundary conditions of equation $(2.1 .5-5)$ yields:

$$
\int_{\Pi_{1}} g\left(\mathbf{r}, \mathbf{r}^{*}\right) \frac{\partial}{\partial n_{1}^{*}} \phi_{0}\left(\mathbf{r}^{*}\right) d s^{*}=\frac{\sigma_{1}}{\sigma_{0}} \int_{\Pi_{1}} g\left(\mathbf{r}, \mathbf{r}^{*}\right) \frac{\partial}{\partial n_{1}^{*}} \phi_{1}\left(\mathbf{r}^{*}\right) d s^{*}=\frac{\sigma_{1}}{\sigma_{0}} \int_{\Pi_{1}} \phi\left(\mathbf{r}^{*}\right) \frac{\partial}{\partial n_{1}^{*}} g\left(\mathbf{r}, \mathbf{r}^{*}\right) d s^{*}(2
$$

For region 2, a similar expression is obtained:

$$
\int_{\Pi_{2}} g\left(\mathbf{r}, \mathbf{r}^{*}\right) \frac{\partial}{\partial n_{1}^{*}} \phi_{0}\left(\mathbf{r}^{*}\right) d s^{*}=\frac{\sigma_{2}}{\sigma_{0}} \int_{\Pi_{2}} \phi \frac{\partial}{\partial n_{1}^{*}} g\left(\mathbf{r}, \mathbf{r}^{*}\right) d s^{*}
$$

Substitution into equation (2.1.5-7) and inclusion of the singular term gives:

$$
\begin{aligned}
\phi_{0}(\mathbf{r})=\phi_{\mathrm{P}}(\mathbf{r})+ & {\left[1-\frac{\sigma_{1}}{\sigma_{0}}\right]\left[\int_{\Pi_{1}} \phi\left(\mathbf{r}^{*}\right) \frac{\partial}{\partial n_{1}^{*}} g\left(\mathbf{r}, \mathbf{r}^{*}\right) d s^{*}+\frac{1}{2} \phi\left(\mathbf{r}^{*}\right) \delta\left(\mathbf{r}, \mathbf{r}^{*}\right)\right] } \\
+ & {\left[1-\frac{\sigma_{2}}{\sigma_{0}}\right]\left[\int_{\Pi_{2}} \phi\left(\mathbf{r}^{*}\right) \frac{\partial}{\partial n_{1}^{*}} g\left(\mathbf{r}, \mathbf{r}^{*}\right) d s^{*}+\frac{1}{2} \phi\left(\mathbf{r}^{*}\right) \delta\left(\mathbf{r}, \mathbf{r}^{*}\right)\right] }
\end{aligned}
$$

where the potentials in the integrals are on the surface of the bodies.

It is apparent from the above derivation that the expression of equation $(2.1 .5-11)$ can be extended to $Q$-bodies. By summing over all the bodies, the multiple bodies problem can be treated like the single body problem. The IE for the potentials that incorporates the interaction and effects of the $Q$-bodies can be expressed as:

$$
\phi_{0}(\mathbf{r})=\phi_{\mathrm{P}}(\mathbf{r})+\sum_{q=1}^{Q}\left[1-\frac{\sigma_{q}}{\sigma_{0}}\right]\left[\int_{\Pi_{q}} \phi\left(\mathbf{r}^{*}\right) \frac{\partial}{\partial n_{q}^{*}} g\left(\mathbf{r}, \mathbf{r}^{*}\right) d s^{*}+\frac{1}{2} \phi\left(\mathbf{r}^{*}\right) \delta\left(\mathbf{r}, \mathbf{r}^{*}\right)\right]
$$

Section (2.3.3) contains the discussion of solving the linear form of the IE which incorporates the interactions of the $Q$-bodies. 


\subsection{Electric Field Volume Integral Equation}

The use of the volume IE to solve for the electric fields of small three-dimensional inhomogeneities was introduced by the studies of Raiche (1974), Hohmann (1975), and Weidelt (1975). The method consists of replacing an inhomogeneity by a volume distribution of point scattering current (densities). The scattering currents can be represented by the product of the anomalous conductivity contrast and the total electric field. This approach also allows an inhomogeneity to have different electrical properties with no modification of the formulation. The scattered fields outside the inhomogeneity are found by integrating the appropriate Green's function with the scattering currents.

The volume IE approach is not commonly used for the DC resistivity problem since this method is more complicated to use than the surface IE method. First, the E-field volume IE is a vector expression that requires three sets of scalar equations that must be solved simultaneously to determine the E-fields. Whereas, the potential or surface charge IE is a scalar expression which results in a smaller set of linear equations to be solved. Second, the volume IE method requires that the volume of the body be discretized versus the surface discretization used for the surface IE method. The volume discretization may be much more involved if the body has a complex shape. If the problem requires only a single component of the E-field or can be reduced to thin objects, the volume IE method becomes practical for solving DC resistivity problems.

Holladay and West (1988) presented the use of the volume IE method to investigate the effects of the well casing on surface resistivity measurements. They simplified the problem by assuming only the vertical E-field existed within the casing. Beasley and Ward (1986) used the volume IE approach to model a fracture in a hydrothermal field where the fracture was modeled as a single row of volume elements. LaBrecque and Ward (1988) combined the two methods to study the effects of well casing on resistivity surveys for fracture location.

All of the above DC resistivity studies assumed that the E-field volume IE existed and 
presented no derivations of this method for the DC problem. This section will formulate the electric field volume integral equation for the DC resistivity problem. To calculate the potential in the medium, two integral equations are needed; one to calculate the electric fields within the body and the other to determine the potential fields. The Poisson's equation for a vector potential function is derived from Maxwell's equations. Since the scattering current density is a vector quantity, the infinite medium Green's function is in a dyadic form. The formulations of multiple inhomogeneities and the half-space boundary will not be presented in this section since these deviations are similar to those of the previous section (surface integral equation).

\subsubsection{Electric Field Equation}

The integral equation is derived from the field equation of electromagnetic theory. The coupled dependence of the magnetic field $\mathbf{H}$ and the electric field $\mathbf{E}$ is described by Maxwell's equations. Using Ampere's law, the magnetic and electric fields due to an impressed (primary) current source, $\mathbf{J}^{i}$ in a homogeneous region is:

$$
\nabla \times \mathbf{H}=\sigma \mathbf{E}+\mathbf{J}^{i}
$$

where $\sigma$ is the conductivity of the medium.

From the solenoidal (magnetic-Gauss) Law, the magnetic field is divergentless, i.e.,

$$
\boldsymbol{\nabla} \cdot \mathbf{H}=\mathbf{0}
$$

Hence, one can represent $\mathbf{H}$ as a curl of a vector function:

$$
\mathbf{H}=\boldsymbol{\nabla} \times \mathbf{A}
$$

where $\mathbf{A}$ is a Schelkunoff vector potential.

Faraday's law for a DC source field shows that the electric field is irrotational,

$$
\nabla \times \mathbf{E}=-\frac{\partial \mathbf{B}}{\partial t} \rightarrow \nabla \times \mathbf{E}=\mathbf{O}
$$

so one can derive the electric field as the gradient of a scalar function:

$$
\mathbf{E}=-\nabla \phi
$$


Substitution of (2.2.1-3) and (2.2.1-5) into (2.2.1-1) yields:

$$
\nabla \times \nabla \times A=-\sigma \nabla \phi+J^{i}
$$

Applying a vector identity to the left side of $(2.2 .1-6)$, results in:

$$
\nabla \nabla \cdot A-\nabla \cdot \nabla A=\nabla \nabla \cdot A-\nabla^{2} A=-\sigma \nabla \phi+\mathbf{J}^{i}
$$

Since $\phi$ is arbitrary, one can arbitrarily impose a restriction. For convenience, impose the Lorentz gauge:

$$
\boldsymbol{\nabla} \cdot \mathbf{A}=-\sigma \phi
$$

This leads to the desired expression:

$$
\nabla^{2} \mathbf{A}=-\mathbf{J}^{i}
$$

which is the vector form of Poisson's equation applied to the homogeneous regions containing the current source.

Equation (2.2.1-9) is the PDE that must be satisfied to solve for the vector potential. This vector equation can be written in terms of the scalar component of $\mathbf{A}$ and the unit base vectors $\left(\mathbf{u}_{k}\right)$ of the coordinate system.

$$
\nabla^{2} \mathbf{A}=-\mathbf{J}^{i} \quad \rightarrow \quad \nabla^{2} \sum_{k} A_{k} \mathbf{u}_{k}=-\sum_{k} J_{k}^{i} \mathbf{u}_{k} \quad \rightarrow \quad \sum_{k} \nabla^{2}\left(A_{k} \mathbf{u}_{k}\right)=\sum_{k}-J_{k}^{i} \mathbf{u}_{k}
$$

The Laplacian operator $\left(\nabla^{2}\right)$ operates on both $A_{k}$ and $\mathbf{u}_{k}$. For the case of the Cartesian coordinates ${ }^{3}$, the base vectors are constant and equation (2.2.1-10) can be written as:

$$
\sum_{k}\left(\nabla^{2} A_{k}\right) u_{k}=\sum_{k}-J_{k}^{i} u_{k}
$$

Equation (2.2.1-11) indicates that a solution to the vector equation is obtained by summing the solutions of its scalar components. Hence, only the scalar equations are needed in order to solve the problem.

$$
\nabla^{2} A_{k}=-J_{k}^{i} \quad(\text { all } k)
$$

Once $A_{k}$ is found, the scalar potential $\phi$ and the electric field $\mathbf{E}$ can be found from the respective equations, (2.2.1-8) and (2.2.1-5).

3 Generally, the Laplacian of a vector is formulated by the vector identity: $\nabla^{2} \mathbf{A}=\nabla(\nabla \cdot \mathbf{A})-\nabla \times \nabla \times \mathbf{A}$. 


\subsubsection{Solution of the Electric Field Equation}

For an impressed current source located within an unbounded homogeneous medium, the general solution for the vector potential of equation $(2.2 .1-11)$ can be found by solving the PDE using a transform technique (Baños, 1966) which is:

$$
\mathbf{A}(\mathbf{r})=\frac{1}{4 \pi} \int_{v^{*}} \frac{\mathbf{J}^{i}\left(\mathbf{r}^{*}\right)}{\left|\mathbf{r}-\mathbf{r}^{*}\right|} d v^{*}=\int_{v^{*}} g\left(\mathbf{r}, \mathbf{r}^{*}\right) \mathbf{J}^{i}\left(\mathbf{r}^{*}\right) d v^{*}
$$

where $g\left(r, r^{*}\right)$ is the whole-space Green's function (see Appendix A).

Thus, if the current density $\mathrm{J}^{i}$ is known then the vector potential $\mathrm{A}$ can be found. By using the relationship of equation (2.2.1-8), the scalar potential can be obtained:

$$
\begin{aligned}
\phi=-\frac{1}{\sigma} \nabla \cdot \mathbf{A} & =-\frac{1}{\sigma} \nabla \cdot\left[\int_{v^{*}} g\left(\mathbf{r}, \mathbf{r}^{*}\right) \mathbf{J}^{i}\left(\mathbf{r}^{*}\right) d v^{*}\right]=-\int_{v^{*}} \frac{1}{\sigma} \nabla \cdot\left[g\left(\mathbf{r}, \mathbf{r}^{*}\right) \mathbf{J}^{i}\left(\mathbf{r}^{*}\right)\right] d v^{*} \\
& =-\int_{v^{*}} \frac{1}{\sigma}\left[g\left(\mathbf{r}, \mathbf{r}^{*}\right) \nabla \cdot \mathbf{J}^{i}\left(\mathbf{r}^{*}\right)+\nabla g\left(\mathbf{r}, \mathbf{r}^{*}\right) \cdot \mathbf{J}^{i}\left(\mathbf{r}^{*}\right)\right] d v^{*}
\end{aligned}
$$

The divergence operation is applied in the field coordinate system $\mathbf{r}$, whereas $\mathbf{J}^{i}$ is in the source system. Therefore, this term is zero and equation $(2.2 .2-2)$ is:

$$
\phi=-\int_{v^{*}} \frac{1}{\sigma} \nabla g\left(\mathbf{r}, \mathbf{r}^{*}\right) \cdot \mathbf{J}^{i}\left(\mathbf{r}^{*}\right) d v^{*}
$$

From equation (2.2.1-5), the electric field is:

$$
\mathbf{E}=-\nabla \phi=\nabla\left[\int_{v^{*}} \frac{1}{\sigma} \nabla g\left(\mathbf{r}, \mathbf{r}^{*}\right) \cdot \mathbf{J}^{i}\left(\mathbf{r}^{*}\right) d v^{*}\right]=\int_{v^{*}} \frac{1}{\sigma} \nabla\left[\nabla g\left(\mathbf{r}, \mathbf{r}^{*}\right) \cdot \mathbf{J}^{i}\left(\mathbf{r}^{*}\right)\right] d v^{*}
$$

Now, expanding the integrand on the right side of equation $(2.2 .2-4)$ gives:

$$
\begin{aligned}
\nabla\left[\nabla g\left(\mathbf{r}, \mathbf{r}^{*}\right) \cdot \mathbf{J}^{i}\left(\mathbf{r}^{*}\right)\right]=\left[\mathbf{J}^{i}\left(\mathbf{r}^{*}\right)\right. & \nabla] \nabla g\left(\mathbf{r}, \mathbf{r}^{*}\right)+\left[\nabla g\left(\mathbf{r}, \mathbf{r}^{*}\right) \cdot \nabla\right] \mathbf{J}^{i}\left(\mathbf{r}^{*}\right) \\
& +\mathbf{J}^{i}\left(\mathbf{r}^{*}\right) \times \nabla \times \nabla g\left(\mathbf{r}, \mathbf{r}^{*}\right)+\nabla g\left(\mathbf{r}, \mathbf{r}^{*}\right) \times \nabla \times \mathbf{J}^{i}\left(\mathbf{r}^{*}\right)
\end{aligned}
$$

The second and last term on the right side of equation (2.2.2-5) are zero since the vector operators and $\mathbf{J}^{i}$ are in different reference (coordinate) systems. The third term is zero by the vector identity: $\nabla \times \nabla \psi=0$. Applying the concept of the the dyadic Green's function (Appendix A) equation (2.2.1-5) can be written as:

$$
\frac{1}{\sigma} \nabla\left[\nabla g\left(\mathbf{r}, \mathbf{r}^{*}\right) \cdot \mathbf{J}^{i}\left(\mathbf{r}^{*}\right)\right]=\frac{1}{\sigma}\left[\mathbf{J}^{i}\left(\mathbf{r}^{*}\right) \cdot \nabla\right] \nabla g\left(\mathbf{r}, \mathbf{r}^{*}\right)
$$




$$
=\mathbf{J}^{i}\left(\mathbf{r}^{*}\right) \cdot \mathbf{G}\left(\mathbf{r}, \mathbf{r}^{*}\right)=\mathbf{G}\left(\mathbf{r}, \mathbf{r}^{*}\right) \cdot \mathbf{J}^{i}\left(\mathbf{r}^{*}\right)
$$

The desired form of the electric field is obtained:

$$
\mathbf{E}(\mathbf{r})=\int_{V^{*}} \mathbf{G}\left(\mathbf{r}, \mathbf{r}^{*}\right) \cdot \mathbf{J}^{i}\left(\mathbf{r}^{*}\right) d v^{*}
$$

The dyadic Green's function is defined for field points outside the inhomogeneity in Appendix A. But when the field point $\mathbf{r}$ is within the volume $V^{*}$, the volume integral is improper. The singularity must be removed from the integration and evaluated separately at the singular point. As shown by Van Bladel (1961 and 1964) and presented by Ward and Hohmann (1988), the dyadic Green's function is:

$$
\vec{G}\left(r, r^{*}\right)=\frac{1}{\sigma}\left\{P\left[\nabla \nabla g\left(r, r^{*}\right)\right]-\frac{1}{3} \delta\left(r-r^{*}\right) \dot{I}\right\}
$$

where $P[\cdot]$ denotes the principal value integration.

\subsubsection{Electric Field Integral Equation Formulation}

The previous section focused on the impressed current source in an unbounded homogeneous medium. However, when there are inhomogeneities within the medium, the impressed current source will "induce" currents within the inhomogeneities. At any point in the medium, the total field observed is the sum of the incident (primary) field caused by the impressed source and the scattered (secondary) field due to the scattering currents in the inhomogeneities with contrasting properties. Since the scattered field can be written in terms of the total field, the integral equation is solved for the total field using a numerical technique.

Consider an unbounded homogeneous medium (host) with volume, $V_{1}$ and conductivity $\sigma_{1}$ and an inhomogeneity (body) with a volume $V_{2}$ and conductivity $\sigma_{2}$ contained within it (Figure 2-5). Let there be a impressed current density source $\mathbf{J}^{i}$ within the host medium. Since the body has a different conductivity than the surrounding medium, some of the incident field (denoted by the superscript $i$ ) will be "deflected" to produce a scattered field (superscript $s)$. The total field observed at a point $\mathbf{r}$ in the host will come from from both the incident and scattered fields. Hence, the electric and magnetic total fields can be decomposed into the 
scattered fields. Hence, the electric and magnetic total fields can be decomposed into the incident and scattered fields:

$$
\begin{aligned}
& E=E^{i}+E^{s} \\
& H=H^{i}+H^{s}
\end{aligned}
$$

Ampere's law for the entire medium is:

$$
\nabla \times \mathbf{H}=\sigma(\mathbf{r}) \mathbf{E}+\mathbf{J}^{i}
$$

where:

$$
\sigma=\sigma(r)= \begin{cases}\sigma_{2}, & \mathbf{r} \text { in } V_{2} \\ \sigma_{1}, & \mathbf{r} \text { in } V_{1}\end{cases}
$$

The incident field that would apply everywhere in the absence of the inhomogeneity would satisfy the following:

$$
\nabla \times H^{i}=\sigma_{1} E^{i}+J^{i}
$$

Subtracting (2.2.3-4) from (2.2.3-2) yields:

$$
\boldsymbol{\nabla} \times\left[\mathbf{H}-\mathbf{H}^{i}\right]=\sigma(\mathbf{r}) \mathbf{E}-\sigma_{1} \mathbf{E}^{i} \rightarrow \nabla \times \mathbf{H}^{s}=\sigma_{1} \mathbf{E}^{s}+\left[\sigma(\mathbf{r})-\sigma_{1}\right] \mathbf{E}
$$

The anomalous conductivity contrast is:

$$
\sigma(\mathbf{r})-\sigma_{1}=\Delta \sigma= \begin{cases}\sigma_{2}-\sigma_{1}, & \mathbf{r} \text { in } V_{2} \\ 0, & \mathbf{r} \text { in } V_{1}\end{cases}
$$

Thus, equation (2.2.3-5) can be written as:

$$
\nabla \times \mathbf{H}^{s}=\sigma_{1} \mathbf{E}^{s}+\mathbf{J}^{s}
$$

and

$$
\boldsymbol{J}^{s}=\Delta \sigma \mathrm{E}
$$

Equation (2.2.4-7) is Ampere's law applied to the scattered fields. On the left side of equation (2.2.4-8), $\mathrm{J}^{s}$ is the equivalent scattered current that replaces the body and is the source of the secondary field. On the right side, $\mathrm{E}$ is the total electric field and $\Delta \sigma$ is the conductivity contrast between the body and host. Adding the primary field, equation (2.2.3-3), to the scattered field, equation (2.2.3-7), yields the expression for the total fields with the primary and equivalent scattered sources. 


$$
\begin{aligned}
\nabla \times \mathbf{H}^{i}+\nabla \times \mathbf{H}^{s} & =\sigma_{1} \mathbf{E}^{i}+\mathbf{J}^{i}+\sigma_{1} \mathbf{E}^{s}+\mathbf{J}^{s} \\
\rightarrow \quad \nabla \times \mathbf{H} & =\sigma_{1} \mathbf{E}+\mathbf{J}^{i}+\mathbf{J}^{s}
\end{aligned}
$$

Using the procedures of Section (2.2.1) will result in the PDE for the vector potential due to the incident and scattered sources:

$$
\nabla^{2} \mathbf{A}=-\mathbf{J}^{i}-\mathbf{J}^{s}
$$

The solution to this PDE is:

$$
\mathbf{A}(\mathbf{r})=\mathbf{A}^{i}+\mathbf{A}^{s}=\int_{v_{1}+v_{2}} g\left(\mathbf{r}, \mathbf{r}^{*}\right) \mathbf{J}^{i}\left(\mathbf{r}^{*}\right) d v^{*}+\int_{v_{2}} g\left(\mathbf{r}, \mathbf{r}^{*}\right) \mathbf{J}^{s}\left(\mathbf{r}^{*}\right) d v^{*}
$$

The left integral is the vector potential due to the incident field which is known. The right is attributed to the scattered field which is dependent on the conductivity contrast and the unknown total electric field.

Using the relations of equation (2.2.1-8) on the incident field and the scattered potential, equations (2.2.2-3) and (2.2.2-8a), yields the desired expression of the scalar potential:

$$
\phi(\mathbf{r})=\phi^{i}(\mathbf{r})+\int_{v_{2}} \nabla^{*} g\left(\mathbf{r}, \mathbf{r}^{*}\right) \cdot \Delta \sigma \mathrm{E}\left(\mathbf{r}^{*}\right) d v^{*}
$$

Applying the relation of (2.2.1-5) on this equation gives the the Fredholm integral equation of the second kind for the electric field:

$$
\mathbf{E}(\mathbf{r})=\mathbf{E}^{i}(\mathbf{r})+\int_{v_{2}} \vec{G}\left(\mathbf{r}, \mathbf{r}^{*}\right) \cdot \Delta \sigma \mathbf{E}\left(\mathbf{r}^{*}\right) d v^{*}
$$




\subsection{Numerical Solution of the Integral Equations}

The next objective of this chapter is to solve the surface IE of Section (2.1) and volume IE of Section (2.2). A common method to solve the functional form IE is to reduce it into a set of linear equations which can be solved by matrix procedures. This is accomplished by an approximation technique known as the method of moments, also called the method of point matching over subsectional bases or method of weighted residual (see Appendix B).

This method involves the expansion of the unknown field function into a series of piecewise constant basis functions and Dirac delta weighting functions at $N$ discrete points in the region of interest. Each basis function exists only over a subsection within the region. The corresponding weighting function will only affect the approximation of the unknown function over that subsection. The integral over the region is then approximated as a summation of integrals over the subsections. This linear form of the equation must now be satisfied at each $N$ discrete points. As a result, a matrix equation is obtained and can be solved to determine the unknown field functions.

\subsubsection{Surface Integral Equation}

The surface IE of Section (2.1):

$$
\phi(\mathbf{r})=\phi_{\mathrm{p}}(\mathbf{r})+C\left[\int_{h}\left(\mathbf{r}^{*}\right) \frac{\partial}{\partial n^{*}} \delta\left(\mathbf{r}, \mathbf{r}^{*}\right) d s^{*}+\frac{1}{2} \phi\left(\mathbf{r}^{*}\right) \delta\left(\mathbf{r}, \mathbf{r}^{*}\right)\right]
$$

where:

$$
C=1-\frac{\sigma_{1}}{\sigma_{0}}
$$

is transformed into the matrix equation by the following procedure. By using $N$ piecewise constant basis functions, equation (2.3.1-1) can be approximated by:

$$
\phi(\mathbf{r})=\phi_{\mathrm{P}}(\mathbf{r})+C\left[\sum_{n=1}^{N} \mathrm{~K}\left(\mathbf{r}, \mathbf{r}_{n}^{*}\right) \phi\left(\mathbf{r}_{n}^{*}\right)+\frac{1}{2} \phi\left(\mathbf{r}_{n}^{*}\right) \delta\left(\mathbf{r}, \mathbf{r}_{n}^{*}\right)\right]
$$

where:

$$
\mathrm{K}\left(\mathbf{r}, \mathbf{r}_{n}^{*}\right)=\int_{\Pi_{n}} \nabla^{*} g\left(\mathbf{r}, \mathbf{r}_{n}^{*}\right) \cdot \mathbf{n}_{\Pi_{n}}\left(\mathbf{r}_{n}^{*}\right) d s^{*}=\int_{\mathrm{n}_{n}} \frac{\partial}{\partial n^{*}} g\left(\mathbf{r}, \mathbf{r}_{n}^{*}\right) d s^{*}
$$


The potential for each $n$-segment can be determined by using equations (2.3.1-2) and (2.3.1-3). If one lets the field point lie on the $m$-th segment (denoted by $\mathbf{r}_{m}$ ), which is equivalent to using the Dirac delta weighting function $\delta\left(r-\mathbf{r}_{m}^{*}\right)$ for all $N$-segments, the integral equation can be written as:

$$
\phi\left(\mathbf{r}_{m}\right)=\phi_{\mathrm{P}}\left(\mathrm{r}_{m}\right)+C\left[\sum_{n=1}^{N} \mathrm{~K}\left(\mathbf{r}_{m}, \mathbf{r}_{n}^{*}\right) \phi\left(\mathbf{r}_{n}^{*}\right)+\frac{1}{2} \phi\left(\mathbf{r}_{n}^{*}\right) \delta\left(\mathbf{r}_{m}, \mathbf{r}_{n}^{*}\right)\right]
$$

Using the property of the Kronecker delta function and rearranging equation (2.3.1-4), yields:

$$
\phi_{\mathrm{P}}\left(\mathrm{r}_{m}\right)=B \phi\left(\mathrm{r}_{m}\right)-C \sum_{n=1}^{N} \mathrm{~K}\left(\mathrm{r}_{m}, \mathrm{r}_{n}^{*}\right) \phi\left(\mathrm{r}_{n}^{*}\right)=\sum_{n=1}^{N}\left[B \delta_{m n}-C \mathrm{~K}_{m n}\right] \phi\left(\mathrm{r}_{n}^{*}\right)
$$

where:

$$
\begin{aligned}
& \delta_{m n}=\delta\left(r_{m}, r_{n}^{*}\right) \\
& \mathrm{K}_{m n}=\mathrm{K}\left(\mathrm{r}_{m}, \mathrm{r}_{n}^{*}\right)
\end{aligned}
$$

and $B=1-1 / 2 C=1 / 2\left(1+\sigma_{1} / \sigma_{0}\right)$.

Equation (2.3.1-5) is the desired form of the matrix equation that can be solved for the potential on each segment.

\subsubsection{Volume Integral Equation}

The volume IE of section (2.2) involving vectors and dyadics instead of scalar quantities can be written as:

$$
\mathbf{E}(\mathbf{r})=\mathbf{E}^{i}(\mathbf{r})+\int_{v^{*}} \mathbf{G}\left(\mathbf{r}, \mathbf{r}^{*}\right) \cdot \Delta \sigma \mathbf{E}\left(\mathbf{r}^{*}\right) d v^{*}
$$

The method of moments procedure to obtain a solution with the vectors is the same as that of the scalar. Using $N$ piecewise constant basis functions, equation (2.3.2-1) can be approximated by:

$$
\mathbf{E}(\mathbf{r})=\mathbf{E}^{i}(\mathbf{r})+\sum_{n=1}^{N} \boldsymbol{P}\left(\mathbf{r}, \mathbf{r}_{n}^{*}\right) \cdot \mathbf{E}\left(\mathbf{r}_{n}^{*}\right)
$$

where:

$$
\vec{\Gamma}\left(\mathbf{r}, \mathbf{r}_{n}^{*}\right)=\Delta \sigma_{n} \int_{V_{n}^{*}} \vec{G}\left(\mathbf{r}, \mathbf{r}_{n}^{*}\right) d v^{*}
$$


The electric field for each $n$-segment can now be determined from equations (2.3.2-2) and (2.3.2-3) by applying the Dirac delta weighting function for each segment. The electric field relation for the $m$-th segment becomes:

$$
\mathbf{E}_{m}=\mathbf{E}_{m}^{i}+\sum_{n=1}^{N} \hat{\Gamma}_{m n} \cdot \mathbf{E}_{n}
$$

where:

$$
\begin{aligned}
\mathbf{E}_{m} & =\mathbf{E}\left(\mathbf{r}_{m}\right) \\
\mathbf{E}_{m}^{i} & =\mathbf{E}^{i}\left(\mathbf{r}_{m}\right) \\
\stackrel{\Gamma}{m n}_{m} & =\hat{F}\left(r_{m}, r_{n}^{*}\right)
\end{aligned}
$$

Rearranging equation (2.3.2-4) gives:

$$
\mathbf{E}_{m}^{i}=\sum_{n=1}^{N} \hat{\Gamma}_{m n} \cdot \mathbf{E}_{n}-\mathbf{E}_{m}=\sum_{n=1}^{N}\left(\dot{\delta}_{m n}-\ddot{P}_{m n}\right) \cdot \mathbf{E}_{n}
$$

The $\delta_{m n}$ is defined by:

$$
\dot{\delta}_{m n}= \begin{cases}\ddot{I}, & m=n \\ \ddot{O}, & m \neq n\end{cases}
$$

where $\boldsymbol{Y}$ is the identity dyadic and $\boldsymbol{\delta}$ is the null dyadic.

Equation (2.3.2-7) is now in the form of a partitioned matrix equation which can be solved for the unknown $E$-field in each segment.

\subsubsection{Matrix Equation}

Equations (2.3.1-5) and (2.3.1-6) can be expressed as a matrix equations of the form:

$$
\mathrm{g}_{m}=\sum_{n=1}^{N} \alpha_{n} l_{m n}
$$

where $g_{m}$ is the known source field, $\alpha_{n}$ is the unknown field, and $l_{m n}$ is the linear integral operator. Table (2-1) shows the relation of the field components of the surface IE and volume IE with the linear matrix equations (2.3.3-1). 


\begin{tabular}{|ccc|}
\hline & Surface IE & Volume IE \\
\cline { 2 - 3 } & $\phi_{\mathrm{P}}\left(\mathbf{r}_{m}\right)$ & $\mathbf{E}^{i}\left(\mathbf{r}_{m}^{*}\right)$ \\
$\alpha_{n}$ & $\phi\left(\mathbf{r}_{n}^{*}\right)$ & $\mathbf{E}\left(\mathbf{r}_{n}^{*}\right)$ \\
$l_{m n}$ & $B \delta_{m n}-C \mathrm{~K}_{m n}$ & $\delta_{m n}-\vec{\Gamma}_{m n}$ \\
\hline
\end{tabular}

Table (2-1)

In vector notation, equation (2.3.3-1) can be expressed as:

$$
\overline{\mathbf{g}}=\bar{\alpha} \tilde{l}
$$

where $\overline{\mathbf{g}}$ and $\bar{\alpha}$ are vectors and $\bar{l}$ is a matrix. From equation (2.3.3-1), the unknown fields of the inhomogeneity $\bar{\alpha}$ are found by:

$$
\bar{\alpha}=\bar{l}^{-1} \overline{\mathbf{g}}
$$

where $\bar{l}^{-1}$ is the inverse of matrix $\bar{l}$.

Equation (2.3.3-3) solves for the unknown field $\alpha_{n}$ for each segment at $r_{n}^{*}$ on/in the inhomogeneity. The field at point $\mathbf{r}$ in the medium can be calculated by substituting $\boldsymbol{\alpha}_{n}$, which represents the potential or electric field of the $n$-th segment of the inhomogeneity, into the respective scattering field of equation (2.3.1-2) or (2.3.2-2).

Since equation (2.3.3-1) is linear, the matrix equation can easily be extended to include multiple bodies (inhomogeneities). For $Q$-inhomogeneities, the matrix equation can be expressed as:

$$
\sum_{q=1}^{Q} \mathbf{g}_{m_{q}}=\sum_{q=1}^{Q}\left[\sum_{n_{q}=1}^{N_{q}} \alpha_{n_{q}} l_{m_{q} n_{q}}\right] \quad \rightarrow \quad \mathbf{G}_{i}=\sum_{j=1}^{\eta} \mathbf{A}_{j} \mathbf{L}_{i j}
$$

where: $\left(i, j=1\right.$ to $\left.\eta=\sum_{q=1}^{Q} N_{q}\right)$ and $\left(m_{q}, n_{q}=1\right.$ to $\left.N_{q}\right)$ are the segment indices and $N_{q}$ is the total number of segments for the $q$-th inhomogeneity. The elements of A can be found by:

$$
\overline{\mathbf{A}}=\tilde{\mathbf{L}}^{-1} \overline{\mathbf{G}}
$$

where $\tilde{\mathbf{L}}^{-1}$ is the inverse of $\mathbf{L}$ and has the dimensions $(\eta \times \eta)$. 
The matrix $\overline{\mathbf{L}}$ of equation (2.3.4) can be thought of as a partitioned matrix composed of $(Q \times Q)$ submatrices in the form of:

$$
\mathbf{L}=\left(\begin{array}{ccc}
\tilde{\mathbf{L}}_{(11)} & \cdots & \tilde{\mathbf{L}}_{(1)} \\
\vdots & \cdot & \vdots \\
\overline{\mathbf{L}}_{(1 \varrho)} & \cdots & \tilde{\mathbf{L}}_{(\varrho)}
\end{array}\right)
$$

This partitioned matrix accounts for the interaction within and between the inhomogeneities. The diagonal matrices $\tilde{\mathbf{L}}_{(q q)}$ incorporate the interaction of the segments of the $q$-th body onto itself and has the dimensions $\left(N_{q} \times N_{q}\right)$. The off-diagonal partitioned matrices $\tilde{\mathbf{L}}_{(p q) \text {, with }}$ $\left(N_{p} \times N_{q}\right)$ elements, represent the interaction between the $p$-th and $q$-th bodies. This matrix contains the effects of the segments for the $q$-body onto the elements of $p$-body, whereas the converse holds for matrix $\overline{\mathbf{L}}_{(q p)}$.

It is worth noting that for the DC resistivity problem the matrix $\bar{l}$ is a full, diagonally dominant, real matrix and can be very large. For an infinite homogeneous medium, this matrix can be symmetric if elements of equal size are used for the segmentation of the inhomogeneity. Additionally, the elements of the matrix $\bar{l}$ contain information on the interaction between the segments of the inhomogeneity and have no primary source field information. Hence, the unknown fields $\bar{\alpha}$ of the inhomogeneity can be solved for any source configuration once the inverse matrix $\tilde{l}^{-1}$ is obtained.

Knowledge of the matrix and the kind of problem being solved is important in solving the linear equations. If a problem involves many different source configurations and a single scatterer configuration, the computational effort can be greatly reduced by saving the inverse matrix $\tilde{l}^{-1}$ and using it to calculate all the source configurations. Since only scatter information is contained in $\tilde{l}$, the matrix only needs to be solved once. Depending on the problem, several matrix inversion algorithms, the Gauss-Jordan elimination, LU decomposition, and singular value decomposition (SVD), are commonly used. 
If the properties of the inhomogencity change during the analysis of the problem, as would occur for an iterative inversion problem, the unknown fields must be calculated and the inverse matrix $\tilde{l}^{-1}$ may not be needed. For these types of problems, the SVD is frequently used to solve for the unknown fields. The iterative techniques, such as the conjugate gradient method (Sarkar and Rao, 1982), are becoming popular for solving large sparse sets of linear equations. Many other methods exist that can be employed to solve linear equations but are beyond the scope of this thesis. 


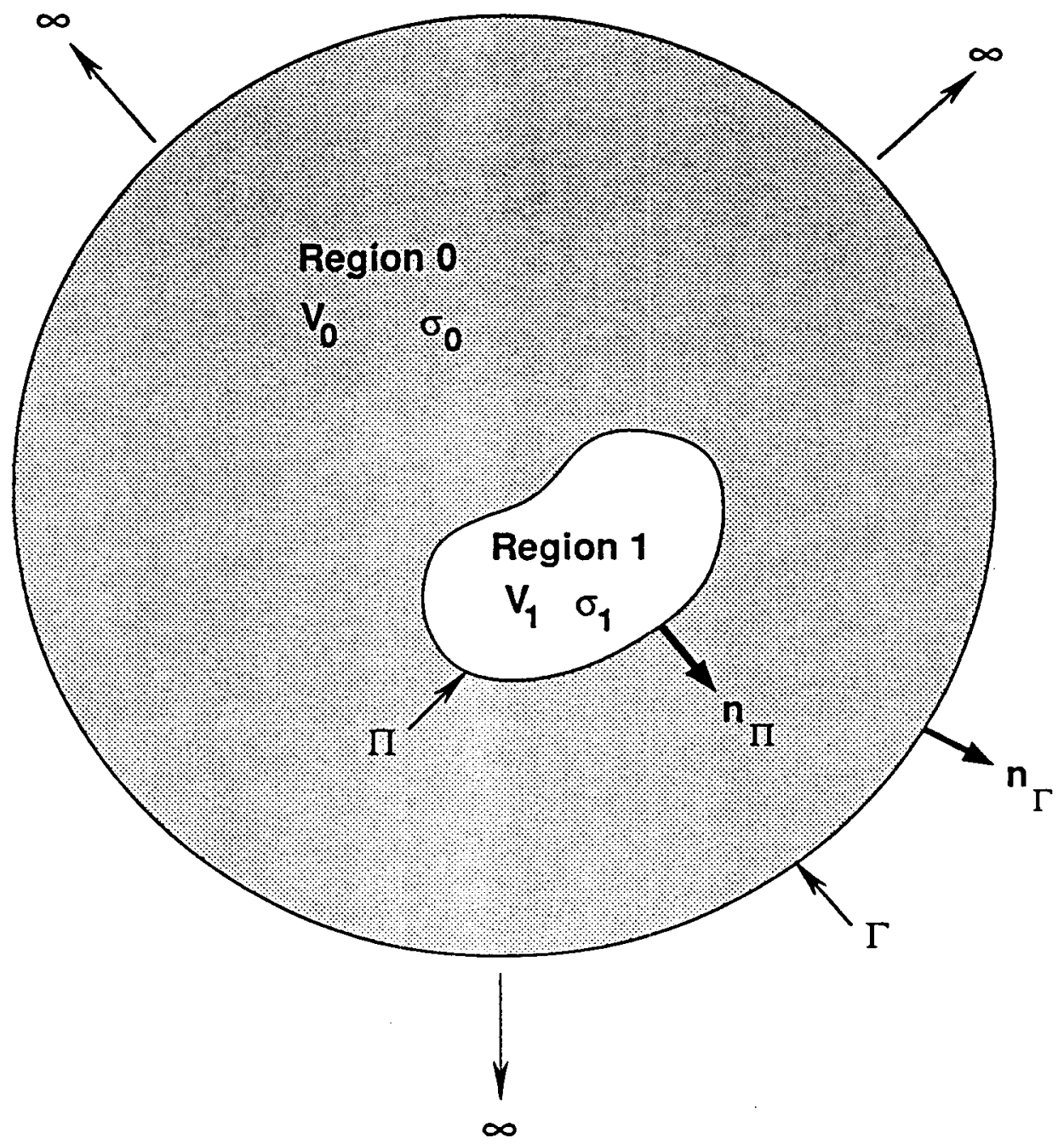

Figure 2-1: The generalized geometrical and electrical configuration of an inhomogeneity within an infinite medium for the potential surface IE method. (Current source position is not shown.) 

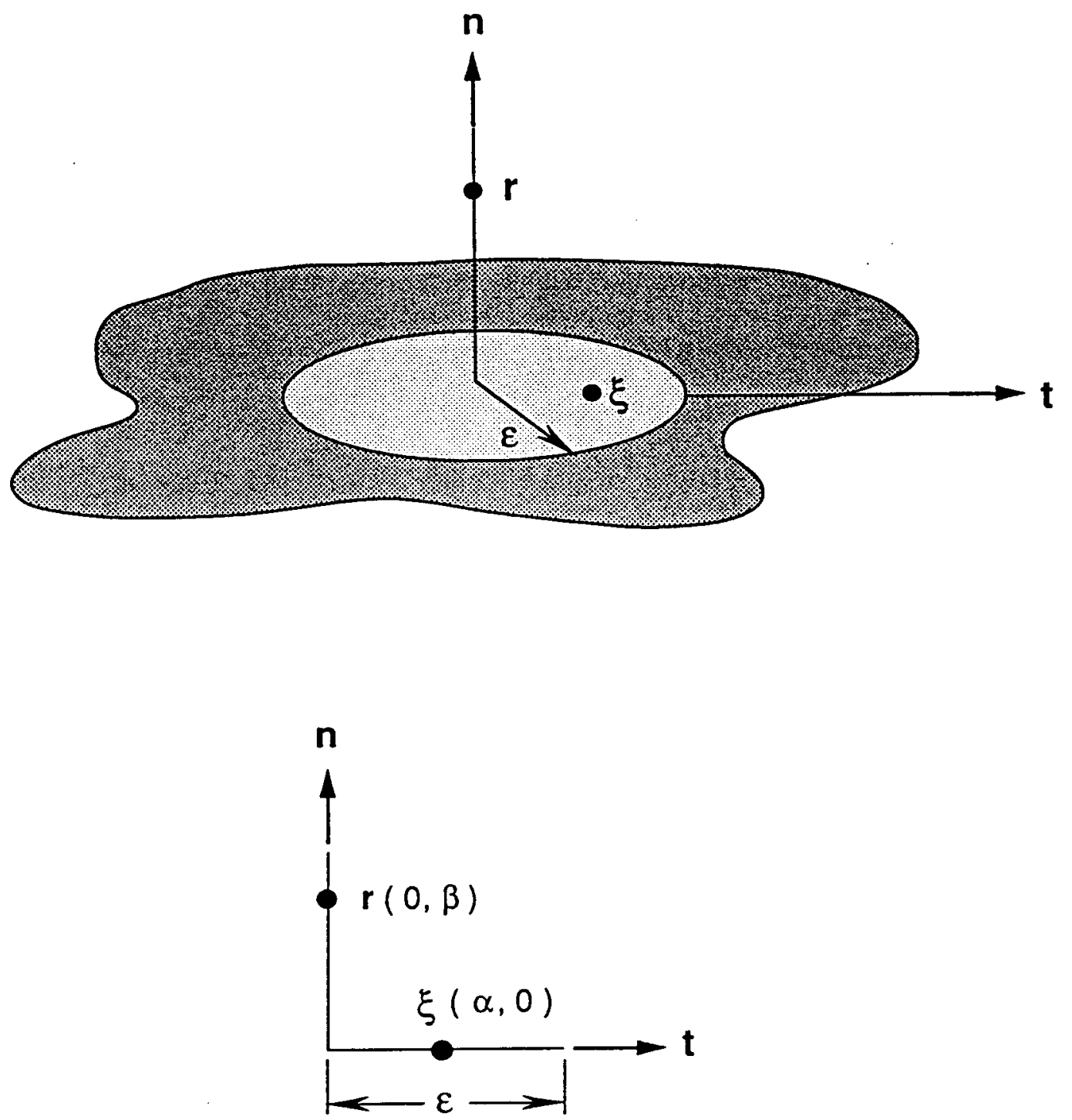

Figure 2-2: The configuration of small surface element of the singular cell for the potential surface IE method. 


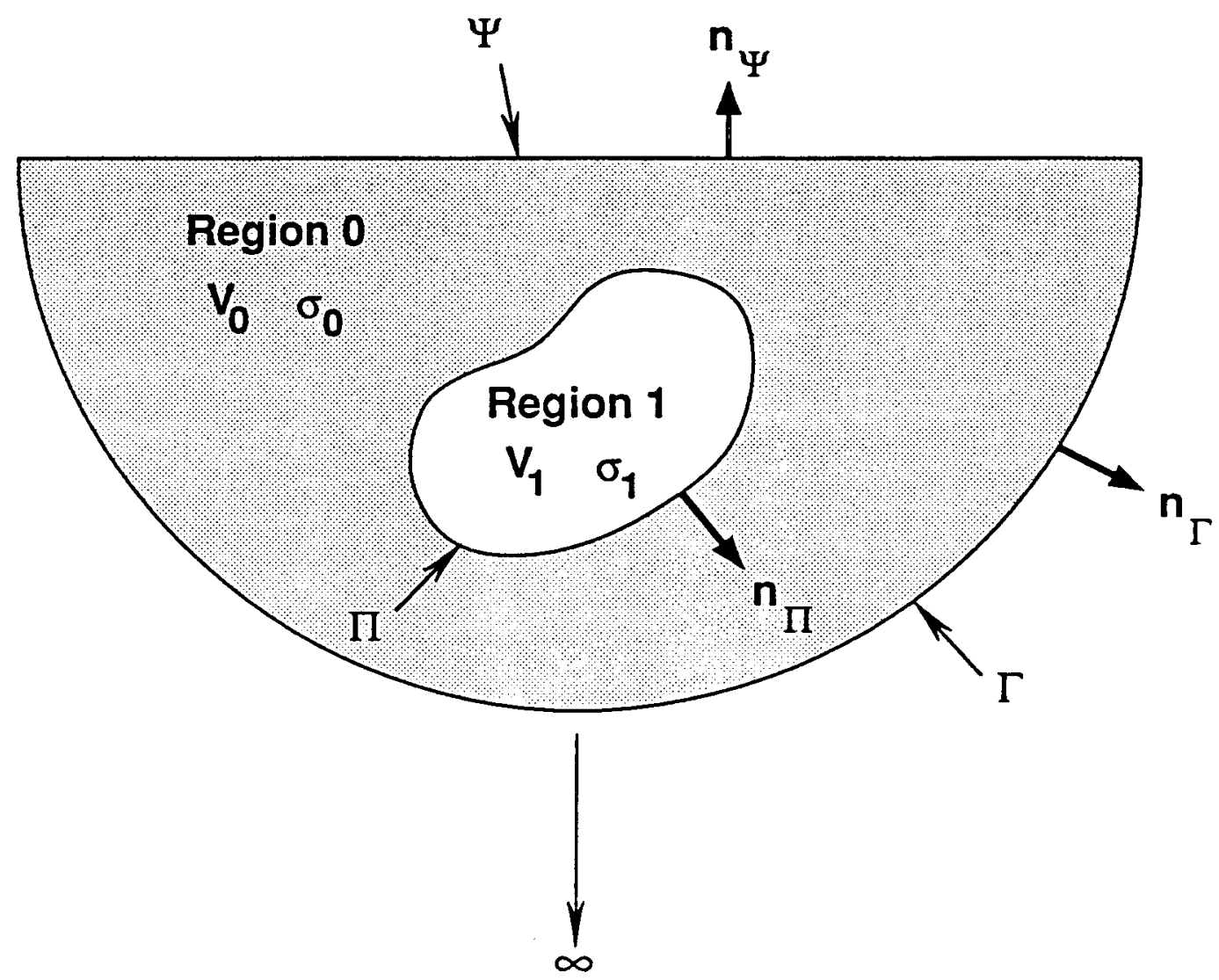

Figure 2-3: The generalized geometrical and electrical configuration of an inhomogeneity within a half-space for the potential surface IE method. (Current source position is not shown.) 


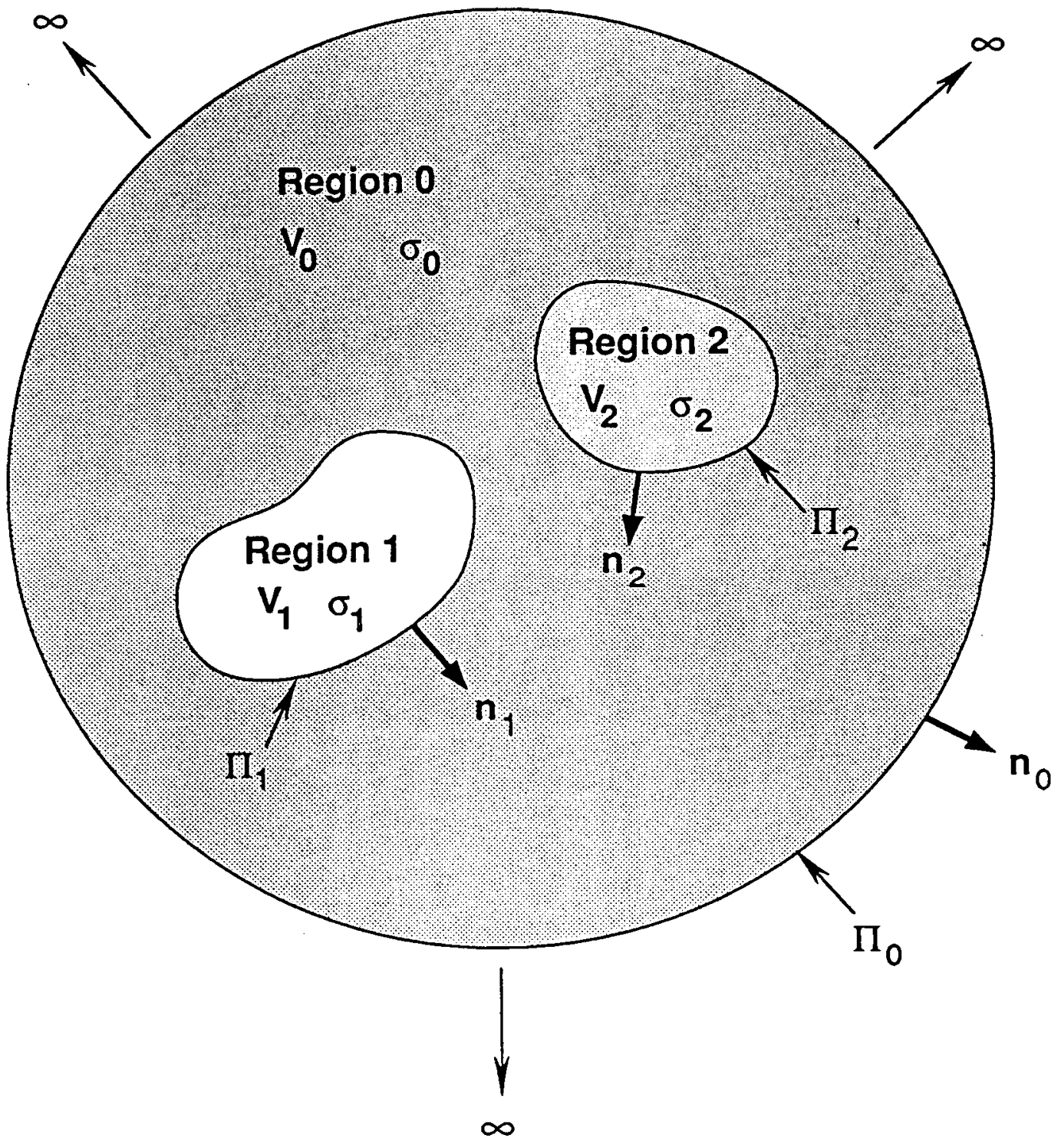

Figure 2-4: The generalized geometrical and electrical configuration of two inhomogeneities within a whole-space for the potential surface IE method. (Current source position is not shown.) 


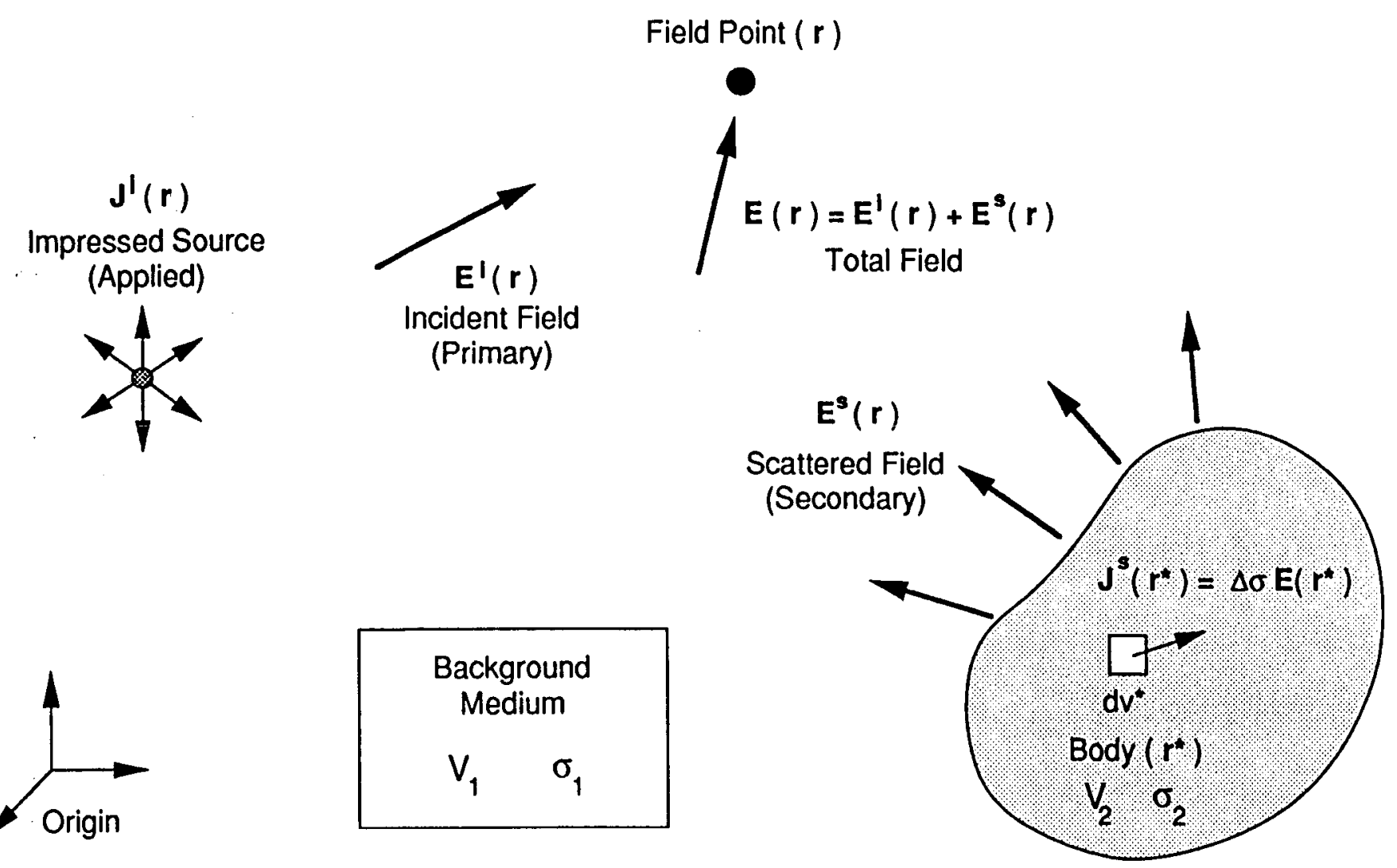

Figure 2-5: The generalized configuration of the impressed current source and the scattering body used for the E-field volume IE method. 


\section{Chapter III}

\section{GREEN'S FunCTION SOLUTIONS}

In the previous chapter, two forms of the IE used to calculate the potential field of an arbitrary inhomogeneity were derived and a numerical method to solve these expressions was introduced. The inhomogeneity produces the scattered field which can be characterized by a source density distribution. The first type of source density is the double-layer potential which is distributed over the surface of the body. The second kind is the volume distribution of current densities within the inhomogeneity. In either case, an integral containing the Green's function must be evaluated in order to calculate the source density. For the remainder of the chapter, this integral will be called the "Green's function integral".

Since the region of interest is a half-space, the image method is used to satisfy the surface boundary condition that no vertical current exist at the half-space surface. The image sources, with the same strengths and polarities as the primary sources, are placed on the opposite side of the half-space boundary. The resulting field is the sum of the primary sources and their images. The same procedure is used to calculate the effects of the half-space on the scattered fields.

The majority of this discussion will deal with a specific inhomogeneity, a vertical annulus or pipe, and the determination of the Green's function integral for this situation. Both the potential surface IE and E-field volume IE approaches are solved for the axial symmetric (axisymmetric) configuration. For the axisymmetric problem, the bodies are discretized into cylindrical rings. The solution of the Green's function integral of the ring elements requires the use of the axisymmetric Green's function.

The Green's function integral of the surface potentials for the three-dimensional (3-D) case will be investigated in both the Cartesian and cylindrical coordinate systems. Flat-surface 
The Green's function integral of the surface potentials for the three-dimensional (3-D) case will be investigated in both the Cartesian and cylindrical coordinate systems. Flat-surface and arc-surface segments are used to describe the body for the Cartesian and cylindrical coordinate systems, respectively. The 3-D Green's functions in both coordinate systems are needed to solve these integrals.

Additional bodies, axisymmetric and off-axis, are incorporated with the pipe model. The fields which are due to the addition of axisymmetric plumes can be obtained by using the axisymmetric Green's function and the multiple body formulation. To determine the fields caused by the pipe and an off-axis body, a 3-D "mixed" coordinate system is used. This mixed system uses arc-surface segments for the pipe and the flat-surface segments for the offaxis body to determine the scattered field. If the body and/or applied sources are a sufficient distance from the pipe, an axisymmetric Green's function can be used to simplify the computations by approximating the response of the pipe with circular ring segments instead of the arc segments. 


\subsection{Axisymmetric Problem}

The Green's function integrals are solved for the axisymmetric ${ }^{\dagger}$ case. This is accomplished by using the axisymmetric Green's function which is derived in Appendix $C$ by satisfying Poisson's equation for the Dirac delta source function in cylindrical coordinates. This Green's function is used to represent the situations where the primary source(s) and axisymmetric objects(s) are coaxial. Along with solving the Green's function integral, a "ring" primary source term is found for the potential and electric field (E-field) using the axisymmetric Green's function.

The first part of this section solves the Green's function surface integral $K\left(r, r^{*}\right)$. This surface integral is comprised of the surface normal derivative of the Green's function integrated over the surface element. The second part finds the Green's function volume integral $\vec{\Gamma}\left(r, r^{*}\right)$ by integrating the components of the dyadic Green's function over the volume of the cell. For the E-field solution, a second IE is required to determine the potential fields in the medium caused by the scattered currents of the circular ring segments.

\subsubsection{K(r, $\left.\mathbf{r}^{*}\right)$ Formulation}

Figure (3-1) illustrates the axisymmetric model configuration of a finite-length pipe and, for simplicity, a single current source coaxially located. Let the surface of the earth be $z=0$ with the positive vertical $z$ axis directed downward. The location of the current electrode on the vertical axis is at $z_{s}$. The origin of the radial direction is at the center of the pipe. The pipe is described by its length: $L$, inner radius: $b$, outer radius: $c$, and a conductivity of $\sigma_{1}$. The earth, host medium, has a conductivity of $\sigma_{0}$. Other bodies, such as the borehole fluid and/or casing cement, may be included in the problem but are not needed in solving for the Green's function.

$\dagger$ In the cylindrical coordinate system, a position of a point can be described by a radial, azimuthal, and vertical coordinates $(\rho, \theta, z)$. The problem has axial symmetry when only the radial and vertical coordinates are required to define the position. 
Recall that the Green's function surface integral for the surface IE, equation (2.3.1-3), is of the form:

$$
\mathbf{K}\left(\mathbf{r}, \mathbf{r}^{*}\right)=\int_{s^{*}} \nabla^{*} g\left(\mathbf{r}, \mathbf{r}^{*}\right) \cdot \mathbf{n}\left(\mathbf{r}^{*}\right) d s^{*}=n \int_{s^{*}} \frac{\partial}{\partial n^{*}} g\left(\mathbf{r}, \mathbf{r}^{*}\right) d s^{*}
$$

where $n$ is the directional cosine of the surface normal unit vector $\mathbf{n}$. For the pipe, or an annular-shaped object, there are two components, i.e., radial and vertical, to the surface integral. Hence the Green's function integral of equation (3.1.1-1) can be written as:

$$
\mathrm{K}\left(\mathbf{r}, \mathbf{r}^{*}\right)=n \int_{s^{*}} \frac{\partial}{\partial n^{*}} g\left(\mathbf{r}, \mathbf{r}^{*}\right) d s^{*}=n_{z} \mathrm{~K}_{z}\left(\mathbf{r}, \mathbf{r}^{*}\right)+n_{\mathrm{p}} \mathrm{K}_{\mathrm{p}}\left(\mathrm{r}, \mathbf{r}^{*}\right)
$$

The first, $\mathrm{K}_{\mathrm{z}}$ defines the flat horizontal ring surface associated with the top and bottom parts of the annulus. The second, $K_{p}$ describes the inner and outer vertical walls of the annulus. Figure (3-2) illustrates the two surfaces associated with an annular object. The components of the directional cosines of the unit surface normal, $n_{z}$ and $n_{\rho}$ are defined as positive downward and outward, respectively. For this problem, they are either \pm one or zero.

\section{Horizontal Surfaces}

The $z$-component of $K\left(r, r^{*}\right)$ is given by the following expression:

$$
\mathrm{K}_{\mathbf{z}}\left(\mathbf{r}, \mathbf{r}^{*}\right)=\int_{s^{*}} \frac{\partial}{\partial z^{*}} g\left(\mathbf{r}, \mathbf{r}^{*}\right) d s^{*}=\int_{0}^{2 \pi} \int_{c}^{b} \frac{\partial}{\partial z^{*}} g\left(\mathbf{r}, \mathbf{r}^{*}\right) \rho^{*} d \theta^{*} d \rho^{*}
$$

The half-space axisymmetric Green's function, appendix equation (C-7), is substituted into equation (3.1.1-3) and is evaluated over the width of the annulus, interval $(b, c)$. The expression now becomes:

$$
\left.\mathrm{K}_{z}=\frac{1}{2} \int_{c}^{b} \frac{\partial}{\partial z^{*}} \int_{0}^{\infty} \mathrm{J}_{0}(\lambda \rho) \mathrm{J}_{0}\left(\lambda \rho^{*}\right)\left[\mathrm{e}^{-\lambda\left|z-z^{*}\right|}+\mathrm{e}^{\lambda\left(z+z^{*}\right.}\right)\right] d \lambda \rho^{*} d \rho^{*}
$$

where the evaluation over the $\theta$-integral from 0 to $2 \pi$ was accomplished.

Since equation (3.1.1-4) is linear, rearranging the operations yields:

$$
\mathrm{K}_{z}=\frac{1}{2} \int_{0}^{\infty} \frac{\partial}{\partial z^{*}}\left[\mathrm{e}^{-\lambda i_{z}-z^{*} 1}+\mathrm{e}^{\lambda\left(z+z^{*}\right)}\right] \int_{c}^{b} \mathrm{~J}_{0}\left(\lambda \rho^{*}\right) \rho^{*} d \rho^{*} \mathrm{~J}_{0}(\lambda \rho) d \lambda,
$$

Evaluating the Bessel integral yields: 


$$
\begin{aligned}
\int_{c}^{b} \mathrm{~J}_{0}\left(\lambda \rho^{*}\right) \rho^{*} d \rho^{*} & =\int_{0}^{b} \mathrm{~J}_{0}\left(\lambda \rho^{*}\right) \rho^{*} d \rho^{*}-\int_{0}^{c} \mathrm{~J}_{0}\left(\lambda \rho^{*}\right) \rho^{*} d \rho^{*} \\
& =\frac{1}{\lambda}\left[b \mathrm{~J}_{1}(\lambda b)-c \mathrm{~J}_{1}(\lambda c)\right]=\frac{1}{\lambda} \sum_{j=1}^{2}(-1)^{j-1} \rho_{j}^{*} \mathrm{~J}_{1}\left(\lambda \rho_{j}^{*}\right)
\end{aligned}
$$

where $\rho_{1}^{*}=b$ and $\rho_{2}^{*}=c$.

Evaluating the $z^{*}$-derivative term yields:

$$
\frac{\partial}{\partial z^{*}}\left[\mathrm{e}^{-\lambda\left|z-z^{*}\right|}+\mathrm{e}^{\lambda\left(z+z^{*}\right)}\right]=\lambda\left[\operatorname{sgn}\left(z-z^{*}\right) \mathrm{e}^{-\lambda \alpha_{1}}-\mathrm{e}^{-\lambda \alpha_{2}}\right]=\lambda \sum_{i=1}^{2}( \pm)_{i} \mathrm{e}^{-\lambda \alpha_{i}}
$$

where: $\alpha_{1}=\left|z-z^{*}\right|, \alpha_{2}=\left(z+z^{*}\right),( \pm)_{1}=\operatorname{sgn}\left(z-z^{*}\right)$, and $( \pm)_{2}=-1$.

Combining the terms yields the solution for $\mathrm{K}_{\mathrm{z}}$ :

$$
\begin{aligned}
\mathrm{K}_{\mathrm{z}} & =\frac{1}{2} \int_{0}^{\infty} \sum_{i=1}^{2}( \pm)_{i} \mathrm{e}^{-\lambda \alpha_{i}} \sum_{j=1}^{2}(-1)^{j-1} \rho_{j}^{*} \mathrm{~J}_{1}\left(\lambda \rho_{j}^{*}\right) \mathrm{J}_{0}(\lambda \rho) d \lambda \\
& =\frac{1}{2} \sum_{i=1}^{2}( \pm)_{i} \sum_{j=1}^{2}(-1)^{j-1} \mathrm{~F}_{100}\left(\rho_{j}^{*}, \rho, \alpha_{i}\right)
\end{aligned}
$$

where $F_{100}$ is found in Luke (1962):

$F_{100}(a, b, p)=a \int_{0}^{\infty} \mathrm{e}^{-\lambda p} \mathrm{~J}_{1}(\lambda a) \mathrm{J}_{0}(\lambda b) d \lambda= \begin{cases}1-\frac{p k \mathrm{~K}(k)}{2 \pi(a b)^{1 / 2}}-\frac{1}{2} \Lambda_{0}(\psi, k), & a>b \\ \frac{1}{2}-\frac{p k \mathrm{~K}(k)}{2 \pi a} & , a=b \\ 0-\frac{p k K(k)}{2 \pi(a b)^{1 / 2}}+\frac{1}{2} \Lambda_{0}(\psi, k), & a<b\end{cases}$

$\Lambda_{0}(\psi, k)$ is the Heuman lambda function,

$$
k^{2}=\left[\frac{4 a b}{p^{2}+(a+b)^{2}}\right] \text { and } \sin ^{2} \psi=\left[\frac{p^{2}}{p^{2}+(a-b)^{2}}\right]
$$

\section{Vertical Surfaces}

The $\rho$-component of $K\left(\mathbf{r}, \mathbf{r}^{*}\right)$ is:

$$
\mathrm{K}_{\rho}\left(\mathbf{r}, \mathbf{r}^{*}\right)=\int_{s^{*}} \frac{\partial}{\partial \rho^{*}} g\left(\mathbf{r}, \mathbf{r}^{*}\right) d s^{*}=\int_{0}^{2 \pi^{2}} \int_{z_{0}^{-}} \frac{\partial}{\partial \rho^{*}} g\left(\mathbf{r}, \mathbf{r}^{*}\right) \rho^{*} d \theta^{*} d z^{*}
$$

Integrating $\theta^{*}$ from 0 to $2 \pi$ and using equation (3.1.1-8) in equation (3.1.1-10) gives:

$$
\mathrm{K}_{\mathrm{\rho}}=\frac{1}{2} \int_{z_{0}^{-}}^{z_{0}^{+}} \frac{\partial}{\partial \rho^{*}} \int_{0}^{\infty} \mathrm{J}_{0}(\lambda \rho) \mathrm{J}_{0}\left(\lambda \rho^{*}\right)\left[\mathrm{e}^{-\lambda\left|z-z^{*}\right|}+\mathrm{e}^{\lambda\left(z+z^{*}\right)}\right] d \lambda \rho^{*} d z^{*}
$$




$$
=\frac{1}{2} \rho^{*} \int_{0}^{\infty} \frac{\partial}{\partial \rho^{*}} \mathrm{~J}_{0}\left(\lambda \rho^{*}\right) \int_{z_{0}^{-}}^{z_{0}^{+}}\left[\mathrm{e}^{-\lambda\left|l_{2}-z^{*}\right|}+\mathrm{e}^{\lambda\left(z+z^{*}\right)}\right] d z^{*} d \lambda
$$

where $z_{0}^{+}=z_{0}+\Delta z$ and $z_{0}^{-}=z_{0}-\Delta z$.

The $\rho^{*}$-derivative is:

$$
\frac{\partial}{\partial \rho^{*}} \mathrm{~J}_{0}\left(\lambda \rho^{*}\right)=-\lambda \mathrm{J}_{1}\left(\lambda \rho^{*}\right)
$$

The evaluation of the $z$-integration in equation (3.1.1-8) yields:

$$
\int_{z_{0}^{-}}^{z_{0}^{+}}\left[\mathrm{e}^{-\lambda\left|z-z^{*}\right|}+\mathrm{e}^{\lambda\left(z+z^{*}\right)}\right] d z^{*}=\int_{z_{0}^{-}}^{z_{0}^{+}} \mathrm{e}^{-\lambda\left|z-z^{*}\right|} d z^{*}+\int_{z_{0}^{-}}^{z_{0}^{+}} \mathrm{e}^{-\left(z+z^{*}\right)} d z^{*}
$$

Three resulting cases occur for the evaluation of the absolute value in the first integral of the right side of equation (3.1.1-11).

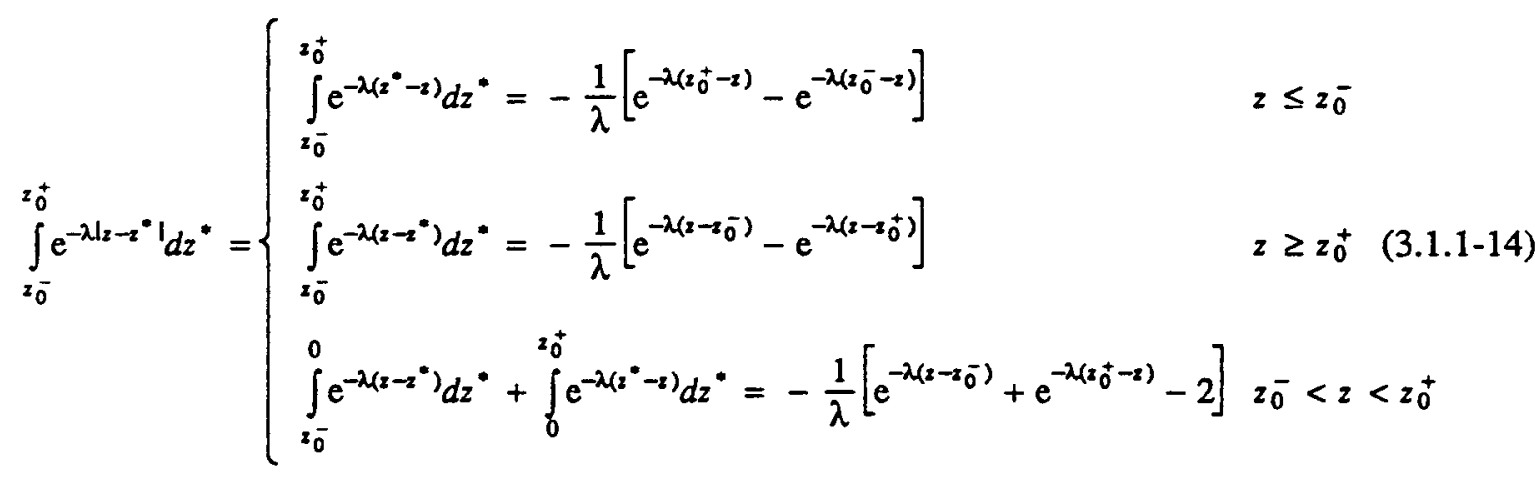

The solution of the second integral in equation (3.1.1-13) is:

$$
\int_{z_{0}^{-}}^{z_{0}^{+}} \mathrm{e}^{-\lambda\left(z+z^{*}\right)} d z^{*}=-\frac{1}{\lambda}\left[\mathrm{e}^{-\lambda\left(z+z_{0}^{+}\right)}-\mathrm{e}^{-\lambda\left(z+z_{0}^{-}\right)}\right]
$$

Combining equations (3.1.1-13) and (3.1.1-14) yields the solution for the $z^{*}$-integration:

$$
\begin{aligned}
& \int_{z_{0}^{-}}^{z_{0}^{+}}\left[\mathrm{e}^{-\lambda\left|z-z^{*}\right|}+\mathrm{e}^{\lambda\left(z+z^{*}\right)}\right] d z^{*} \\
& =-\frac{1}{\lambda}\left\{\left[\begin{array}{l}
+ \\
- \\
+
\end{array}\right] \mathrm{e}^{-\lambda \alpha_{1}}+\left[\begin{array}{l}
- \\
+ \\
+
\end{array}\right] \mathrm{e}^{-\lambda \alpha_{2}}+\mathrm{e}^{-\lambda \alpha_{3}}-\mathrm{e}^{-\lambda \alpha_{4}}-\left[\begin{array}{l}
0 \\
0 \\
2
\end{array}\right] \text { for }\left[\begin{array}{c}
z \leq z_{0}^{-} \\
z \geq z_{0}^{+} \\
z<z<z_{0}^{+}
\end{array}\right]\right. \\
& =-\frac{1}{\lambda} \sum_{k=1}^{4}( \pm)_{k} \mathrm{e}^{-\lambda \alpha_{k}}-2 \delta\left(z, z_{0}^{ \pm}\right)
\end{aligned}
$$


where $\alpha_{1}=\left|z-z_{0}^{+}\right|, \quad \alpha_{2}=\left|z-z_{0}^{-}\right|, \quad \alpha_{3}=\left(z+z_{0}^{+}\right), \quad \alpha_{4}=\left(z+z_{0}^{-}\right)$, and $\delta\left(z, z_{0}^{ \pm}\right)$equals unity for $z_{0}^{-}<z<z_{0}^{+}$or zero otherwise. Combining the terms of equations (3.1.1-15) and (3.1.1-16) gives:

$$
\begin{aligned}
\mathrm{K}_{\mathrm{p}} & =\frac{1}{2} \rho^{*} \int_{0}^{-} J_{1}\left(\lambda \rho^{*}\right) J_{0}(\lambda \rho)\left[\sum_{k=1}^{4}( \pm)_{k} \mathrm{e}^{-\lambda \alpha_{k}}-2 \delta\left(z, z_{0}^{ \pm}\right)\right] d \lambda \\
& =\frac{1}{2} \sum_{k=1}^{4}( \pm)_{k} F_{100}\left(\rho^{*}, \rho, \alpha_{k}\right)-F_{100}\left(\rho^{*}, \rho, 0\right) \delta\left(z, z_{0}^{ \pm}\right)
\end{aligned}
$$

Equations (3.1.1-8) and (3.1.1-17) are the expressions for the components of the Green's function surface integral used to evaluate the response of the annulus surfaces in a half-space. To calculate the potential fields on the surface of the annulus and in the medium, these two expressions, equations (3.1.1-8) and (3.1.1-17), must be used in the scattering term of the surface IE, equation (2.3.1-3).

\subsection{2 $\overleftrightarrow{\Gamma}\left(r, r^{*}\right)$ Formulation}

The same model configuration, Figure (3-1), is used where the pipe of length $(L)$, thickness $(t)$, inner $(b)$ and outer $(c)$ radii is coaxial with the single current source which is located on the vertical axis at $z_{s}$. As in the previous section, the field point is represented by $\mathbf{r}$ and the scattered position is defined as $r^{*}$. Usually the conductivity of the borehole fluid is much closer to the conductivity of the surrounding host than the casing conductivity.

$$
\sigma_{\text {fluid }}, \sigma_{\text {host }} \ll \sigma_{\text {pipe }}
$$

For this section, the problem is simplified by assuming that $\sigma_{\text {fluid }}=\sigma_{\text {has }}=\sigma_{0}$ when comparing with the casing conductivity, $\sigma_{1}$. Figure (3-3) shows a segmented cell of the pipe used for the volume integral equation approximation. The scattering current can be decomposed into two components: $J_{\rho}^{s}$ and $\mathbf{J}_{z}^{s}$. The location of the center of the cell is $\left(0, z_{0}\right)$ and has a center radius, half-height, and half-thickness of $a, \Delta z=h_{z} / 2$ and $\Delta a=t / 2$, respectively.

The expression of the Green's function volume integral for the volume IE, equation (2.3.2-3), is in a dyadic form: 


$$
\vec{\Gamma}\left(r, r^{*}\right)=\Delta \sigma \int_{v^{*}} \vec{G}\left(r, r^{*}\right) d v^{*}
$$

where $\Delta \sigma=\sigma_{1}-\sigma_{0}$ and the axisymmetric dyadic Green's function is found in equation (3G-9) in Appendix 3G. For the axisymmetric problem, there are four elements to $\vec{F}$ :

$$
\left[\hat{\Gamma}\left(r, r^{*}\right)\right]=\left[\begin{array}{ll}
\Gamma_{p p^{*}} p p^{*} & \Gamma_{p z^{*}} p z^{*} \\
\Gamma_{z \rho^{*}} z p^{*} & \Gamma_{z z^{*}} z^{*}
\end{array}\right]
$$

where the $\stackrel{F}{ }$ component, $\Gamma_{a b}$ relates the $a$-component of the field at point $(r)$ to the $b$ component of the scattering current at $\mathbf{r}^{*}$. For the axisymmetric problem, equation (3.1.2-1) is:

$$
\vec{\Gamma}\left(\mathbf{r}, \mathbf{r}^{*}\right)=\Delta \sigma \iint_{z^{*} \rho^{*}} \int_{\theta^{*}} \vec{G}\left(\mathbf{r}, \mathbf{r}^{*}\right) \rho^{*} d \theta^{*} d \rho^{*} d z^{*}=2 \pi \Delta \sigma \iint_{z^{*}} \int_{\rho^{*}} \vec{G}\left(\mathbf{r}, \mathbf{r}^{*}\right) \rho^{*} d \rho^{*} d z^{*}
$$

Using appendix equation (A-11), equation (3.1.2-3) can be written as:

$$
\vec{\Gamma}\left(\mathbf{r}, \mathbf{r}^{*}\right)=-\frac{2 \pi \Delta \sigma}{\sigma_{0}} \int_{z^{*} \rho^{*}} \int_{0} g\left(\mathbf{r}, \mathbf{r}^{*}\right) \rho^{*} d \rho^{*} d z^{*}
$$

The elements of the dyadic $\stackrel{F}{F}$ function are:

$$
\left[\begin{array}{ll}
\Gamma_{\rho p^{*}} & \Gamma_{\rho z^{*}} \\
\Gamma_{z \rho^{*}} & \Gamma_{2 z^{*}}
\end{array}\right]=-\frac{2 \pi \Delta \sigma}{\sigma_{0}} \int_{z^{*} \rho^{*}}\left[\begin{array}{ll}
\frac{\partial}{\partial \rho} \frac{\partial}{\partial \rho^{*}} & \frac{\partial}{\partial \rho} \frac{\partial}{\partial z^{*}} \\
\frac{\partial}{\partial z} \frac{\partial}{\partial \rho^{*}} & \frac{\partial}{\partial z} \frac{\partial}{\partial z^{*}}
\end{array}\right] g\left(\mathbf{r}, \mathbf{r}^{*}\right) \rho^{*} d \rho^{*} d z^{*}
$$

where:

$$
g\left(\mathbf{r}, \mathbf{r}^{*}\right)=\frac{1}{4 \pi} \int_{0}^{\infty} J_{0}\left(\lambda \rho^{*}\right) J_{0}(\lambda \rho)\left[e^{-\lambda\left|z-z^{*}\right|}+e^{-\lambda\left(z+z^{*}\right)}\right] d \lambda
$$

is the expression for the axisymmetric Green's function, equation (3G-7) in Appendix C.

The solutions for the $\boldsymbol{F}$ components are derived in Appendix $D$ and are:

$$
\begin{aligned}
& \Gamma_{\mathrm{p \rho ^{* }}}=\frac{a \Delta a \Delta \sigma}{\sigma_{0}} \sum_{i=1}^{4}\left\{( \pm)_{i}^{\rho} \frac{\alpha_{i} k_{i}}{4 \pi(\rho a)^{3 / 2}}\left[\frac{1+k^{\prime 2}}{k^{\prime 2}} \mathbf{E}\left(k_{i}\right)-2 \mathbf{K}\left(k_{i}\right)\right]-\varepsilon_{\rho}\right\} \\
& \Gamma_{2 \rho^{*}}=\frac{a \Delta a \Delta \sigma}{\sigma_{0}} \sum_{i=1}^{4}(3.1 .2-1)^{i} \frac{k_{i}}{2 \pi a(\rho a)^{1 / 2}}\left[\mathbf{K}\left(k_{i}\right)-\frac{\alpha_{i}{ }^{2}+\rho^{2}-a^{2}}{\alpha_{i}{ }^{2}+(\rho-a)^{2}} \mathbf{E}\left(k_{i}\right)\right]
\end{aligned}
$$




$$
\begin{aligned}
& \Gamma_{\rho z^{*}}=\frac{a \Delta a \Delta \sigma}{\sigma_{0}} \sum_{i=1}^{4}(3.1 .2-1)^{i+1} \frac{k_{i}}{2 \pi \rho(\rho a)^{1 / 2}}\left[\mathrm{~K}\left(k_{i}\right)-\frac{\alpha_{i}^{2}+a^{2}-\rho^{2}}{\alpha_{i}^{2}+(a-\rho)^{2}} \mathbf{E}\left(k_{i}\right)\right] \\
& \Gamma_{z^{*}}=\frac{a \Delta a \Delta \sigma}{\sigma_{0}} \sum_{i=1}^{4}( \pm)_{i}^{2} \frac{\alpha_{i}}{4 \pi(\rho a)^{3 / 2}} \frac{k_{i}{ }^{3}}{\left(1-k_{i}\right)^{2}} \mathbf{E}\left(k_{i}\right)
\end{aligned}
$$

where $\mathbf{K}(k)$ and $\mathbf{E}(k)$ are the complete elliptic integrals of the first and second kind, respectively, and $k_{i}$ was defined in equation (3.1.1-9b). The values of $( \pm)_{i}^{p}= \pm 1$, in equation (3.1.2$6 \mathrm{a}$, and $( \pm)_{i}^{z}= \pm 1$, in equation (3.1.2-6d), are dependent on $z, z_{0}$, and $\Delta z$ in appendix equations (D4-4) and (D1-12), respectively. In equation (3.1.2-6a), the $\varepsilon_{p}$ is equal to $1 / a^{2}$ in the singular cell and zero otherwise.

\section{Highly Conductive Pipe}

At this point, consider the behavior of E-fields within the pipe. Figure (3-4) shows the interface between the host medium and pipe with conductivities of $\sigma_{0}$ and $\sigma_{1}$, respectively. The boundary condition (b.c.) of the continuity of the tangential E-fields requires that the tangential components of the E-fields on both sides of the interface be equal, i.e.,

$$
E_{T_{0}}=E_{T_{1}}
$$

The b.c. of the continuity of the normal component of the current densities $\mathbf{J}$ insists that:

$$
\mathrm{J}_{N_{0}}=\mathrm{J}_{N_{1}} \quad \rightarrow \quad \mathrm{E}_{N_{1}}=\frac{\sigma_{0}}{\sigma_{1}} \mathrm{E}_{N_{0}}
$$

Typically, the conductivity of a metal pipe $\left(\sigma_{1}\right)$ is $6-10$ orders of magnitude larger than that of the host medium $\left(\sigma_{0}\right)$. Hence, the normal E-field within the pipe essentially is nil, i.e., $\mathrm{E}_{N_{1}} \approx 0$. Figure (3-5) is a plot comparing the components of the E-fields within a infinitelength pipe with the conductivity ratio $\sigma_{0} / \sigma_{1}=10^{-8}$. In terms of Figure (3-4), $E_{p}$ represents the normal component and $\mathrm{E}_{2}$ is the tangential component of the E-field. Except for the positions very near the source depth, the $E_{z}$-field is much greater than the $E_{p}$-field. This shows that the vertical E-field within the pipe dominates the response for source-receiver separations greater than $0.1 \mathrm{~m}$. 
Recall that the E-fields within the pipe are found by solving the linear matrix equation which approximates the IE. This matrix equation is formed by letting the field point $\mathbf{r}_{m}$ approach the scattering source position $r_{n}^{*}$ for all segments. Since only the $E_{z}$-field exists within the pipe, the $\Gamma_{z^{*}}$ is the only component needed to form the matrix equation. For the highly conductive pipe, the dyadic $\vec{F}$ function integral can be reduced to the scalar quantity $\Gamma_{2 x^{*}}$, equation $(3.1 .2-6 \mathrm{~d})$.

\section{Potential Field}

Unfortunately, equation (3.1.2-6d) is limited to calculating the $E_{2}$-fields within the pipe segments. To determine the potential fields in the medium from the scattered currents within the pipe, the integral equation $(2.2 .3-12)$ is required.

$$
\phi(\mathbf{r})=\phi_{i}(\mathbf{r})+\phi_{s}(\mathbf{r})
$$

The scattered potential of equation (3.1.2-9) can be expressed as:

$$
\begin{aligned}
\phi_{s}(\mathbf{r}) & =-\frac{1}{\sigma_{0}} \int_{v^{*}} \nabla g\left(\mathbf{r}, \mathbf{r}^{*}\right) \cdot \Delta \sigma \mathrm{E}\left(\mathbf{r}^{*}\right) d v^{*} \\
& =-\frac{\Delta \sigma}{\sigma_{0}} \int_{v^{*}} \frac{\partial}{\partial z} g\left(\mathbf{r}, \mathbf{r}^{*}\right) E_{\mathbf{z}}\left(\mathbf{r}^{*}\right) d v^{*} \\
& =-\frac{\Delta \sigma}{\sigma_{0}} E_{\mathbf{z}}\left(\mathbf{r}^{*}\right)\left[\frac{\partial}{\partial z} \int_{v^{*}} g\left(\mathbf{r}, \mathbf{r}^{*}\right) d v^{*}\right]
\end{aligned}
$$

The bracketed term in equation (3.1.2-10) is formulated in Appendix E and is:

$$
\frac{\partial}{\partial z} \int_{v^{*}} g\left(\mathbf{r}, \mathbf{r}^{*}\right) d v^{*}=a \Delta a \sum_{i=1}^{4}(3.1 .2-1)^{i} \frac{k_{i}}{\pi(\rho a)^{1 / 2}} \mathbf{K}\left(k_{i}\right)
$$

Substituting this into equation (3.1.2-10), one can calculate the scattered potential in the medium caused by the scattered currents of the ring segments, and hence, the total potential in the half-space from equation (3.1.2-9). 


\subsection{Three-Dimensional Problem}

The solution of the Green's function surface integral: $K\left(r, r^{*}\right)$, equation (2.3.1-3), for the three-dimensional problem follows steps similar to those of the axisymmetric case. The problem now assumes no geometric symmetry and must be modeled by using flat or curved surface elements. In this section, the procedures necessary to describe the pipe/annulus by flat surface elements or curved quadrilaterals are given. The Cartesian coordinate system is used to derive $K\left(r, r^{*}\right)$, for the flat triangular and rectangular surface elements. The solution of the Green's function surface integral for the curved quadrilateral segments is derived in the cylindrical coordinate system.

\subsubsection{Cartesian Coordinate System $K\left(r, r^{*}\right)$ Formulation}

Figure 3-6 illustrates two surface elements used to approximate the two shapes encountered when defining the pipe surface. The top and bottom horizontal surfaces of the pipe can be described by triangular elements. Two triangles are required to approximate this curvilinear quadrilateral. To approximate the curved vertical surface of the outer and inner pipe walls, a series of rectangles is used. The number of surface elements needed to describe the side surfaces is reduced by a factor of two by using rectangular elements instead of triangles.

In this section, the Green's function surface integral for the triangular cells will be derived first followed by the solution using rectangular elements. The derivations are based on the method described by Barnett $(1972,1976)$ and Paul (1974) where the surface integrals over triangular domains are solved in Cartesian coordinates for the IE based on surface charge distribution. Unlike the solution using triangular cells, $K\left(r, r^{*}\right)$ for the rectangular elements are exact and require no numerical integration.

The formulation for the triangular cells is very useful since the surface of any 3-D body can be approximated by a set of triangular segments. Assume that the triangular segments that define the surface of the body are arbitrarily oriented with respect to the $x, y$, and $z$ axes (Figure 3-7). By using the 3-D Green's function, appendix equation (A-6), the Green's 
function surface integral, equation (2.3.1-3), can be expressed by the following:

$$
\mathbf{K}\left(\mathbf{r}, \mathbf{r}^{*}\right)=\int_{f} \nabla^{*} g\left(\mathbf{r}, \mathbf{r}^{*}\right) \cdot \mathbf{n}\left(\mathbf{r}^{*}\right) d s^{*}=-\frac{1}{4 \pi} \int_{\xi} \frac{\left(\mathbf{r}-\mathbf{r}^{*}\right)}{\left|\mathbf{r}-\mathbf{r}^{*}\right|^{3}} \cdot \mathbf{n}\left(\mathbf{r}^{*}\right) d s^{*}
$$

Expanding this in terms of the $x, y$, and $z$ components yields:

$$
\mathbf{K}\left(\mathbf{r}, \mathbf{r}^{*}\right)=-\frac{1}{4 \pi} \int_{s} \frac{\left(x-x^{*}\right) n_{z}+\left(y-y^{*}\right) n_{y}+\left(z-z^{*}\right) n_{z}}{\left[\left(x-x^{*}\right)^{2}+\left(y-y^{*}\right)^{2}+\left(z-z^{*}\right)^{2}\right]^{3 / 2}} d s^{*}\left(x^{*}, y^{*}, z^{*}\right)
$$

where $n_{x}, n_{y}$, and $n_{z}$ are the directional cosines of the surface normal unit vector $n$.

Since the segment is arbitrarily oriented, evaluating all of the surface integrals that describe the pipe is difficult. The evaluation is made much easier by rotating the coordinate system such that one of the coordinate axes is perpendicular to the surface of the segment and another coordinate axis is parallel to a side of the triangular segment.

If the apices of the triangular cell are numbered as shown in Figure (3-7), the following unit vectors can be defined:

$$
I=\frac{s_{13}}{\left|s_{13}\right|} \quad n=\frac{s_{12} \times s_{13}}{\left|s_{12} \times s_{13}\right|} \quad m=n \times l
$$

where:

$$
\mathbf{s}_{i j}=\left(x_{j}-x_{i}\right) \hat{\mathbf{x}}+\left(y_{j}-y_{i}\right) \hat{\mathbf{y}}+\left(z_{j}-z_{i}\right) \hat{\mathbf{z}}
$$

Now the desired coordinate transformation can be achieved by letting:

$$
\begin{array}{lll}
\boldsymbol{u}=1 \cdot \mathbf{r} & \boldsymbol{v}=\mathbf{m} \cdot \mathbf{r} & \boldsymbol{w}=\mathbf{n} \cdot \mathbf{r} \\
\boldsymbol{u}^{*}=1 \cdot \mathbf{r}^{*} & \boldsymbol{v}^{*}=\mathbf{m} \cdot \mathbf{r}^{*} & \boldsymbol{w}^{*}=\mathbf{n} \cdot \mathbf{r}^{*}
\end{array}
$$

Using these relations, the following are true:

$$
\begin{aligned}
\left(\mathbf{r}-\mathbf{r}^{*}\right) \cdot \mathbf{n}\left(\mathbf{r}^{*}\right) & =\left(w-w^{*}\right) \\
\left|\mathbf{r}-\mathbf{r}^{*}\right| & =\left[\left(u-u^{*}\right)^{2}+\left(v-v^{*}\right)^{2}+\left(w-w^{*}\right)^{2}\right]^{1 / 2} \\
d s^{*} & =d u^{*} d v^{*}
\end{aligned}
$$

Equation (3.2.1-1) can now be written as:

$$
\begin{aligned}
\mathbf{K}\left(\mathbf{r}, \mathbf{r}^{*}\right) & =-\frac{1}{4 \pi} \int_{S} \frac{\left(\mathbf{r}-\mathbf{r}^{*}\right)}{\left|\mathbf{r}-\mathbf{r}^{*}\right|^{3}} \cdot \mathbf{n}\left(\mathbf{r}^{*}\right) d s^{*} \\
& =-\frac{1}{4 \pi} \int_{u} \int_{v} \frac{\left(w-w^{*}\right)}{\left[\left(u-u^{*}\right)^{2}+\left(v-v^{*}\right)^{2}+\left(w-w^{*}\right)^{2}\right]^{3 / 2}} d u^{*} d v^{*}
\end{aligned}
$$

A shift of the origin to the field point location is done for convenience so equation (3.2.1-5) 
is:

$$
\mathbf{K}\left(\mathbf{r}, \mathbf{r}^{*}\right)=\frac{1}{4 \pi} \iint_{q} \frac{r}{\left(p^{2}+q^{2}+r^{2}\right)^{3 / 2}} d p d q=\frac{1}{4 \pi} \int_{q_{1}}^{q_{2}} \int_{p_{1}}^{p_{3}} \frac{r}{\left(p^{2}+q^{2}+r^{2}\right)^{3 / 2}} d p d q
$$

where:

$$
p=u^{*}-u \quad q=v^{*}-v \quad r=w^{*}-w
$$

Integrating with respect to $p$-variable yields:

$$
\mathbf{K}\left(\mathbf{r}, \mathbf{r}^{*}\right)=\frac{1}{4 \pi} \int_{q_{1}}^{q_{2}}\left[\frac{r p}{\left(q^{2}+r^{2}\right)\left(p^{2}+q^{2}+r^{2}\right)^{1 / 2}}\right]_{p_{1}}^{p_{3}} d q
$$

The $p$-variable in equation (3.2.1-7) is a linear function of $q$, i.e.,

$$
p_{i}=p_{i}(q)=g_{i} q+h_{i} \quad(\text { for } i=1 \text { and } 3)
$$

where $g_{i}$ (slope) and $h_{i}$ (intercept) are the following:

$$
\begin{array}{ll}
g_{1}=\frac{p_{2}-p_{1}}{q_{2}-q_{1}} & h_{1}=\frac{p_{1} q_{2}-p_{2} q_{1}}{q_{2}-q_{1}} \\
g_{3}=\frac{p_{2}-p_{3}}{q_{2}-q_{1}} & h_{3}=\frac{p_{3} q_{2}-p_{2} q_{2}}{q_{2}-q_{1}}
\end{array}
$$

The $q$-integral is accomplished by using a Gauss-Legendre method for numerical integration.

For the rectangular surface element, the $K\left(r, r^{*}\right)$ formulation follows the same procedures as was done for the triangular segment. The coordinate system is rotated and translated so as to make the integral evaluation simple. If the apices of the rectangular element are numbered as in Figure (3-8), the previous procedures up to and including equation (3.2.1-7), are the same as above. Unlike the triangular element, the $p$-variable integration is independent of $q$. Hence, the integration with respect to the $q$-variable can be done analytically. After some manipulations and algebra, the solution to the general form of the integral is:

$$
\int \frac{r p}{\left(q^{2}+r^{2}\right)\left(p^{2}+q^{2}+r^{2}\right)^{1 / 2}} d q=\tan ^{-1}\left[\frac{q p}{r\left(p^{2}+q^{2}+r^{2}\right)^{1 / 2}}\right]
$$

Hence, the Green's function surface integral for the rectangular elements reduces to four arctangent functions and can be expressed as:

$$
\mathbf{K}\left(\mathbf{r}, \mathbf{r}^{*}\right)=\left.\left.\frac{1}{4 \pi} \tan ^{-1}\left[\frac{q p}{r\left(p^{2}+q^{2}+r^{2}\right)^{1 / 2}}\right]\right|_{p_{1}} ^{p_{3}}\right|_{q_{1}} ^{q_{2}}
$$




\subsubsection{Cylindrical Coordinate System $\mathrm{K}\left(r, r^{*}\right)$ Formulation}

To approximate the surface of the pipe using the flat rectangles and triangles, many elements are required. This results in a very large linear system of equations which much be solved to determine the unknown fields on the surface of the elements. One could reduce the size of the matrix of unknowns by describing the surface with curved-quadrilateral elements. Figure (3-9) shows the two surface elements that are used to approximate the pipe surface. The vertical walls can be enclosed with a series of curved-rectangular surface elements. A flat-surfaced, curve-sided quadrilateral segment is used to define the horizontal pipe surface.

By using the cylindrical coordinate system, $K\left(r, r^{*}\right)$ for the quadrilateral segments can be derived. for completeness, two formulations will be presented in the section. The first formulation is for the "blue collar" form of $K\left(r, r^{*}\right)$ which is in terms of trigonometric functions and can easily be calculated on a computer. The second derivation is an elegant form which is expressed as elliptic integrals and can be used for limiting approximations.

If the coordinates of the field and source points are $(\rho, \theta, z)$ and $\left(\mathbf{r}^{*}, \theta^{*}, z^{*}\right)$, respectively, then for an arbitrary azimuthal component, $\phi$, the following relations exist:

$$
\mathbf{r}-\mathbf{r}^{*}=\left[\rho \cos \left(\theta-\theta^{*}\right)-\rho^{*} \cos \left(\phi-\theta^{*}\right)\right] \rho+\left[\rho \sin (\theta-\phi)+\rho^{*} \sin \left(\phi-\theta^{*}\right)\right] \hat{\phi}+\left(z-z^{*}\right) \hat{\mathbf{z}}(3.2 .2-1)
$$

Since $\hat{\phi}$ is arbitrary, let $\hat{\theta}=\hat{\phi}$ so equation (3.2.2-1) is:

$$
\begin{gathered}
\mathbf{r}-\mathbf{r}^{*}=\left[\rho-\rho^{*} \cos \left(\theta-\theta^{*}\right)\right] \hat{\rho}+\rho^{*} \sin \left(\theta-\theta^{*}\right) \hat{\theta}+\left(z-z^{*}\right) \hat{\mathbf{z}} \\
\left|\mathbf{r}-\mathbf{r}^{*}\right|=\left[\rho^{2}+\rho^{* 2}-2 \rho \rho^{*} \cos \left(\theta-\theta^{*}\right)+\left(z-z^{*}\right)^{2}\right]^{1 / 2}
\end{gathered}
$$

The first formulation of the Green's function surface integral is given below. Using equations (3.2.2-2) and (3.2.2-3), the expression for the $K\left(r, r^{*}\right)$ is:

$$
\begin{aligned}
& \mathbf{K}\left(\mathbf{r}, \mathbf{r}^{*}\right)=\int_{\xi} \nabla^{*} g\left(\mathbf{r}, \mathbf{r}^{*}\right) \cdot \mathbf{n}\left(\mathbf{r}^{*}\right) d s^{*}=-\frac{1}{4 \pi} \int_{f} \frac{\left(\mathbf{r}-\mathbf{r}^{*}\right)}{\left|\mathbf{r}-\mathbf{r}^{*}\right|^{3}} \cdot \mathbf{n}\left(\mathbf{r}^{*}\right) d s^{*} \\
& =-\frac{1}{4 \pi} \int_{S} \frac{\left[\rho-\rho^{*} \cos \left(\theta-\theta^{*}\right)\right] n_{\rho}+\rho^{*} \sin \left(\theta-\theta^{*}\right) n_{\theta}+\left(z-z^{*}\right) n_{z}}{\left[\rho^{2}+\rho^{* 2}-2 \rho \rho^{*} \cos \left(\theta-\theta^{*}\right)+\left(z-z^{*}\right)^{2}\right]^{3 / 2}} d s^{*}\left(\rho^{*}, \theta^{*}, z^{*}\right)
\end{aligned}
$$

where $n_{\rho}, n_{\theta}$, and $n_{z}$ are the directional cosines of the surface normal vector, $\mathbf{n}$. 
For the pipe, no surfaces exist such that $n_{\theta} \neq 0$. The horizontal surfaces have $n_{p}=0$ and $n_{z}= \pm 1$ and the vertical surfaces have $n_{p}= \pm 1$ and $n_{z}=0$. Hence, equation (3.2.2-4) can be decomposed into two components:

$$
\begin{aligned}
& \mathrm{K}_{H}\left(\mathrm{r}, \mathrm{r}^{*}\right)=\frac{1}{4 \pi} \int_{\theta^{*}}\left[-\int_{\rho^{*}} \frac{\left(z-z^{*}\right)}{\left|\mathbf{r}-\mathbf{r}^{*}\right|^{3}} \rho^{*} d \rho^{*}\right] d \theta^{*} \\
& K_{V}\left(r, r^{*}\right)=\frac{1}{4 \pi} \int_{\theta^{*}}\left[-\int_{z^{*}}^{\rho^{* 2}-\rho^{*} \cos \left(\theta-\theta^{*}\right)} \frac{\left|r-r^{*}\right|^{3}}{\mid z^{*}}\right] d \theta^{*}
\end{aligned}
$$

where $\left|\mathbf{r}-\mathbf{r}^{*}\right|$ is defined by equation (3.2.2-2b) and $K_{H}$ and $K_{V}$ are the horizontal $(H)$ and vertical $(V)$ components of the Green's function surface integral, respectively. The solutions to the bracketed terms in equation (3.2.2-5) are:

$$
\begin{aligned}
& I_{H}\left(\theta-\theta^{*}\right)=-\int_{\rho^{*}} \frac{\left(z-z^{*}\right)}{\left|\mathbf{r}-\mathbf{r}^{*}\right|^{3}} \rho^{*} d \rho^{*}=\left.\frac{\left[\rho^{2}-\rho \rho^{*} \cos \left(\theta-\theta^{*}\right)+\left(z-z^{*}\right)^{2}\right]}{\left[\rho^{2}-\rho^{2} \cos ^{2}\left(\theta-\theta^{*}\right)+\left(z-z^{*}\right)^{2}\right]} \frac{\left(z-z^{*}\right)}{\left|\mathbf{r}-\mathbf{r}^{*}\right|}\right|_{\rho_{1}} ^{\rho_{2}} \\
& I_{V}\left(\theta-\theta^{*}\right)=-\int_{z^{*}} \frac{\left[\rho^{* 2}-\rho \rho^{*} \cos \left(\theta-\theta^{*}\right)\right]}{\left|\mathbf{r}-\mathbf{r}^{*}\right|^{3}} d z^{*}=\left.\frac{\left[\rho^{* 2}-\rho \rho^{*} \cos \left(\theta-\theta^{*}\right)\right]}{\left[\rho^{2}+\rho^{* 2}-2 \rho \rho^{*} \cos ^{2}\left(\theta-\theta^{*}\right)\right]} \frac{\left(z-z^{*}\right)}{\left|\mathbf{r}-\mathbf{r}^{*}\right|}\right|_{z_{1}} ^{z_{2}}
\end{aligned}
$$

In the above formulation, there are no complicated functions involved and both integrands can easily be calculated. To obtain $K\left(r, r^{*}\right)$, equation (3.2.2-5) must be numerically integrated with respect to $\theta^{*}$-variable. To simplify the integration, let $\psi^{*}=\theta^{*}-\theta_{0}$ where $\theta_{0}$ is the azimuthal location of the center of the surface arc-element (see Figure 3-10). The general form of the components of $K\left(\mathbf{r}, \mathbf{r}^{*}\right)$ can be expressed as:

$$
\mathrm{K}_{k}=\frac{1}{4 \pi} \int_{\theta_{1}}^{\theta_{2}} I_{k}\left(\theta-\theta^{*}\right) d \theta^{*}=\frac{1}{4 \pi} \int_{\psi_{1}=-\Delta \theta}^{\psi_{2}=\Delta \theta} I_{k}\left(\theta-\theta_{0}-\psi^{*}\right) d \psi^{*}=\frac{1}{4 \pi} \int_{-\Delta \theta}^{\Delta \theta} I_{k}\left(\psi-\psi^{*}\right) d \psi^{*}
$$

where $\psi=\theta-\theta_{0}, \Delta \theta$ is the half arc-length of the surface element, and the subscript $k$ represents either the horizontal or vertical component of $K\left(r, \mathbf{r}^{*}\right)$.

The following procedure is the second formulation of the Green's function surface integral. The general form of the $K\left(r, r^{*}\right)$ can be written as:

$$
\begin{aligned}
\mathbf{K}\left(\mathbf{r}, \mathbf{r}^{*}\right)=\int_{S^{*}} \nabla^{*} g\left(\mathbf{r}, \mathbf{r}^{*}\right) \cdot \mathbf{n}\left(\mathbf{r}^{*}\right) d s^{*} & =\frac{1}{4 \pi} \int_{\Gamma^{*}} \int_{\theta^{*}} \frac{\partial}{\partial n^{*}} \frac{1}{\left|\mathbf{r}-\mathbf{r}^{*}\right|} \rho^{*} d \theta^{*} d \Gamma^{*} \\
& =\frac{1}{4 \pi} \int_{\Gamma^{*}} \frac{\partial}{\partial n^{*}}\left[\int_{\theta^{*}} \frac{1}{\left|\mathbf{r}-\mathbf{r}^{*}\right|} d \theta^{*}\right] \rho^{*} d \Gamma^{*}
\end{aligned}
$$


where $\left|r-r^{*}\right|$ is defined by equation $(3.2 .2-3)$ and $\Gamma^{*}$ is the integration variable which is perpendicular to the surface normal vector, $n$. The integral in the brackets can be expressed as:

$$
I_{\theta}\left(\rho^{*}, z^{*}\right)=\int_{\theta^{*}} \frac{1}{\left|\mathbf{r}-\mathbf{r}^{*}\right|} d \theta^{*}=\frac{2}{a}[\mathrm{~F}(\Psi, k)]_{\Psi_{1}}^{\Psi_{2}}=\frac{2 k}{b}[\mathrm{~F}(\Psi, k)]_{\Psi_{1}}^{\Psi_{2}}
$$

where $F(\psi, k)$ is the incomplete elliptic integral of the first kind and:

$$
\begin{aligned}
& k^{2}=\frac{b^{2}}{a^{2}}=\frac{4 \rho \rho^{*}}{\left(\rho+\rho^{*}\right)^{2}+\left(z-z^{*}\right)^{2}} \\
& \Psi_{2}=\frac{1}{2}\left(\theta-\theta_{0}+\Delta \theta\right) \\
& \Psi_{1}=\frac{1}{2}\left(\theta-\theta_{0}-\Delta \theta\right)
\end{aligned}
$$

Again, there are two surface elements for the pipe: horizontal and vertical. Hence, the Green's function integral is expressed as the horizontal $(H)$ and vertical $(V)$ components:

$$
\begin{aligned}
& K_{H}\left(r, r^{*}\right)=\frac{1}{4 \pi} \int_{\rho^{*}} \frac{\partial}{\partial z^{*}} I_{\theta}\left(\rho^{*}, z^{*}\right) \rho^{*} d \rho^{*} \\
& K_{V}\left(r, r^{*}\right)=\frac{1}{4 \pi} \int_{z^{*}} \frac{\partial}{\partial z^{*}} I_{\theta}\left(\rho^{*}, z^{*}\right) \rho^{*} d z^{*}
\end{aligned}
$$

Evaluating the partial derivatives yields the integrands:

$$
\begin{aligned}
& I_{H}\left(\rho^{*}, z^{*}\right)=2\left(z-z^{*}\right) \frac{k}{b R^{2}}\left[\mathrm{E}(\Psi, k)-\frac{k^{2} \sin \psi \cos \psi}{\left(1-k^{2} \sin ^{2} \psi\right)^{1 / 2}}\right]_{\Psi_{1}}^{\Psi_{2}} \\
& I_{V}\left(\rho^{*}, z^{*}\right)=\left[\frac{\gamma^{2}}{\rho^{*}} \frac{k}{b R^{2}}\left[\mathrm{E}(\Psi, k)-\frac{k^{2} \sin \psi \cos \psi}{\left(1-k^{2} \sin ^{2} \psi\right)^{1 / 2}}\right]-\frac{k}{b \rho^{*}} \mathrm{~F}(\Psi, k)\right]_{\Psi_{1}}^{\Psi_{2}}
\end{aligned}
$$

where $\mathrm{E}(\psi, k)$ is the incomplete elliptic integral of the second kind and:

$$
\begin{aligned}
& \gamma^{2}=\rho^{2}-\rho^{* 2}+\left(z-z^{*}\right)^{2} \\
& R^{2}=\left(\rho-\rho^{*}\right)^{2}+\left(z-z^{*}\right)^{2}
\end{aligned}
$$

The evaluation of the equation (3.2.2-14) may be computationally time consuming. In each step, one needs to evaluate the integral of the Green's function where the elliptical integrals is needed. The calculation of these elliptical integrals requires a routine involving an infinite series and many time consuming conditional statements. Thus, using equation (3.2.26) may result in a computationally faster evaluation of the $K\left(r, r^{*}\right)$ than using equation (3.2.2$15)$. 


\subsection{Off-axis Geometry and Asymmetric Approximations}

Thus far, the method to determine the responses of several coaxially located or threedimensional bodies has been presented. Now, the objective is to present a method which can calculate the coupled response of a pipe/well casing (annular-shaped body) and an arbitrarily located 3-D body (see Figure 3-11).

For the majority of the configurations involving the pipe and off-axis bodies, the surface IE approach is simple and convenient. With the exception of thin objects, the volume IE method may be quite cumbersome to discretize the body. For thin objects, like fractures, the volume IE approach can model the fracture as a single layer of rectangular blocks. But for a larger body, the number of volume elements needed to approximate it greatly increases and the process of volume discretization becomes complicated. The procedure used to discretize a surface is simpler than discretizing a volume. Additionally, the number of unknowns that must be solved are fewer using the surface IE method. For the surface IE approach, the unknowns are the potentials which are scalar quantities versus the vector quantities, the E-fields, used in the volume IE method. Thus, the potential surface IE formulation described in Chapter 2 may be a better method to calculate the coupled response of the pipe and an offaxis body.

In Section (3.2.1), the formulation of the Green's function surface integral $K\left(\mathbf{r}, \mathbf{r}^{*}\right)$ was derived for any arbitrary body. The Green's function integral for triangular surface elements, equation (3.2.1-7), can be used to calculate the fields due to the 3-D body. The casing response is approximated by either the Cartesian or cylindrical coordinate formulation of $\mathbf{K}\left(\mathbf{r}, \mathbf{r}^{*}\right)$. By substituting the appropriate Green's function surface integral into equation (2.61), the response of any body that is near or in contact with the pipe can be calculated. Using the procedure in Section 2.6 for multiple bodies, the potentials in the medium can be determined.

Discretizing the surface of the body and pipe with triangular cells requires a tremendous number of segments. This results in a very large set of linear equations that must be solved 
in order to determine the unknown field for each segment. If the pipe surface can be approximated with annular segments, as in the axisymmetric method, the number of unknowns for the pipe will be reduced. This reduction is proportional to the number of ring segments needed to replace the arc segments.

The model study of LaBrecque and Ward (1988) used such a method in their volume IE formulation to determine the electric field responses of fractures located near the steel cased well (see Chapter 1). They combined the integral equation formulation for the axisymmetric and 3-D problems. The electric fields in the well casing were assumed to be radially symmetric. Thus, the axisymmetric Green's function was applied to the casing. The fracture was modeled as an off-axis tabular body which was discretized with rectangular blocks. The 3-D Green's function of Beasley and Ward (1986) was used for the tabular body.

The use of the axisymmetric Green's function for the pipe was based on the work of Holladay and West (1984). They indicated that if the off-axis source distances are "much greater" than the radius of the pipe, then an axisymmetric Green's function may substitute for the 3-D Green's function. Their approximation was numerically tested by comparing the zero and first order solution coefficients for a variety of source positions with an infinite-cylinder model.

The axisymmetric approximation is based on the assumption that the annular region surrounding any point on the pipe is at the same potential (equipotential). This phenomenon may be possible because of the extremely large pipe conductivity. If a surface segment of pipe is an equipotential surface, then the arc element of equation (3.2.2-13), see Figure (3-10) can be evaluated about the circumference of the pipe, i,e, $\Delta \theta=\pi$. Since the integration is evaluated about the entire ring segment, the azimuthal center of the arc can be arbitrarily chosen. For convenience, let $\theta_{0}=\theta$ and then equation (3.2.2-13) becomes:

$$
I_{\theta}=\frac{2 k}{b}[\mathrm{~F}(\psi, k)]_{\psi_{1}=-\pi / 2}^{\psi_{2}=\pi / 2}=\frac{2 k \mathrm{~K}(k)}{\left(\rho \rho^{*}\right)^{1 / 2}}
$$

where the following two identities were used: 


$$
\begin{aligned}
& \mathrm{F}(-\psi, k)=-\mathrm{F}(\psi, k) \\
& \mathrm{F}(\pi / 2, k)=\mathrm{K}(k)
\end{aligned}
$$

From Luke (1962), equation (3.3-1) can be written as:

$$
I_{\theta}=2 \pi\left[\frac{k \mathbf{K}(k)}{\pi\left(\rho \rho^{*}\right)^{1 / 2}}\right]=2 \pi \int_{0}^{\infty} J_{0}\left(\lambda \rho^{*}\right) J_{0}(\lambda \rho) e^{-\lambda l_{z-z^{*}} \mid} d \lambda=\int_{0}^{2 \pi}\left[\int_{0}^{\infty} J_{0}\left(\lambda \rho^{*}\right) J_{0}(\lambda \rho) e^{-\lambda l_{z-z^{*}} \mid} d \lambda\right] d \theta^{*}(3.3-3)
$$

The Green's function surface integral becomes:

$$
\begin{aligned}
\mathrm{K}_{k}\left(\mathrm{r}, \mathrm{r}^{*}\right) & =\frac{1}{4 \pi} \int_{\Gamma^{*}} \frac{\partial}{\partial n^{*}} I_{\theta} \rho^{*} d \Gamma^{*}=\frac{1}{4 \pi} \int_{\Gamma^{*}} \frac{\partial}{\partial n^{*}} \int_{0}^{2 \pi}\left[\int_{0}^{\infty} J_{0}\left(\lambda \rho^{*}\right) J_{0}(\lambda \rho) e^{-\left.\lambda\right|_{z}-z^{*}} d \lambda\right] d \theta^{*} \rho^{*} d \Gamma^{*} \\
& =\int_{\Gamma^{*}}^{2 \pi} \frac{\partial}{\partial n^{*}}\left[\frac{1}{4 \pi} \int_{0}^{-} J_{0}\left(\lambda \rho^{*}\right) J_{0}(\lambda \rho) e^{-\lambda k_{z}-z^{*}} d \lambda\right] \rho^{*} d \theta^{*} d \Gamma^{*}=\int_{s^{*}} \frac{\partial}{\partial n^{*}} g\left(\mathrm{r}, \mathrm{r}^{*}\right) d s^{*}
\end{aligned}
$$

where $g\left(r, r^{*}\right)$ is the axisymmetric Green's function.

From this derivation, if the annular surface is a near-equipotential surface, then the axisymmetric approximation of the pipe segment may be used. The validity of this assumption will be investigated in the next chapter by comparing the solutions of the 3-D Cartesian and the axisymmetric formulations. 


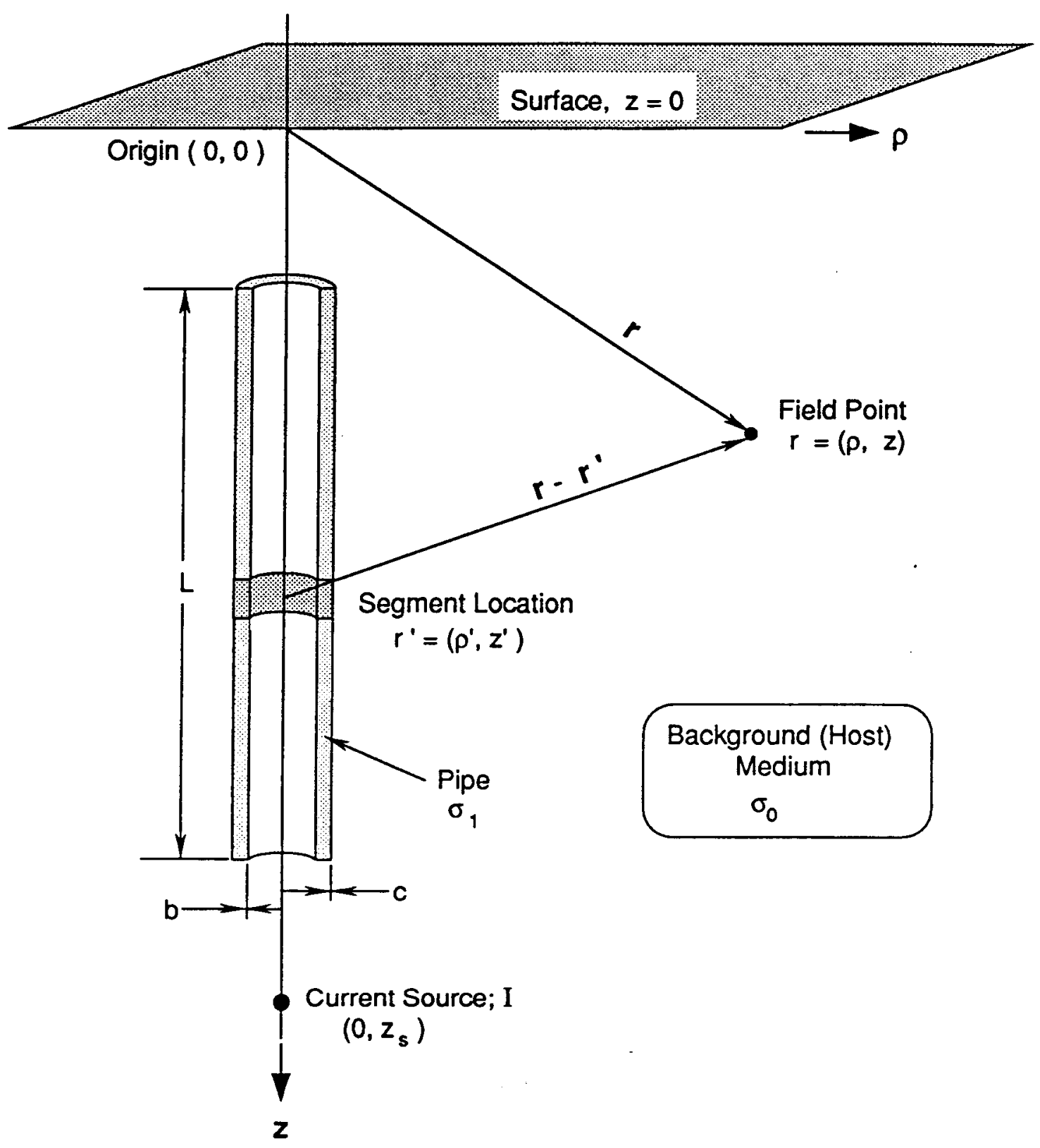

Figure 3-1: A generalized model of the finite-length pipe coaxial with a current source within a homogeneous half-space. 

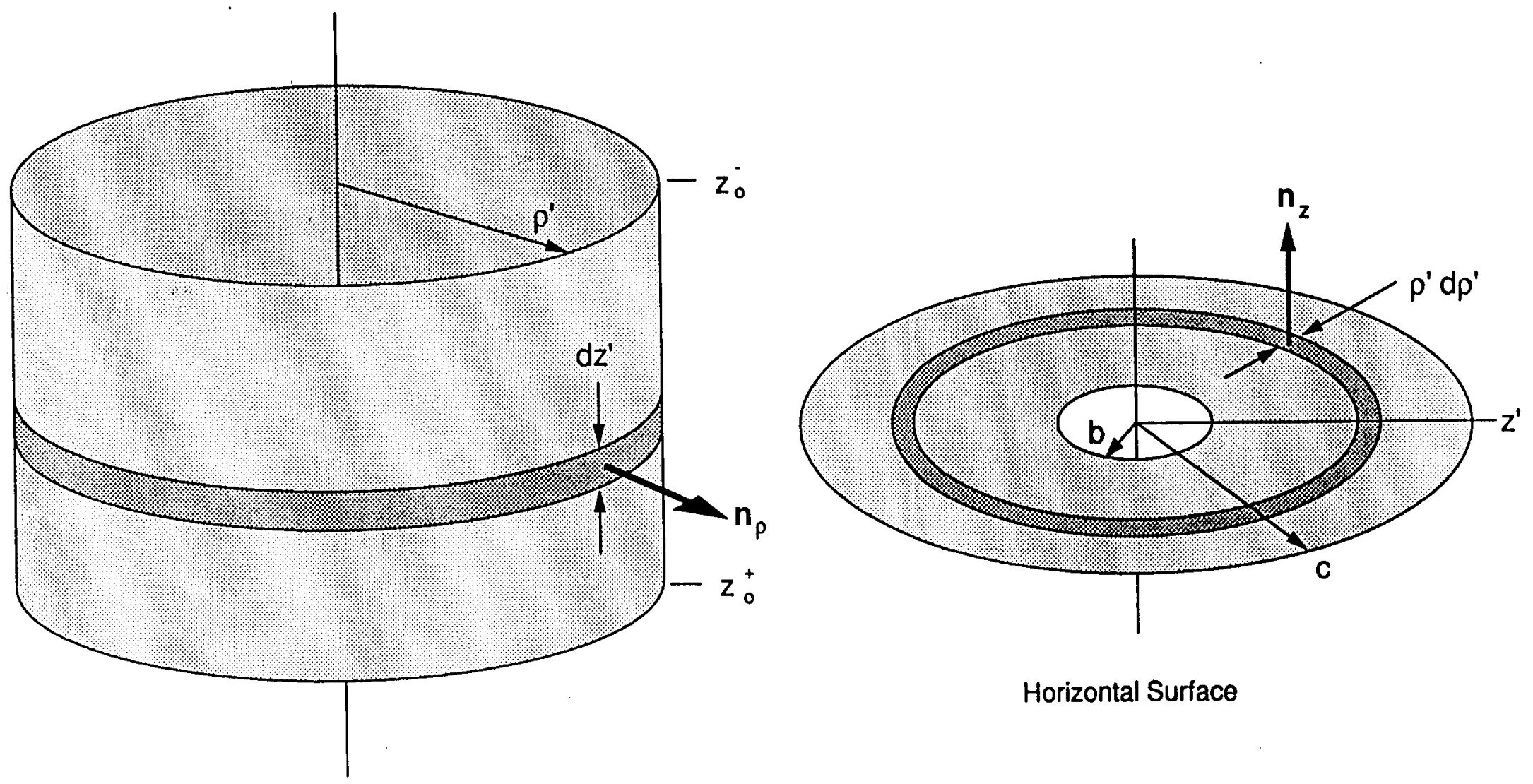

Vertical Surface

Horizontal Surface

Figure 3-2: The surface elements associated with the ring segment of the pipe. The inner and outer surfaces are described by the vertical cylindrical shell (left). The top and bottom surfaces are approximated by the horizontal disk (right) 


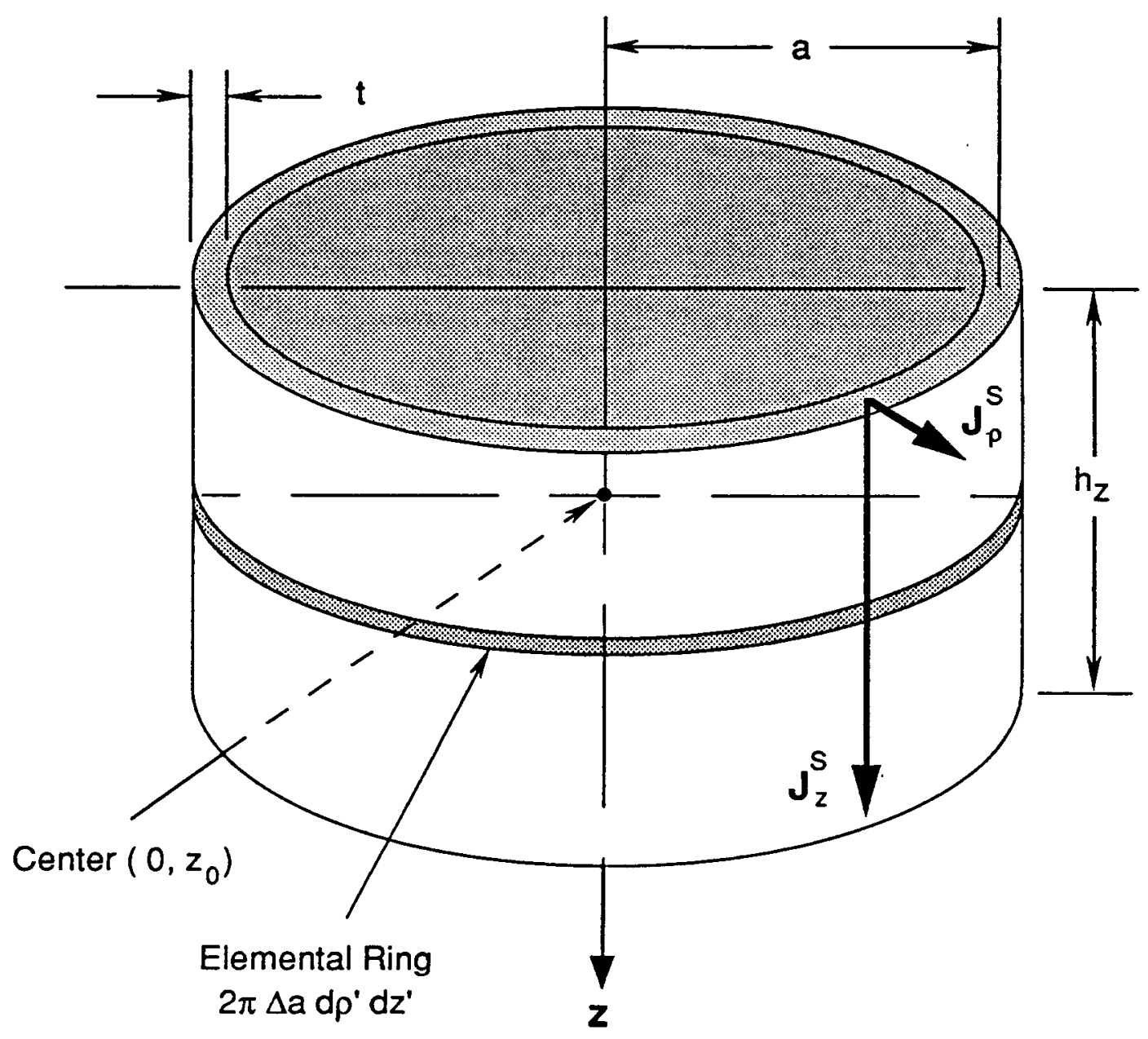

Figure 3-3: The volume segment used to define the pipe for the volume IE method. The scattered current densities are decomposed into two components: radial $\left(\mathrm{J}_{\rho}\right)$ and vertical $\left(\mathrm{J}_{z}\right)$. 


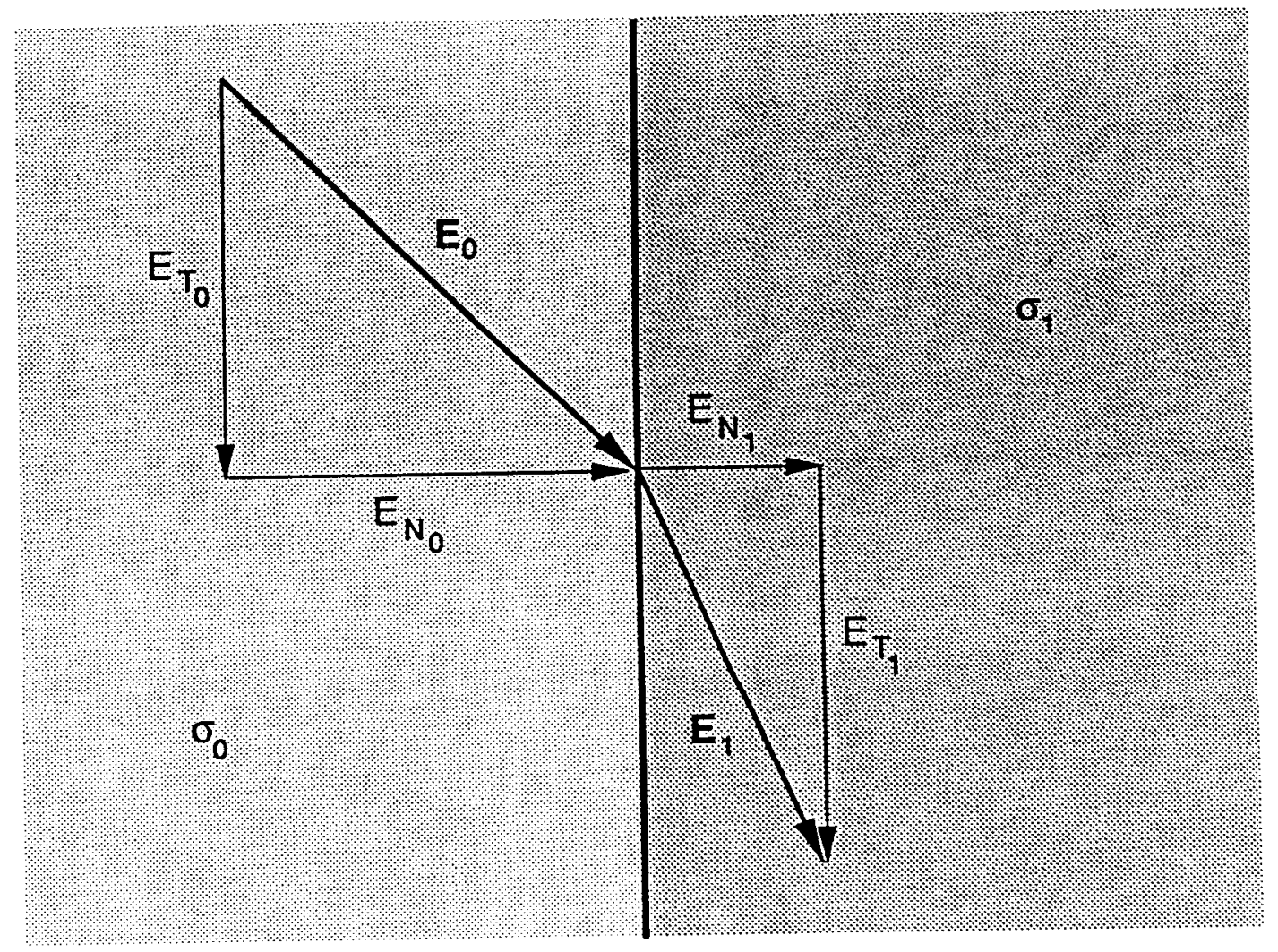

\section{Boundary Conditions:}

1) $E_{T_{1}}=E_{T_{0}}$

2) $J_{N_{1}}=J_{N_{0}} \rightarrow \sigma_{0} E_{N_{0}}=\sigma_{1} E_{N_{1}} \rightarrow E_{N_{1}}=\frac{\sigma_{0}}{\sigma_{1}} E_{N_{0}}$

Figure 3-4: The E-field behavior in the two mediums is dependent on the boundary condition at the interface: tangential electric fields and normal current densities are continuous. 


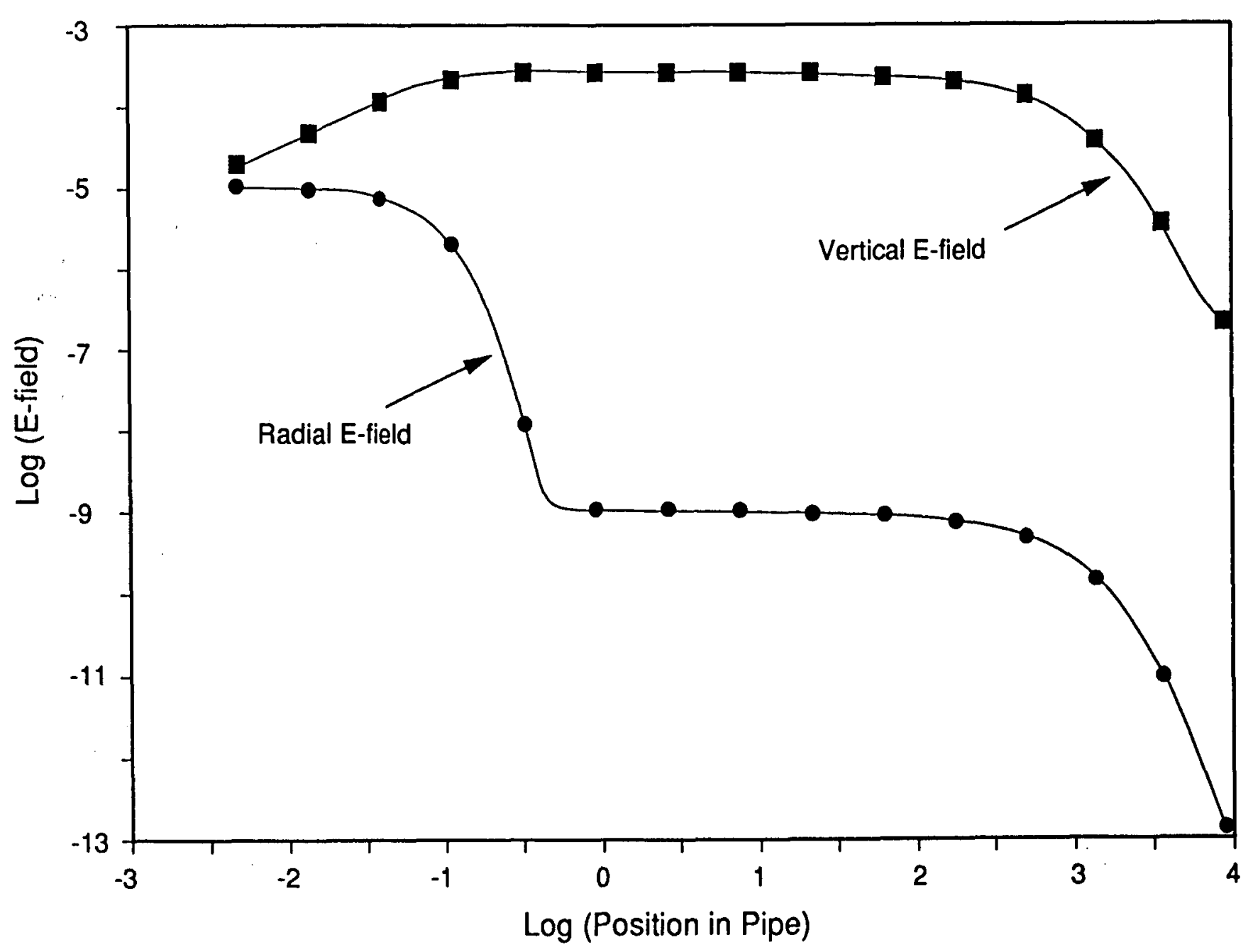

Figure 3-5: The log plot of the radial and vertical E-fields within a semi-infinite pipe coaxial with a current point source located at the half-space surface. 


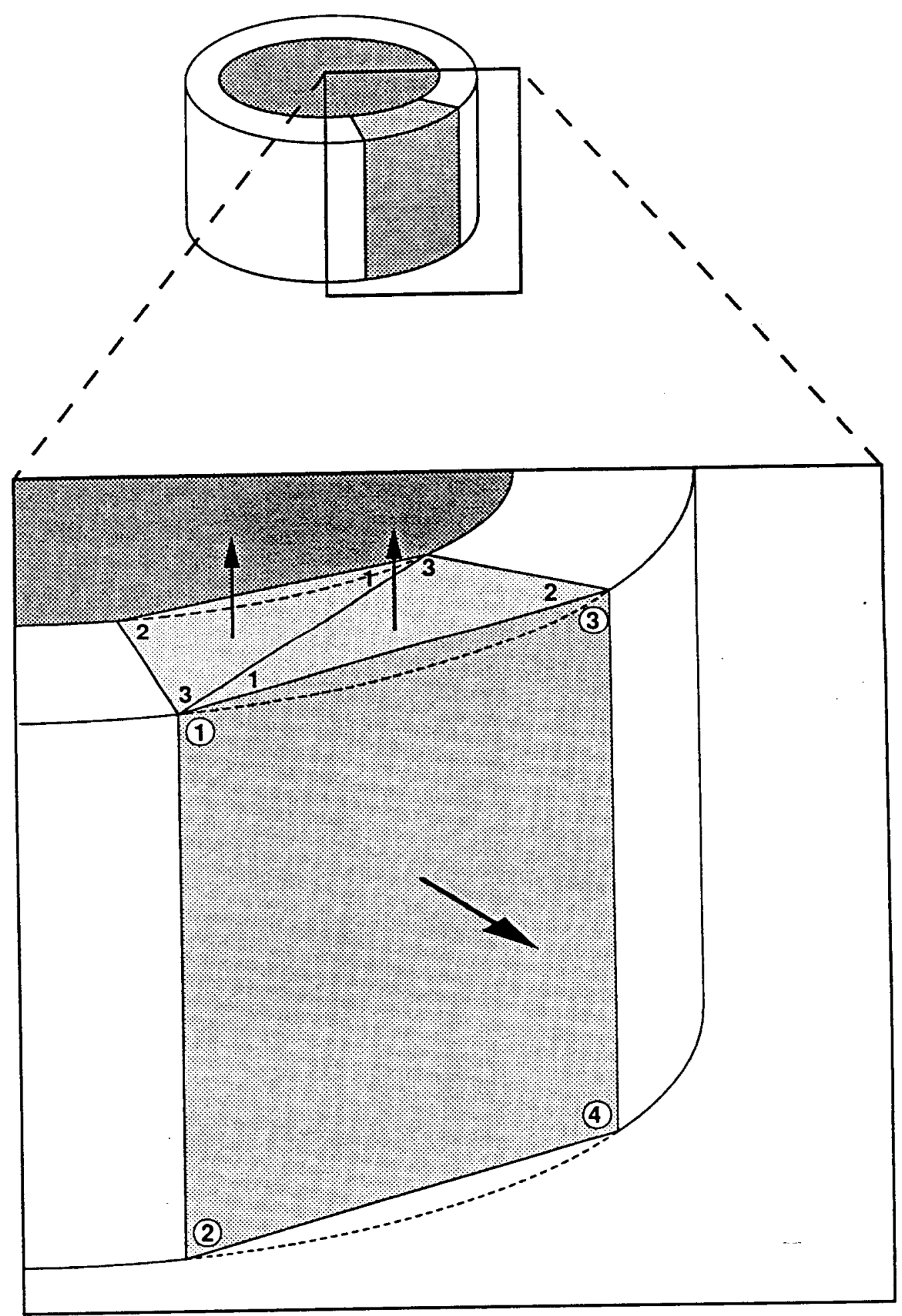

Figure 3-6: The surface elements used to approximate an arc sections of the pipe. For the 3-D Cartesian coordinate formulation, triangular and rectangular surface elements are used. The arrows represent the surface normals of the surface elements. 

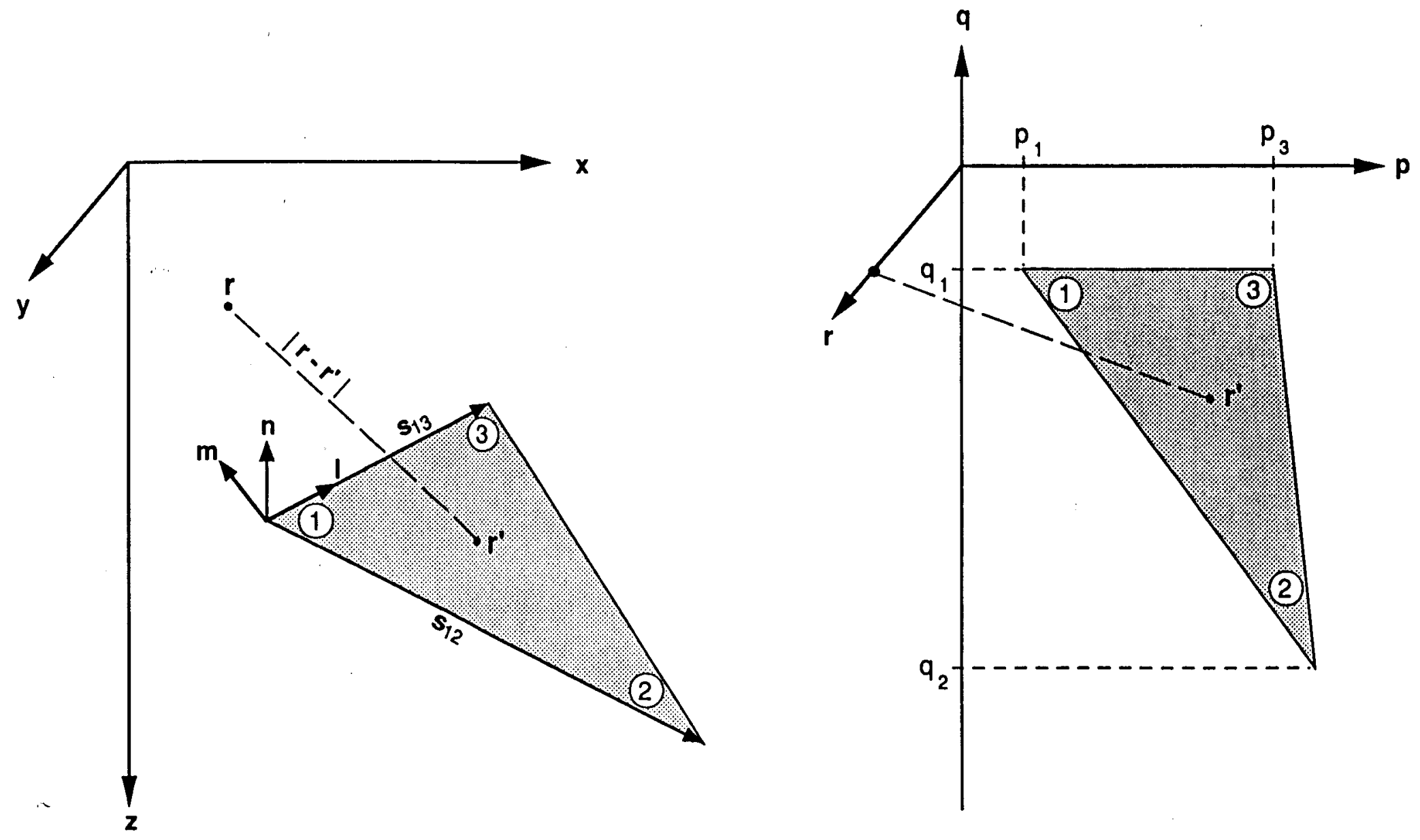

Figure 3-7: A graphical representation of the transform of the rectangular surface elements from the $x, y, z$ - to the $p, q, r$-coordinate system. 

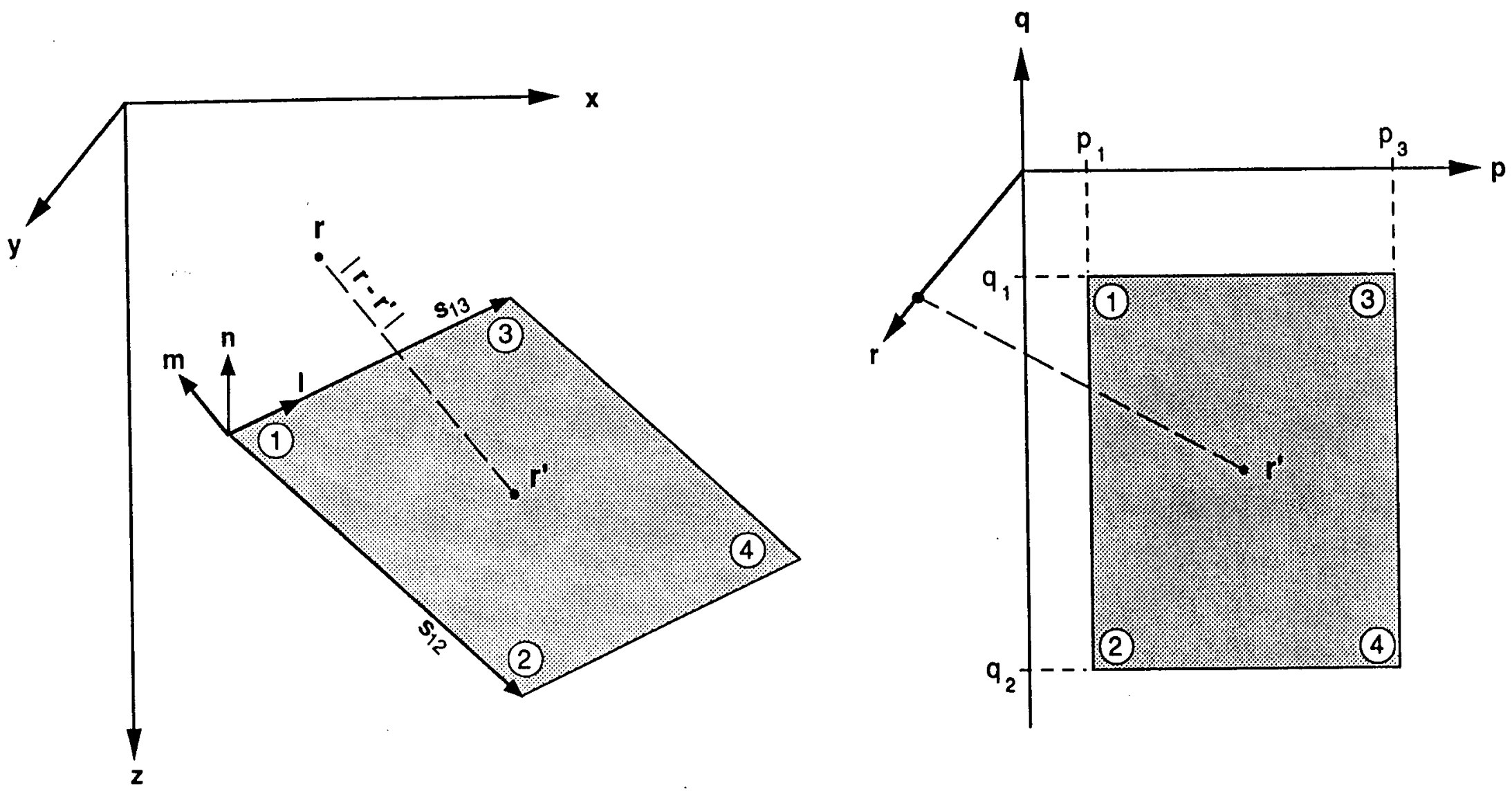

Figure 3-8: A graphical representation of the of the transform of the rectangular surface element from the $x, y, z$ - to the $p, q, r$-coordinates. 


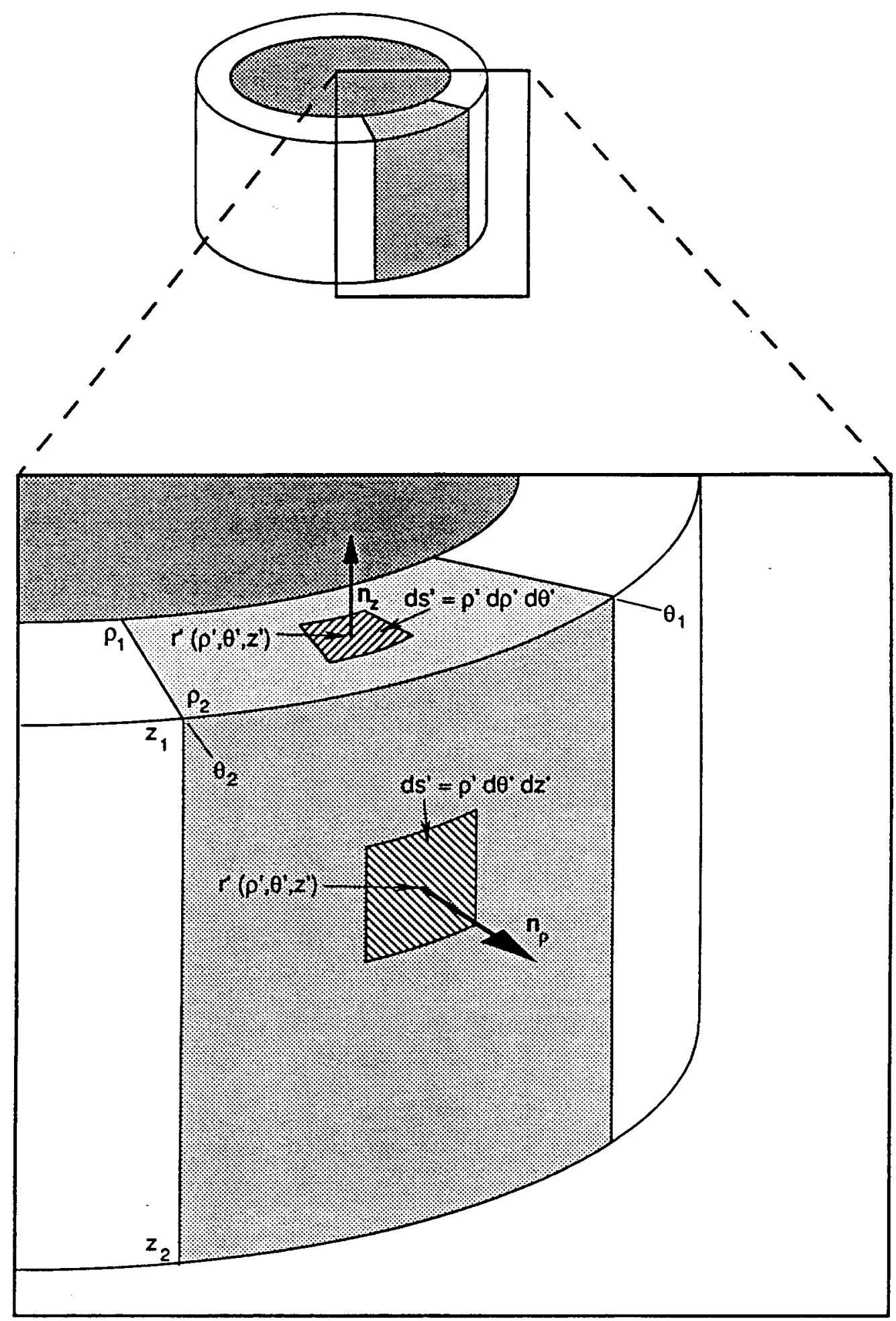

Figure 3-9: The surface elements used to approximate an arc sections of the pipe. For the 3-D cylindrical coordinate formulation, curvilinear-quadilateral and curvilinear-rectangular surface elements are used. The arrows represent the surface normals of the surface elements. 


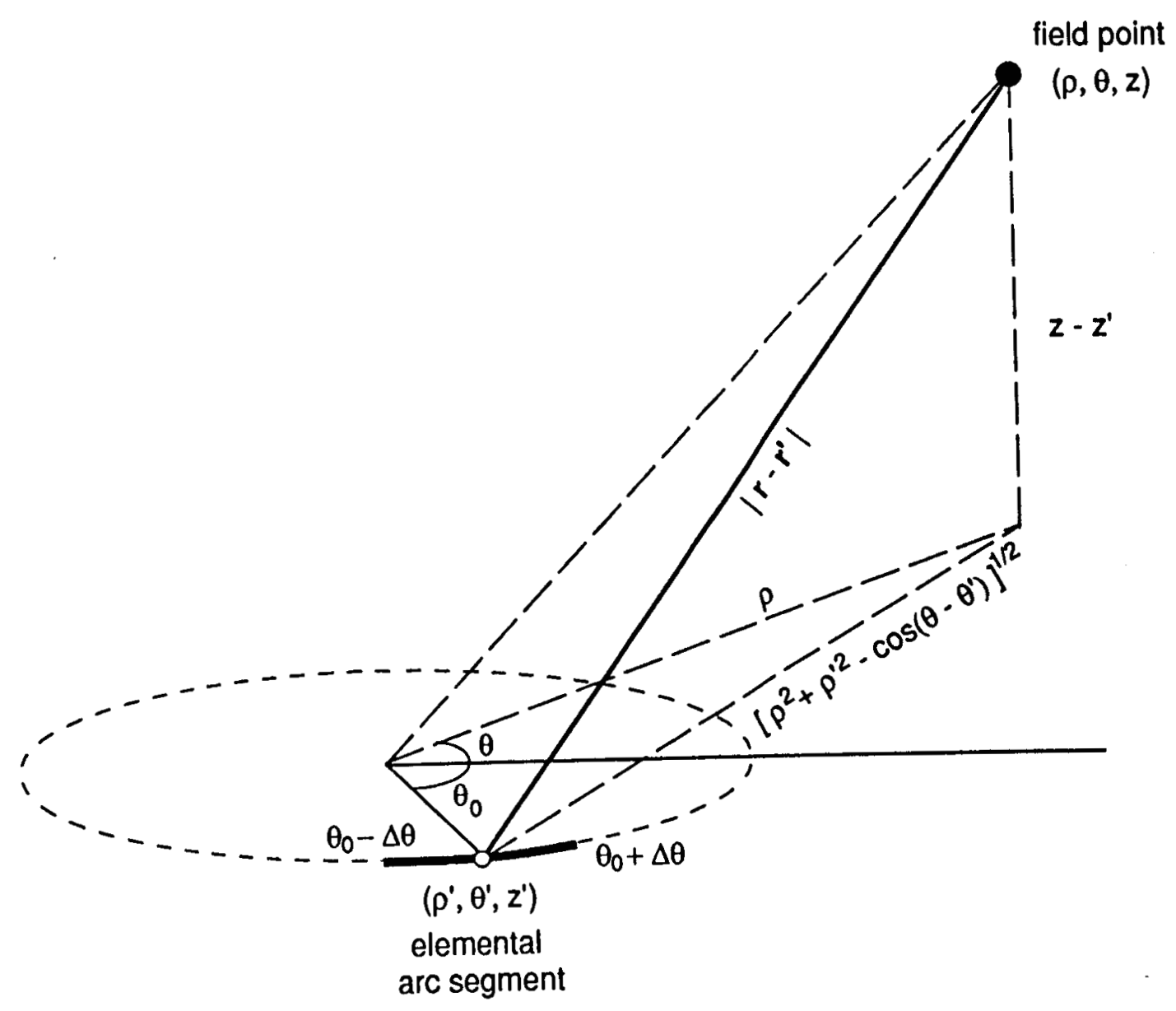

Figure 3-10: The generalized representation of the elemental arc-segment located at $r$ ', which is integrated about $\theta^{\prime}$, and field point placed at $r$ in the cylindrical coordinate system. 


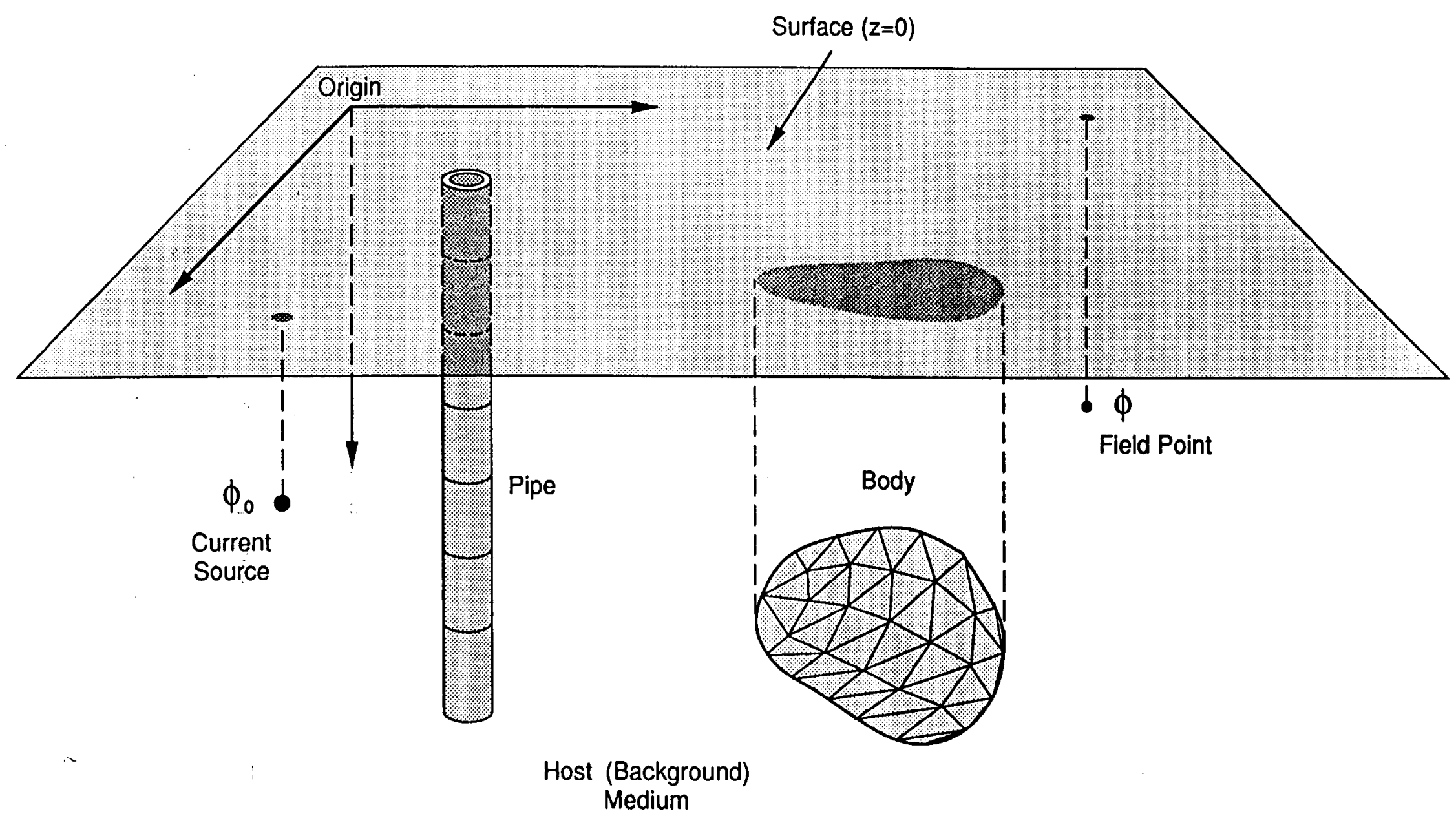

Figure 3-11: The generalized configuration of the 3-D problem with an arbitrarily located pipe, body, current source, and field point in a half-space. 


\section{Chapter IV}

\section{CONSISTENCY, ACCURACY, AND VALIDITY}

The theoretical formulation and programming of the numerical algorithms that solve the IE problem are checked by evaluating the consistency, accuracy, and validity of the results. Two numerical algorithms were developed to calculate the potentials for the axisymmetric problem based on the two IE approaches. The computer code founded on the volume IE uses the vertical E-field approximation and calculates the potentials in the half-space, whereas the routine based on the surface IE method is more general and can compute the potential anywhere in the medium.

The method of moments, used to solve both integral equations, approximates the integral over the body (region) by summing subsectional integrals located at discrete points. The shape of the body may also be approximated at these points with surface or volume elements. For each subsection, the unknown "continuous" function is assumed constant. A linear matrix equation for the unknown function is produced and then solved. By increasing the number of discrete points, a better approximation of the unknown function and shape of the body can be achieved. If there were an infinite number of points, an exact solution could be obtained.

Since the number of points is limited to the size and speed of the available computer, "truncation" errors will occur due to the approximation, and render the solution unreliable. In Section 4.1, the self-consistency and accuracy are investigated by evaluating the convergence and reciprocity properties of the numerical results. Since errors can also occur during the theoretical formulation and programming of the computer algorithm, the solution must be checked before it can be considered valid. In Section 4.2 , the validity of the method will be evaluated by the comparison of analytic and published solutions with the IE results. 


\subsection{Self-Consistency and Accuracy}

A valuable test for the self-consistency of the numerical method is the convergence of the solution as the number of model subsections increases. By decreasing the size of the segments, the IE must be satisfied at more points on the body resulting in a better approximation of the unknown function. If the solutions vary greatly for the different cell sizes, then the numerical technique is not consistent and may not be reliable. The convergence of the solution for an increasing number of segments indicates that the method is self-consistent which is necessary for reliability.

The convergence of the solution does not imply a reliable solution. Another check on the numerical technique is based on the theorem of reciprocity. Applying reciprocity to this problem, the same results should be obtained if the source and receiver are interchanged. Since reciprocity was not enforced in the development of the algorithm, this theorem can be used to test the accuracy of the numerical results.

Figure (4-1) illustrates the model used for the convergence and reciprocity tests. The vertical annulus or pipe with length $L=50 \mathrm{~m}$, inner radius $b=10.16 \mathrm{~cm}$, and thickness $t=1.27 \mathrm{~cm}$ is divided into $N$ equal length segments of height $\Delta L$. It is coaxial with a point current source (unit strength) which is located at the surface. The resistivities of the pipe and background medium are $\rho_{1}=10^{-6} \Omega \cdot \mathrm{m}$ and $\rho_{0}=10 \Omega \cdot \mathrm{m}$, respectively.

\section{Convergence Check}

The convergences of the potentials at three locations (top, middle, and bottom) on the surface of the pipe are evaluated for the surface IE approach and shown in Figure (4-2). The number of the equidimensional cells varied from 1 cell $(\Delta L=50 \mathrm{~m})$ to 500 cells $(\Delta L=0.1 \mathrm{~m})$. The total number of surface elements needed to describe the surface of the pipe is $2\left(N_{z}+N_{\rho}\right)$ where $N_{z}$ and $N_{\rho}$ are the number of elements in the vertical and radial directions, respectively. Each vertical cell requires two surface elements, and the horizontal segments need one surface element each. A convergent solution was obtained at about 200 cells $(\Delta L=0.25 \mathrm{~m})$, while a 
reasonable result could be obtained at 250 cells $(\Delta L=0.2 \mathrm{~m})$.

Figure (4-3) illustrates the convergences of the E-fields within the pipe where the E-fields are calculated with the volume IE method. With the use of volume elements, only $N$ segments are necessary to define the pipe. The number of cells ranged from 10 segments $(\Delta L=5 \mathrm{~m})$ to 500 segments $(\Delta L=0.05 \mathrm{~m})$. The figure illustrates that the convergence of $E$-fields is very rapid. A good approximation can be obtained with about 50 segments $(\Delta L=1.0 \mathrm{~m})$.

The convergence of the fields appear to be dependent upon the pipe dimensions and the model geometry, but was most influenced by the conductivity contrast between the pipe and host. A cell size of approximately two pipe radii was sufficient to give accurate results for the surface potential IE method. The E-field volume IE method converges much more rapidly and requires a cell size of 5-10 pipe radii to produce an accurate response.

\section{Reciprocity Check}

For the reciprocity check, two pole-pole array configurations were used (Figure 4-4). Since the source was placed off-axis from the pipe, the results were calculated using the axisymmetric approximation. The first pole-pole electrode combines a surface radial line and downhole array. The surface line (\#1-\#5) has the electrodes placed every $5 \mathrm{~m}$ from $25 \mathrm{~m}$ to $5 \mathrm{~m}$ from the pipe. However, the downhole array (\#6-\#11) has an interval of $10 \mathrm{~m}$ which starts at the top and terminates at the bottom of the pipe. The second array configuration is set off-axis from the pipe $5 \mathrm{~m}$ in the $y$-direction and extends from $-25 \mathrm{~m}(\# 1)$ to $+25 \mathrm{~m}(\# 11)$ on the $x$-axis with a $5 \mathrm{~m}$ interval. The tables in this section are tabulated such that each row represents the source position on the array and the columns are the receiver locations.

Table (4-1) presents the results using the combination array of the calculations for the surface IE method. The table of the potentials is nearly diagonally symmetric indicating that reciprocity for this problem is upheld. Most of the deviation between two reciprocal values was less than one percent. The largest $(\approx 1.5 \%)$ occurred for the case when either the source or receiver was at the end of the pipe. 
When the volume E-field IE approach was used, the calculated results (Table 4-2) were very similar. The largest deviation $(\approx 3 \%)$ occurred for the array with an electrode at the bottom of the pipe. Additionally, a 3\% deviation was observed for the reciprocal pair, electrodes of \#5 and \#6, (the top of the pipe and the first point away from the pipe). This deviation may be due to the vertical component approximation used for the E-field in the pipe. When the field point is adjacent to the source location, the majority of the field contribution is due to the radial component of the E-field which is neglected in the single component E-field approximation.

Table (4-3) represents the potentials calculated for the off-axis line. The comparison of the corresponding potentials of the two methods shows little difference. Both are diagonally symmetric and have nearly identical values for the potential at every source receiver combination. Although not shown, the deviations of all reciprocal pairs for both methods were less than one percent.

These results indicate that both methods uphold the reciprocity theorem and are accurate for nearly all array configurations. The largest deviations occurred for the situation where electrodes are placed at the ends of the pipe. The axisymmetric approximation is accurate if the off-axis source is not located too close $(>5 \mathrm{~m})$ to the pipe for downhole receivers. For the surface IE method, this source-pipe separation may be smaller. 


\subsection{Validity}

The validity of the axisymmetric formulation and computer program is checked by comparing the numerical results with the analytical and numerical solutions for three situations. The first check, which verifies the situation of radial boundaries, is the comparison of the potentials due to a conductive semi-infinite vertical annulus at the surface, $z=0$. The second test compares the apparent resistivities for a lateral log configuration in a layered medium to validate the numerical solution for the horizontal boundaries case. The third comparison is the calculated apparent resistivity for a normal $\log$ configuration in a medium involving both radial and horizontal boundaries.

\section{Radial Boundaries}

An expression for the potentials and E-fields can be obtained by modifying the solution for the infinite vertical annulus which can be found in Wait (1982). The method of images is used to produce the semi-infinite annulus solution. The solutions are in the form of cosine and sine transforms and can easily be coded into an algorithm. Because of the long length of the pipe, the limited number of surface elements, and the surface position of the source and receiver points, the length of the vertical surface elements increased with depth. This segmentation seems appropriate since the major interactions between the elements and source are greatest near the surface. For the semi-infinite annulus and numerical pipe models, a thickness of $1.27 \mathrm{~mm}$ and an inner radius of $10.16 \mathrm{~cm}$ were used. For the numerical model, the pipe was $10 \mathrm{~km}$ long. The resistivities were $10^{-6} \Omega \cdot \mathrm{m}$ and $1 \Omega \cdot \mathrm{m}$ for the pipe and the host medium, respectively. The coaxial current source was placed on the surface, $z=0$, and had a strength of 1 Ampere.

Figure (4-5) is the plot of potentials on the half-space surface for the analytical and 1400 elements numerical solutions. The numerical results differ $(\approx 40 \%)$ from the analytical solutions near the pipe and quickly converge to the analytical values for increasing radial distance. The differences are due to the lack of surface elements needed to approximate the highly conductive pipe. Because of the high conductivity contrast, very small surface elements are 
needed to obtain a good approximation of the potentials on the pipe. For a long pipe model, a large number of the small elements is required. This makes the numerical calculation intractable since a very large matrix must be inverted to obtain the potentials.

\section{Horizontal Boundaries}

The layered medium solution can be obtained from Van Nostrand and Cook (1966). For this case, the solution is in the form of a zero order Hankel transform. An algorithm for this problem, called "POTWELL", can be found in the U.C. Berkeley's Engineering Geoscience computer library. Figure (4-6a) is the three-layer model used for this comparison. The top and bottom layers have a resistivity of $10 \Omega \cdot \mathrm{m}$. The $100 \Omega \cdot \mathrm{m}$ target (middle) layer is $3 \mathrm{~m}$ thick located $49 \mathrm{~m}$ below the surface. For the numerical calculation, the infinite extent of the target layer was approximated by placing the outer boundary at $5.5 \mathrm{~km}$. A lateral $\log$ configuration was used where the current electrode $(A)$ is $1 \mathrm{~m}$ from the center $(O)$ of the two potential electrodes ( $M$ and $N$ ). The separation of the potential electrodes is $20 \mathrm{~cm}$. Like the semi-infinite annulus model, the target layer was segmented such that the lengths of the surface elements increased with radial distance away from the source.

Two types of numerical solutions were checked: a point solution and a (10cm radius) ring solution. The ring solution is used in situations where the source and/or potential electrodes are in contact with a cylindrical object, such as a pipe. Figure (4-6b) is a plot of the apparent resistivities for the analytical and the two numerical solutions. All three curves are essentially the same for this situation.

\section{Dual Boundaries}

A numerical technique that determines the potentials for the situation involving both the radial and horizontal boundaries is described by Gianzero and Anderson (1982). Their method obtains the solution for the potentials by iteratively solving a system of singular integral equations. This system is the result of suitably matching the boundary conditions of the problem. Gianzero and Anderson illustrated their work by applying it to several logging configurations. 
One such case is shown in Figure (4-7a) which simulates a 16 inch $(0.4064 \mathrm{~m})$ normal log in a two layer medium with an 8 inch $(0.2032 \mathrm{~m})$ diameter mud-filled borehole. The mud resistivity in the borehole, assumed infinite in extent, is $1 \Omega \cdot \mathrm{m}$. The top and bottom layers, also assumed infinite in the radial and vertical directions, have resistivities of $10 \Omega \cdot \mathrm{m}$ and $100 \Omega \cdot \mathrm{m}$, respectively. The model used for the surface IE technique described in Chapter 3 has a $100 \Omega \cdot \mathrm{m}$ layer with a radius of $5 \mathrm{~km}$, placed $5 \mathrm{~km}$ below the surface in a $10 \Omega \cdot \mathrm{m}$ host medium, and a $10 \mathrm{~km}$ long cylinder with a resistivity of $1 \Omega \cdot \mathrm{m}$. The large dimensions are used to approximate the infinite extent of the layers and borehole.

Figure (4-7b) illustrates a comparison between the computed response of the Gianzero and Anderson (GA) method, the measured response obtained by a resistor network model, ${ }^{1}$ and the calculated values of the surface integral equation (SIE) method. The results of the SIE method are nearly equivalent to that of the GA method. The comparison to the network method shows minor differences but still has excellent agreement.

\section{Axisymmetric Approximation}

In addition to these checks, the validity of the axisymmetric approximation for both forms of the IE methods is tested by comparing the potentials calculated on a radial array with the 3D formulation for a vertical finite-length pipe. The two types of cell segmentation used to represent the pipe are shown in Figure (4-8). The first is the 3-D Cartesian coordinate approximation where the surfaces are substituted by rectangular and triangular segments. The other applies the axisymmetric approximation and uses the circular ring cells.

The dimensions of the pipe are $L=25 \mathrm{~m}, t=10.16 \mathrm{~cm}$, and $b=1.27 \mathrm{~cm}$. The resistivities are $\rho_{0}=10 \Omega \cdot \mathrm{m}$ and $\rho_{1}=10^{-6} \Omega \cdot \mathrm{m}$. The potential electrode array starts at $2 \mathrm{~m}$ away from the pipe and has an interval of $2 \mathrm{~m}$. Figure (4-9) shows the potentials on the radial array for two current electrode positions: within the pipe and $4 \mathrm{~m}$ orthogonal to the electrode array.

1 The resistor network values are taken from the Gianzero and Anderson paper which references the study of Segesman (1962). 
The plots indicate that the axisymmetric approximation for the off-axis source will give a good result. The volume IE approach shows some discrepancy for the near-pipe electrodes. However, the differences diminished when the number of volume cells was increased.

\subsection{Summary}

The tests applied in this section indicate that the IE methods will give correct results. The discrepancies encountered are due to the numerical approximation caused by the discretization and can be reduced by decreasing the segmentation size. The axisymmetric approximation will give an accurate solution provided that the pipe is discretized with small enough segments compared to the given field and source positions. 


\begin{tabular}{|c|c|c|c|c|c|c|c|c|c|c|c|}
\hline \multicolumn{12}{|c|}{ SIE Potentials (mV) } \\
\hline Sources & 1 & 2 & 3 & 4 & 5 & $\begin{array}{c}\text { seivers } \\
6\end{array}$ & 7 & 8 & 9 & 10 & 11 \\
\hline 1 & - & 317 & 157 & 103 & 75 & 46 & 46 & 45 & 45 & 45 & 41 \\
\hline 2 & 317 & $\cdots$ & 316 & 155 & 99 & 52 & 52 & 52 & 51 & 51 & 46 \\
\hline 3 & 157 & 316 & $\cdots$ & 312 & 148 & 61 & 60 & 60 & 59 & 59 & 53 \\
\hline 4 & 103 & 155 & 312 & - & 299 & 74 & 73 & 72 & 71 & 70 & 62 \\
\hline 5 & 75 & 99 & 148 & 299 & -- & 96 & 094 & 92 & 91 & 91 & 78 \\
\hline 6 & 45 & 52 & 60 & 73 & 96 & --- & 199 & 199 & 198 & 197 & 164 \\
\hline 7 & 45 & 52 & 60 & 72 & 93 & 199 & -- & 199 & 198 & 197 & 164 \\
\hline 8 & 45 & 51 & 60 & 71 & 92 & 199 & 199 & --- & 198 & 198 & 164 \\
\hline 9 & 45 & 51 & 59 & 71 & 91 & 198 & 198 & 198 & -- & 198 & 165 \\
\hline 10 & 45 & 51 & 59 & 71 & 91 & 197 & 198 & 198 & 198 & -.- & 166 \\
\hline 11 & 42 & 47 & 54 & 63 & 80 & 167 & 167 & 167 & 167 & 168 & -- \\
\hline
\end{tabular}

\begin{tabular}{|c|c|c|c|c|c|c|c|c|c|c|c|}
\hline \multirow{3}{*}{ Sources } & \multicolumn{6}{|c|}{ Reciprocal Pairs Percent Deviation } & \multicolumn{2}{|c|}{$\frac{2|s-r|}{(s+r)} \times 100 \%$} & \multirow[b]{3}{*}{9} & \multirow[b]{3}{*}{10} & \multirow[b]{3}{*}{11} \\
\hline & \multicolumn{8}{|c|}{ Receivers } & & & \\
\hline & 1 & 2 & 3 & 4 & 5 & 6 & 7 & 8 & & & \\
\hline 1 & ---- & & & & & & & & & & \\
\hline 2 & 0.00 & --- & & & & & & & & & \\
\hline 3 & 0.00 & 0.00 & ---. & & & & & & & & \\
\hline 4 & 0.01 & 0.01 & 0.00 & --- & & & & & & & \\
\hline 5 & 0.05 & 0.05 & 0.04 & 0.03 & ---- & & & & & & \\
\hline 6 & 0.54 & 0.67 & 0.83 & 0.96 & 0.07 & --- & & & & & \\
\hline 7 & 0.43 & 0.52 & 0.62 & 0.75 & 0.90 & 0.01 & -.. & & & & \\
\hline 8 & 0.18 & 0.19 & 0.18 & 0.14 & 0.12 & 0.02 & 0.02 & ---. & & & \\
\hline 9 & 0.09 & 0.14 & 0.20 & 0.26 & 0.24 & 0.06 & 0.05 & 0.04 & - & & \\
\hline 10 & 0.35 & 0.41 & 0.48 & 0.54 & 0.47 & 0.15 & 0.14 & 0.12 & 0.09 & --.- & \\
\hline 11 & 1.05 & 1.19 & 1.36 & 1.51 & 1.58 & 1.51 & 1.51 & 1.50 & 1.48 & 1.49 & $\cdots$ \\
\hline
\end{tabular}

Table 4-1: Potentials (top) calculated with the surface IE method for sources and receivers located on the positions numbered in Figure (4-4). Percent deviation (bottom) of the reciprocal pairs of the electrodes from the top table. Pipe model: $L=50 \mathrm{~m}, b=10.16 \mathrm{~cm}, t=1.27 \mathrm{~cm}$, and $N=502\left(N_{z}=250, N_{\rho}=1\right)$. Resistivity: $\rho_{1}=1.0^{-6} \Omega \cdot \mathrm{m}$ and $\rho_{0}=10 \Omega \cdot \mathrm{m}$. 


\begin{tabular}{|c|c|c|c|c|c|c|c|c|c|c|c|}
\hline \multicolumn{12}{|c|}{ VIE Potentials (mV) } \\
\hline Sources & 1 & 2 & 3 & 4 & 5 & $\begin{array}{c}\text { eceive } \\
6\end{array}$ & 7 & 8 & 9 & 10 & 11 \\
\hline 1 & $\ldots$ & 317 & 157 & 103 & 75 & 44 & 45 & 45 & 45 & 45 & 45 \\
\hline 2 & 317 & - & 316 & 155 & 99 & 50 & 51 & 51 & 51 & 51 & 51 \\
\hline 3 & 157 & 316 & $\ldots$ & 312 & 148 & 58 & 59 & 60 & 60 & 60 & 59 \\
\hline 4 & 103 & 155 & 312 & -- & 299 & 69 & 71 & 72 & 72 & 72 & 71 \\
\hline 5 & 75 & 99 & 148 & 299 & --- & 83 & 99 & 93 & 93 & 93 & 91 \\
\hline 6 & 45 & 51 & 59 & 69 & 86 & $\ldots$ & 212 & 211 & 210 & 210 & 204 \\
\hline 7 & 45 & 51 & 59 & 71 & 91 & 211 & --- & 211 & 210 & 210 & 205 \\
\hline 8 & 45 & 51 & 60 & 71 & 92 & 210 & 211 & $\cdots$ & 211 & 210 & 205 \\
\hline 9 & 45 & 51 & 60 & 72 & 93 & 210 & 210 & 211 & -- & 211 & 205 \\
\hline 10 & 45 & 52 & 60 & 72 & 93 & 210 & 210 & 211 & 211 & -.- & 206 \\
\hline 11 & 46 & 52 & 60 & 72 & 93 & 211 & 211 & 211 & 212 & 213 & -- \\
\hline
\end{tabular}

\begin{tabular}{|c|c|c|c|c|c|c|c|c|c|c|c|}
\hline \multirow{3}{*}{ Sources } & \multicolumn{6}{|c|}{ Reciprocal Pairs Percent Deviation } & \multicolumn{2}{|c|}{$\frac{2|s-r|}{(s+r)} \times 100 \%$} & & & \\
\hline & \multicolumn{11}{|c|}{ Receivers } \\
\hline & 1 & 2 & 3 & 4 & 5 & 6 & 7 & 8 & 9 & 10 & 11 \\
\hline 1 & --- & & & & & & & & & & \\
\hline 2 & 0.00 & - & & & & & & & & & \\
\hline 3 & 0.00 & 0.00 & -..- & & & & & & & & \\
\hline 4 & 0.01 & 0.01 & 0.00 & --- & & & & & & & \\
\hline 5 & 0.04 & 0.04 & 0.04 & 0.03 & $\ldots$ & & & & & & \\
\hline 6 & 0.25 & 0.28 & 0.25 & 0.04 & 2.84 & --- & & & & & \\
\hline 7 & 0.22 & 0.25 & 0.29 & 0.38 & 0.63 & 0.00 & -..- & & & & \\
\hline 8 & 0.10 & 0.11 & 0.13 & 0.15 & 0.24 & 0.02 & 0.01 & --.- & & & \\
\hline 9 & 0.05 & 0.07 & 0.09 & 0.09 & 0.01 & 0.04 & 0.04 & 0.03 & --- & & \\
\hline 10 & 0.21 & 0.24 & 0.27 & 0.28 & 0.16 & 0.10 & 0.10 & 0.08 & 0.06 & -.-- & \\
\hline 11 & 1.76 & 1.99 & 2.24 & 2.50 & 2.66 & 3.17 & 3.17 & 3.16 & 3.14 & 3.10 & ---- \\
\hline
\end{tabular}

Table 4-2: Potentials (top) calculated with the volume IE method for sources and receivers located on the positions numbered in Figure (4-4). Percent deviation (bottom) of the reciprocal pairs of the electrodes from the top table. Pipe model: $L=50 \mathrm{~m}, b=10.16 \mathrm{~cm}, t=1.27 \mathrm{~cm}$, and $N=250$. Resistivity: $\rho_{1}=1.0^{-6} \Omega \cdot \mathrm{m}$ and $\rho_{0}=10 \Omega \cdot \mathrm{m}$. 


\begin{tabular}{|c|c|c|c|c|c|c|c|c|c|c|c|}
\hline \multicolumn{12}{|c|}{ SIE Potentials (mV); $N=402(N z=200, N p=1)$} \\
\hline \multirow{2}{*}{ Sources } & \multicolumn{11}{|c|}{ Receivers } \\
\hline & 1 & 2 & 3 & 4 & 5 & 6 & 7 & 8 & 9 & 10 & 11 \\
\hline 1 & -- & 317 & 158 & 104 & 76 & 56 & 49 & 43 & 38 & 34 & 31 \\
\hline 2 & 317 & $\ldots$ & 316 & 156 & 101 & 73 & 58 & 50 & 43 & 38 & 34 \\
\hline 3 & 158 & 316 & -- & 313 & 151 & 96 & 72 & 59 & 50 & 43 & 38 \\
\hline 4 & 104 & 156 & 313 & -- & 306 & 142 & 94 & 72 & 59 & 50 & 43 \\
\hline 5 & 76 & 101 & 151 & 306 & -- & 290 & 138 & 94 & 72 & 58 & 49 \\
\hline 6 & 59 & 73 & 96 & 142 & 290 & $\ldots$ & 290 & 142 & 96 & 73 & 59 \\
\hline 7 & 49 & 58 & 72 & 94 & 138 & 290 & $\ldots$ & 306 & 151 & 101 & 76 \\
\hline 8 & 43 & 50 & 59 & 72 & 94 & 142 & 306 & --. & 313 & 156 & 104 \\
\hline 9 & 38 & 43 & 50 & 59 & 72 & 96 & 151 & 313 & --- & 316 & 158 \\
\hline 10 & 34 & 38 & 43 & 50 & 58 & 73 & 101 & 156 & 316 & -- & 317 \\
\hline 11 & 31 & 34 & 38 & 43 & 49 & 60 & 76 & 104 & 158 & 317 & --- \\
\hline
\end{tabular}

\begin{tabular}{|c|c|c|c|c|c|c|c|c|c|c|c|}
\hline \multicolumn{12}{|c|}{ VIE Potentials (mV); $N=200$} \\
\hline \multirow{2}{*}{ Sources } & \multicolumn{11}{|c|}{ Receivers } \\
\hline & 1 & 2 & 3 & 4 & 5 & 6 & 7 & 8 & 9 & 10 & 11 \\
\hline 1 & -.- & 317 & 158 & 104 & 76 & 59 & 49 & 43 & 38 & 34 & 31 \\
\hline 2 & 317 & -- & 316 & 156 & 101 & 73 & 58 & 50 & 43 & 38 & 34 \\
\hline 3 & 158 & 316 & -.. & 313 & 151 & 96 & 72 & 59 & 50 & 43 & 38 \\
\hline 4 & 104 & 156 & 313 & -.- & 306 & 142 & 94 & 72 & 59 & 50 & 43 \\
\hline 5 & 76 & 101 & 151 & 306 &.-- & 290 & 138 & 94 & 72 & 58 & 49 \\
\hline 6 & 59 & 73 & 96 & 142 & 290 & $\ldots$ & 290 & 142 & 96 & 73 & 59 \\
\hline 7 & 49 & 58 & 72 & 94 & 138 & 290 & --- & 306 & 151 & 101 & 76 \\
\hline 8 & 43 & 50 & 59 & 72 & 94 & 142 & 306 & --. & 313 & 156 & 104 \\
\hline 9 & 38 & 43 & 50 & 59 & 72 & 96 & 151 & 313 & ... & 316 & 158 \\
\hline 10 & 34 & 38 & 43 & 50 & 58 & 73 & 101 & 156 & 316 & $\cdots$ & 317 \\
\hline 11 & 31 & 34 & 38 & 43 & 49 & 59 & 76 & 104 & 158 & 317 & -.. \\
\hline
\end{tabular}

Table 4-3: Potentials calculated for the off-axis electrode array for sources and receivers located on the electrodes numbered in Figure (4-4). The top and bottom tables are calculated with the surface and volume IE methods, respectively. Pipe model: $L=50 \mathrm{~m}, b=10.16 \mathrm{~cm}$, and $t=1.27 \mathrm{~cm}$. Resistivity: $\rho_{1}=1.0^{-6} \Omega \cdot \mathrm{m}$ and $\rho_{0}=10 \Omega \cdot \mathrm{m}$. 


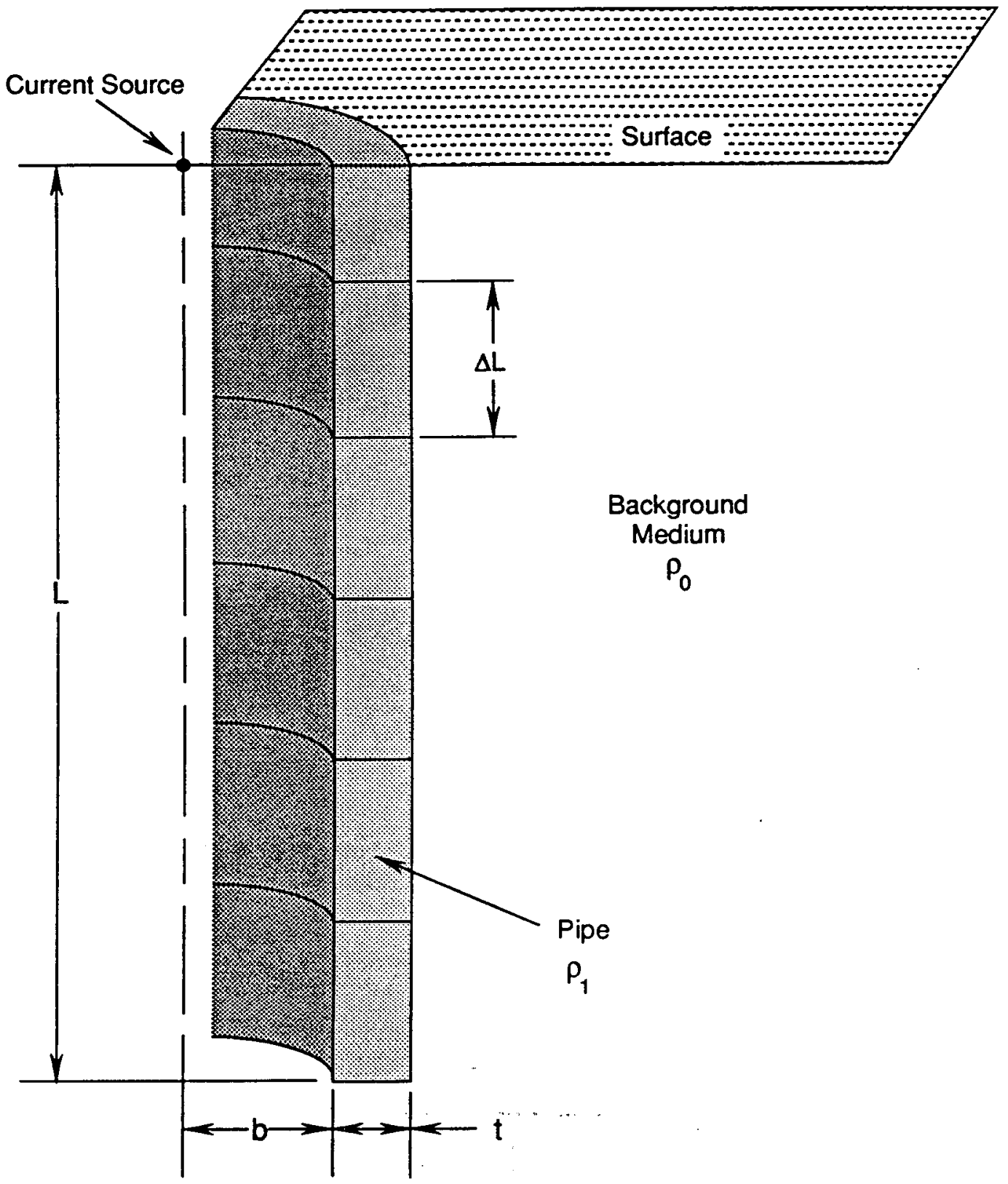

Figure 4-1: The generalized model of the pipe coaxial with a point source, in a half-space. 


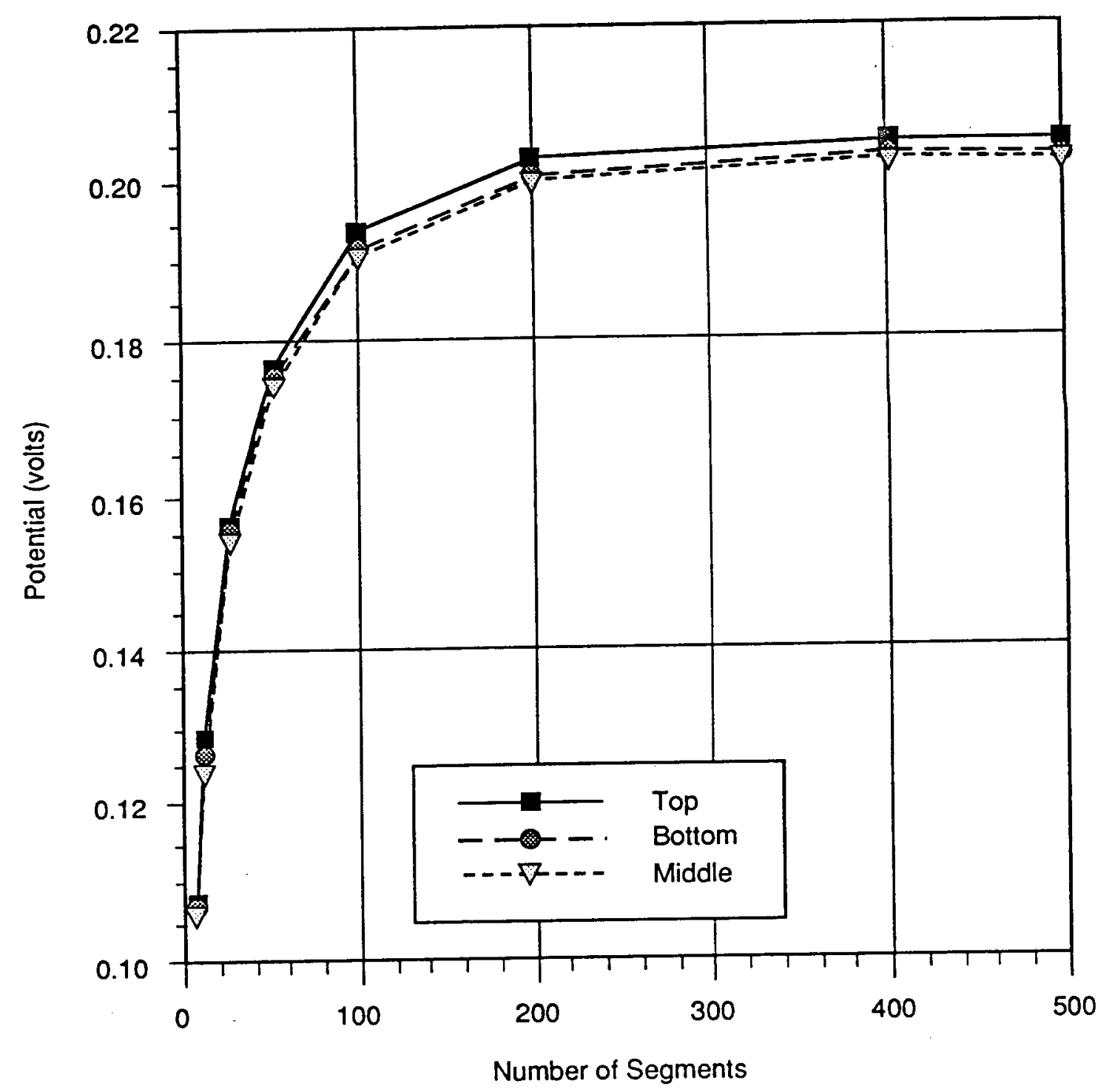

Figure 4-2: The plot of the potentials at three locations (top, middle, and bottom) on the pipe surface for increasing number of cells. The axisymmetric surface IE formulation calculated the potentials. 


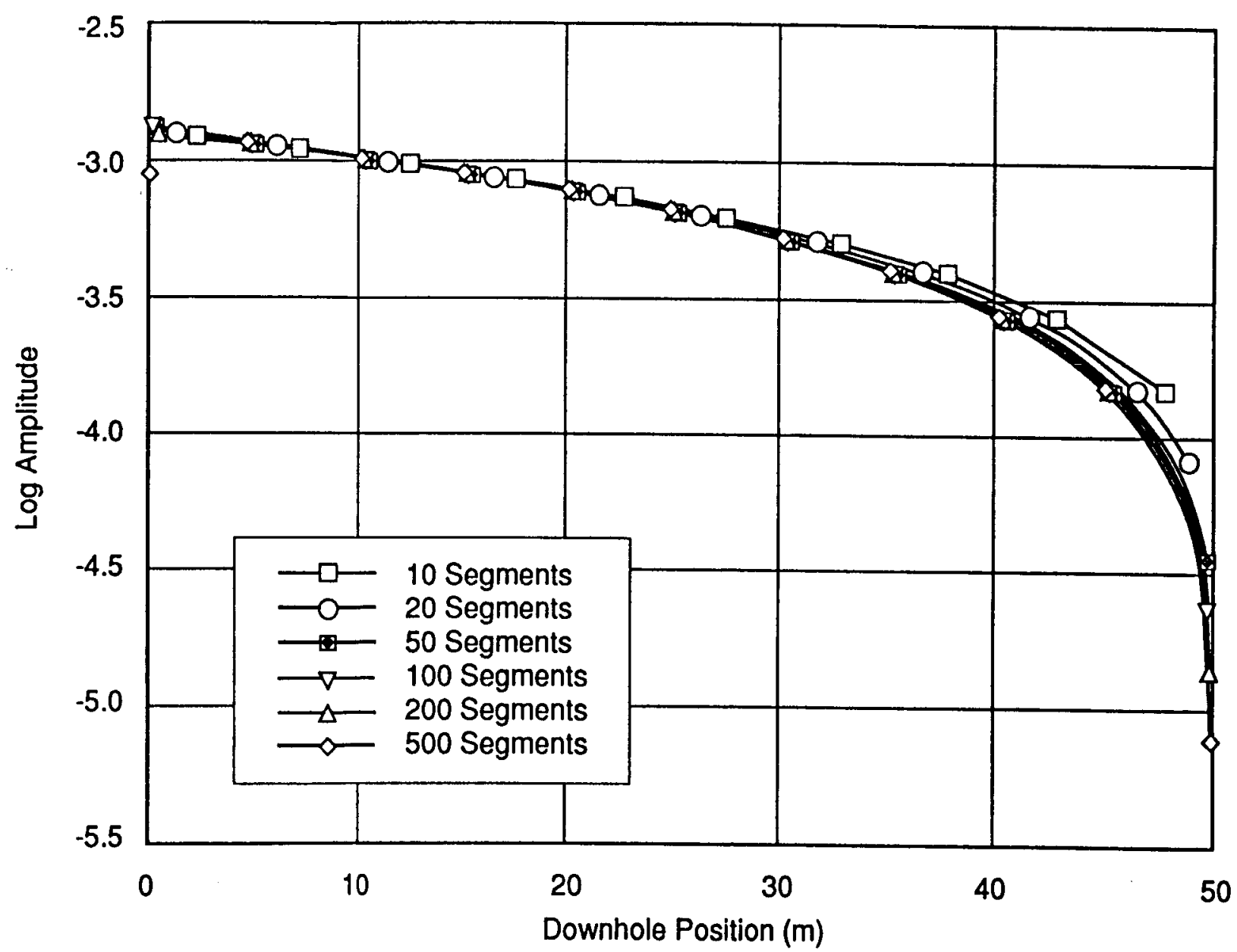

Figure 4-3: The semi-log plot of the vertical E-fields in the pipe for increasing numbers of volume segments. The axisymmetric volume IE method was used. 


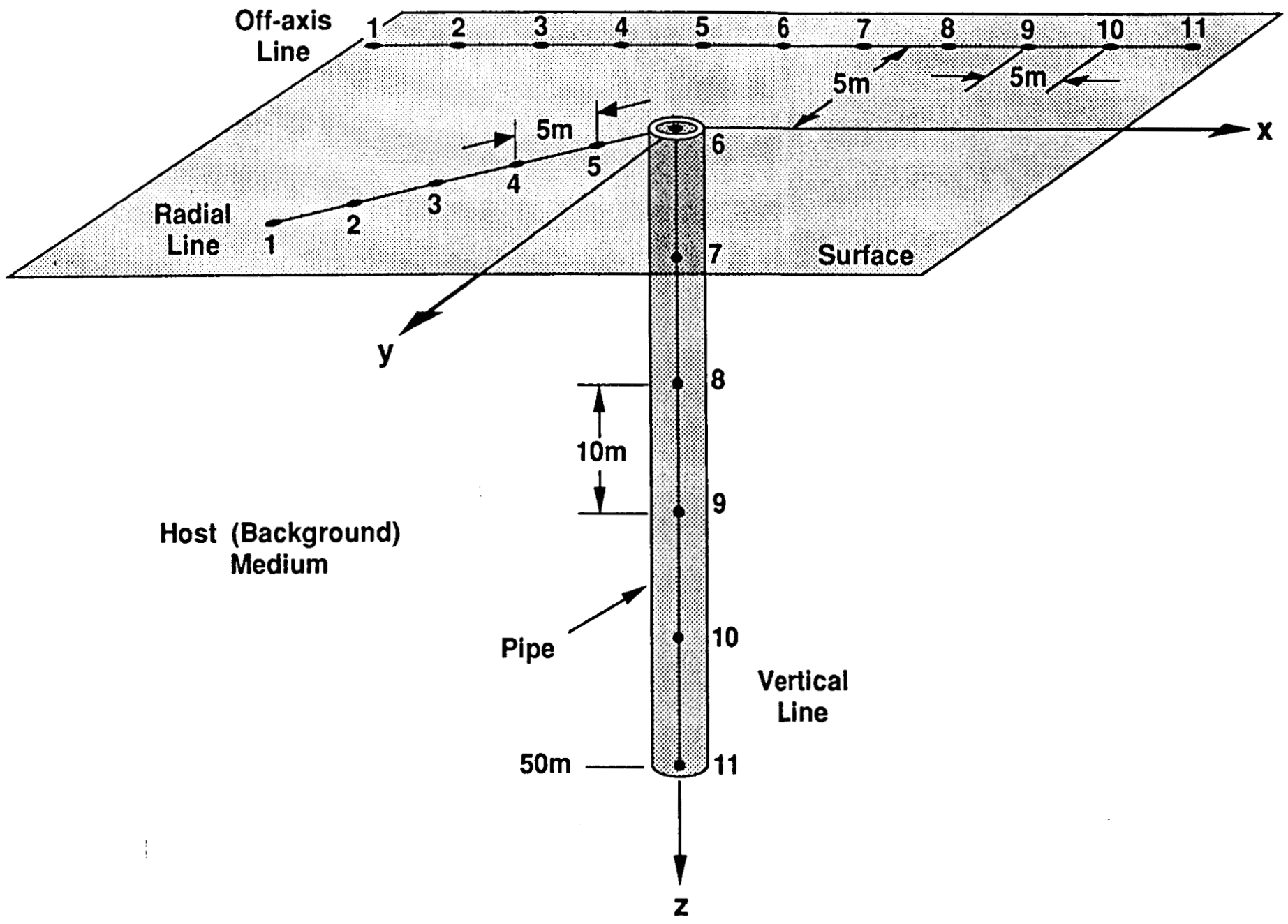

Figure 4-4: The array configurations used for the reciprocity check. One electrode array combines the radial surface and downhole lines and the other is an off-axis line. 


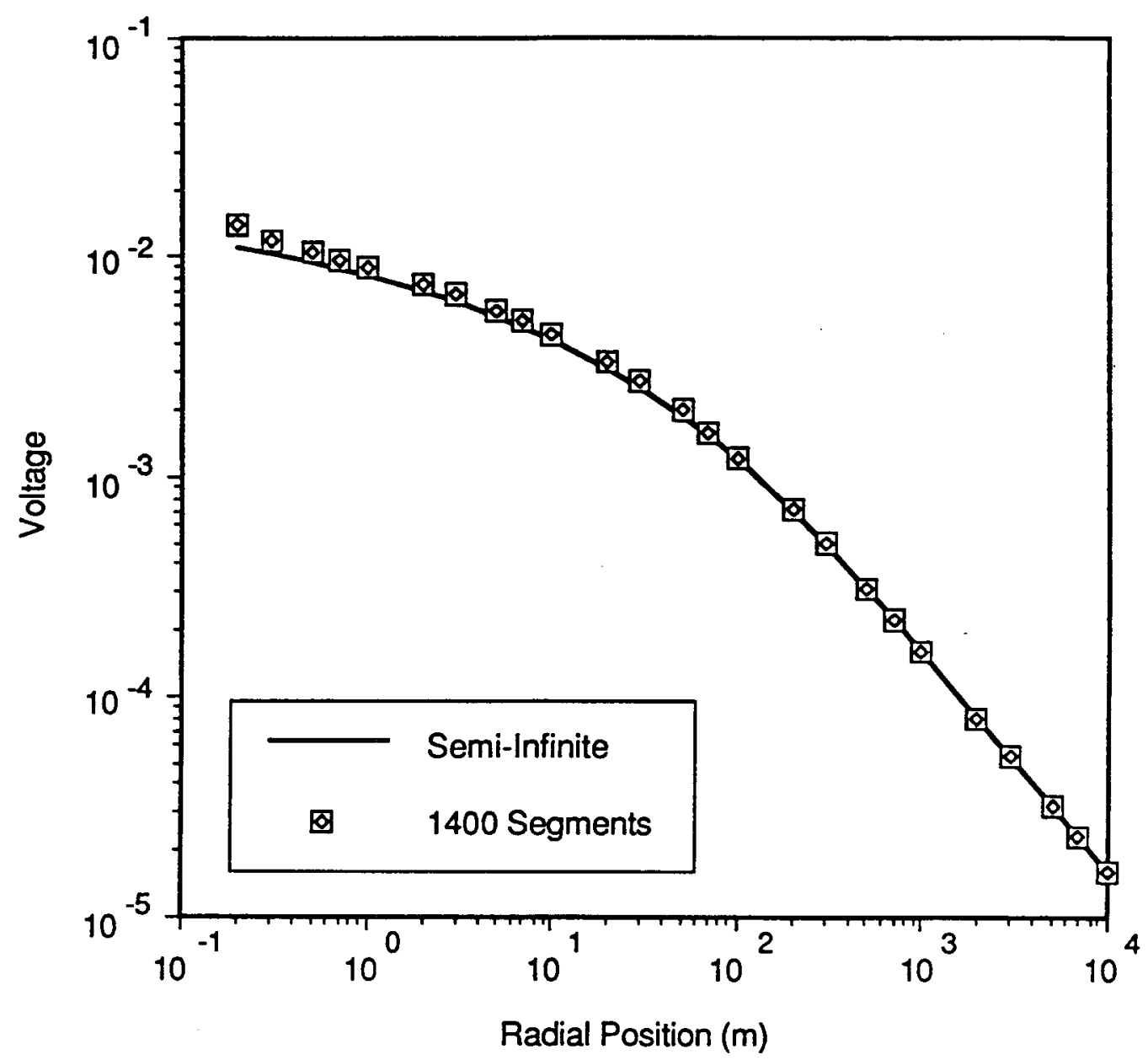

Pipe info:

Location: $0 \mathrm{~m}$

Length: $10 \mathrm{~km}$

Thk: $1.27 \mathrm{~mm}$

Inner R: $10.16 \mathrm{~cm}$

No. of Seg: 1400
Source Info:

Location: Om

Strength: $1 \mathrm{Amp}$

Radius: Om
Besistivity Inio:

Host: $1 \Omega \mathrm{m}$

Pipe: 1e-68m

Figure 4-5: A log plot of the potentials vs. the radial surface position for a vertical semiinfinite length annulus. The 1400 cell numerical model calculated the potentials using the surface IE method. 

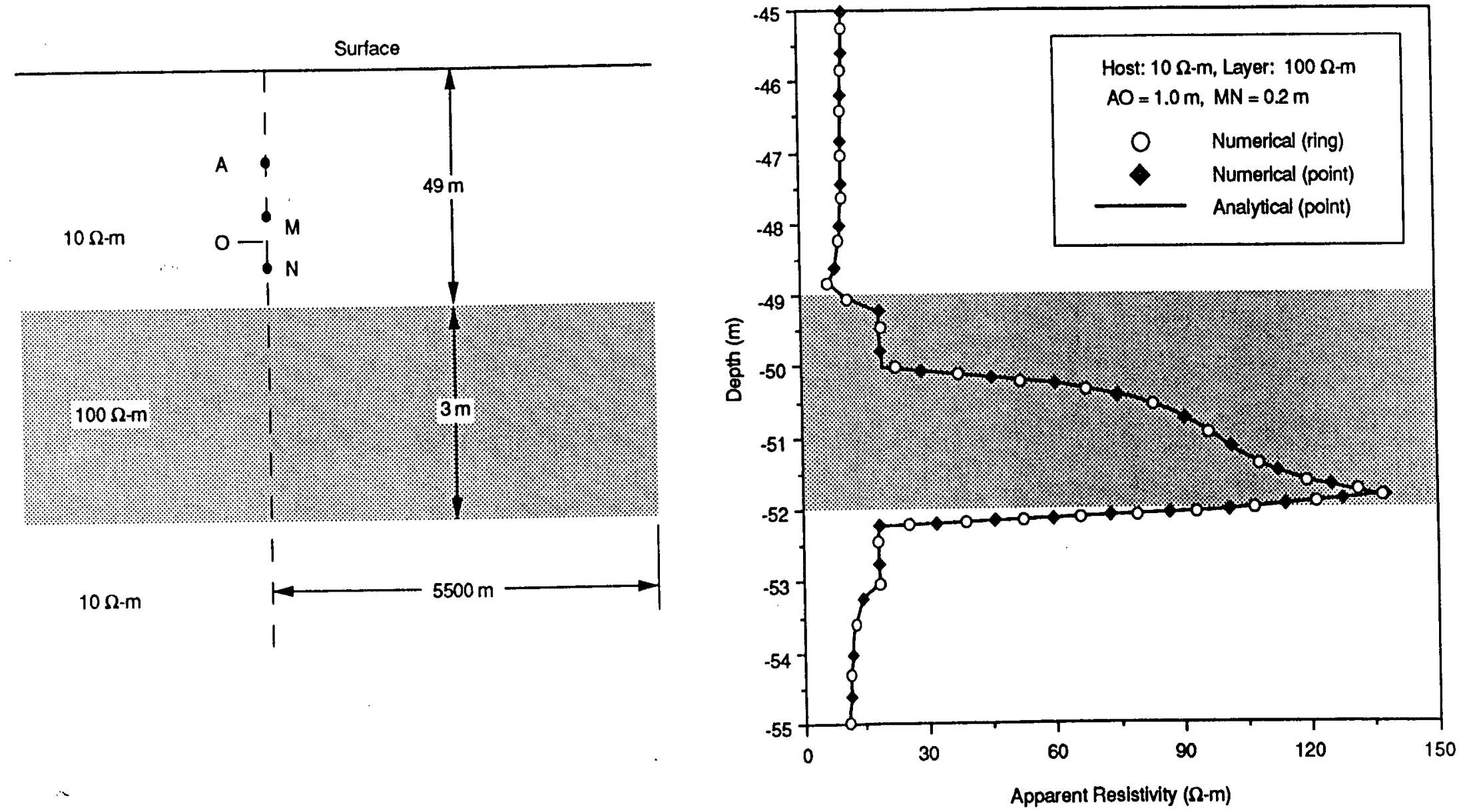

Figure 4-6: A three-layer model and lateral log configuration (left) used to calculate the apparent resistivities (right).

Two numerical results for a ring and point source are compared with the analytic values for a layered medium. 

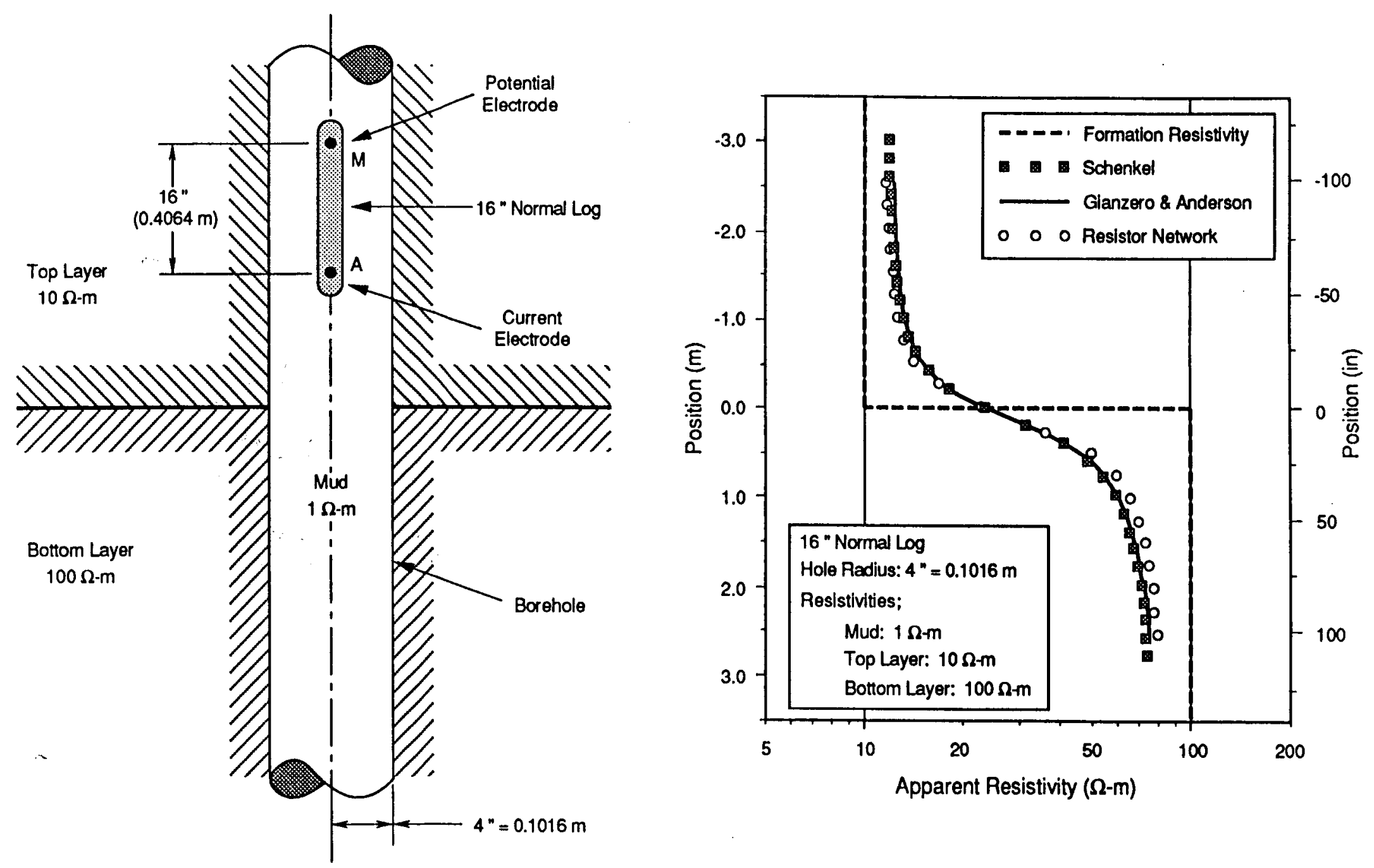

Figure 4-7: The model configuration (left) simulating a 16" normal log near a layer boundary with an 8 " diameter mud-filled borehole. The computed results of the apparent resistivities are compared with several other methods (right). 

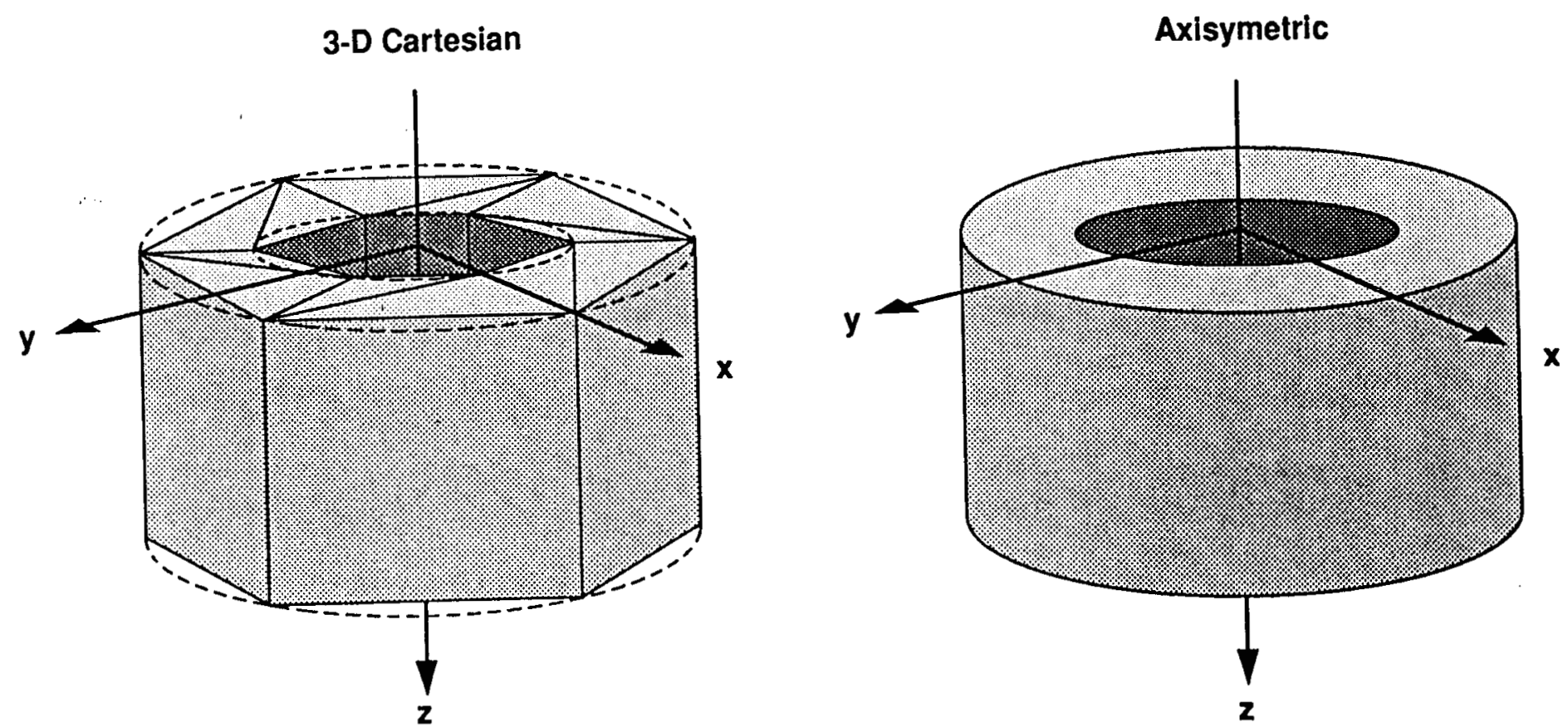

Figure 4-8: The discretization of a generalized circular ring cell using the 3-D Cartesian coordinate formulation (left) and the axisymmetric approximation (right). 

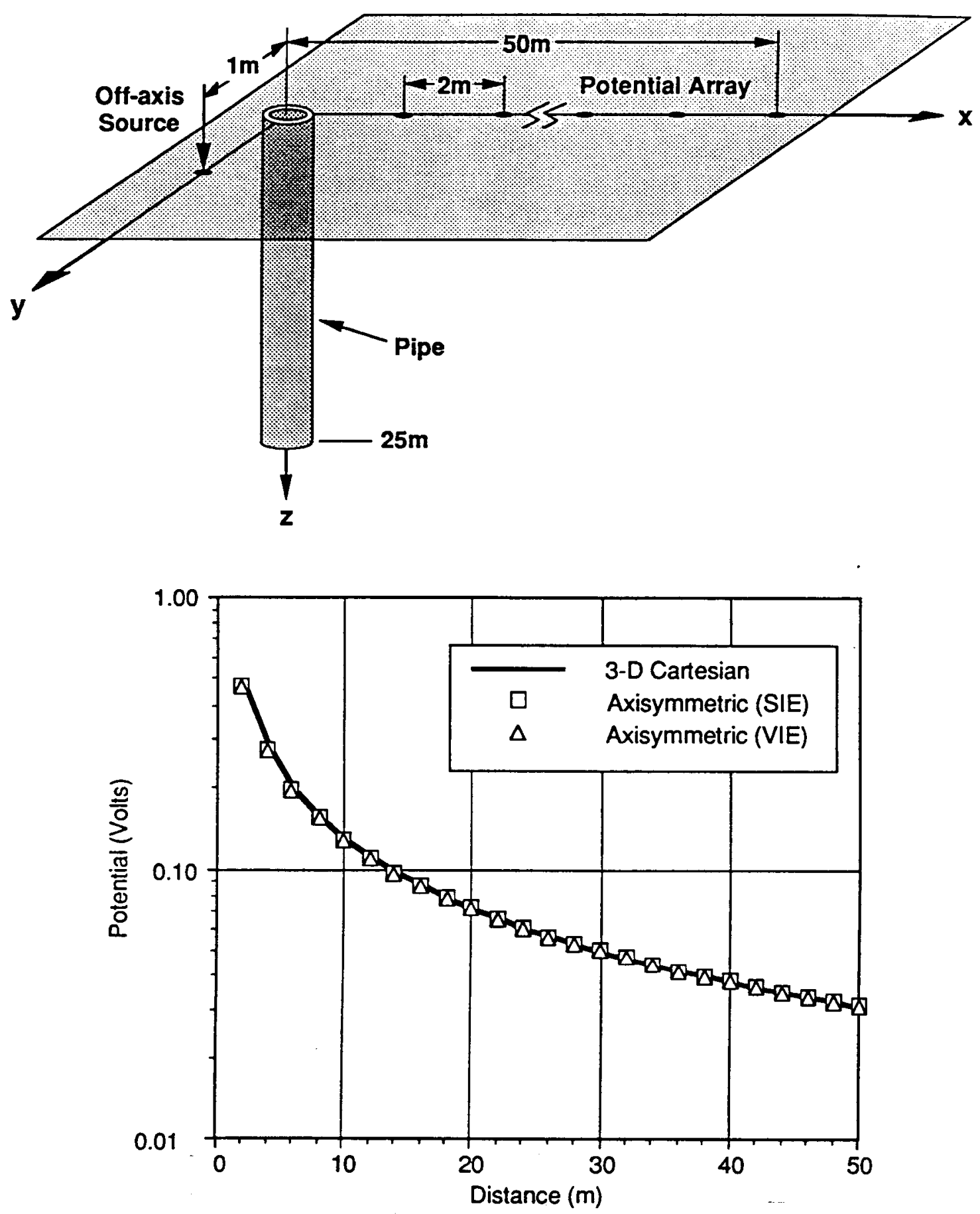

Figure 4-9: The source and potential array configuration (top) used for the semi-log plot of the potentials (bottom) calculated with the 3-D Cartesian coordinate, axisymmetric surface, and axisymmetric volume IE formulations. 


\section{Chapter V}

\section{NUMERICAL ANALYSIS OF CASING EFFECTS}

In Chapters II and III, the IE solutions were developed for a finite-length vertical annulus (pipe) in a conductive medium with arbitrarily located current sources. In Chapter IV, the computer algorithms, based on the surface and volume IE formulations, were examined and validated by performing convergence tests, reciprocity checks, and comparisons. The algorithm was proven accurate in situations involving boundaries that are horizontal, vertical, or a combination of both. In this chapter, the algorithms will be used to investigate several geophysical situations involving resistivity methods in steel-cased wells.

The validity of the line source approximation, used by Sill and Ward (1978), Kauahikaua et al. (1980), Sill (1983), and Rocroi and Koulikov (1985), is investigated. These authors considered the casing as a line source of current. The actual situation is simulated here by placing a current source in contact with the inner wall of the casing. By placing the current electrode beyond the end of the pipe, the casing effects and the spatial extent of the distortion are evaluated for downhole to surface and cross-hole measurements. The determination of the casing effects is important to correctly interpret field measurements acquired in the presence of the steel casing. The coupling effects between adjacent pipe segments separated by insulating segments are studied next. If the coupling is small, the separated casing segments can be used as downhole current and potential electrodes for DC tomography (Daily and Yorkey, 1988 and Shima and Saito, 1988). Further, there is the intriguing possibility that the separated segments, used as current electrodes, may be able to direct or focus currents (Jackson, 1981 and Parra et al., 1986). A dipole gap at the end of the pipe can simulate the DC limit for certain types of electromagnetic signal telemetry through the earth for measurement-while-drilling 
(MWD). One can also use the algorithm to model a MWD resistivity logging operation. Lastly, the algorithm is used to simulate several field situations which involve steel cased wells.

While examining these situations, the factors or parameters that influence the fields will be identified. The geometrical and electrical variables; position, thickness, diameter, and length of the pipe, as well as conductivities of the casing and background medium appear to be important factors that influence the pipe response. The effects of these variables are determined by examining their effects on the vertical electric fields $\left(E_{z}\right)$ in the pipe. During this analysis, several of the geometrical and electrical variables will be combined into characteristic parameters that describe the fields. 


\subsection{Source on or in the Casing}

As indicated in the introduction, several field studies have been conducted using an energized casing or pipe as a line source. For this situation, the casing or pipe is energized by placing a current source in contact with the casing. Kauahikaua et al. in their mise-a-la-masse experiment to map a geothermal reservoir noted that the current appeared not to extend to the lower sections of the casing. Rocroi and Koulikov, using two energized casings to delineate a hydrocarbon deposit, showed in their models that the apparent resistivities were dependent on the location of the current source within the casing.

The objectives here are to examine the use of the casing as a source, to study the effects of the casing parameters on the fields, and to determine the situations when the line source approximation is valid. The parameters that influence the fields are identified by using several configurations of the pipe and current source. A semi-infinite pipe configuration examines half-space boundary effects on the $E_{z}$ behavior. The whole-space and half-space problems are studied with a finite length pipe to determine the effect of boundaries at both ends of the pipe. The potentials calculated for a finite-length casing are compared to the potentials of a point source to investigate the region distorted by the casing and to a line source to evaluate the line source approximation for an energized casing.

\subsubsection{Semi-Infinite Length Pipe}

Consider a highly conductive semi-infinite length vertical annulus with thickness $t$, center radius $a$, and conductivity $\sigma_{c}$ in a homogeneous half-space with conductivity $\sigma_{0}$. A current source of strength $I$ is applied to the top of the pipe (Figure 5.1-1). An expression for the $E_{z}$ can be obtained for an elemental section of the pipe located sufficiently far from the source such that the radial E-field is negligible (see Figure (3-5) in Chapter III).

By using Ohm's law, the potential difference $\Delta \phi$ across the section can be expressed as:

$$
\Delta \phi=I_{c} R_{c}=\frac{I_{c} \Delta L}{S_{c}}
$$


where $\Delta L$ is the segment length, $I_{c}$ is the current within the segment, $S_{c}$ is the pipe conductance ${ }^{1}$ defined by Kaufman (1990) as:

$$
S_{c}=2 \pi a t \sigma_{c}=4 \pi a \Delta a \sigma_{c}
$$

and $\Delta a=t / 2$ is the half-thickness of the pipe. Multiplying equation (5.1-1) by $\Delta L^{-1}$ and letting $\Delta L$ shrink to zero about some point in the section, the $\mathrm{E}_{\mathbf{z}}$-field can be approximated:

$$
\lim _{\Delta L \rightarrow 0} \frac{\Delta \phi}{\Delta L}=\mathrm{E}_{z}=\frac{I_{c}}{S_{c}}
$$

The current in the elemental section $I_{c}$ is the portion of the applied current $I$ which has not dissipated into the surrounding formation. The amount of current leakage is dependent on the host medium conductivity, pipe conductance, and distance from the current source. Therefore, $E_{z}$ is proportional to the applied current source and inversely proportional to the pipe conductance, i.e., $\mathrm{E}_{z} \propto I / S_{c}$ and can be normalized by the factor $\left(I / S_{c}\right)$.

Kaufman (1990) indicated that for an infinite pipe the $E_{z}$ can be approximated by the transmission line equivalent (Sunde, 1968):

$$
\mathrm{E}_{z}=\frac{I}{2 S_{c}} \exp \left[-\frac{\Delta z}{\left(S_{c} / \sigma_{0}\right)^{1 / 2}}\right] \quad \text { for } \quad \Delta z \leq\left(S_{c} / \sigma_{0}\right)^{1 / 2}(5.1-4)
$$

where $\Delta z$ is the source-receiver separation distance and is defined, in terms of vertical position of the source $z$ os and field (receiver) $z$, as:

$$
\Delta z=\left|z-z_{0 s}\right|
$$

In equation (5.1-4), the factor of one-half accounts for equal distribution of current flow in both directions of the infinite pipe. For a semi-infinite pipe and a source placed on the half-space surface, the factor is unity instead of one-half since all the current in the pipe would flow downward due to the boundary. The term in the denominator of the exponent is defined as the "(effective) conduction length" of the (infinite) pipe $\delta_{L}$ and is given as:

1 The pipe conductance seems to be a misnomer since its units are [ $S \cdot m$ ] instead of [ $S$ ], the units of conductance. It is actually, although not mentioned in Kaufman's paper, the inverse of the unit length longitudinal resistance. Hence, the units of the pipe (longitudinal) conductance are $\left[(\Omega / m)^{-1}\right]=[S \cdot m]$. 


$$
\delta_{L}=\left(S_{c} / \sigma_{0}\right)^{1 / 2}=\left[\frac{4 \pi a \Delta a \sigma_{c}}{\sigma_{0}}\right]^{1 / 2}
$$

The conduction length of the pipe relates the pipe conductance with the conductivity of the background medium and is an indicator of the pipe's ability to carry current along its length. When the conduction length is short, the current in the pipe quickly leaks into the surrounding medium. A long conduction length indicates that the pipe conductance is large compared to the surrounding medium conductivity so that the current travels in the pipe with little leakage into the formation. The conduction length is similar in definition to the skin depth used in electromagnetics. At $\Delta z=\delta_{L}$, the field amplitude has dissipated to approximately $37 \%$ $\left(\mathrm{e}^{-1}\right)$ of the field at $\Delta z=0$.

Figure (5.1-2) shows the normalized $E_{z}$ variations for a semi-infinite pipe with the current source placed at the surface $\left(z_{0 s}=0\right)$. This plot shows that the decay of the field is dependent only upon the conduction lengths. All the field curves have decayed to approximately $e^{-1}$ of their original values at $\Delta z=\delta_{L}$. For an infinite annulus, all curves would converge to $1 / 2$ at $\Delta z=0$. The only difference between the two sets of plots is a factor of onehalf due to the half-space boundary.

The $E_{z}$ in the semi-infinite pipe can be characterized by a parameter $\beta_{s}$ that normalizes the conduction length by the source depth. It is given by the following:

$$
\beta_{S}=\frac{\delta_{L}}{z_{a s}}=\frac{\left(S_{c} / \sigma_{0}\right)^{1 / 2}}{z_{a s}}
$$

The vertical position is also normalized and defined as:

$$
z_{s}=\frac{\Delta z}{z_{0 s}}
$$

Figure (5.1-3) shows two plots of the normalized $E_{2}$ in a semi-infinite pipe for $\beta_{s}<1$ and $\beta_{S} \geq 1$. All curves to the right of the $z_{S}=1$ represent downgoing $E_{z}$ which is positive. To the left of $z_{s}=1$, the upgoing $E_{z}$ is negative. Since current densities are proportional to the $E$ field, these plots also represent the amount of current flowing in that portion of the pipe. 
These curves indicate that the half-space boundary and the $\beta_{S}$-ratios dictate the distribution of the fields or current flow in the pipe. The amplitudes of the upgoing and downgoing $E_{z}$ are dependent on the longitudinal resistance of the pipe for the sections above and below the source $\left(z_{s}=1\right)$. Since the half-space surface has infinite resistance, the $\beta_{s}$-ratio (conduction length to source depth ratio) will determine the amplitudes of the upgoing and downgoing fields.

For large $\beta_{s}$-ratios $\left(\beta_{s} \geq 2\right)$, most of the current is traveling downward due to the surface boundary effect on the upgoing fields. The upgoing curves which are impeded by the surface boundary resemble the fields for $\beta_{s}=1.75$ with a shift of the vertical axis. As the $\beta_{s}$-ratios decreases, the disparity between the upgoing and downgoing fields decreases and their amplitudes tend toward one-half at $z_{s}=1$. The half-space boundary has less effect so that more current is able to flow upwards and leak into the surrounding medium. For small $\beta_{s}$-ratios $\left(\beta_{s} \leq 0.3\right)$, there is very little difference between the amplitudes of the upgoing and downgoing fields at the source depth. The downgoing fields behave like those of the infinite-length pipe. However, the upgoing fields are still affected by the half-space boundary. The deviation from the infinite-length field curves is dependent upon the $\beta_{S}$-ratios.

\subsubsection{Finite-Length Pipe}

When the pipe is finite in length (see Figure 5.1-4), the behavior of the fields will be dependent upon the length of the pipe and the relative source position. The parameters that characterize the fields should reflect the finite length. This can be achieved by dividing the numerator and denominator in the exponential in equation $(5.1-4)$ by the length of the pipe (L). Thus, the approximation in equation (5.1-4) which describes the behavior of the $E_{z}$ will not be affected. The parameter called the conduction ratio, $\alpha_{L}$, is introduced. It is the ratio of the conduction length to pipe length and is given as:

$$
\alpha_{L}=\frac{\delta_{L}}{\left(z_{B}-z_{T}\right)}=\frac{\delta_{L}}{L}
$$

The normalized vertical position, $z_{L}$, is: 


$$
z_{L}=\frac{\Delta z}{\left(z_{B}-z_{T}\right)}=\frac{\Delta z}{L}
$$

Two length variables, $\Delta L_{T}$ and $\Delta L_{B}$, will be used to define the lengths from the source to the top and bottom of the pipe, respectively.

$$
\Delta L_{T}=\left|z_{\text {OS }}-z_{T}\right| \quad \text { and } \quad \Delta L_{B}=\left|z_{B}-z_{\text {OS }}\right|
$$

where $z_{T}$ and $z_{B}$ are the depth to the pipe top and bottom, respectively. Another parameter is introduced to account for the source position in the pipe relative to the pipe length. This parameter is called the relative source position, $S / L$-ratio, and is defined as:

$$
S / L=\frac{\left(z_{0 S}-z_{T}\right)}{\left(z_{B}-z_{T}\right)}=\frac{\Delta L_{T}}{L}=1-\frac{\Delta L_{B}}{L}
$$

Figure (5.1-5a) shows the normalized whole-space $E_{z}$ of several $\alpha_{L}$-ratios with the source located at the top of the pipe $(S / L=0)$. At first glance, these fields resemble the downgoing fields of the semi-infinite pipe. But when compared to the semi-infinite pipe curves, the whole-space fields fall off more rapidly due to the boundary conditions at the bottom of the pipe. The boundaries have the greatest effect on the fields when $\alpha_{L}>1.75$ ( $\delta_{L}>1.75 L$ ) so that the normalized $E_{z}$ in the pipe appears to have the same response.

Evaluating the effects of source position on the $E_{z}$ (Figures 5.1-5b to 5.1-5d) indicates that the behavior of the fields in the pipe is dictated by the longitudinal resistance of the upper and lower sections. The longitudinal resistance is the equivalent resistance of the pipe section observed at the source and is related to the inverse of the section length. Since the pipe is in the whole-space, the $E_{z}$-fields for $0.5 \geq S / L \geq 1$ are the mirror image of $0 \leq S / L \leq 0.5$. As the $\alpha_{L}$-ratio decreases, the boundary has less influence on the fields in the pipe. Hence, the differences between the upgoing and downgoing fields at the source decrease and the $E_{z}$ value approaches one-half.

The effects of the half-space boundary on the finite-length pipe is seen by comparing the normalized $E_{z}$ for $S / L=0.5$ of the whole-space (Figure 5.1-5d) and the half-space (Figure 5.1-6) cases. Due to the surface boundary condition $E_{z}=0$ at $z_{L}=0$, the upgoing half-space 
$E_{z}$-fields are smaller than the whole-space fields. The effect of the half-space is the greatest in the upper portion of the pipe. The downgoing half-space fields are similar to those of the whole-space since both have the same boundary condition. At the bottom end of the pipe, the boundary condition is that $\mathrm{E}_{z}=0$ due to the large conductivity contrast between the pipe and medium. The results indicate the the fields are influenced by the pipe depth. However, this effect is minor compared to the effects caused by the conduction ratio and relative source position.

\subsubsection{Potentials in the Medium}

The potentials $\phi$ in the half-space can be characterized by the same parameters defined in the previous section. Recall that the relationship of the conductivities between the background host medium and the pipe is incorporated in the conduction length. The potentials in the medium are presented as logarithmic-contour plots.

From the analysis of the current source within the pipe and $\alpha_{L}>1.75\left(\delta_{L}>1.75 L\right)$, it was found that the potentials were proportional to the current $I$ and inversely proportional to the background conductivity $\sigma_{0}$ and pipe length $L$. For a given conduction length, pipe depth, and source position, the normalized potential is given as:

$$
\phi_{L}=\phi\left[\frac{I}{4 \pi \sigma_{0} L}\right]^{-1}
$$

For current sources located within the pipe, the normalized field positions, $\rho_{L}$ and $z_{L}$, and conduction ratio $\alpha_{L}$ are used since the fields are independent of the pipe length. The radial $\rho_{L}$ and vertical $z_{L}$ components of the normalized field position are given as:

$$
\rho_{L}=\rho / L \quad \text { and } \quad z_{L}=z / L
$$

where $\rho$ and $z$ are the radial and vertical coordinates of the field point, respectively. The area of interest is within the bounds $z_{L}=(0,2)$ and $\rho_{L}=(0.01,2.01)$. The pipe is placed at the surface $\left(z_{T}=0\right)$ and several current source positions $S / L$ are used. 
Contour plots of the potentials calculated for a current source located within the pipe $(S / L<1)$ are presented in Figures (5.1-7) to (5.1-10). Each figure is composed of several plots which are the normalized total field for several $\alpha_{L}$-ratios and the normalized primary field. From these figures, there are certain cases $\left(\alpha_{\ell}<1.75\right)$ where the potentials appear similar to those produced for $\alpha_{L}>1.75$. For these situations, let:

$$
\alpha_{R}=1.75 \frac{\max [\Delta L]}{L}
$$

where $\Delta L_{T}$ and $\Delta L_{B}$ are defined by equation (5.1-11) and $\max [\Delta L]$ is the maximum length of $\Delta L_{T}$ and $\Delta L_{B}$.

For $\alpha_{L}>\alpha_{R}$, all the normalized potentials have nearly identical contour plots and are independent of the source position. The pipe alters the fields such that the potentials appear as fields produced by a line source showing no evidence of the primary source position. When $\alpha_{L}<\alpha_{R}$, the normalized potentials are dependent on the source position. The source position is evident from the curvature of the equipotential lines. The values of the total field approaches the primary field due to the reduced pipe effect. For very small $\alpha_{L}$-ratios $\left(\alpha_{L}<0.05\right)$, the currents in the pipe leak quickly into the surrounding medium. The pipe has almost no influence on the potentials, and thus the total and primary potentials are nearly the same.

The distortion of the potentials caused by the pipe can be analyzed by observing the behavior of the ratio between the total and primary fields:

$$
R_{\phi}=\frac{\phi}{\phi_{0}}
$$

For a non-distorted field, $R_{\phi}$ is unity. A $R_{\phi}$ greater than one indicates that the pipe effects add to the primary field, and a $R_{\phi}$ less than one implies that the pipe contribution reduces the field. Coincidentally, the $R_{\text {- }}$-ratio is also the ratio of the apparent resistivity to that of the background medium for a pole-pole survey.

The half-space primary field from a single current source is given as: 


$$
\phi_{0}=\frac{I}{4 \pi \sigma_{0}}\left[\frac{1}{\left|\mathbf{r}-\mathbf{r}_{0}\right|}+\frac{1}{\left|\mathbf{r}-\mathbf{r}_{0}^{\prime}\right|}\right]=\frac{I}{4 \pi \sigma_{0}} G
$$

where $\mathbf{r}, \mathbf{r}_{0}$, and $\mathbf{r}_{0}^{\prime}$ are the location of the field (measurement) point, current source, and source image, respectively. Solving for the array geometric factor $G$ yields:

$$
G=4 \pi \sigma_{0} \frac{\phi_{0}}{I}
$$

The apparent resistivity for a pole-pole survey is:

$$
\rho_{a}=4 \pi \frac{\phi}{I}\left[\frac{1}{\left|r-r_{0}\right|}+\frac{1}{\left|r-r_{0}^{\prime}\right|}\right]=4 \pi \frac{\phi}{I} G^{-1}
$$

where $\phi$ is the (total) potential measurement at the field position. Substituting for $G$ in equation (5.1-19) with equation (5.1-18) yields:

$$
\rho_{a}=\frac{1}{\sigma_{0}} \frac{\phi}{\phi_{0}} \quad \rightarrow \quad \frac{\rho_{a}}{\rho_{0}}=\frac{\phi}{\phi_{0}}=R_{\phi}
$$

where $\rho_{0}=1 / \sigma_{0}$.

Figures (5.1-11) is a series of contour plots for $\alpha_{L}=2$ and various source positions within the pipe. The model configuration is similar to that of the previous section. The shaded areas in the plots are the locations where the total field is within $5 \%$ of the primary field, i.e., the distortion of the potential due to the pipe is $5 \%$ or less.

When the $R_{\phi}$-ratios are calculated, the results indicate that the area affected by the pipe is dependent upon the source position. Large pipe effects are observed for situations where the current source is near the pipe ends $(S / L \approx 0.0$ and 1.0). The largest distortion occurs when the source is near the bottom of the pipe $(S / L>0.8)$. The configuration which shows the least overall distortion is where sources are located very near the pipe center $(S / L \approx 0.5)$. Surface and cross-hole measurements would be the least affected by the pipe. The distortion of greater than $5 \%$ occurs within $0.4 L$ on the surface and $0.6 L$ at the source depth. The source positions slightly below the pipe center $(S / L=0.65-0.70)$ appear to give optimum results for measurements below the pipe. The non-distorted area begins approximately $0.2-$ $0.4 L$ beyond the pipe end. 


\subsubsection{Line Source Approximation}

To evaluate the line source approximation, the ratio between the total potential and the potential due to a line source is calculated. A ratio near unity indicates that the energized casing behaviors like a line source. The potential produced by a finite-length equipotential conductor in a half-space is:

$\phi(\rho, z)=\frac{I}{4 \pi \sigma_{0}\left(z_{B}-z_{T}\right)} \ln \left[\frac{\left.\left[\left(z-z_{T}\right)^{2}+\rho^{2}\right]^{1 / 2}+\left(z-z_{T}\right)\right]}{\left.\left[\left(z-z_{B}\right)^{2}+\rho^{2}\right]^{1 / 2}+\left(z-z_{B}\right)\right]} \times \frac{\left.\left[\left(z+z_{B}\right)^{2}+\rho^{2}\right]^{1 / 2}+\left(z+z_{B}\right)\right]}{\left.\left[\left(z+z_{T}\right)^{2}+\rho^{2}\right]^{1 / 2}+\left(z+z_{T}\right)\right]}\right]$

where $z_{X}$ and $z_{B}$ are depths to the top and bottom of the casing, respectively.

Figures (5.1-12) to (5.1-16) are the contour plots of the potential ratio for several source positions. Each figure has four plots for different conduction ratios. The shaded areas represent the locations where the total field is within $1 \%$ of the potentials produced by a finite-length line source. The figures show that the line source approximation is dependent on the $\alpha_{L}$-ratio.

Except for the area near the surface and within one pipe length, a $1 \%$ or less deviation occurs for $\alpha_{L}>\alpha_{R}$. The largest deviations occurs when the source is placed at the ends of the pipe for $\alpha_{L} \leq 0.5$. When the source is placed near the middle of the pipe, the region of distortion is the smallest. A $1 \%$ or less deviation occurs for field points that are at least one-half pipe length away from the casing.

For a resistive host (large $\alpha_{L}$-ratio), the line source approximation of the casing is valid at all field positions. The deviation is less than $5 \%$ at all points within the medium for any source depth within the casing. The spatial distortion increases as the conduction ratio decreases. In a conductive medium, the line source approximation greatly distorts the potentials and is dependent on the source position within the casing. For $5 \%$ or less deviations, surface measurements cannot be less than one casing length away from the pipe when $\alpha_{L}=0.5$. Cross-hole surveys can be conducted as close as $1 / 2$ pipe length to the casing if the field points are at least $1 / 2$ casing length beneath the surface. 


\subsubsection{Summary}

The $E_{z}$-fields in the pipe are found to be proportional to the applied current source and inversely proportional to the pipe thickness, radius, and conductivity, i.e., the pipe conductance. Like $E_{z}$, the potentials are also proportional to the current source, but are inversely proportional to the background conductivity and pipe length.

In general, $\mathrm{E}_{\mathrm{z}}$ and potentials can be characterized by the following four parameters: the conduction length of the pipe $\left(\delta_{L}\right)$, position of the current source $(S)$, the length of the pipe $(L)$, and depth to the pipe $(T)$. All the parameters contain some geometric aspect of the problem, i.e., the positions, distances, and dimensions. Of the four parameters, only the conduction length of the pipe is based on the electric properties of the problem. It relates the pipe conductance with the background medium conductivity and is an indicator of the pipe's ability to carry current along its length. When the current source is located within the pipe, all parameters and spatial variables can be normalized by the pipe length reducing the number of characteristic parameters to three. They are the conduction ratio $\left(\alpha_{L}\right)$, relative source position $(S / L)$, and pipe depth to pipe length ratio $\left(z_{T} / L\right)$.

When the current source is within the pipe boundaries, the conduction ratio appears to be the most important parameter that characterizes the response of the pipe. The behavior of the $E_{z}$ is mainly affected by the conduction ratio and source location. The depth to the pipe has only a minor effect on the $E_{z}$ in the pipe. 


\subsection{Source Beyond Casing End}

In many instances, the wells are only steel cased in the upper portion of the borehole and open at depth. Bevc and Morrison (1990) conducting a monitoring experiment of a steam injection, and Schenkel and Morrison (1990b) in another field study (see Chapter 7) have reported anomalously high apparent resistivities at locations near the well. Both experiments, downhole to surface resistivity surveys, were conducted in wells that were partially cased with steel. The steel casing can strongly distort the response of the desired signals which may lead to erroneous interpretation of the field data (Holladay and West, 1984).

The main objective of this section is to evaluate the distortion caused by the casing on borehole to surface and cross-hole resistivity surveys for current sources placed beyond the end of the pipe. The areas where the potentials are greatly distorted must be determined and avoided. If it is not possible to avoid these regions, then the pipe influence must be determined to distinguish casing effects from the target response. The electric fields within the casing are studied, as are the potentials in the medium.

\subsection{1 $\mathrm{E}_{\mathrm{z}}$-Fields in the Casing}

For current sources located beyond the pipe end, the pipe-source separation $(\Delta s)$, is used to describe the current source location and is defined as:

$$
\Delta s=\left|z_{O S}-z_{B}\right|
$$

Figure (5.2-1) illustrates the $E_{z}$ for a constant pipe-source separation with the conduction length varying. The sharp fall-offs at the top and bottom of the pipe are caused by the boundary conditions at the ends of the pipe. The $E_{z}$ values near the bottom of the pipe are similar

for all conduction lengths. But along the pipe, the fields decay according to its conduction length.

Figure (5.2-2) are plots of the $E_{z}$ in the pipe for increasing separation distance from the pipe end These values correspond to $\alpha_{L}$-ratios of $2,0.5,0.2$, and 0.1 , respectively. using 
$600 \mathrm{~m}, 150 \mathrm{~m}, 60 \mathrm{~m}$, and $30 \mathrm{~m}$ for the conduction lengths. ${ }^{2}$ For these plots, the top of the pipe was located at the surface and had a length of 300 meters. From the figures, the fields asymptote to zero towards the surface and decrease with increasing pipe-source separation distance. For large conduction lengths, the fields become more symmetric and uniform near the pipe center as the separation increases.

When the source is located beyond the end of the pipe, an equivalent $S / L$-ratio will not produce the same $E_{z}$ for different pipe lengths. Figure (5.2-3) shows normalized $E_{z}$ values for different source separation distances calculated from a $30 \mathrm{~m}$ pipe which has a conduction length of $60 \mathrm{~m}$ ( $\alpha_{L}=2$ for this configuration). Comparing Figure (5.2-3b) to Figure (5.2-2a) $\left(\delta_{L}=600 \mathrm{~m}\right.$ or $\left.\alpha_{L}=2\right)$ shows that several of the curves match each other if the spatial variables are normalized by the pipe length. This indicates that a relationship exist between the $\mathrm{E}_{z}$ in the pipe and the pipe length, conduction length (conductivities), and current source position. ${ }^{3}$ But this relation is much more complex than the simplified approximation of equation (5.14). Hence, the pipe length must be included as a parameter for a current source beyond the pipe end.

\subsubsection{Potentials in the Medium}

The potentials $\phi$ in the half-space can be characterized by the same parameters defined in the previous section. For this analysis, the top of the finite-length pipe is located at the surface and several conduction lengths are used. The field position $(\rho, z)$ and pipe location $\left(z_{T}\right)$ cannot be normalized by the pipe length. The current source position, pipe length, and conduction length $\delta_{L}$ are needed to calculate the fields. A $300 \mathrm{~m}$ long pipe is placed at the surface $\left(z_{T}=0\right)$ and several conduction lengths are used. The area of interest is from $0 \mathrm{~m}$ to $600 \mathrm{~m}$ in the vertical direction and from $3 \mathrm{~m}$ to $603 \mathrm{~m}$ in the radial direction. The source depth, which

2 For sources beyond the pipe end, the $E_{z}$ in the pipe is dependent on the pipe length, so that the conduction ratio $\alpha_{L}$ cannot be used.

3 Although not shown, changing the variables of conduction length for both pipe lengths resulted in proportional variations of the $\mathrm{E}_{\mathrm{z}}$-fields. Hence, the E-fields are still dependent on the conduction length. 
also cannot be normalized, is described in terms of pipe-source separation $\Delta s$, given in equation (5.2-1).

Several source-pipe separations ranging from $0.1 \mathrm{~m}$ to $10 \mathrm{~m}$ are used to evaluate the distortion caused by the pipe (Figures $5.2-4$ to $5.2-8$ ). For a given separation distance, the calculation of the fields using $\delta_{L}>1.75 L$ produces the same normalized potentials. As can be expected, the area of the non-distorted field decreases as the conduction length decreases for all source locations. For large conduction lengths and current source slightly below the pipe end, the equipotential lines are still parallel to the pipe. However, the potential is not completely masked by the pipe effect since the equipotential lines enclose the current source. As the separation distance increases, the total field asymptotes to the primary field since the pipe effects become less pronounced. At approximately $10 \mathrm{~m}$ separation, the maximum amplitude of the two are equivalent and the pipe has a very small influence on the potentials except at field positions very near the pipe. With decreasing conduction lengths, the effects of the pipe are even further reduced. At $\delta_{L}=15 \mathrm{~m}$, the pipe becomes essentially transparent for $\Delta s \geq 2 \mathrm{~m}$ and the equipotential lines intersecting the pipe are nearly perpendicular to it.

As in Section (5.1), the distortion of the potentials caused by the pipe is evaluated by the ratio between the total and primary fields, $R_{\phi}$. Recall that $R_{\phi}$ is unity for a non-distorted field and is also the ratio of the apparent resistivity to that of the background medium for a polepole survey.

Figures (5.2-9) and (5.2-10) are logarithmic-contour plots of the normalized primary field for source positions beyond the end of the pipe. The model configuration for Figure (5.2-9) has the pipe located at the surface with a length of $300 \mathrm{~m}$ and a conduction length of $600 \mathrm{~m}$. For this situation, the pipe-source separation distance used in the field calculation ranged from $0.1 \mathrm{~m}$ to $20 \mathrm{~m}$. For the results of Figure (5.2-10), the pipe-source separation is fixed at $0.5 \mathrm{~m}$ and the conduction length varied from $60 \mathrm{~m}$ to $600 \mathrm{~m}$. The shaded areas in the plots are the locations where the total field is within $5 \%$ of the primary field, i.e., the distortion of the potential due to the pipe is $5 \%$ or less. 
Figure (5.2-9) indicates that the influence of the pipe decreases as the separation distance increases. For small separation distances, $R_{\phi}$ is similar to that produced by the source at the pipe end. Only areas far from the pipe have little distortion of the fields. As the source moves farther from the pipe, the distorted areas decrease in spatial extent and the amplitudes of the potentials approach the primary field values. At $15 \mathrm{~m}$ and greater, only the area adjacent the pipe shows large pipe effects. The area between the pipe and source shows minor changes due to the pipe. Little casing effects are seen in the areas below the source.

Figure (5.2-10) shows a decrease of the distortion as the conduction length decreases. The $5 \%$ or more distortion extends approximately two casing lengths beneath the casing and radially outward from the well at the surface. For decreasing conduction lengths, the two distorted areas near the surface and below the casing are significantly reduced. When compared with the $600 \mathrm{~m}$ conduction length, the extent of the distortion is reduced by approximately a factor of two for the $60 \mathrm{~m}$ conduction length.

\subsubsection{Summary}

Once the current source is beyond the end of the pipe, the $E_{z}$-fields and potentials are dependent on the pipe length and the characteristic parameters cannot be normalized by the pipe length. For this situation, the source position was conveniently defined in terms of the pipe-current source separation distance instead of the source depth. Both $E_{z}$-fields in the pipe and potentials in the medium are largely influenced by the pipe-current source separation distance, conduction length, and pipe length. The effect of the pipe decreases as the pipe-current source separation distance increases and as the conduction length becomes smaller.

A $10^{-6} \Omega \cdot \mathrm{m}$ casing with a $10 \mathrm{~cm}$ inner radius and $6 \mathrm{~m}$ thickness will have a conduction length of $623 \mathrm{~m}$ in a $100 \Omega \cdot \mathrm{m}$ half-space. The potentials in the region very near the pipe are substantially influenced by the pipe for a source 100 casing diameters below the end of the casing. For a field distortion 5\% or less, the surface measurements must not be closer than $1 / 2$ pipe length. In cross-hole resistivity surveys, the affected area is greatly reduced; surveys 
can be conducted as close as $1 / 6$ the casing length for with a distortion of $5 \%$ or less. In a conductive $1 \Omega \cdot \mathrm{m}$ half-space, the pipe conduction length is reduced to $62 \mathrm{~m}$. surface measurements can be as close as $1 / 4$ pipe length for $5 \%$ or less distortion of the fields, while the cross-hole survey can be conducted as close as 1/10 pipe length from the pipe. 


\subsection{Multiple Pipe Segments}

By separating several segments in a borehole (Figure 5.3-1), as was done by Bevc and Morrison (1990), multiple sources or receivers can be used to image the target in a downhole survey. Downhole current electrodes can be created by energizing the isolated segments. Likewise, the insulated segments could be used as potential electrodes. If additional wells are drilled with multiple segments, then cross-hole measurements can be made and processed using DC imaging techniques (Daily and Yorkey, 1988 and Shima and Saito, 1988). The imaging technique requires that the interaction between segments is such that the sources and receivers show point-like behavior. Thus, the main purpose of this section is to evaluate the coupling effects of the adjacent casing segments and determine when the point-like approximation may be used.

The interaction between separate coaxial pipe segments is investigated by using a long upper segment (fixed casing) and short lower segments (electrodes). Figure (5.3-2) illustrates the configuration of the two pipe segments with lengths, $L_{1}$ and $L_{2}$, separated by a distance $\Delta d$. The behavior of $E_{2}$ in the pipe segments and the potentials in the half space was investigated by varying the pipe separation distances $\Delta d$, segment lengths $L$, conduction lengths $\delta_{L}$, and current source positions within the active segment. ${ }^{4}$ The $E_{z}$-fields and potentials are normalized with the same factors used in the previous sections, $I / S_{c}$ and $I / 4 \pi \sigma_{0} L_{1}$, respectively. The parameters and spatial variables are not normalized, thereby eliminating confusion regarding which of the pipe lengths is used for the normalization.

For the following analysis, the pipe segments will have the same conductance with the upper segment, $C_{1}$, is fixed at the surface and of length $L_{1}=300 \mathrm{~m}$. Several lower segment lengths $L_{2}$, conduction lengths $\delta_{L}$, and separation distances $\Delta d$ are used to illustrate the distortion caused by the passive segment.

4 The term "active" is used to describe the pipe segment containing the current source. Whereas, "passive" refers to the source free casing segment. 


\subsubsection{E-fields in the Casing}

Figures (5.3-3) to (5.3-6) are the normalized $E_{z}$ in the passive $C_{1}$. In Figure (5.3-3), the $E_{z}$-fields are calculated for several conduction lengths for $L_{2}=5 \mathrm{~m}, \Delta d=5 \mathrm{~m}$, and the current source placed in the center of the lower segment. In Figure (5.3-4), the $E_{z}$-fields in $C_{1}$ are plotted when the source is placed at the top of the $5 \mathrm{~m}$ lower segment and several separation distances are used. The influence of different lengths for the lower segment, $2 \mathrm{~m}$ below the upper segment, is seen in Figure (5.3-5). The effects of current source position in the $5 \mathrm{~m}$ active segment, that is separated $2 \mathrm{~m}$ from the upper casing, are illustrated in Figure (5.3-6).

The $\mathrm{E}_{\mathrm{z}}$-fields in passive $C_{1}$ are similar to the fields produced by a current source below a single pipe. The fall-off of the fields is mainly dominated by the conduction length as seen in Figure (5.3-3). Figure (5.3-4) shows that the $\mathrm{E}_{z}$-fields in $C_{1}$ become smaller and more symmetric for longer separation distances. For a given separation distance and conduction length, the longer length of the active pipe segment results in smaller $\mathrm{E}_{\mathrm{z}}$ (Figure 5.3-5). Since the current is distributed along the length of $C_{2}$, the effect on $C_{1}$ is reduced for long $C_{2}$ segments. Figure (5.3-6) illustrates that the $\mathrm{E}_{z}$ in $C_{1}$ is independent of the current source position in $C_{2}$. Its effect can be approximated by a current source placed at a distance $\Delta d+L_{2} / 2$ from the end of $C_{1}$ in absence of $C_{2}$.

Since the length of the lower active segment is short, the $\mathrm{E}_{2}$ in the active segment $C_{2}$ resembles the field for a current source placed within the pipe that is below the half-space surface. Hence, the $E_{z}$ in the active segment is not shown. For a given conduction length, the fields of different active pipe lengths $L_{2}$ appear the same if the position and separation are normalized with the respective pipe length. The $E_{z}$-fields for all $\delta_{L}>1.75 L_{2}$ are the same for a known separation distance and pipe length.

\subsubsection{Potentials in the Medium}

Contour plots of the log-potential and the total-primary potential ratio are used to determine the region affected by the addition of the passive segment. If the distortion of the fields 
caused by the passive segment is small, the ratio of the potentials is close to unity. The greater the deviation from one, the larger the distortion. The potentials and distortion due to the lower segment are illustrated for various lengths of $C_{2}$ (Figure 5.3-7), separation distances, and conduction lengths (Figures 5.3-8 to 5.3-11).

In general, the behavior of the potentials is similar to that of the current source located beneath a single casing. For short separation distance and large conduction lengths, the equipotential lines are nearly parallel to the upper casing. As the active segment length increases, separation distance increases, or conduction length decreases the total potentials asymptote to the values of the primary fields. The extent of the affected area near the surface is reduced for longer $L_{2}$, whereas the distorted region near the lower segment shows a larger reduction for longer segment lengths. For short separation distances, there is a large distorted region near the surface adjacent to the upper casing and in the vicinity of the lower segment. When the separation distance is increased, there is a reduction of the distorted areas indicating that there is less interaction between the two pipes. For large conduction lengths, the potentials adjacent to the lower pipe appear similar to those produced by a line source although this is difficult to observe from the figures. The potentials along the upper segment are affected such that the equipotential lines are nearly parallel to the pipe. The distortion increases along the passive casing towards the surface and radially outward. The areal extent of the distortion is downwards along the lower segment and extents far below the active lower segment. As the conduction length decreases, the coupling between the pipes is small. Thus, the upper segment carries less current and the equipotential lines along $C_{1}$ merge into the the pipe and the distorted area is significantly reduced in the region.

\subsubsection{Additional Segments}

Next, a $5 \mathrm{~m}$ segment is placed below the long and short segments to evaluate the variations of the potentials due to the additional segment. This configurations can be represented by the upper three segments in Figure (5.3-1). Contour plots of the log-potentials and total to 
primary ratio for the two $5 \mathrm{~m}$ segments placed below the $300 \mathrm{~m}$ casing. For the analysis, a current source placed in either of the two $5 \mathrm{~m}$ segments.

Figures (5.3-12) to (5.3-15) are the contour plots with the source in the upper short segment using several conduction lengths and separation distances. Comparing these plots to the results of the single casing and the casing and short segment configurations show only slight differences of the potentials in the medium between the three situations. The distorted area is nearly the same as that for the single casing. This indicates that the lower passive segment has negligible effects on the fields.

Figures (5.3-16) to (5.3-19) are contour plots for the same situation except that the current source is located in the lower short segment. These figures show that the response is slightly different from the single casing situation. Since the center passive segment lies between the bottom active segment and the upper passive casing, the center segment acts as a conduit for the currents to flow into the upper casing. Thus, the spatial distortion is larger than that for the situation of the single casing.

\subsubsection{Summary}

The behavior of the fields is dependent on any factors that increase the effective conductance of the passive segment. Short separation distances, as well as long segments and conduction lengths appear to have the most influence on the behavior of the fields. The location of the current source within the active segment has no influence on the fields. Placing the source anywhere on the active segment is equivalent to a current source located at a distance of $\Delta d+L_{2} / 2$ below the upper casing. The length of the active pipe has a minor influence on the fields.

The interaction between the two pipe segments results in the reduction of potentials adjacent to the upper segment, with a corresponding increase in the fields below this segment. The separation distance between the two segments and the conductance length have the most influence on the potentials. When the separation distance is short, the interaction between the 
two pipes is great, distorting the region outward from the casing near the surface and downward below the segments. This effect is accentuated when the conduction length is large.

For the two segment configuration, the major contribution to the distortion is due to the long surface casing. For additional segments, the effects are small if they are located below the long surface casing and short active segment. If a segment is between the active segment and surface casing, its addition would effectively increase the conductance between the active segment and surface casing. This causes the spatial distortion of the potentials to be larger that that of the single casing or double segment configurations. 


\subsection{Dipole Current Source}

Consider a dipole current source located at the end of the casing where one pole is located in or near the casing end and the other below. This configuration may be used for downhole to surface or cross-hole surveys as will be shown in the next section. By attaching the polarized current source to insulated segments, measurement-while-drilling (MWD) operations can be simulated.

The fields from a grounded dipole source can be calculated by the superposition of positive and negative pole sources. Thus, the analyses from the previous sections can be applied to this situation. The $E_{z}$ in the casing and the potentials in the medium are examined to deter-

mine the effects of the conduction length, dipole gap ${ }^{5}$ length, and a lower segment on the fields. Throughout this section, the $E_{2}$-fields and the potentials are normalized by the factors $I / S_{c}$ and $I / 4 \pi \sigma_{0}$, respectively. Additionally, the potentials are plotted at logarithmic contour intervals of approximately one-half.

\subsubsection{Conduction Length}

Consider a fixed dipole, with a gap length $\Delta z_{0}=0.2 \mathrm{~m}$, located at the end of a $300 \mathrm{~m}$ casing (Figure 5-4.1). Several conduction lengths $(600,300,150$, and $60 \mathrm{~m})$ are used for the casing which extends to the surface. For this analysis, no lower segment is used.

Figure (5.4-2) shows that the $\mathrm{E}_{\mathbf{z}}$-fields in the casing for various conduction lengths are similar to those produced by a source at the end of the pipe. The figure indicates that the fall-off of $E_{2}$ is strongly dependent on the conduction length and is slightly greater than that of the single source due to the effect of the negative pole below the pipe.

For this situation, the primary potentials due to the dipole source are the same for all conduction lengths since only the medium conductivity is varied and this is incorporated into the normalization (Figure 5.4-3). Figure (5.4-4) shows the total potentials produced by the

5 The dipole gap length is the distance between the two current source poles. 
dipole source in the presence of the casing. For large conduction lengths, the behavior of the potentials is similar to that produced by a line and pole source of opposite polarity. In the vicinity of the casing, the zero potential line is shifted up towards the pipe. Away from the casing, the equipotential line asymptotes to the zero potential of the dipole field. As the conduction length decreases, the potentials become more symmetric about the source such that the equipotential lines merge into the adjacent casing.

The most intriguing aspect of the casing effect is that the amplitudes of the potentials are much larger than the fields of the primary potentials. This amplification effect is the largest near the casing and is at least two orders of magnitude. Away from the casing, the effect is reduced by a factor of 10 . For a fixed dipole gap length, this distortion is reduced for smaller conduction lengths.

\subsubsection{Dipole Gap Length}

The effects of the dipole gap length $\left(\Delta z_{0}\right)$ on the $E_{z}$-fields in the pipe is shown in Figure (5.4-5). The model used for this evaluation is a $300 \mathrm{~m}$ casing extending to the surface with a conduction length of $600 \mathrm{~m}$.

The behavior of the curves is similar to those of the $\mathrm{E}_{z}$-fields from the single source at the end of the casing. The single source configuration is the limiting case of the infinite gap length. The amplitudes of all the $E_{z}$ curves produced by a finite gap length will be less than this limiting case. The amplitudes of the $E_{z}$ are dependent on the gap length of the dipole. For a long gap length, the negative pole is situated far below the casing and has little influence on the fields in the casing. Hence, the shorter gap lengths will result in smaller $E_{z}$ fields within the casing.

Figure (5.4-6) illustrates the effects of the dipole gap length on the primary potentials in the medium. The plots show that the primary fields are dependent on the dipole gap length. The potentials away from the source are proportional to the dipole length $\Delta z_{0}$. The presence of the casing for the different gap lengths are illustrated in Figure (5.4-7). As with the pri- 
mary fields, the total potentials increase with increasing dipole length although it appears that this relationship is not linear. The potentials are similar to those described in the previous case for the conduction length effects. The total potentials appear to be caused by a line and pole source which are much larger than the primary fields of a dipole source.

\subsubsection{Lower Segment Length}

The effects of the length of an separated segment attached to the negative pole of the source is examined. Both the $300 \mathrm{~m}$ upper surface casing and lower separated segment with a length of $L_{2}$ have a conduction length of $600 \mathrm{~m}$ and are separated by a distance of $0.2 \mathrm{~m}$ (Figure 5.4-8). For this problem, the positive pole is placed at the bottom of the casing and the negative pole is located at the top of the segment.

The $E_{z}$-fields in the upper casing and lower segment are shown in Figure (5.4-9) for various lengths of the lower segment. The fields in the upper casing increase as the lower segment length increases. The asymptotic limit for these curves is the $\mathrm{E}_{\mathbf{z}}$-field produced by a pole. This behavior is due to the current distribution in the lower segment which reduces the influence of the pole in the segment on the upper casing. The response of the $E_{z}$-fields in the lower segment is similar to that in an active segment of the single source case. As with the other dipole configurations, the fall-off of the fields is slightly greater than the decay of the single pole in the upper casing.

All of the primary fields in Figure (5.4-10) are the same since the dipole gap $\left(\Delta z_{0}=0.2 \mathrm{~m}\right)$ and conduction length $\left(\delta_{L}=600 \mathrm{~m}\right)$ did not vary. Figure (5.4-11) is a series of contour plots of the total potentials for several lengths of the lower segment. The fields in the medium behave like those produced by two line sources of opposite polarity. The potentials near the casing and the segment increase as the lower segment length increases. Also, the potentials are more symmetric about the current source as the lower segment length increases. Again, the total potentials are much greater than the primary fields of the dipole. 


\subsubsection{Surface Response}

The potentials and the total-primary ratio are calculated on the half-space surface to illustrate the magnification of the potentials caused by the casing with the dipole source as opposed to the potentials from a dipole source with no casing. Several dipole gaps and lower segment lengths are used to illustrate the effect. A $300 \mathrm{~m}$ upper casing with a conductance of $3883 \mathrm{~S} \cdot \mathrm{m}$ is placed in a $100 \Omega \cdot \mathrm{m}$ half-space (this is equivalent to a conduction length of approximately two).

Figure (5.4-12) illustrates the effects of the dipole gap length on the potentials. The curves for different gap lengths are nearly parallel to each other. The fall-off of the potentials is greater than that of the single pole curve. As the gap length increases, the potentials increase and approach the single pole case. However, the total-primary ratio is reduced as the gap length increases. For surface positions near the casing, the total fields can be $2-3$ orders of magnitude greater than the primary field. In the far-field, the potentials are reduced by a factor of 5 to 10 .

The influence of the lower segment length on the potentials and the total-primary ratio is shown in Figure (5.4-13). For this problem, the casing separation (dipole gap) is $0.2 \mathrm{~m}$. From this figure, both the potentials and the total-primary ratio increase as the lower segment lengthens. The potentials are magnified by the presence of the upper casing and lower segment. Near the casing, the potentials are 300-3000 times larger than the primary fields. The distortion is reduced by an order of magnitude when the field point is approximately one casing length away from the well.

\subsubsection{Summary}

In the presence of the casing and/or a segment of casing below upper casing, the potentials in the medium are much larger than the fields from the dipole source alone. The fields are influenced by the dipole gap, conduction length, and separated segment length. The potentials in the half-space are increased when any of these factors is increased. Although the far- 
fields are greatly affected, the largest deviations of the potentials occurs near the casing and segment where they are approximately 100-1000 times greater than the primary fields. 


\subsection{Numerical Examples}

In this section, two examples are used to simulate field situations that may be encountered with steel cased wells. The first model simulates the monitoring of an injection experiment; the cross-hole and downhole to surface surveys are considered. The last example is directed to the low frequency limit of an electromagnetic telemetry scheme used in measurement while drilling (MWD).

\subsubsection{Injection Monitoring Simulations}

The principle behind the monitoring of an injection experiment can be illustrated in the simple cross-section in Figure (5.5-1). Consider electric current injected into the earth between a downhole electrode $\mathrm{A}$ and a remote surface electrode $\mathrm{B}$. In a homogeneous medium, the currents from the source (position $A_{1}$ ) radiate away from the hole producing a predictable set of voltages at measuring potential electrodes located at the surface or in an adjacent well. When the electrode is located in a zone of high conductivity, caused for example by the injection of conductive fluids, the current pattern is distorted by the zone as shown by the current flow lines when the electrode is at position $A_{2}$. This distortion produces a perturbation in the voltages observed at the measuring arrays. This method can be used to monitor the changes associated with an injection over time.

For many injection situations, the wells are cased with steel. The fluids are usually injected through a perforated zone in the casing or out the bottom of the pipe into the surrounding formation. Not only does the steel casing distort the voltages at the potential electrodes, but there will be a coupling effect between the injected fluid and steel casing which can also distort the voltages. The objectives of this study are two fold: (1) to determine if the spatial changes associated with the injection can be measured at the potential electrodes and (2) to determine if the casing will reduce or distort these changes so that they may not be measurable or distinguishable. The cross-hole and downhole to surface surveys are used for the monitoring simulations. 


\section{Cross-Hole}

Consider a well cased with steel and perforated at some depth. Conductive fluids are injected through the perforated section into the surrounding media producing, under ideal conditions, a cylindrical zone which is often referred to as a plume. As the fluid is continuously injected, this plume will increase in size over time. For this problem, the steel casing is energized by a downhole source and an array of potential electrodes is located in an open-hole adjacent to the injection well.

Two models are used for this numerical example. The first model is the injected plume in a homogeneous half-space with the downhole source placed within the plume (mise-a-lamasse). The second model, shown in Figure (5.5-2), consists of the plume and the casing in the host medium. The current source is placed inside the casing at the same depth $(200 \mathrm{~m})$ as in the mise-à-1a-masse configuration. ${ }^{6}$

The $300 \mathrm{~m}$ casing which has a resistivity of $10^{-6} \Omega \cdot \mathrm{m}$ is embedded in a $100 \Omega \cdot \mathrm{m}$ homogeneous medium. The $1 \Omega \cdot \mathrm{m}$, disk-like plume is injected into the formation at a depth of $200 \mathrm{~m}$. A radius $R$ represents the spatial extent of the $2 \mathrm{~m}$ thick plume during the injection process. The cross-hole measuring array, which is located $100 \mathrm{~m}$ from the injection well, has the potential electrodes spaced $5 \mathrm{~m}$ apart and extends to a depth of $400 \mathrm{~m}$.

Figures (5.5-3) and (5.5-4) are plots of the potentials and $E_{z}$-fields in the monitoring well for the plume only (circles) and plume with casing (squares). The background or preinjection fields, i.e., the fields in absence of the plume, are represented by the dashed (casing) and solid (no casing) lines. Radii of $25 \mathrm{~m}$ (black) and $50 \mathrm{~m}$ (white) are used for the plume. Both figures consist of two plots: one is the field (potential or $E_{z}$ ) and the other is the difference between the injection and pre-injection fields.

Figure (5.5-3a) shows that the pre-injection potentials dominate the signature of the

6 This depth is used for convenience. For the parameters used in this example, the analysis of Section 5.1 indicates that the potentials in the medium are independent of the source location within the casing. 
fields. The plume produces small changes or perturbations on the background in the uncased model. An interesting phenomenon occurs when the casing is incorporated in the model. The perturbations caused by the plume are enhanced when the casing is present (Figure 5.5-3b). However, the near-surface response is also quite large and may be misinterpreted as an anomaly.

Since pole measurements may be difficult to acquire in field situations, the $E_{z}$-fields were calculated from differences of adjacent electrodes. Figure (5.5-4a) has the $\mathrm{E}_{\mathbf{z}}$-fields for non-casing and casing models. The background $\mathrm{E}_{\mathbf{z}}$-fields for the mise-a-la-masse case are much larger than those in a cased well, and the injected plume produces perturbations on the background fields in both cases. The differences in the $E_{z}$-field due to the plume with the casing present are much larger than those of the mise-à-la-masse configuration (Figure 5.5-4b).

To understand the response of the potentials for the two situations, the current patterns in the medium and conductive plume are illustrated in Figure (5.5-5). For the mise-à-la masse configuration (top), the currents are first channeled into the plume and then dissipated into the surrounding formation. The perturbations observed at the cross-hole array have a larger peak and greater fall-off. The plume effectively moves the source closer to the potential array.

When the casing is present, two differences in current patterns are observed. First, the pipe energized by the pole current source it behaves like a line source; in the absence of any inhomogeneity, the currents would flow in a radial direction away from the casing. Second, the currents radiating from the casing are channeled into the conductive plume. Away from the casing, the currents in the plume leak into the formation as in the uncased mise-a-la-masse situation. The effects of current channeling into the plume from a line source provides a relatively greater focusing effect at the cross-hole array than the simple current channeling in the plume for a source in an uncased well.

\section{Downhole to Surface}

In the next example, consider the conductive fluid to be injected at the bottom of the 
steel casing. The measuring array of electrodes is placed on the surface radially away from the injection well and the current source is located below the casing. The values of the parameters were chosen such that the numerical results simulated a field injection test. ${ }^{7}$

Figure (5.5-6) illustrates the models used for this numerical analysis. The first model, which excluded the pipe and fluid in the borehole, had an injection plume in a homogeneous half-space. The second figure shows a model of a plume and a pipe filled with fluid embedded in a homogeneous half-space. The fluid resistivity is $1 \Omega \cdot m$ while the host had a resistivity of $1000 \Omega \cdot \mathrm{m}$. The pipe has a length of $750 \mathrm{~m}(\approx 2460 \mathrm{ft})$ and two conduction lengths of $13.5 \mathrm{~m}$ and $426.7 \mathrm{~m}$ are used to represent an insulated and conductive pipe, respectively. The disklike plume emerging from the end of the pipe has a height of $2 \mathrm{~m}$ and its radius varies to simulate its growth during the fluid injection. The values of the plume radius are $0.1 \mathrm{~m}, 3 \mathrm{~m}$, $10 \mathrm{~m}, 30 \mathrm{~m}, 100 \mathrm{~m}$, and $300 \mathrm{~m}$.

Two source configurations are used in the modeling. The first is the downhole source placed $1 \mathrm{~m}$ beyond the end of the pipe within the plume (mise-à-la-masse). The remote electrode is assumed to be at infinity. The second is the downhole dipole with its center located $3 \mathrm{~m}$ beyond the pipe's end and an electrode separation of $4 \mathrm{~m}$. One electrode is within the body and the other is located in the host below the plume. The surface array has a potential electrode spacing of $15 \mathrm{~m}$. The voltage differences between these electrodes are used to calculate the apparent resistivity.

The next six figures (5.5-7) to (5.5-12) are the apparent resistivity (top plot) and the percent difference (bottom plot) for the three models: plume only, resistive pipe and fluid, and conductive pipe and fluid. The apparent resistivities for the pipe models are plotted on a semi-logarithmic scale; all other plots are linear. The downhole pole-source is used for Figures (5.5-7) to (5.5-9) and Figures (5.5-10) to (5.5-12) have a dipole as the current source. The percent difference is used to monitor changes in apparent resistivities due to the increase

7 Chapter VII discusses the downhole-surface resistivity survey conducted at DuPont's Hemby Branch test well near Waveriy, Tennessee in early October 1989. 
in size of the plume. The percent difference, $\delta$, is calculated by the following expression:

$$
\delta_{i}=\frac{\left(\rho_{0}-\rho_{i}\right)}{\rho_{0}} \times 100 \%
$$

where $\rho_{0}$ and $\rho_{i}$ are the apparent resistivities of the background (no plume) and with the inhomogeneity (plume), respectively.

The plots of Figure (5.5-7) (plume and pole-source) indicate that the plume must be of significant size ( $>30 \mathrm{~m}$ ) to to be distinguishable from the background measurements. Only the $100 \mathrm{~m}$ and $300 \mathrm{~m}$ apparent resistivity curves show significant deviations from the background value of $1000 \Omega \cdot \mathrm{m}$. The percent difference curves show this more clearly: the $100 \mathrm{~m}$ plume shows a maximum difference of only $3 \%$, and the difference increases to $23 \%$ for the $300 \mathrm{~m}$ plume.

Figure (5.5-8) shows responses for the resistive pipe filled with fluid and for the polesource. The pipe effects are seen on the near-well apparent resistivities. The percent difference plot show values over $2 \%$ (near-well electrodes) for all the plume radii. The pipe and fluid appears to accentuate the surface response of the plume especially at the near-well electrodes. The apparent resistivity and the percent difference for the conductive pipe with fluid are shown in Figure (5.5-9). The pipe significantly distorts the apparent resistivities. This effect extends along the entire array but has a much smaller effect at the far-field electrodes. The presence of the conductive pipe greatly increases the percent differences for all radii of the plume.

Figures (5.5-10) to (5.5-12) are equivalent to the plots of Figures (5.5-6) to (5.5-8) with a dipole current source instead of a pole source. All the responses show sizable differences from the background values. With this electrode configuration, even the small radius $(3 \mathrm{~m})$ show deviations from the background level. The percent difference plot of Figure (5.5-12) shows that the $100 \mathrm{~m}$ and $300 \mathrm{~m}$ curves are nearly the same for the distances shown in the figure. Thus for the conductive pipe, the size of the large plumes may not be distinguishable from one another if the electrode array does not radially extend far from the well. 


\section{Conclusion}

This results suggest that a cross-hole and downhole to surface surveys to monitor in time an injection experiment in cased wells may be possible. Using an energized casing as a source or placing a dipole source at the end of the casing enhances the response of the injected plume. When compared to background measurements, the anomalies produced by the plume with the casing present are significantly larger than those without the casing. Thus, using the casing in monitoring of an injection may be advantageous to determine the extent of a plume.

\subsubsection{Measurement While Drilling}

Measurement while drilling (MWD) is a method that provides realtime downhole information during drilling operations. Commonly, the MWD tool is composed of two systems that are assembled in a drill collar and placed above the drill bit (Figure 5.5-13). The first is the sensor package that acquires the data. The second is the telemetry system which relays the information to the surface.

The information collected by the sensors can be divided into two categories: drilling information and formation properties. Drilling information includes hole direction and inclination used for directional drilling. Other information, such as downhole weight-on-bit, torque, temperature, and pressure can be acquired. Formation characteristics, natural gamma ray emissions and electrical resistivity, are also typically gathered with MWD sensors.

There are several telemetry methods by which signals are sent from downhole to the surface (Gravley, 1983). One method transmit the signals by generating pressure waves in the drilling mud. The pressure waves can be frequency modulated or pulsed. Another technique transmits the information through electrical conductors inside the pipe. The pipes are prewired such that the circular conductor is insulated from the drill pipe by two concentric dielectric rings (Holbrook, 1985). A third method sends an acoustic signal through the drill string. Since these acoustic waves have high attenuation, repeaters are needed in the drill string to 
relay the signal to the surface.

The last transmission technique, which is the topic of this section, is the electromagnetic telemetry method. The signals are transmitted through the earth to surface receivers by a EM source near the drill bit. The attenuation of the signals is dependent, in part, on the electrical properties of the medium. The DC limit of the EM telemetry is investigated in this example. If the DC responses are small for a configuration, the EM signal are probably not measurable since the DC value is the upper asymptotic limit. Two major factors that affect the signal amplitudes are the depth of the transmitter and the conduction length of the drill stem. Recall that the conduction length relates the drill stem conductance and the formation conductivity.

Figure (5.5-14) illustrates the simplified model used for the EM telemetry problem. The transmitter is simulated by a current dipole $(I=1 \mathrm{Amp})$ placed across an insulated gap at the end of the drill string. Thus, the drill string will act as a long line source and the lower collar and bit behave like a short line source of opposite polarity. For this example, a model with short dipole gap $\Delta z_{0}=0.2 \mathrm{~m}$ and no lower drill collar $L_{2}=0.0 \mathrm{~m}$ is considered. The $10^{-6} \Omega \cdot \mathrm{m}$ drill string, which varies in length $L_{1}$ from $100 \mathrm{~m}$ to $3000 \mathrm{~m}$, has a thickness of $6 \mathrm{~mm}$, has an inner radius of $10 \mathrm{~cm}$, and is embedded in a homogeneous half-space. Resistivities of 1,10 , and $100 \Omega \cdot m$ are used for the half-space which correspond to conduction lengths of 62,197 , and $623 \mathrm{~m}$, respectively.

Figure $(5.5-15)$ shows the potential difference or voltage $(\Delta V)$ on the surface measured between the drill string and radial surface electrodes. Figure (5.5-16) plots the voltage measured between the well and an electrode at the $500 \mathrm{~m}$ as a function of drill string length $L_{1}$. For short string lengths, the voltages quickly reach their asymptotic values. The voltages are proportional to the host resistivity and the inverse of the string length. As the drill string is lengthened, the signals in the $1 \Omega \cdot \mathrm{m}$ formation are significantly reduced $\left(\Delta V=10^{-7}-10^{-8} \mathrm{~V}\right.$ for $\left.L_{1}>1000 \mathrm{~m}\right)$. The signals transmitted in the $10 \Omega \cdot \mathrm{m}$ host begin to deteriorate $\left(\Delta V<10^{-6} \mathrm{~V}\right)$ for lengths greater than $3000 \mathrm{~m}$. In a resistive environment $(100 \Omega \cdot \mathrm{m})$, the signals are relatively large even for the $3000 \mathrm{~m}$ drill pipe $\left(\Delta V=10^{-3} \mathrm{~V}\right)$. For great lengths, the voltages are 
proportional $\mathrm{L}^{-4}$. The transition length to this fall off appears to occur at approximately $1-3$ conduction lengths.

For this example, a "worst case" scenario was considered. The analysis in Section 5.4 indicates that the voltages can be improved by lengthening the dipole gap or introducing a lower collar. Figure (5.5-17) illustrates that the voltage can be increased by increasing the dipole gap or lower collar length. These values correspond to a model with a $300 \mathrm{~m}$ drill string and $100 \Omega \cdot m$ host formation. The maximum increase appears to asymptote to 4-5 times that of a model with no collar and $0.2 \mathrm{~m}$ dipole gap.

Lastly, this configuration can be used as a MWD resistively tool. The current pattem in the medium is shown in Figure (5.5-18) for an insulated collar separated from the drill string with a dipole source placed across the gap. For this configuration, the currents are emitted from the collar into the formation and return to the upper drill pipe. Its current pattern appears similar to that produced by an open-hole focus log. Other current electrodes may be added to the adjust the focusing of the current, to further resemble the current pattem of a focus log. By placing potential electrodes near the center of the lower collar, a MWD focus $\log$ can be simulated.

These results indicate that EM telemetry through the earth is possible in resistive formations. For a conductive earth, the signals transmitted at depth may be greatly attenuated and may be difficult to receive at the surface. The signal strength at the surface can be improved by increasing the dipole gap or lengthening the the drill collar. Additionally, this configuration has a current pattern resembling those produced by an open-hole focus log. Thus, one may be able to simulate MWD resistivity logging configurations. 


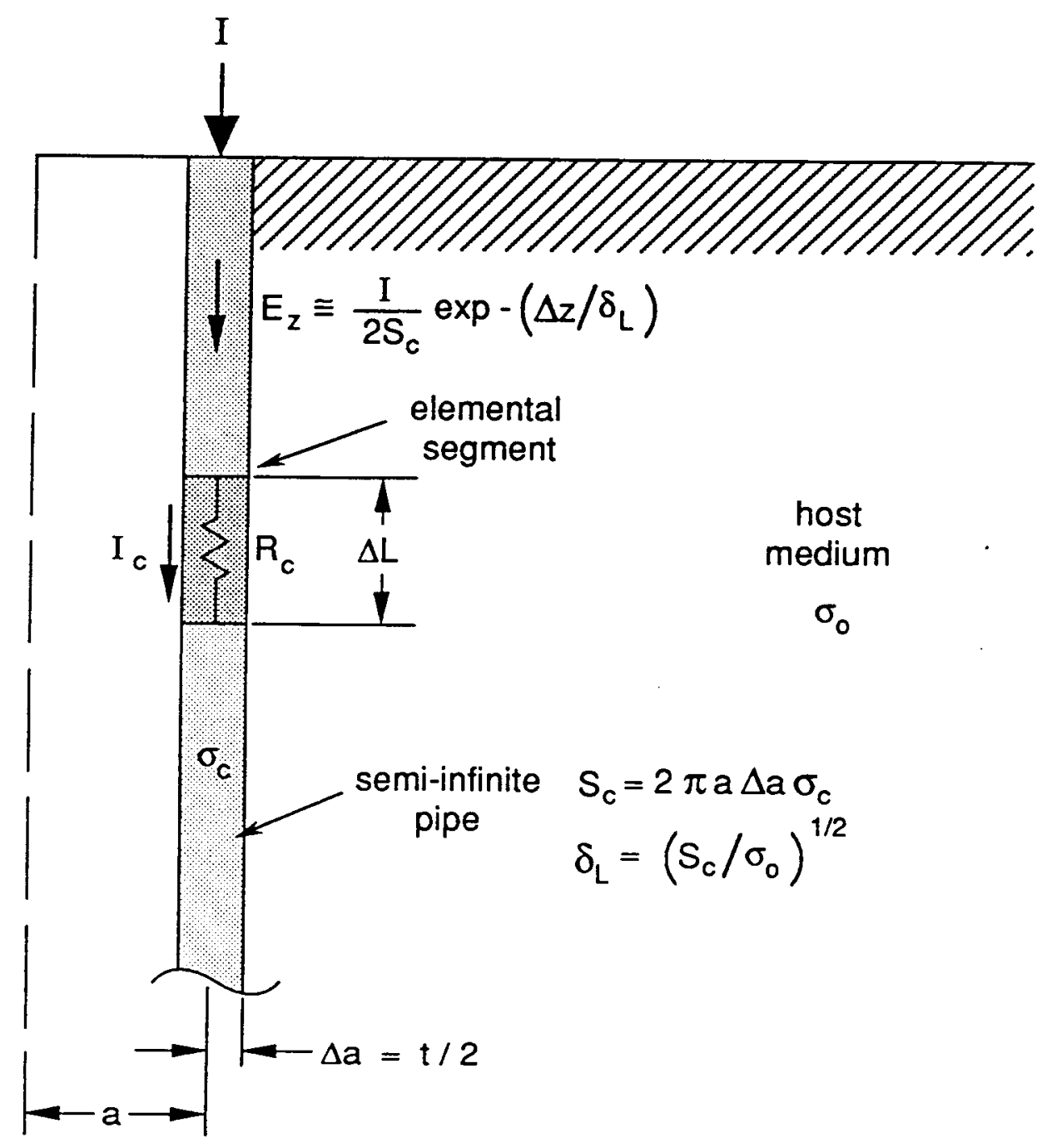

Figure 5.1-1: Generalized configuration of the semi-infinite vertical annulus in a homogeneous half-space, applied current source, and field position. 


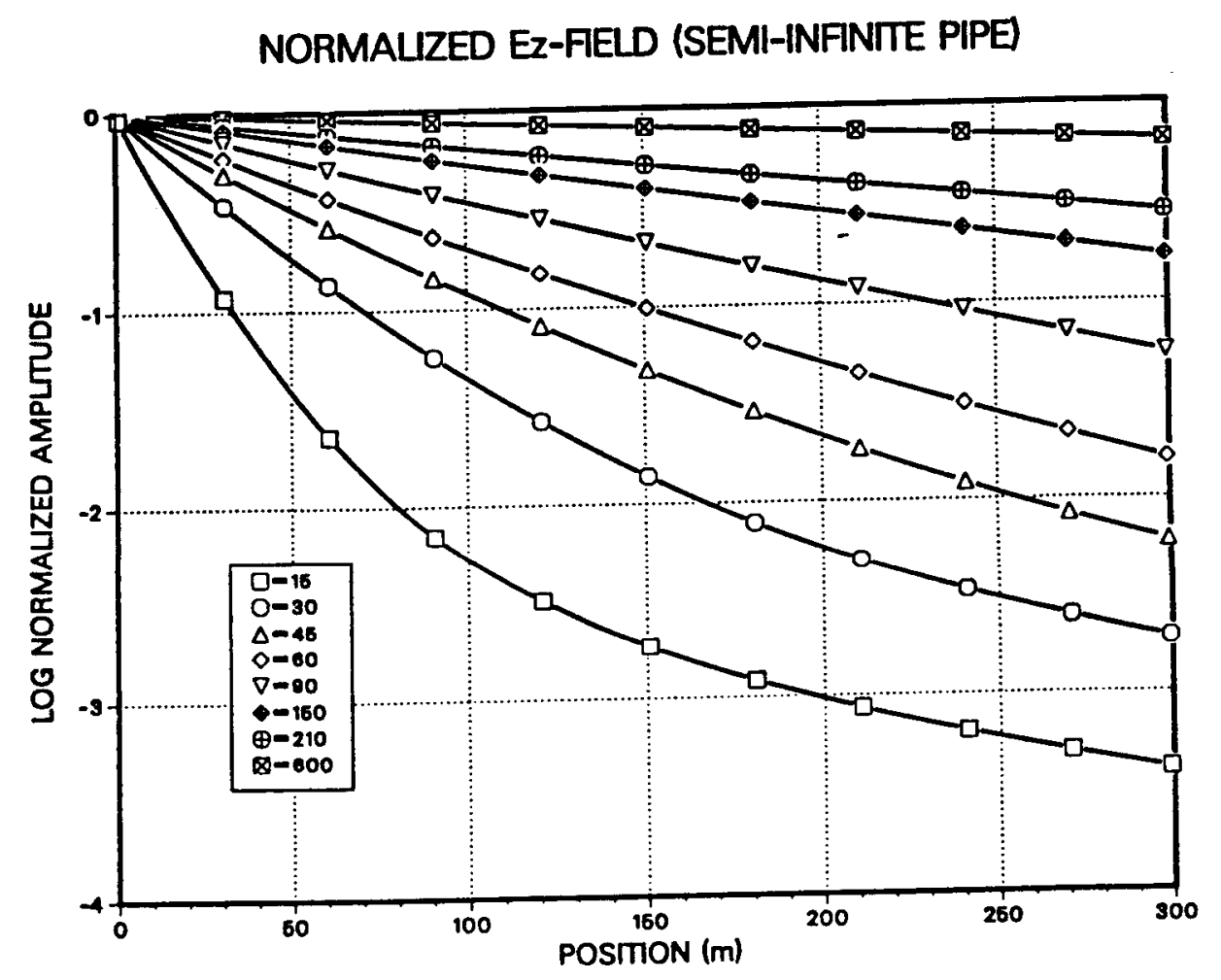

Figure 5.1-2: Semi-log plot of the normalized $E_{z}$ in a semi-infinite pipe for several $\delta_{L}$ values with current source placed at the surface. $E_{z}$ is normalized by the factor $I / S_{c}$. 

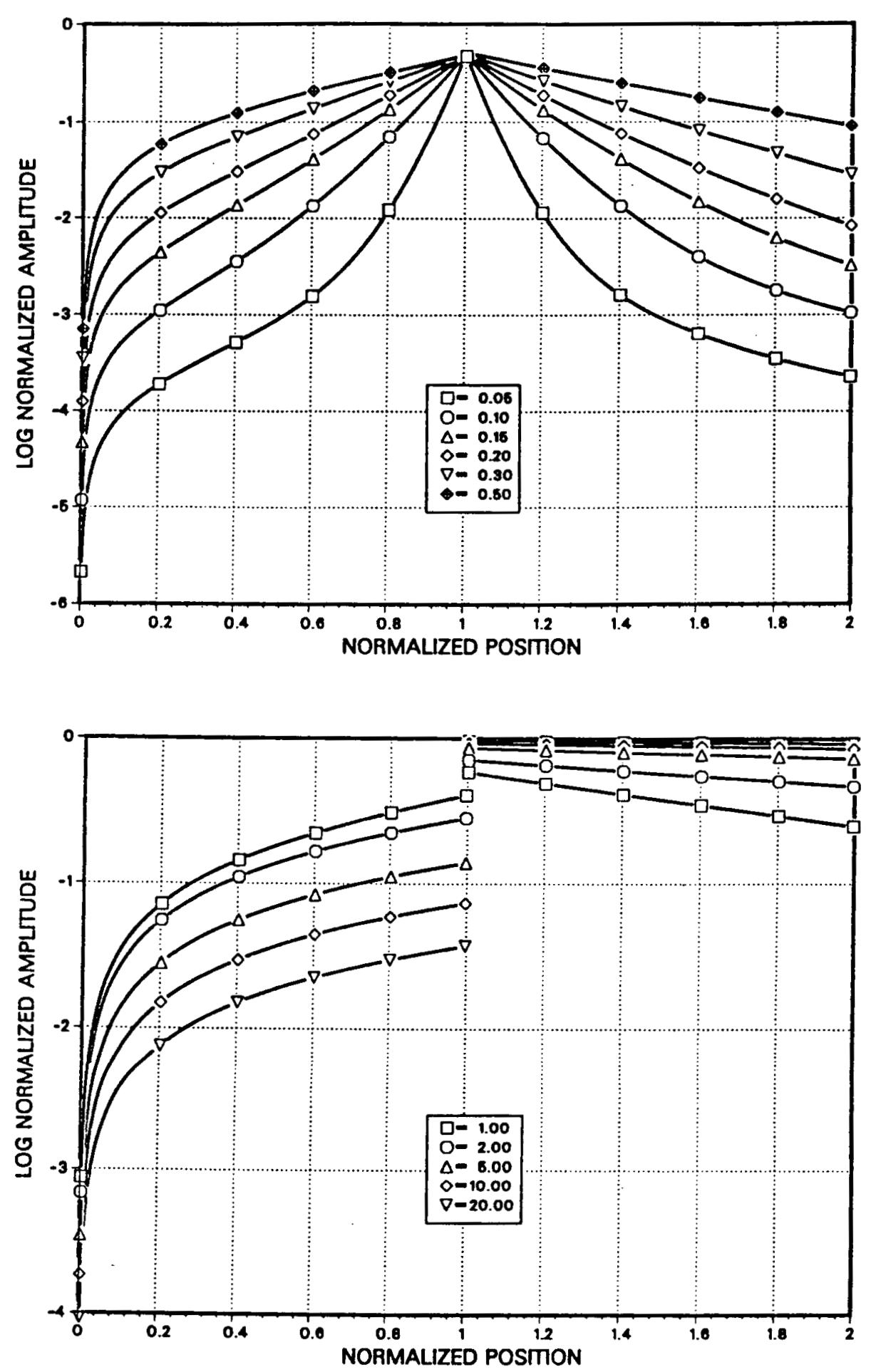

Figure 5.1-3: Semi-log plots of the normalized $E_{2}$ in a semi-infinite pipe for $\beta_{S}<1$ (top) and $\beta_{s} \geq 1$ (bottom) with current source located at $z_{s}=1$. The spatial variables and $E_{z}$ are normalized by the source depth $z_{0 s}$ and factor $I / S_{c}$, respectively. 


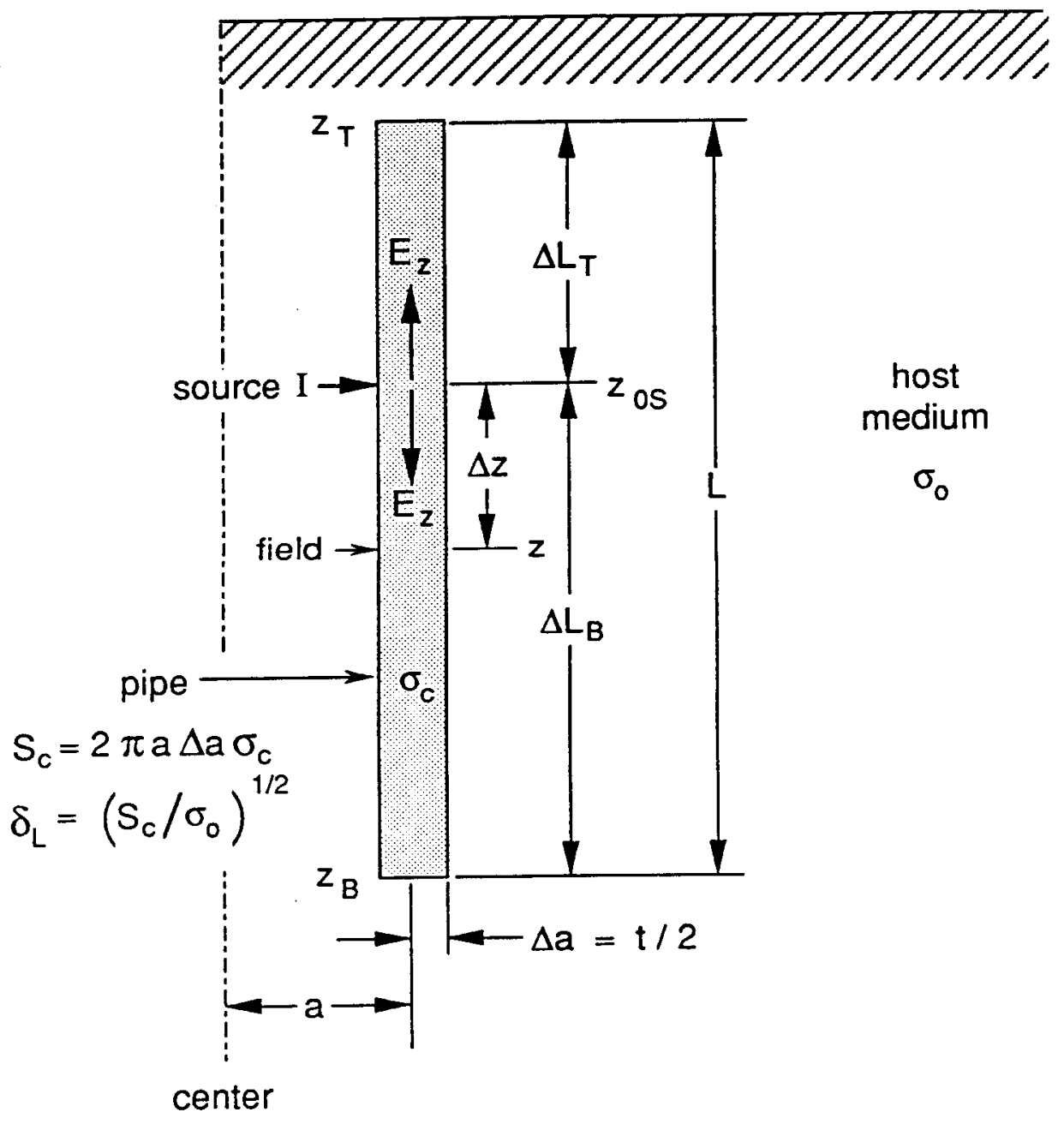

Figure 5.1-4: Generalized configuration of the finite length pipe in a homogeneous half-space, applied current source, and field position. 


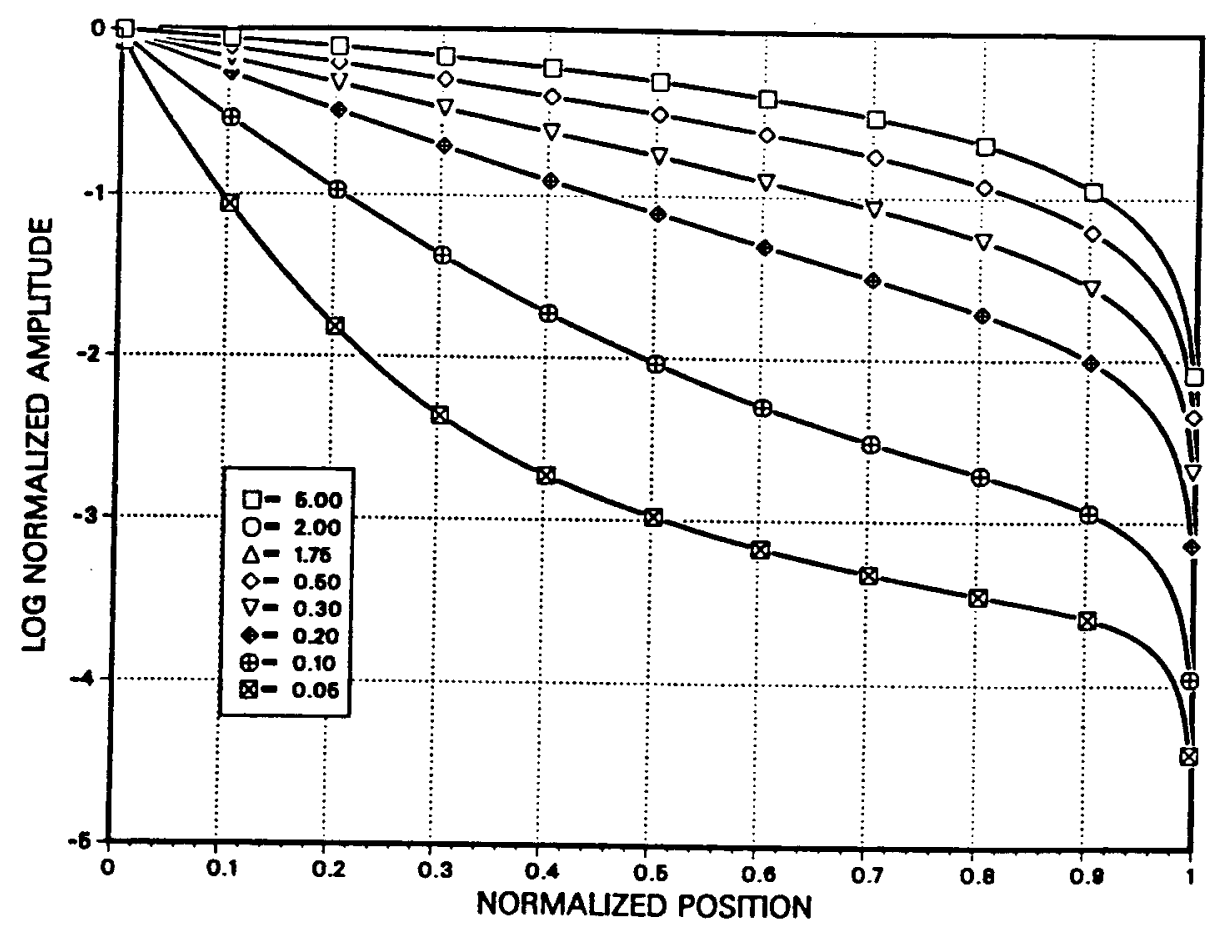

Figure 5.1-5a: Semi-log plots of the normalized whole-space $E_{z}$ in a pipe for several $\alpha_{L}$-ratios with current source located at $S / L=0.0$. The spatial variables and $E_{z}$ are normalized by the pipe length $L$ and factor $I / S_{c}$, respectively.

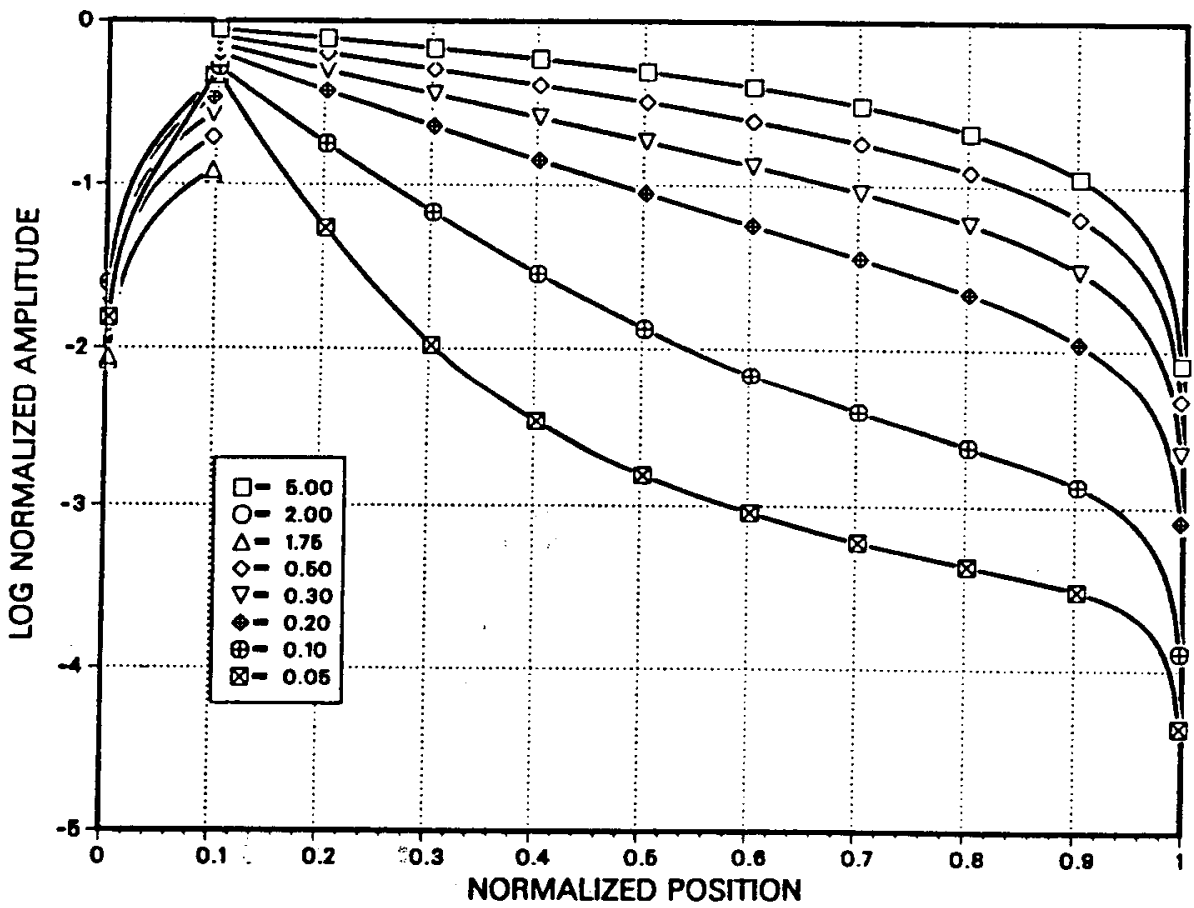

Figure 5.1-5b: Semi-log plots of the normalized whole-space $E_{z}$ in a pipe for several $\alpha_{L}$ ratios with current source located at $S / L=0.1$. The spatial variables and $E_{z}$ are normalized by the pipe length $L$ and factor $I / S_{c}$, respectively. 


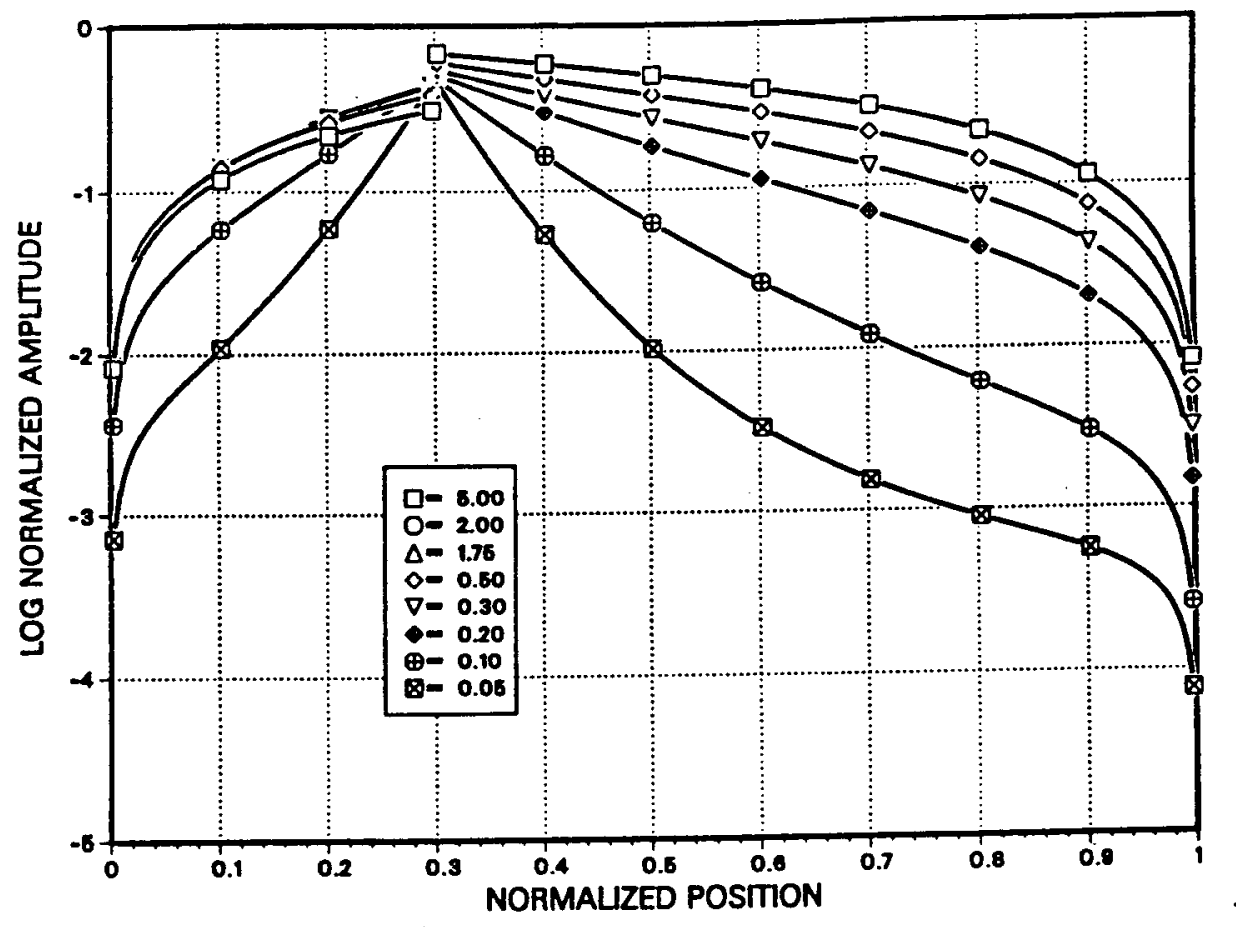

Figure 5.1-5c: Semi-log plots of the normalized whole-space $E_{z}$ in a pipe for several $\alpha_{L}$-ratios with current source located at $S / L=0.3$. The spatial variables and $E_{z}$ are normalized by the pipe length $L$ and the factor $I / S_{c}$, respectively.

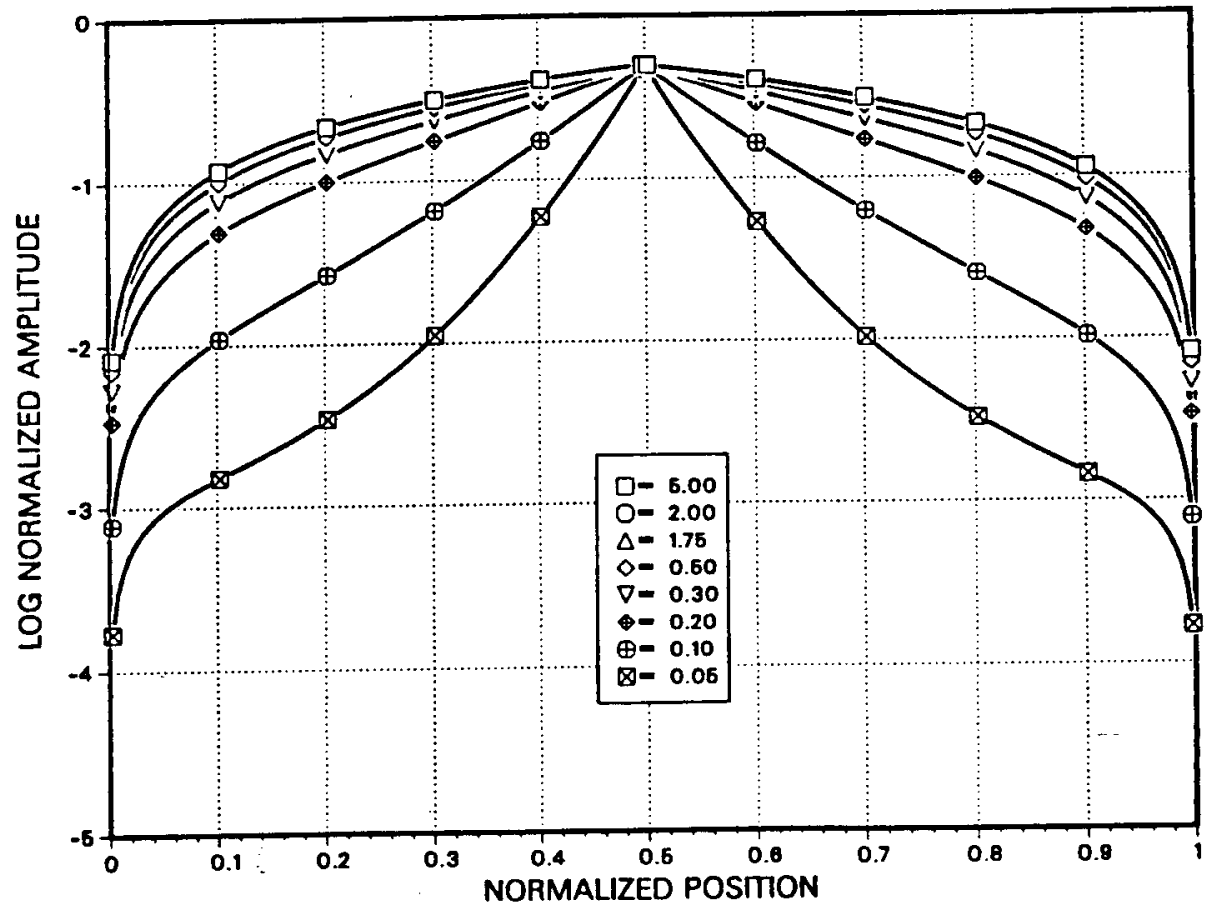

Figure 5.1-5d: Semi-log plots of the normalized whole-space $E_{z}$ in a pipe for several $\alpha_{L}$ ratios with current source located at $S / L=0.5$. The spatial variables and $E_{z}$ are normalized by the pipe length $L$ and the factor $I / S_{c}$, respectively. 


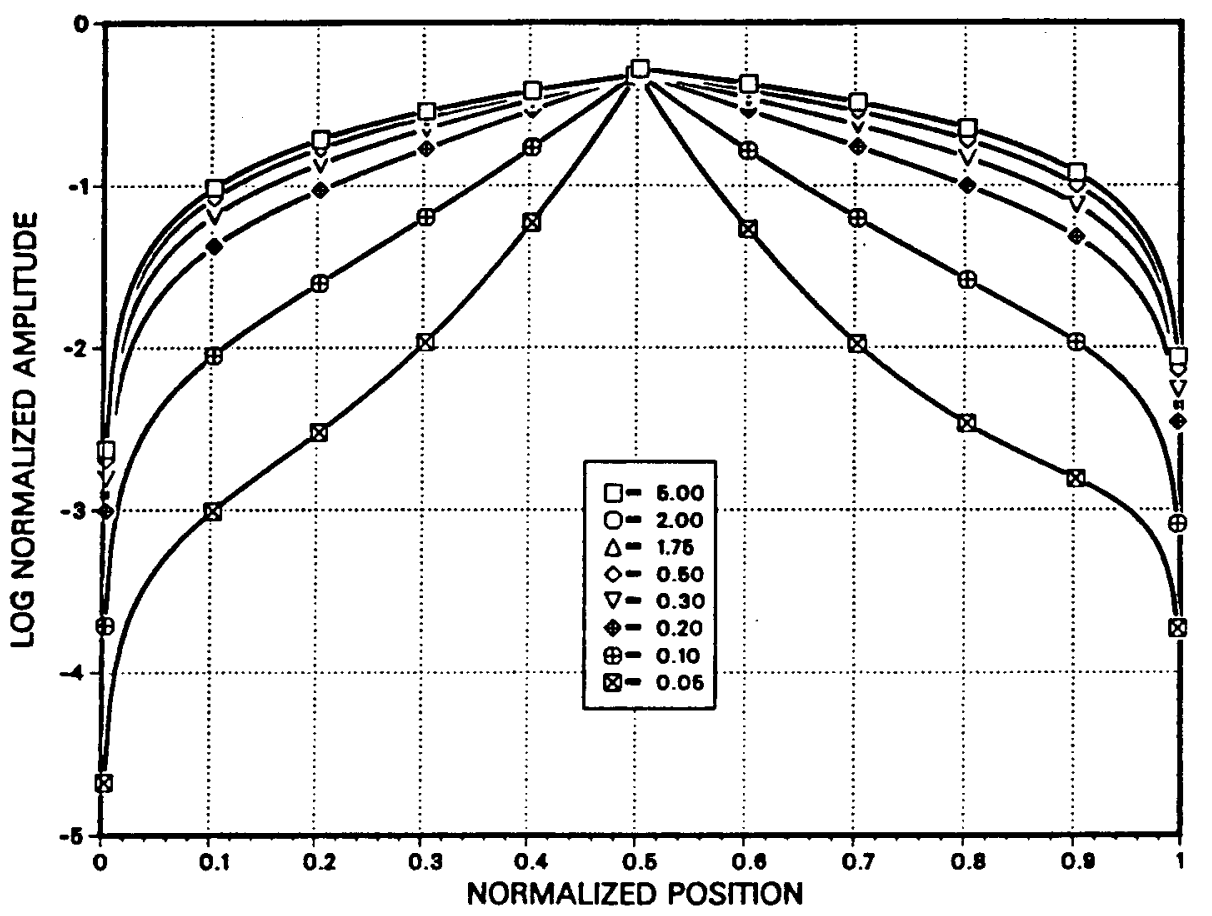

Figure 5.1-6: Semi-log plots for several $\alpha_{L}$-ratios of the normalized half-space $E_{z}$ with current source located at $S / L=0.5$. The spatial variables and $E_{z}$ are normalized by the pipe length $L$ and the factor $I / S_{c}$, respectively. 

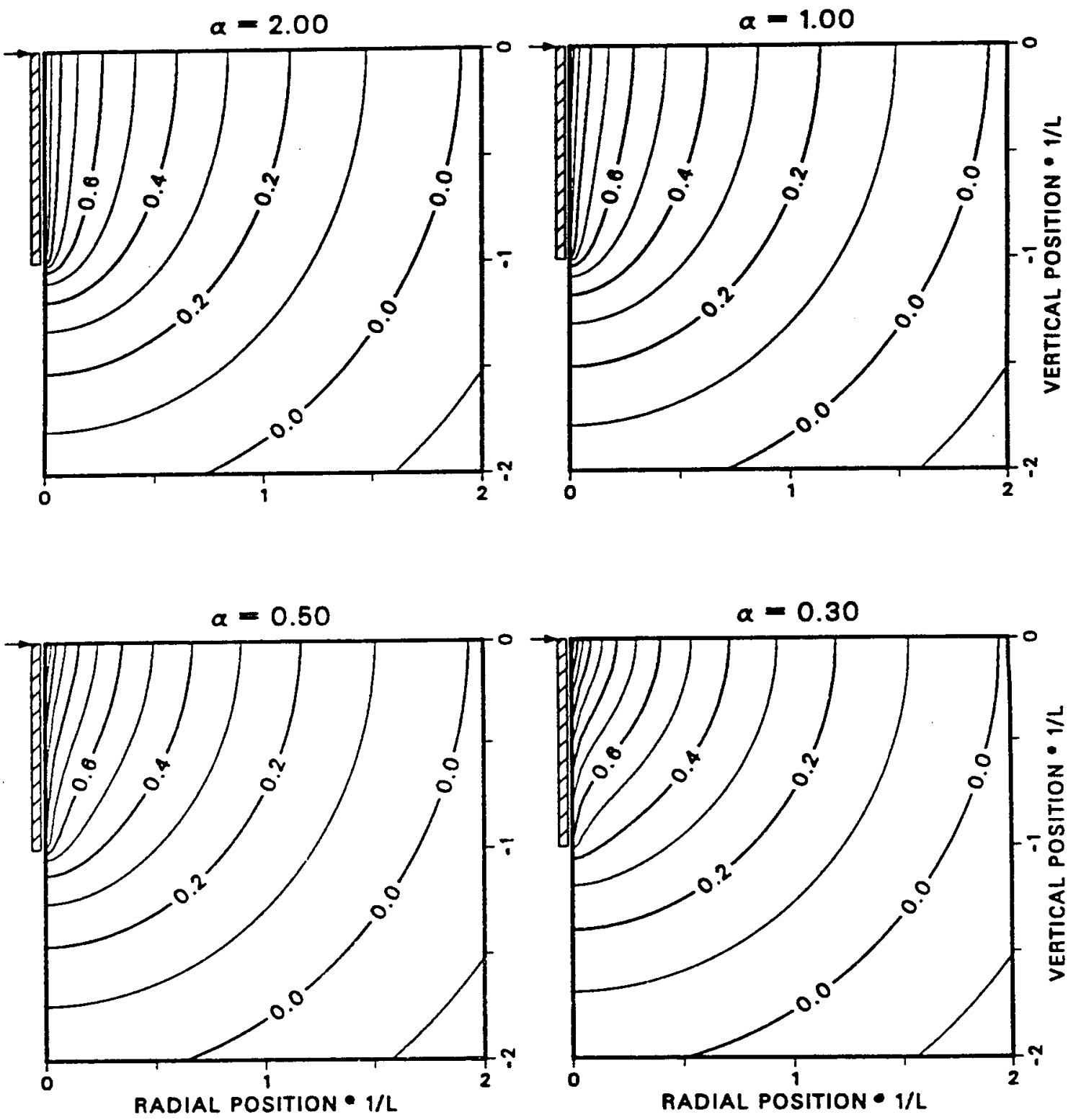

Figure 5.1-7a: Log-contour plots of the normalized half-space potentials due to a pipe for several $\alpha_{L}$-ratios with current source located at $S / L=0.0$. The spatial variables and potentials are normalized by the pipe length $L$ and the factor $I / 4 \pi \sigma_{0} L$, respectively. 

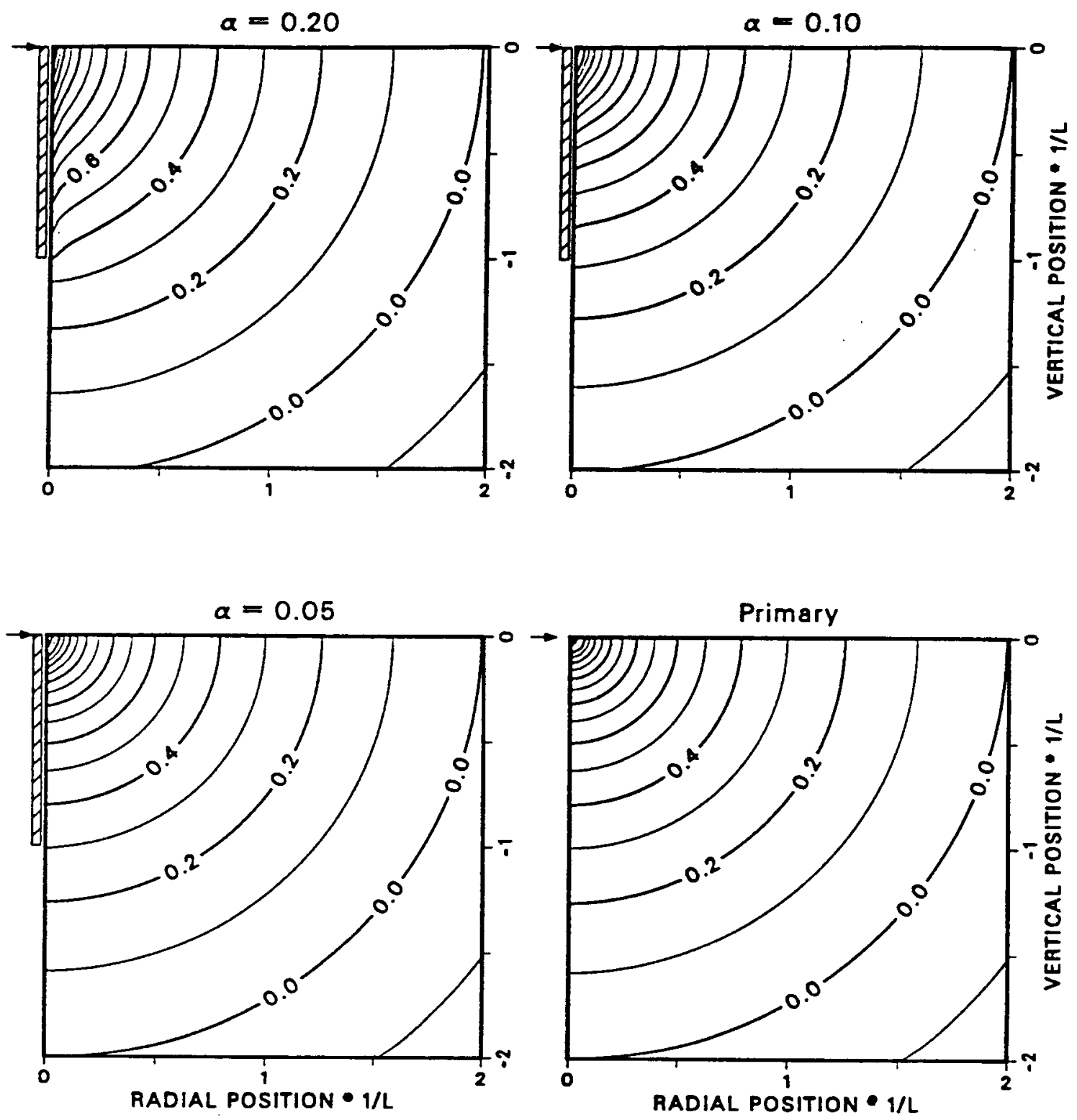

Figure 5.1-7b: Log-contour plots of the normalized half-space potentials for a pipe using several $\alpha_{L}$-ratios and the primary field (lower right) with current source located at $S / L=0.0$. The spatial variables and potentials are normalized by the pipe length $L$ and the factor $I / 4 \pi \sigma_{0} L$, respectively. 

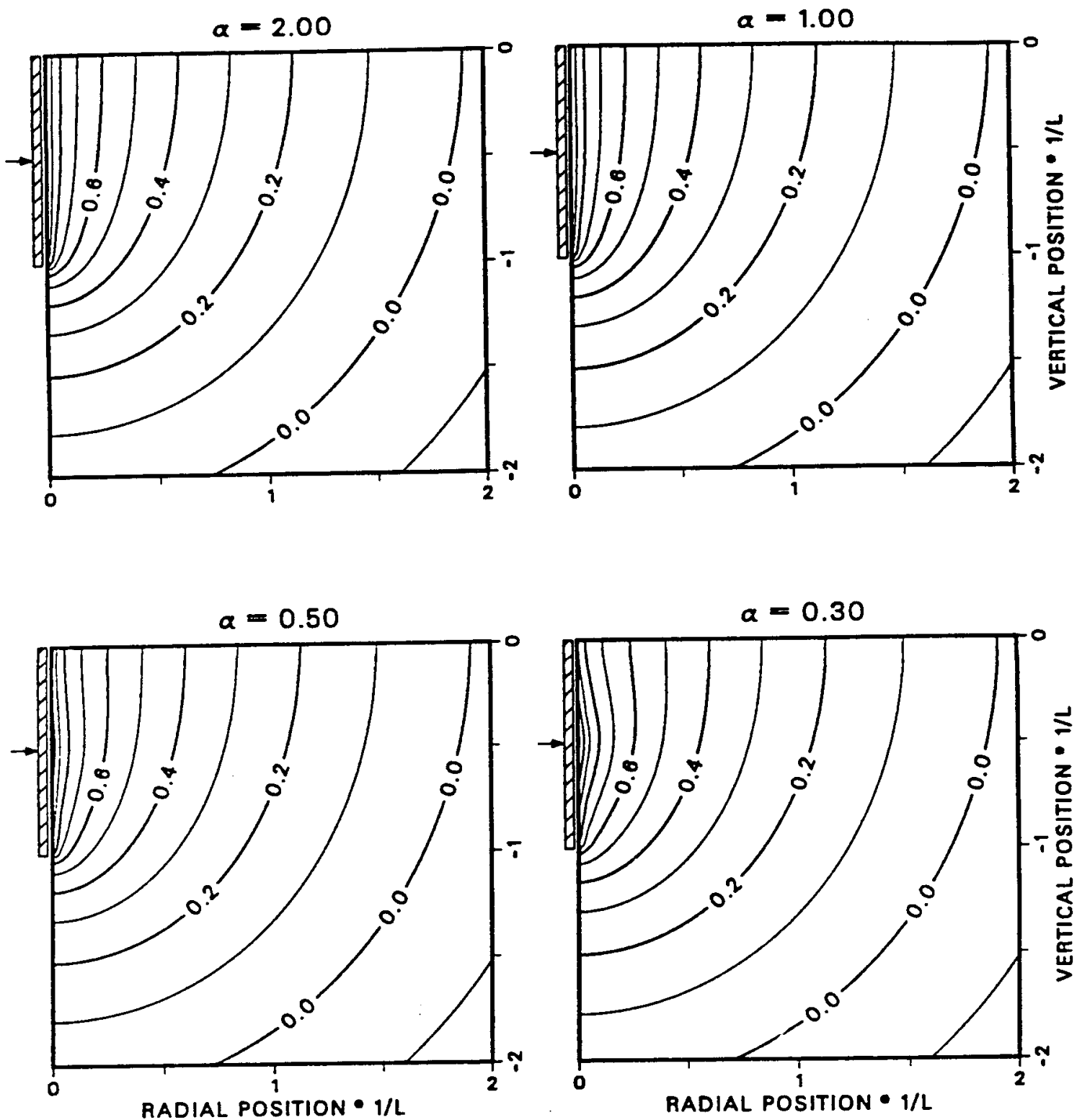

Figure 5.1-8a: Log-contour plots of the normalized half-space potentials due to a pipe for several $\alpha_{L}$-ratios with current source located at $S / L=0.5$. The spatial variables and potentials are normalized by the pipe length $L$ and the factor $I / 4 \pi \sigma_{0} L$, respectively. 

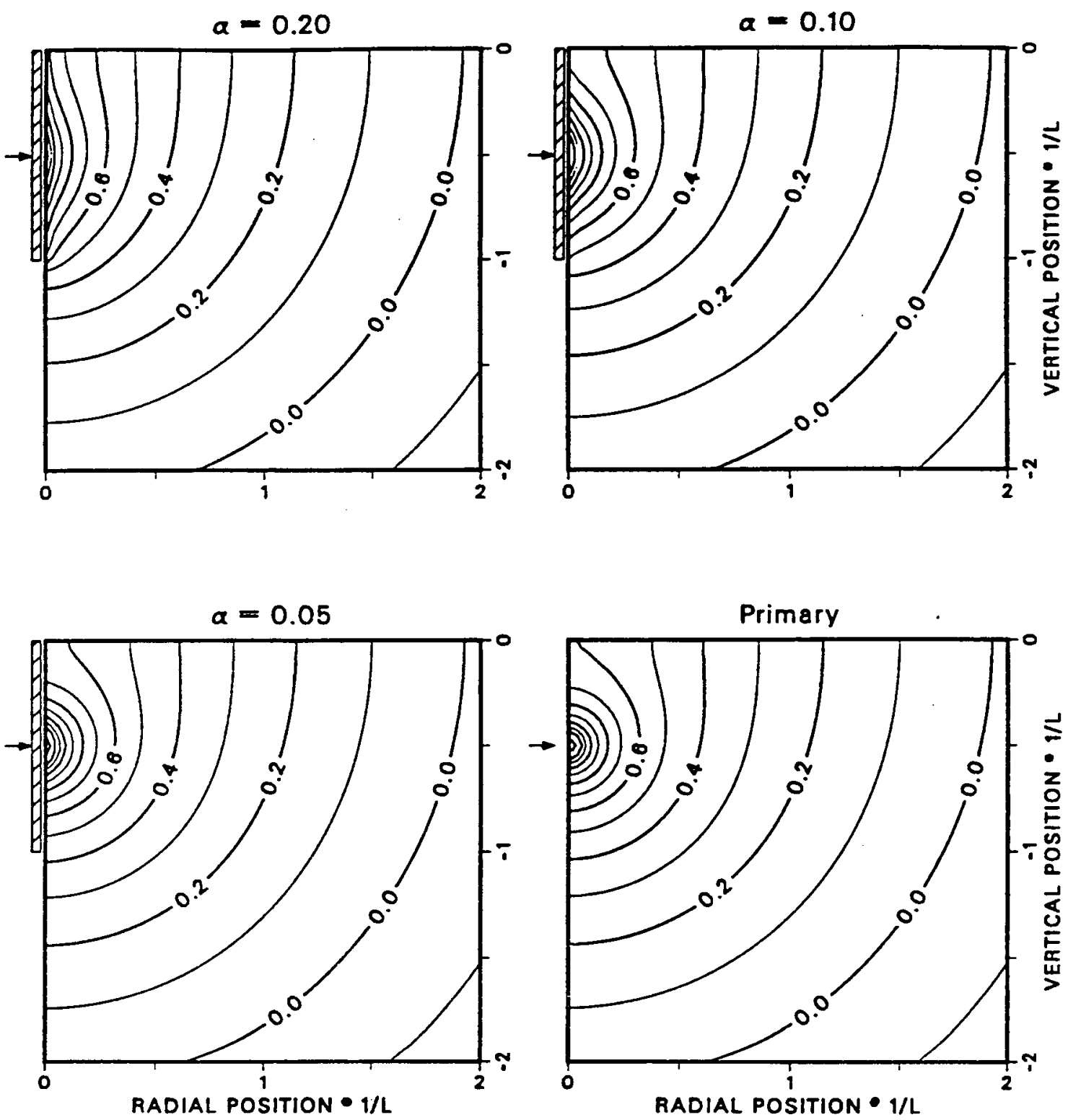

Figure 5.1-8b: Log-contour plots of the normalized half-space potentials for a pipe using several $\alpha_{L}$-ratios and the primary field (lower right) with current source located at $S / L=0.5$. The spatial variables and potentials are normalized by the pipe length $L$ and the factor $I / 4 \pi \sigma_{0} L$, respectively. 

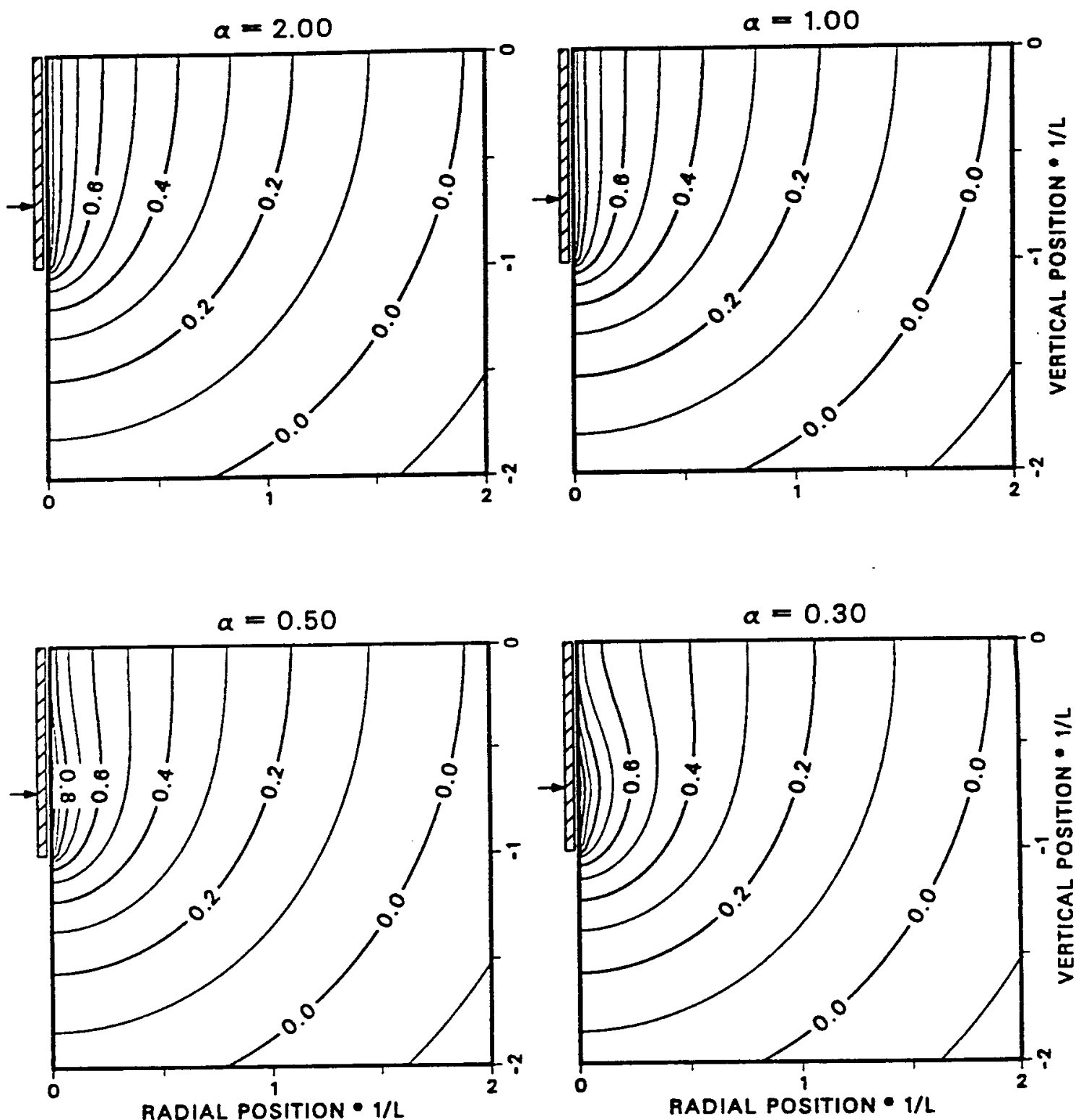

Figure 5.1-9a: Log-contour plots of the normalized half-space potentials due to a pipe for several $\alpha_{L}$-ratios with current source located at $S / L=0.7$. The spatial variables and potentials are normalized by the pipe length $L$ and the factor $I / 4 \pi \sigma_{0} L$, respectively. 

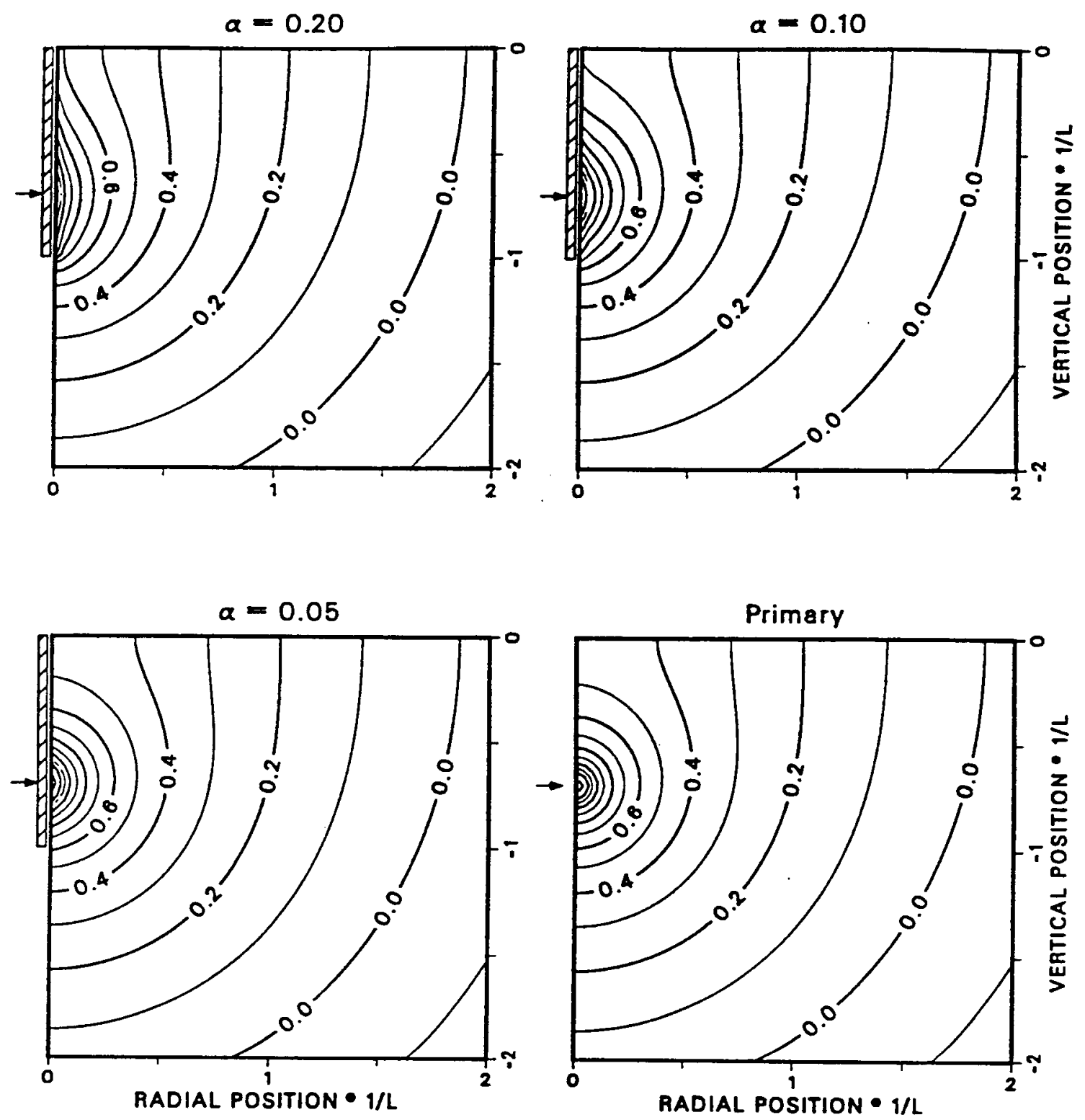

Figure 5.1-9b: Log-contour plots of the normalized half-space potentials for a pipe using several $\alpha_{L}$-ratios and the primary field (lower right), with current source located at $S / L=0.7$. The spatial variables and potentials are normalized by the pipe length $L$ and the factor $I / 4 \pi \sigma_{0} L$, respectively. 

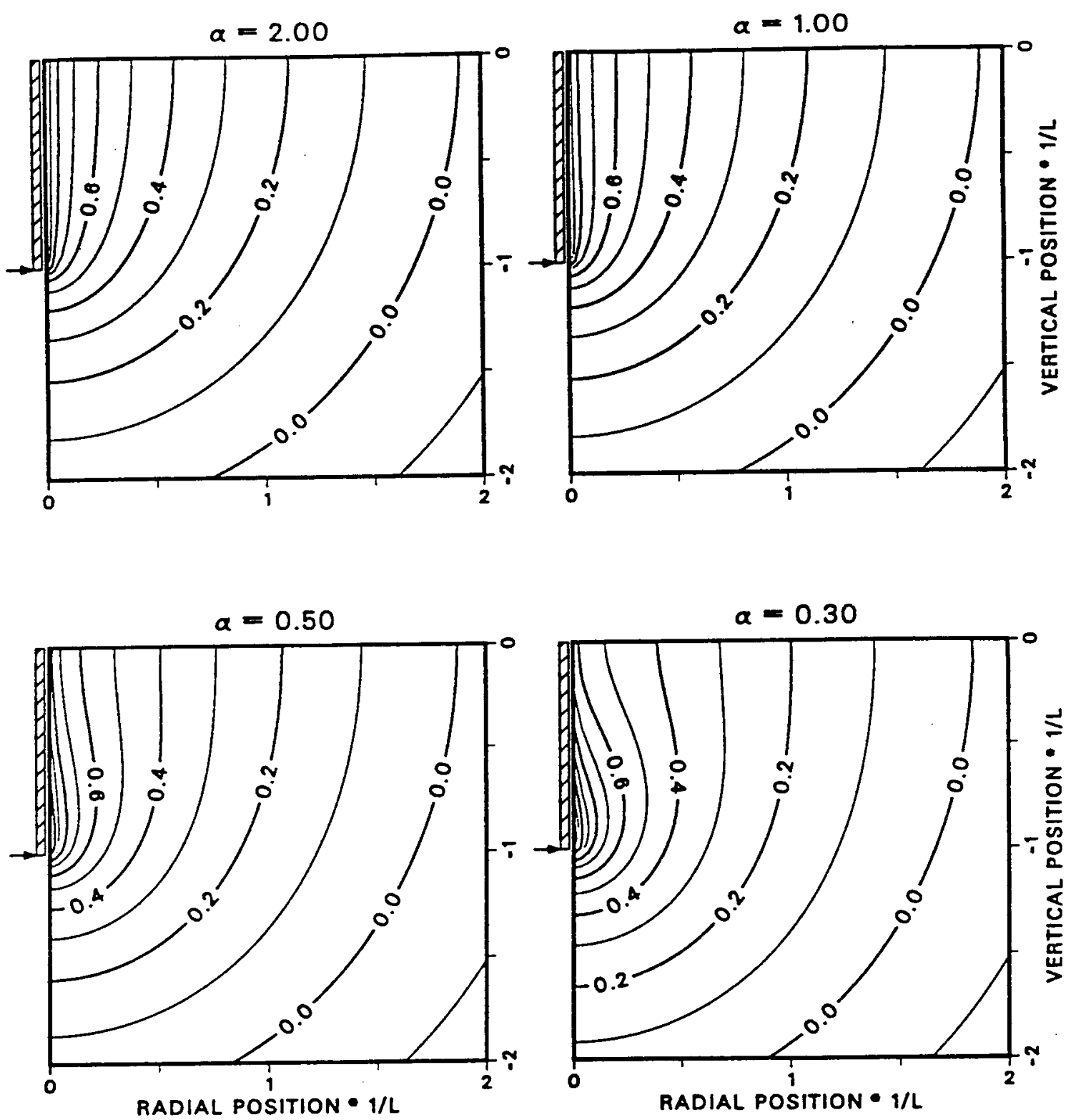

Figure 5.1-10a: Log-contour plots of the normalized half-space potentials due to a pipe for several $\alpha_{L}$-ratios with current source located at $S / L=1.0$. The spatial variables and potentials are normalized by the pipe length $L$ and the factor $I / 4 \pi \sigma_{0} L$, respectively. 

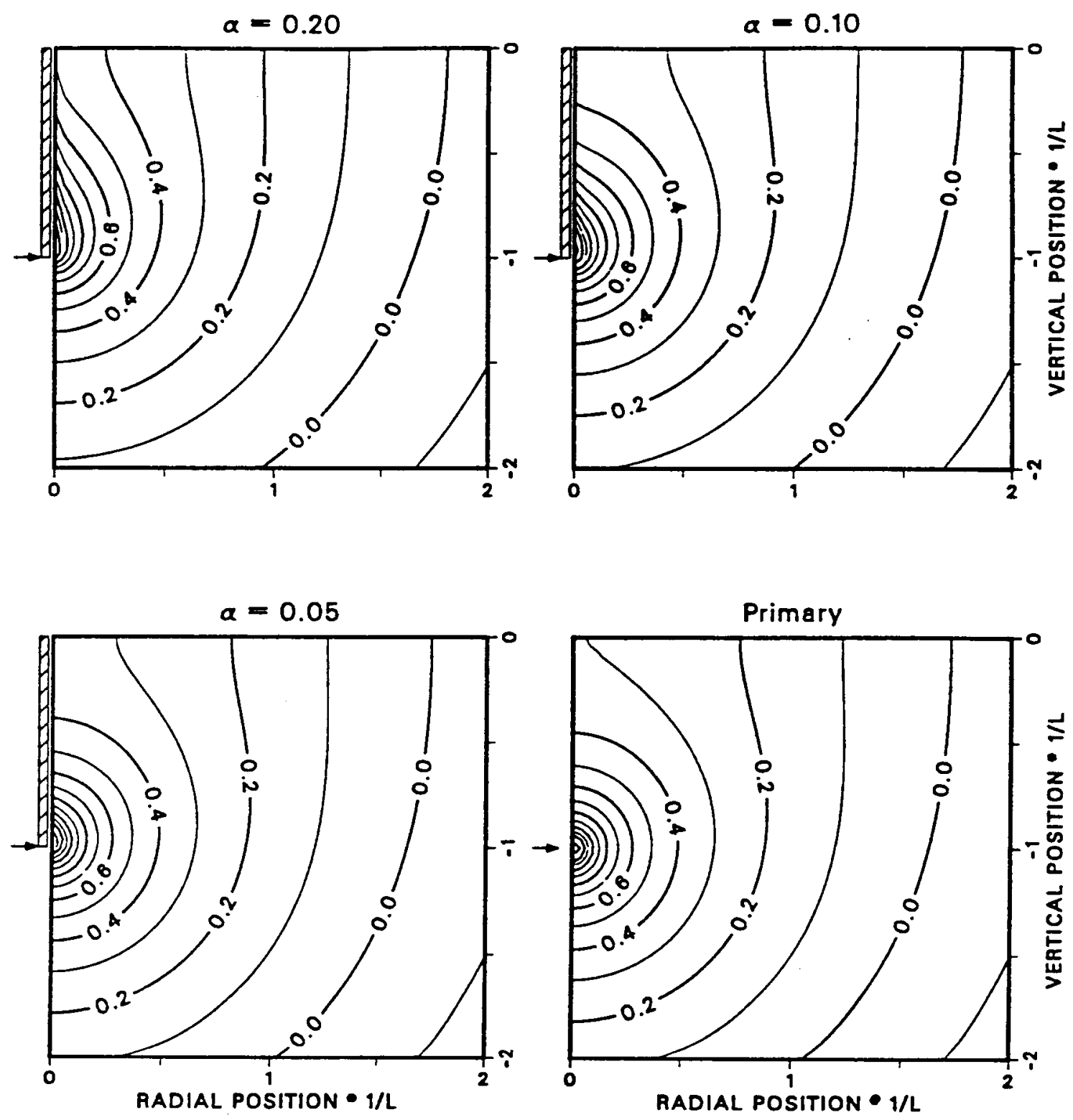

Figure 5.1-10b: Log-contour plots of the normalized half-space potentials for a pipe using several $\alpha_{L}$-ratios and the primary field (lower right) with current source located at $S / L=1.0$. The spatial variables and potentials are normalized by the pipe length $L$ and the factor $1 / 4 \pi \sigma_{0} L$, respectively. 

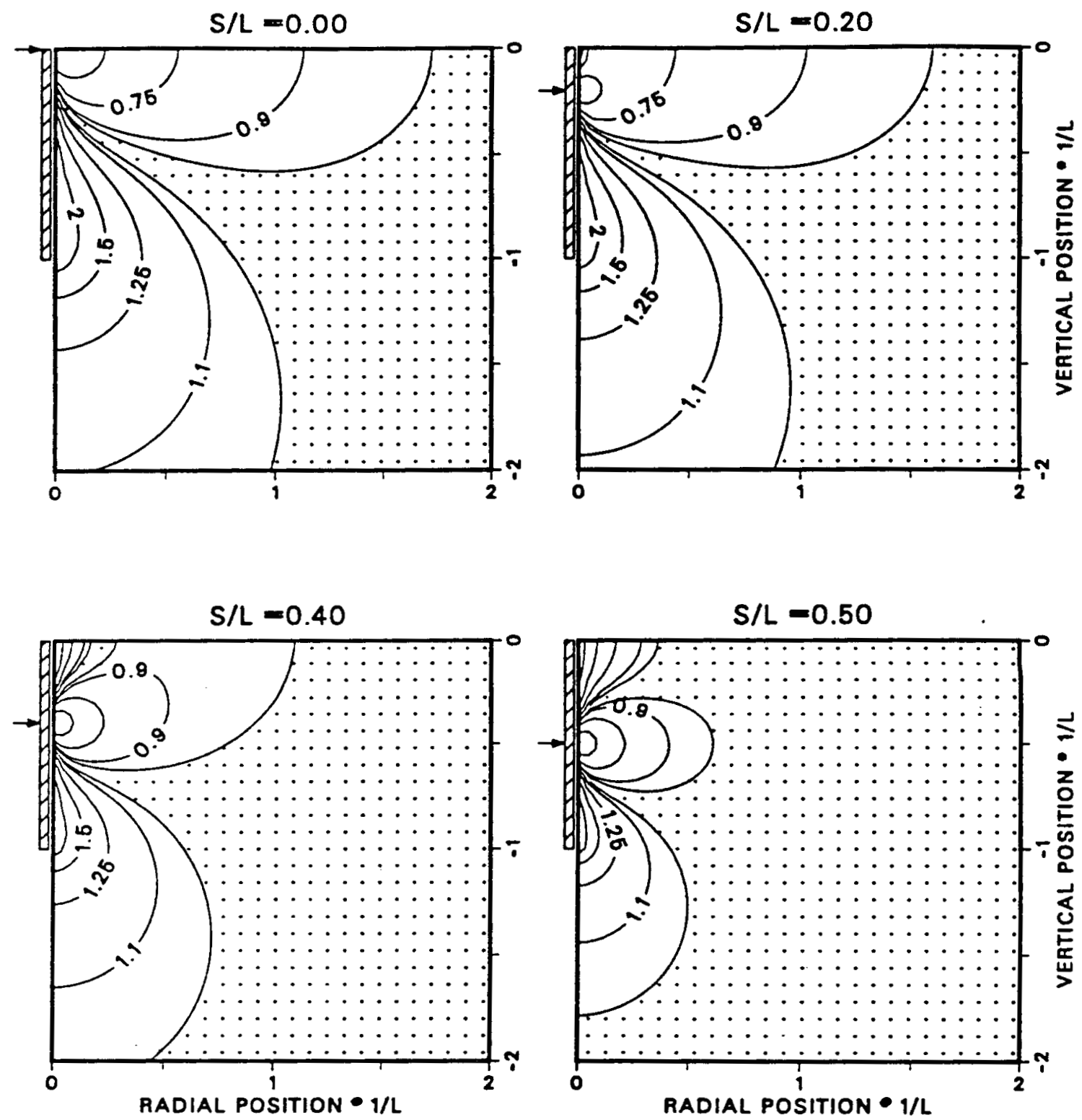

Figure 5.1-11a: Contour plots of the ratio of the total to primary fields due to a pipe and $\alpha_{L}=2$ with current source located within the pipe: $0.0 \leq S / L \leq 0.5$. The spatial variables and potentials are normalized by the pipe length $L$ and the factor $I / 4 \pi \sigma_{0} L$, respectively. The dotted area represents $5 \%$ or less deviation of the total field from the primary field. Coincidentally, these plots are the ratio of the apparent and background resistivities for a pole-pole survey. 

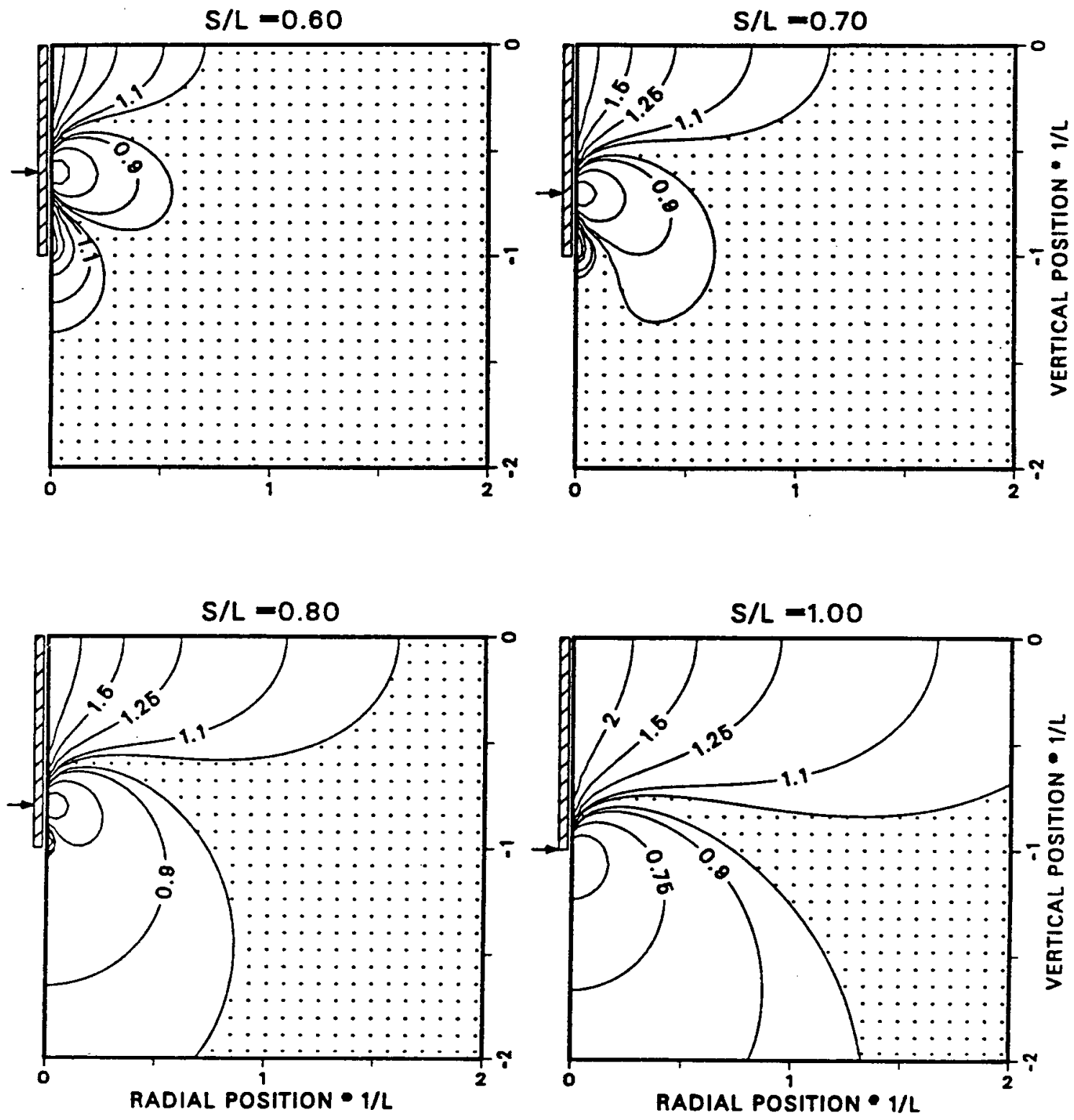

Figure 5.1-11b: Contour plots of the ratio of the total to primary fields due to a pipe and $\alpha_{L}=2$ with current source located within the pipe: $0.5<S / L \leq 1.0$. The spatial variables and potentials are normalized by the pipe length $L$ and the factor $I / 4 \pi \sigma_{0} L$, respectively. The dotted area represents $5 \%$ or less deviation of the total field from the primary field. Coincidentally, these plots are the ratio of the apparent and background resistivities for a pole-pole survey. 

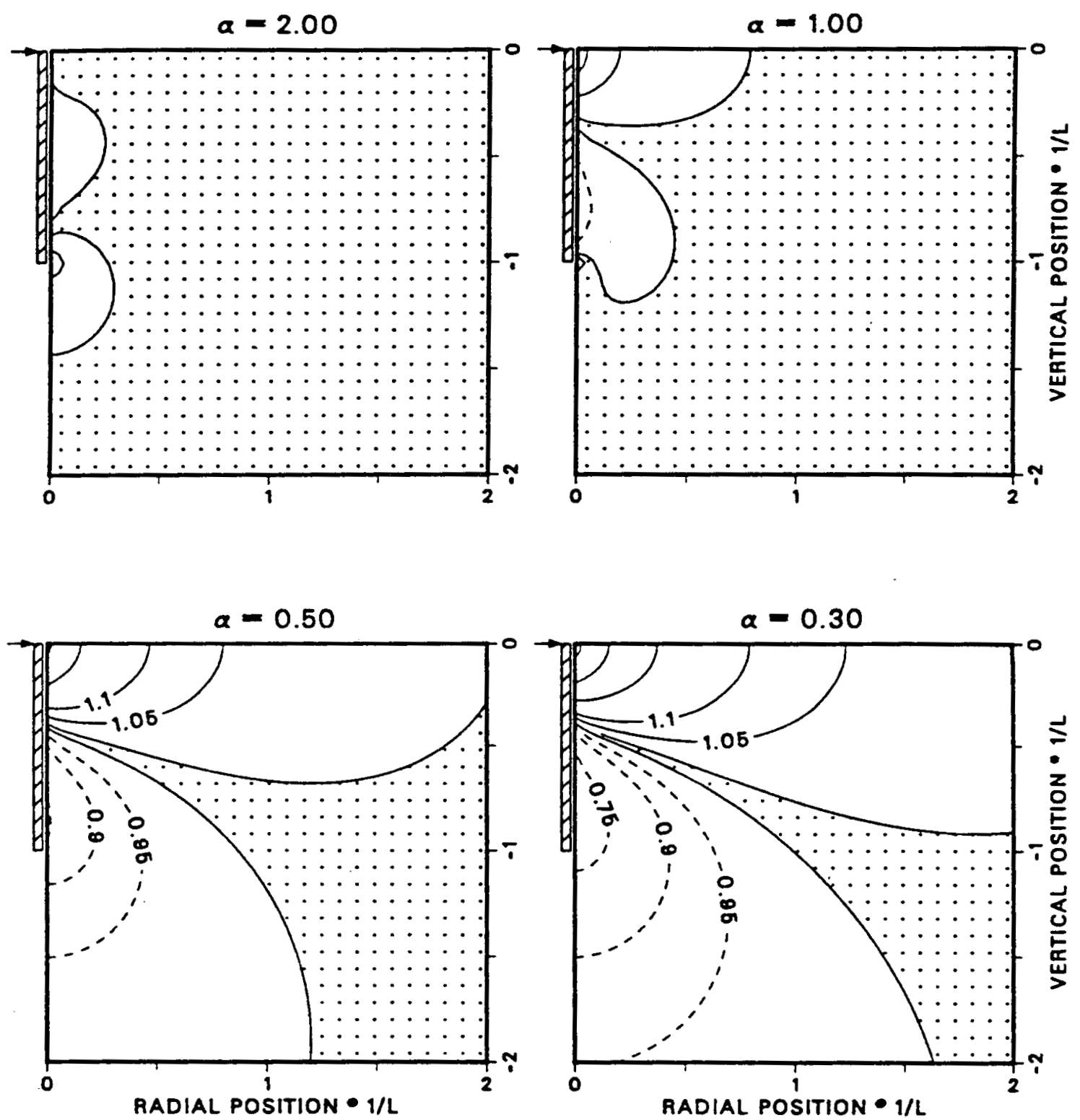

Figure 5.1-12: Contour plots of the ratio of the total to finite-length line source potential for several $\alpha_{L}$-ratios with the current source located at $S / L=0.0$. The dotted area represents locations where the total potential is within $1 \%$ of the line source potentials. 

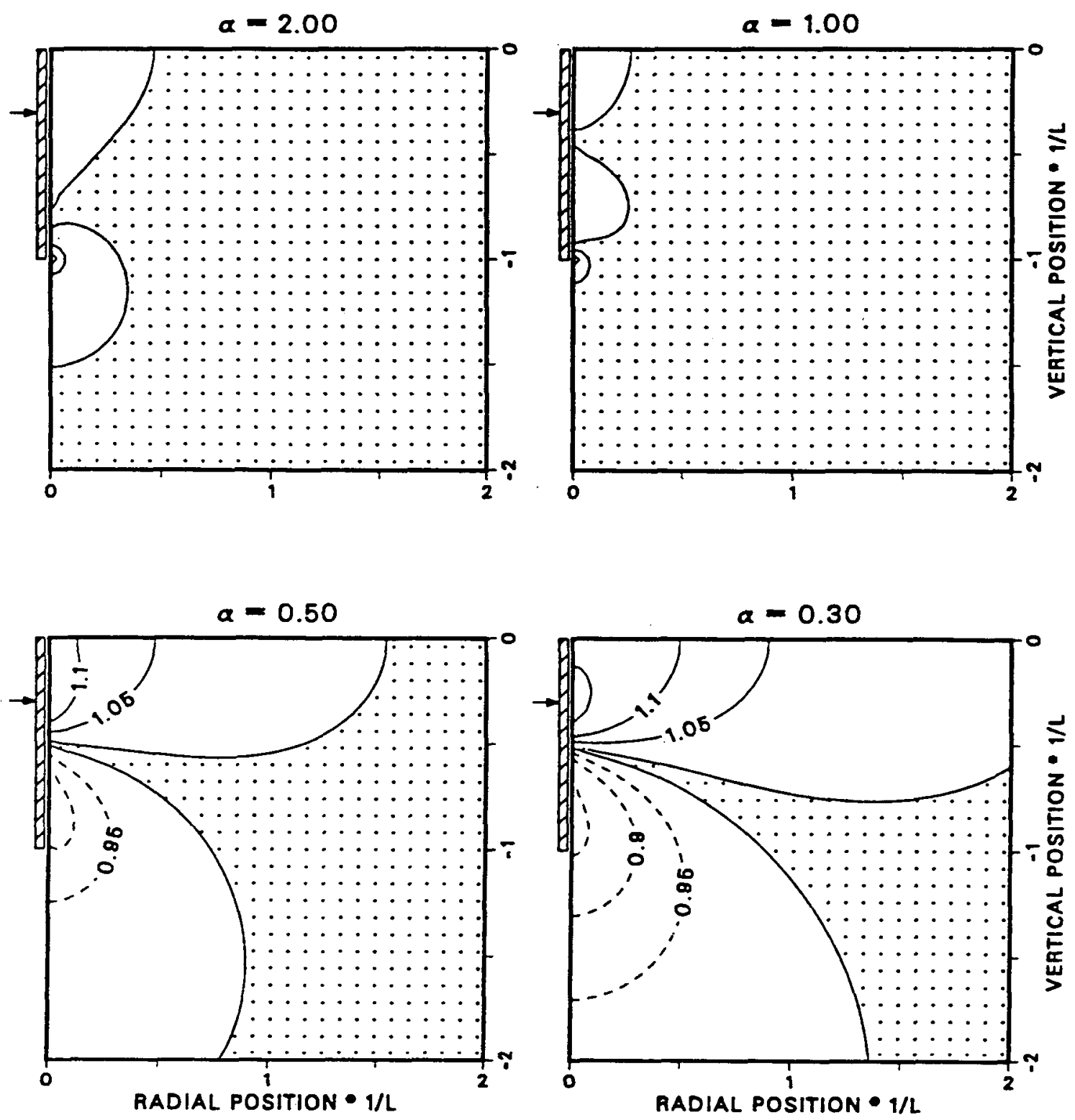

Figure 5.1-13: Contour plots of the ratio of the total to finite-length line source potential for several $\alpha_{L}$-ratios with the current source located at $S / L=0.3$. The dotted area represents locations where the total potential is within $1 \%$ of the line source potentials. 

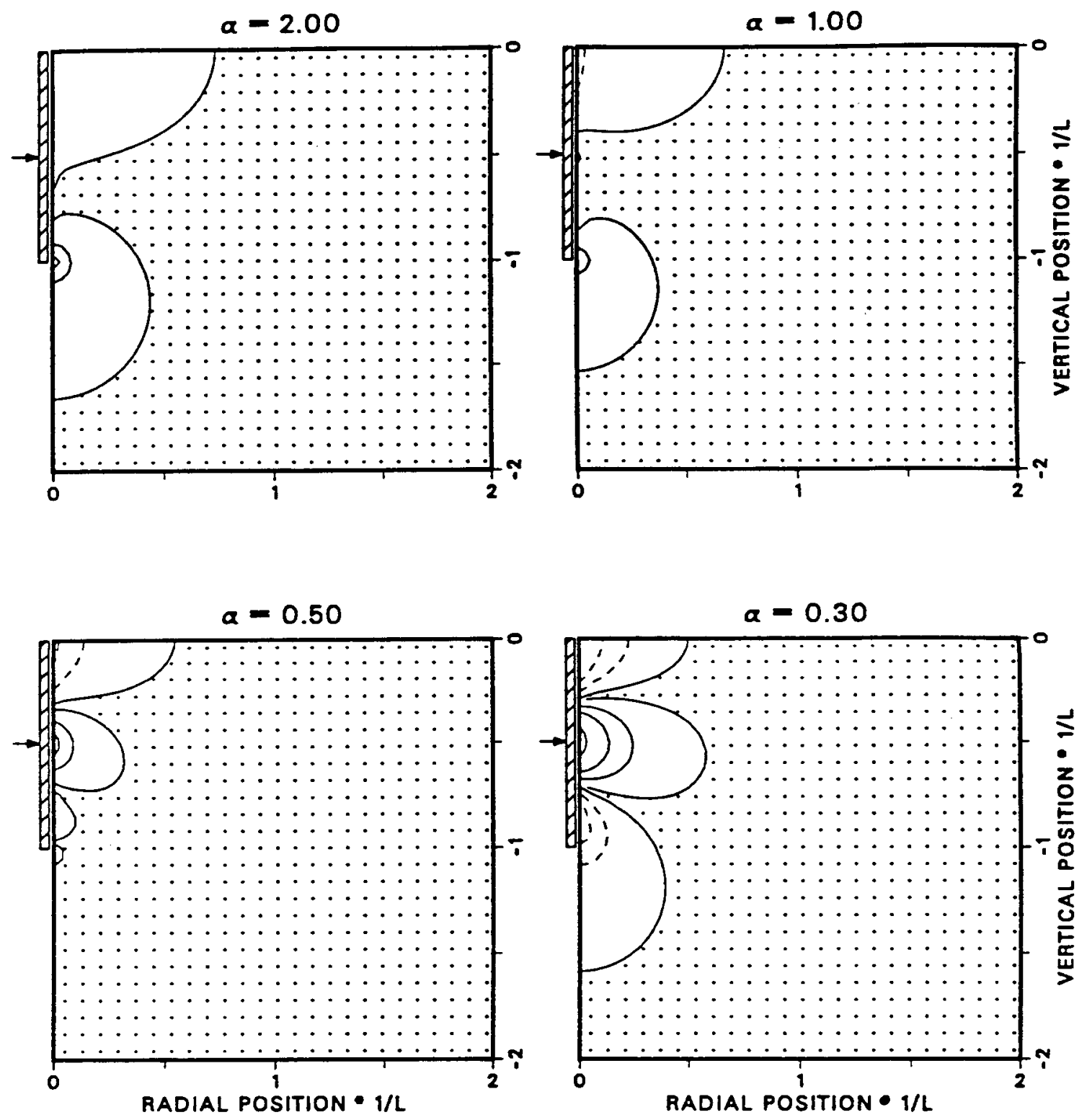

Figure 5.1-14: Contour plots of the ratio of the total to finite-length line source potential for several $\alpha_{L}$-ratios with the current source located at $S / L=0.5$. The dotted area represents locations where the total potential is within $1 \%$ of the line source potentials. 

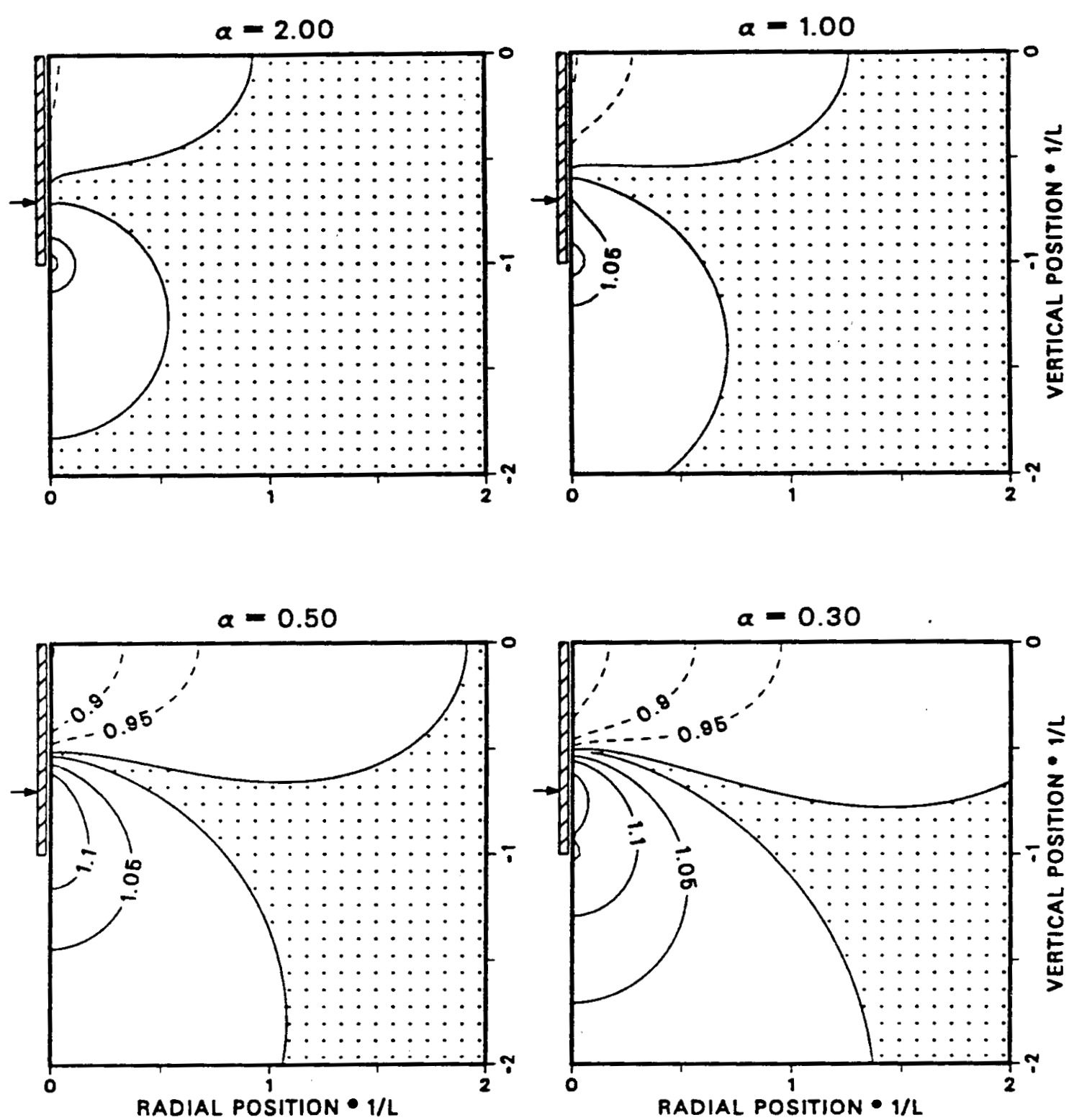

Figure 5.1-15: Contour plots of the ratio of the total to finite-length line source potential for several $\alpha_{L}$-ratios with the current source located at $S / L=0.7$. The dotted area represents locations where the total potential is within $1 \%$ of the line source potentials. 

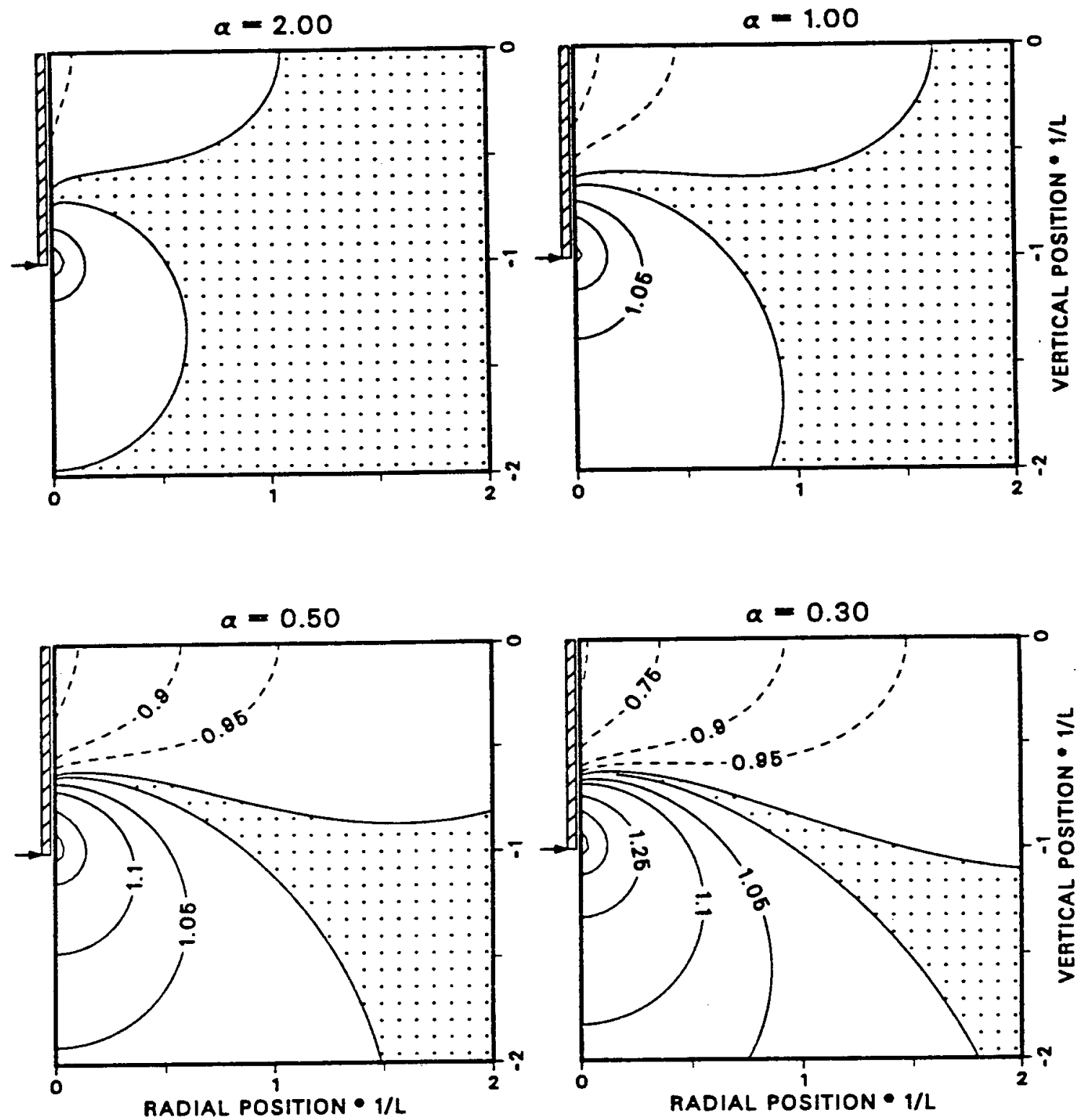

Figure 5.1-16: Contour plots of the ratio of the total to finite-length line source potential for several $\alpha_{L}$-ratios with the current source located at $S / L=1.0$. The dotted area represents locations where the total potential is within $1 \%$ of the line source potentials. 


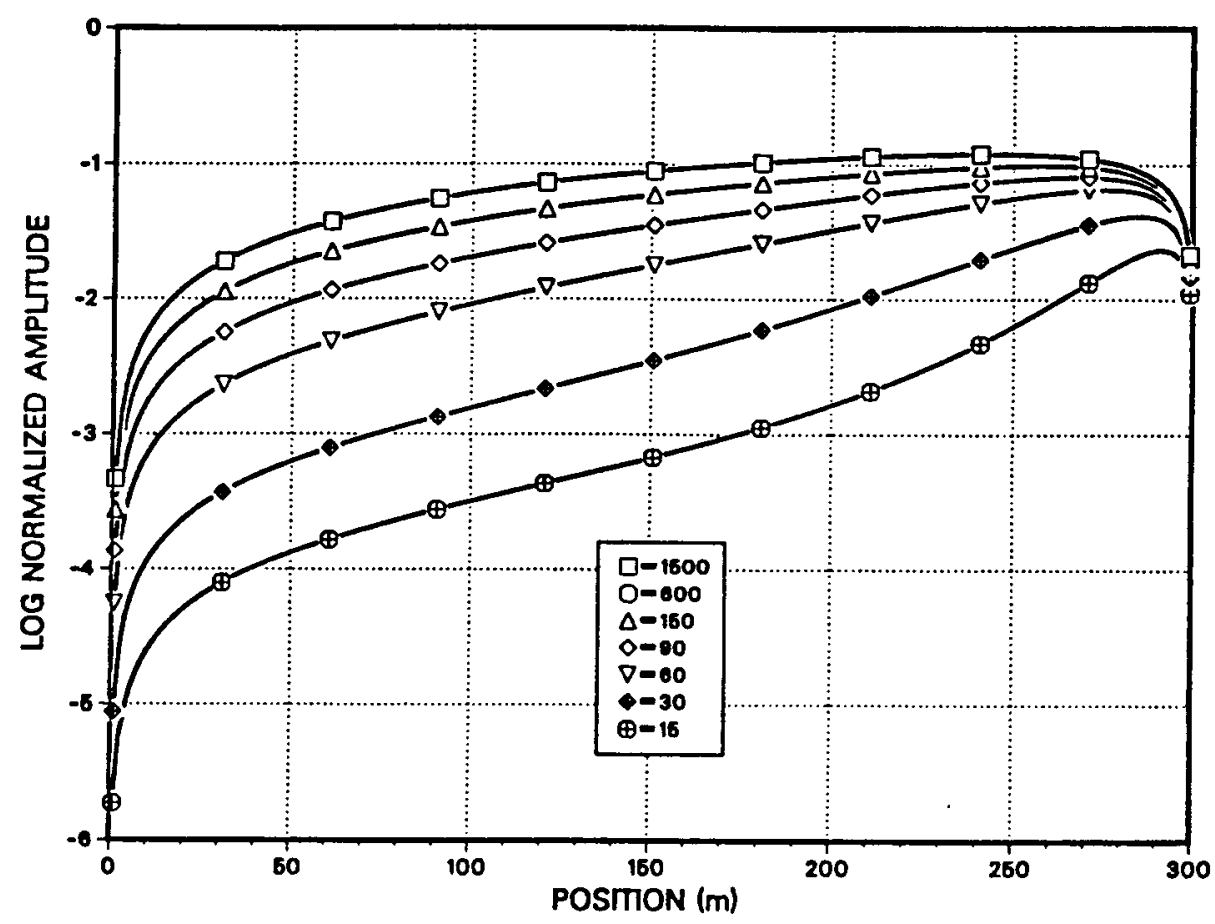

Figure 5.2-1: Semi-log plots of the normalized half-space $E_{z}$ in a $300 \mathrm{~m}$ pipe for several $\delta_{L}$ with $\Delta s=10 \mathrm{~m}$. The $\mathrm{E}_{z}$ is normalized by the factor $I / S_{c}$. 


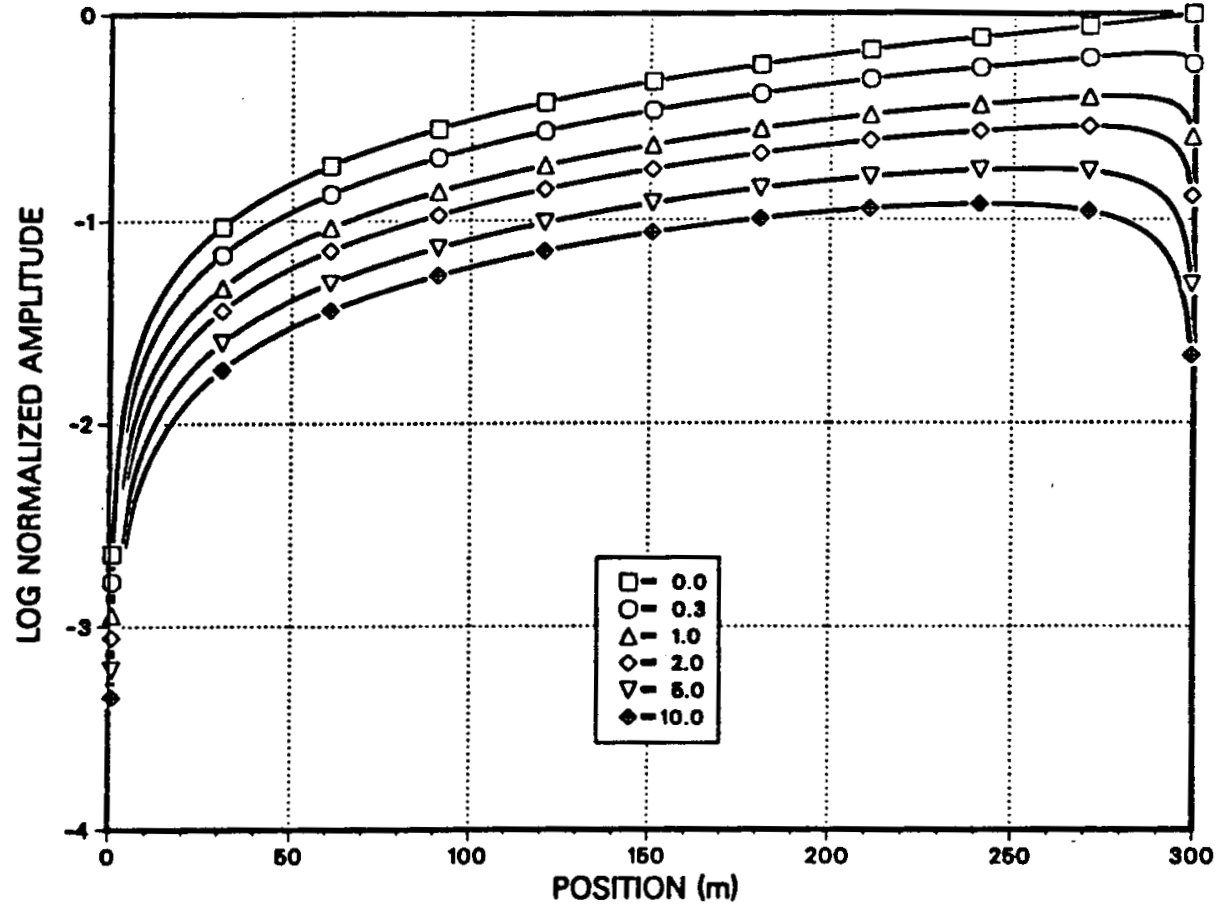

Figure 5.2-2a: Semi-log plots of the normalized half-space $E_{z}$ in a $300 \mathrm{~m}$ pipe for several $\Delta s$ with $\delta_{L}=600 \mathrm{~m}$. The $\mathrm{E}_{z}$ is normalized by the factor $I / S_{c}$.

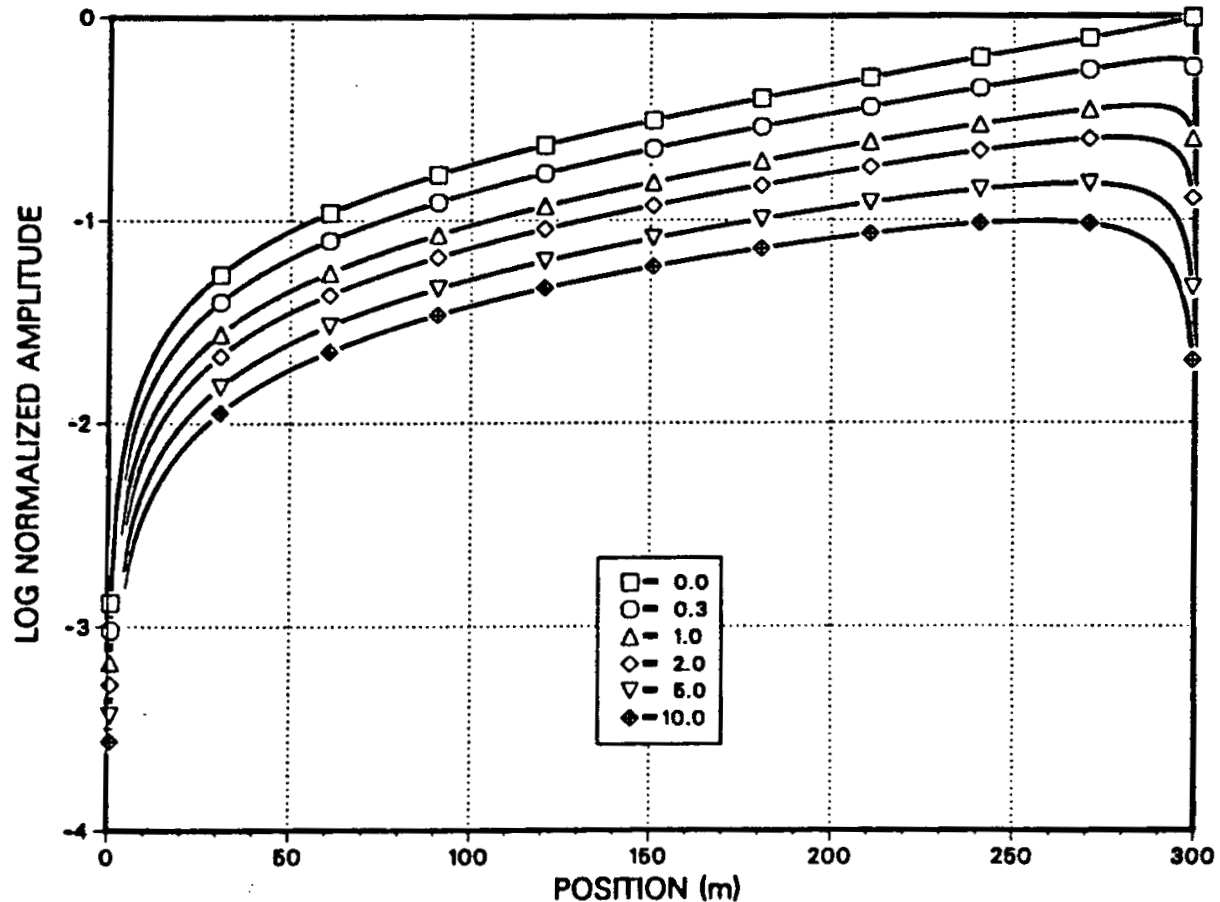

Figure 5.2-2b: Semi-log plots of the normalized half-space $E_{z}$ in a $300 \mathrm{~m}$ pipe for several $\Delta s$ with $\delta_{L}=150 \mathrm{~m}$. The $\mathrm{E}_{\mathrm{z}}$ is normalized by the factor $I / S_{c}$. 


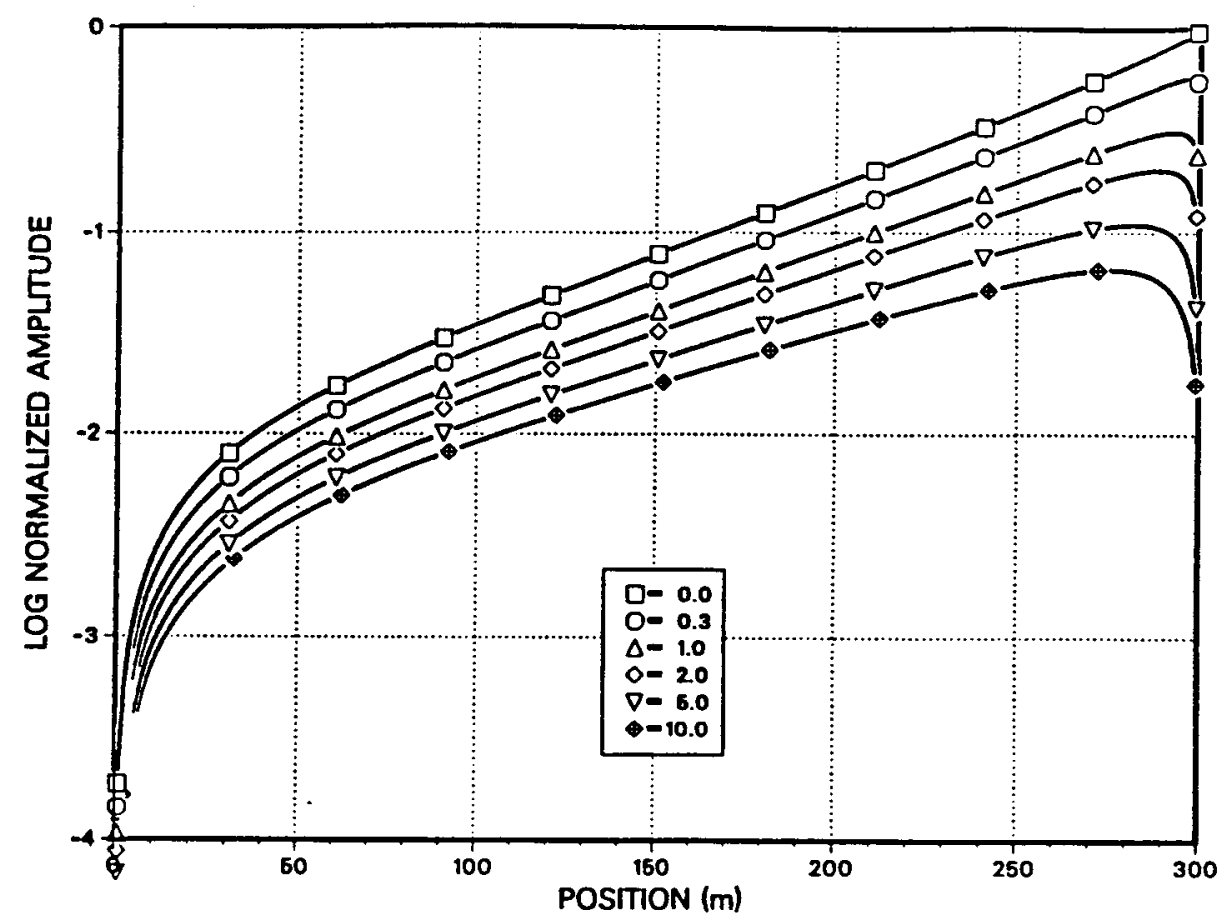

Figure 5.2-2c: Semi-log plots of the normalized half-space $E_{z}$ in a $300 \mathrm{~m}$ pipe for several $\Delta s$ with $\delta_{L}=60 \mathrm{~m}$. The $E_{z}$ is normalized by the factor $I / S_{c}$.

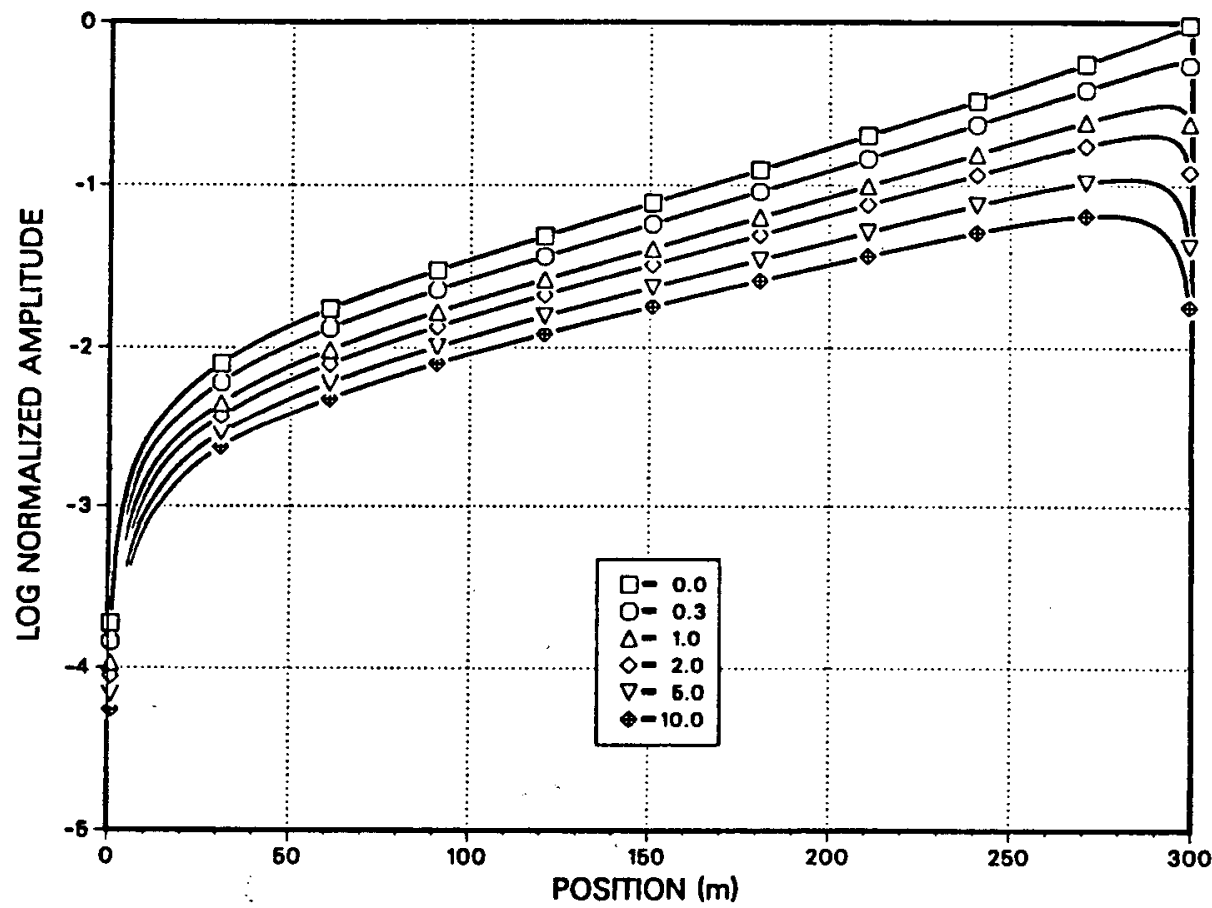

Figure 5.2-2d: Semi-log plots of the normalized half-space $E_{\mathbf{z}}$ in a $300 \mathrm{~m}$ pipe for several $\Delta s$ with $\delta_{L}=30 \mathrm{~m}$. The $E_{z}$ is normalized by the factor $I / S_{c}$. 

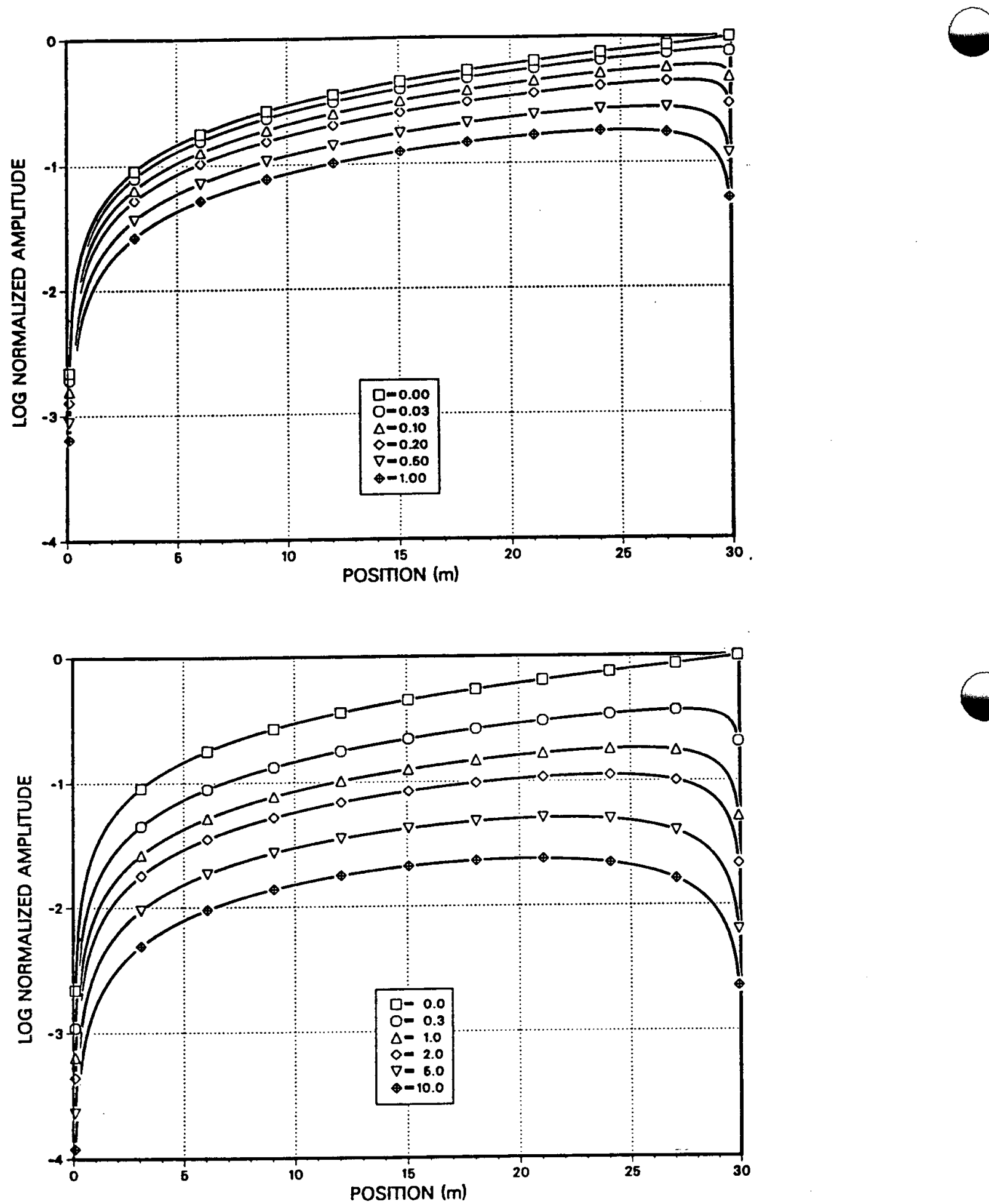

Figure 5.2-3: Semi-log plots of the normalized half-space $E_{z}$ in a $30 \mathrm{~m}$ pipe for $\Delta s \leq 1 \mathrm{~m}$ (top) and $\Delta s \geq 1 \mathrm{~m}$ (bottom) with $\delta_{L}=60 \mathrm{~m}$. The $E_{z}$ is normalized by the factor $I / S_{c}$. 

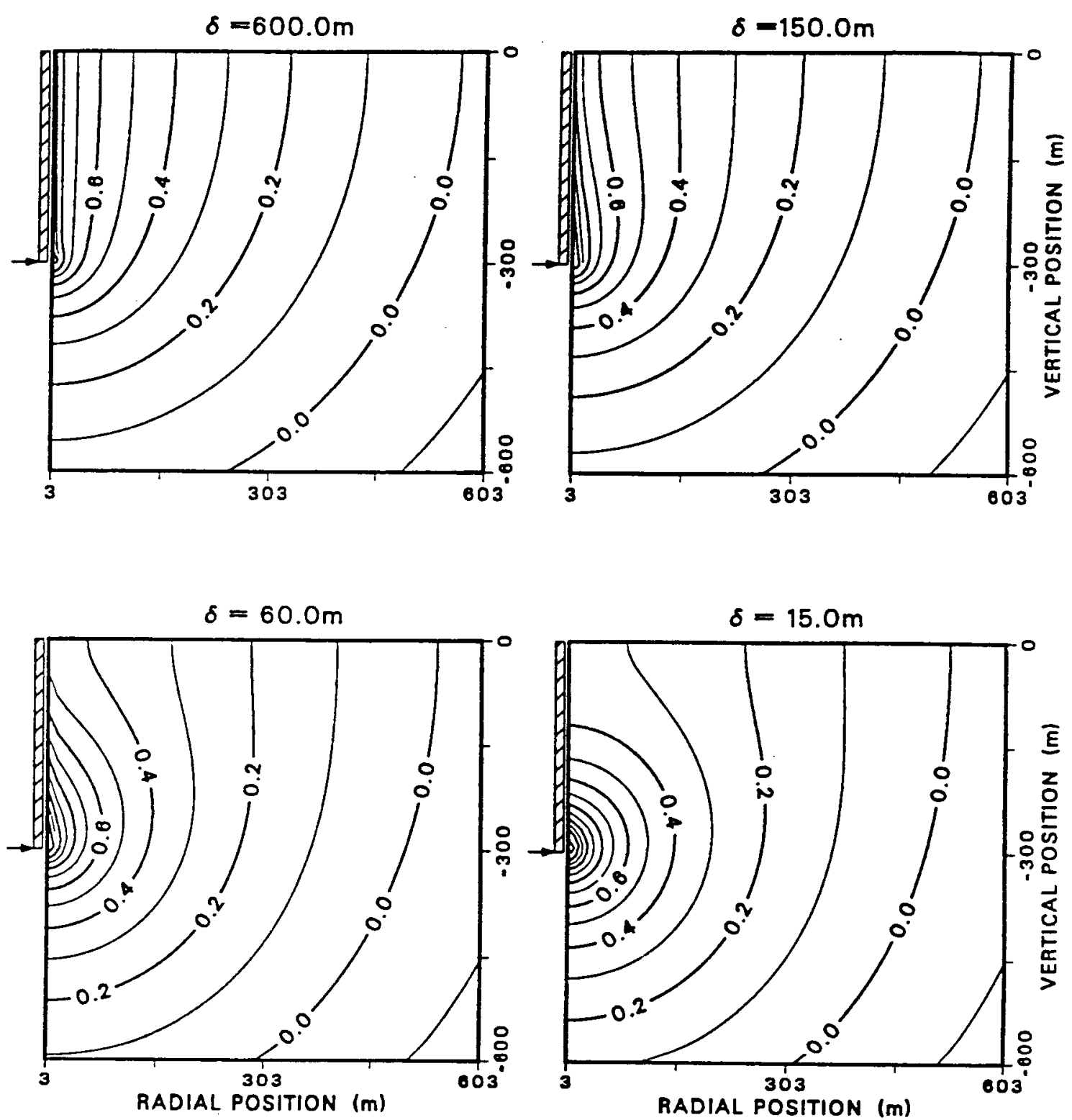

Figure 5.2-4: Log contour plots of the normalized half-space potentials due to a $300 \mathrm{~m}$ pipe for several $\delta_{L}$ with current source located at $\Delta s=0.10 \mathrm{~m}$. The potential is normalized by the factor $I / 4 \pi \sigma_{0} L$. 

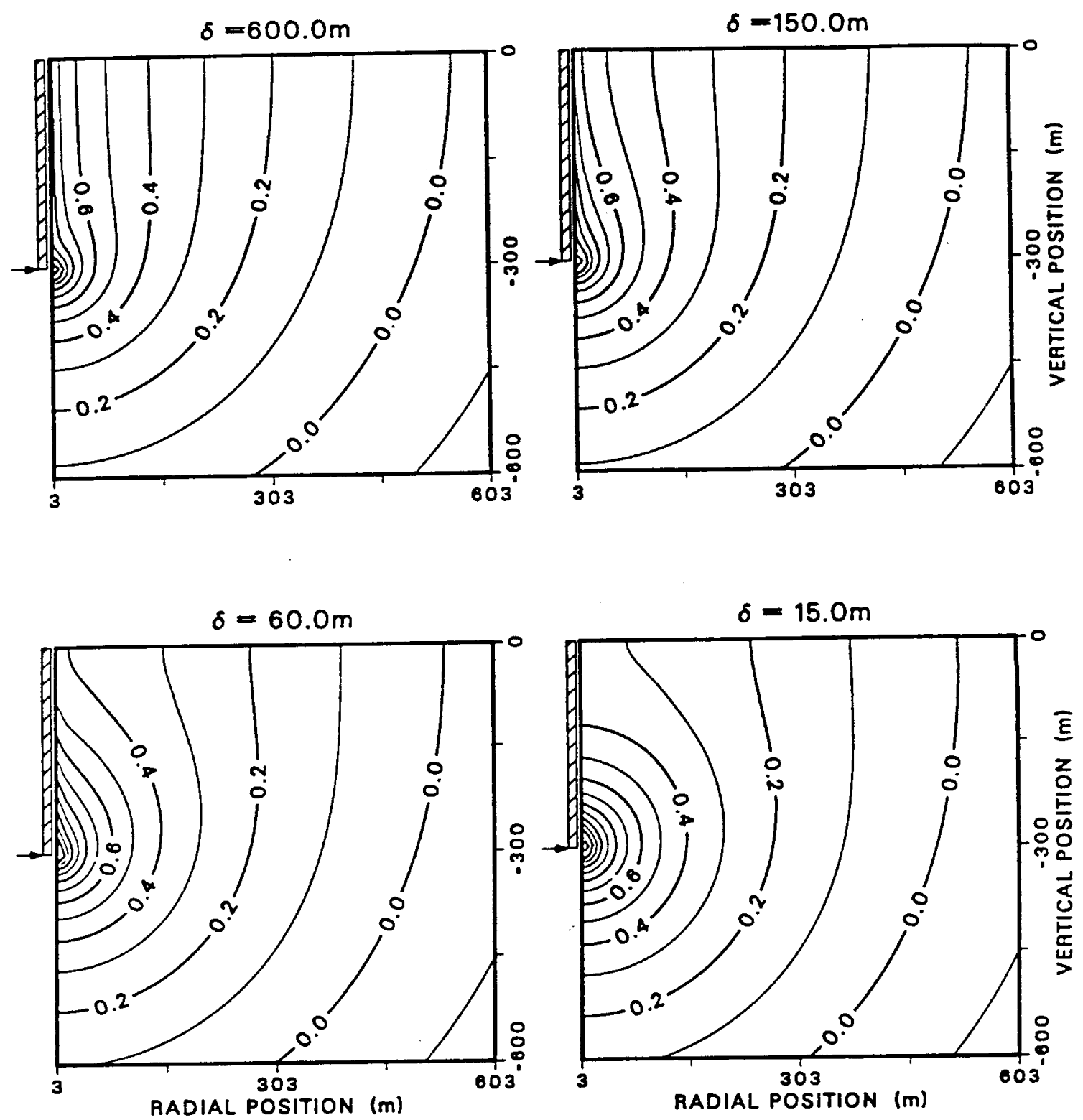

Figure 5.2-5: Log contour plots of the normalized half-space potentials due to a $300 \mathrm{~m}$ pipe for several $\delta_{L}$ with current source located at $\Delta s=0.50 \mathrm{~m}$. The potential is normalized by the factor $I / 4 \pi \sigma_{0} L$. 

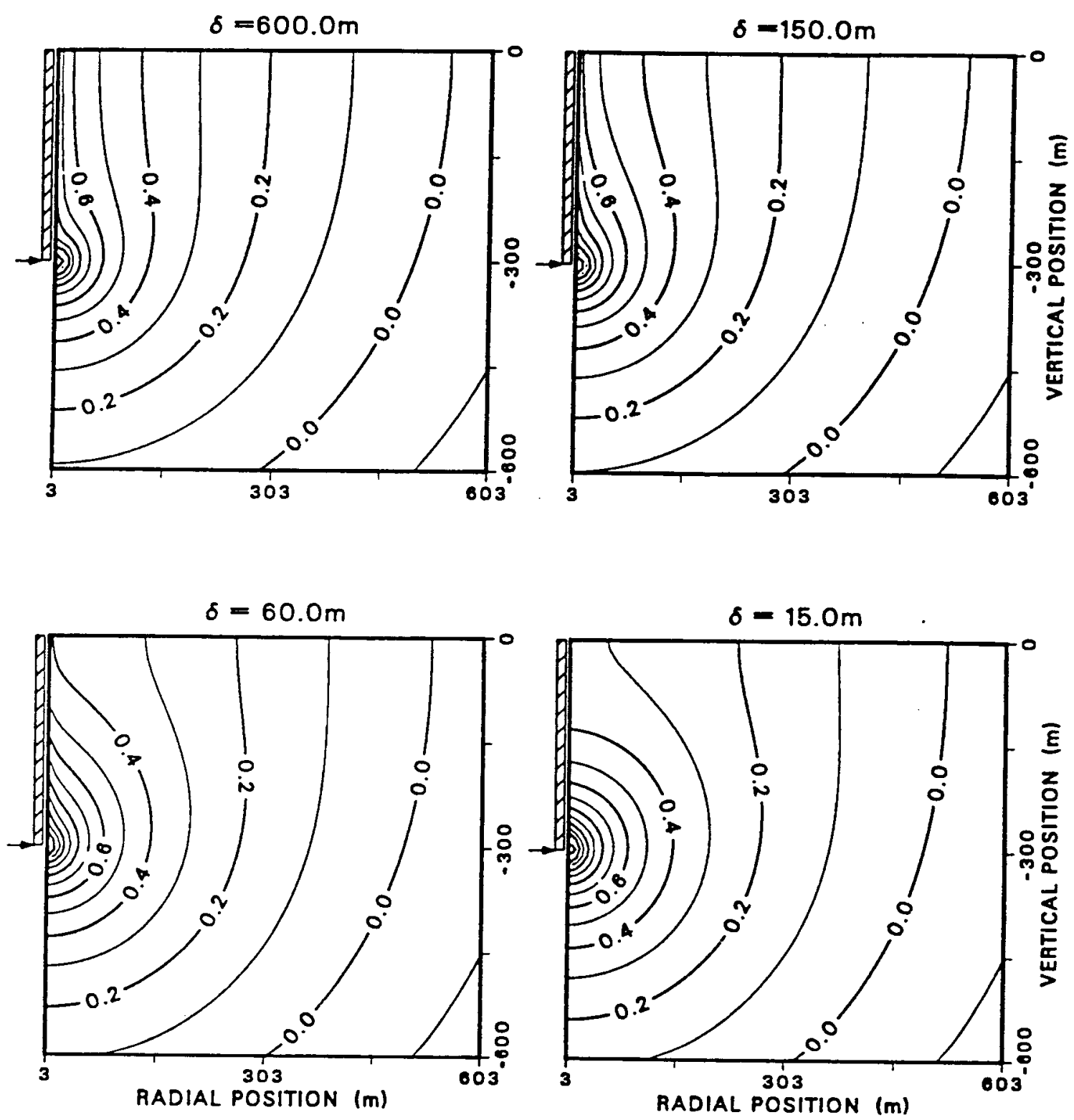

Figure 5.2-6: Log contour plots of the normalized half-space potentials due to a $300 \mathrm{~m}$ pipe for several $\delta_{L}$ with current source located at $\Delta s=1.0 \mathrm{~m}$. The potential is normalized by the factor $1 / 4 \pi \sigma_{0} L$. 

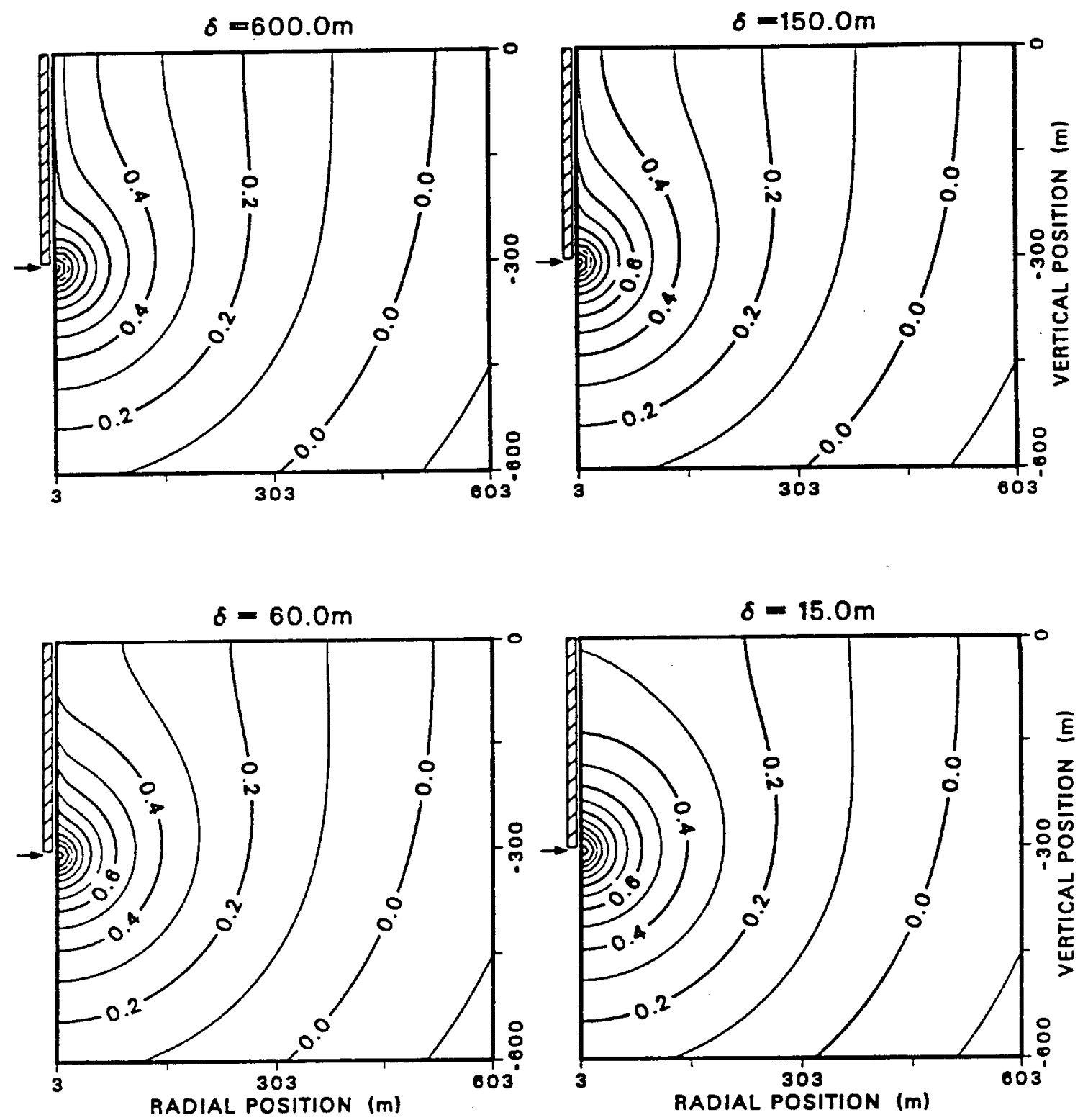

Figure 5.2-7: Log contour plots of the normalized half-space potentials due to a $300 \mathrm{~m}$ pipe for several $\delta_{L}$ with current source located at $\Delta s=5.0 \mathrm{~m}$. The potential is normalized by the factor $I / 4 \pi \sigma_{0} L$. 

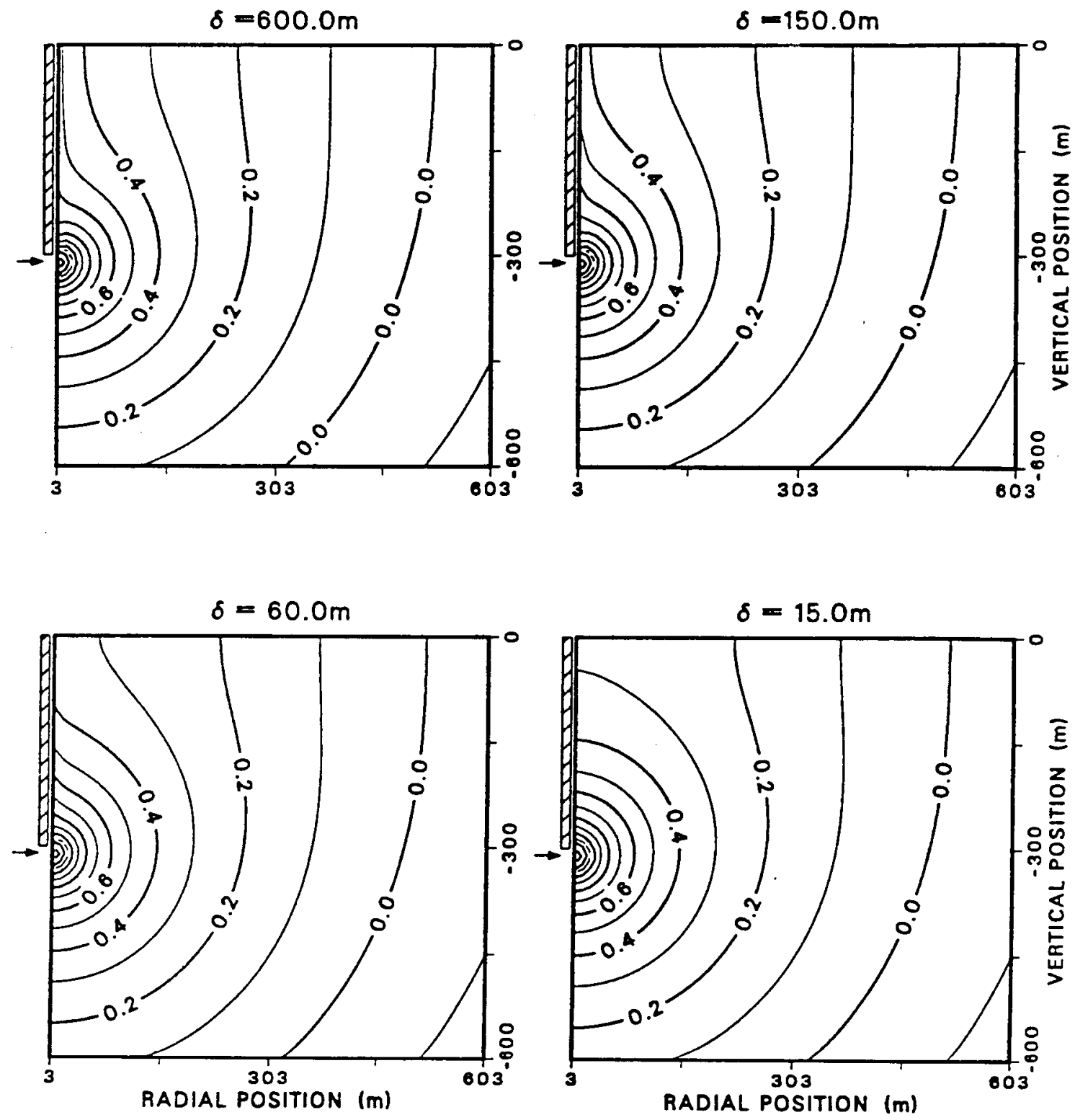

Figure 5.2-8: Log contour plots of the normalized half-space potentials due to a $300 \mathrm{~m}$ pipe for several $\delta_{L}$ with current source located at $\Delta s=10.0 \mathrm{~m}$. The potential is normalized by the factor $I / 4 \pi \sigma_{0} L$. 

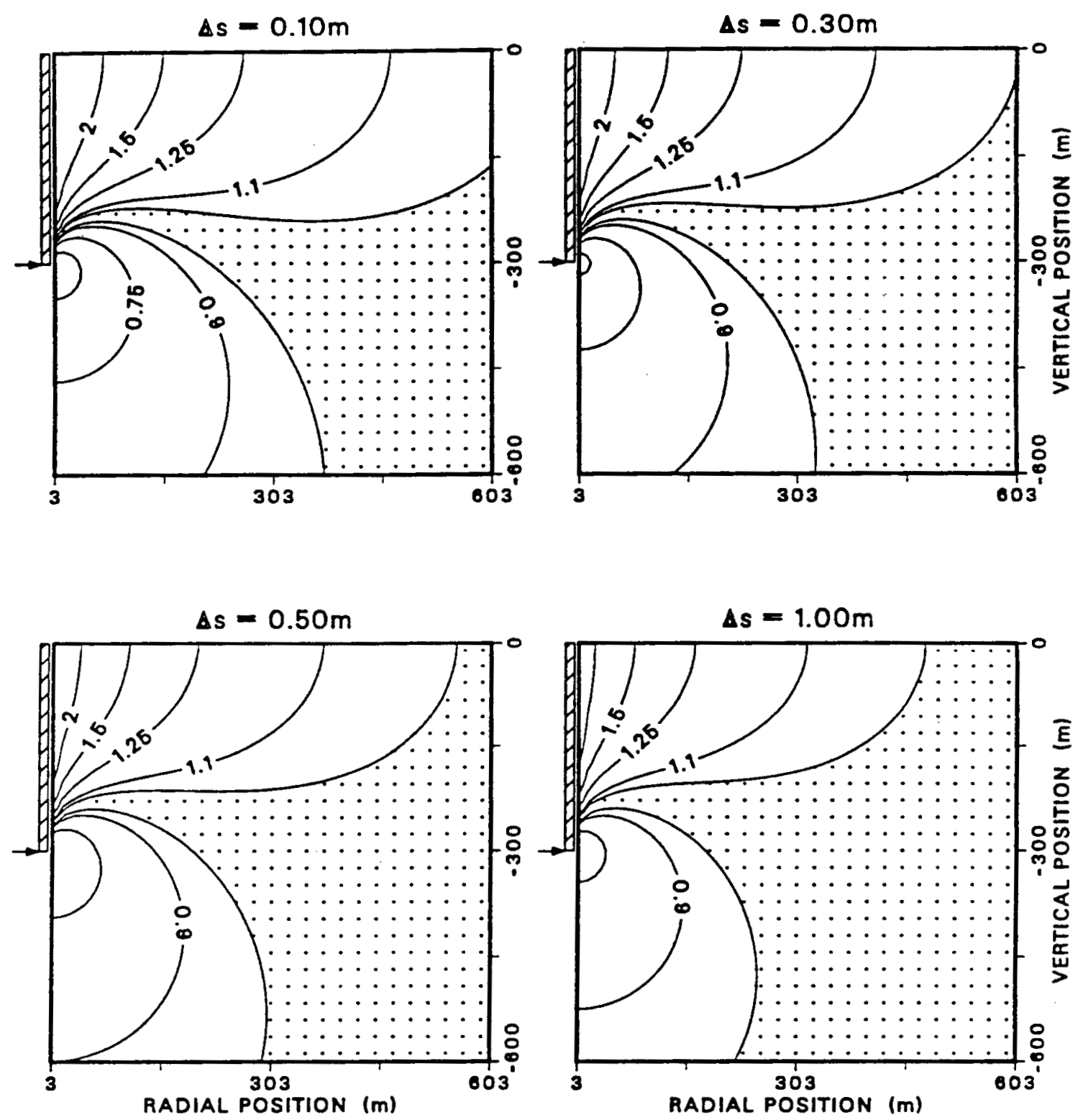

Figure 5.2-9a: Contour plots of the ratio of the total to primary fields due to a $300 \mathrm{~m}$ pipe for $\delta_{L}=600 \mathrm{~m}$ and current source located below the pipe at $\Delta s=0.1 \mathrm{~m}$ to $1 \mathrm{~m}$. The potential is normalized by the factor $I / 4 \pi \sigma_{0} L$. The dotted area represents $5 \%$ or less deviation of the total field from the primary field. Coincidentally, these plots are the ratio of the apparent and background resistivities for a pole-pole survey. 

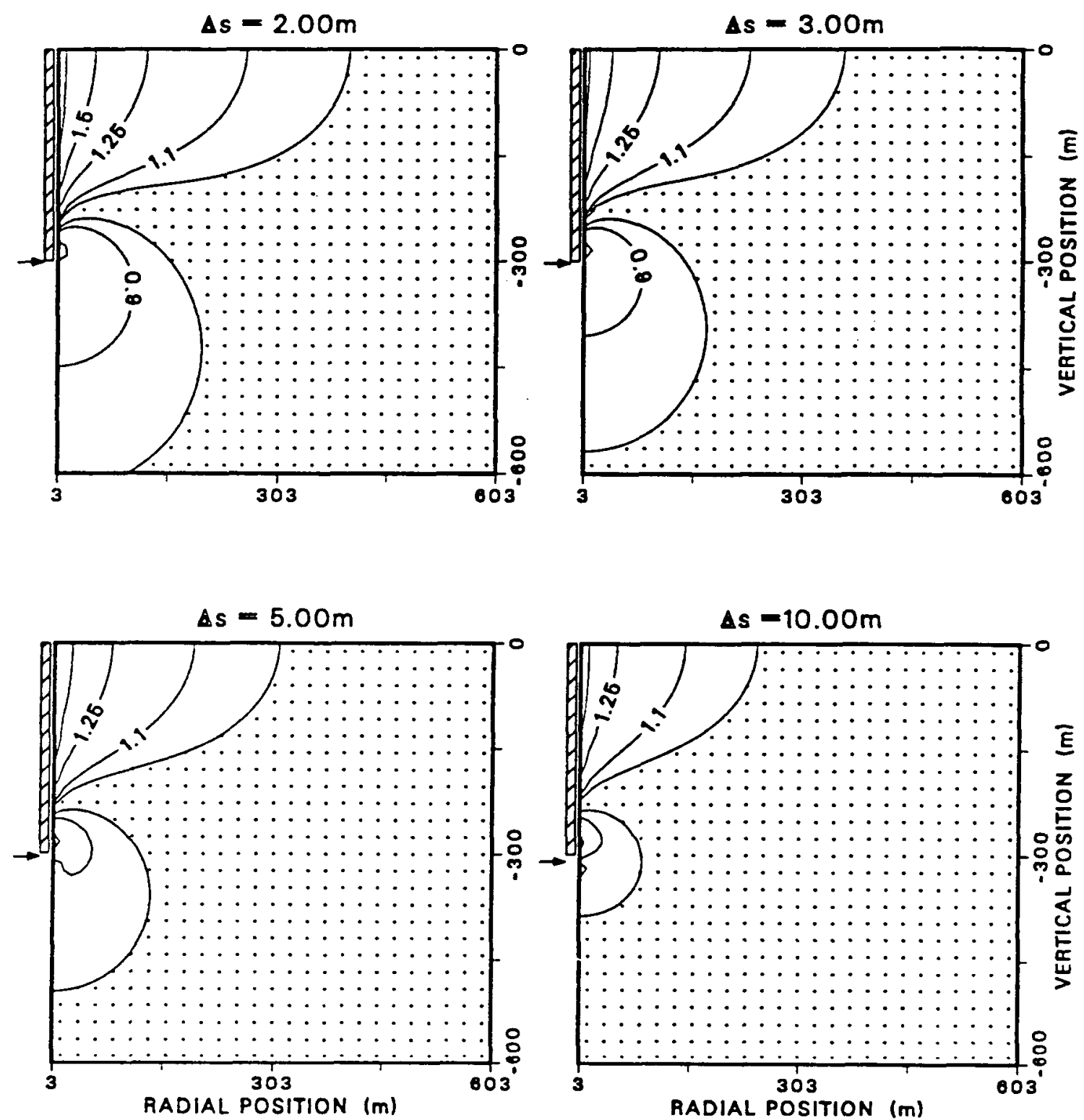

Figure 5.2-9b: Contour plots of the ratio of the total to primary fields due to a $300 \mathrm{~m}$ pipe for $\delta_{L}=600 \mathrm{~m}$ with current source located below the pipe at $\Delta s=2 \mathrm{~m}$ to $10 \mathrm{~m}$. The potential is normalized by the factor $I / 4 \pi \sigma_{0} L$. The dotted area represents $5 \%$ or less deviation of the total field from the primary field. Coincidentally, these plots are the ratio of the apparent and background resistivities for a pole-pole survey. 


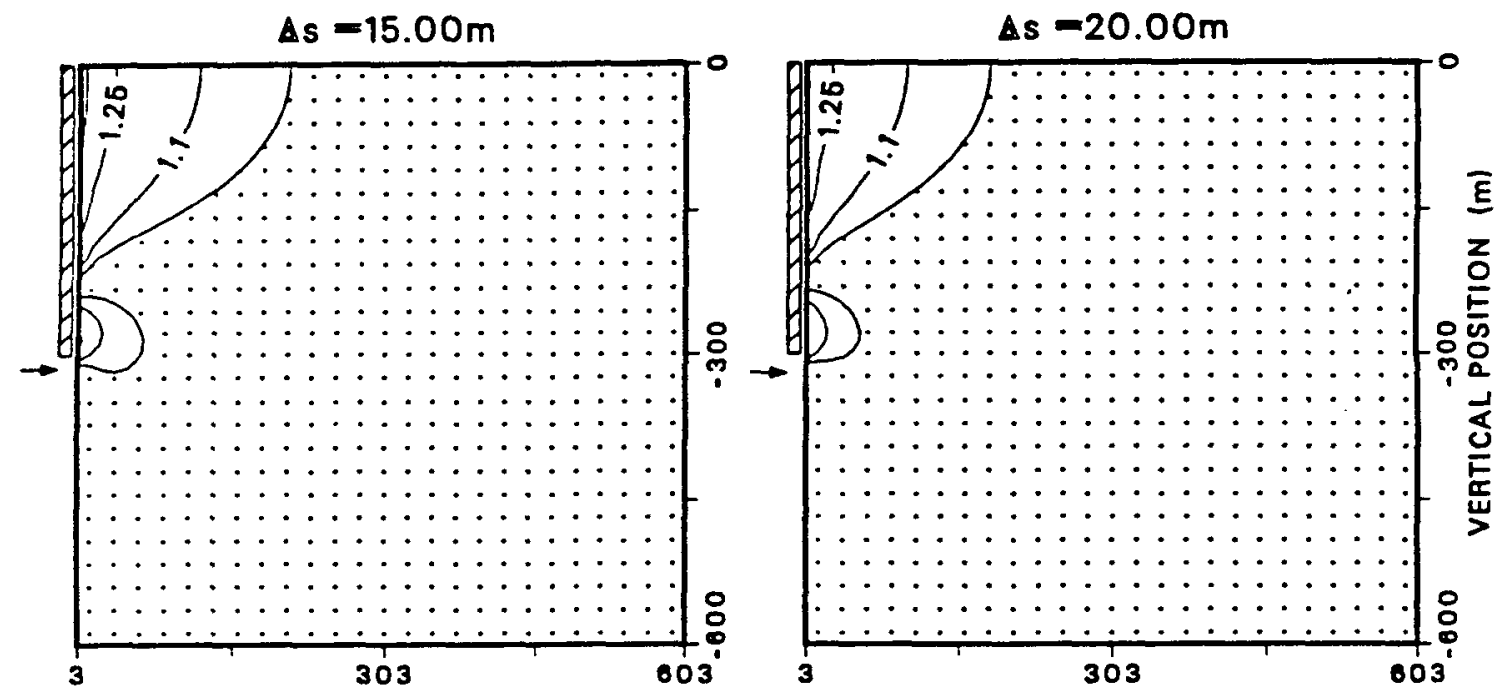

Figure 5.2-9c: Contour plots of the ratio of the total to primary fields due to a $300 \mathrm{~m}$ pipe for $\delta_{L}=600 \mathrm{~m}$ with current source located below the pipe at $\Delta s=15 \mathrm{~m}$ to $20 \mathrm{~m}$. The potential is normalized by the factor $I / 4 \pi \sigma_{0} L$. The dotted area represents $5 \%$ or less deviation of the total field from the primary field. Coincidentally, these plots are the ratio of the apparent and background resistivities for a pole-pole survey. 

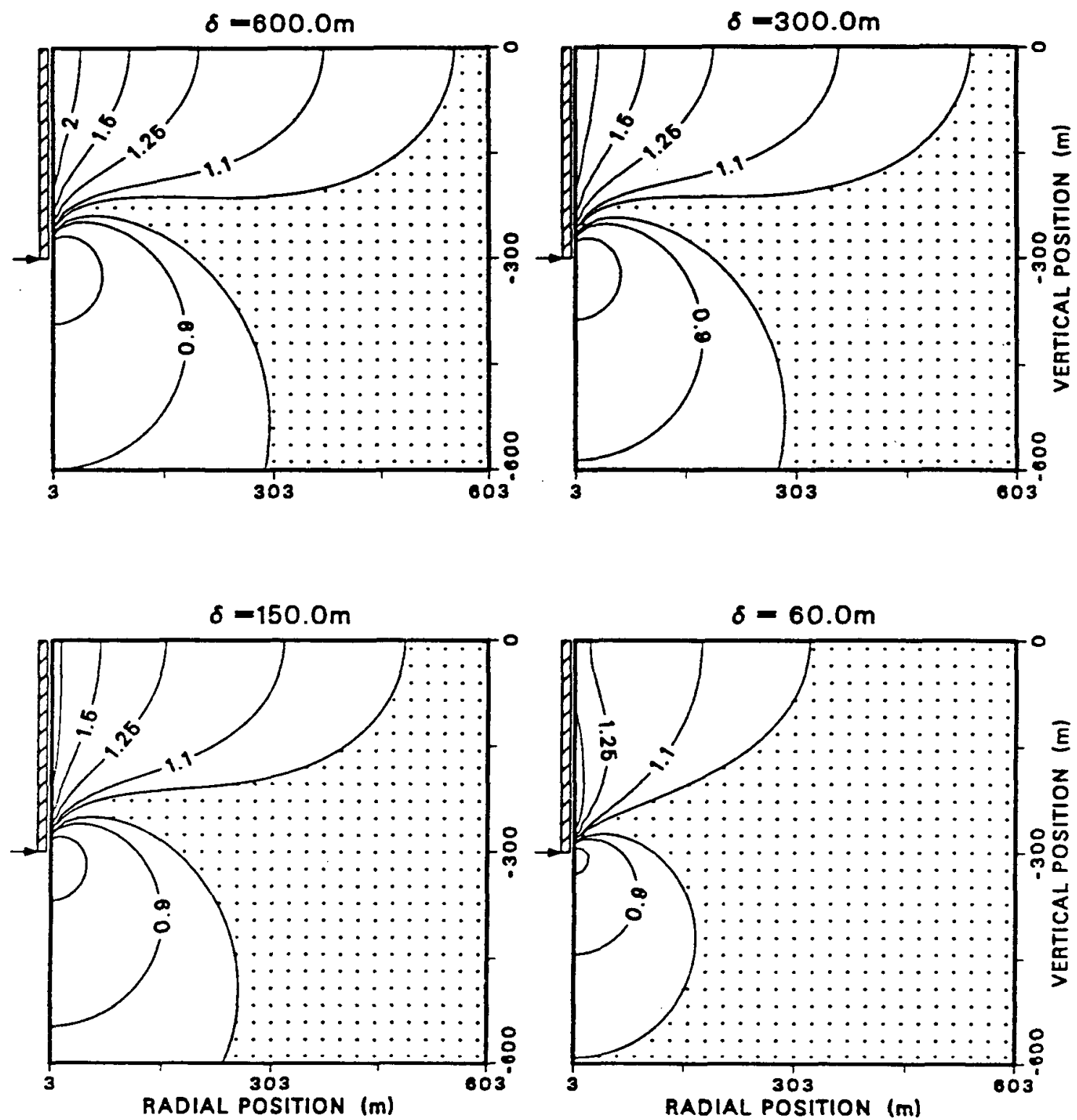

Figure 5.2-10: Contour plots of the ratio of the total to primary fields due to a $300 \mathrm{~m}$ pipe for several $\delta_{L}$ with the current source located $0.5 \mathrm{~m}$ below the pipe. The potential is normalized by the factor $I / 4 \pi \sigma_{0} L$. The dotted area represents $5 \%$ or less deviation of the total field from the primary field. Coincidentally, these plots are the ratio of the apparent and background resistivities for a pole-pole survey. 


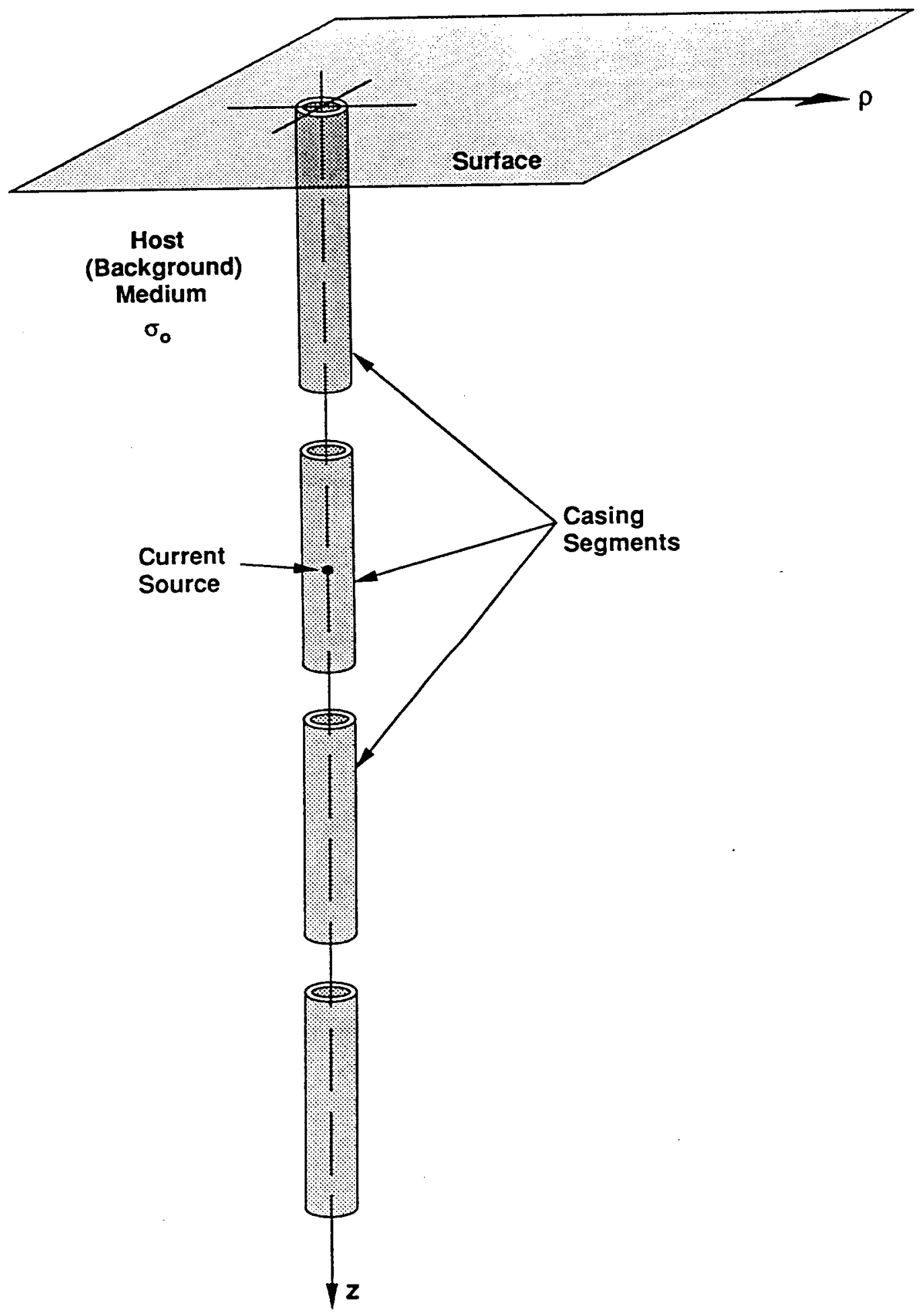

Figure 5.3-1: Generalized configuration of the multiple casing segments coaxial with an arbitrarily located pole current source in a homogeneous half-space. 


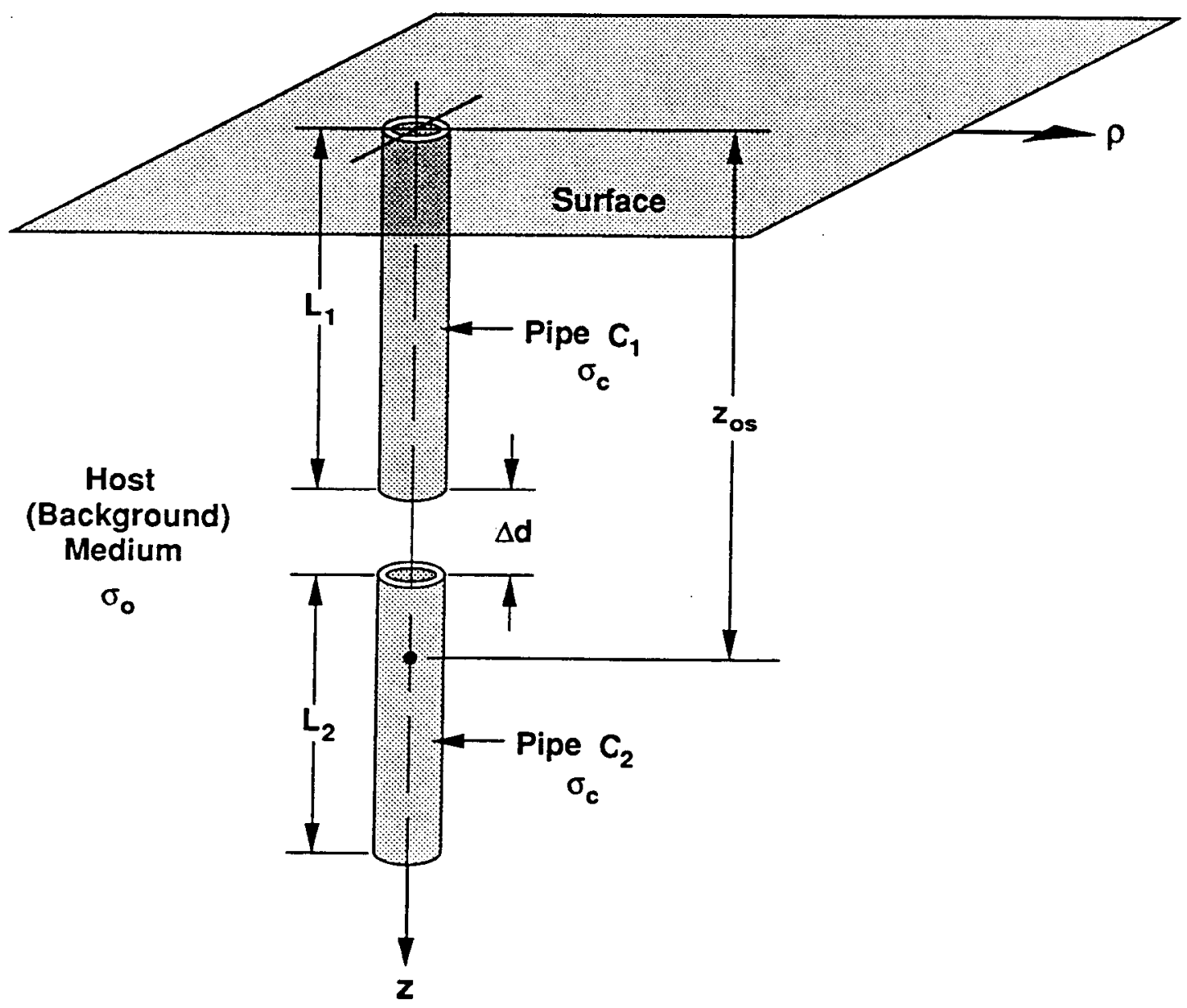

Figure 5.3-2: Model of the two pipe segments coaxial with an arbitrarily located pole current source in a homogeneous half-space. For the analysis, $L_{1}>L_{2}$. 


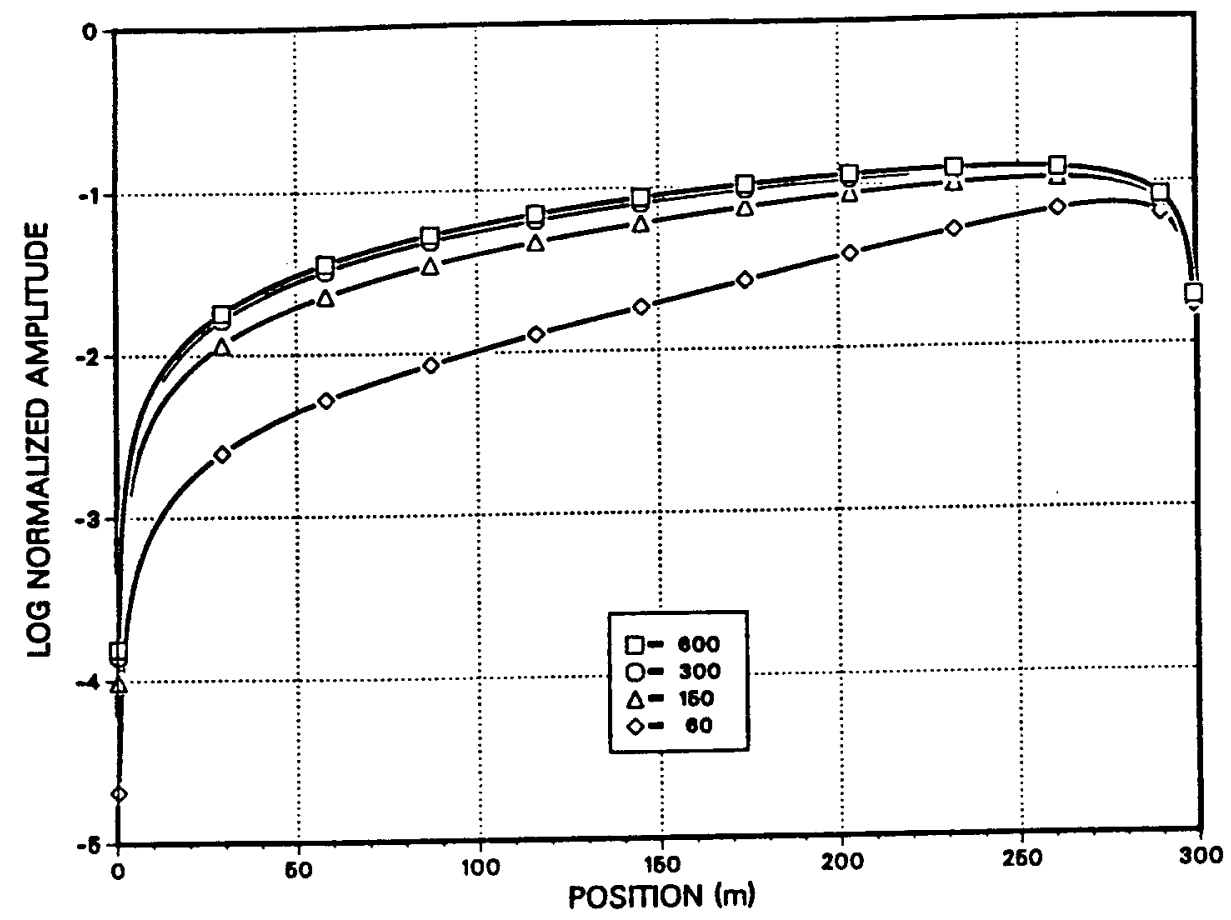

Figure 5.3-3: Semi-log plots of the normalized $E_{z}$ in the $300 \mathrm{~m} C_{1}$ for several $\delta_{L}$ with $\Delta d=0.2 \mathrm{~m}, L_{2}=5 \mathrm{~m}$, and current source located at the center of $C_{2} . E_{z}$ is normalized by the factor $I / S_{c}$.

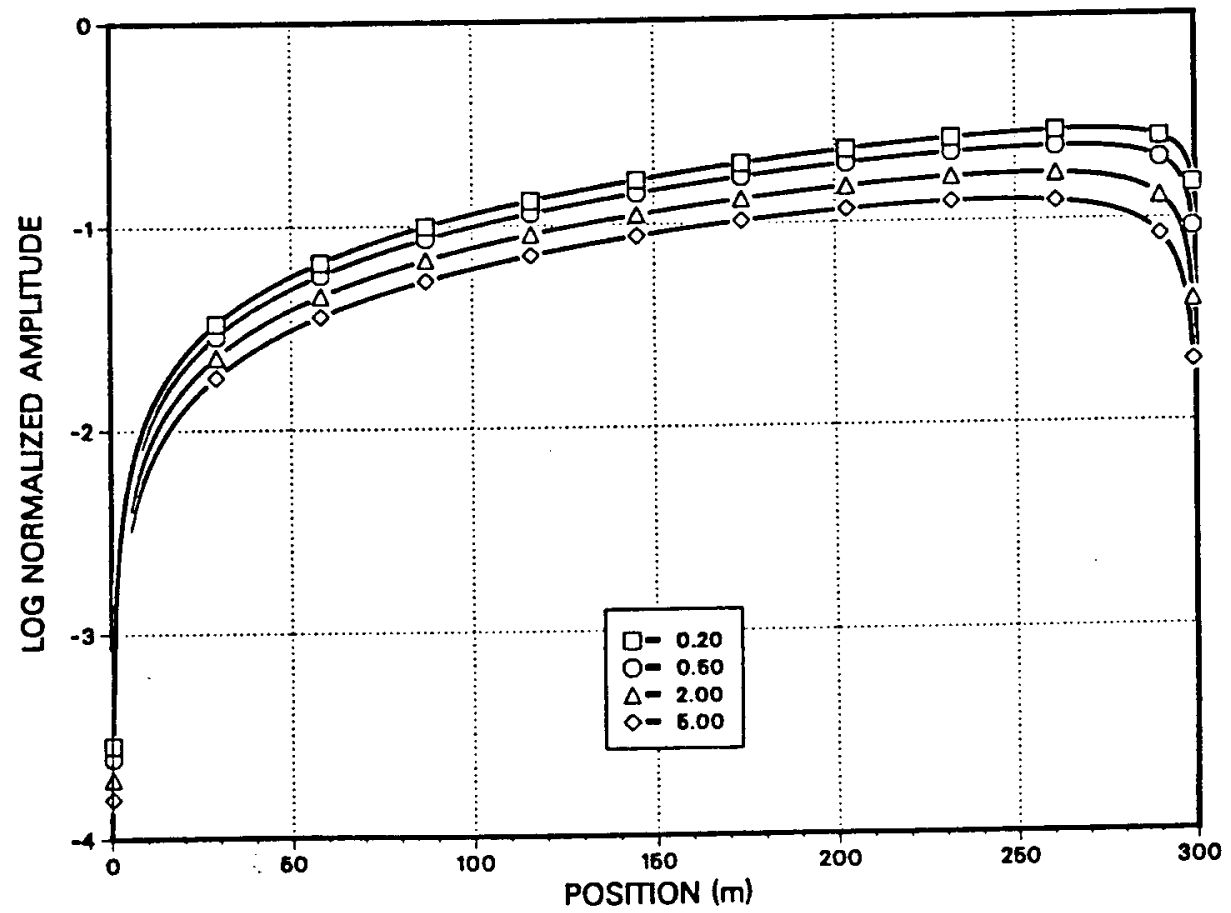

Figure 5.3-4: Semi-log plots of the normalized $E_{2}$ in the $300 \mathrm{~m} C_{1}$ for several $\Delta d$ with $\delta_{L}=600 \mathrm{~m}, L_{2}=5 \mathrm{~m}$, and current source located at the top of $C_{2} . \mathrm{E}_{2}$ is normalized by the factor $I / S_{c}$. 


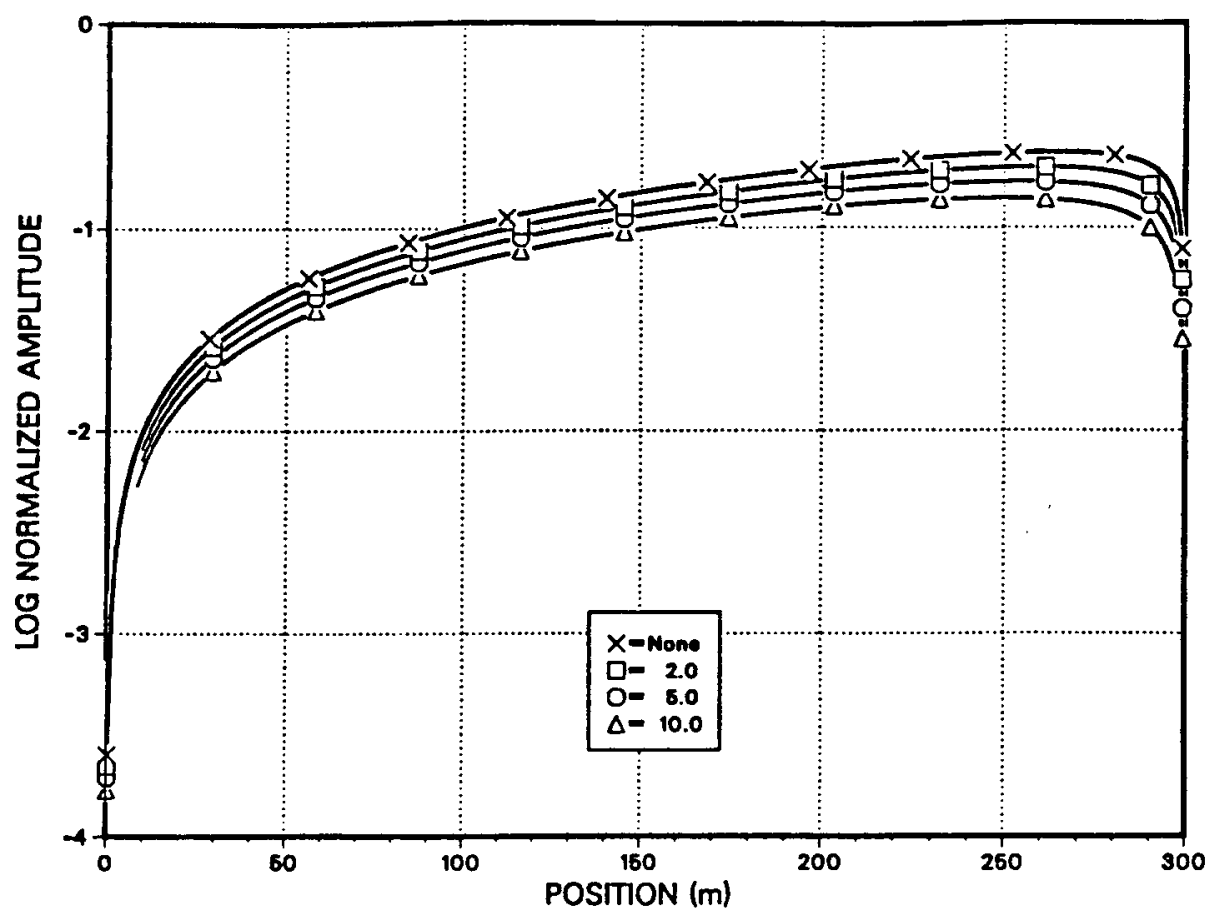

Figure 5.3-5: Semi-log plots of the normalized $\mathrm{E}_{\mathrm{z}}$ in the $300 \mathrm{~m} C_{1}$ for several $L_{2}$ with $\delta_{L}=600 \mathrm{~m}, \Delta d=2 \mathrm{~m}$, and current source located at the top of $C_{2} . E_{z}$ is normalized by the factor $I / S_{c}$.

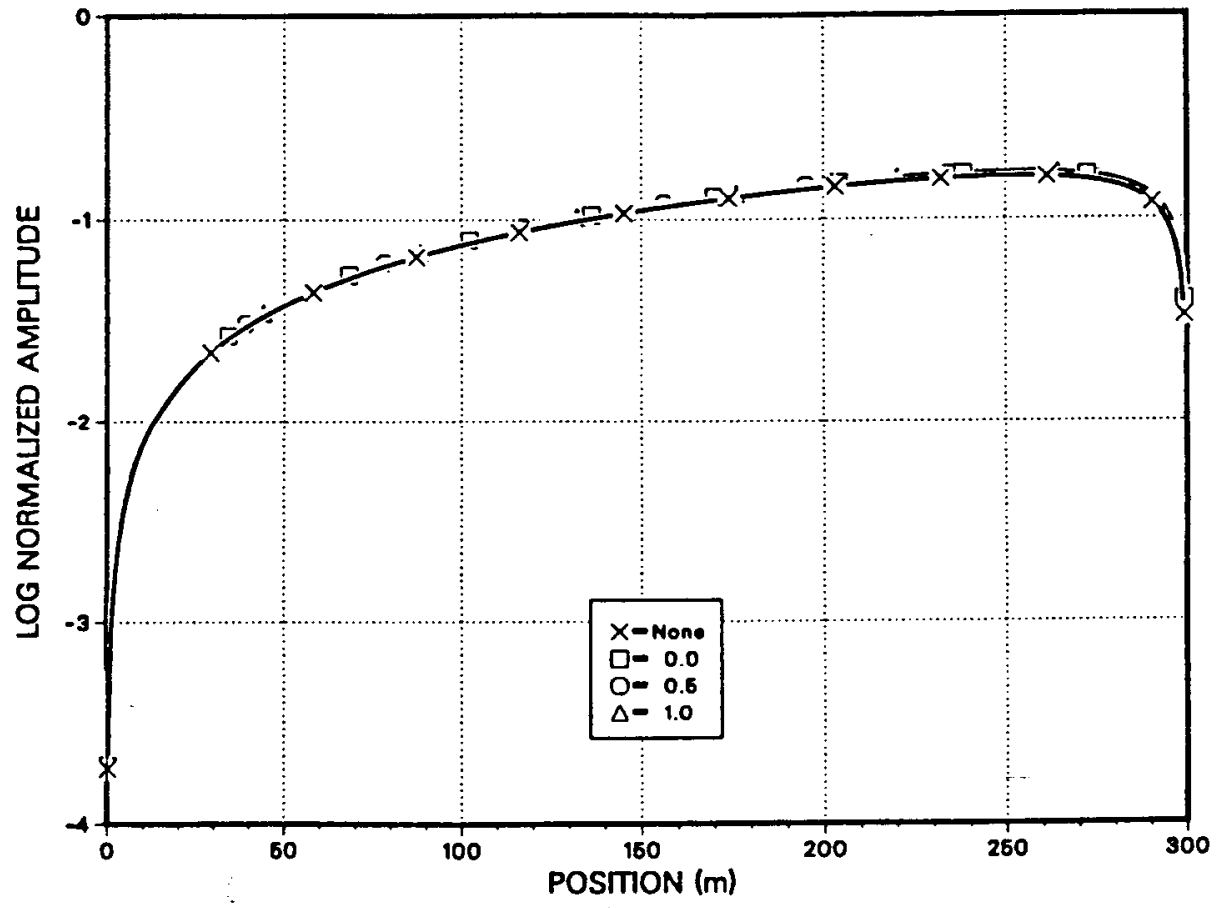

Figure 5.3-6: Semi-log plots of the normalized $E_{z}$ in the $300 \mathrm{~m} C_{1}$ for several $z_{0 S}$ with $\delta_{L}=600 \mathrm{~m}, \Delta d=2 \mathrm{~m}$, and $L_{2}=5 \mathrm{~m}$. The top, center, and bottom positions of the source within $C_{2}$ are represented by $0.0,0.5$, and 1.0 , respectively. "None" is the source placed at 0.5 position with no $C_{2}$. $E_{z}$ is normalized by the factor $I / S_{c}$. 

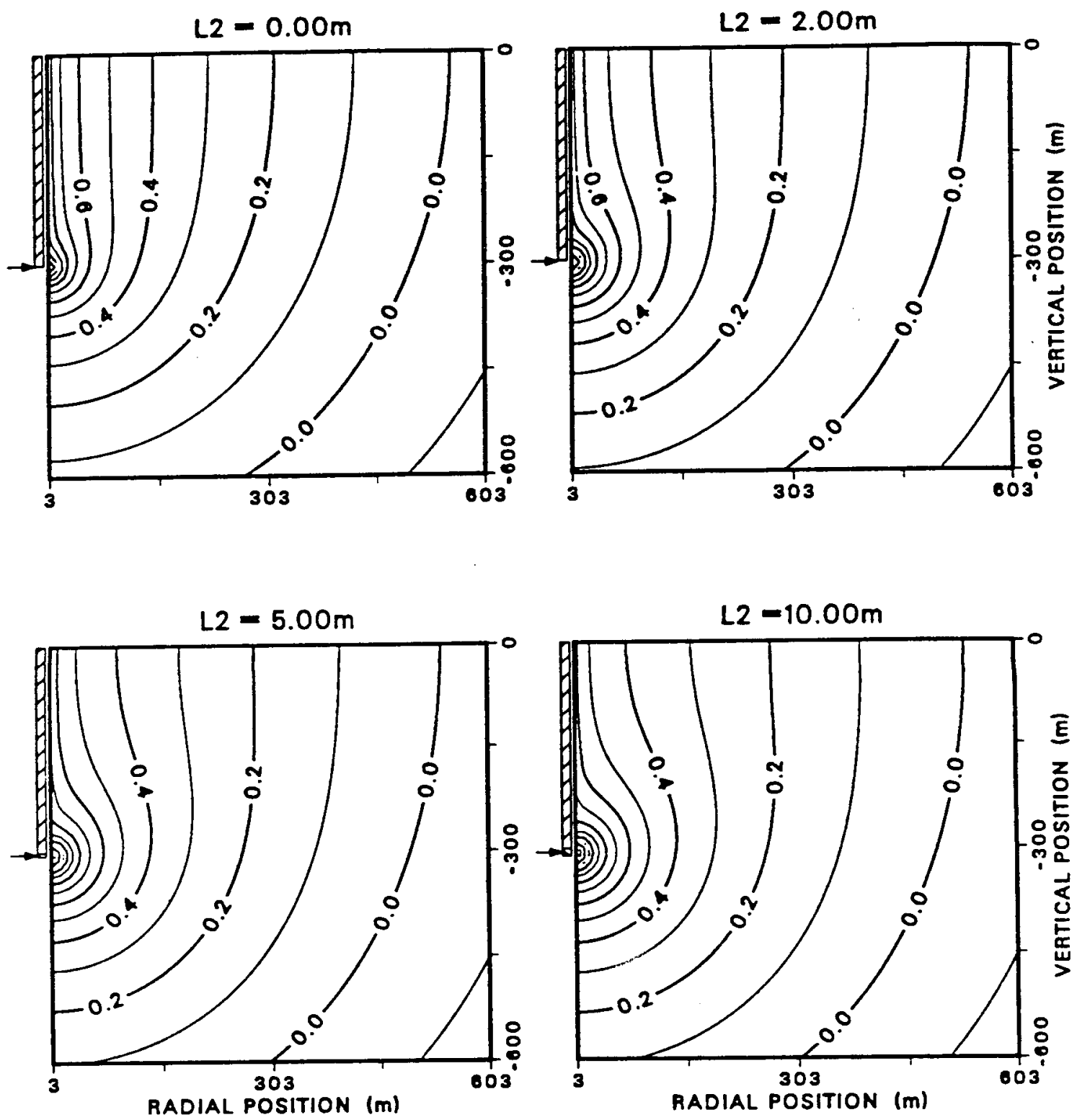

Figure 5.3-7a: Log-contour plots of the normalized half-space potentials due to the $300 \mathrm{~m} C_{1}$ for several $L_{2}$ with $\delta_{L}=600 \mathrm{~m}, \Delta d=0.2 \mathrm{~m}$ and current source located at the center of $C_{2}$. The potentials are nomalized by the factor $I / 4 \pi \sigma_{0} L_{1}$. 

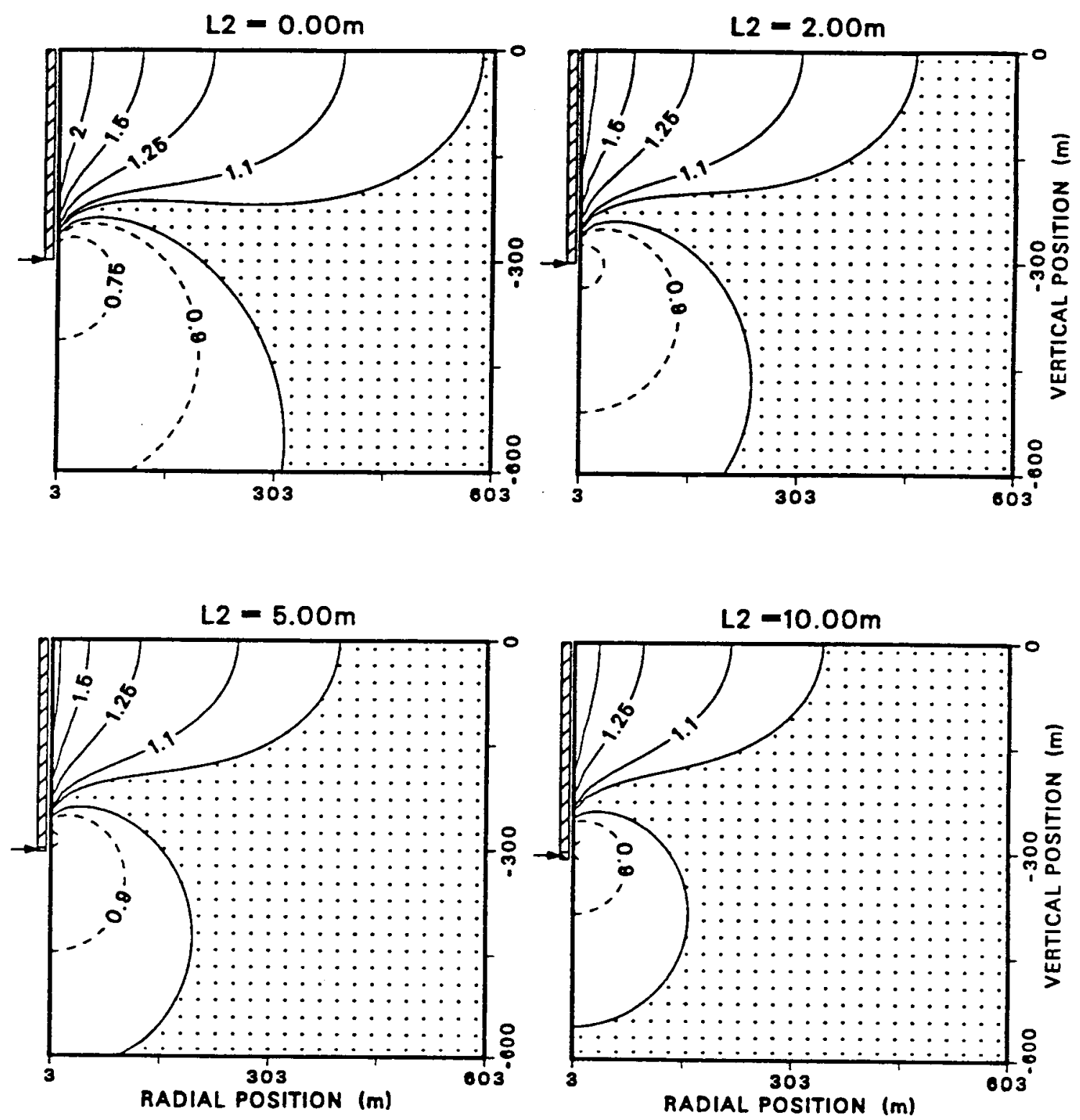

Figure 5.3-7b: Log-contour plots of the total-primary ratio for the same model as Figure 5.3$7 \mathrm{a}$. The irregularly spaced contour levels are: $\pm 2, \pm 1.5, \pm 1.25$, and \pm 1 . The dotted area represents $0.5 \%$ or less deviation. 

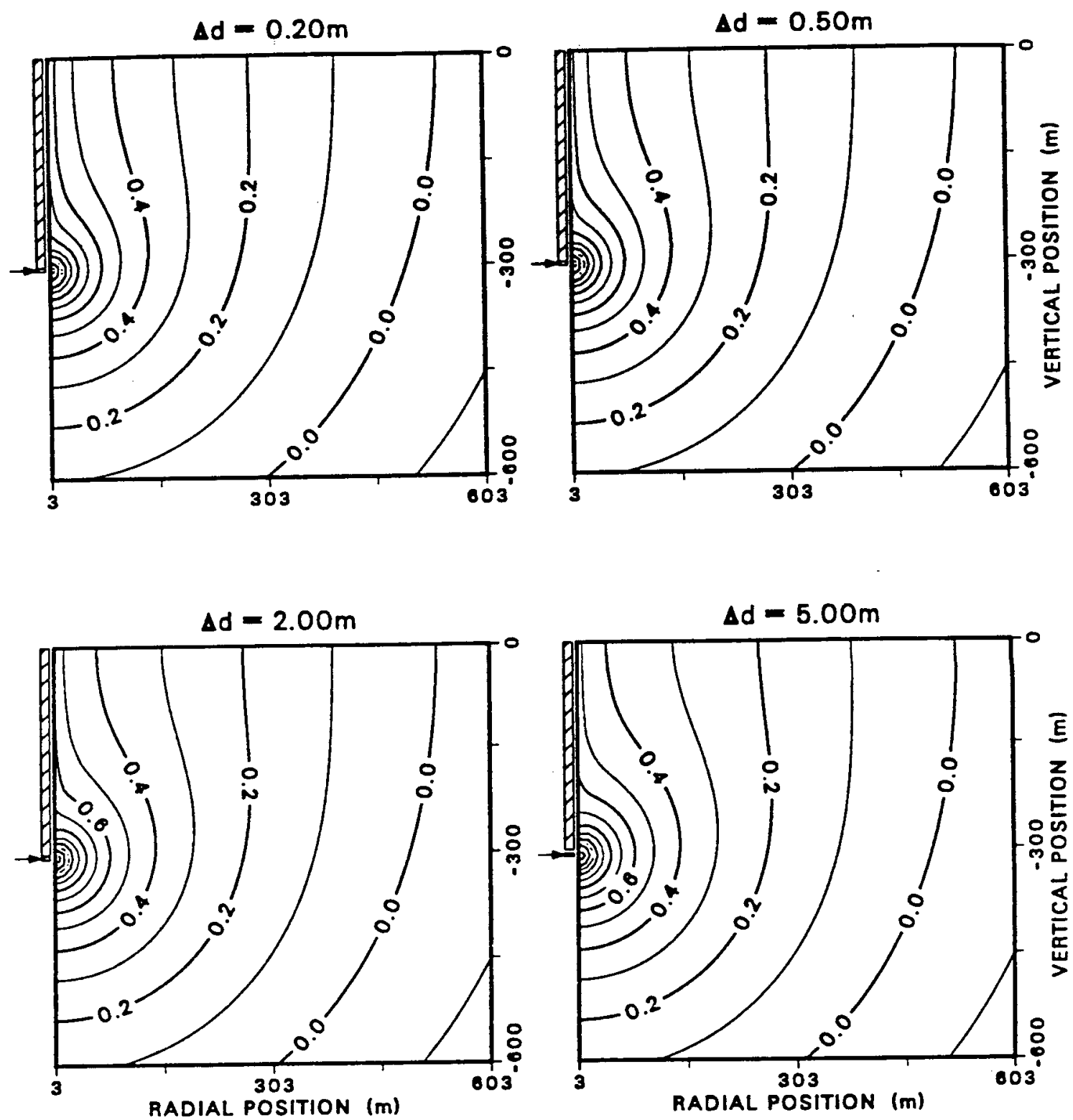

Figure 5.3-8a: Log-contour plots of the normalized half-space potentials due to the $300 \mathrm{~m} C_{1}$ for several $\Delta d$ with $\delta_{L}=600 \mathrm{~m}, L_{2}=5.0 \mathrm{~m}$ and current source located at the center of $C_{2}$. The potentials are normalized by the factor $I / 4 \pi \sigma_{0} L_{1}$. 

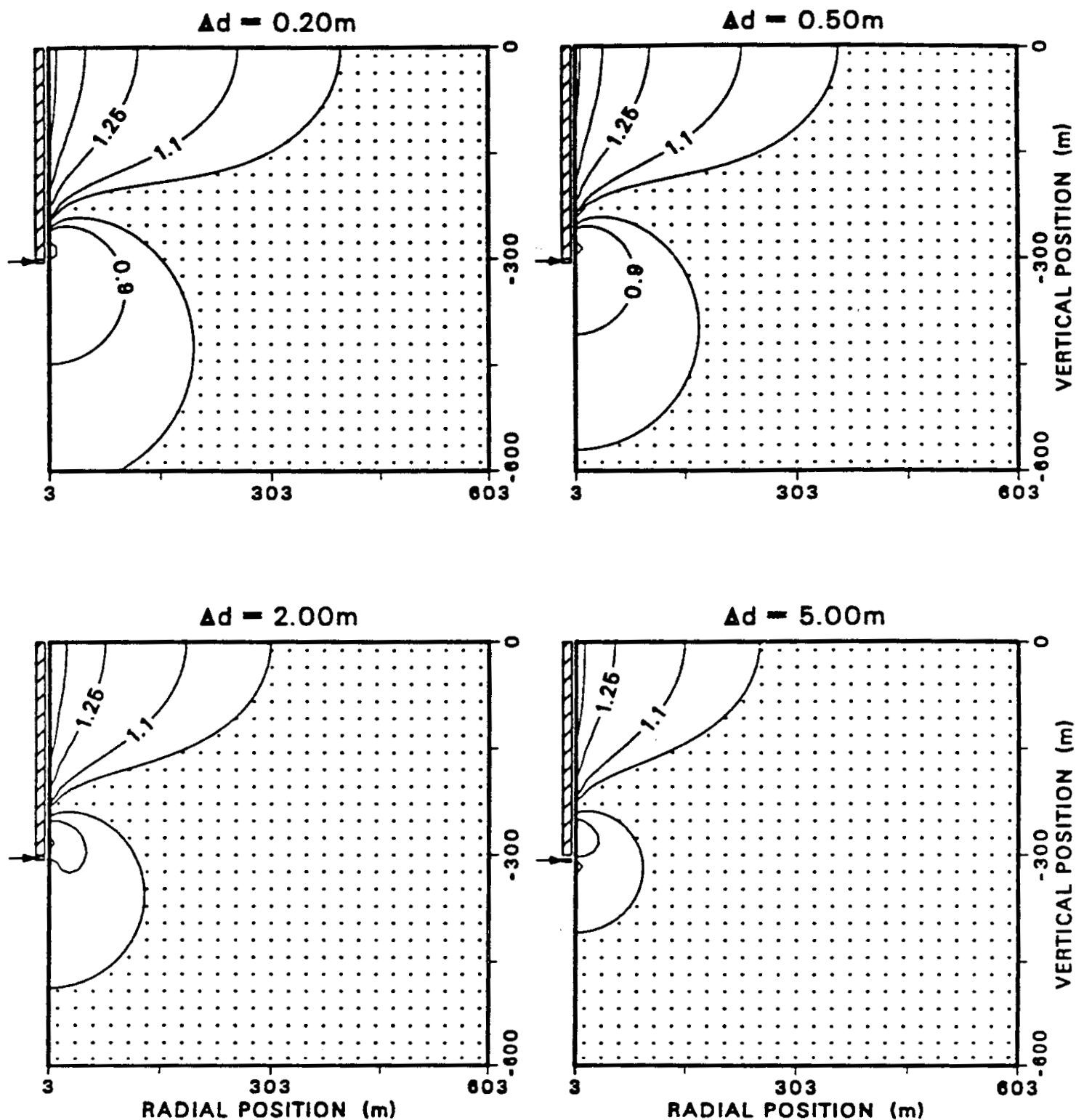

Figure 5.3-8b: Log-contour plots of the total-primary ratio for the same model as Figure 5.38 a. The irregularly spaced contour levels are: $\pm 2, \pm 1.5, \pm 1.25$, and \pm 1 . The dotted area represents $0.5 \%$ or less deviation. 

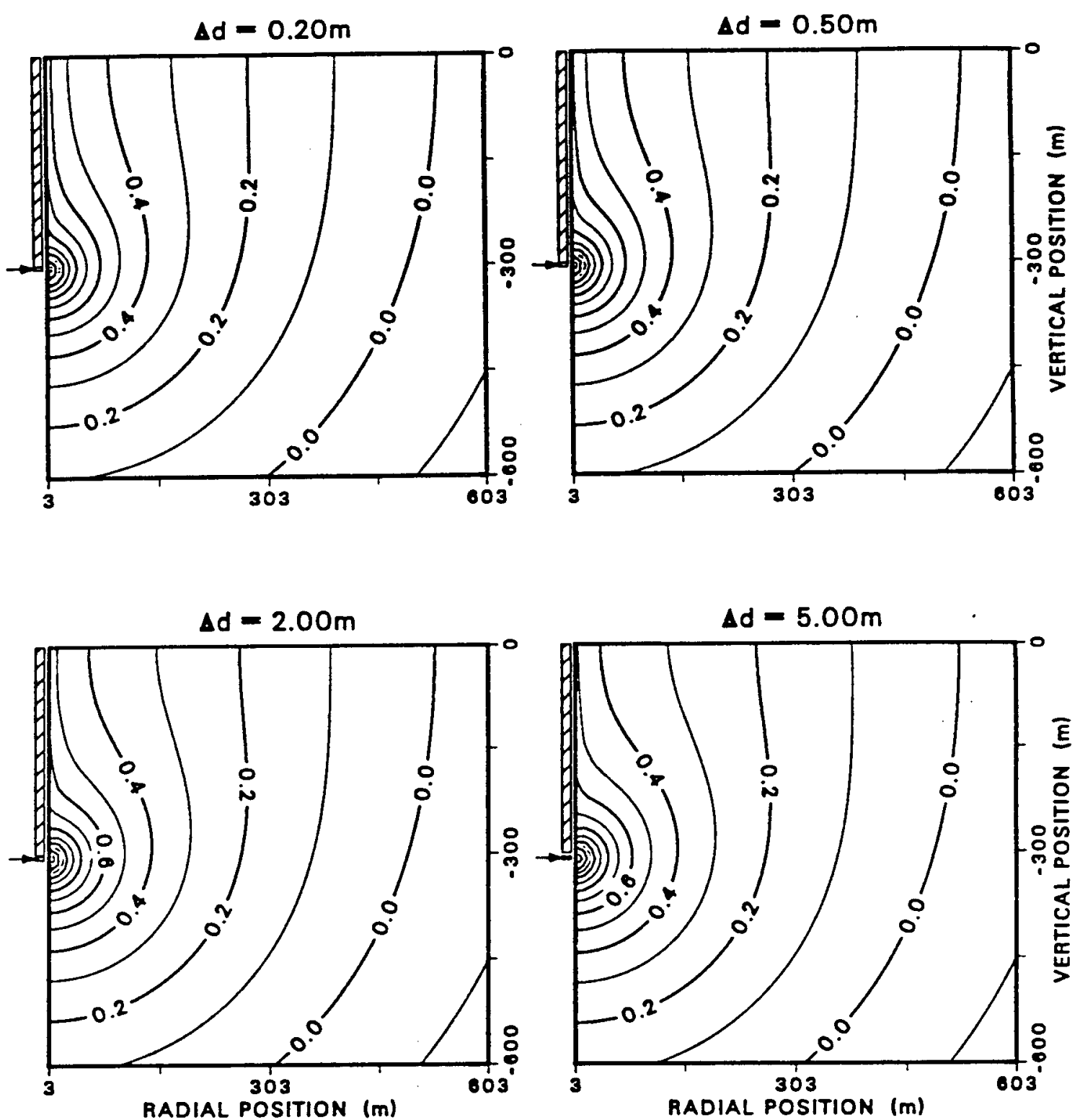

Figure 5.3-9a: Log-contour plots of the normalized half-space potentials due to the $300 \mathrm{~m} C_{1}$ for several $\Delta d$ with $\delta_{L}=300 \mathrm{~m}, L_{2}=5.0 \mathrm{~m}$ and current source located at the center of $C_{2}$. The potentials are normalized by the factor $I / 4 \pi \sigma_{0} L_{1}$. 

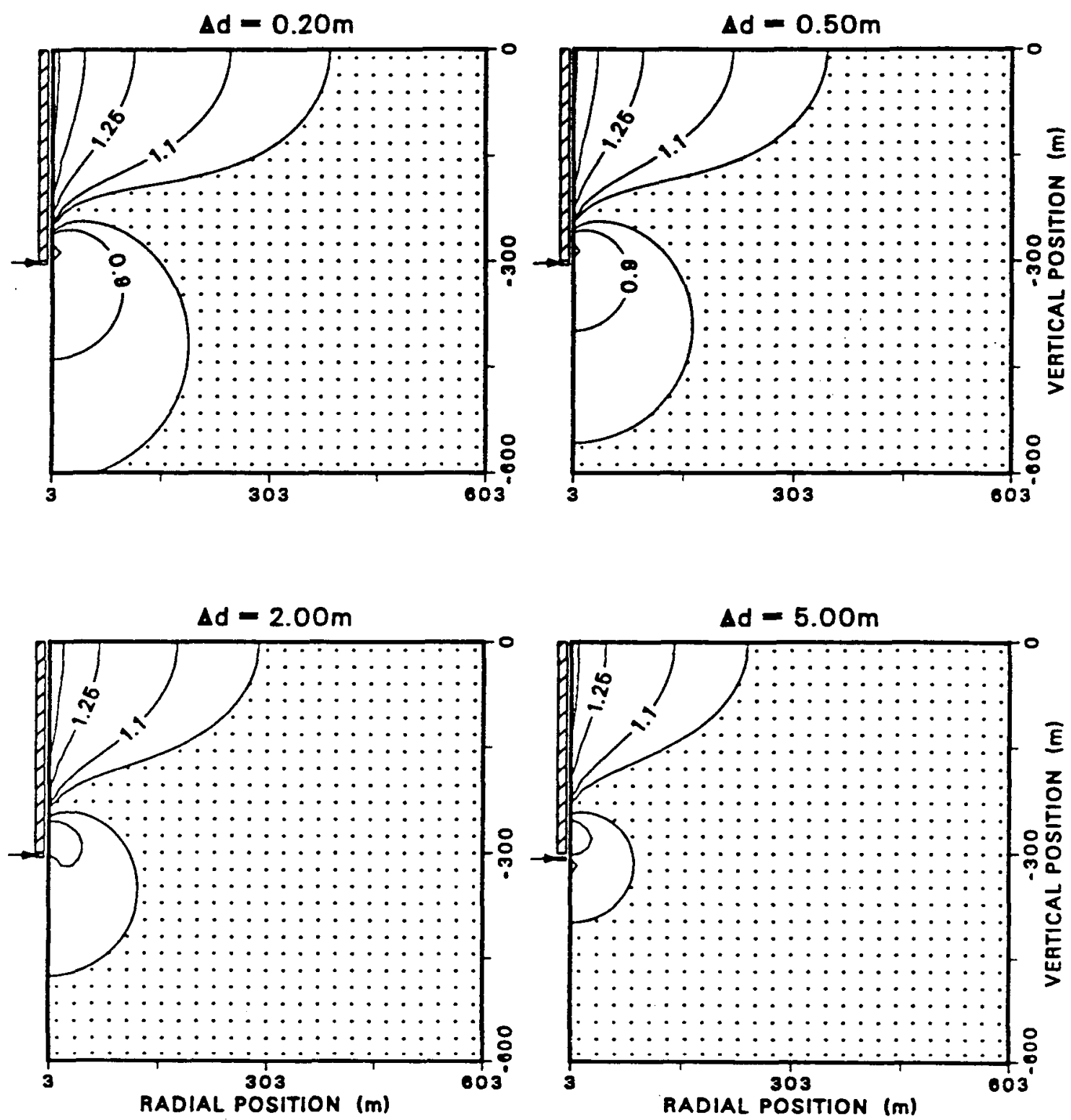

Figure 5.3-9b: Log-contour plots of the total-primary ratio for the same model as Figure 5.3$9 \mathrm{a}$. The irregularly spaced contour levels are: $\pm 2, \pm 1.5, \pm 1.25$, and \pm 1 . The dotted area represents $0.5 \%$ or less deviation. 

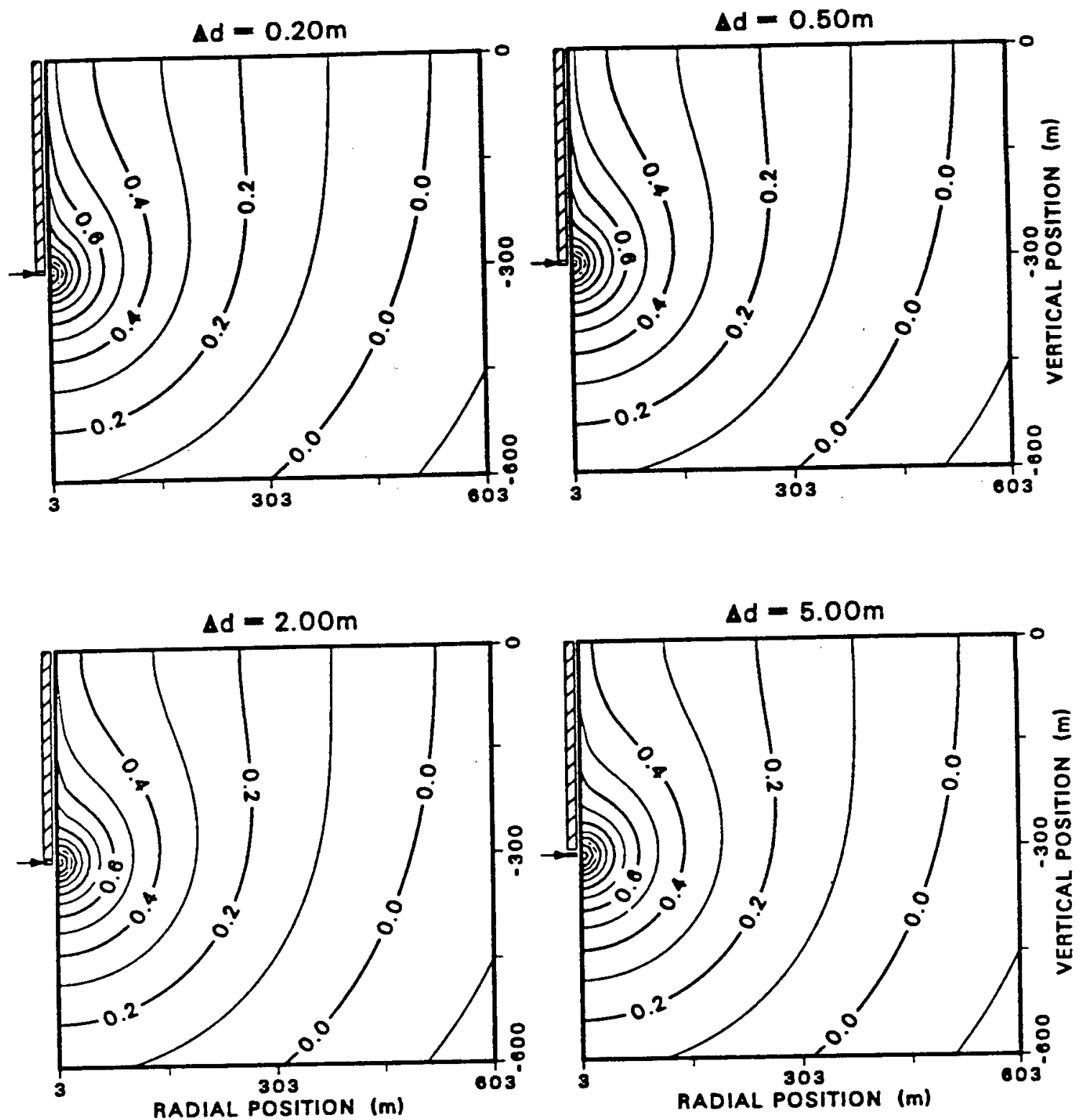

Figure 5.3-10a: Log-contour plots of the normalized half-space potentials due to the $300 \mathrm{~m} C_{1}$ for several $\Delta d$ with $\delta_{L}=150 \mathrm{~m}, L_{2}=5.0 \mathrm{~m}$ and current source located at the center of $C_{2}$. The potentials are normalized by the factor $I / 4 \pi \sigma_{0} L_{1}$. 

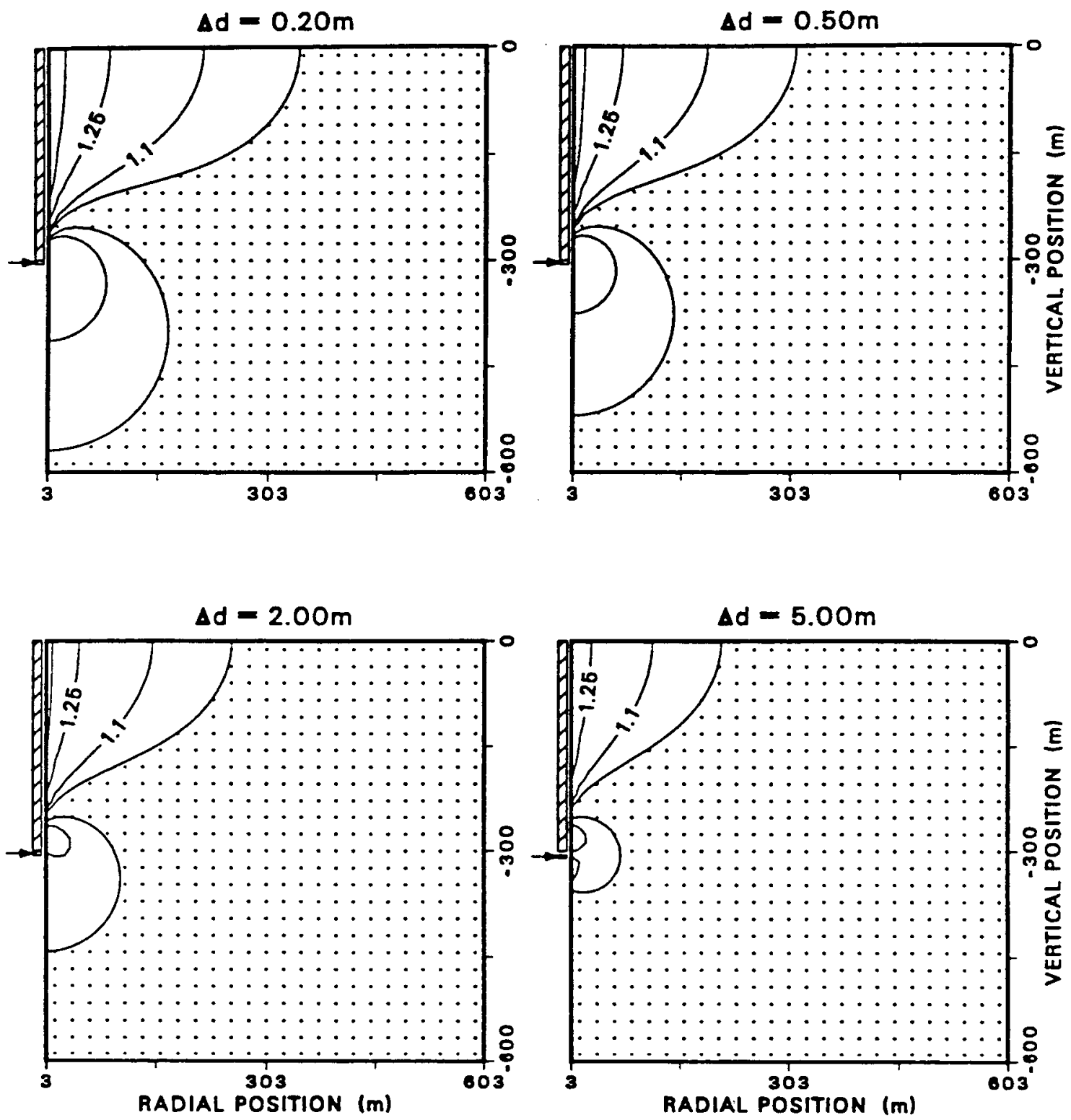

Figure 5.3-10b: Log-contour plots of the total-primary ratio for the same model as Figure 5.3-10a. The irregularly spaced contour levels are: $\pm 2, \pm 1.5, \pm 1.25$, and \pm 1 . The dotted area represents $0.5 \%$ or less deviation. 

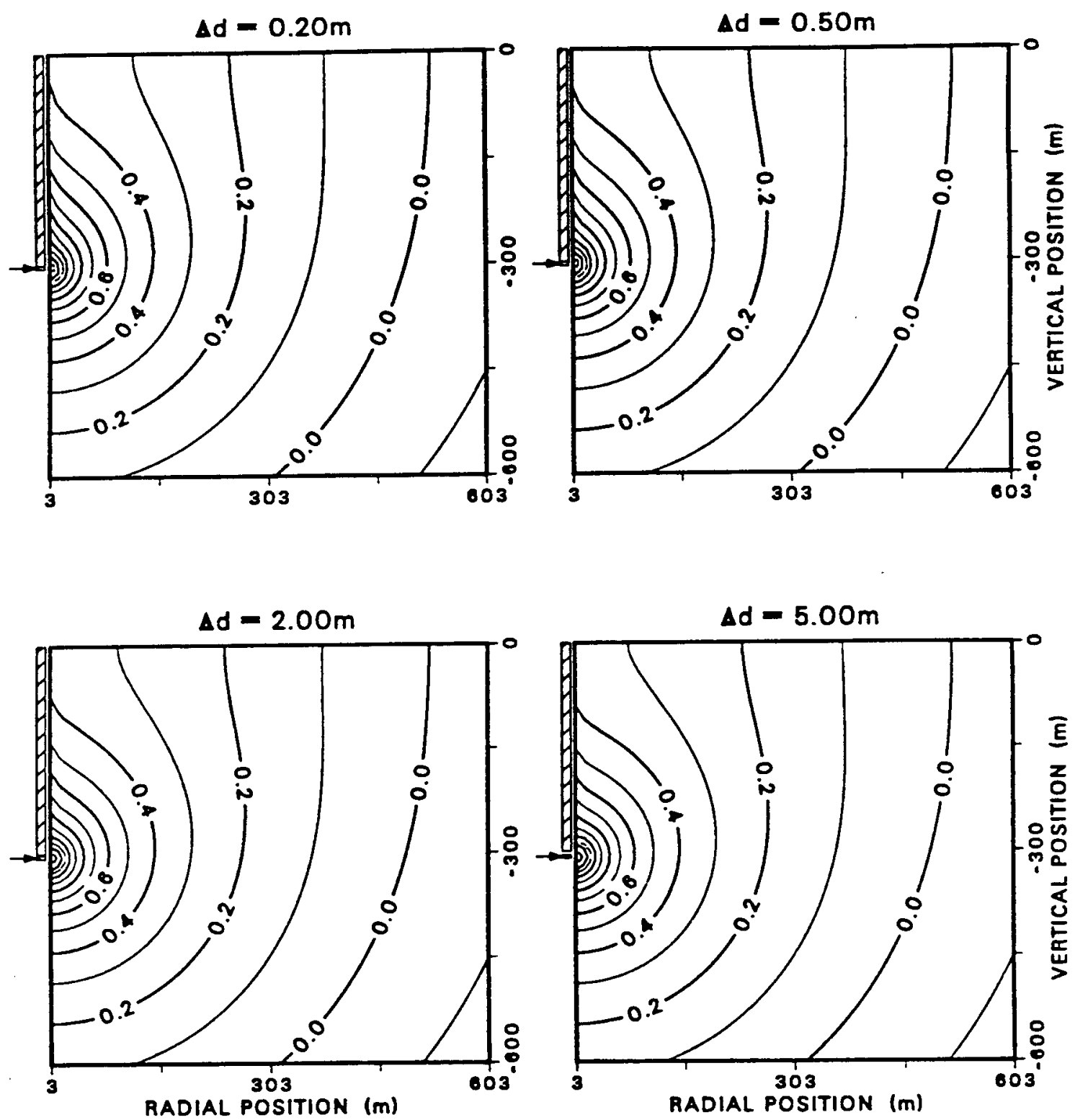

Figure 5.3-11a: Log-contour plots of the normalized half-space potentials due to the $300 \mathrm{~m} C_{1}$ for several $\Delta d$ with $\delta_{L}=60 \mathrm{~m}, L_{2}=5.0 \mathrm{~m}$ and current source located at the center of $C_{2}$. The potentials are normalized by the factor $I / 4 \pi \sigma_{0} L_{1}$. 

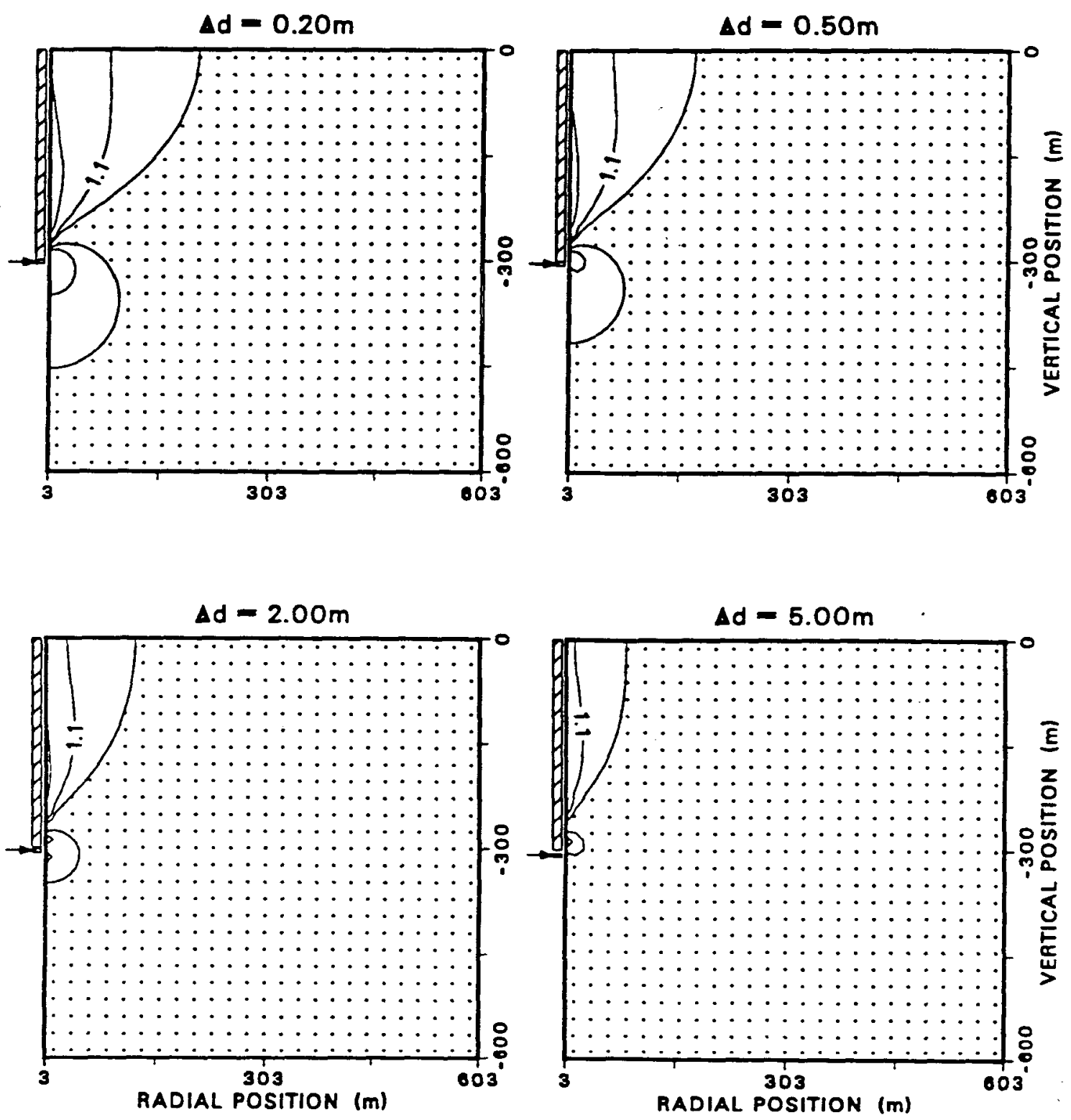

Figure 5.3-11b: Log-contour plots of the total-primary ratio for the same model as Figure 5.3-11a. The irregularly spaced contour levels are: $\pm 2, \pm 1.5, \pm 1.25$, and \pm 1 . The dotted area represents $0.5 \%$ or less deviation. 

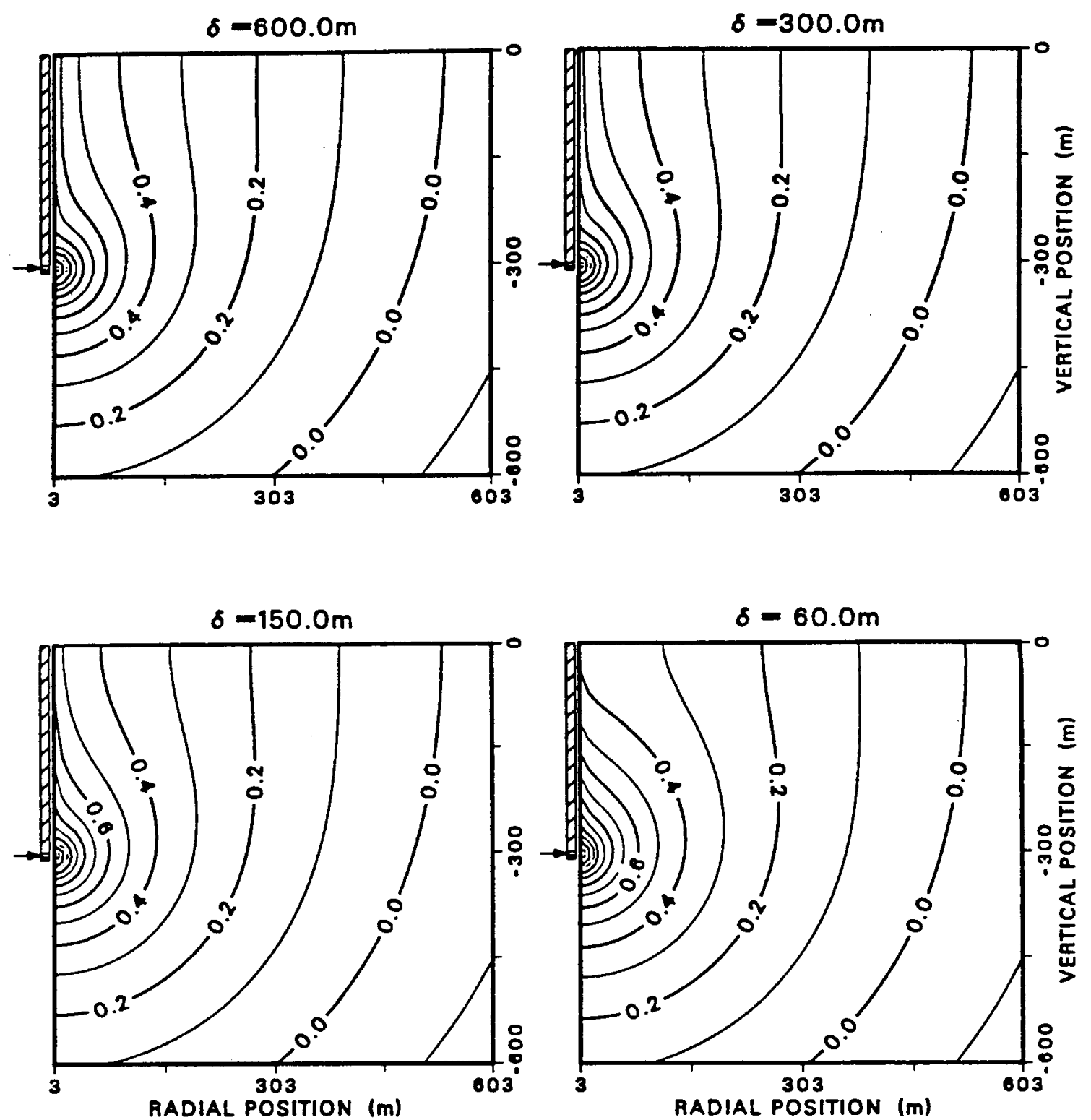

Figure 53-12a: Log-contour plots of the normalized half-space potentials due to the $300 \mathrm{~m} C_{1}$ and two underlying $5 \mathrm{~m}$ segments $\left(C_{2}\right.$ and $\left.C_{3}\right)$ for several $\delta_{L}$ with $\Delta d=0.2 \mathrm{~m}$ and current source located at the center of $C_{2}$. The potentials are normalized by the factor $I / 4 \pi \sigma_{0} L_{1}$. 

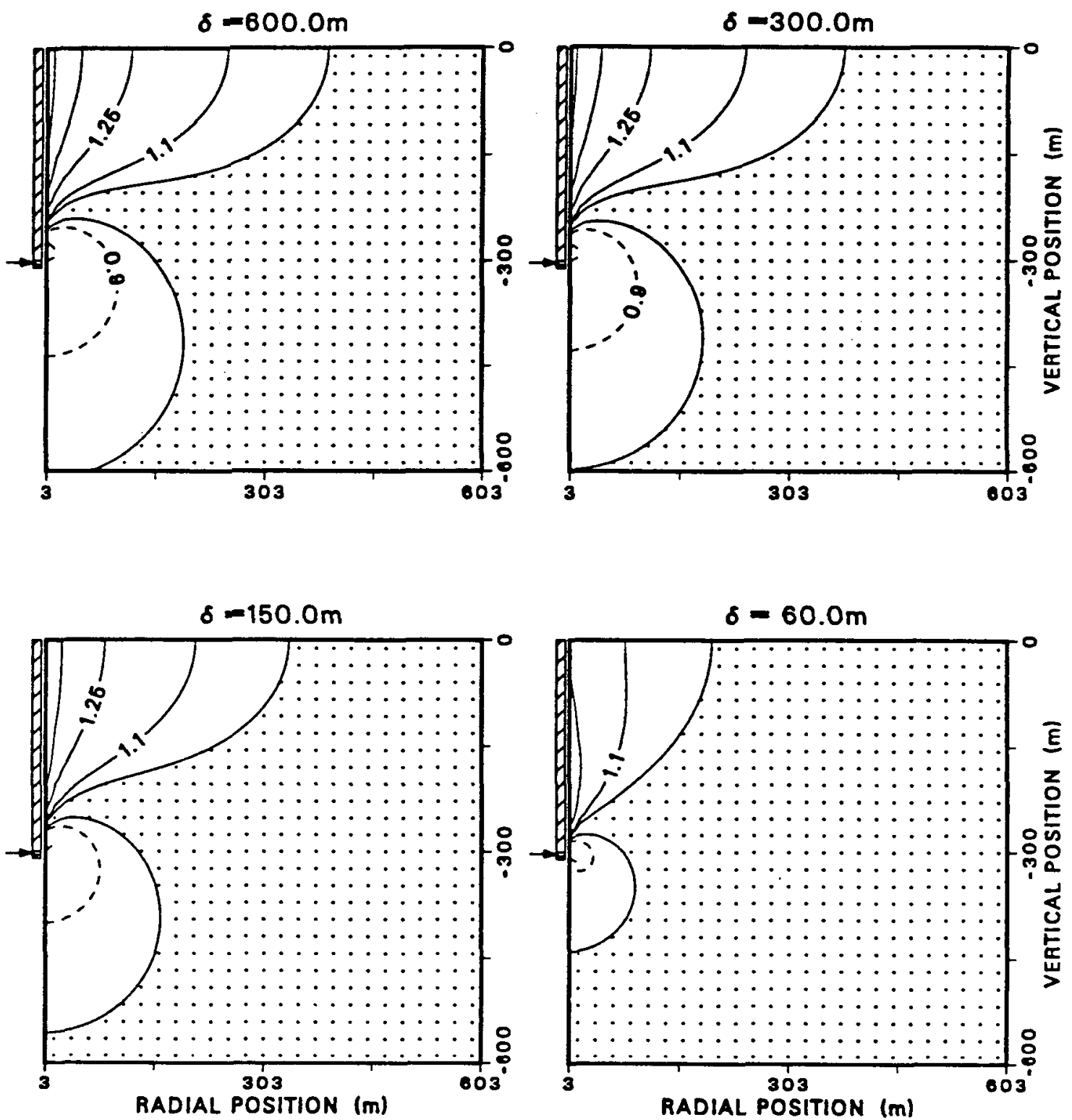

Figure 5.3-12b: Log-contour plots of the total-primary ratio for the same model as Figure 5.3-12a. The irregularly spaced contour levels are: $\pm 2, \pm 1.5, \pm 1.25$, and \pm 1 . The dotted area represents $0.5 \%$ or less deviation. 

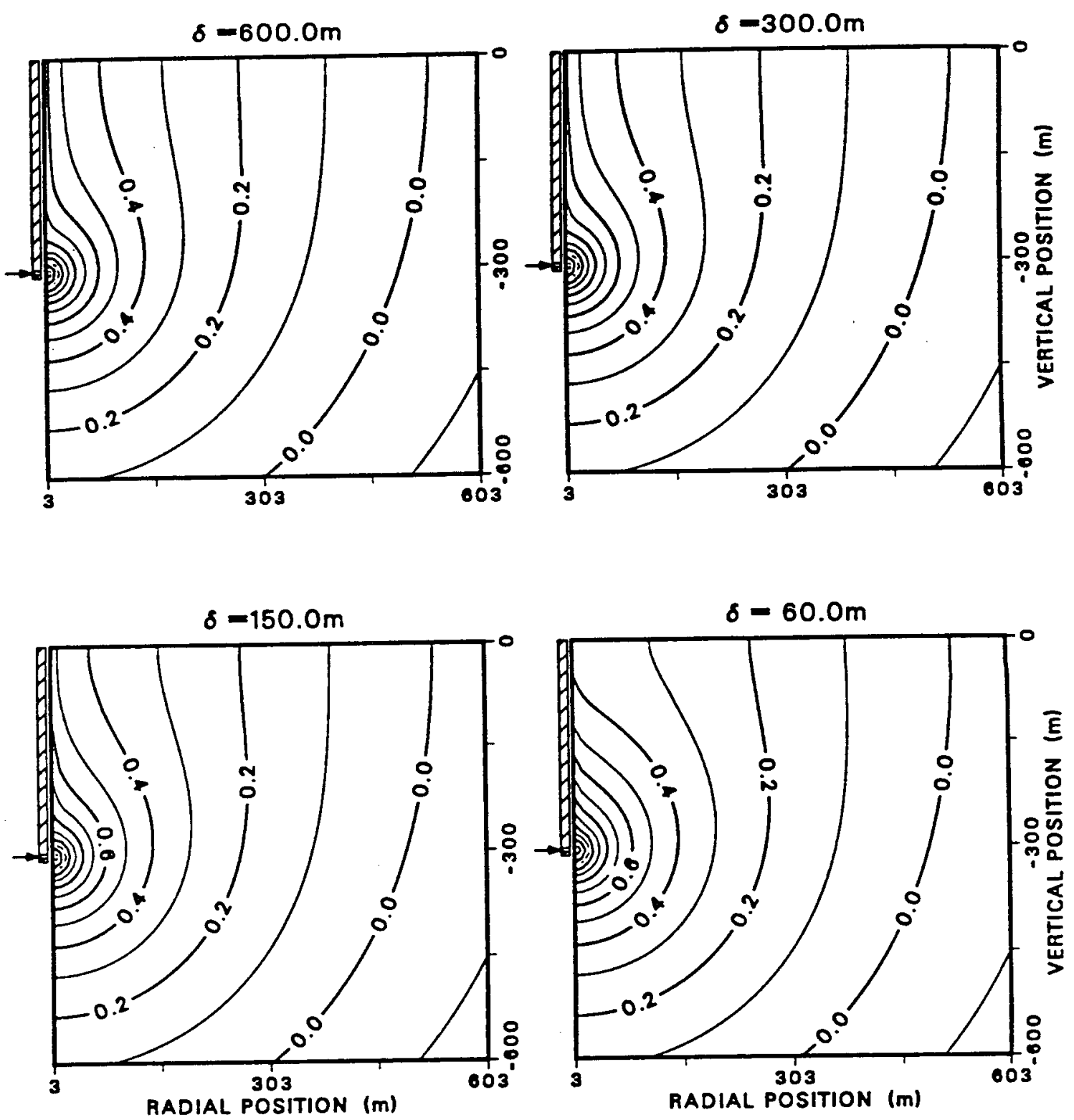

Figure 5.3-13a: Log-contour plots of the normalized half-space potentials due to the $300 \mathrm{~m} C_{1}$ and two underiying $5 \mathrm{~m}$ segments $\left(C_{2}\right.$ and $\left.C_{3}\right)$ for several $\delta_{L}$ with $\Delta d=0.5 \mathrm{~m}$ and current source located at the center of $C_{2}$. The potentials are normalized by the factor $I / 4 \pi \sigma_{0} L_{1}$. 

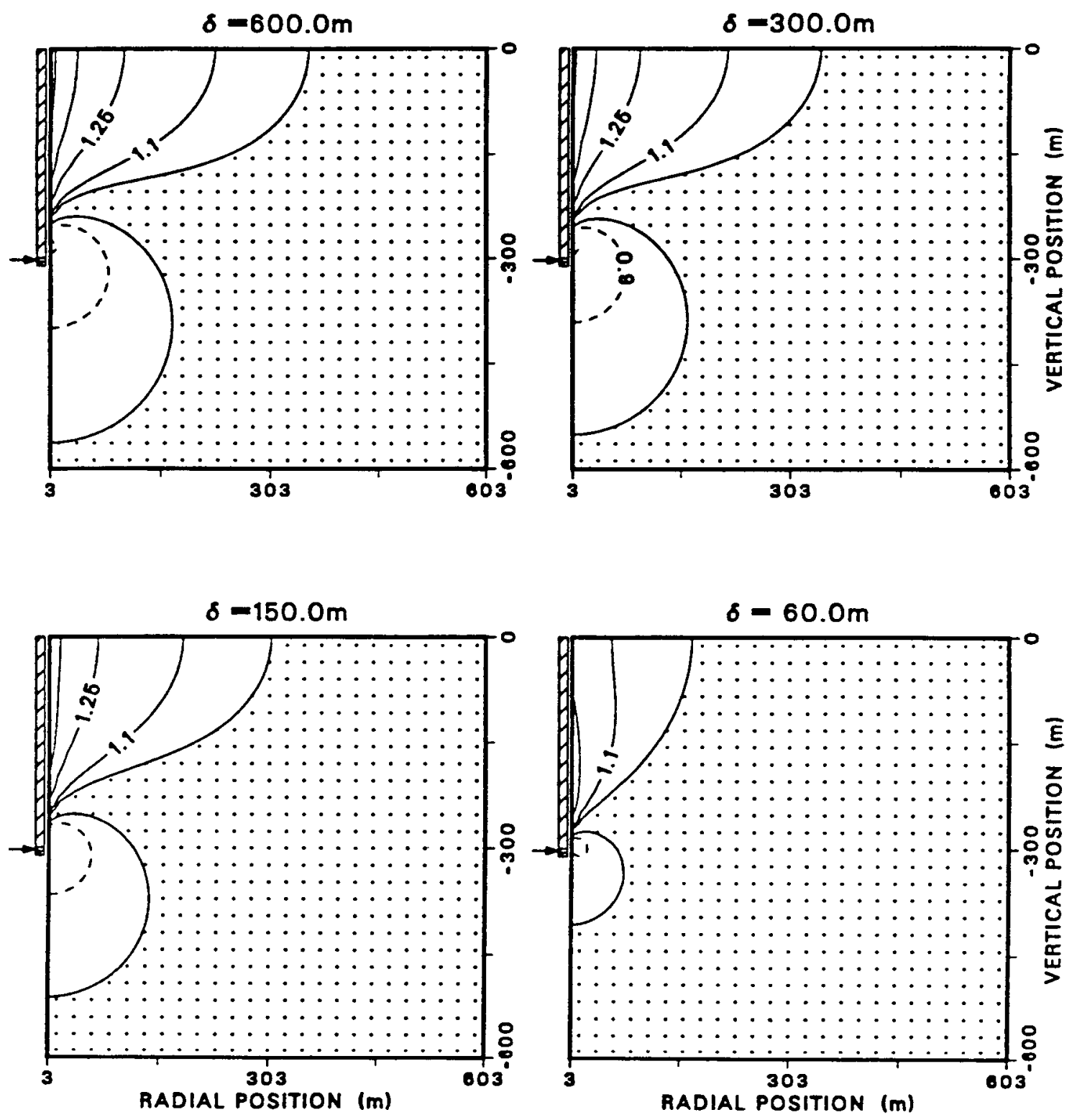

Figure 5.3-13b: Log-contour plots of the total-primary ratio for the same model as Figure 5.3-13a. The irregularly spaced contour levels are: $\pm 2, \pm 1.5, \pm 1.25$, and \pm 1 . The dotted area represents $0.5 \%$ or less deviation. 

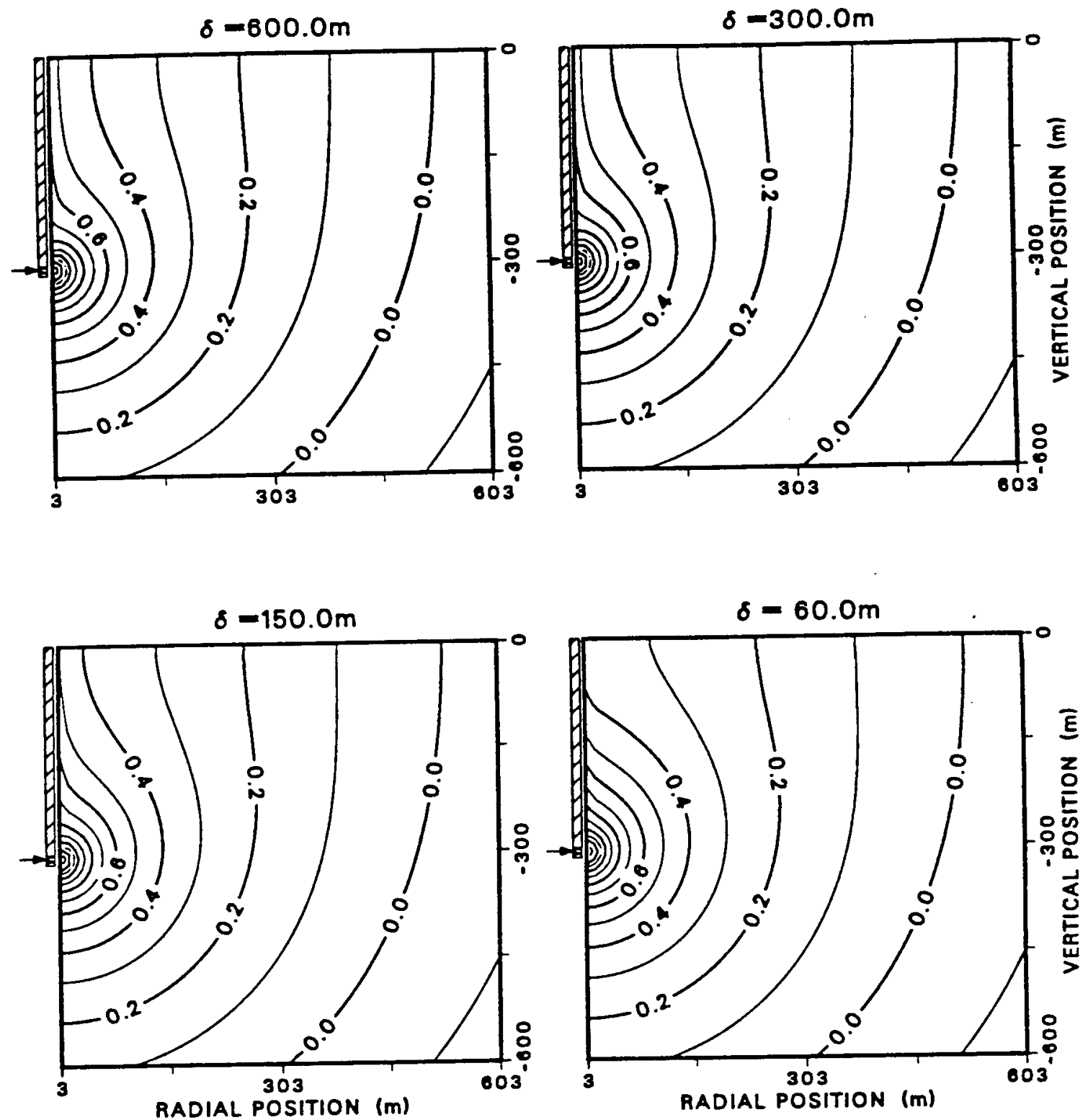

Figure 5.3-14a: Log-contour plots of the normalized half-space potentials due to the $300 \mathrm{~m} C_{1}$ and two underlying $5 \mathrm{~m}$ segments $\left(C_{2}\right.$ and $\left.C_{3}\right)$ for several $\delta_{L}$ with $\Delta d=2.0 \mathrm{~m}$ and current source located at the center of $C_{2}$. The potentials are normalized by the factor $I / 4 \pi \sigma_{0} L_{1}$. 

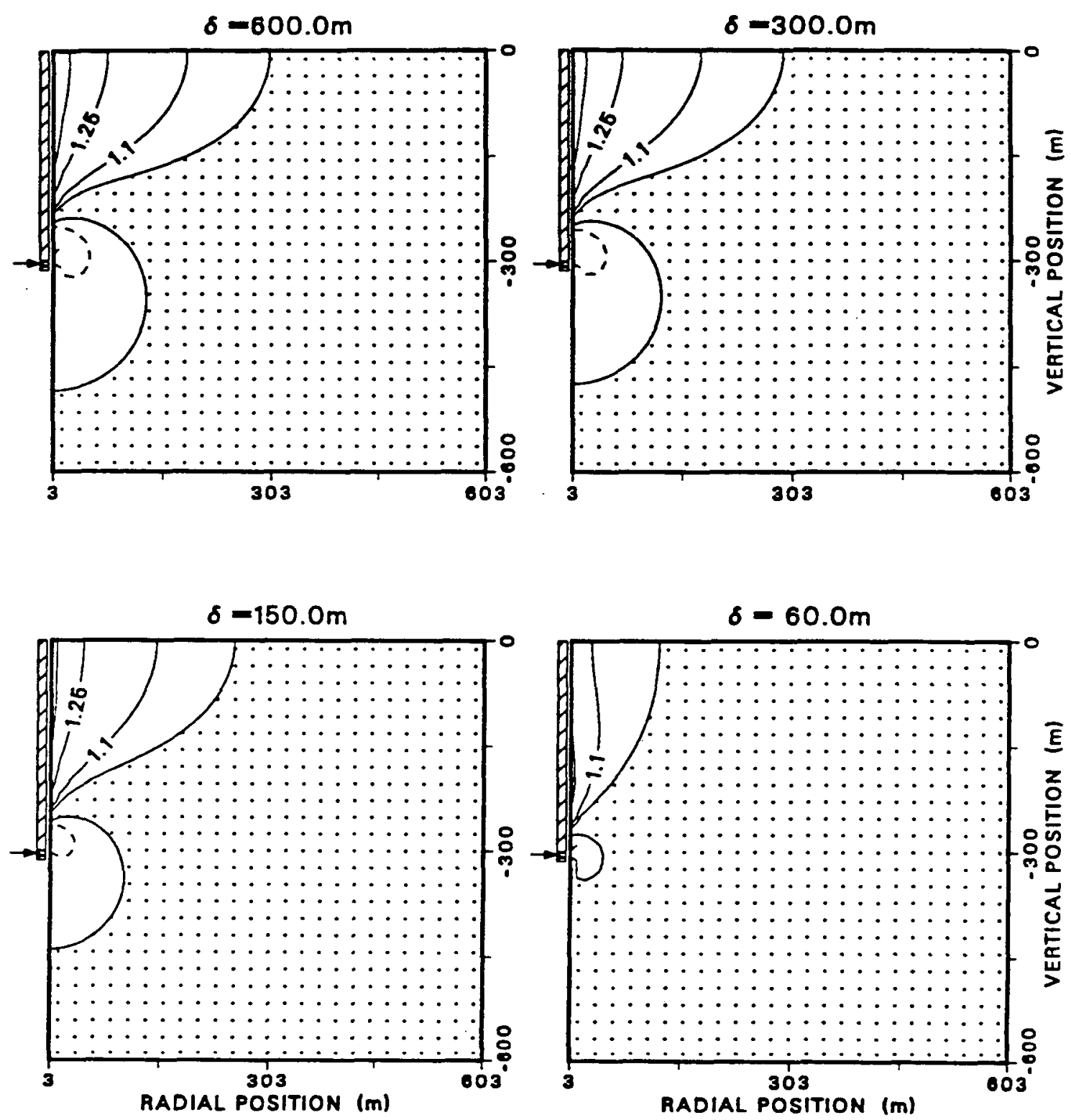

Figure 5.3-14b: Log-contour plots of the total-primary ratio for the same model as Figure 5.3-14a. The irregularly spaced contour levels are: $\pm 2, \pm 1.5, \pm 1.25$, and \pm 1 . The dotted area represents $0.5 \%$ or less deviation. 

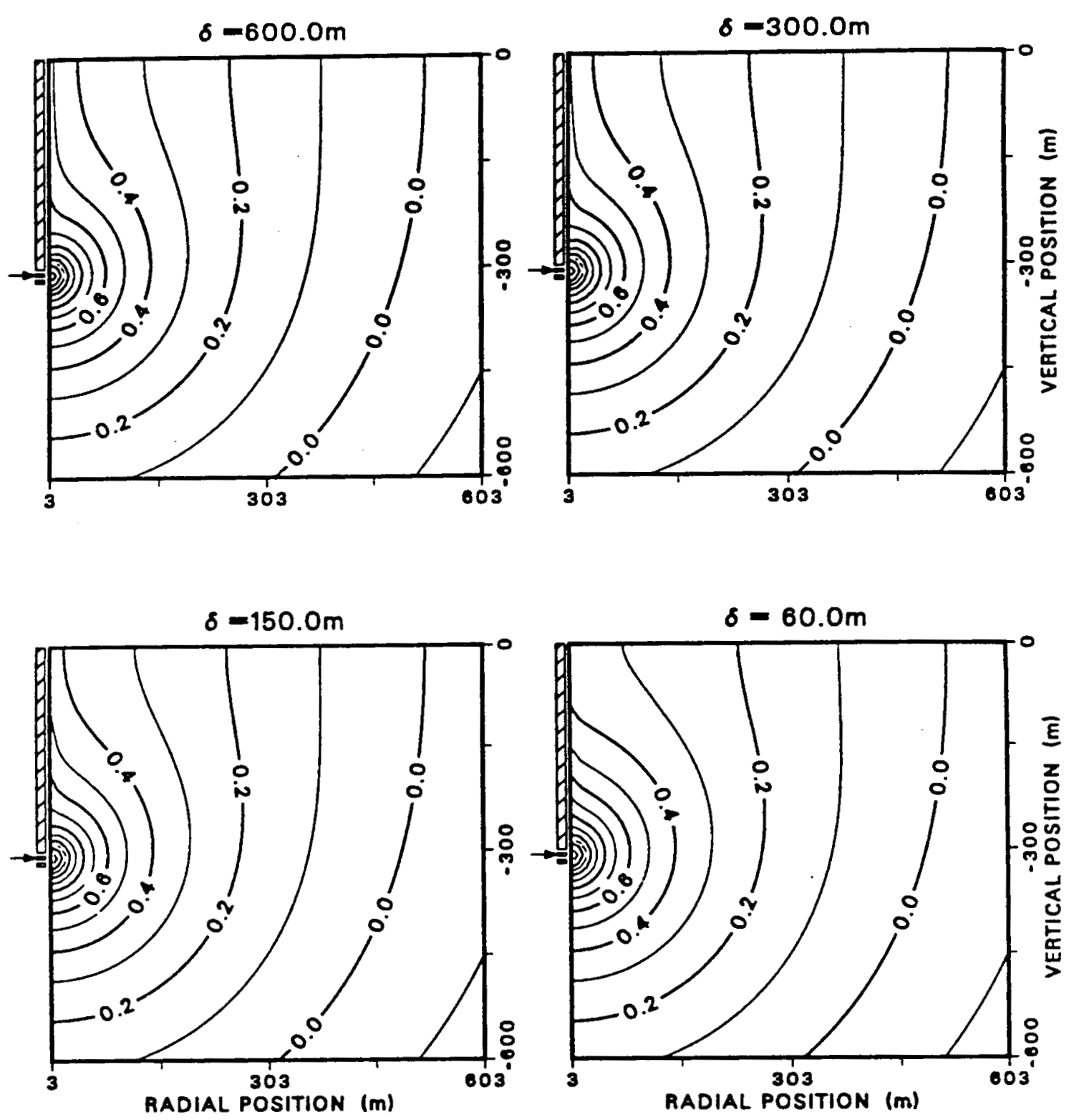

Figure 5.3-15a: Log-contour plots of the normalized half-space potentials due to the $300 \mathrm{~m} C_{1}$ and two underlying $5 \mathrm{~m}$ segments $\left(C_{2}\right.$ and $\left.C_{3}\right)$ for several $\delta_{L}$ with $\Delta d=2.0 \mathrm{~m}$ and current source located at the center of $C_{2}$. The potentials are normalized by the factor $I / 4 \pi \sigma_{\alpha_{1}}$. 

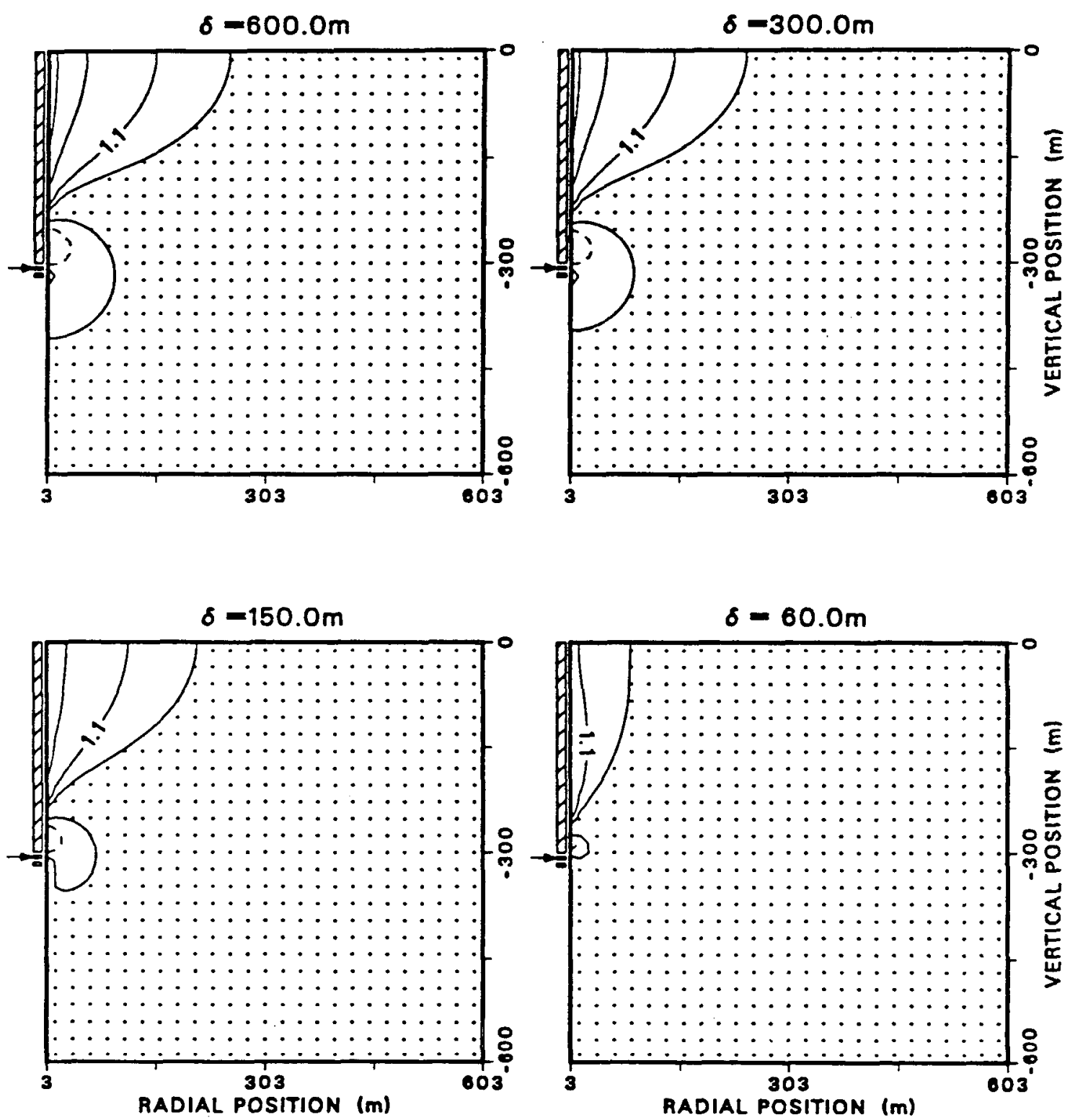

Figure 5.3-15b: Log-contour plots of the total-primary ratio for the same model as Figure 5.3-15a. The irregularly spaced contour levels are: $\pm 2, \pm 1.5, \pm 1.25$, and \pm 1 . The dotted area represents $0.5 \%$ or less deviation. 

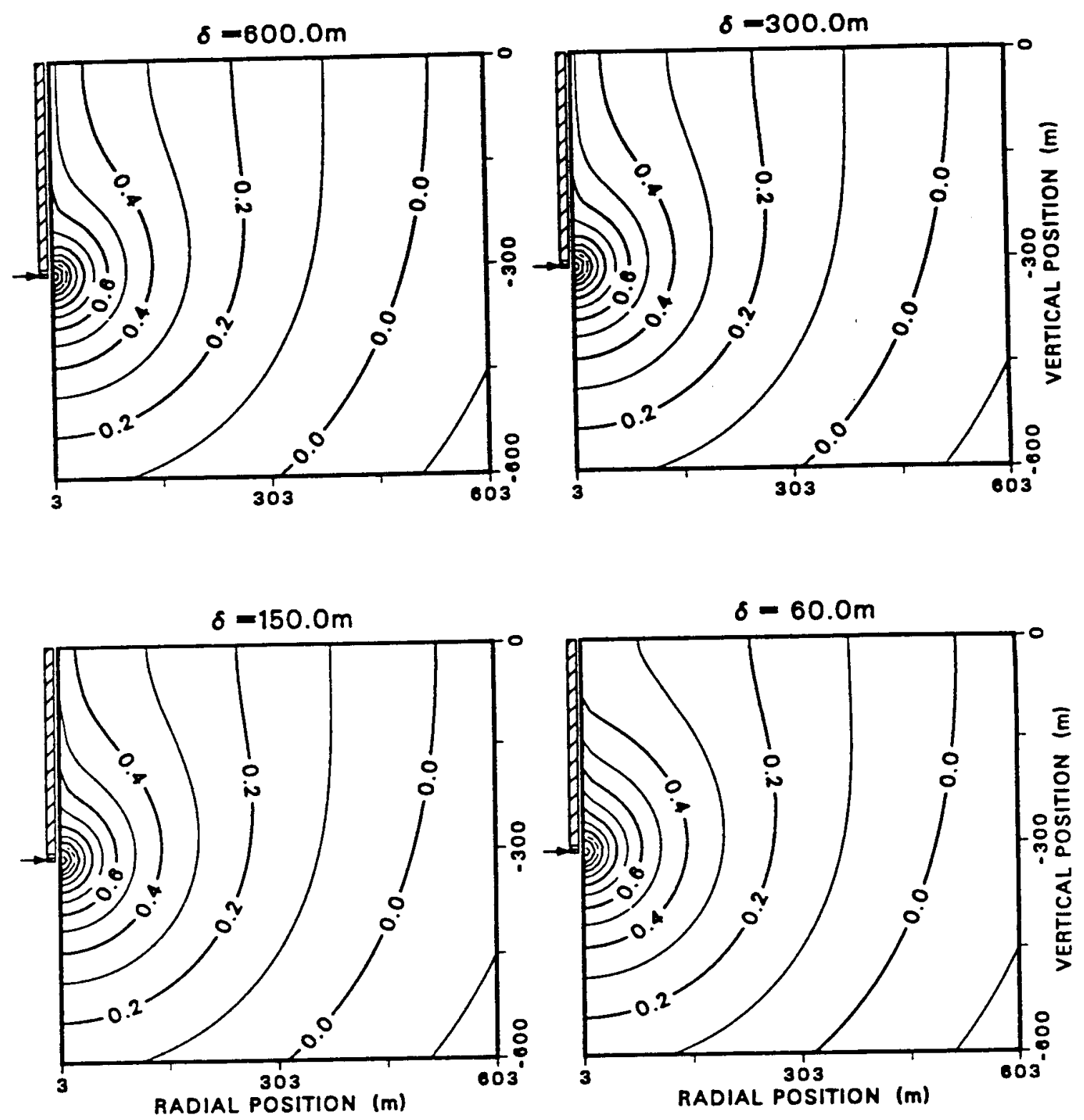

Figure 5.3-16a: Log-contour plots of the normalized half-space potentials due to the $300 \mathrm{~m} C_{1}$ and two underlying $5 \mathrm{~m}$ segments $\left(C_{2}\right.$ and $\left.C_{3}\right)$ for several $\delta_{L}$ with $\Delta d=0.2 \mathrm{~m}$ and current source located at the center of $C_{3}$. The potentials are normalized by the factor $I / 4 \pi \sigma_{0} L_{1}$. 

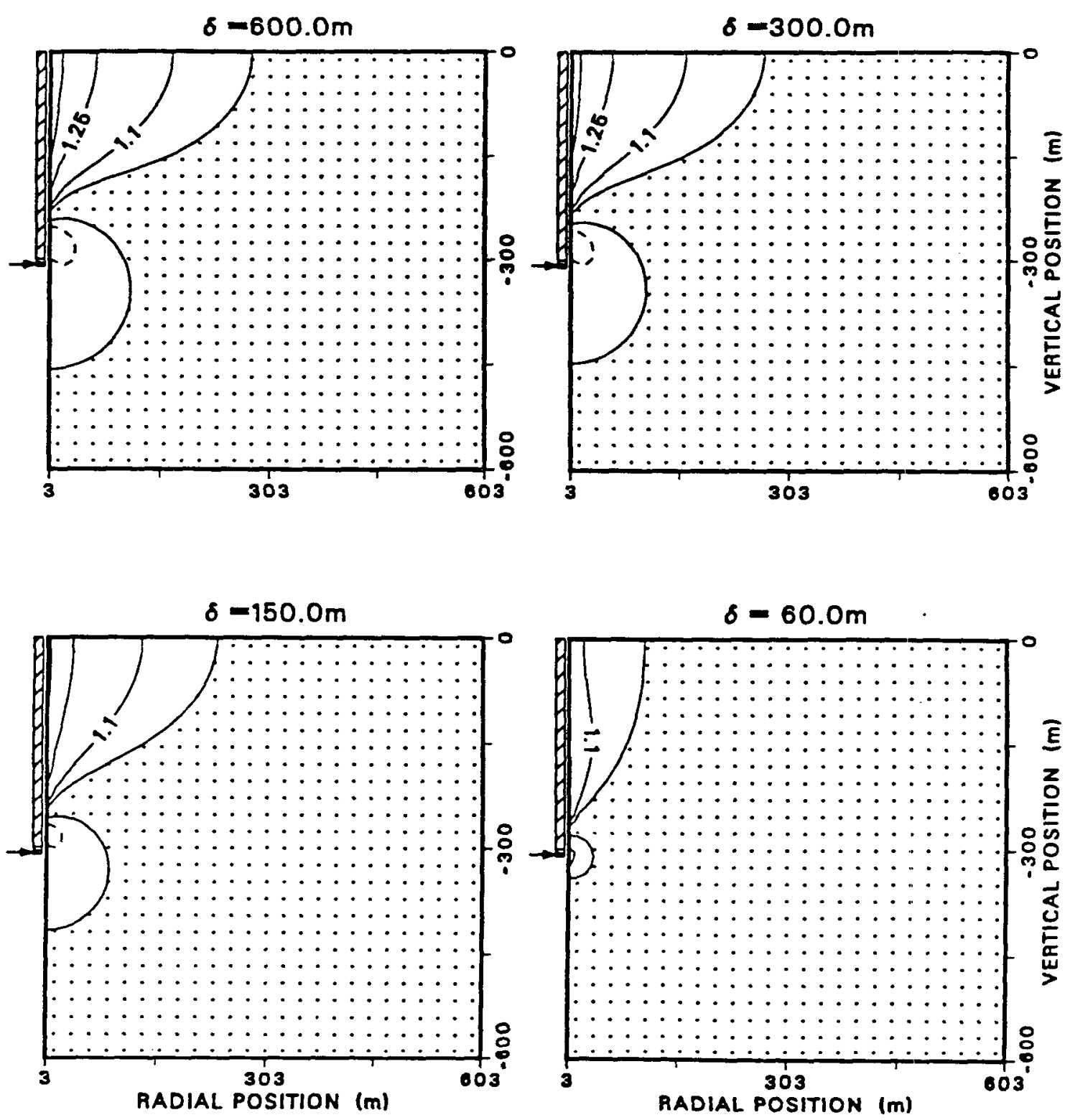

Figure 5.3-16b: Log-contour plots of the total-primary ratio for the same model as Figure 5.3-16a. The irregularly spaced contour levels are: $\pm 2, \pm 1.5, \pm 1.25$, and \pm 1 . The dotted area represents $0.5 \%$ or less deviation. 

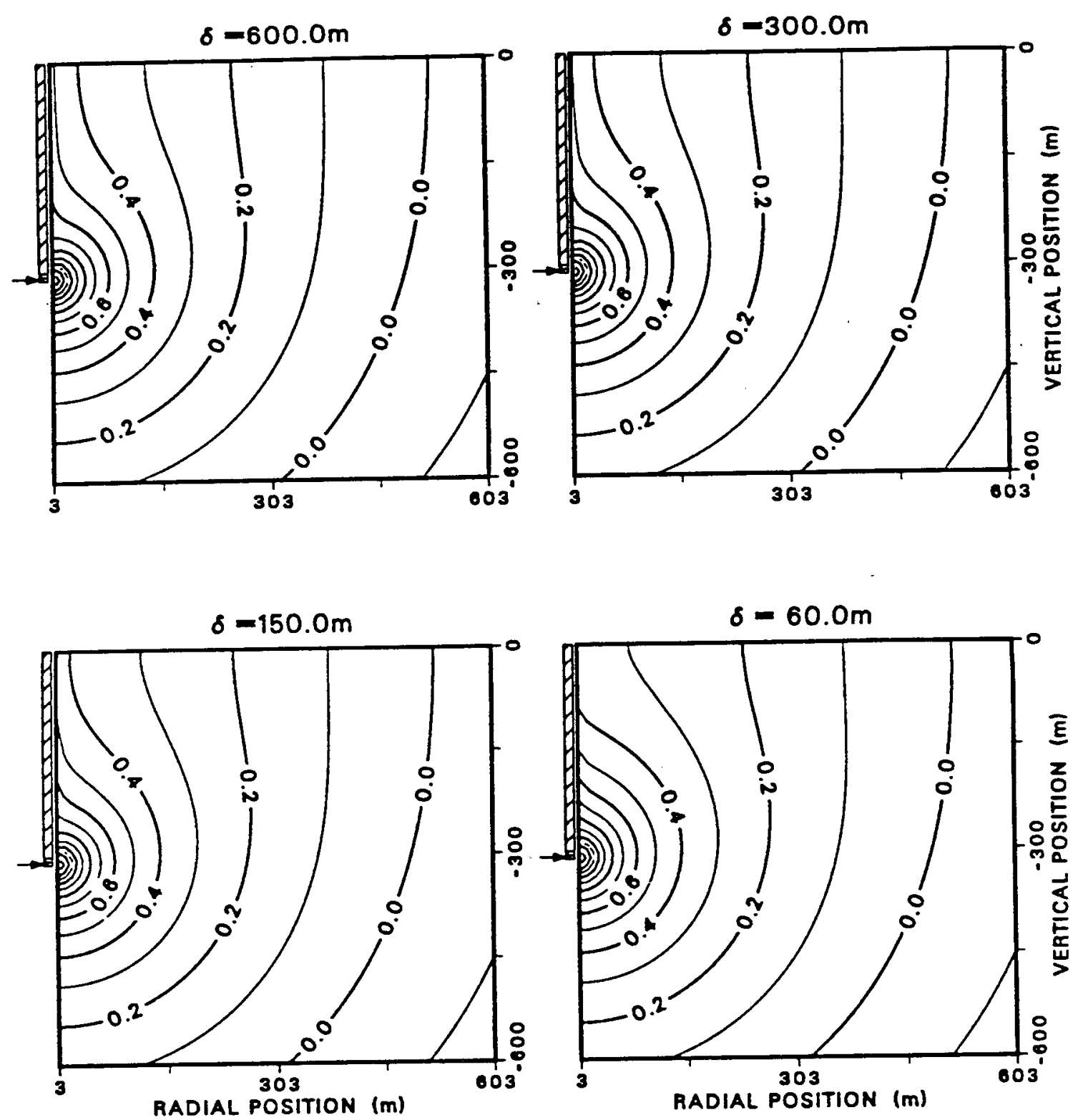

Figure 5.3-17a: Log-contour plots of the normalized half-space potentials due to the $300 \mathrm{~m} C_{1}$ and two underlying $5 \mathrm{~m}$ segments $\left(C_{2}\right.$ and $\left.C_{3}\right)$ for several $\delta_{L}$ with $\Delta d=0.5 \mathrm{~m}$ and current source located at the center of $C_{3}$. The potentials are normalized by the factor $I / 4 \pi \sigma_{0} L_{1}$. 

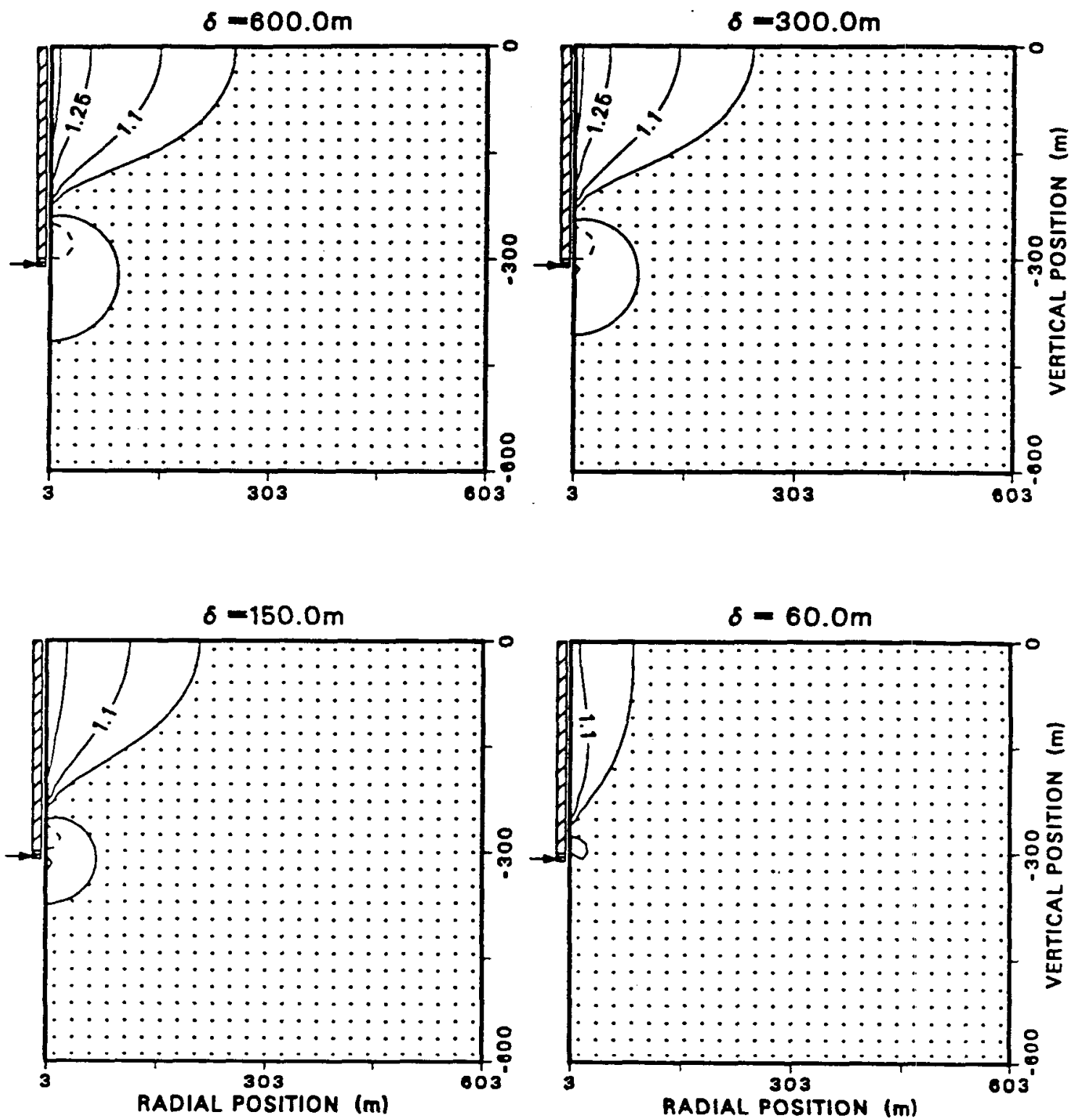

Figure 5.3-17b: Log-contour plots of the total-primary ratio for the same model as Figure 5.3-17a. The irregularly spaced contour levels are: $\pm 2, \pm 1.5, \pm 1.25$, and \pm 1 . The dotted area represents $0.5 \%$ or less deviation. 

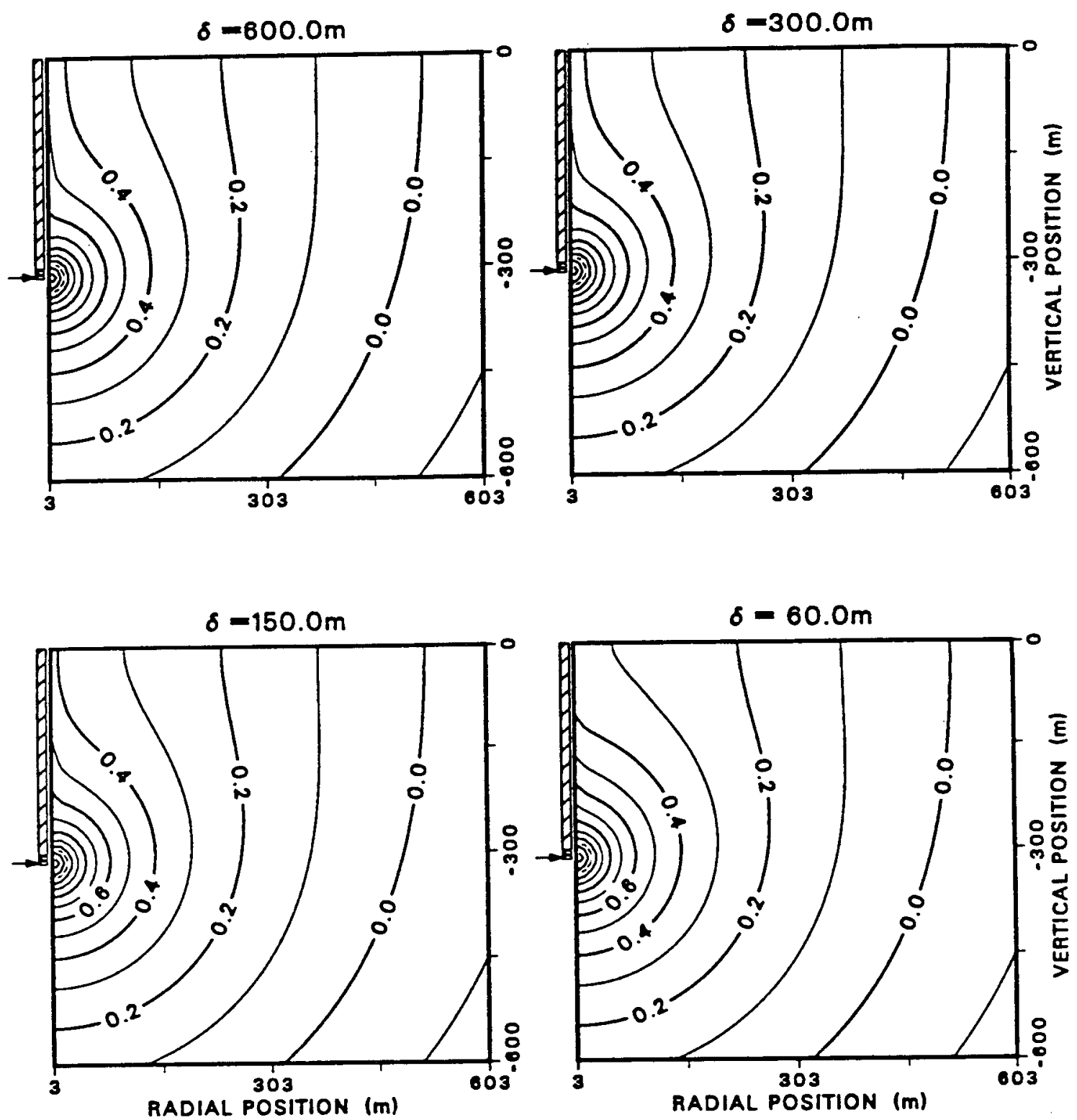

Figure 5.3-18a: Log-contour plots of the normalized half-space potentials due to the $300 \mathrm{~m} C_{1}$ and two underlying $5 \mathrm{~m}$ segments $\left(C_{2}\right.$ and $\left.C_{3}\right)$ for several $\delta_{L}$ with $\Delta d=2.0 \mathrm{~m}$ and current source located at the center of $C_{3}$. The potentials are normalized by the factor $I / 4 \pi \sigma_{0} L_{1}$. 

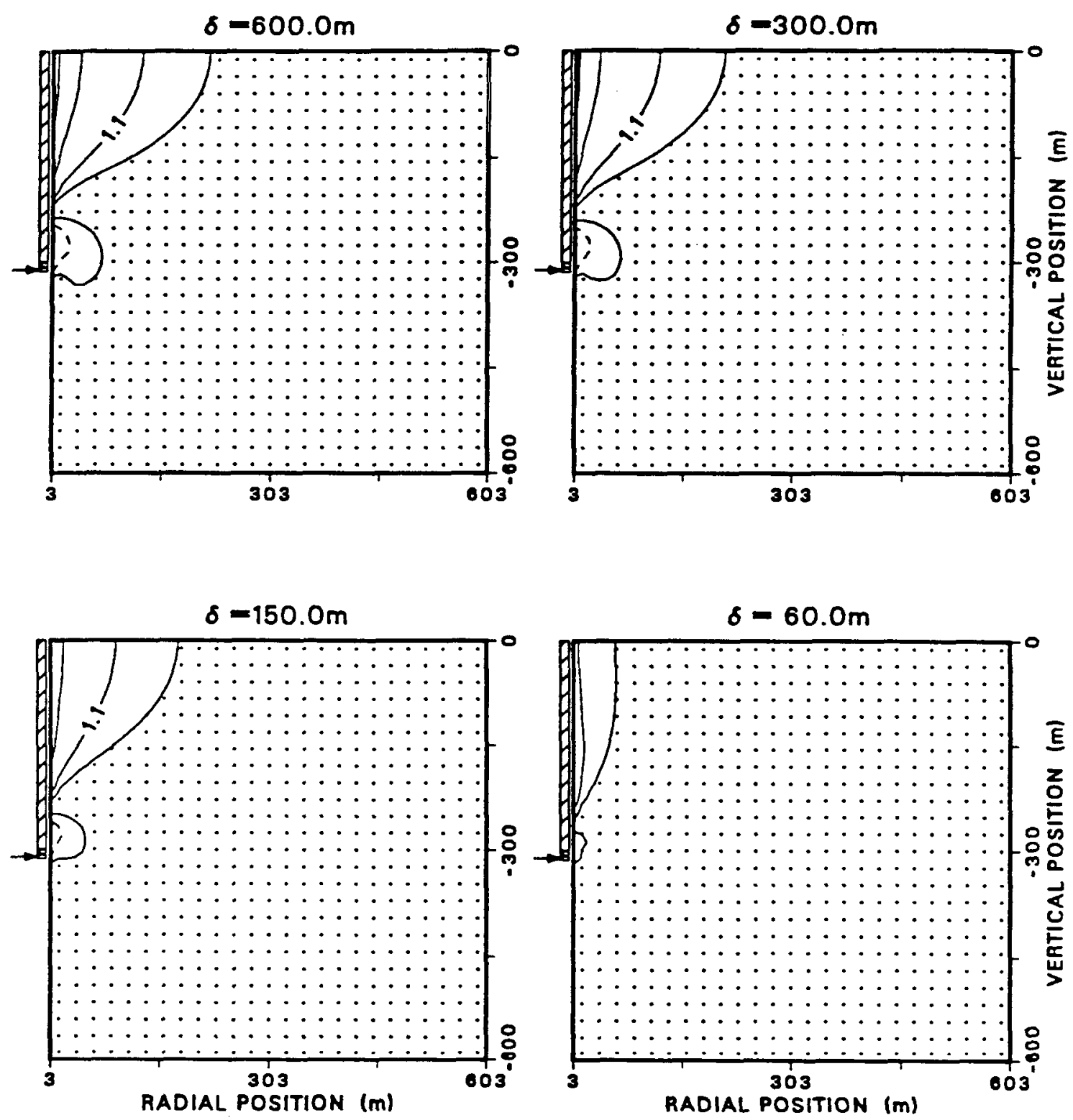

Figure 5.3-18b: Log-contour plots of the total-primary ratio for the same model as Figure $5.3-18 \mathrm{a}$. The irregularly spaced contour levels are: $\pm 2, \pm 1.5, \pm 1.25$, and \pm 1 . The dotted area represents $0.5 \%$ or less deviation. 

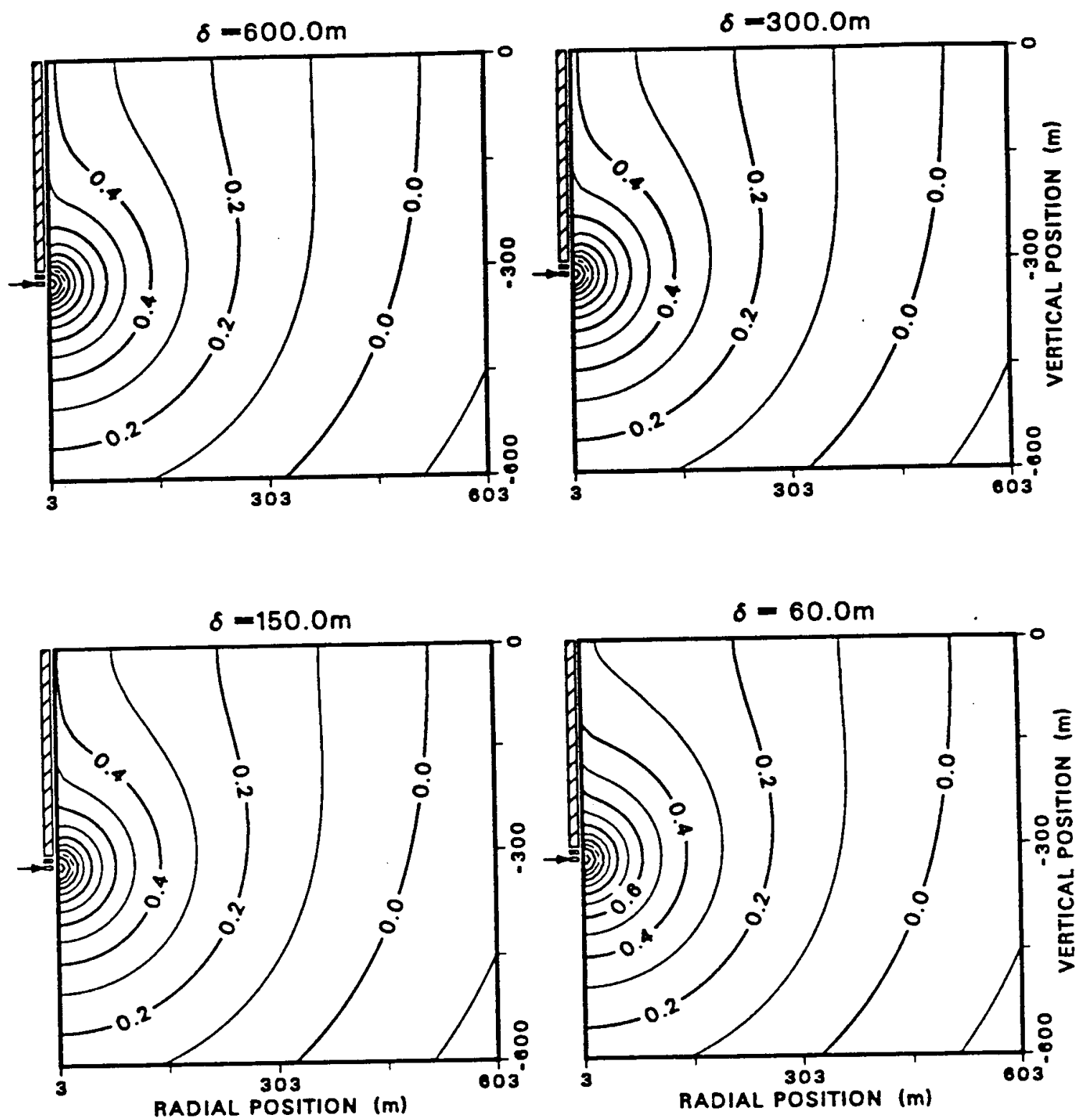

Figure 5.3-19a: Log-contour plots of the normalized half-space potentials due to the $300 \mathrm{~m} C_{1}$ and two underlying $5 \mathrm{~m}$ segments $\left(C_{2}\right.$ and $\left.C_{3}\right)$ for several $\delta_{L}$ with $\Delta d=2.0 \mathrm{~m}$ and current source located at the center of $C_{3}$. The potentials are normalized by the factor $I / 4 \pi \sigma_{0} L_{1}$. 

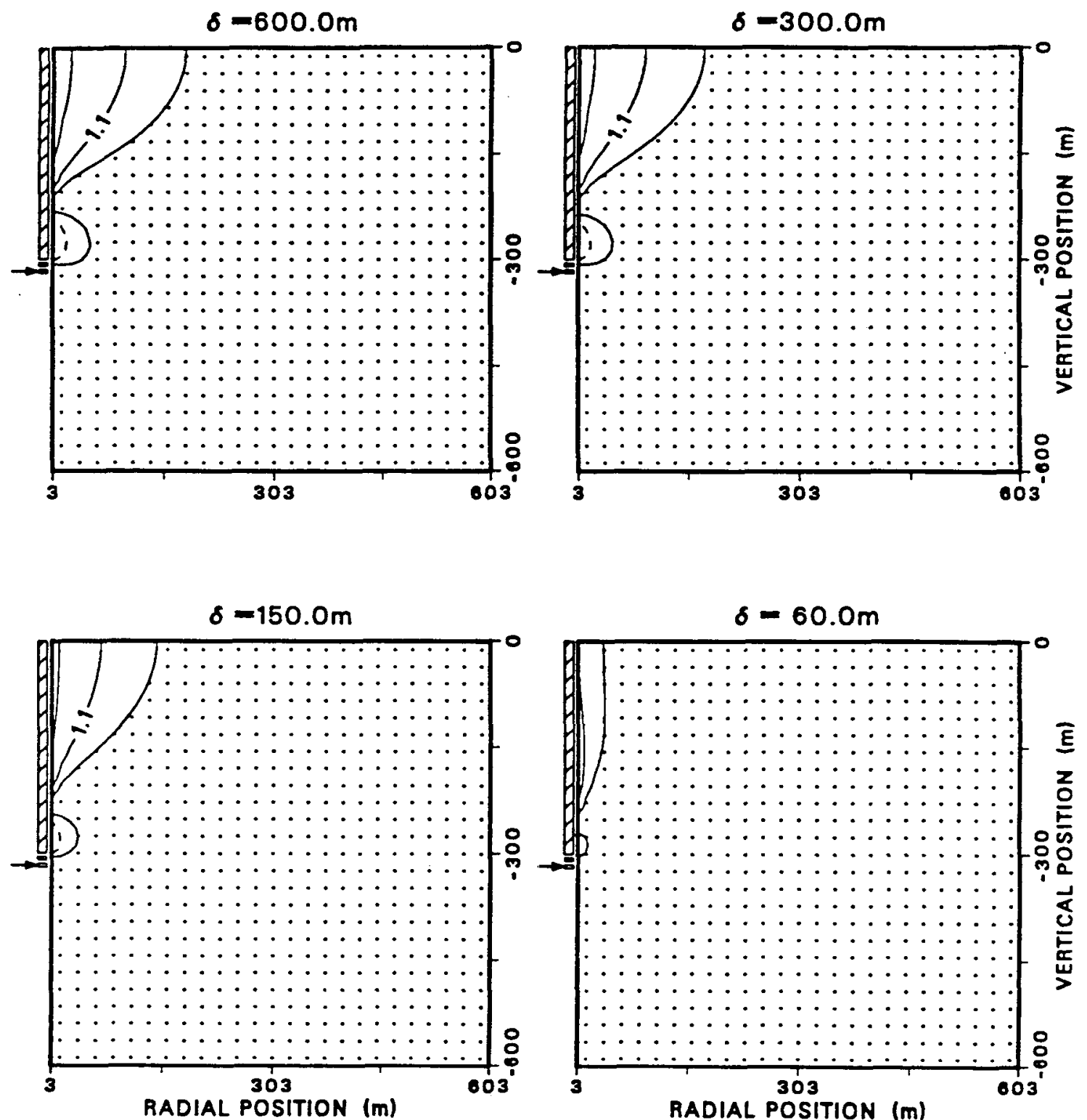

Figure 5.3-19b: Log-contour plots of the total-primary ratio for the same model as Figure $5.3-19$ a. The irregularly spaced contour levels are: $\pm 2, \pm 1.5, \pm 1.25$, and \pm 1 . The dotted area represents $0.5 \%$ or less deviation. 


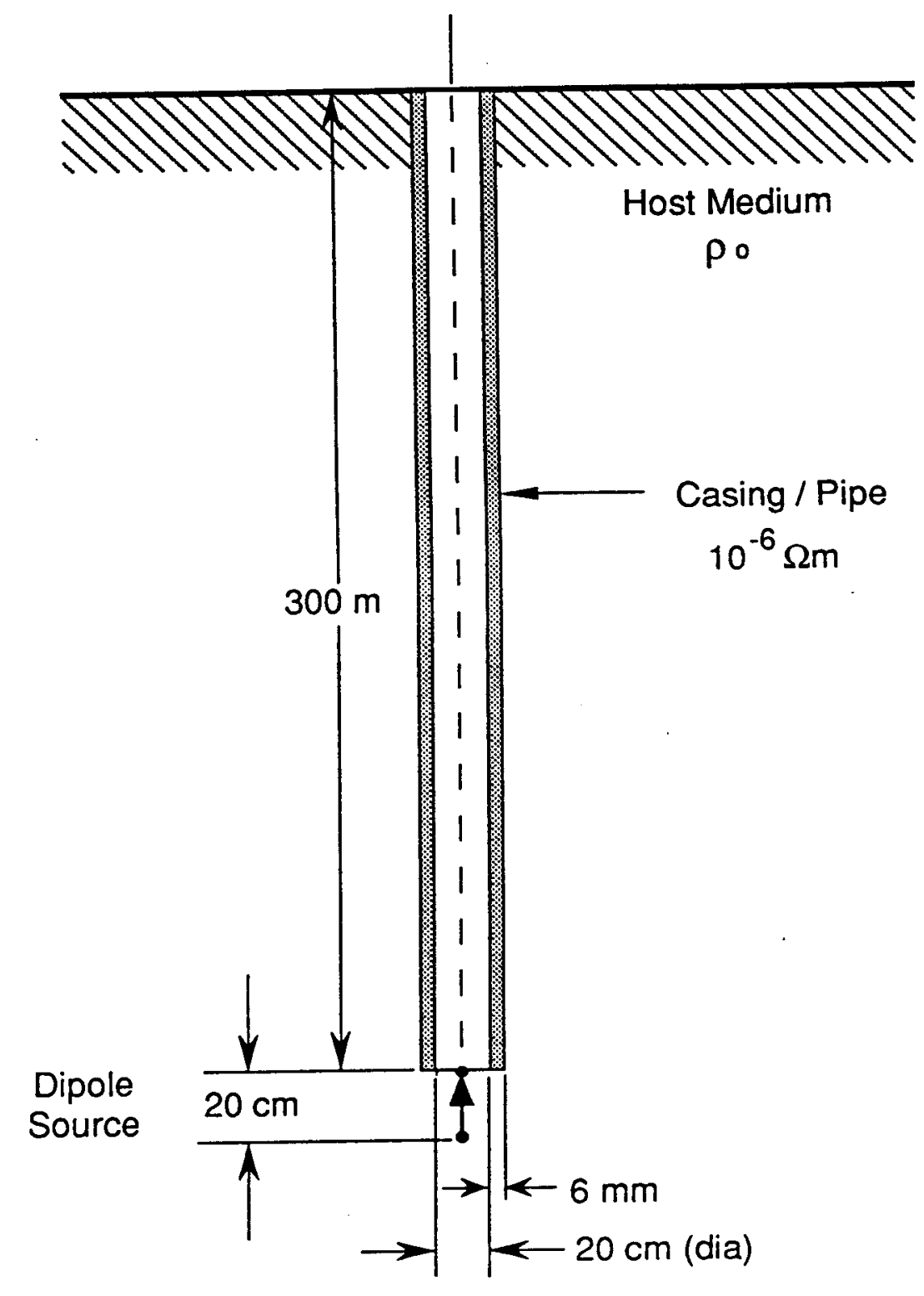

Figure 5.4-1: Generalized configuration of a $300 \mathrm{~m}$ casing coaxial with a dipole current source in a homogeneous half-space. 


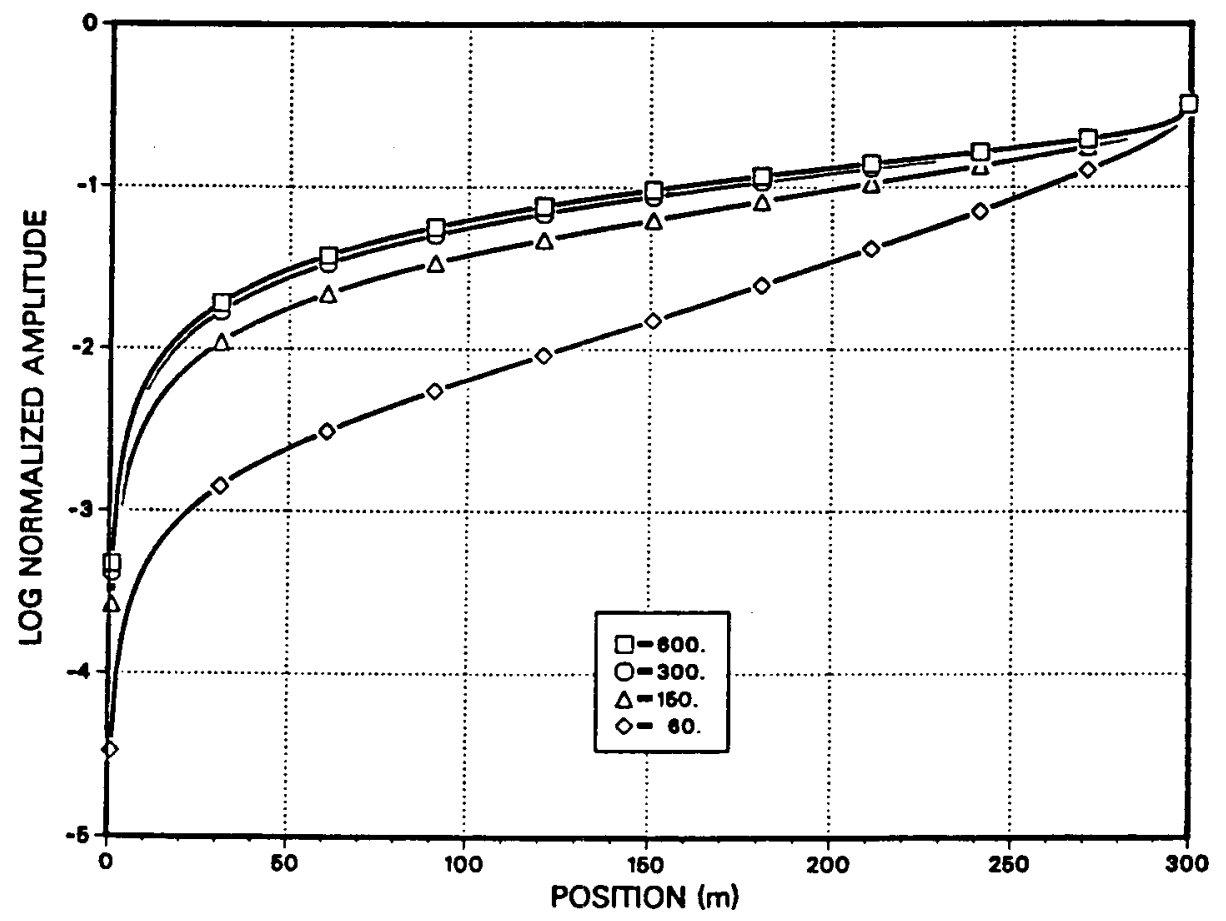

Figure 5.4-2: Semi-log plots of the normalized $E_{z}$ in the $300 \mathrm{~m} C_{1}$ for several $\delta_{L}$ with $\Delta z_{0}=0.2 \mathrm{~m}$. The $\mathrm{E}_{z}$ is normalized by the factor $I / S_{c}$. 

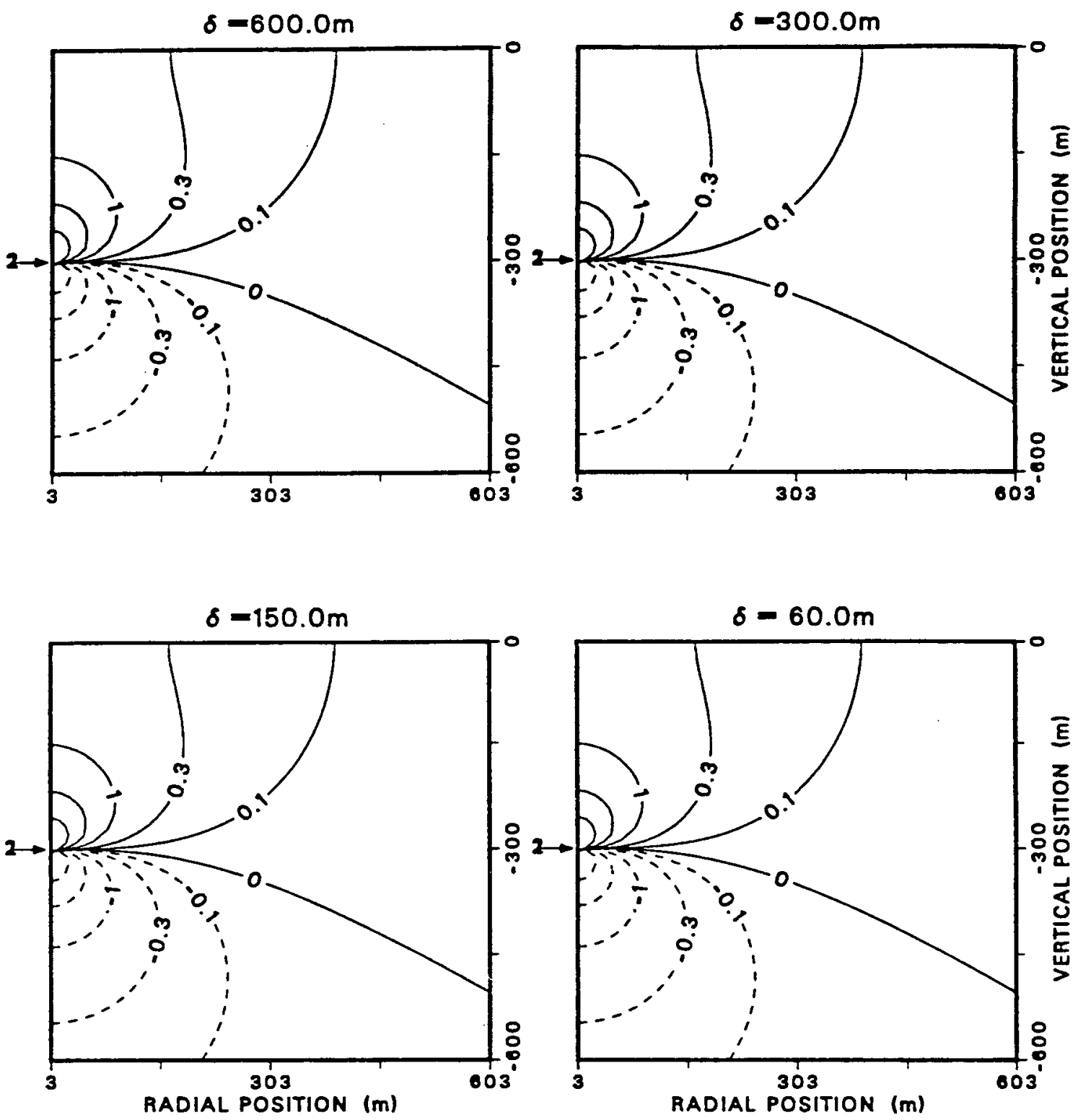

Figure 5.4-3: Contour plots of the normalized half-space potentials of a dipole source with $\Delta z_{0}=0.2 \mathrm{~m}$ located $300 \mathrm{~m}$ below the surface for several $\delta_{L}$. A logarithmic contour interval of 0.5 is used. The potentials are normalized by the factor $I / 4 \pi \sigma_{0} \times 10^{-5}$. 

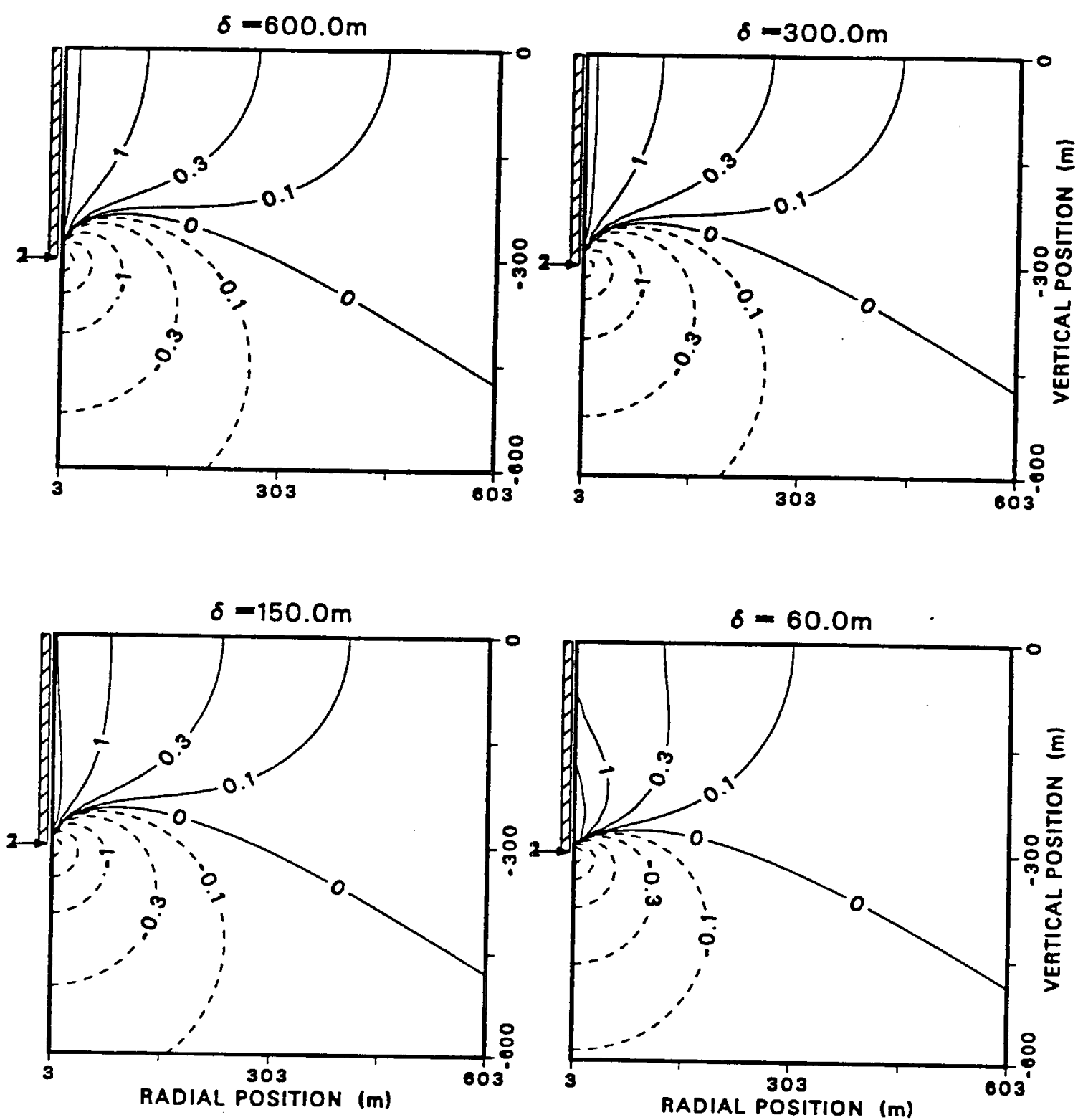

Figure 5.4-4: Contour plots of the normalized half-space potentials due to the $300 \mathrm{~m} C_{1}$ for several $\delta_{L}$ with $\Delta z_{0}=0.2 \mathrm{~m}$. A logarithmic contour interval of 0.5 is used. The potentials are normalized by the factor $I / 4 \pi \sigma_{0} \times 10^{-3}$. 


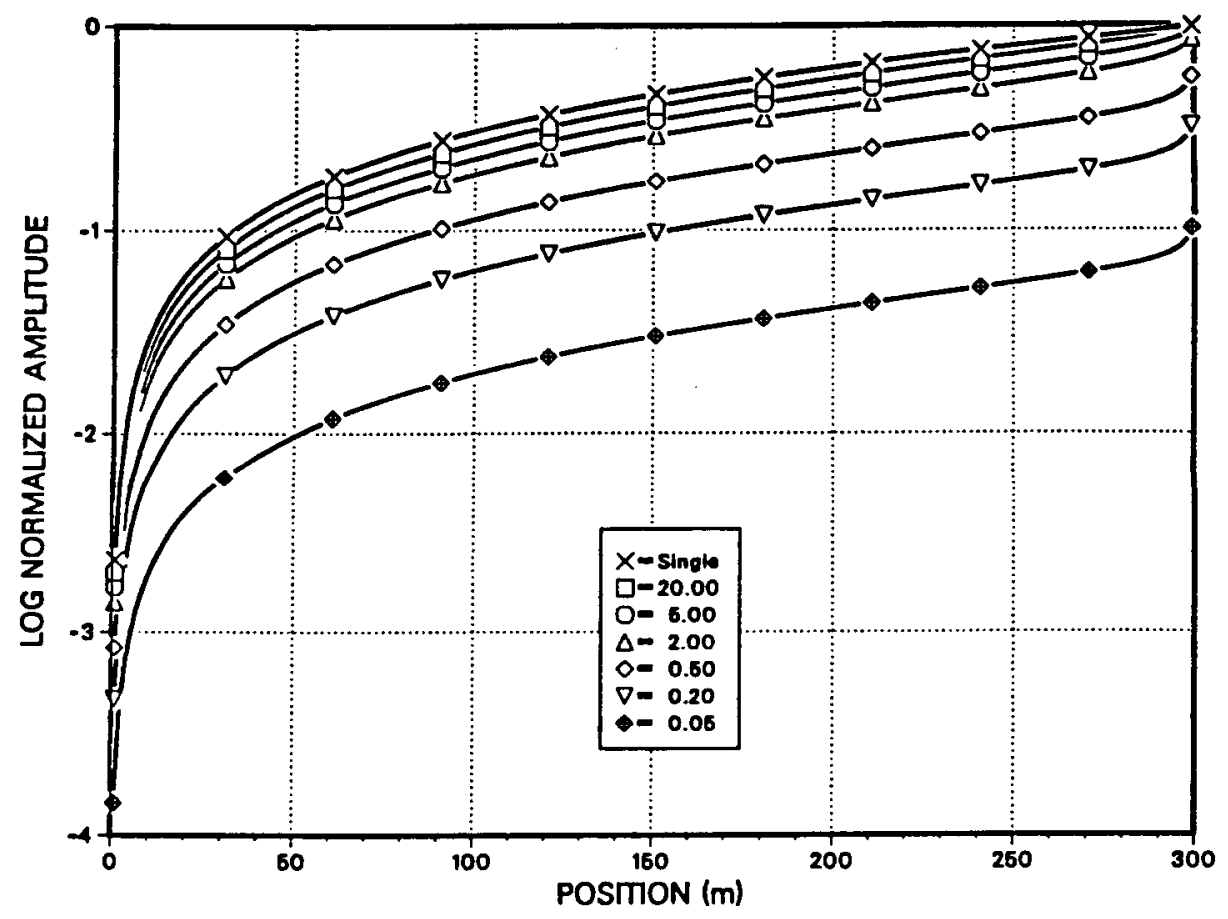

Figure 5.4-5: Semi-log plots of the normalized $E_{z}$ in the $300 \mathrm{~m} C_{1}$ for several $\Delta z_{0}$ with $\delta_{L}=600 \mathrm{~m}$. The $E_{z}$ is normalized by the factor $I / S_{c}$. 

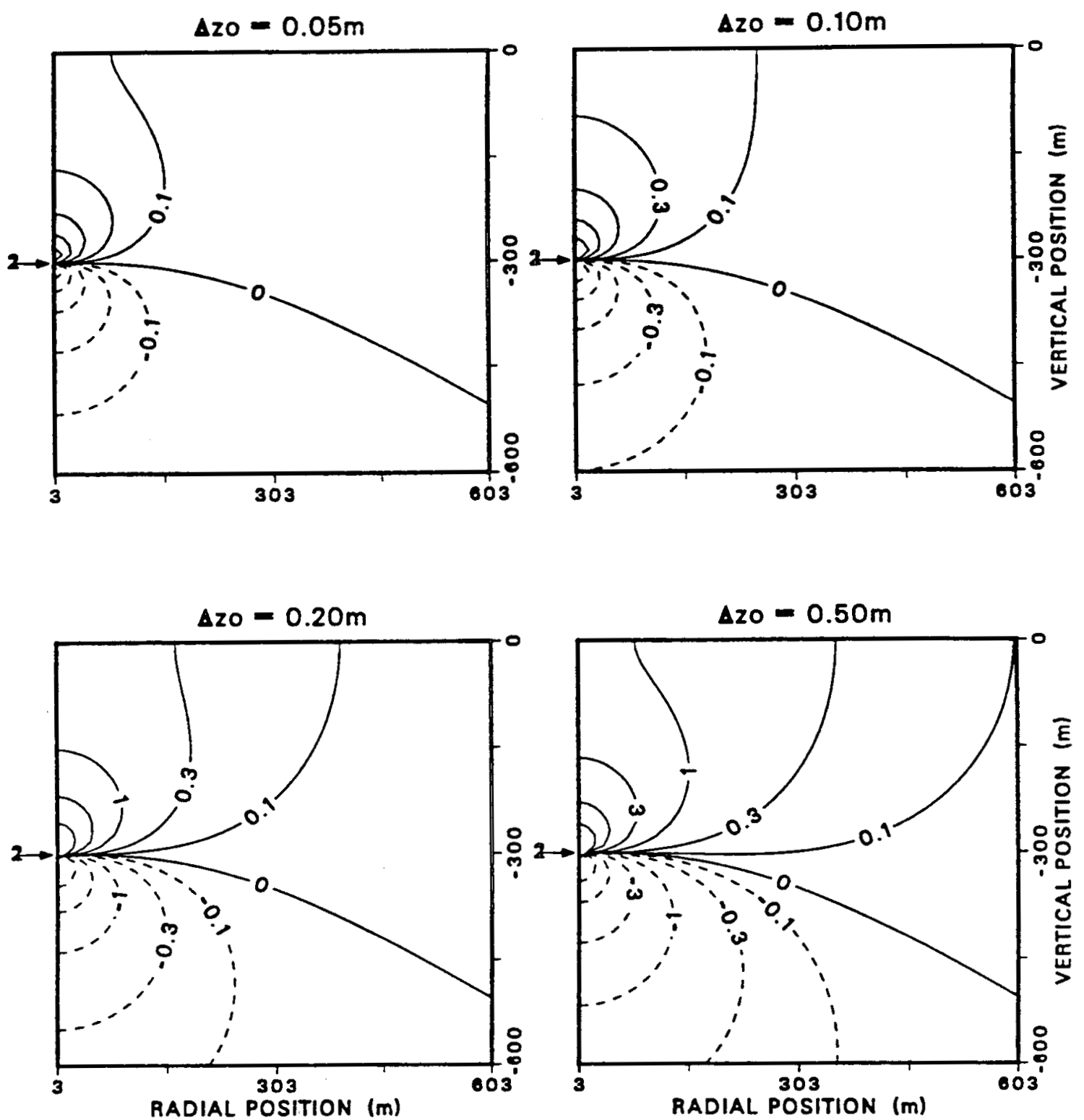

Figure 5.4-6a: Contour plots of the normalized half-space potentials using a dipole source with $\Delta z_{0}=0.05,0.1,0.2$, and $0.5 \mathrm{~m}$ located $300 \mathrm{~m}$ below the surface with $\delta_{L}=600 \mathrm{~m}$. A logarithmic contour interval of 0.5 is used. The potentials are normalized by the factor $I / 4 \pi \sigma_{0} \times 10^{-5}$. 

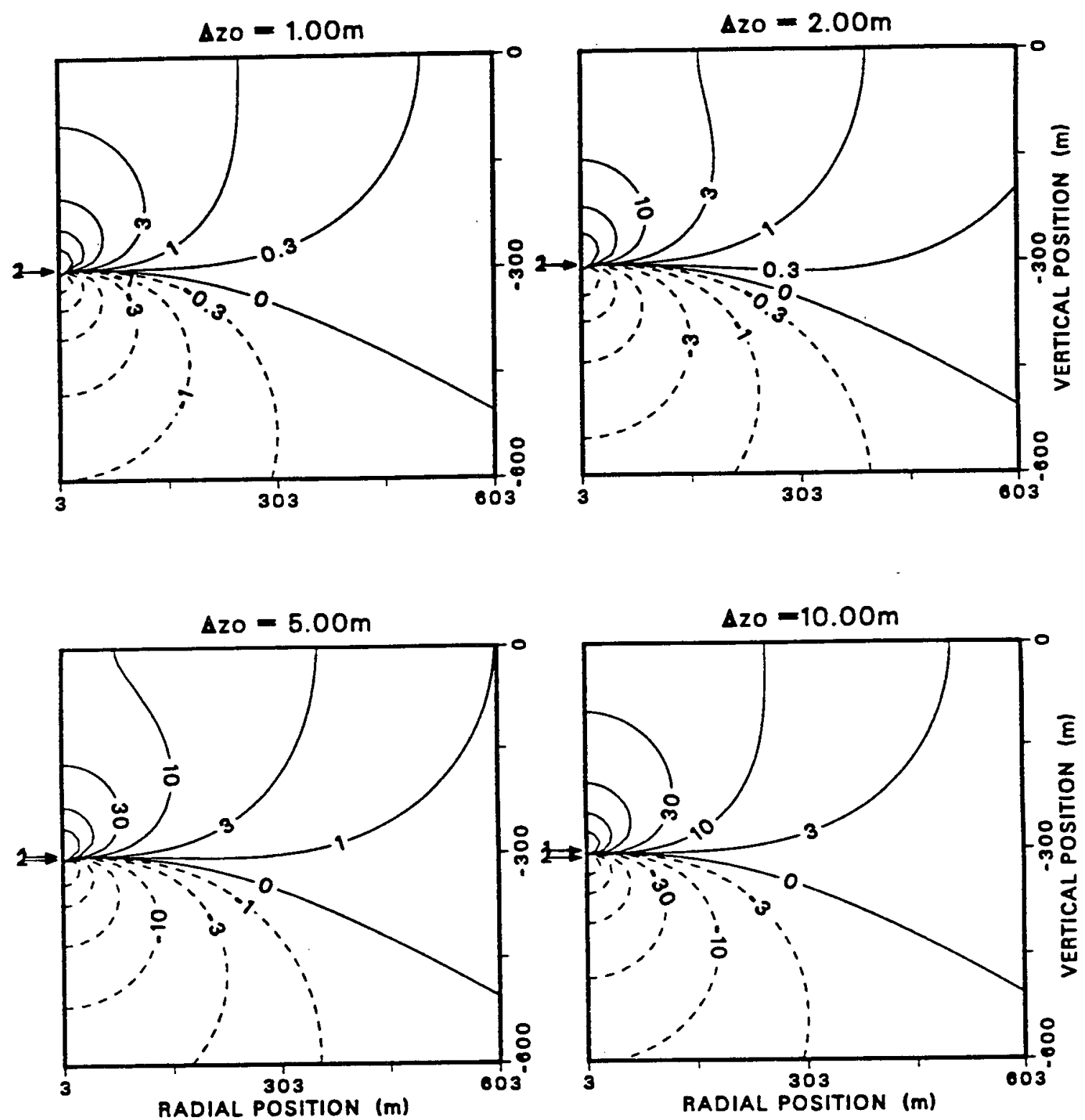

Figure 5.4-6b: Contour plots of the normalized half-space potentials using a dipole source with $\Delta z_{0}=1.0,2.0,5.0,10.0 \mathrm{~m}$ located $300 \mathrm{~m}$ below the surface with $\delta_{L}=600 \mathrm{~m}$. A logarithmic contour interval of 0.5 is used. The potentials are normalized by the factor $I / 4 \pi \sigma_{0} \times 10^{-5}$. 

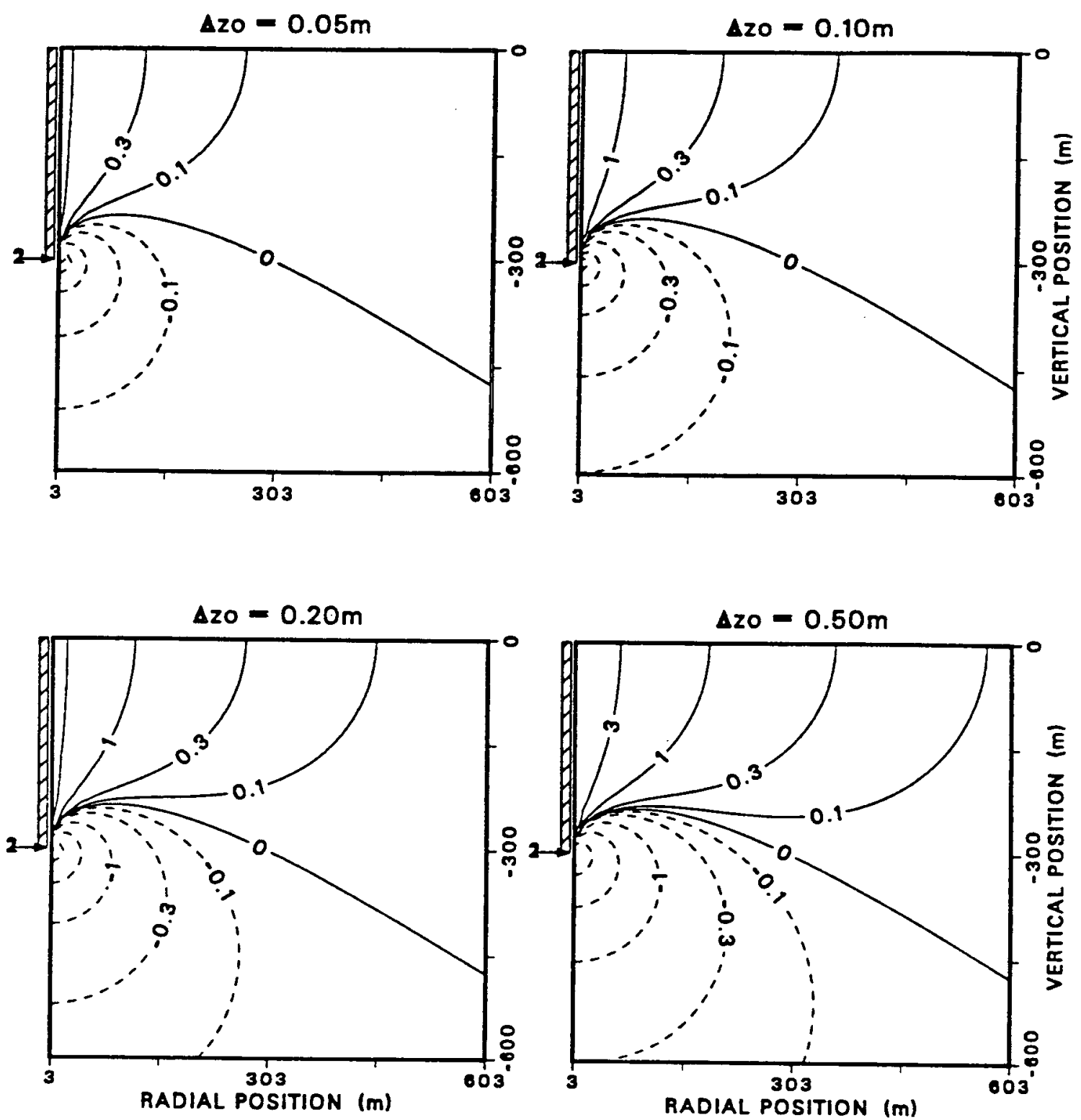

Figure 5.4-7a: Contour plots of the normalized half-space potentials due to the $300 \mathrm{~m} C_{1}$ for $\Delta z_{0}=0.05,0.1,0.2$, and $0.5 \mathrm{~m}$ with $\delta_{L}=600 \mathrm{~m}$. A logarithmic contour interval of 0.5 is used. The potentials are normalized by the factor $I / 4 \pi \sigma_{0} \times 10^{-3}$. 

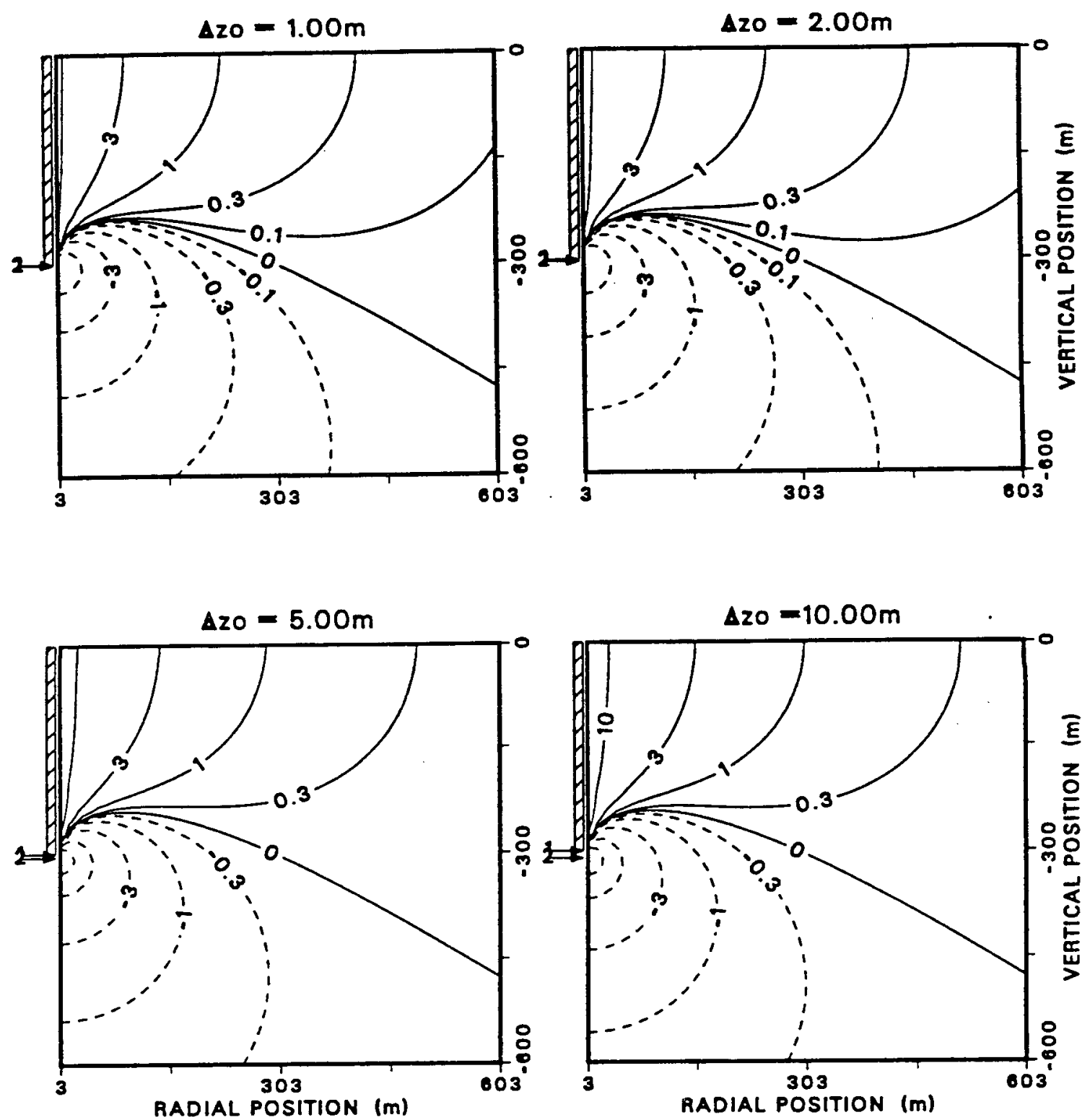

Figure 5.4-7b: Contour plots of the normalized half-space potentials due to the $300 \mathrm{~m} C_{1}$ for $\Delta z_{0}=1.0,2.0,5.0$, and $10.0 \mathrm{~m}$ with $\delta_{L}=600 \mathrm{~m}$. A logarithmic contour interval of 0.5 is used. The potentials are normalized by the factor $1 / 4 \pi \sigma_{0} \times 10^{-3}$. 


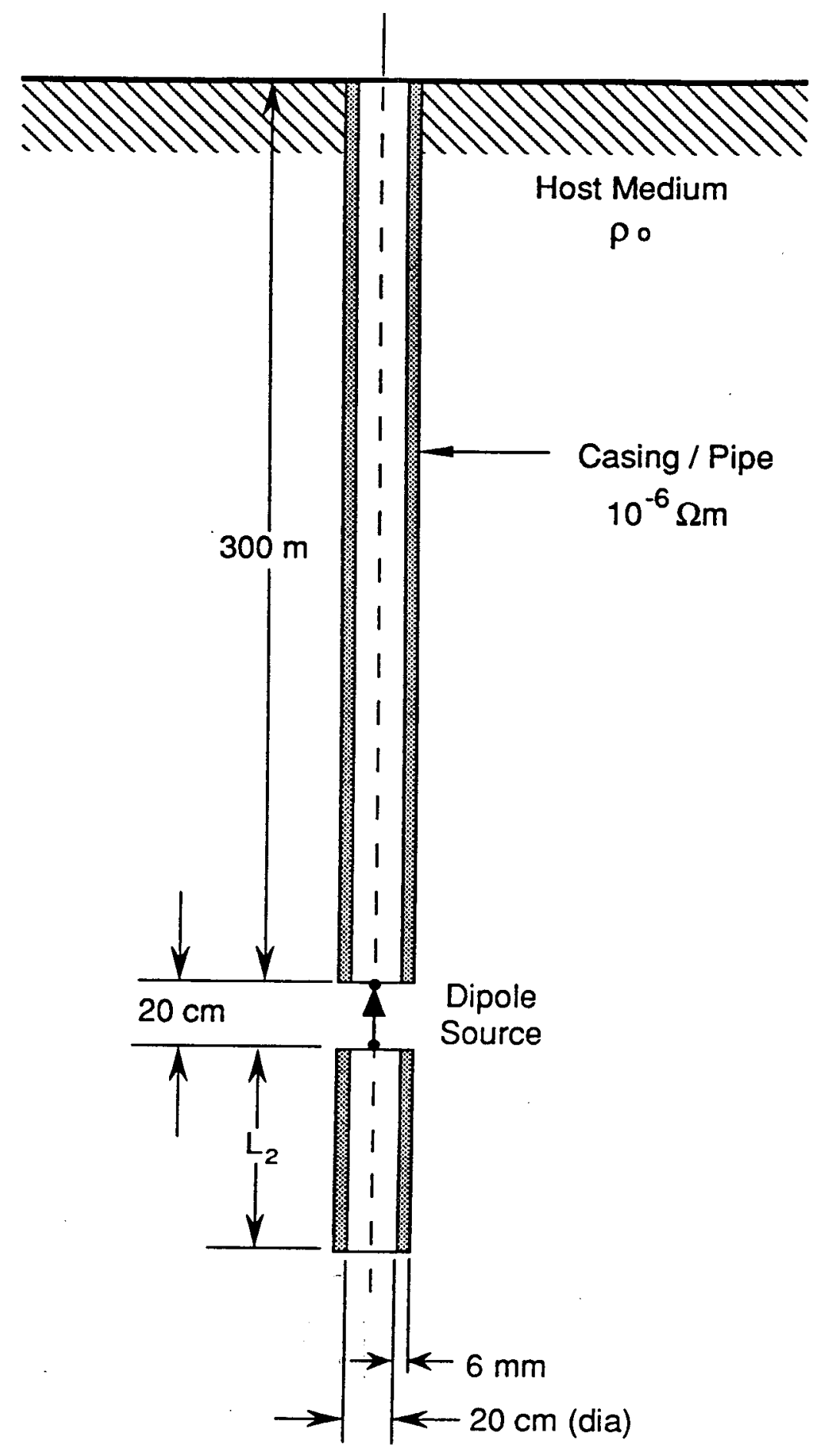

Figure 5.4-8: Configuration for the two casing segments and a coaxial dipole source. 


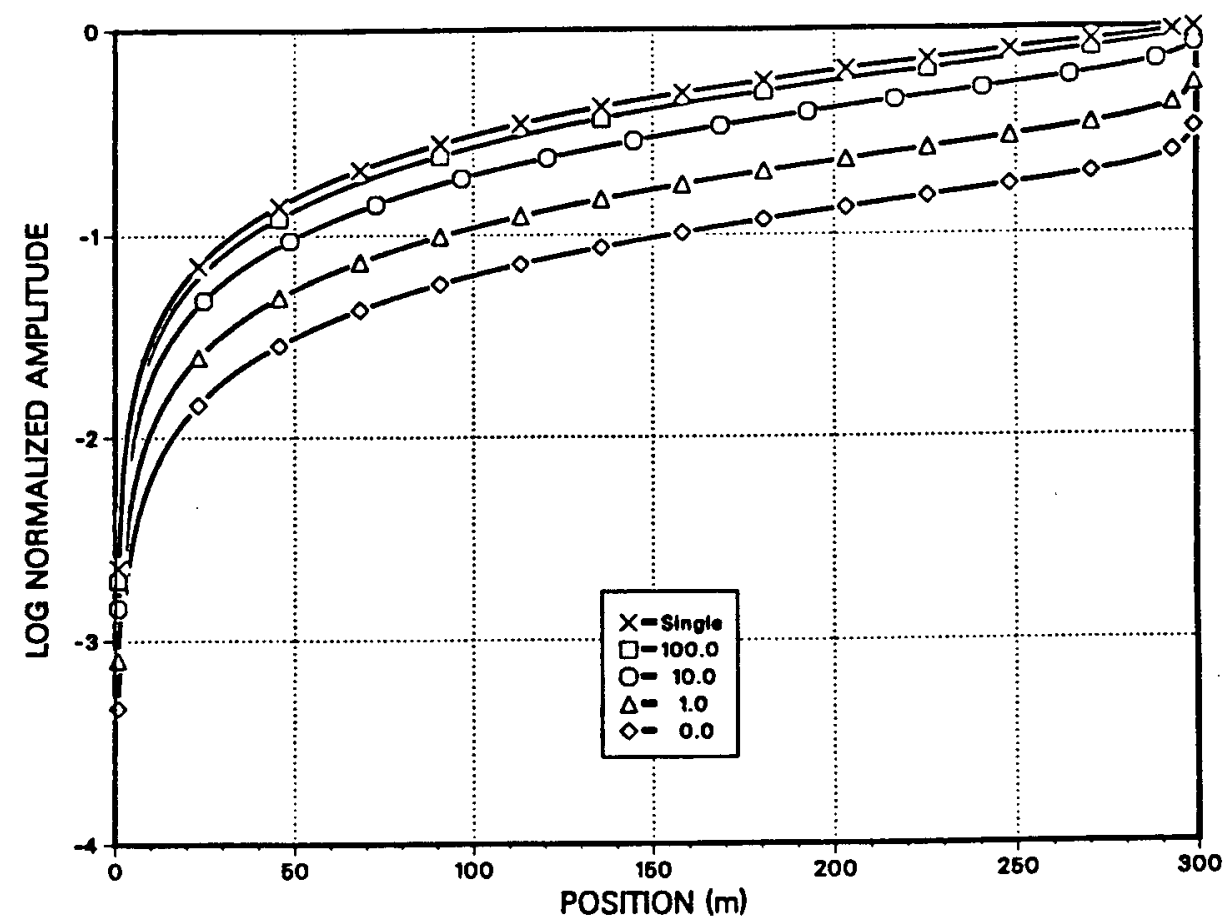

Figure 5.4-9a: Semi-log plots of the normalized $\mathrm{E}_{z}$ in the $300 \mathrm{~m} C_{1}$ for several $L_{2}$ with $\delta_{L}=600 \mathrm{~m}$ and $\Delta z_{0}=0.2 \mathrm{~m}$. The $\mathrm{E}_{z}$ is normalized by the factor $I / S_{c}$.

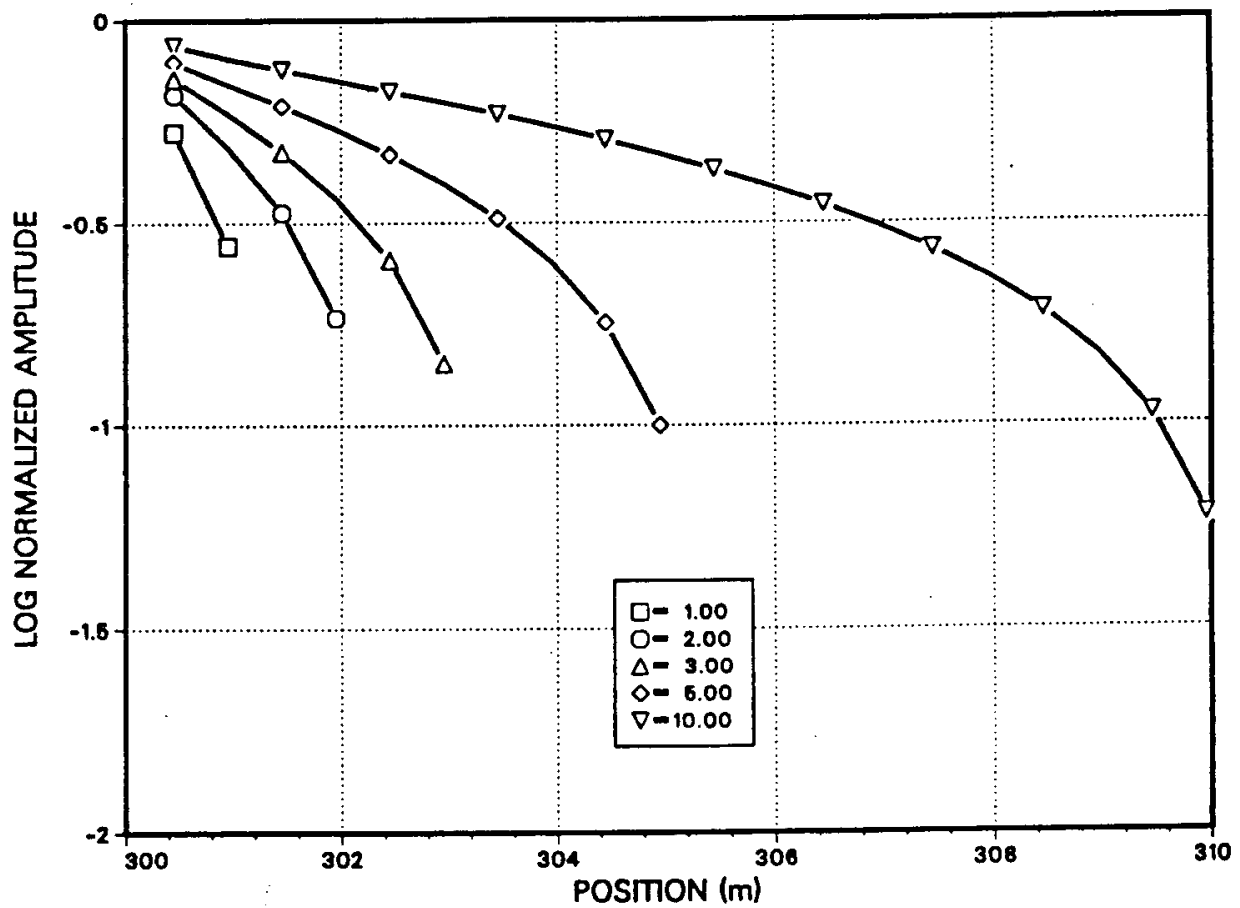

Figure 5.4-9b: Semi-log plots of the normalized $E_{z}$ in $C_{2}$ for several $L_{2}$ with $L_{1}=300 \mathrm{~m}$, $\delta_{L}=600 \mathrm{~m}$, and $\Delta z_{0}=0.2 \mathrm{~m}$. The $E_{z}$ is normalized by the factor $I / S_{c}$. 

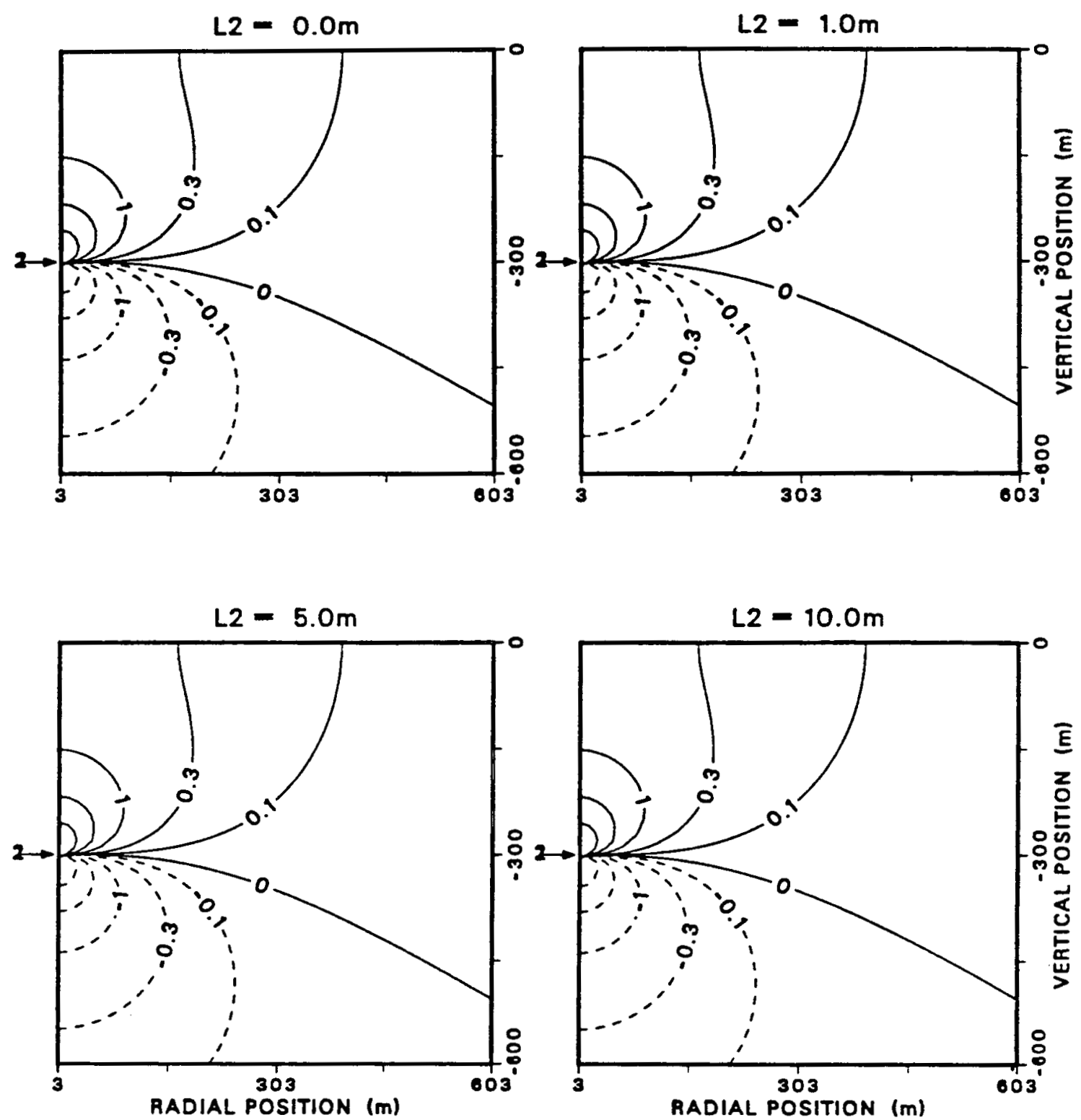

Figure 5.4-10a: Contour plots of the normalized half-space potentials of the dipole source with of $\Delta z_{0}=0.2 \mathrm{~m}$ located $300 \mathrm{~m}$ below the surface with $\delta_{L}=600 \mathrm{~m}$ for $L_{2}=0,1,2$, and $10 \mathrm{~m}$. A logarithmic contour interval of 0.5 is used. The potentials are normalized by the factor $I / 4 \pi \sigma_{0} \times 10^{-5}$. 

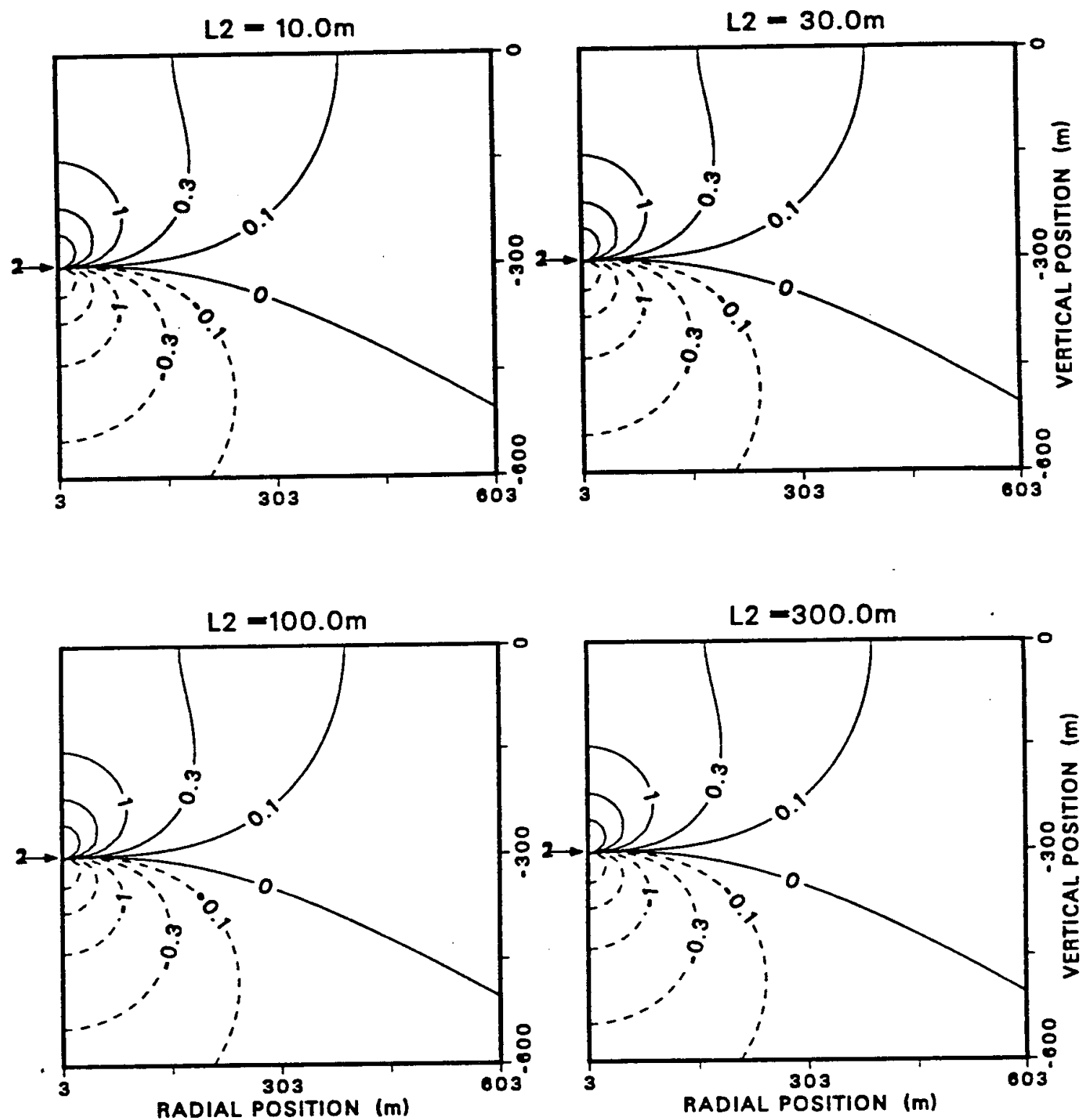

Figure 5.4-10b: Contour plots of the normalized half-space potentials using a dipole source with $\Delta z_{0}=0.2 \mathrm{~m}$ located $300 \mathrm{~m}$ below the surface with $\delta_{L}=600 \mathrm{~m}$ for $L_{2}=10,30,100$, and $300 \mathrm{~m}$. A logarithmic contour interval of 0.5 is used. The potentials are normalized by the factor $1 / 4 \pi \sigma_{0} \times 10^{-5}$. 

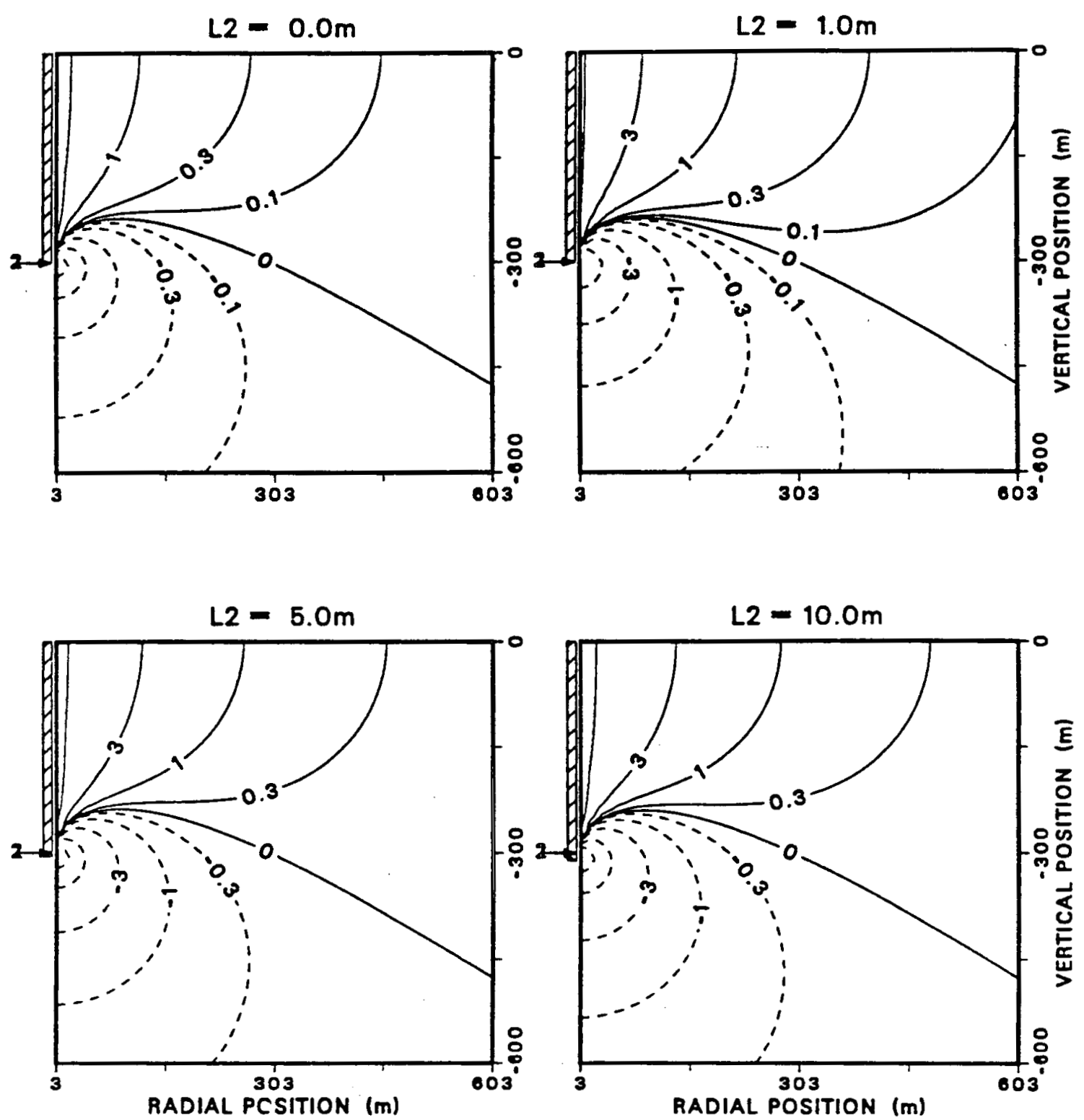

Figure 5.4-11a: Contour plots of the normalized half-space potentials due to the $300 \mathrm{~m} C_{1}$ for $L_{2}=0,1,2,10 \mathrm{~m}$ with $\delta_{L}=600 \mathrm{~m}$ for $\Delta z_{0}=0.2 \mathrm{~m}$. A logarithmic contour interval of 0.5 is used. The potentials are normalized by the factor $I / 4 \pi \sigma_{0} \times 10^{-3}$. 

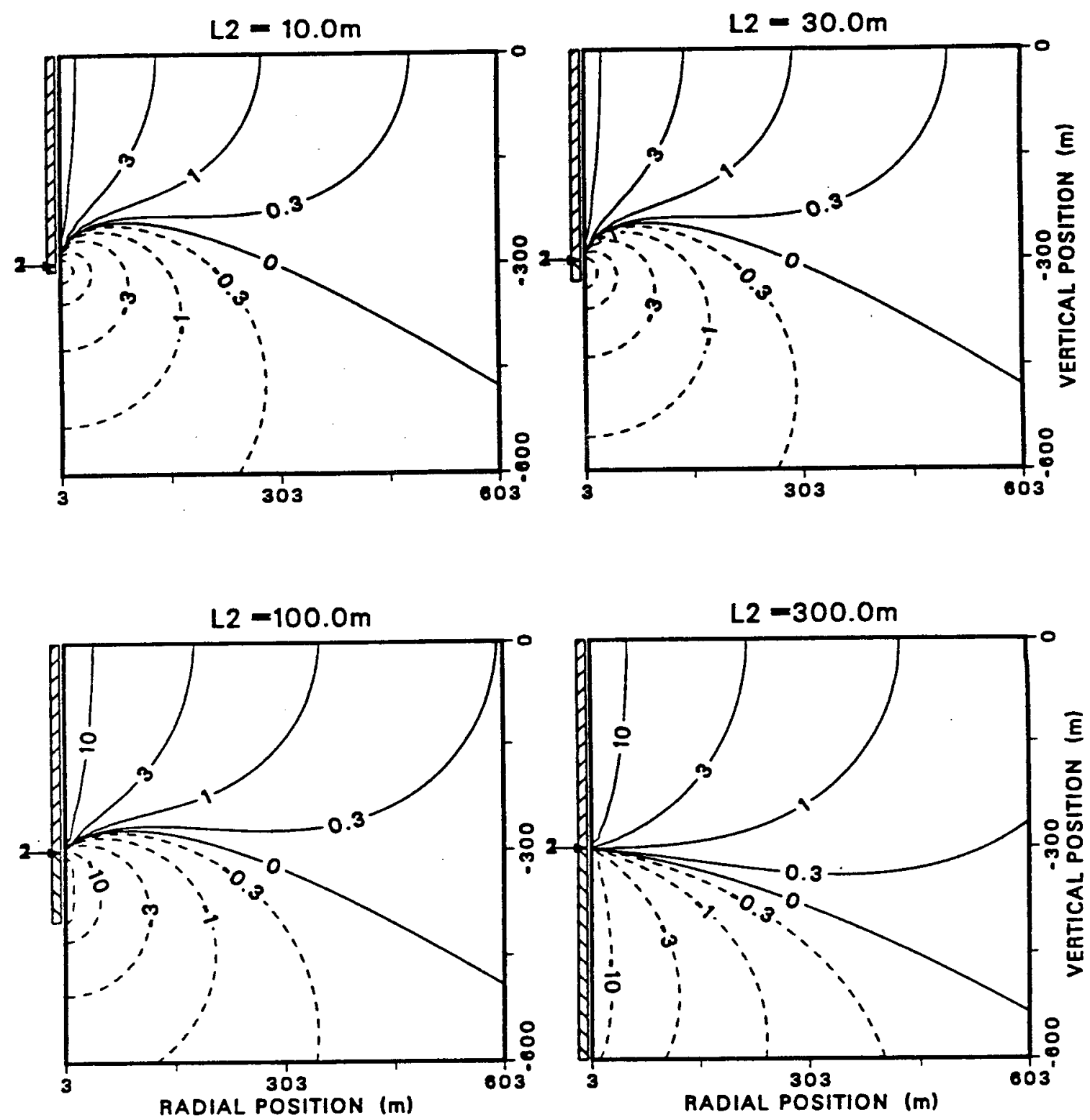

Figure 5.4-11b: Contour plots of the normalized half-space potentials due to the $300 \mathrm{~m} C_{1}$ for $L_{2}=10,30,100,300 \mathrm{~m}$ with $\delta_{L}=600 \mathrm{~m}$ and $\Delta z_{0}=0.2 \mathrm{~m}$. A logarithmic contour interval of 0.5 is used. The potentials are normalized by the factor $I / 4 \pi \sigma_{0} \times 10^{-3}$. 

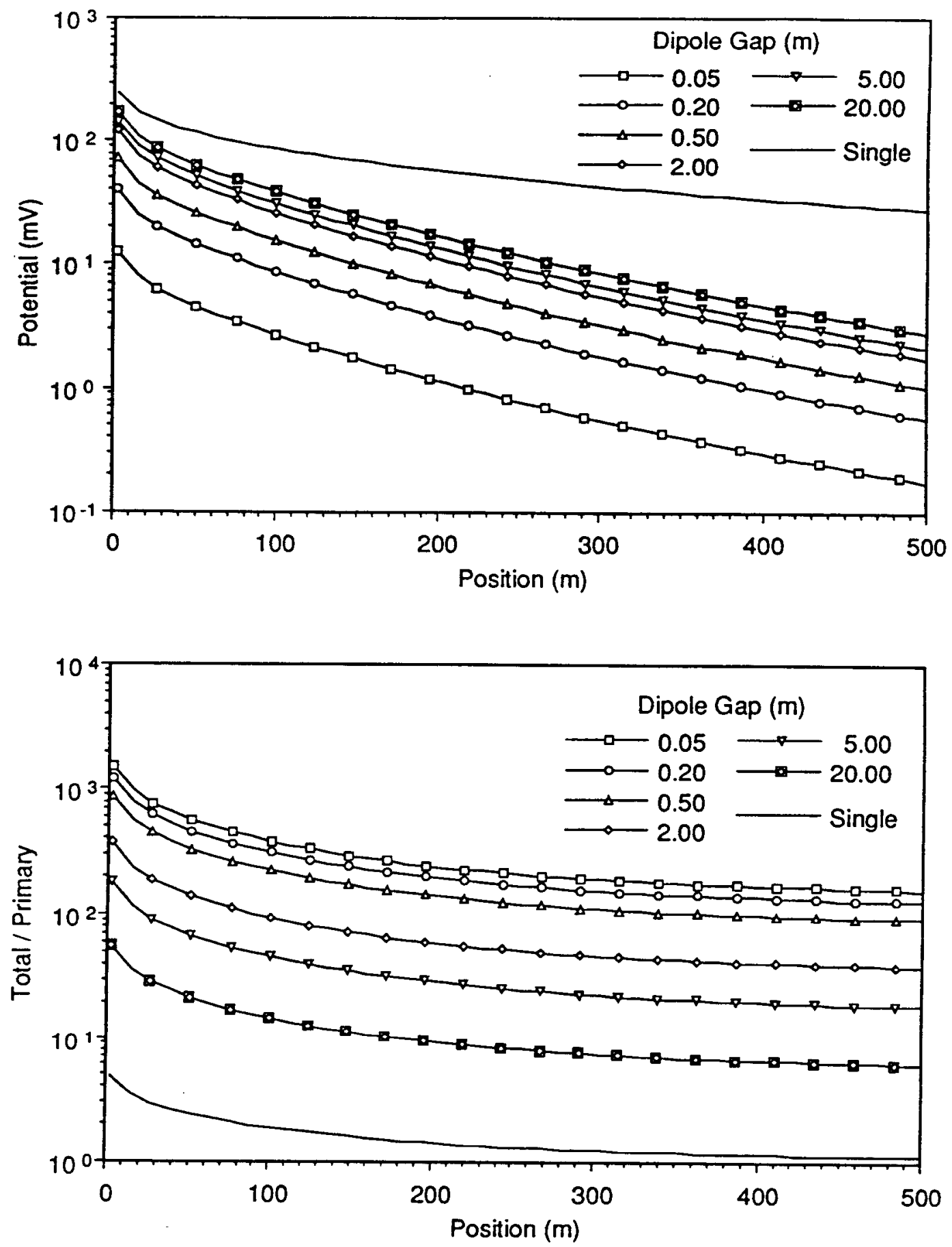

Figure 5.4-12: Semi-logarithmic plots of the total (top) and total-primary ratio (bottom) at the surface for a $300 \mathrm{~m}$ casing with conductance of $3883 \mathrm{~S} \cdot \mathrm{m}$ in a $100 \Omega \cdot \mathrm{m}$ medium for several dipole gap lengths. 

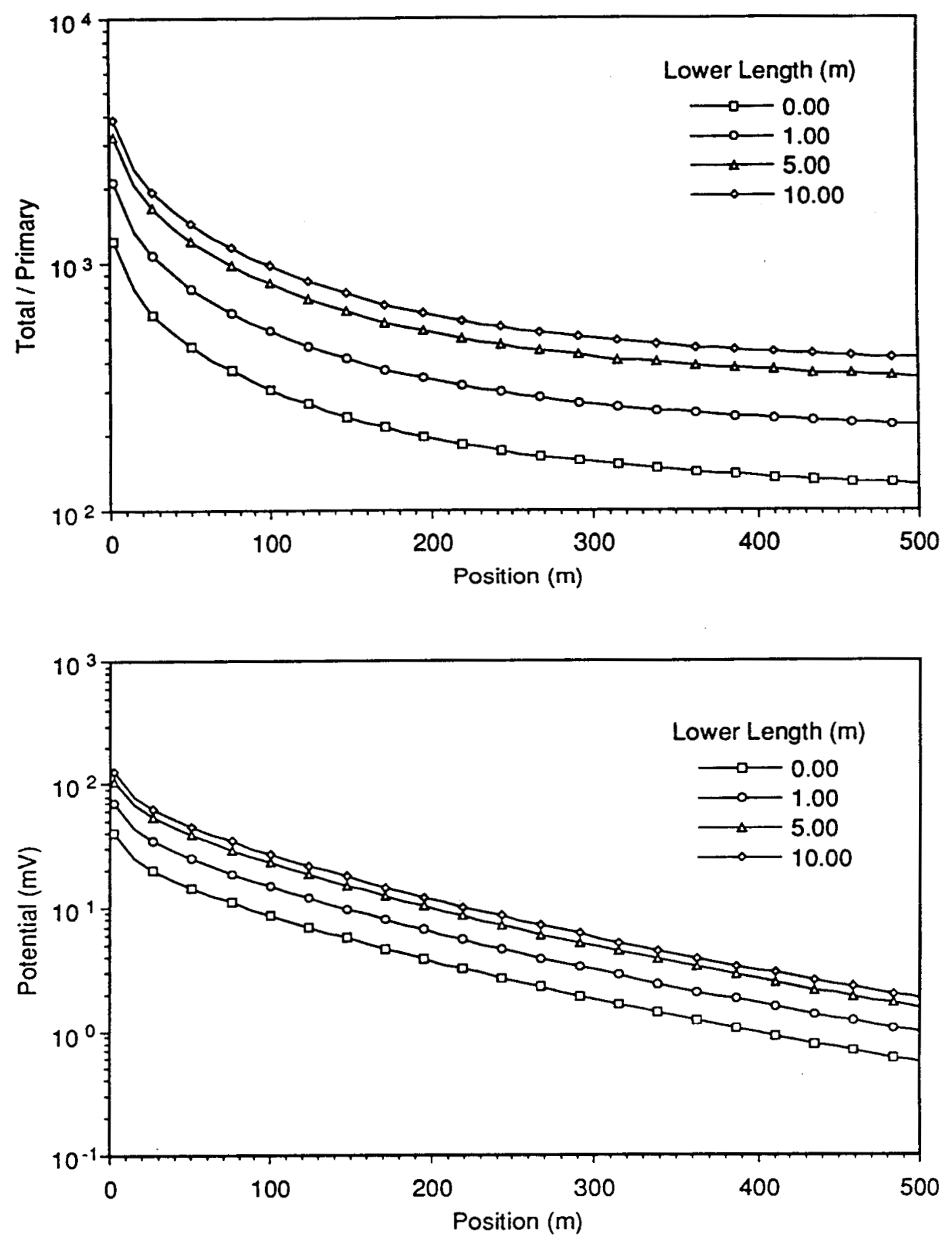

Figure 5.4-13: Semi-logarithmic plots of the total (top) and total-primary ratio (bottom) at the surface for a $300 \mathrm{~m}$ casing with conductance of $3883 \mathrm{~S} \cdot \mathrm{m}$ in a $100 \Omega \cdot \mathrm{m}$ medium for several lower segment lengths. The separation between the pipes and the dipole gap is $\Delta d=\Delta z_{0}=0.2 \mathrm{~m}$. 


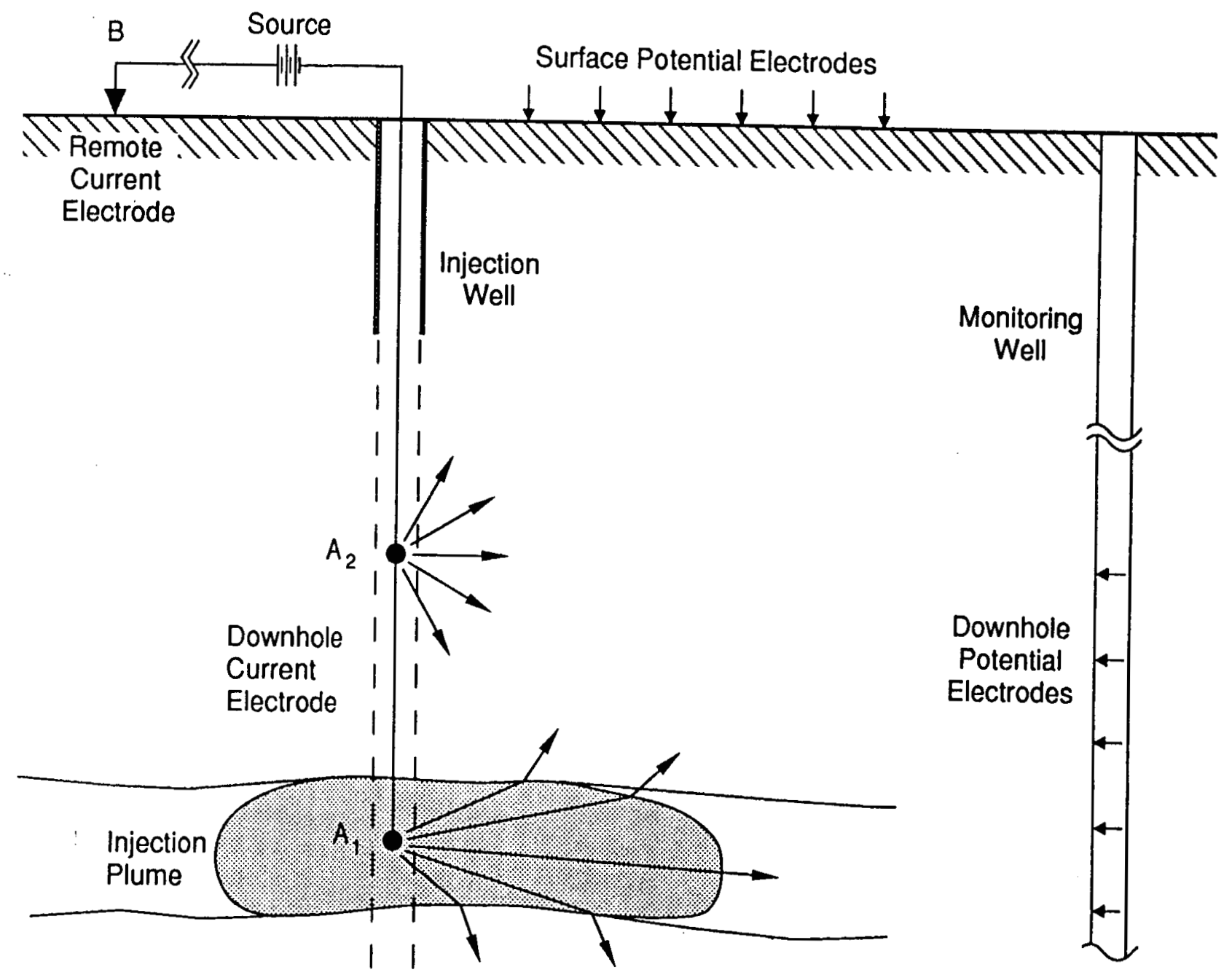

Figure 5.5-1: The generalized cross-section for the resistivity monitoring of an injection zone. 


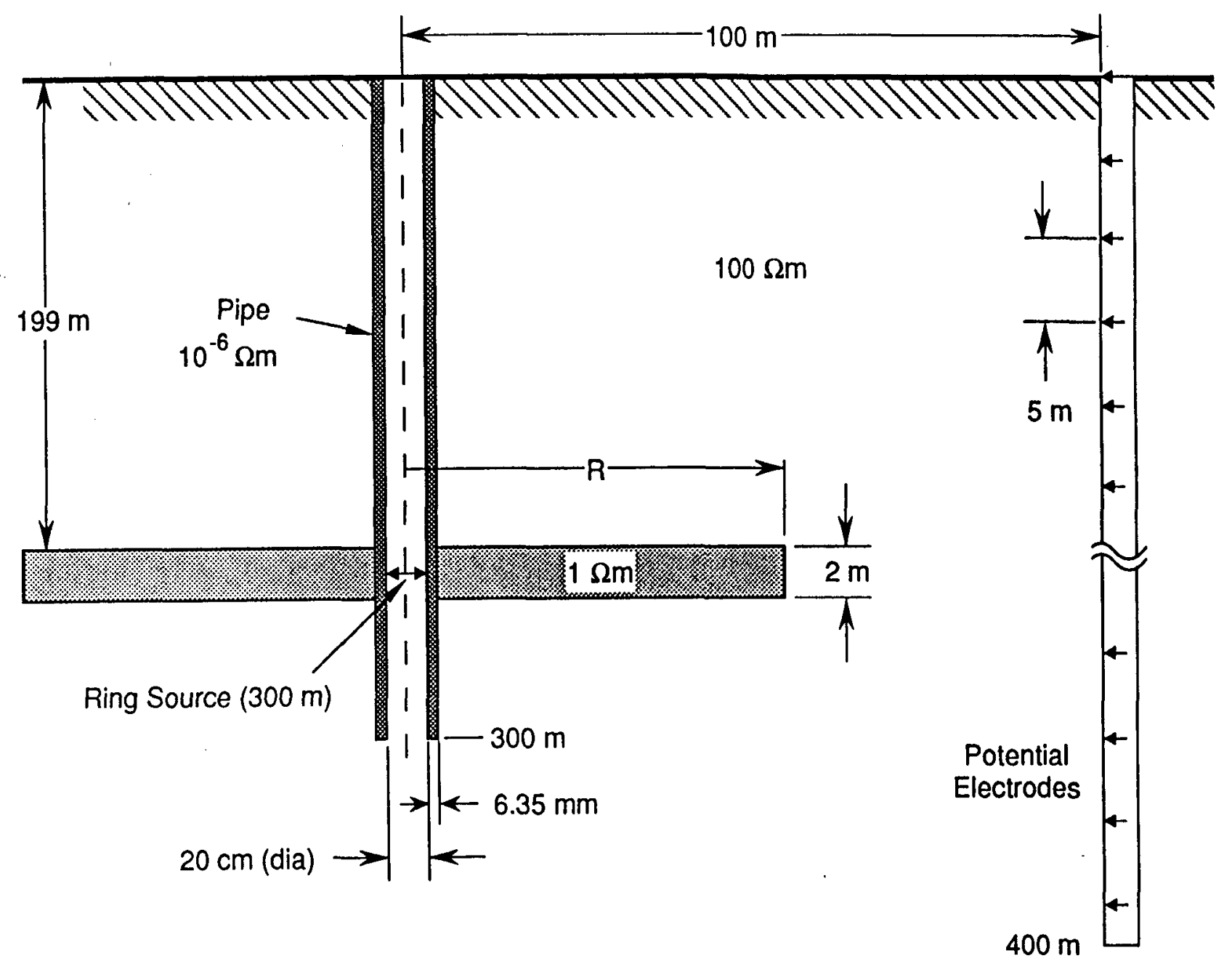

Figure 5.5-2: The configuration for the cross-hole resistivity monitoring simulation of an injection process. 

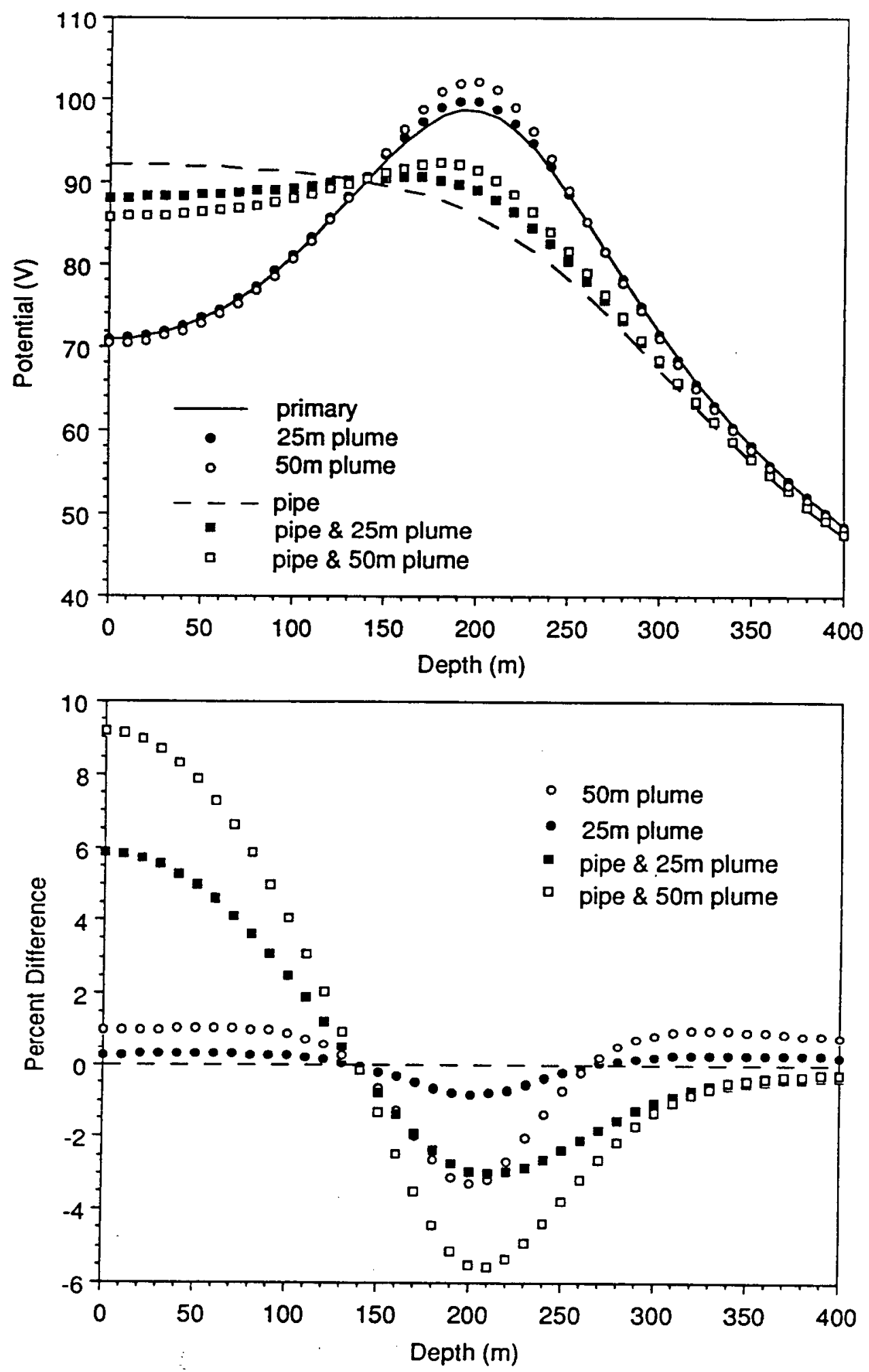

Figure 5.5-3: Plots of the potentials (top) and percent difference between the background and injection potentials (bottom) for plume only (circle): and plume/pipe (square) for $25 \mathrm{~m}$ (black) and $50 \mathrm{~m}$ (white) plumes. The pre-injection potentials are the dashed lines (with casing) and solid lines (without casing). 

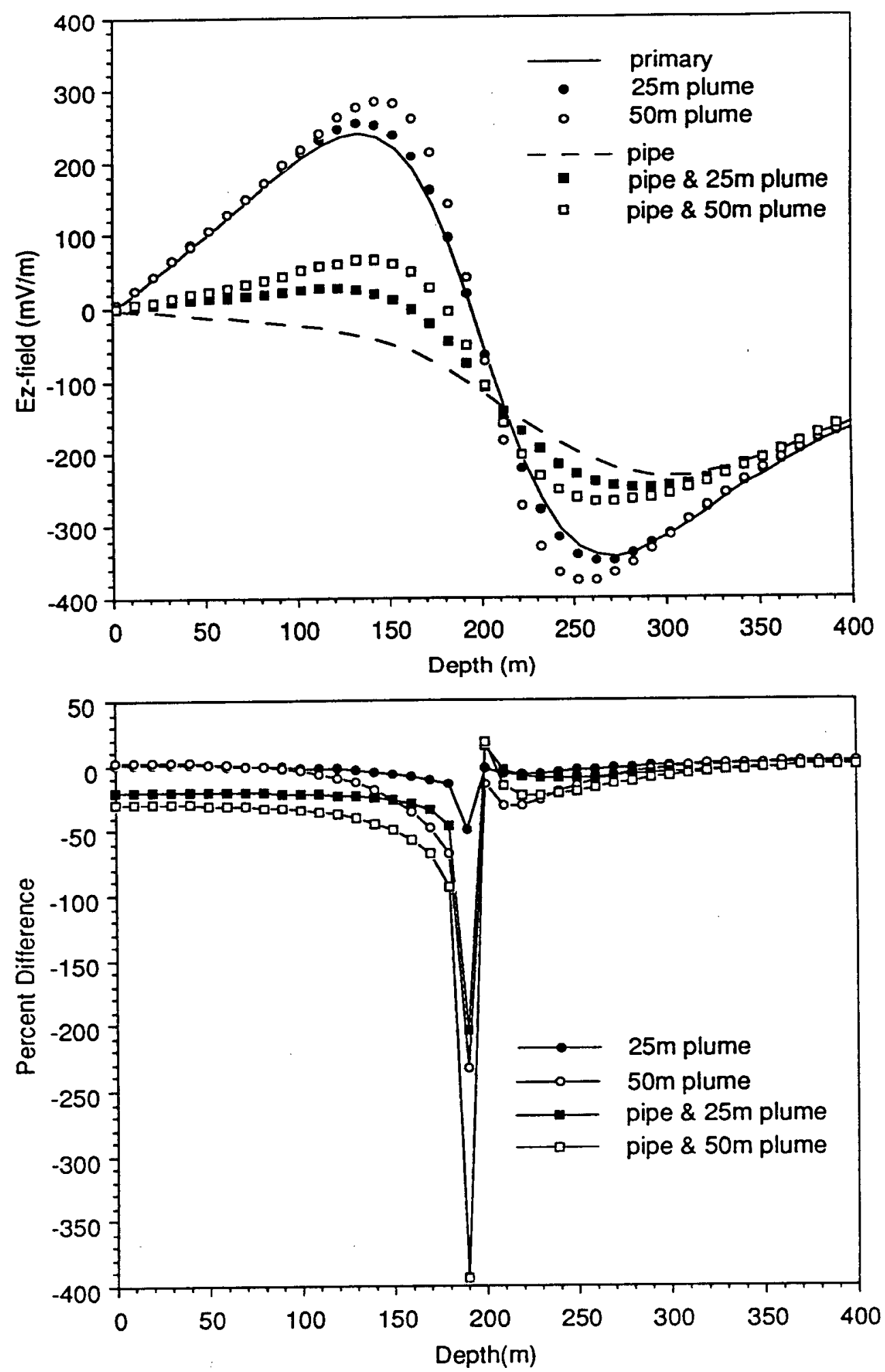

Figure 5.5-4: Plots of the $E_{z}$ fields (top) and percent difference between the background and injection $E_{z}$-fields (bottom) for plume only (circle) and plume/pipe (square) for $25 \mathrm{~m}$ (black) and $50 \mathrm{~m}$ (white) plumes. The pre-injection potentials are the dashed lines (with casing) and solid lines (without casing). 

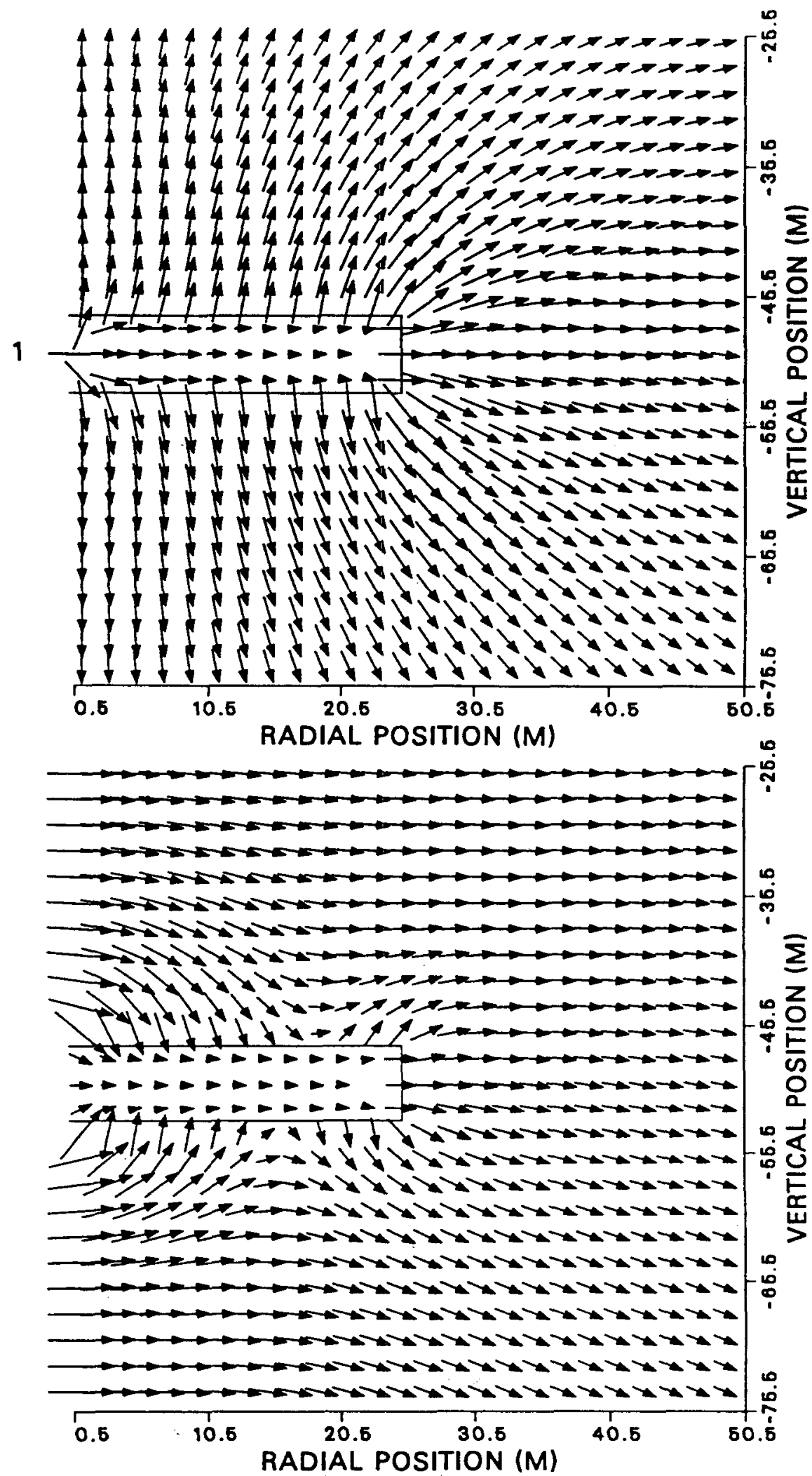

Figure 5.5-5: a) Current patterns in the medium and conductive plume for the mise-à-la-masse configuration (top). b) Current patterns in the medium and conductive plume for the energized casing configuration (bottom). 

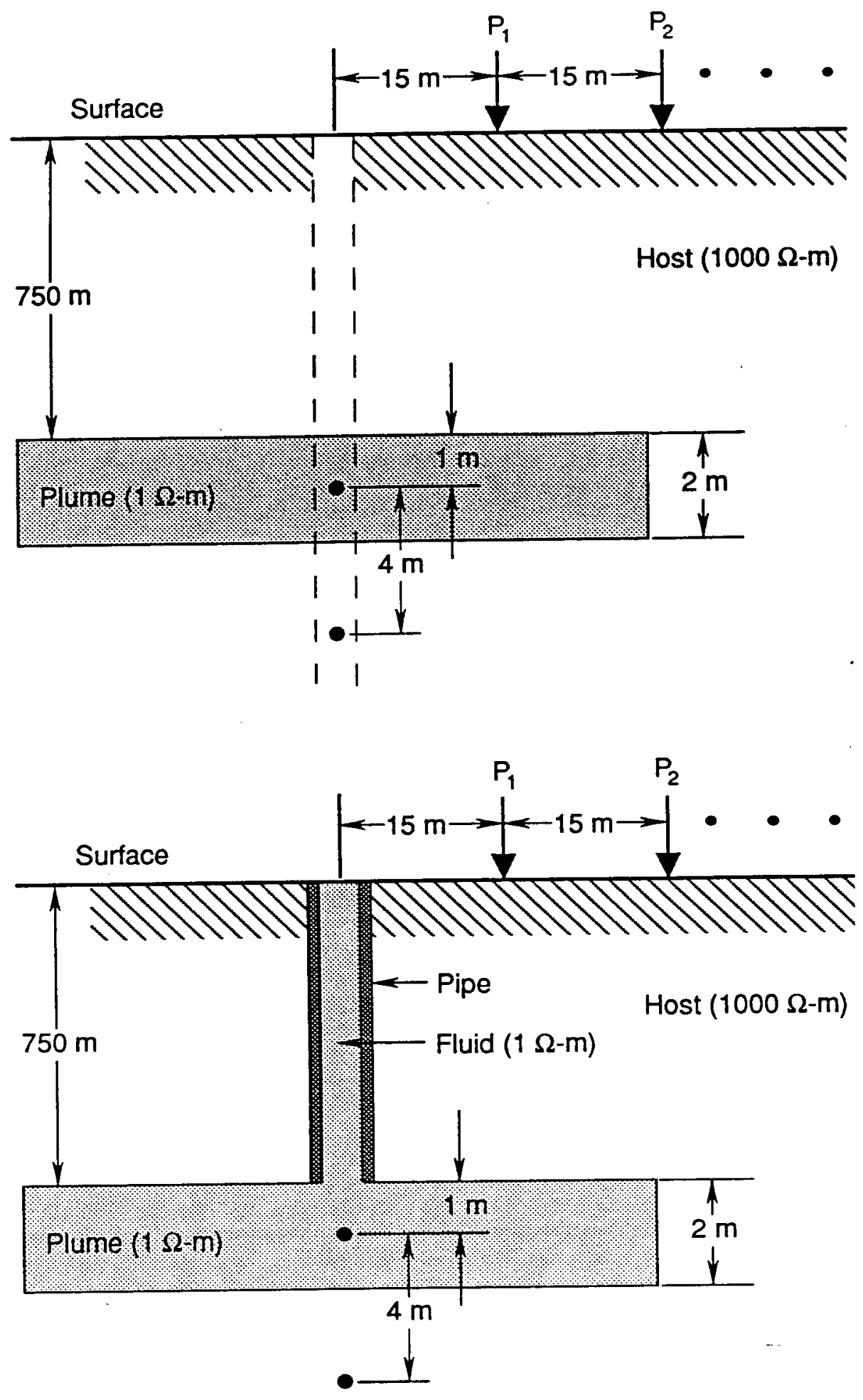

Figure 5.5-6: Illustration of the simulated injection used for the numerical model: plume-only (top) and casing, fluid, and plume (bottom). 

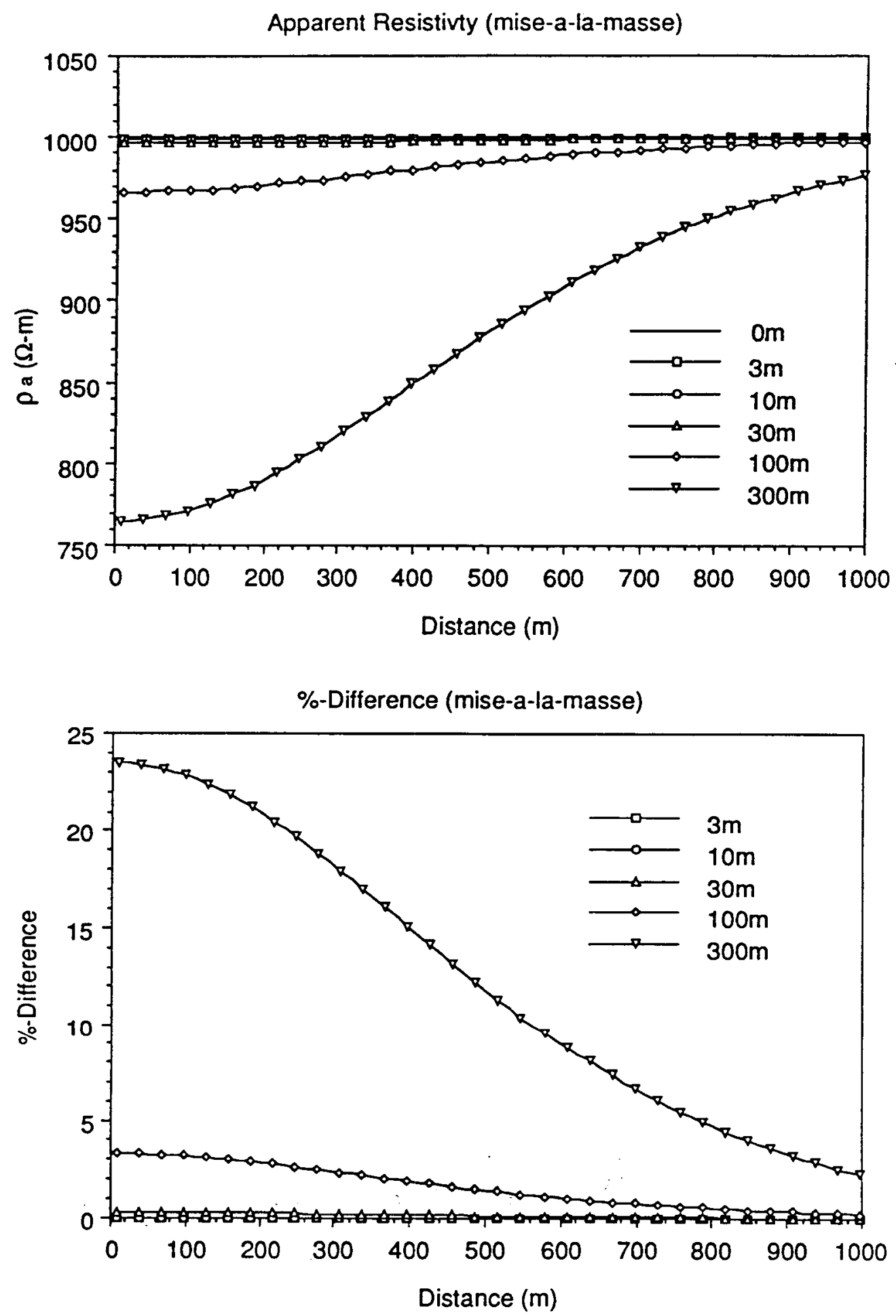

Figure 5.5-7: Apparent resistivity (top) and percent difference (bottom) for the plume-only with the pole source model. Legend shows the length of the plume. 

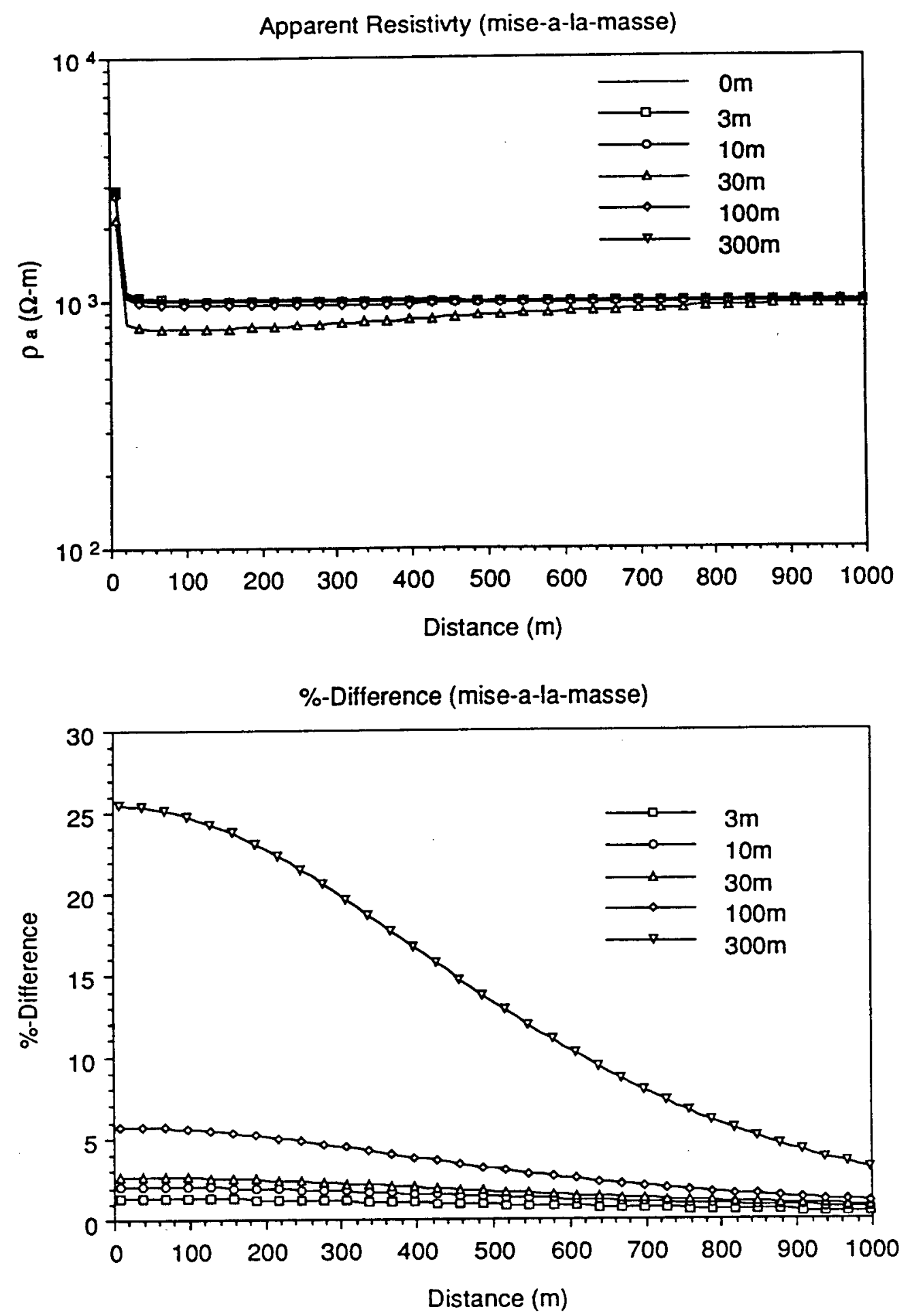

Figure 5.5-8: Apparent resistivity (top) and percent difference (bottom) for the resistive casing, fluid, and plume with the pole source model. Legend shows the length of the plume. 

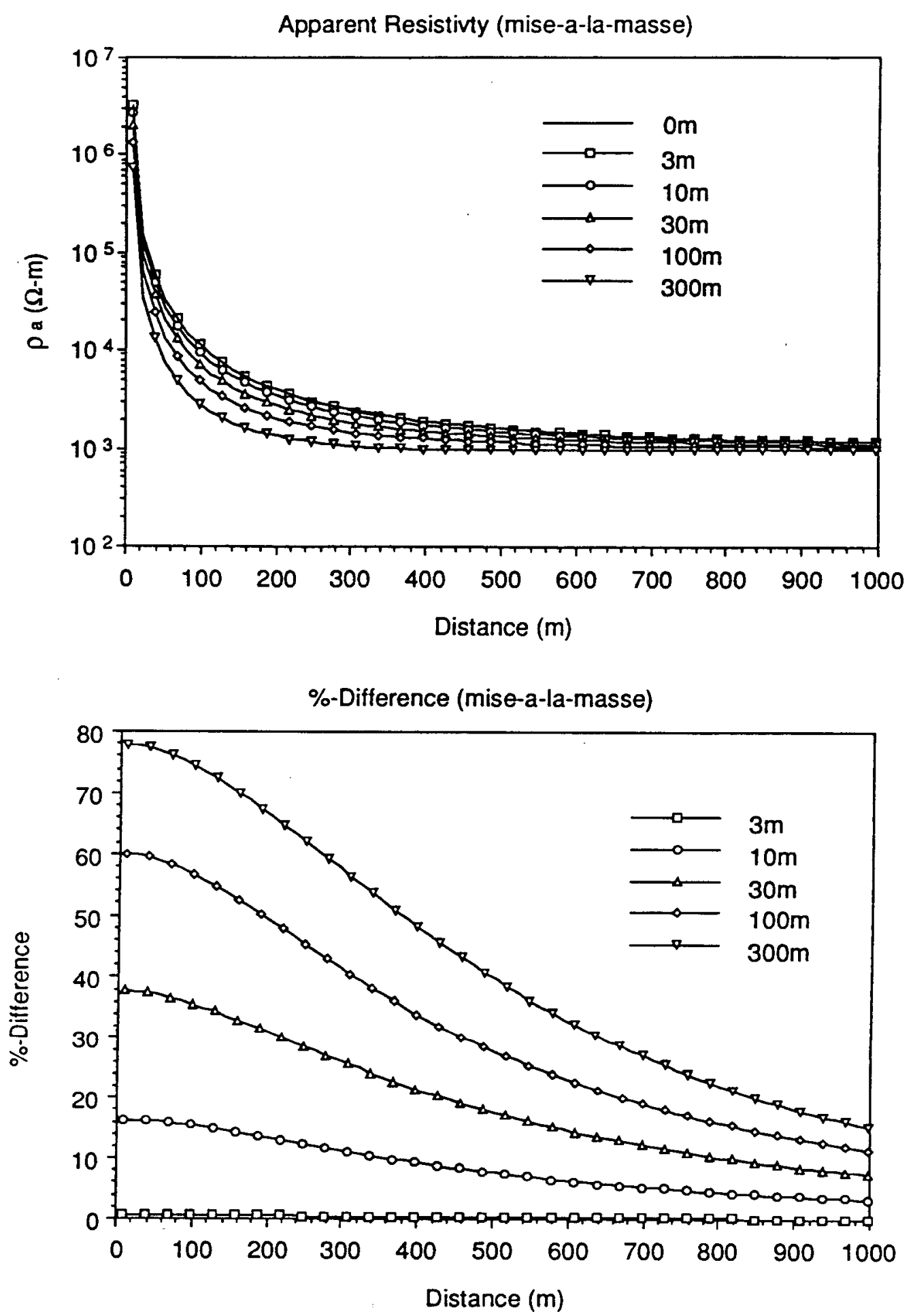

Figure 5.5-9: Apparent resistivity (top) and percent difference (bottom) for the conductive casing, fluid, and plume with the pole source model. Legend shows the length of the plume. 

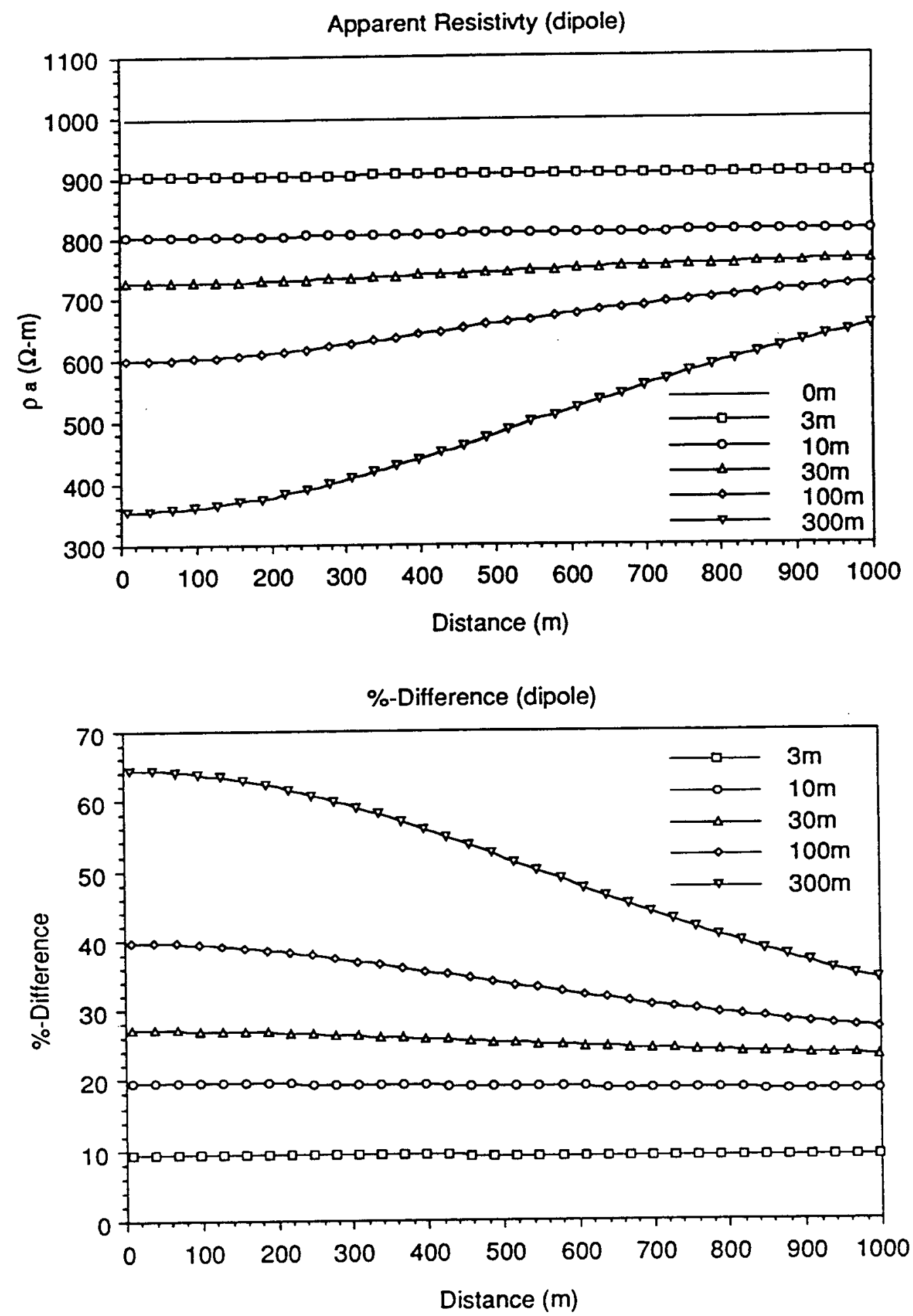

Figure 5.5-10: Apparent resistivity (top) and percent difference (bottom) for the plume-only with the dipole source model. Legend shows the length of the plume. 

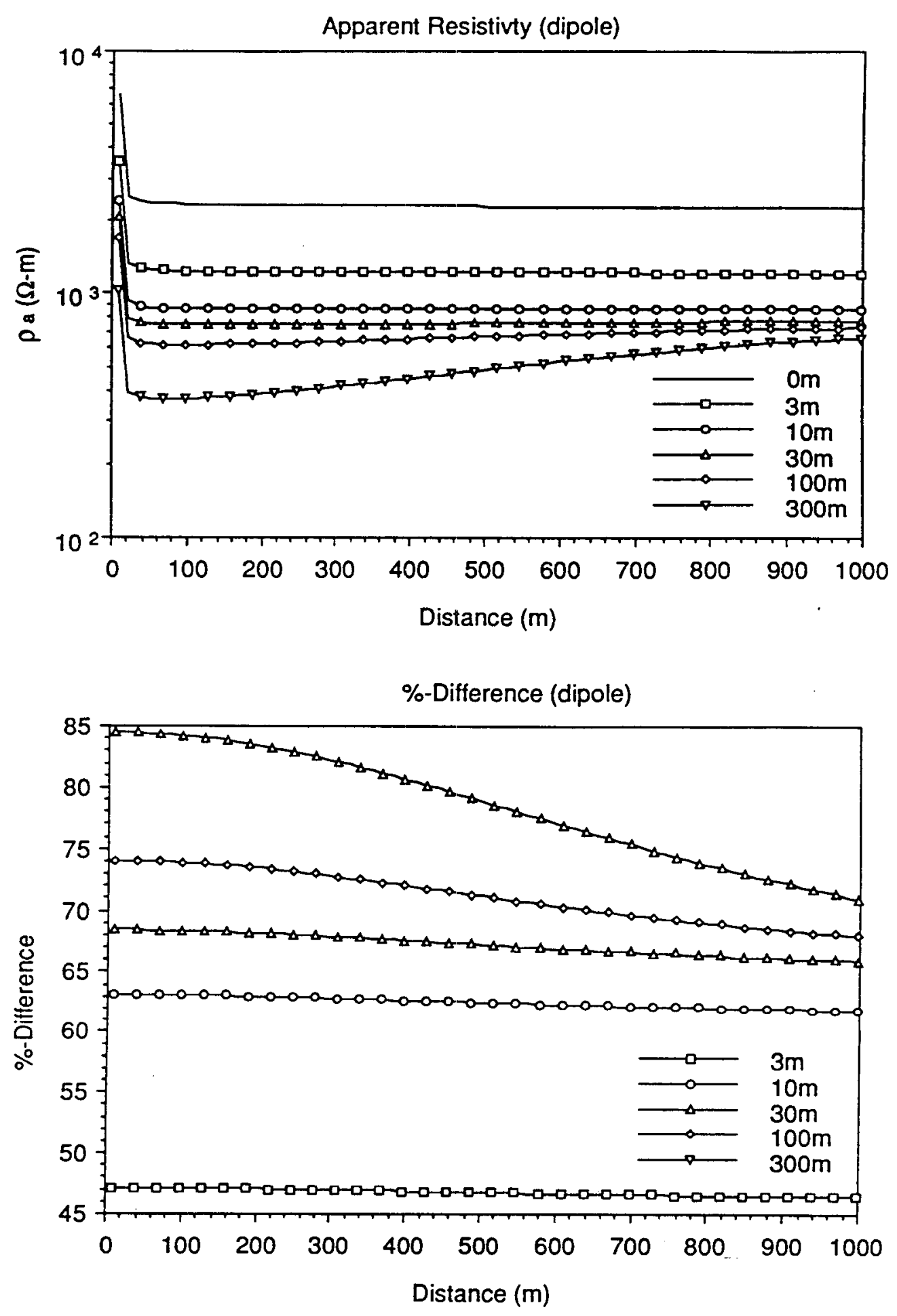

Figure 5.5-11: Apparent resistivity (top) and percent difference (bottom) for the resistive casing, fluid, and plume with the dipole source model. Legend shows the length of the plume. 

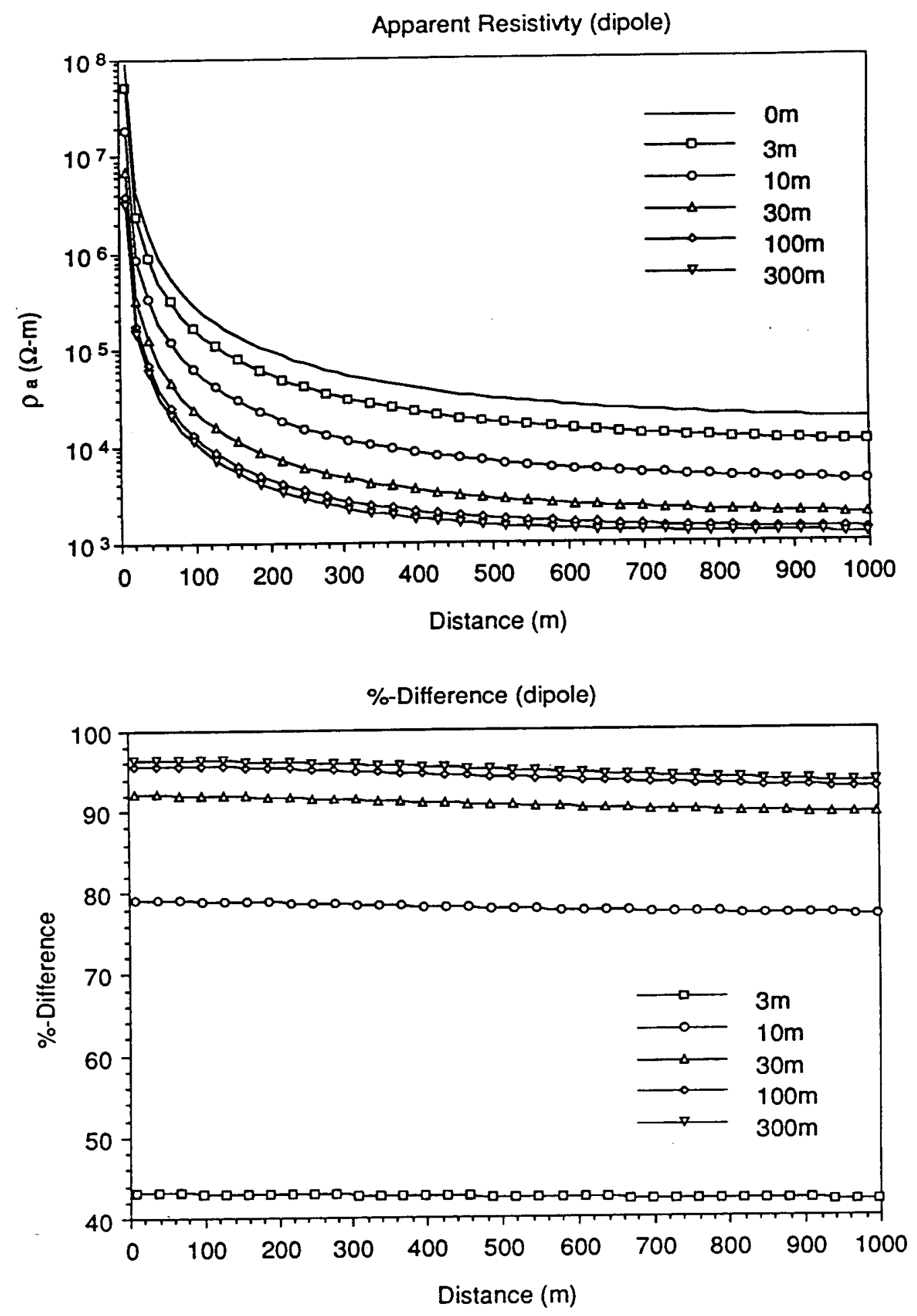

Figure 5.5-12: Apparent resistivity (top) and percent difference (bottom) for the conductive casing, fluid, and plume with the dipole source model. Legend shows the length of the plume. 


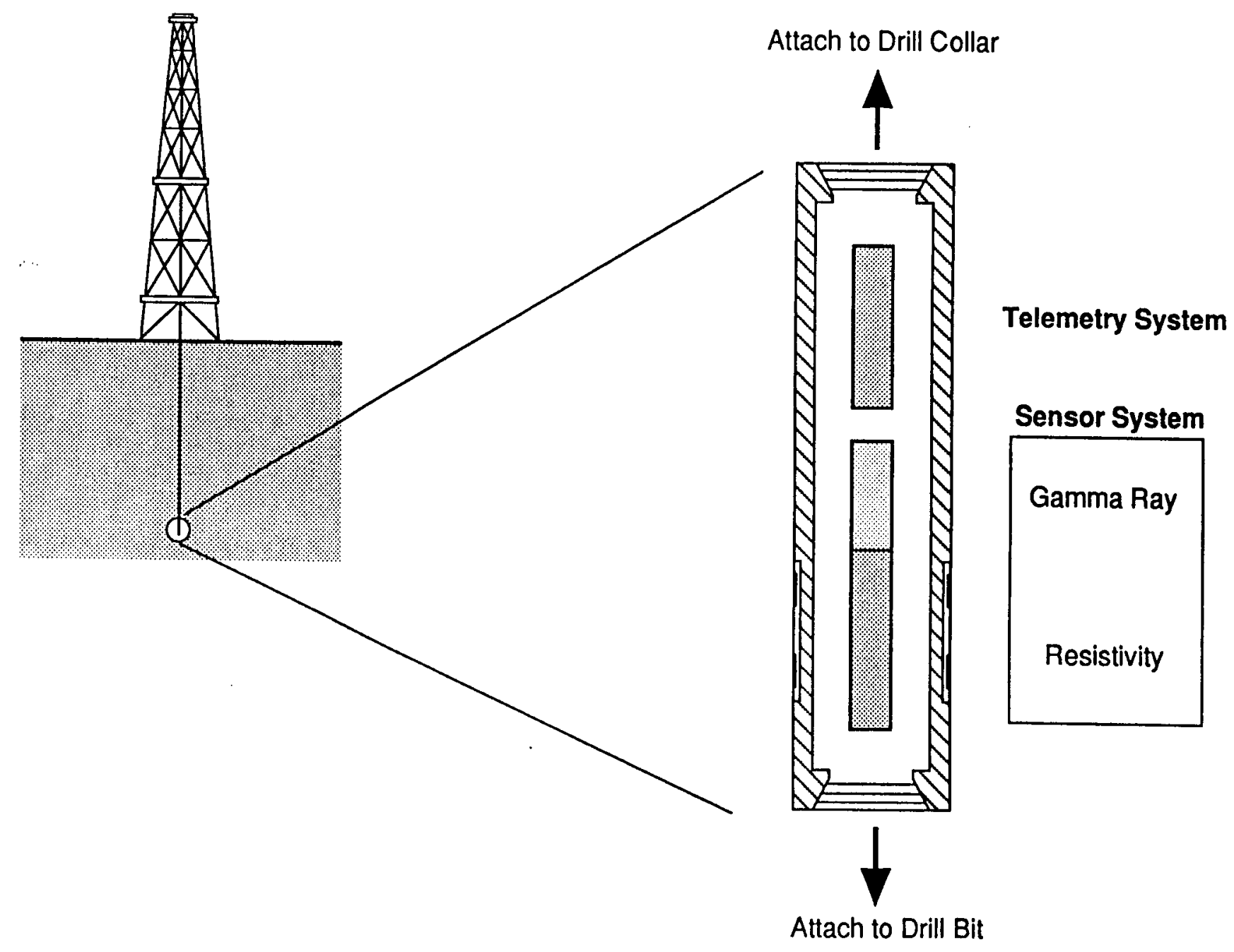

Figure 5.5-13: A typical MWD tool composed of a telemetry system ans a sensor system. The sensor system shown here measures gamma rays and the electrical resistivity. 


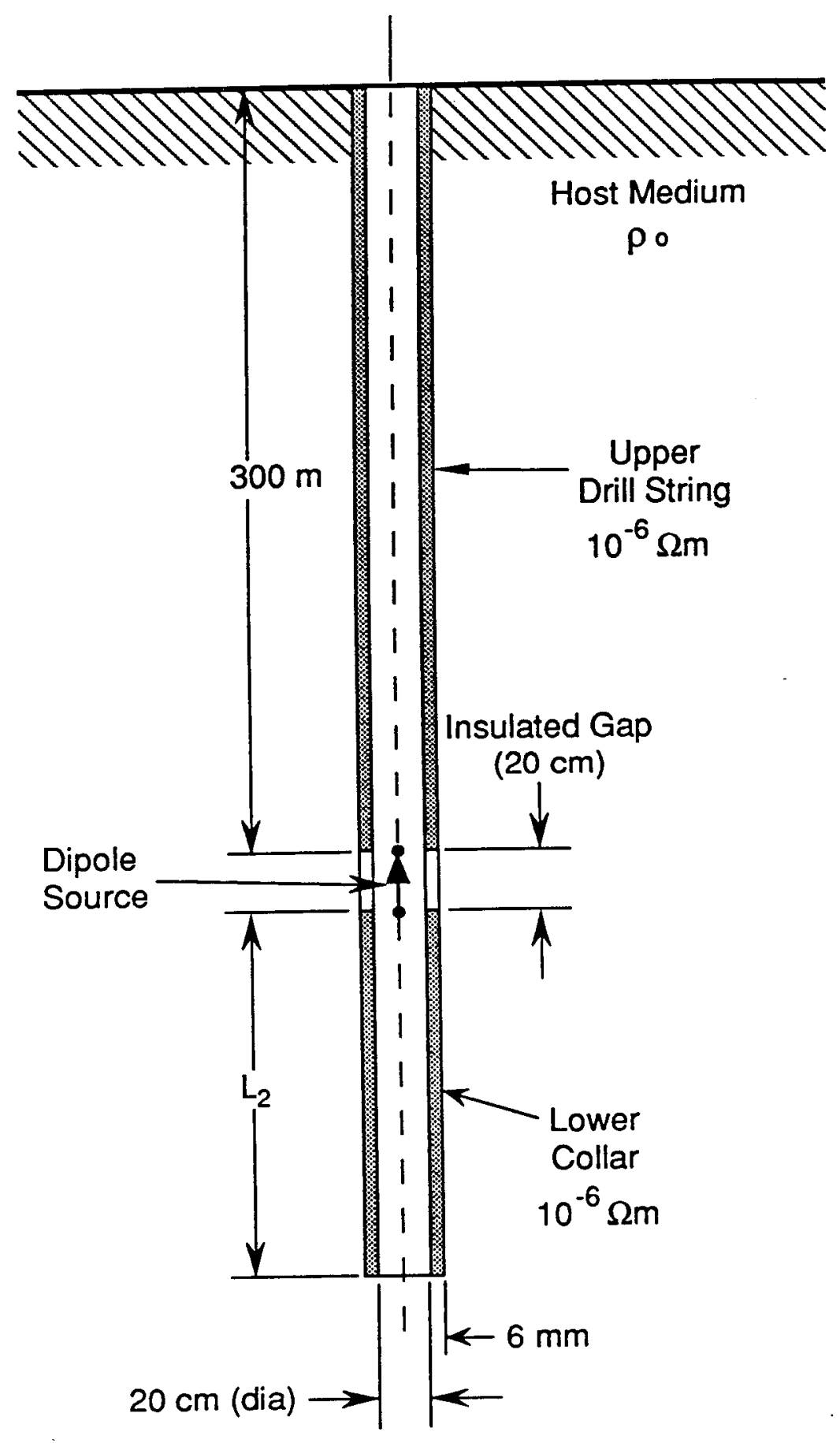

Figure 5.5-14: A simplified model used for the EM telemetry problem. The EM source is simulated with a dipole source between two pipe segments. The upper segment represents the drill string and the lower models the MWD tool and drill bit. 

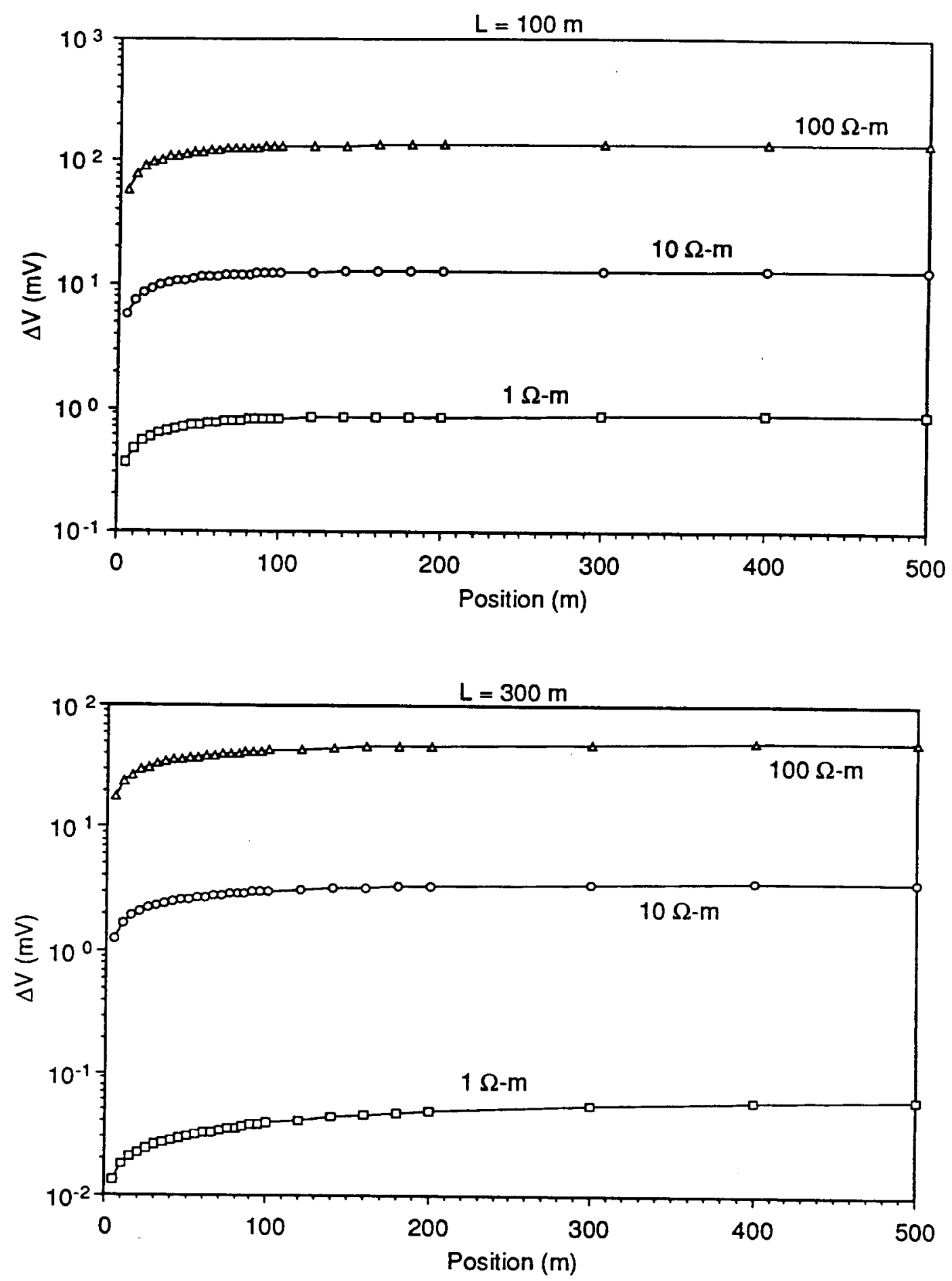

Figure 5.5-15a: Plots of the potential difference measured between the casing and radial position on the surface for a drill string of $100 \mathrm{~m}$ (top) and $300 \mathrm{~m}$ (bottom). No lower collar and a dipole gap $\Delta z_{0}=0.2 \mathrm{~m}$ were used. 

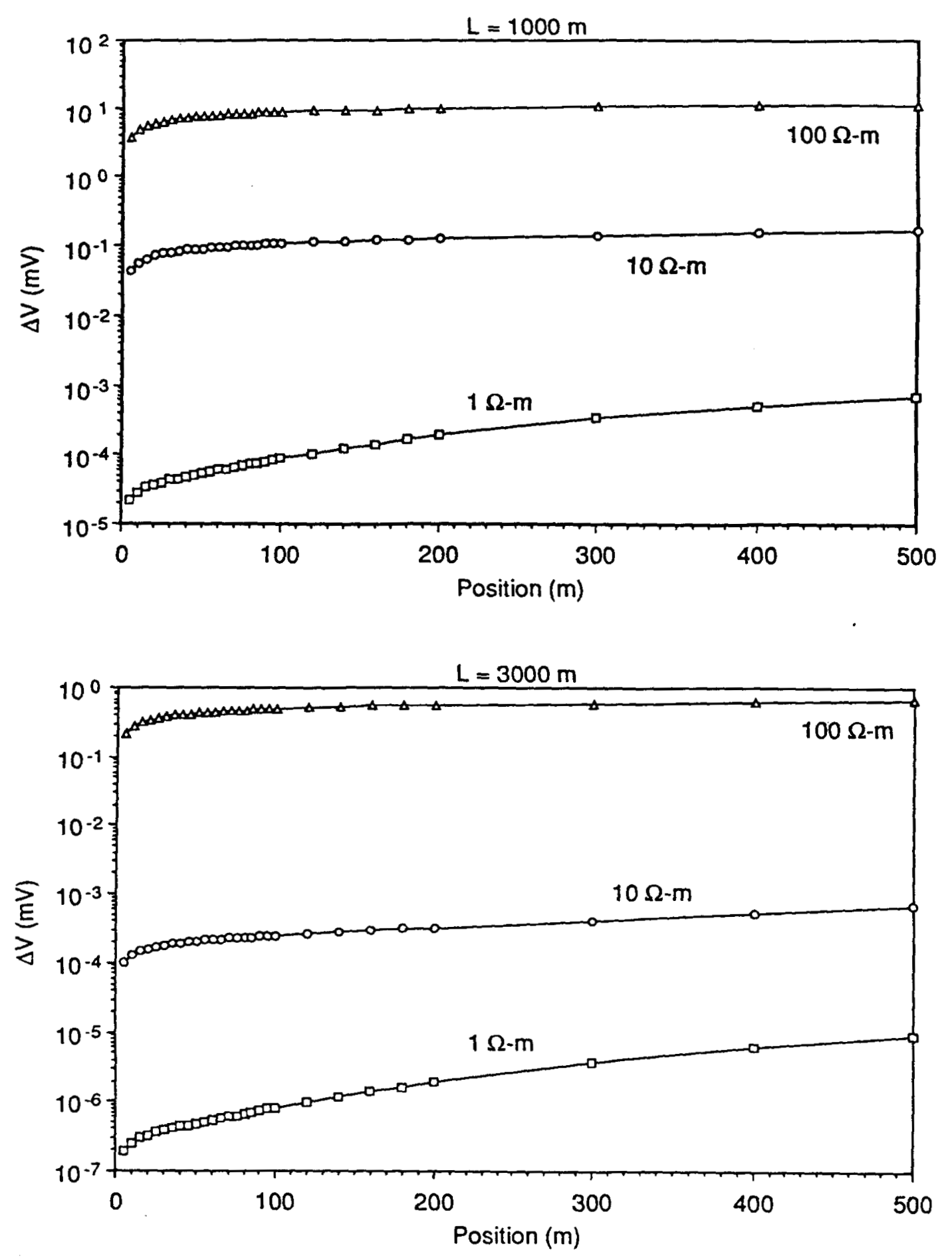

Figure 5.5-15b: Plots of the potential difference measured between the casing and radial position on the surface for a drill string of $1000 \mathrm{~m}$ (top) and $3000 \mathrm{~m}$ (bottom). No lower collar and a dipole gap $\Delta z_{0}=0.2 \mathrm{~m}$ were used. 


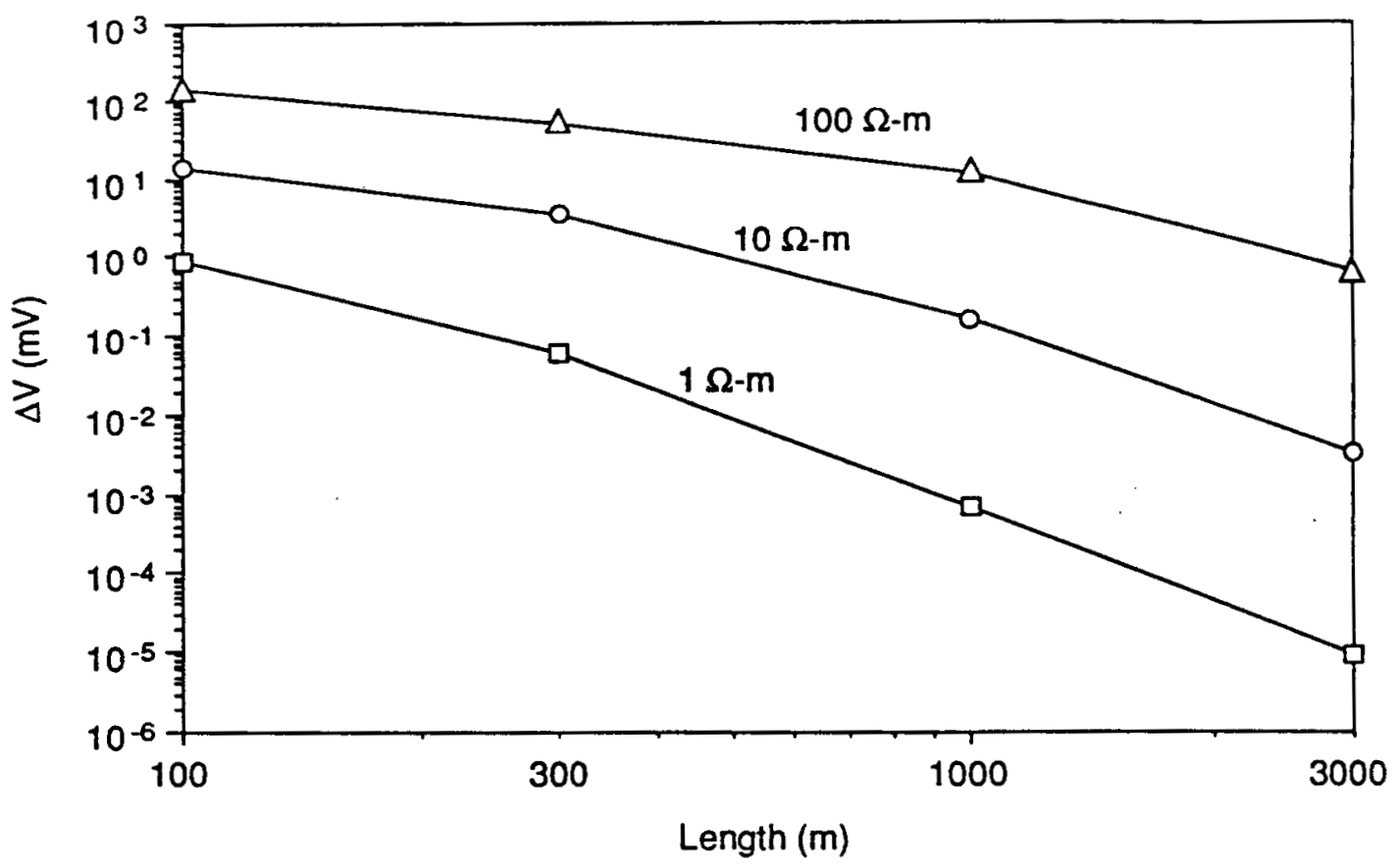

Figure 5.5-16: Plot of the potential difference measured between the casing and radial position on the surface as a function of the drill string length. No lower collar and a dipole gap $\Delta z_{0}=0.2 \mathrm{~m}$ were used.

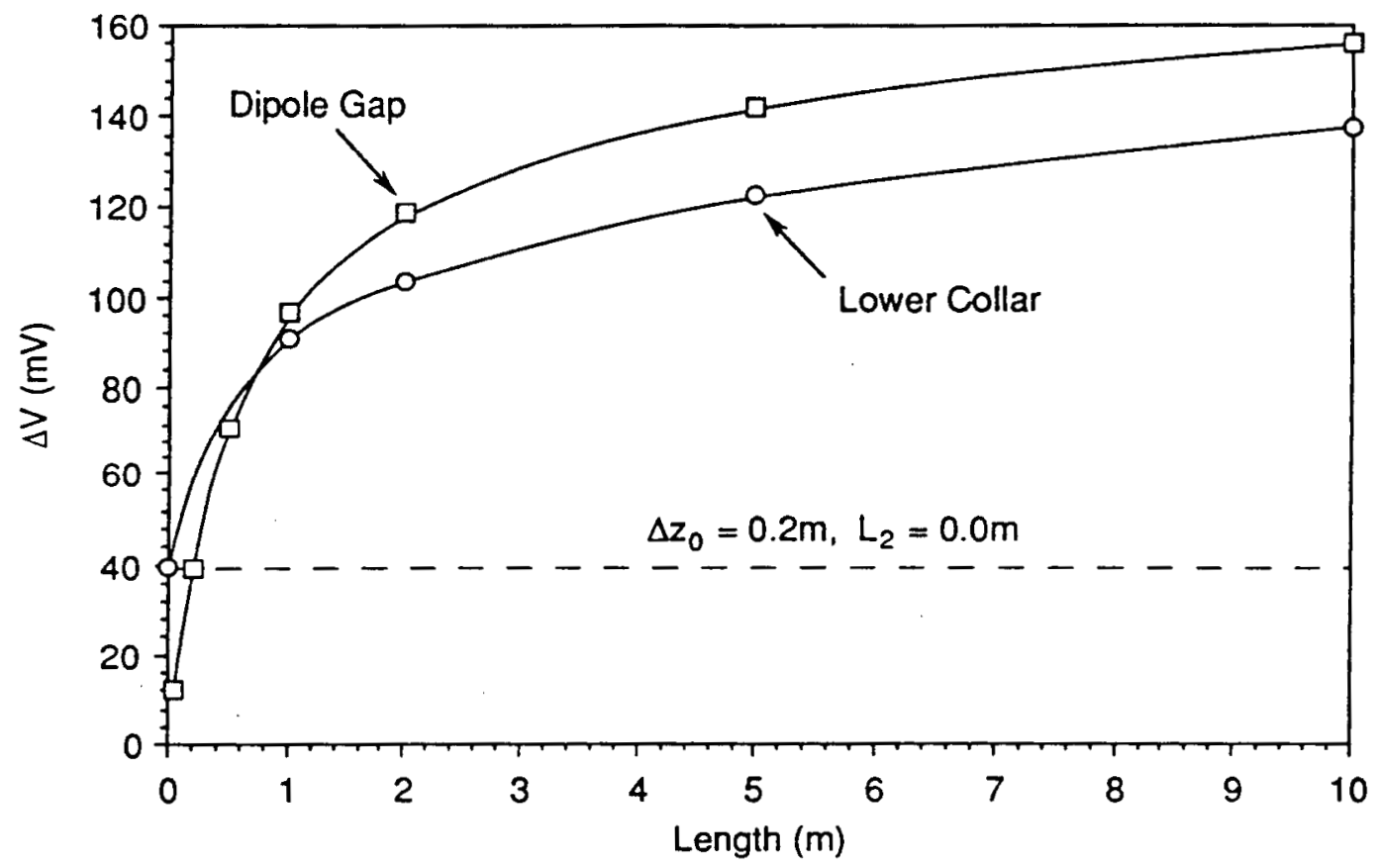

Figure 5.5-17: Plot of the potential difference measured between the casing and radial position on the surface for a $300 \mathrm{~m}$ drill string in a $100 \Omega \cdot \mathrm{m}$ half-space as a function of the dipole gap and lower collar lengths. The dashed line is equivalent to the $300 \mathrm{~m}$ drill string length, $100 \Omega \cdot \mathrm{m}$ point in Figure 5.5-16. 


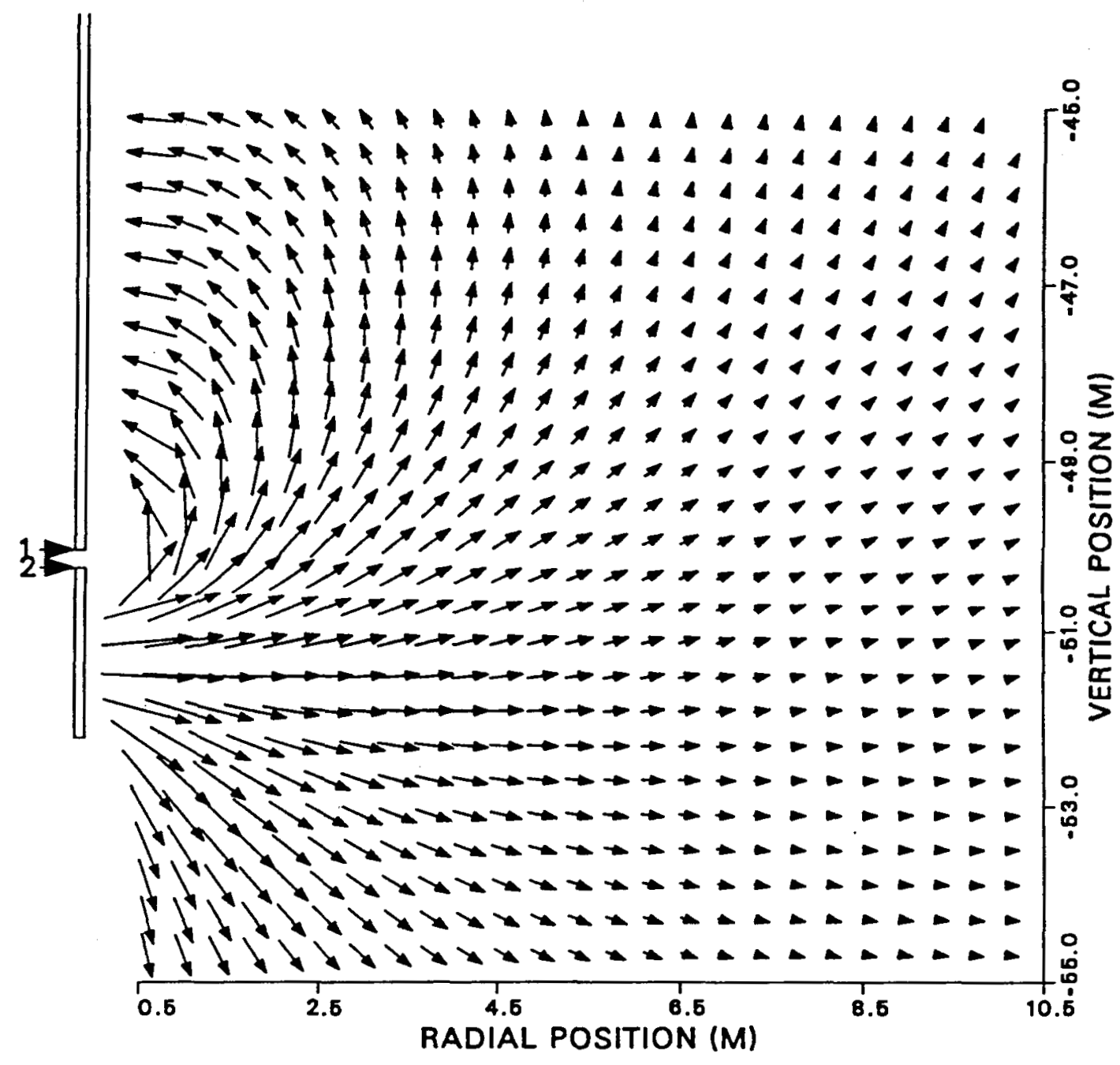

Figure 5.5-18: Current patterns in the medium for the MWD simulation. 


\section{CHAPTER VI}

\section{Resistivity Measurements Through Metal Casing}

In recent years, there has been increased interest in measuring formation resistivity through metal casing. Measurements made through casing can aid in characterizing existing reservoirs for effective recovery of oil and gas, as well as geothermal heat, without the cost and time of drilling new wells. Measuring the resistivity of adjacent formations may be useful to locate oil that was missed during the original logging prior to insertion of the casing. The method may also monitor changes in resistivity caused by subsurface processes such as injection or leakage of contaminants from a waste site, flooding operations for enhanced oil recovery, or processes of geothermal production.

In the past, logging in cased wells has been limited to measuring parameters of downhole flow, casing conditions, and cement integrity. Information on formation properties was mainly obtained through gamma ray or neutron scattering methods, but the radius of investigation of these techniques is limited to tens of centimeters. Several seismic techniques are capable of measuring some formation parameters but resistivity or induction logs appeared impossible to acquire since the highly conductive metal casing short circuits any current. Several patents however, have recently been issued which describe methods and devices that are capable of measuring the formation resistivity through casing (Kaufman 1989, Vail 1989a and 1989b, Gard et al. 1989). Currently, Vail is known to have developed and tested such a device, called Through-Casing Resistivity Apparatus (TCRA).

The patents of Kaufman and Vail discuss the use of two pairs of voltage measurements (three-point measurement) in contact with the casing to calculate the formation resistivity. Gard et al., using a single pair of voltage measurements also in contact with the casing, sug- 
gested that the formation can be continuously logged through the casing by making two traverses. All these methods use a compensation technique to "correct" for the unknown casing conductance, i.e., wall thickness and conductivity.

Kaufman (1990) investigated the behavior of the potential and its derivatives for a borehole with casing based on models of an infinite-length conductive pipe in a homogeneous medium. He showed that the second vertical derivative of the potential is proportional to the casing conductance and formation resistivity for receivers located in the "intermediate zone" from the current source. He concluded that for a known casing conductance $S_{c}$, the formation resistivity $\rho_{f}$ can be calculated at the depth where the potential $\phi$ and its second derivative are measured by the expression:

$$
\phi=\rho_{f} S_{c} \frac{d^{2} \phi}{d z^{2}}
$$

The objective of this chapter is to investigate the validity of Kaufman's and Vail's approach to determine the formation resistivity through metal casing for a finite length pipe and surrounding annulus in a layered medium. The annulus surrounding the casing can represent contact resistance or a cement layer. The IE approaches, which were derived in Chapter II and III, are used to calculate the potentials and E-fields on the casing and to simulate the resistivity measurements through casing (MTC) logging technique for a variety of pipe and layer parameters.

In addition to logging simulations, the effects of variations of the casing conductance and surrounding cement layer are studied. Variations in either the casing or cement layer may alter the result of the measurements since the currents tend to leak out in areas where the resistances are small. The patents, that were previously cited, claim to be able to compensate for variations in the casing conductance, but actually can only account for variations that occur over distances greater than the array dimension. It will be shown that compensation for short distance variations (less than the electrode separation) requires an additional term. 
The effects of potential electrode separation on the layer boundary resolution and radius of investigation are also examined. Kaufman suggested that the resolution of the layer boundary is dependent on the ability of the electrode array to approximate the second derivative. The radius of investigation needs to be studied, as it may also be dependent on the electrode spacing. If this is the case, one may be able to compensate for its influence and determine the formation resistivity as function of distance from the well. 


\subsection{Resistor Circuit Relation}

The second order differential equation (DE) given from Kaufman can be derived by resistor circuit relations. Figure (6-1) illustrates the resistor circuit for the casing and adjacent formation. The circuit can be consider as a series of resistors, representing the casing, intersected by shunt resistors that depict the formation resistance. The resistance of the formation at the $i$-th node can be determined by measuring the current leaking from the casing into the adjacent formation at the node by measuring the potentials across the two, $i$ and $i+1$, sections above and below the $i$-th node (at three points) on the casing.

Ohm's law, $V=I R$, is used to determine the relation between the current $I_{i}$, voltage $V_{i}$, and casing resistance $R_{i}$ in the $i$-th section of the casing:

$$
V_{i}=U_{i}-U_{i-1}=I_{i} R_{i} \quad \rightarrow \quad I_{i}=\frac{V_{i} S_{i}}{\Delta z_{i}}
$$

where $U_{i}$ is the potential measured at the $i$-th node and $S_{i}$, the casing conductance for the $i$-th section, is defined by Kaufman (1990) as:

$$
S_{i}=\left[\frac{R_{i}}{\Delta z_{i}}\right]^{-1}=\left[\frac{1}{\Delta z_{i}} \frac{\rho_{i} \Delta z_{i}}{A_{i}}\right]^{-1}=2 \pi a_{i} t_{i} \sigma_{i}
$$

and $a_{i}, t_{i}$, and $\sigma_{i}$ are the center radius, thickness, and conductivity in the $i$-th casing section, respectively. To obtain the relation for the current $I_{f i}$ flowing from the $i$-th node into the formation, Ohm's law is again applied:

$$
V_{f i}=U_{i}-U_{-}=I_{f i} R_{f i} \quad \rightarrow \quad I_{f i}=\frac{U_{i}-U_{\infty}}{R_{f i}}
$$

Kaufman defines the term "transverse resistance" ${ }^{2} T_{i}$ of the medium as:

$$
T_{i}=L_{i} R_{f i}
$$

where $L_{i}$ is the height of a cylindrical layer used to represent the formation adjacent to the $i$-th node. This height is one-half the sum of the lengths of the sections above and below the $i$-th

1 The casing (longitudinal) conductance is discussed in Chapter V, Section 5.1

2 Transverse resistance appears to be a misnomer since its units are [ $\Omega \cdot m]$ instead of [ $\Omega$ ], the units of resistance. It is actually the inverse of the unit length leakage conductance. Thus, the units of the transverse resistance are $\left[(\mathrm{S} / \mathrm{m})^{-1}\right]=[\Omega \cdot \mathrm{m}]$. 
node, i.e.,

$$
L_{i}=\frac{\Delta z_{i}+\Delta z_{i+1}}{2}
$$

The factor one-half is used since the adjacent $i-1$ and $i+1$ nodes account for the other halves of the section lengths needed to calculate the transverse resistance at those nodes. Substituting equations (6-5) and (6-6) into (6-4) yields:

$$
I_{f i}=\frac{\left(U_{i}-U_{-}\right)}{T_{i}} \frac{\left(\Delta z_{i}+\Delta z_{i+1}\right)}{2}=\frac{U_{i}}{T_{i}} \frac{\left(\Delta z_{i}+\Delta z_{i+1}\right)}{2}
$$

where $U_{-}$is measured at a point sufficiently far from the source and $U_{i}$ such that $U_{i}-U_{-} \approx U_{i}$. The use of Kirchoff's current law at the $i$-th, $I_{f i}=I_{i}-I_{i+1}$, yields:

$$
\frac{U_{i}}{T_{i}} \frac{\left(\Delta z_{i}+\Delta z_{i+1}\right)}{2}=\frac{V_{i} S_{i}}{\Delta z_{i}}-\frac{V_{i+1} S_{i+1}}{\Delta z_{i+1}}
$$

Let $\Delta s_{i}=S_{c}-S_{i}$ represent the deviation of the casing conductance $S_{i}$ from the average conductance, where the average conductance $S_{c}=\left(S_{i}+S_{i+1}\right) / 2$ is measured across the threeelectrode array. Now, equation (6-8) can be written as:

$$
\frac{U_{i}}{T_{i}} \frac{\left(\Delta z_{i}+\Delta z_{i+1}\right)}{2}=\left[\frac{V_{i}}{\Delta z_{i}}-\frac{V_{i+1}}{\Delta z_{i+1}}\right] S_{c}-\left[\frac{V_{i} \Delta s_{i}}{\Delta z_{i}}-\frac{V_{i+1} \Delta s_{i+1}}{\Delta z_{i+1}}\right]
$$

If the electrode separation distances are the same, i.e., $\Delta z_{i}=\Delta z_{i+1}=\Delta z$, then equation (6-10) becomes:

$$
U_{i}=T_{i}\left[S_{c} \frac{\left(V_{i}-V_{i+1}\right)}{\Delta z^{2}}-\frac{\left(V_{i} \Delta s_{i}-V_{i+1} \Delta s_{i+1}\right)}{\Delta z^{2}}\right]
$$

If the casing conductance between both sets of electrodes is the same, then $\Delta s_{i}=\Delta s_{i+1}=0$ and equation (6-10) is:

$$
U_{i}=T_{i} S_{c} \frac{\left(V_{i}-V_{i+1}\right)}{\Delta z^{2}}
$$

Equation (6-11) is the discrete form of the second order DE, equation (6-1) given by Kaufman. It suggests that if the casing conductance is known, the transverse resistance can be calculated from the potential and its second derivative. This expression cannot account for casing conductance variations, such as changes in thickness and conductivity, that span dis- 
tances shorter than the potential array length $2 \Delta z$.

To account for the shorter variations of the casing conductance in the transverse resistance calculations, the second term in equation (6-10) which compensates for casing conductance variations between the electrode pairs is required. It accounts for the differences in the voltages due to the variations of the casing conductances between the two sets of potential electrodes. This term is similar to Vail's "second order error" term that he indicates is due to the product of the differences in resistances between the sets of electrodes and leakage current flowing into the formation.

There are two interpretations of the relationship between the transverse resistance and formation resistivity. Kaufman indicates that the currents flowing outside the casing are radial due to charge build-up on the outer casing surface. Hence, an annular disk, representing the region where the radial current flows, could be used to calculate the transverse resistance which is:

$$
T_{i}=\rho_{f} \frac{1}{2 \pi} \ln \left[\frac{c}{b}\right]
$$

where $b$ and $c$ are the inner edge (outer pipe radius) and outer edge of the annular disk, respectively.

Equation (6-12) shows that the value of the transverse resistance is dependent on the formation resistivity and the logarithm of the ratio between the outer and inner radius of the annular disk. Kaufman compared the approximation of the E-fields in the borehole with the solution of an analogous transmission line problem and deduced that the formation resistivity could replace the transverse resistance in the transmission line solution, i.e.,

$$
T_{i}=\rho_{f}
$$

In his analysis, Kaufman compared equation (6-12) and (6-13) and stated that the outer edge of the annular disk with zero potential would be located at a sufficient distance from the borehole, "sufficient" being defined by the equation:

$$
c=b \mathrm{e}^{2 \pi}
$$


It is not obvious that equations (6-13) and (6-14) are valid. A comparison with an approximation is not sufficient to validate the relation of the inner and outer radii in equation (6-14). According to equation (6-14), if the inner radius of the annulus is $0.1 \mathrm{~m}=4 "$, then $c=54 \mathrm{~m}$ is sufficient so that the outer edge of the annular disk has zero potential. In practice, this distance may be adequately far from the casing so that the potential at this radius is effectively zero. However, the choice of sufficient distance for zero potential greatly dictates the value of the transverse resistance of equation (6-12). If one selects the outer radius of the disk to be at infinity, where the potential is actually zero, the transverse resistance will become infinitely large. With no theoretical evidence to support equation (6-14), this expression for sufficient distance appears to be an accommodating relation to obtain the formation resistivity directly from the transverse resistance.

Equation (6-11) is also the general form of Vail's equation. In his formulation, the transverse resistance is based on the approximation given by Tagg (1964) for the resistance of a grounded electrode in a half-space. ${ }^{3}$ Vail's expression, which has been misinterpreted from Tagg's formula, is:

$$
T_{i}=\rho_{f} \frac{1}{2 \pi} \ln \left[\frac{2 \Delta z}{a}\right]
$$

where $\Delta z$ is the electrode separation and $a$ is the radius of the pipe.

Equations (6-12) and (6-15) appear similar in form but have different representations. Kaufman's formulation of the transverse resistance is based only on the properties of the formation, whereas Vail incorporates the geometrical properties of the measuring array in his description of the transverse resistance. Intuitively, one would expect that the transverse resistance is similar to the apparent resistivity and is dependent on the formation properties, as well as the geometry of the array. The factors that influence the transverse resistance will be presented later in this chapter.

3 See equation (6-20) in Section 6.3.1 for Tagg's approximation 


\subsection{Electrode Arrays for Unknown Casing Conductance}

For an unknown casing conductance, two independent sets of measurements are needed to determine the transverse resistance by equation (6-10) or (6-11). Figure (6-2) illustrates the two electrode configurations that estimate the casing conductance and measure the potential and its second derivative. To compensate for the variations in the casing conductance and to approximate the second derivative, three potential electrodes, $M, N$, and $M^{\prime}$, are needed.

For the first array configuration, the calibration or compensation state, the current $I$ is applied at the source electrodes A and B which are in close proximity to the potential electrodes. Due to the large contrast in conductivities between the casing and adjacent formation, essentially all of the current will flow within the casing. Thus, the voltages $V_{1}$ and $V_{2}$ measured across their respective electrode pairs, MN and NM', will reflect the conductance of the casing between the electrode pairs. By measuring $V_{1}$ and $V_{2}$ separately and knowing the current strength, the conductance for each section can be estimated from Ohm's law:

$$
S_{i}=\frac{I \Delta z}{V_{i}} \quad(\text { for } i=1,2)
$$

This expression assumes no current leakage into the formation. There may be some leakage if the potential electrodes are located "too far" from the current source in a highly conductive formation.

With the second electrode configuration, the measurement state, the current electrode $B$ is moved to a remote position (infinity in theory). Now the current must not only flow in the casing but also through the formation. The measured voltages $V_{1}$ and $V_{2}$, as well as the potential $U_{N}$, will reffect both the casing and formation resistivities. By subtracting the two voltages, an approximation of the second derivative is obtained. With the estimate of the casing conductance and second derivative, the transverse resistance is calculated from equation (6-10) or (6-11).

Equation (6-16) suggests that the transverse resistance can be described in terms of the E-fields of the calibration and measurement states. The casing conductance for the $i$-th section 
can be determined from the calibration state, and written in terms of the E-field:

$$
E_{i}^{c}=\frac{V_{i}}{\Delta z_{i}} \rightarrow S_{i}=\frac{I_{i}^{C}}{E_{i}^{C}}
$$

Substituting for $S_{i}$ in equation (6-8) and noting that $V_{i}$ is the voltage difference across the $i$-th section in the measurement state, the transverse resistance can be expressed as:

$$
T_{i}=\frac{U_{i}}{I_{i}{ }^{C}}\left[\frac{E_{i}^{M}}{E_{i}^{C}}-\frac{E_{i+1}^{M}}{E_{i+1}^{C}}\right]^{-1}
$$

where $E^{C}$ and $E^{M}$ are the E-fields determined from the calibration and measurement states, respectively. This expression shows that the transverse resistance is inversely proportional to the difference between the measurement-calibration E-field ratio of the two sets of electrodes.

\subsection{Casing Effects}

The effects of the geometrical and electrical properties of the casing on the transverse resistance are now investigated. A $10^{-6} \Omega \cdot \mathrm{m}$ casing with length $L$, (inside) diameter $\mathrm{ID}$, and thickness, $t$ is embedded in a homogeneous half-space. For this analysis, the separation between the current and center potential electrode is AN $=4 \mathrm{~m}$. The potential electrode spacing is set at $\mathrm{MN}=\mathrm{NM}^{\prime}=2 \mathrm{~m}$.

Figure (6-3) is the transverse resistance for a $200 \mathrm{~m}$ casing with thicknesses ${ }^{4}$ of $1 / 4$ ", $3 / 8^{\prime \prime}$, and $1 / 2 "$ and $\mathrm{ID}^{5}$ of $4 ", 6 "$, and $8 "$ in a $10 \Omega \cdot \mathrm{m}$ medium. Variations due to the different casing thickness only resulted in a change of approximately 1.5 percent in the transverse resistance. The casing diameter appears to have a greater influence on the transverse resistance where the larger casing diameters decrease the transverse resistance. Variations of about 3-7 percent occur for different diameters depending on the thickness.

The transverse resistance appears to be related to the inverse of the diameter. Large diameter casing produces the small transverse resistance, whereas the small diameter pipe

4 The thicknesses of $1 / 4,3 / 8$, and $1 / 2$ " correspond to $0.635,0.9525$, and $1.27 \mathrm{~cm}$, respectively.

5 These inside diameters correspond to inner radii of 2,3 , and 4 " or $5.08,7.62$, and $10.16 \mathrm{~cm}$, respectively. 
results in large resistance. For small casing diameters, the casing thickness has some influence on the transverse resistance. For large diameter casing, different thicknesses have negligible changes of the transverse resistance.

Figures (6-4) to (6-6) are plots of the transverse resistance calculated for a $20.32 \mathrm{~cm}\left(8^{\prime \prime}\right)$ ID, $1.27 \mathrm{~cm}(1 / 2 ")$ thick casing using several casing lengths in a host formation with resistivities of 1,10 , and $100 \Omega \cdot \mathrm{m}$. The figures indicate that the transverse resistance is dependent on the formation resistivity and the casing length. Near the half-space surface, the larger formation resistivity and longer casing length result in a greater deviation of the transverse resistance from the formation resistivity. Away from the surface, the transverse resistance is dependent on the conduction ratio of the casing. Recall that the conduction ratio is inversely proportional to the casing length.

When the conduction ratio is greater than one-half, the transverse resistance is mainly affected by the length of the casing. The deviation of the transverse resistance from the true resistivity is proportional to the logarithm of the casing length. This is especially true for high formation resistivities. All the transverse resistance curves are convex in shape where the values slowly decrease with depth in the upper portion of the casing and then rapidly decay near the bottom.

For conduction ratios less than one-half, the transverse resistance near the surface is dependent on the logarithm of the casing length and partially on the formation resistivity. Near the casing ends, the transverse resistances are large at the surface and small at the bottom of the casing. Toward the center, the curve becomes concave and the transverse resistance approaches the formation resistivity.

The current leakage is strongly influenced by the boundary conditions at the ends of the casing. When the conduction ratio is greater than one-half, current leakage is nearly the same along the casing. Thus, the transverse resistance will strongly depend on the casing length and position. When this ratio is less than one-half, the casing length is effectively much greater than the length of conduction. Away from the ends, the effects of the boundaries are 
reduced since much of the current can dissipate into the formation. Hence, the transverse resistance gives a better estimate of the formation resistivity.

\subsubsection{Geometric Variations}

These results suggest that a correction factor $G$ must be applied to the transverse resistance $T$ to obtain the formation resistivity. This relation can be expressed as:

$$
T=\rho_{f} G
$$

For a conduction ratio greater than one-half, the factor $G$ compensates for the geometric parameters, such as casing diameter, length, and measuring (field) position. When the ratio falls below one-half, the electrical parameters and the source position must be included in the compensation.

As discussed previously, Tagg's approximation the resistance of a grounded electrode in the half-space with an "average" resistance calculated for an ellipsoid of revolution where its length $L$ is much greater than the radius $a$. Using Tagg's result, the correction factor can be written as:

$$
G_{t}=\frac{1}{2 \pi} \ln \left(\frac{2 L}{a}\right)
$$

Equation (6-20) shows that this factor is dependent on the logarithm of the ratio of the length to radius. This expression assumes a uniform average value along the entire conductor. It will only shift the transverse resistance curve and cannot compensate for the variations along the casing as seen in Figures (6-4) to (6-4).

Sunde (1968) presented a formula for the potential along a conductor in a half-space based on constant leakage of current. From this expression, a geometric factor can be written as:

$$
G_{s}=\frac{1}{2 \pi} \ln \left[\frac{\left[(z+L / 2)^{2}+a^{2}\right]^{1 / 2}+(z+L / 2)}{\left[(z-L / 2)^{2}+a^{2}\right]^{1 / 2}+(z-L / 2)}\right]
$$


Equation (6-21) incorporates the field position on the conductor, as well as the length and radius. However, for this formulation, the location of the current source is assumed to be at the surface.

Figures (6-7) to (6-9) are the formation resistivities calculated from the transverse resistances using the correction factor, equation (6-21). These plots show that for large conduction ratios, the variations of the transverse resistance due to position can be partially corrected. The curves are flattened and the deviations from the true formation resistivity are slightly reduced. However, for the long casing length $(600 \mathrm{~m})$, one tends to grossly overcorrect the transverse resistance, whereas for the short length $(50 \mathrm{~m})$, one severely underestimates the correction. For small conduction ratios, the variations of the transverse resistance curves are not accurately compensated. The curves are overcorrected and the deviations are larger than the values without the application of the correction factor.

Since $G_{s}$ assumes a fixed source location and constant leakage of current, it can only account for the geometrical effects for large conduction ratios. It cannot compensate the transverse resistance along the casing for small conduction ratios since the boundary effects are greatly reduced. Thus, the source location and electrical parameters must be incorporated into the formulation of the correction factor. This may be done by assuming the currents within the casing behave like the those in a transmission line problem. The current can be represented as a superposition of upgoing and downgoing currents in the form:

$$
I(z)=I_{0}\left[I^{D} \exp \left[\frac{-\left(z-z_{0}\right)}{\delta_{L}}\right)+I^{U} \exp \left(\frac{\left(z-z_{0}\right)}{\delta_{L}}\right)\right]
$$

where $\delta_{L}$ is the conduction length, $\left(z-z_{0}\right)$ is the distance from the current source depth, $I_{0}$ is the current strength, $I^{U}$ and $I^{D}$ are the upgoing and downgoing coefficients that are found by solving a transmission line boundary value problem. Once the current is found, the correction factor $G$ can be obtained by integrating the current over the length of the conductor:

$$
G=\frac{1}{2 \pi I_{0}} \int_{-L}^{L} I\left(z^{\prime}\right)\left[(z-z)^{2}+a^{2}\right]^{-1 / 2} d z^{\prime}
$$


where $z^{\prime}$ is the depth of the elemental segment.

Incorporating the source position and electrical properties into the correction factor may be more complicated than solving the actual problem. Hence, at this time it may be better to note that these parameters must be included in the correction factor in order to effectively compensate for the effects of the casing and the formation interaction.

\subsubsection{Conductance Variations}

The effects of variations in the casing conductance are investigated. Gard et al. indicated that variations of the conductance are due to manufacturing tolerances, chemical composition of the metal, corrosion, and other environmental factors. Often, several casing segments with different radii and thicknesses are inserted in a single hole. The use of casing segments with different conductance properties may cause problems at the places where the two segments meet. Additionally, the collars which join the casing segments will change the conductance locally.

Equations (6-11) and (6-10) are used to illustrate the effects of conductance variations and the errors associated with this situation. Recall that equation (6-11) assumes no variations of the conductance within the electrode array, whereas equation (6-10) can incorporate small scale variations. Figure (6-10) is a model of two segments with different conductances used to study the variation effects on the E-field, measured conductance, and transverse resistance. Both segments have equal lengths of $100 \mathrm{~m}$ and inner radii of $0.1016 \mathrm{~m}$. The conductivitythickness products of the top and bottom segments are $\sigma_{1} t_{1}$ and $\sigma_{2} t_{2}$, respectively. The threeelectrode array is used for the two independent sets of measurements needed to simulate an unknown casing conductance situation. The potentials are measured at electrodes $M, N$, and $\mathrm{M}^{\prime}$ and the current is injected at electrodes A and B.

Figures (6-11) to (6-13) illustrate differences of equations (6-11) and (6-10) for a casing that has a conductance discontinuity. Equation (6-11) is not corrected for casing variations, whereas equation (6-10) compensates for these variations. The spacings of the electrodes used 
for this analysis are $M N=N^{\prime}=2 m$ and $A N=B N=4 m$. A discontinuity of the casing conductance occurs at the interface between the two segments due to the difference of the thickness. The thicknesses of the upper and lower segments are $12.7 \mathrm{~cm}$ and $6.35 \mathrm{~cm}$, respectively. The positions along the casings are relative to the interface with the increasing distances going downward. The conductivities of the segments and host medium are $10^{6} \mathrm{~S} / \mathrm{m}$ and $1.0 \mathrm{~S} / \mathrm{m}$, respectively.

The calibration E-fields and the estimate of the casing conductance are shown is Figure (6-11). The E-field across electrodes $\mathrm{MM}^{\prime}$ represents that of the uncompensated measurement and is the average value of the E-fields for $\mathrm{MN}$ and NM'. From the E-fields, the casing conductance can be calculated and it is inversely proportional to these fields. These estimates are slightly larger (2-5 percent) than the actual value used for the model.

The potentials and E-fields for the measurement state are illustrated in Figure (6-12). The potentials are essentially constant showing very small variations. The E-fields are similar to those of the calibration state. There are slight variations between the two measurement Efields due to the distance the measuring electrodes are from the current electrode. This difference is larger on the positive side of the interface indicating that the casing conductance is smaller than that of the negative side.

Figure (6-13) is the estimate of the second derivative calculated from the E-fields of the measurement state. Theoretically, the second derivative for this situation is a spike located at the discontinuity and zero everywhere else. The plot shows that the spike is approximated by a triangular-shaped response. This function is flat at $|d| \geq 2 m$, is linear from $|d| \leq 2 m$, and has a minimum at $\mathrm{d}=0 \mathrm{~m}$. The width of this "triangle" is the same as the length of the three-electrode array. These results indicate that smaller electrode spacing produces better resolution of a casing junction.

The transverse conductance, which is the inverse of the transverse resistance, would have a large error in the vicinity of the interface if the effects of the change in casing conductance were not included in the calculations (Figure 6-13b). Curve G1 is equivalent to the first term 
in equation (6-10), or equation (6-11), which cannot compensate for the discontinuity of the conductance. Curve G2 represents the second term in equation (6-10) which corrects for these variations of the conductance. Curve $G c$ is the difference of $G 1$ and G2 which is the transverse conductance compensated for the casing conductance discontinuities. For locations away from the interface $(\mathrm{d} \mid>2 \mathrm{~m})$, the casing segments may be corrected with equation (611). If a discontinuity lies within the electrode array, equation (6-10) must be used to compensate for the changing conductance.

This analysis suggests that small spatial variations in the casing conductance may be compensated by applying equation (6-10). The effects of the discontinuity only occur at the locations where the three-electrode array lies over the interface. When the measurements are beyond one array length from the interface, the measurements are not affected by the discontinuity. The situation of a collar joining two casing segments would produce similar results. Since the spatial width of the discontinuities due to the collar are usually much smaller than the size of the array spacing, the deviations caused by the collar should be much like those of the two adjoining segments situation.

\subsection{MTC Logging - Layers}

The resistivity measurement through casing method is simulated with a of a target layer and an unknown casing conductance. The E-fields and transverse resistance are determined for several resistivities of the target layer. Since the casing conductance is unknown, two electrode configurations are used: one to estimate the casing conductance and another to calculate the transverse resistance. The second derivative is approximated with a three point measurement. Using equation (6-10), the transverse resistance is obtained from the three-point measurement and casing conductance estimate.

Figure (6-14) illustrates the model used for this analysis. The simplified model consists of a finite-length conductive casing filled with fluid embedded in a three-layer medium. For simplicity, the resistivity of $10 \Omega \cdot \mathrm{m}$ is used for the top layer, bottom layer, and borehole fluid. 
The casing has a resistivity of $10^{-6} \Omega \cdot \mathrm{m}$ and length of $100 \mathrm{~m}$. The target layer is $3 \mathrm{~m}$ thick with its top located $49 \mathrm{~m}$ below the surface. To approximate a layer of infinite extent, the outer boundary of the layer is placed at $5000 \mathrm{~m}$. The equally spaced potential electrodes, $\mathrm{M}, \mathrm{N}$, and M', are straddled by two current electrodes, A and B. All electrodes are placed in contact with the casing. For models with a single current source, electrode B is placed at "infinity", the remote position.

The calculated voltage difference for a lateral-log configuration is shown in Figure (615). These voltage differences, which are normalized by the potential electrode separation $(\mathrm{MN}=0.5 \mathrm{~m})$, represent an estimate of the electric fields on the casing and are proportional to the current leakage. The separation from the source to center potential array, AO, was $2.0 \mathrm{~m}$. The resistivities of $1 \Omega \cdot \mathrm{m}$ and $100 \Omega \cdot \mathrm{m}$ are used for the target layer. The discontinuity of the curves correspond to the change in resistivity. For the conductive layer, the increased rate of voltage drop is due to the increased current leakage into the adjacent formation. A resistive formation has the opposite effect and a decreased rate of change is observed through the target layer.

A resistivity log of through casing measurements with unknown casing conductivity is simulated using the parameters in Figure (6-14) and the numerical results for several resistivities of the target layer are illustrated in Figure (6-16). The resistivity values for the target layer ranged from $1 \Omega \cdot \mathrm{m}$ to $100 \Omega \cdot \mathrm{m}$. For this analysis, the separation between current and center potential electrode, AN, was $2.25 \mathrm{~m}$. The potential electrode spacings, MN and NM', were $1.0 \mathrm{~m}$.

Figure (6-16) shows that the resolution of the layer boundaries is about $2.0 \mathrm{~m}$ which correspond to the length of potential electrode spacing. ${ }^{6}$ The transverse resistance curves respond to the resistivity boundaries of the model remarkably well. The value of the transverse resistivity is slightly larger than the resistivity of the homogeneous (no layer)

6 The resolution of the layer boundary will be investigated later in this chapter. 
model. This deviation $(=10 \%)$ is probably due to the effects of the casing and array geometries discussed in the previous section. When the target layer is resistive, the transverse resistance is larger then the true formation resistivity. This discrepancy increases for increasing layer resistivity: for a $100 \Omega \cdot \mathrm{m}$ layer, the transverse resistance is $20-30 \%$ greater than the layer resistivity. For a conductive target layer, the transverse resistance underestimates the layer resistivity. This effect is contrary to the discrepancies associated with the geometric variations. This behavior of the transverse resistances indicates that the currents in the casing are channeled towards and leaks out into the formations with low resistivity. Greater current leakage results in a larger E-fields, and thus a smaller transverse resistance.

When the electrode array spans across the interface, the conductive layer has a greater influence on the transverse resistance than the resistive layer. The thickness of resistive beds may be underestimated, whereas conductive layers thickness may be overestimated in an MTC log. One would assume that the transverse resistance value at the interface should be approximately equal to the average of the resistivities of the two layers. Actually, the transverse resistance for an array that spans an interface is inversely proportional to the weighted average of E-field rates of change. The weighting is proportional to the fractional length of the array in a particular formation. Hence, the transverse resistance of a resistive layer in the vicinity of an interface will always appear more conductive than its true resistivity.

\subsection{Borehole Fluid Effects}

The influence of the borehole fluid on the transverse resistance for the MTC method is examined using several resistivities for the fluid. The unknown casing conductance is simulated, thus two sets of measurements are required. Figure (6-17a) is the cross-section of the casing filled with fluid in a layered medium. A $3 \mathrm{~m}$ target layer is placed between an overlying $49 \mathrm{~m}$ layer and basal half-space. The resistivity of the target layer is $1 \Omega \cdot \mathrm{m}$ while $10 \Omega \cdot \mathrm{m}$ is used for the resistivity of the other two formations. The $100 \mathrm{~m}$ casing has a thickness of $1.27 \mathrm{~cm}$, inner radius of $10.16 \mathrm{~cm}$, and resistivity of $10^{-6} \Omega \cdot \mathrm{m}$. The electrodes are placed in 
contact with the inner wall of the casing. The separation between the current and center potential electrodes is $2.25 \mathrm{~m}$, while $1.0 \mathrm{~m}$ is used for the potential electrode spacing.

The effects on the transverse resistance for various borehole fluids is illustrated in Figure (6-17b). The plot shows that all of the transverse resistance curves are the same for different borehole fluid resistivities. The resistivity of borehole fluid ranged from $1 \Omega \cdot m$ to $20 \Omega \cdot m$. It appears that the borehole fluid resistivity has a negligible influence on the transverse resistivity measurements. Some minor effects may appear if the electrodes were not in contact with the casing, since the current has a chance to dissipate before reaching the casing.

\subsection{Electrode Separation}

In this section, the effects of electrode separation on the resolution of the layer boundary and radius of investigation are examined. In Section 6.3, the results indicate that the vertical resolution is dependent on electrode spacing. The radius of investigation (RI) may also be dependent on electrode separation. Currently, there is no information about the investigation distance for the MTC method.

\subsubsection{Vertical Resolution}

Equation (6-11) indicates that the transverse resistance can be obtained from the ratio of the potential and its second derivative provided the casing conductance is known. This relation shows that the resolution of the transverse resistance is limited to the electrode spacing needed to approximate the second derivative.

Figure (6-18) illustrates the effects of the potential electrode separation on the layer boundary resolution. The model used consist of a $100 \mathrm{~m}$ casing embedded in a two-layer medium. The resistivities of the upper layer and basal half-space are $1 \Omega \cdot \mathrm{m}$ and $10 \Omega \cdot \mathrm{m}$, respectively. The casing has a resistivity of $10^{-6} \Omega \cdot \mathrm{m}$, thickness of $1.27 \mathrm{~cm}$, and inner radius of $10.16 \mathrm{~cm}$. The distance from the current electrodes to the center potential electrode is $2.25 \mathrm{~m}$. The potential electrode spacings of $0.2 \mathrm{~m}, 0.5 \mathrm{~m}$, and $1.0 \mathrm{~m}$ show that the boundary resolution improves as the separation becomes smaller. The resolution distance is 
approximately the length of the three-electrode array $2 \Delta z$ which is needed to estimate the second derivative.

Figure (6-18) confirms the results of Section 6.4 that the conductive layer has a greater influence on the transverse resistance in the vicinity of the interface than the resistive layer. The value at the interface is much closer to the resistivity of the conductive layer. On the resistive side of the interface, the transverse resistance approaches the true resistivity value slightly less than one electrode spacing from the boundary. For shorter electrode separations, this effect still exists but appears less pronouned since the vertical resolution has improved.

\subsubsection{Radius of Investigation}

For typical resistivity logging methods, increasing the separation of the electrodes increases the radius of investigation (RI). Thus, annular boundaries, which are not detected for a short electrode separation, may be discemible with longer spacing. A general rule of thumb for the RI of resistivity methods is approximately one-half the separation distance. In this section, the effects of the electrode separation on the RI are examined for the TCL method.

Figure (6-19) illustrates the model configuration used for this evaluation. A $50 \mathrm{~m}$ length of casing with a resistivity of $10^{-6} \Omega \cdot \mathrm{m}$ is surrounded by an annulus with thickness $\Delta t$ and resistivity $\rho_{c a}$ and a $10 \Omega \cdot \mathrm{m}$ homogeneous medium. Three potential electrodes are placed on the inner wall at the center of the casing with a separation $\Delta z$. The current electrodes are placed $2 \mathrm{~m}$ from the outer potential electrodes. Resistivities of $5 \Omega \cdot \mathrm{m}$ and $20 \Omega \cdot \mathrm{m}$ are used for the annulus to represent a conductive and resistive annular layer.

The result of increasing the electrode separation $\Delta z$ is shown in Figure (6-20). The solid and dashed lines represent the transverse resistance for a homogeneous medium, no annulus. The other curves are the transverse resistance for different values of $\Delta t$. Note that all the curves are essentially parallel to one another and somewhat flat, with approximately $10 \%$ variation from the $1 \mathrm{~m}$ to $20 \mathrm{~m}$ separations. The oscillation of the curves for small separations is 
due to poor discretization of the casing indicating that smaller segments are needed for short electrode separations.

These results indicate that the $\mathrm{RI}$ is independent of the potential electrode separation distance. If the RI was dependent on the electrode spacing, the curves in Figure (6-20) would start near the annulus resistivity for short $\Delta z$ and then asymptote to background resistivity as $\Delta z$ increases. The rate at which the curves approach $\rho_{f}$ is dependent on the thickness of the center annulus. For a radially thin annulus, the curves in Figure (6-20) would approach the background resistivity for shorter electrode separations than a thick annulus.

For a borehole with no casing, the equipotential lines from a pole current source are spherical in a homogeneous medium. When a vertical boundary is present, these equipotential lines are perturbed and can be detected by the electrodes in the well. A long electrode spacing can to detect perturbations caused by an annular boundary that is radial far from the well. If the well is cased in steel, the equipotential lines are now parallel to the vertical boundary of the annulus. Although the potentials are affected, the potential lines are not distorted by the annular boundary. Thus, all arrays with different electrode spacing will essentially measure the same response.

\subsection{Cement Annulus}

The results at the end of the previous section make determining the formation resistivity in the presences of an annular layer, like a cement layer, very difficult. In an uncased borehole, a tool with short electrode spacing can acquire data related to the resistivity of the annular zone. With this information, the formation resistivity may be approximated from measurements made with long electrode spacing. The resistivity measured in a well with an annular zone is a combination of the annular layer and the formation. When the hole is cased, the combined resistivity measured in the presence of the annular layer cannot be distinguished from a formation which has an equivalent resistivity since modifying the electrode spacing does not change the radius of investigation. 
Kaufman (1990) determines the transverse resistance in the presence of an annular layer by assuming only a radial current distribution outside the casing. ${ }^{7}$ Thus, the transverse resistance can be considered as the series resistance of a medium, an annular layer and host background (Figure 6-21a). Kaufman expresses this as: 8

$$
T=\rho_{f}+\frac{\rho_{c a}-\rho_{f}}{2 \pi} \ln \left[\frac{\Delta t}{a_{c a}}+1\right]
$$

where $a_{c a}$ is the inner radius, $\Delta t$ is the thickness, and $\rho_{c a}$ is the resistivity of the cement annulus and $\rho_{f}$ is the resistivity of the host medium.

The series resistance, calculated with equation (6-24), are compared to transverse resistance in Figure (6-20). The resistances as a function of the annulus thickness for selected electrode separation distances (Figure 6-21b). Although the transverse resistances are larger than the series resistances, the behavior of the resistances generally agree with each other. For small thickness of the annulus, the resistance approaches the background medium resistivity. As $\Delta t$ increases, the transverse resistance asymptotes to the center annulus resistivity. The larger transverse resistance can be expected since it is dependent on the casing conduction length (see Section 6.5.1). This figure also confirms that the transverse resistance is independent of the electrode spacing. Similar responses of the transverse resistance are obtained for different electrode separation.

The effects of thickness variations of the cement annulus in a homogeneous medium are illustrated in Figure (6-22a). The model used for this simulation is a $50 \mathrm{~m}$ long casing surrounded by a $20 \Omega \cdot \mathrm{m}$ cement annulus which has two thicknesses. The upper $25 \mathrm{~m}$ is $1.0 \mathrm{~m}$ thickness and the lower portion is $0.2 \mathrm{~m}$ thick. Figures $(6-22 \mathrm{~b})$ to $(6-22 \mathrm{~d})$ correspond to the three host resistivities of $1 \Omega \cdot \mathrm{m}, 5 \Omega \cdot \mathrm{m}$, and $10 \Omega \cdot \mathrm{m}$. Two electrode spacings are used to determine if variations of the thickness produce any significant changes of the transverse resistance.

7 A radial current pattem can only exist for an infinite-length perfect conductor.

8 This expression is based on equation 52 in Kaufman (1990), but his equation does not correspond to the notation in his Figure $1 d$. 
For small host resistivities, the transverse resistances agree with the series resistances calculated using equation (6-24). There is essentially no difference between measurements made with either electrode spacing. As the host medium resistivity increases, two observations are apparent. First, the transverse resistances of the two electrode spacings are slightly different where the longer spacing results in smaller values. Unfortunately, the deviations are about $2 \%$ on either side of the thickness discontinuity which are insignificant. Second, there is a large discrepancy of the transverse and series resistances on the thick side (shaded area) of the cement annulus, whereas the resistance values are about the same on the thin side (unshaded). This difference increases as the resistivity of the host medium increases.

The effects of the cement layer on the resolution of a target of a layer are shown in Figure (6-23). A uniform $20 \Omega \cdot \mathrm{m}$ cement annulus with thickness $\Delta t$ is sandwiched between a $50 \mathrm{~m}$ casing and a three-layer medium (Figure 6-23a). The upper layer and basal half-space have resistivities of $10 \Omega \cdot \mathrm{m}$, while the $10 \mathrm{~m}$ target layer has a resistivity of $5 \Omega \cdot \mathrm{m}$. In comparison with the series resistances, the discrepancies between the values tend to decrease in the resistive formation and increase in the conductive layer as the cement thickness increases. Thus, the target response tends to "blend in" with the background as the annulus becomes thicker.

Figure (6-24) illustrates the effects of thickness variation of the cement annulus on the transverse resistance response for a layered medium. The same model of Figure (6-23a) is used for this analysis, except the thickness of the cement in the target zone was allowed to vary. Two thicknesses, $20 \mathrm{~cm}$ and $0 \mathrm{~cm}$, are used for the cement annulus. For a curve (A-B) in the figure, $\mathrm{A}$ is the thickness (in centimeters) of the cement adjacent to the host and $\mathrm{B}$ is the thickness in the target layer.

The curve (00-00), representing no cement annulus, is $10-20 \%$ larger than true formation resistivity which is probably due to geometric variations (Section 6.5.1). When a uniform $20 \mathrm{~cm}$ cement annulus is placed, curve (20-20), the transverse resistance increases due to the resistive cement layer. When a $20 \mathrm{~cm}$ cement layer is present only within the target zone, 
curve $(00-20)$, the transverse resistance in the target layer slightly smaller the resistivity of the host medium. From the transverse resistance curve (00-20), the layer is almost indistinguishable from the background. Reversing the situation, cement adjacent to the host and no annulus in the target layer, results in the opposite effect. Now, the transverse resistance outside the target layer is much larger compared with the other curves. The response in the target zone is smaller that those of the no annular layer case. The anomalous response corresponding to the presence or absence of the resistive cement annulus indicates that the currents in the casing are channeling toward and leak out into the zones where the contact resistance is low. This may, in part, explain the large discrepancies associated with the thick cement annulus in Figure (6-22c).

Acquiring measurements with different electrode separations in a cased well provides no additional information about the conductance of the cement. If the thickness or resistivity of the cement zone is known, then the series resistance representation, equation (6-21), can be used to estimate the transverse resistance for a uniform annulus. However, this expression tends to undercompensate the transverse resistance for an annulus that has variable thickness. These results indicate again that the currents in the casing leak into the formations where the contact resistance is small.

\subsection{Summary}

The numerical results clearly show the feasibility of determining formation resistivity through metal casing. When the casing conductance is unknown, two measurements with different electrode configurations are needed to determine the formation resistivity at a given depth. One is used to calculate the casing conductance and the other to-estimate the second derivative of the potential. A three-point measurement on the inner surface of the metal casing is needed to approximate the second derivative of the potentials. Using equation (6-11), the formation resistivity can be calculated from the three-point measurement, the potential, and the estimate of the casing conductance. 
An analysis of electrical logging through metal casing was done for the case of a finitelength casing in a layered or half-space medium. A surface integral equation approach was used to determine the potentials on the casing. The casing, borehole fluid, and layers were modeled as inhomogeneities in the background medium. For the simulations, all electrodes were in contact with the inner wall of the casing. The length and radius of the casing, as well as the location within the casing are geometric factors that influence the measurements. Variations of the casing conductance also have an effect on the transverse resistance. The borehole fluid appears to have negligible influence on the formation resistivity measurements. Variations of the casing conductance only affect measurements where the electrode array lies over the discontinuity. Equation (6-11) can only compensate for spatial variations of the conductance that are longer than the electrode array span. The small spatial variations can be compensated by using equation (6-10).

Numerical results appear to confirm that the discontinuities of the E-fields correspond to the boundaries of the layer and the rate of change of the field is inversely proportional to the resistivity of the adjacent formation. The calculated values of transverse resistance compared closely to the resistivities of the layer model. The behavior of the transverse resistance indicates that the currents in the casing leaks out into the formations with low resistivity. Thus, the resistivity of formations that are more conductive than the surrounding medium is underestimated, whereas the transverse resistance for resistive layers is larger than the true layer resistivity. When the electrode array spans across the interface, the conductive layer has a greater influence on the transverse resistance than the resistive layer since the transverse resistance is inversely proportional to the weighted average of the rates of change of the E-fields. Hence, the transverse resistance of a resistive layer in the vicinity of an interface will always appear more conductive than its true resistivity.

The vertical resolution of the layer boundaries is limited to the potential electrode spacing needed to approximate the second derivative. The response of the MTC log in the vicinity of an interface of two electrically contrasting beds is influenced by the more conductive layer. 
Whereas conductive layers may be overestimated, thicknesses of resistive beds may be underestimated.

Variations in parameters of a the cement annulus have a great influence on the estimate of the formation resistivity. As with the layered medium simulation, the current in the casing tends to leak out in areas where the contact resistance is small. However, using several electrode lengths does not provide any additional information about the cement conductance since the radius of investigation appears to be independent of the electrode spacing. Thus, determining the true formation resistivity in the presence of a cement annulus may not be possible without prior knowledge of the thickness or resistivity of the cement layer. 


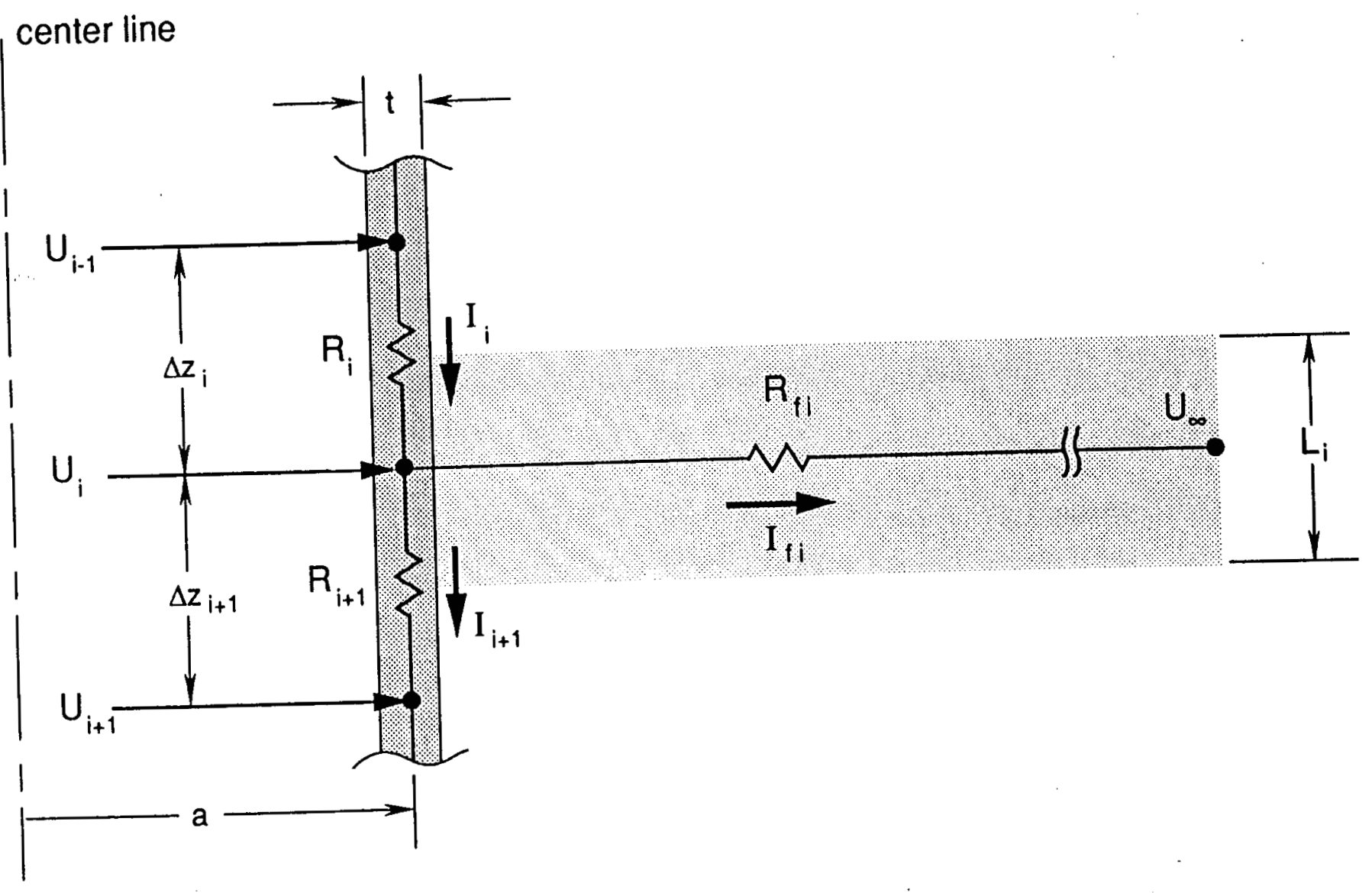

Figure 6-1: Resistor circuit analogy of the casing (series resistors) and adjacent formation (shunt resistor). 


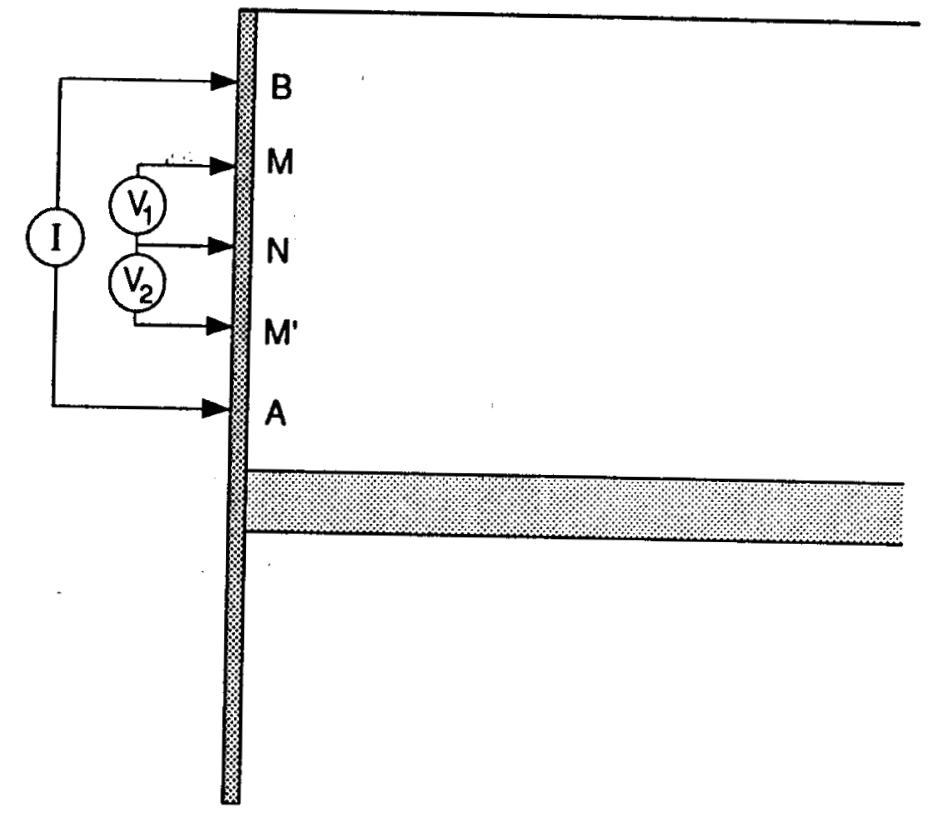

Calibration State

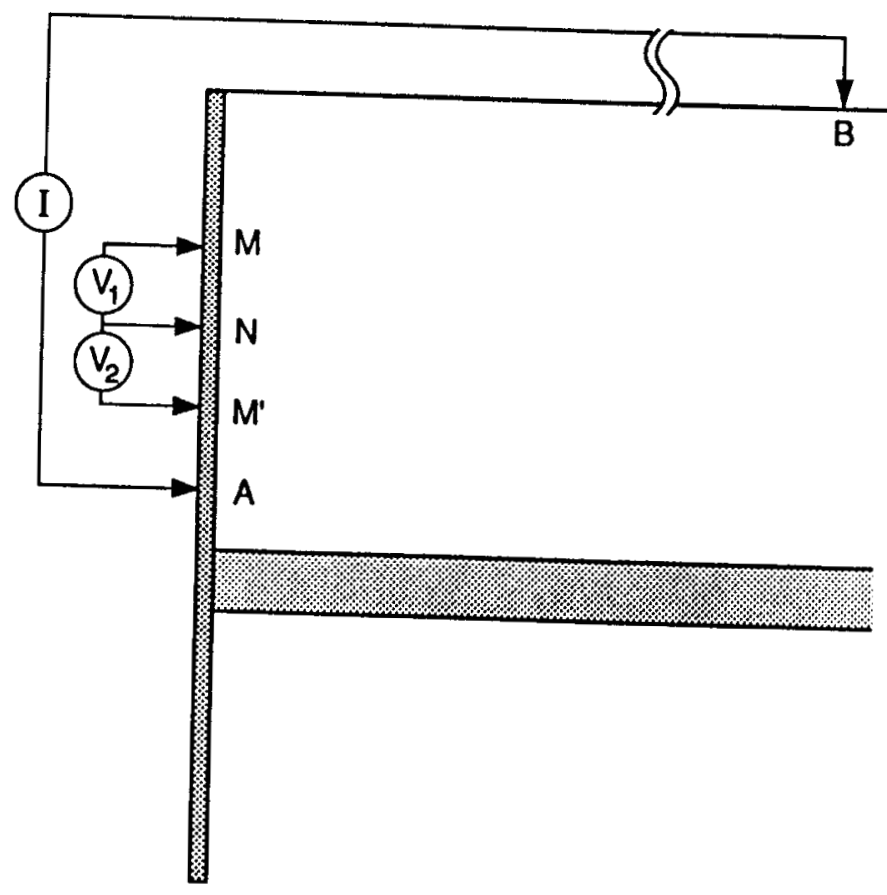

Measurement State

Figure 6-2: Electrode array used to calculate formation resistivity. The casing conductance is estimated from the left electrode array and ratio of the potential to its second derivative is approximated with the right electrode array. 

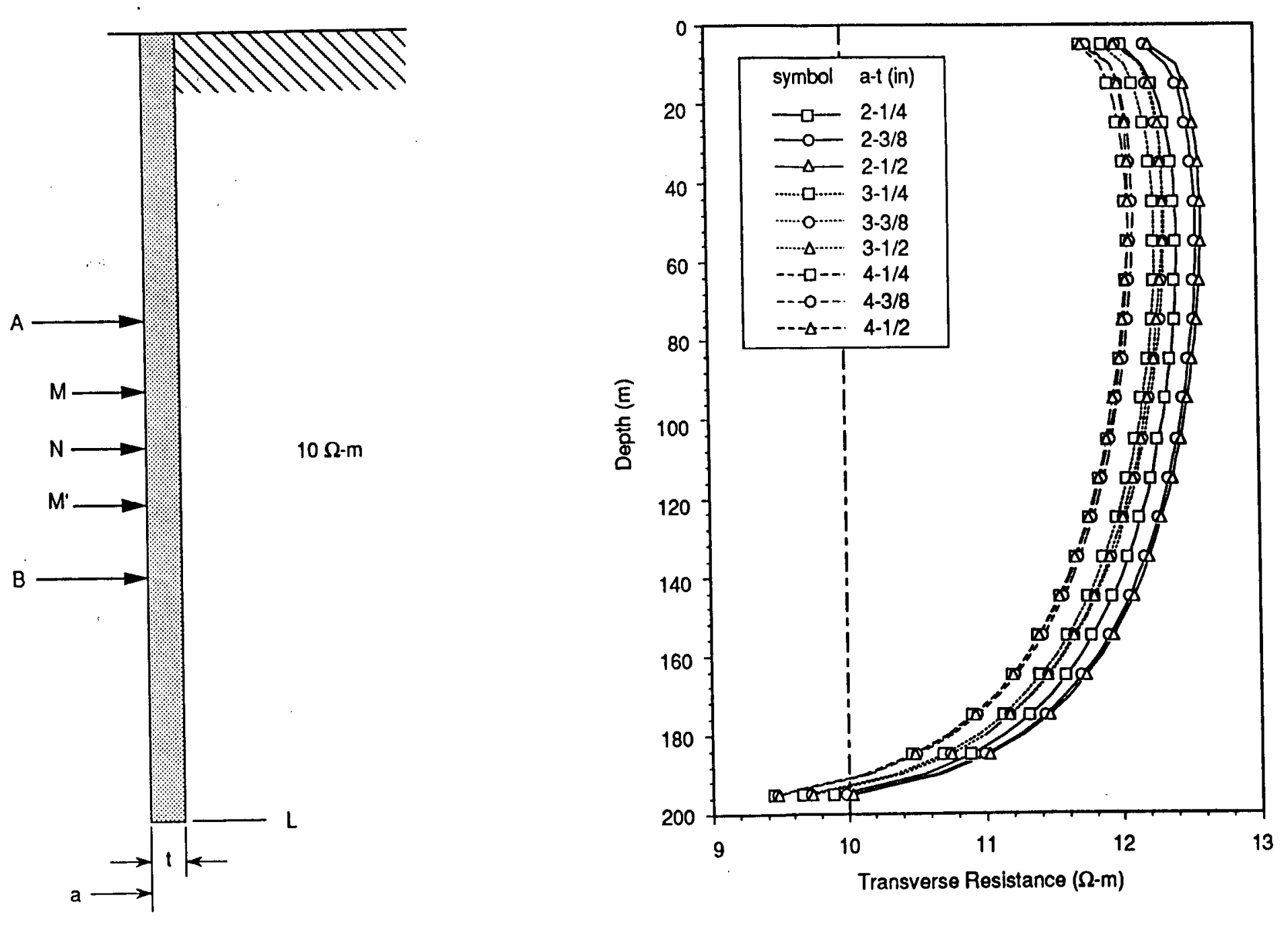

Figure 6-3: Model of a $200 \mathrm{~m}$ casing in a half-space and array configuration (left) used to calculate the transverse resistance (right) for several casing thicknesses ( $t$ ) and radii (a). The radius and thickness are in inches. 


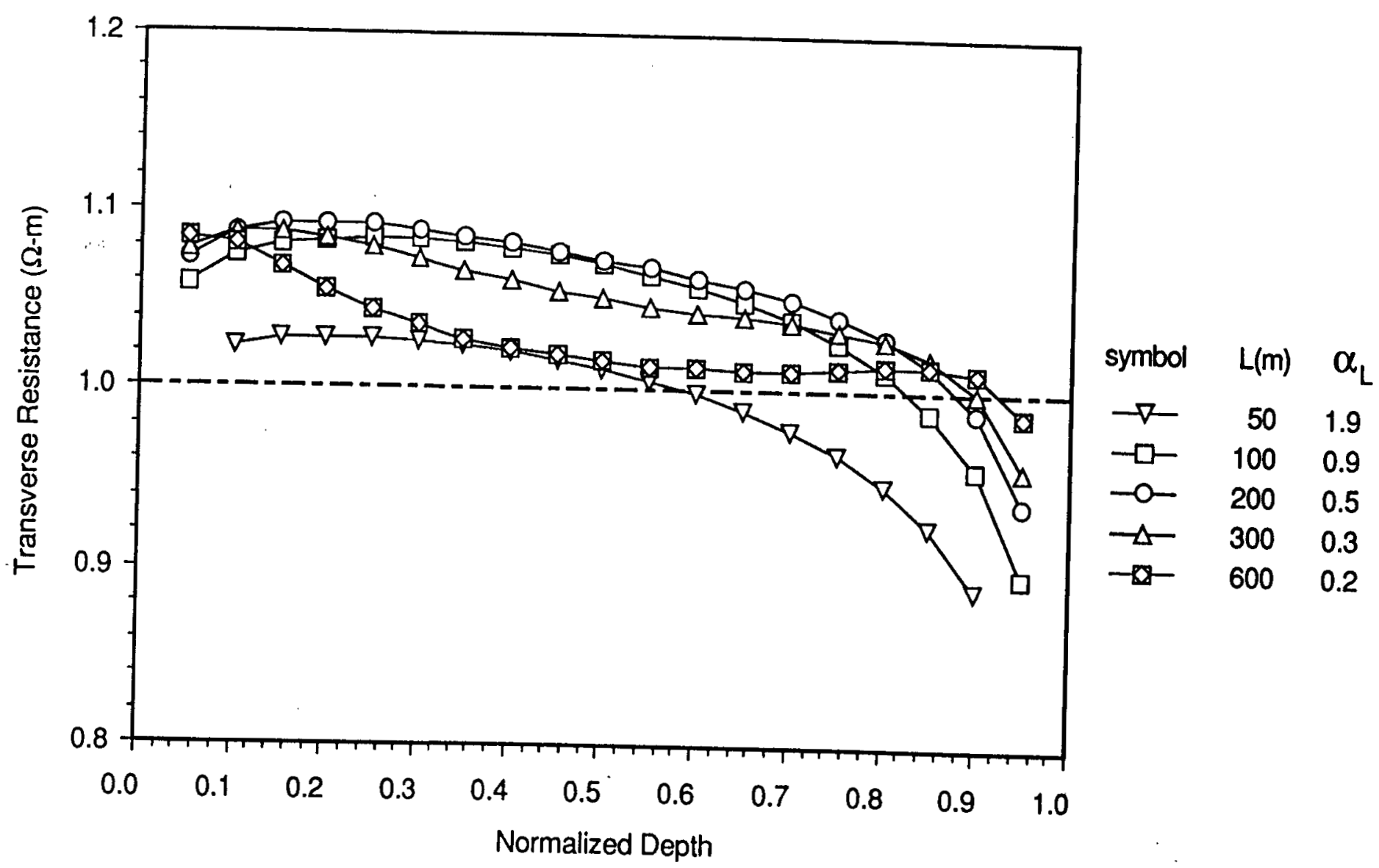

Figure 6-4: Transverse resistances calculated for several casing lengths or conduction lengths. The casing which has a thickness of $1.27 \mathrm{~cm}$ and radius of $10.16 \mathrm{~cm}$ is in a $1 \Omega-\mathrm{m}$ half-space. 


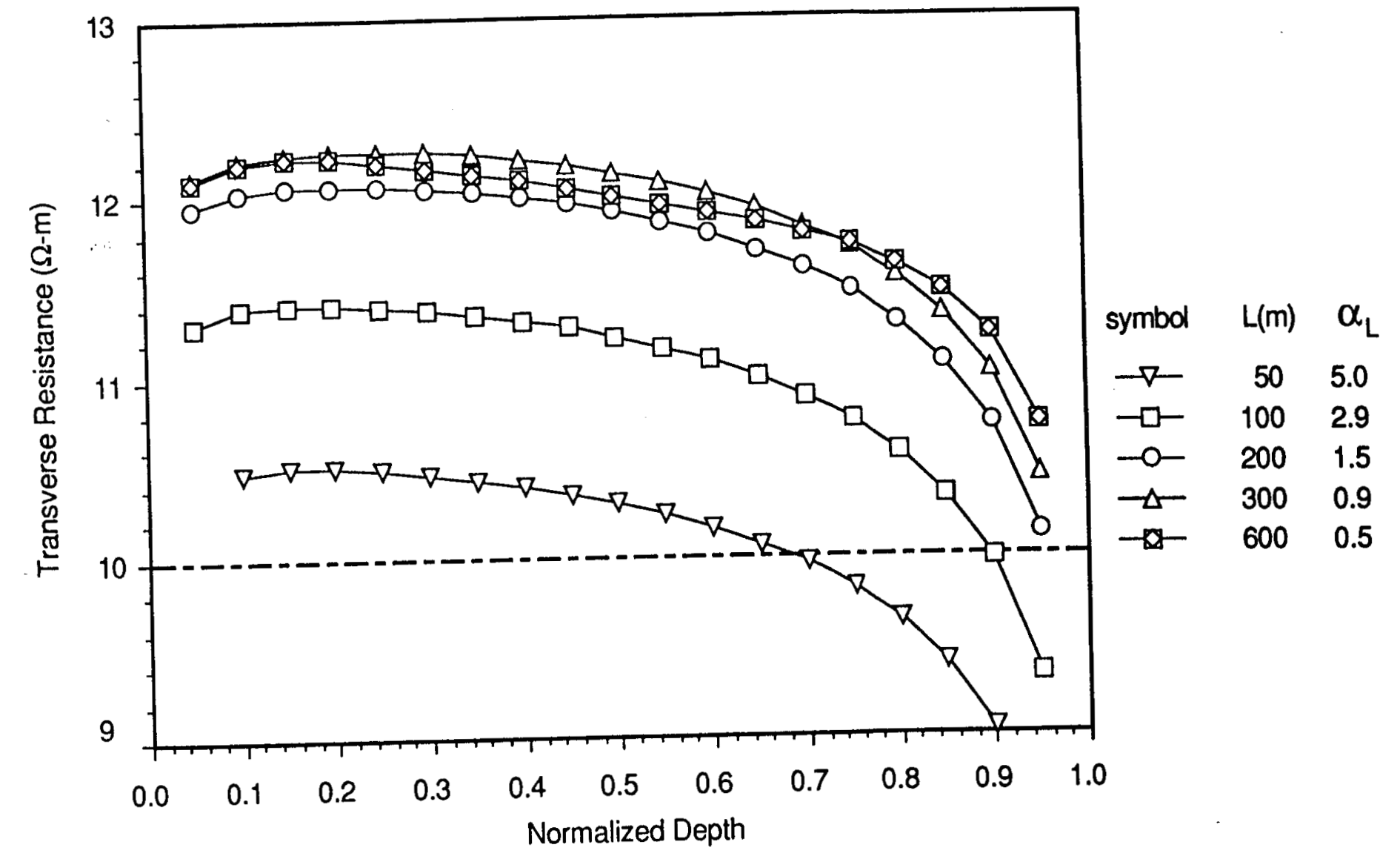

Figure 6-5: Transverse resistances calculated for several casing lengths or conduction lengths. The casing which has a thickness of $1.27 \mathrm{~cm}$ and radius of $10.16 \mathrm{~cm}$ is in a $10 \Omega-\mathrm{m}$ half-space. 


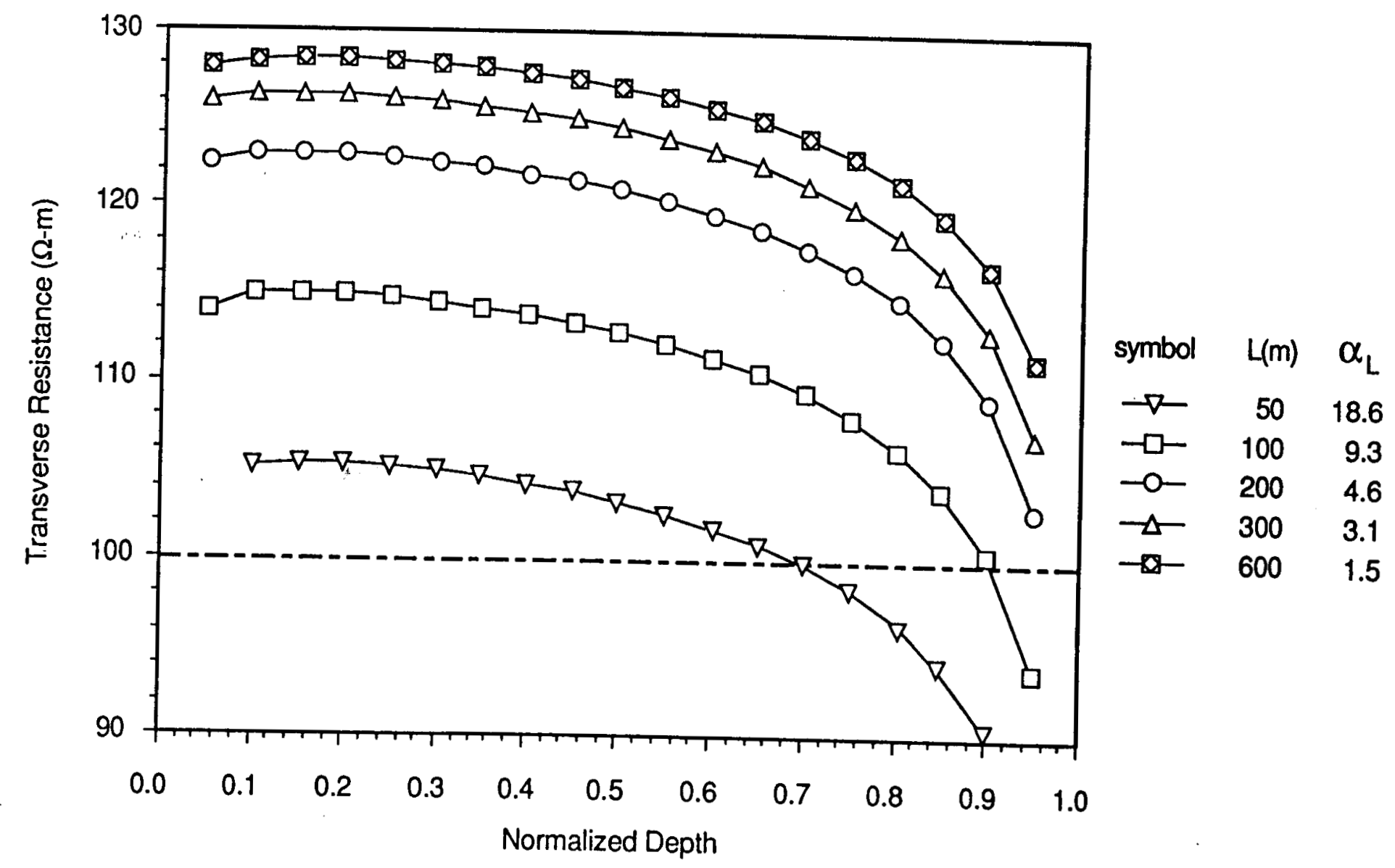

Figure 6-6: Transverse resistances calculated for several casing lengths or conduction lengths. The casing which has a thickness of $1.27 \mathrm{~cm}$ and radius of $10.16 \mathrm{~cm}$ is in a $100 \Omega$-m half-space. 


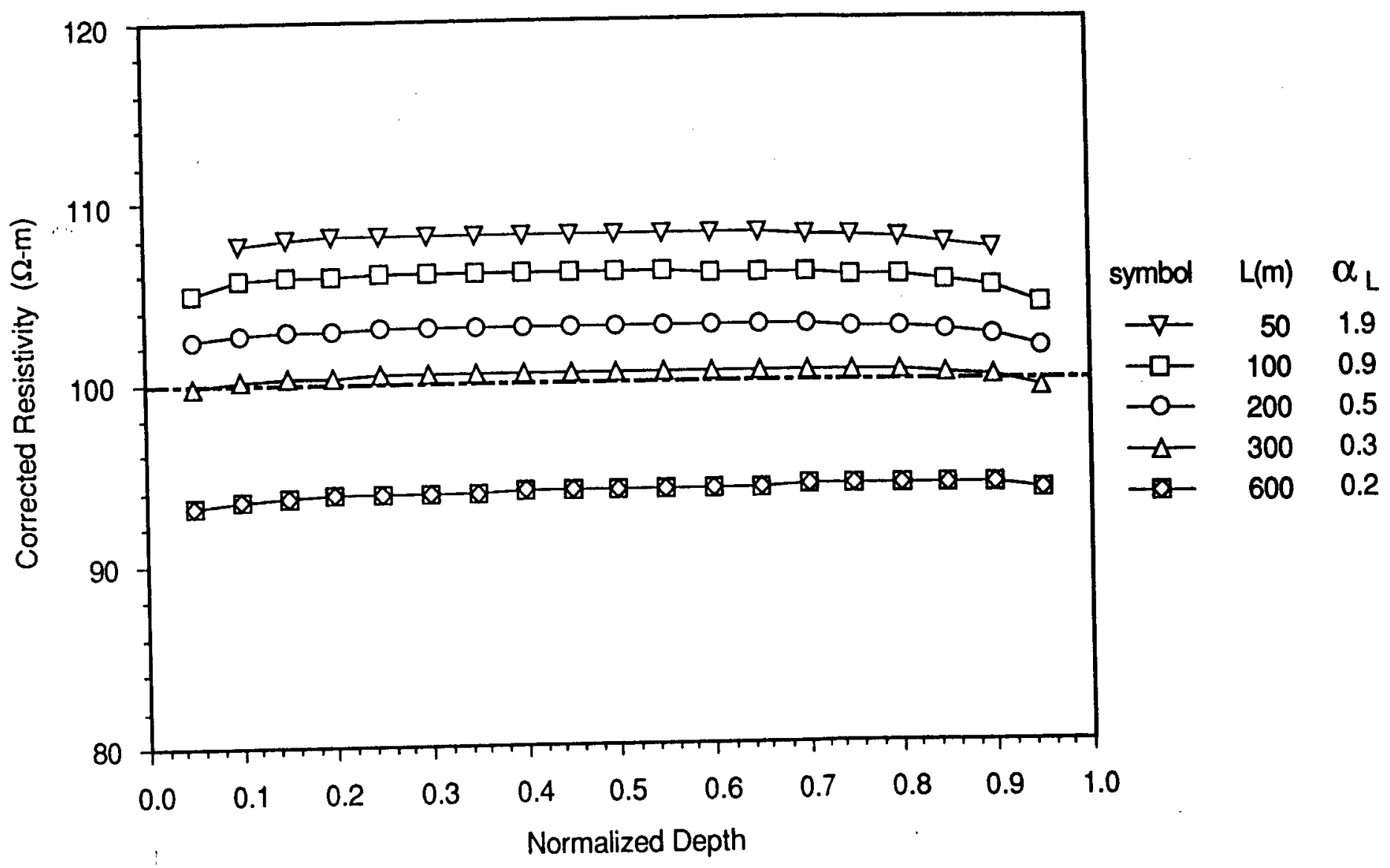

Figure 6-9: The corrected transverse resistance of Figure (6-6) that has been compensated for geometric variations by equation (6-18). 


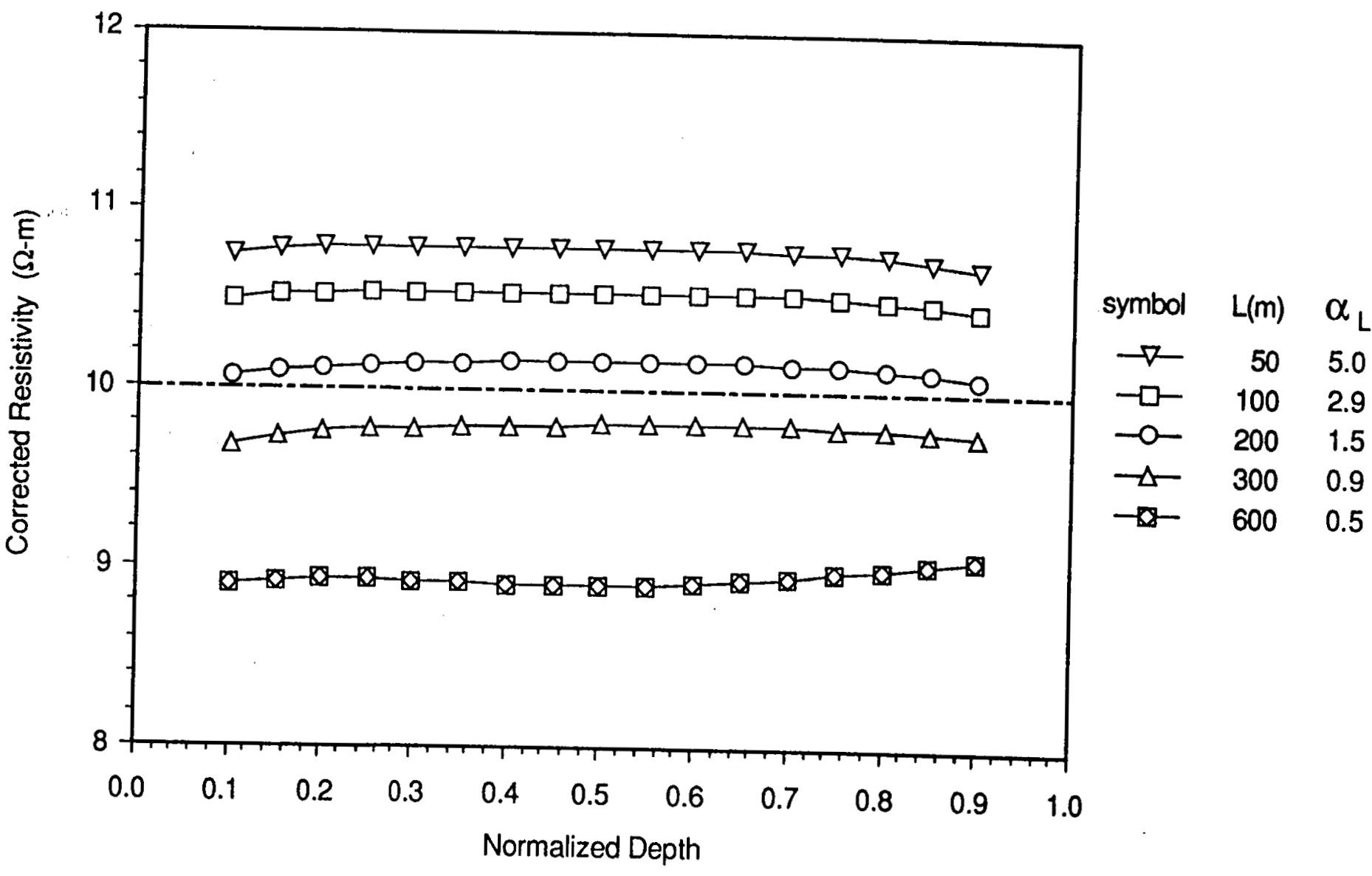
Figure 6-8: The corrected transverse resistance of Figure (6-5) that has been compensated for geometric
variations by equation (6-18). 


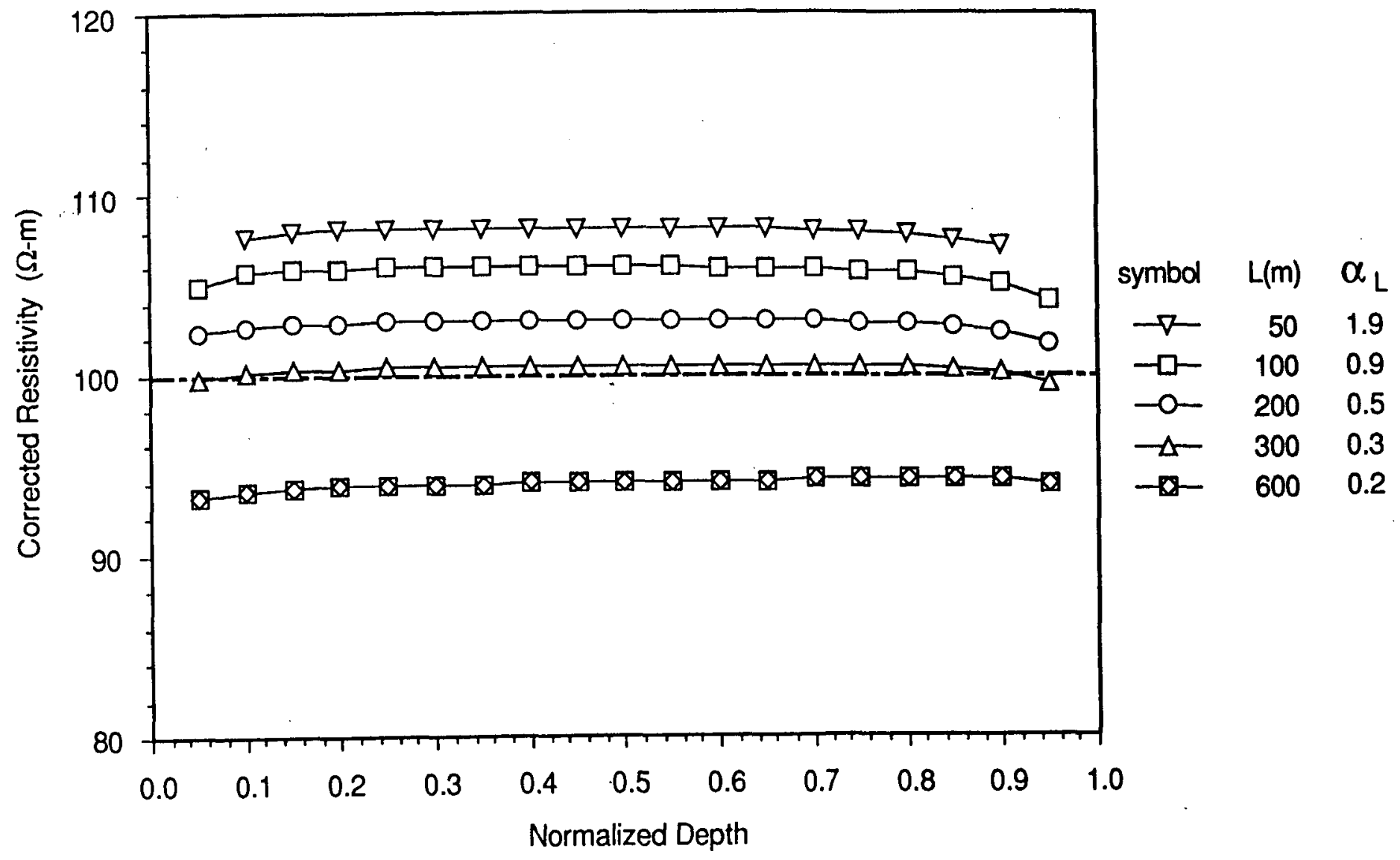

Figure 6-9: The corrected transverse resistance of Figure (6-6) that has been compensated for geometric variations by equation (6-18). 


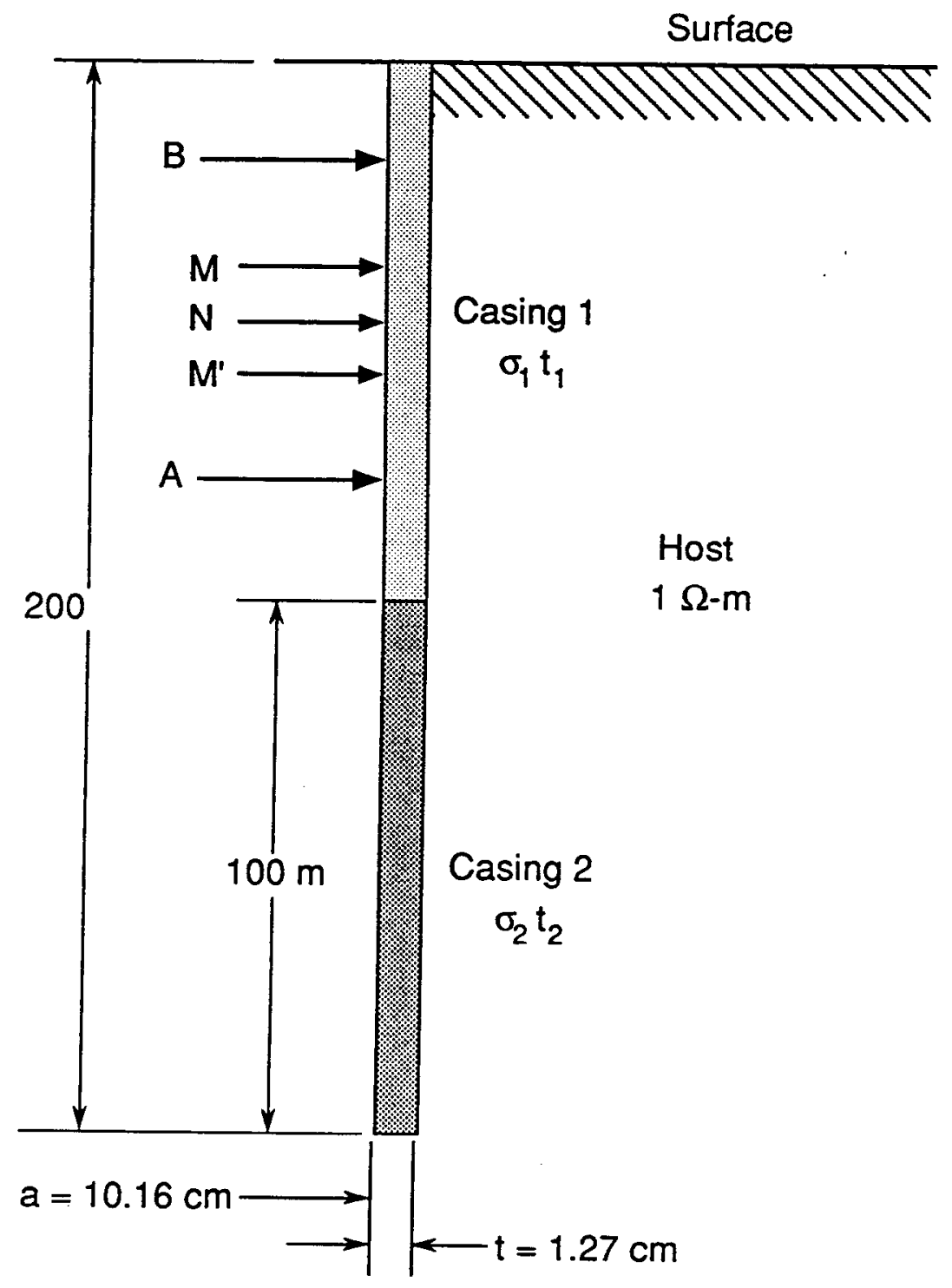

Figure 6-10: Model of two adjacent casing segments with different conductance properties in a half-space and electrode array. 

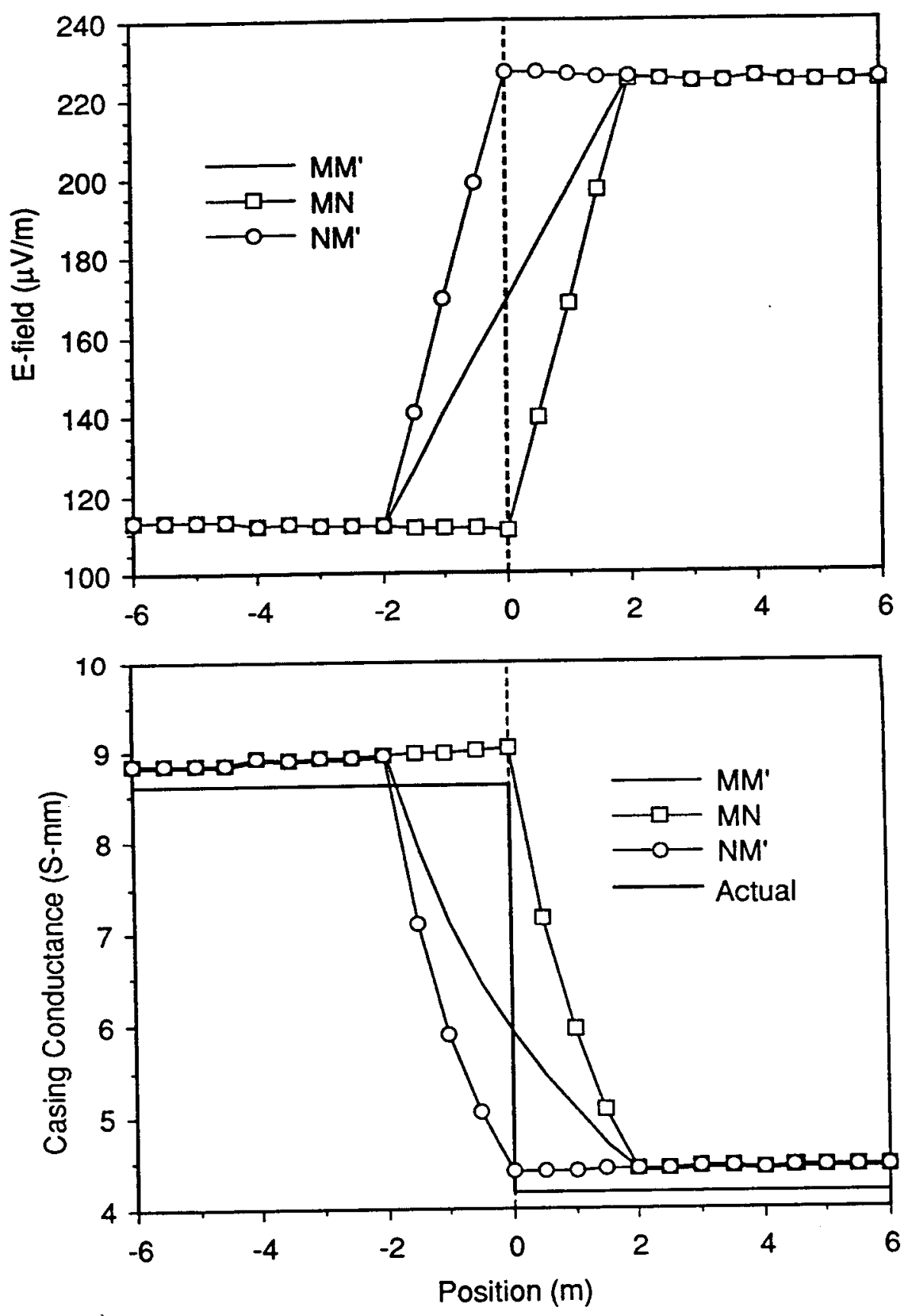

Figure 6-11: The electric fields (top) and casing conductance (bottom) of the calibration state array (see Figure 6-2) calculated for electrode pairs: MN, NM', and MM'. ' 

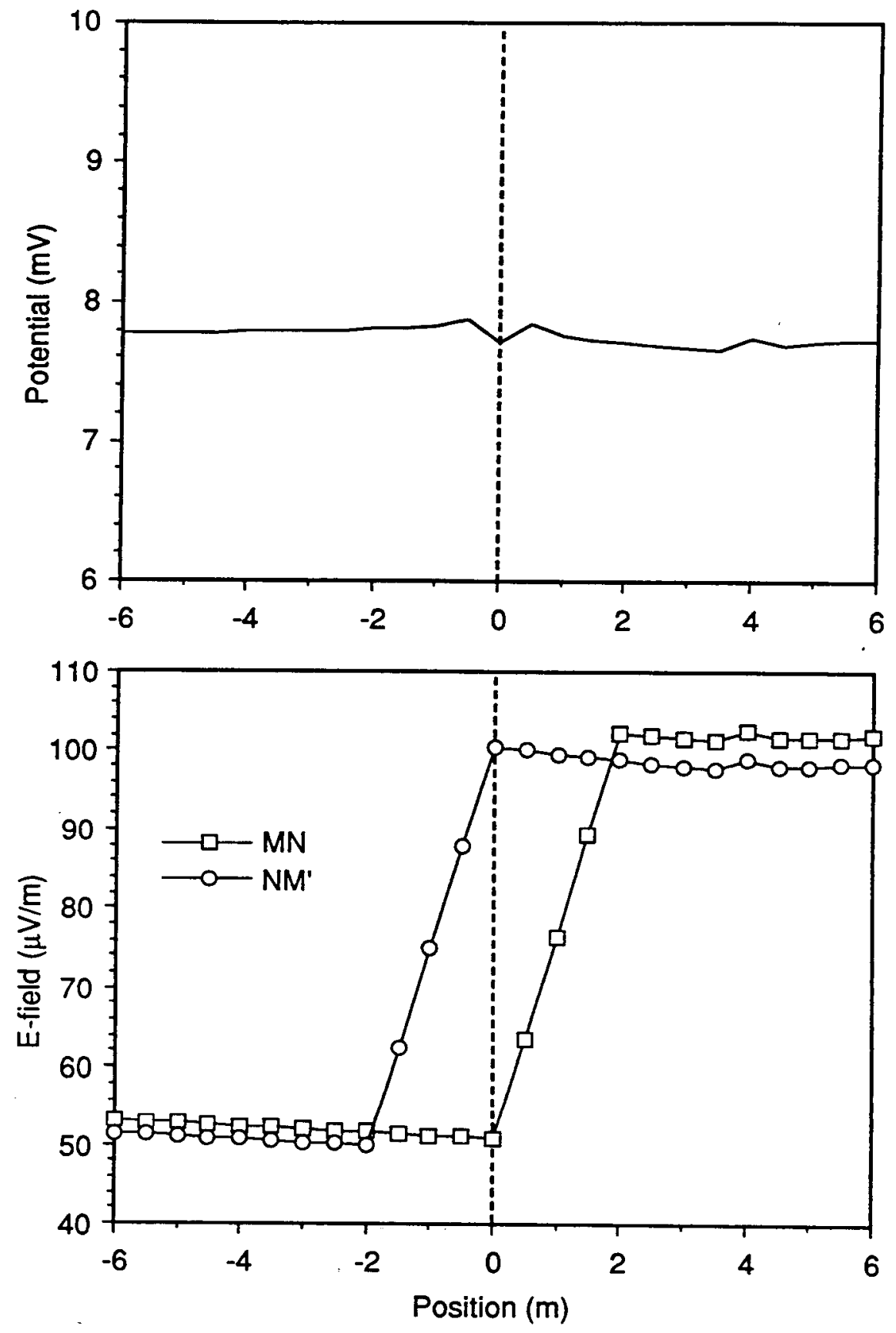

Figure 6-12: The potentials (top) and electric fields (bottom) of the measurement state array (see Figure 6-2) for electrode pairs: MN, NM', and MM'. 

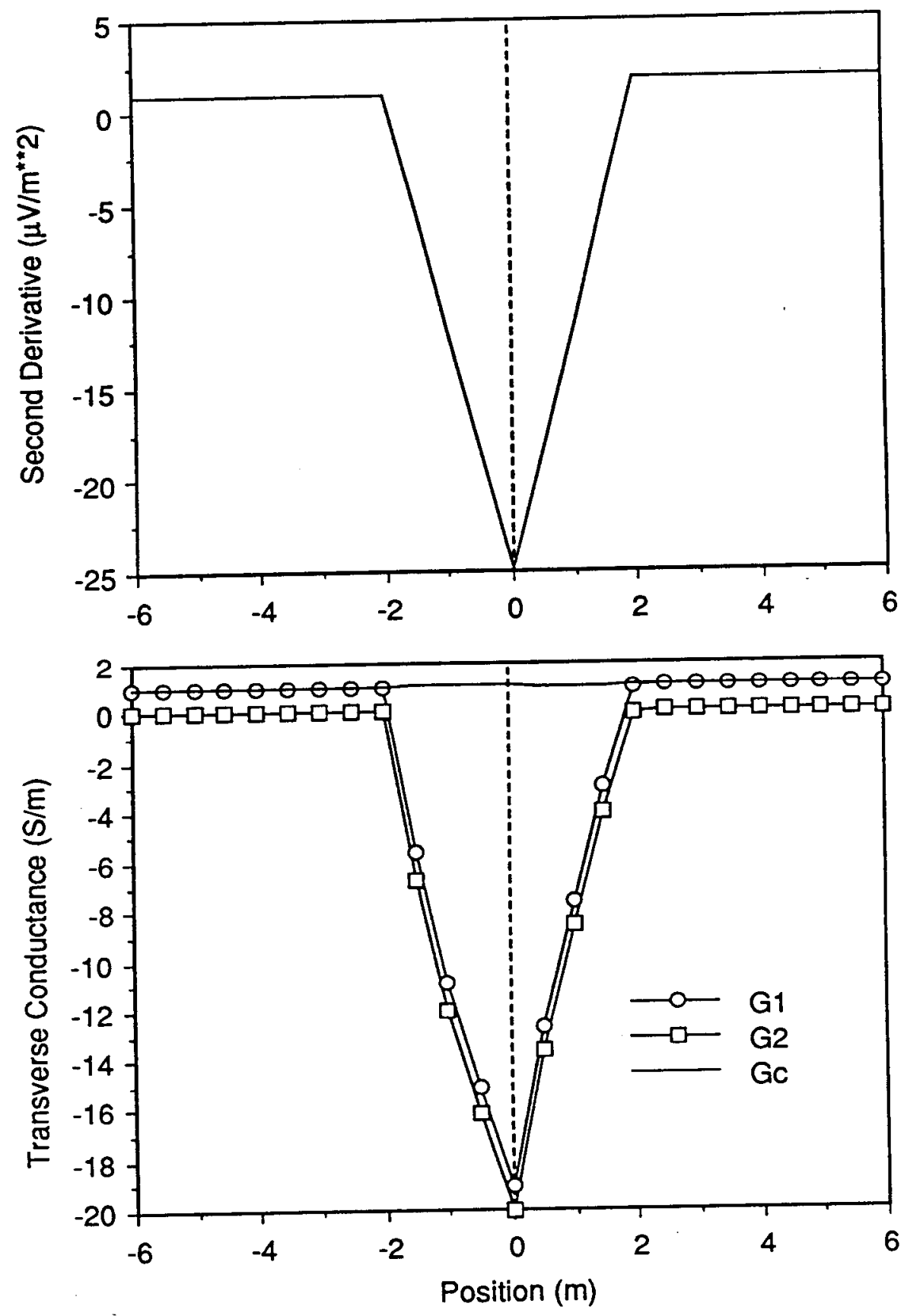

Figure 6-13: The estimate of the second derivative (top) and transverse conductance (bottom). Curves $G 1$ and $G 2$ are equivalent to the first and second terms of equation (6-10), curve Gc. 


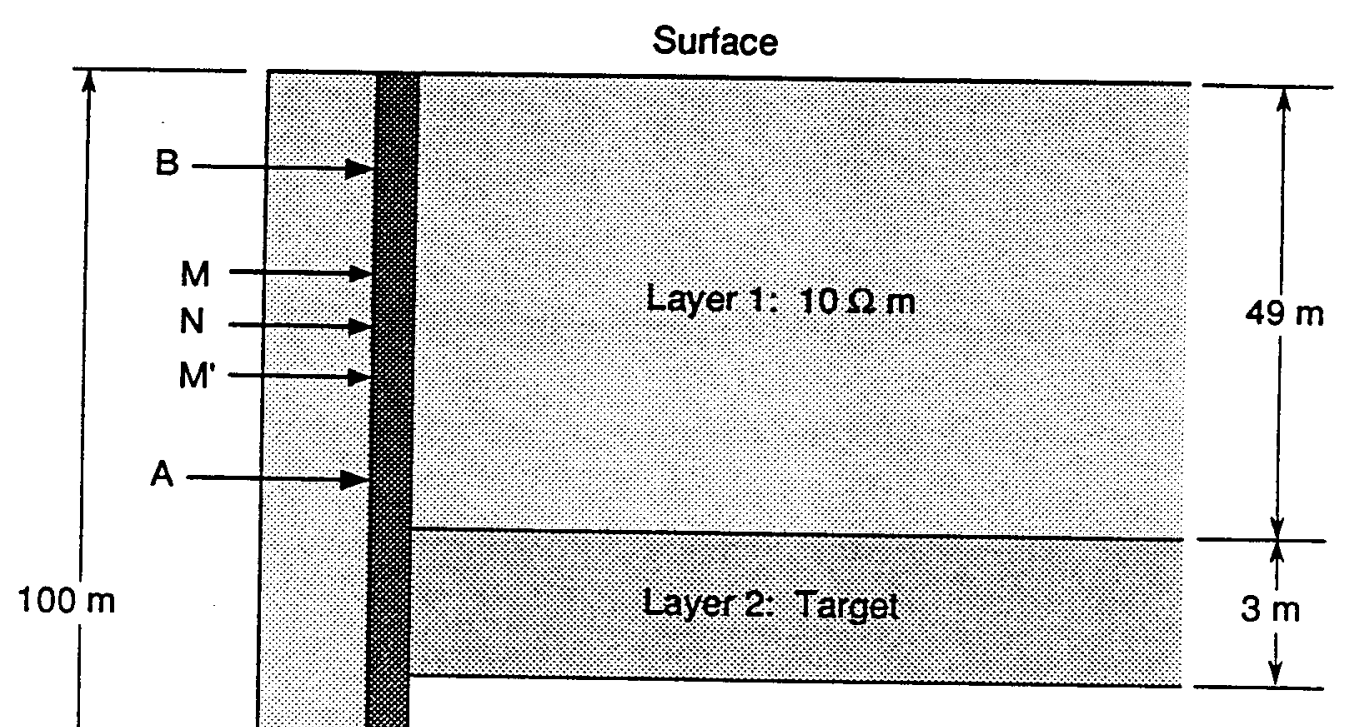

Layer 3: $10 \Omega \mathrm{m}$

Figure 6-14: Model of a filled-fluid casing in a layered medium and electrode configuration. 

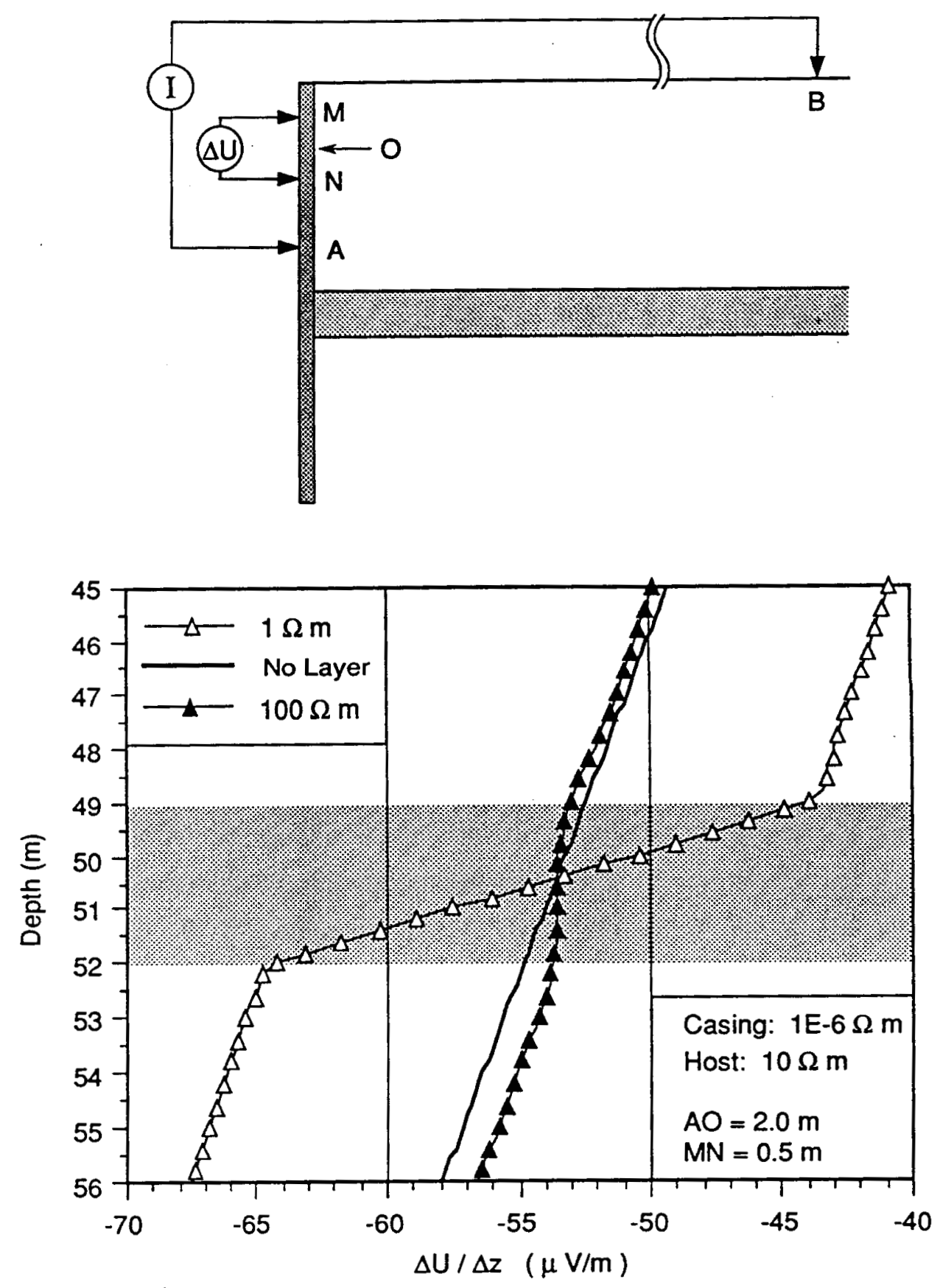

Figure 6-15: Lateral log electrode array (top) used for E-field calculations. The E-field response (bottom) with increasing depth for a $1 \Omega \cdot \mathrm{m}$ and $100 \Omega \cdot \mathrm{m}$ layer (shaded area). 


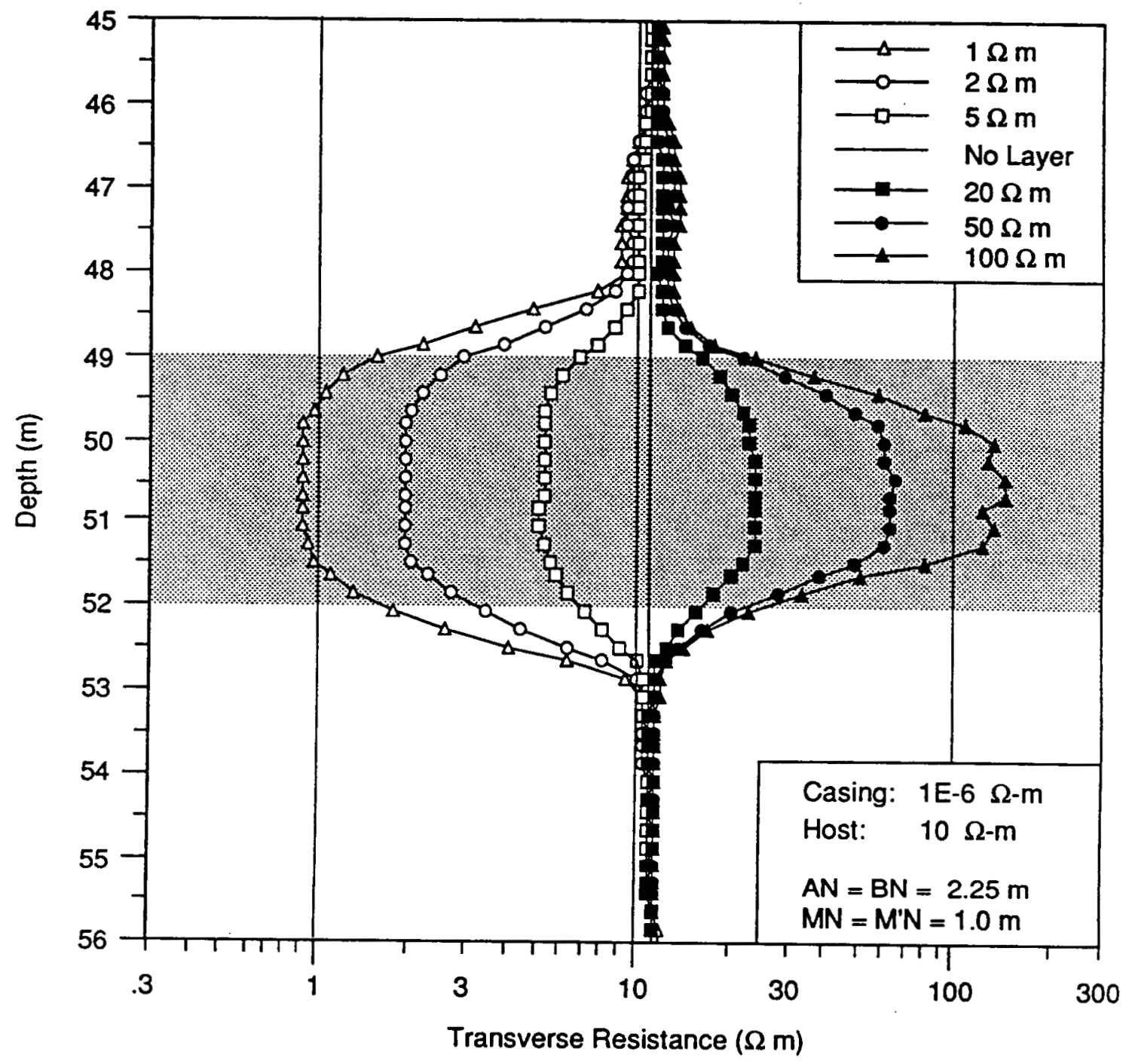

Figure 6-16: Transverse resistance calculated using equation (6-11) for the model of Figure (6-14). Several resistivities are used for the target layer (shaded area). 


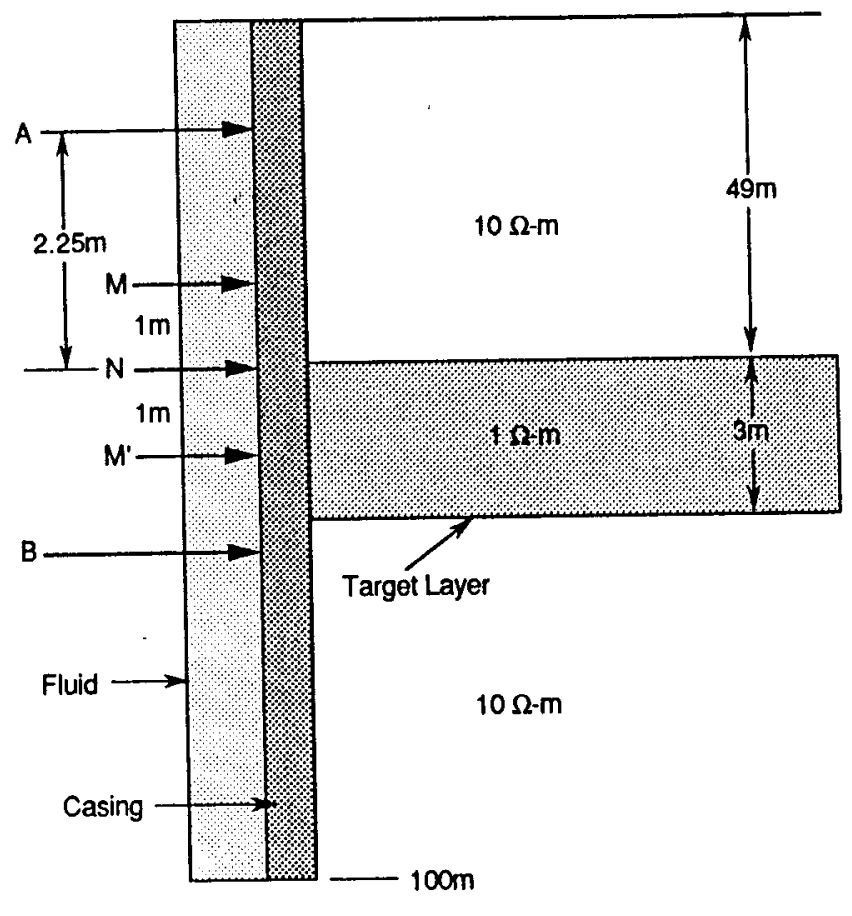

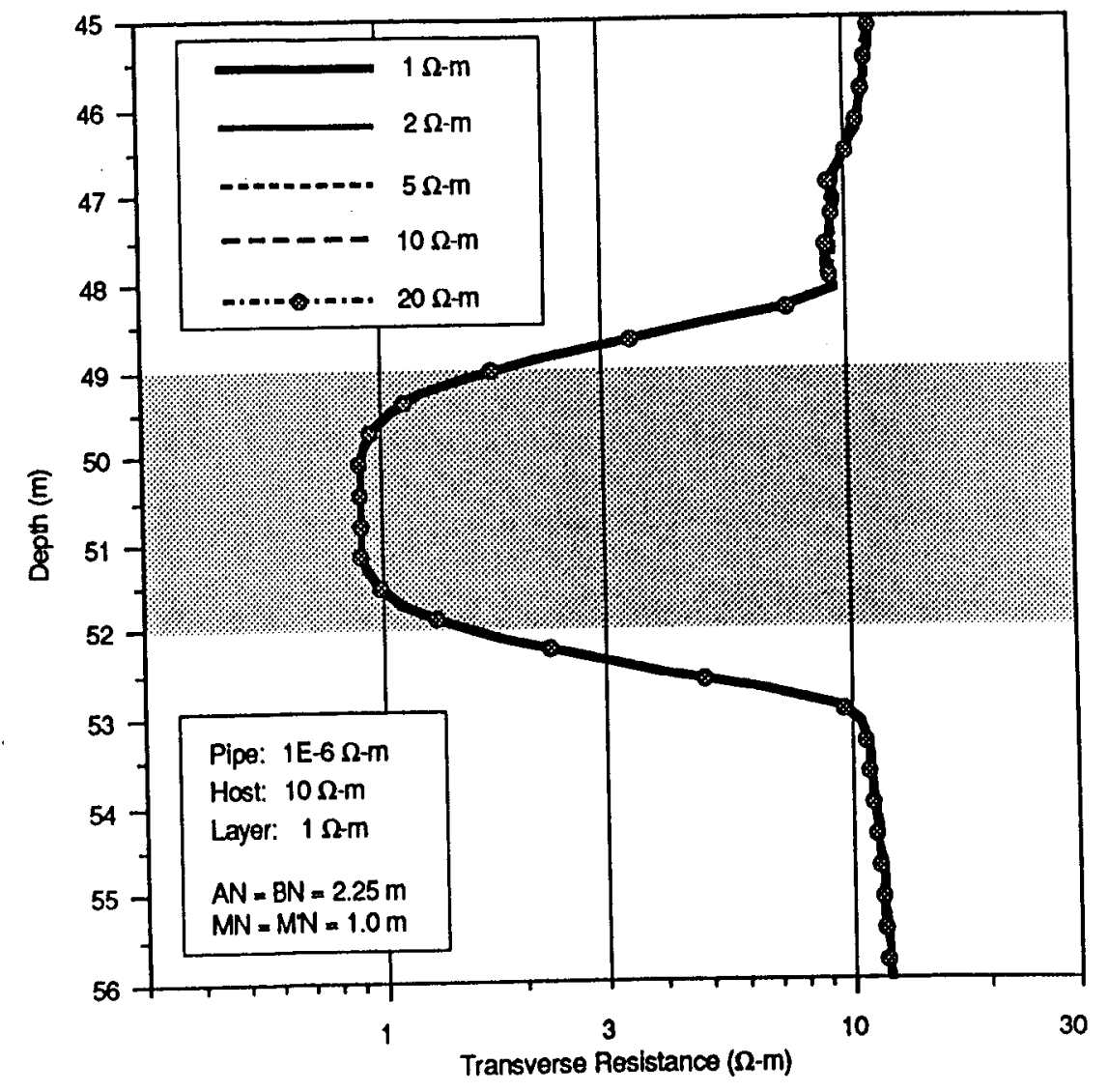

Figure 6-17: a) Model of fluid-filled casing in a layered medium and electrode array (left).

b) Transverse resistance for several resistivities of the borehole fluid (right). 

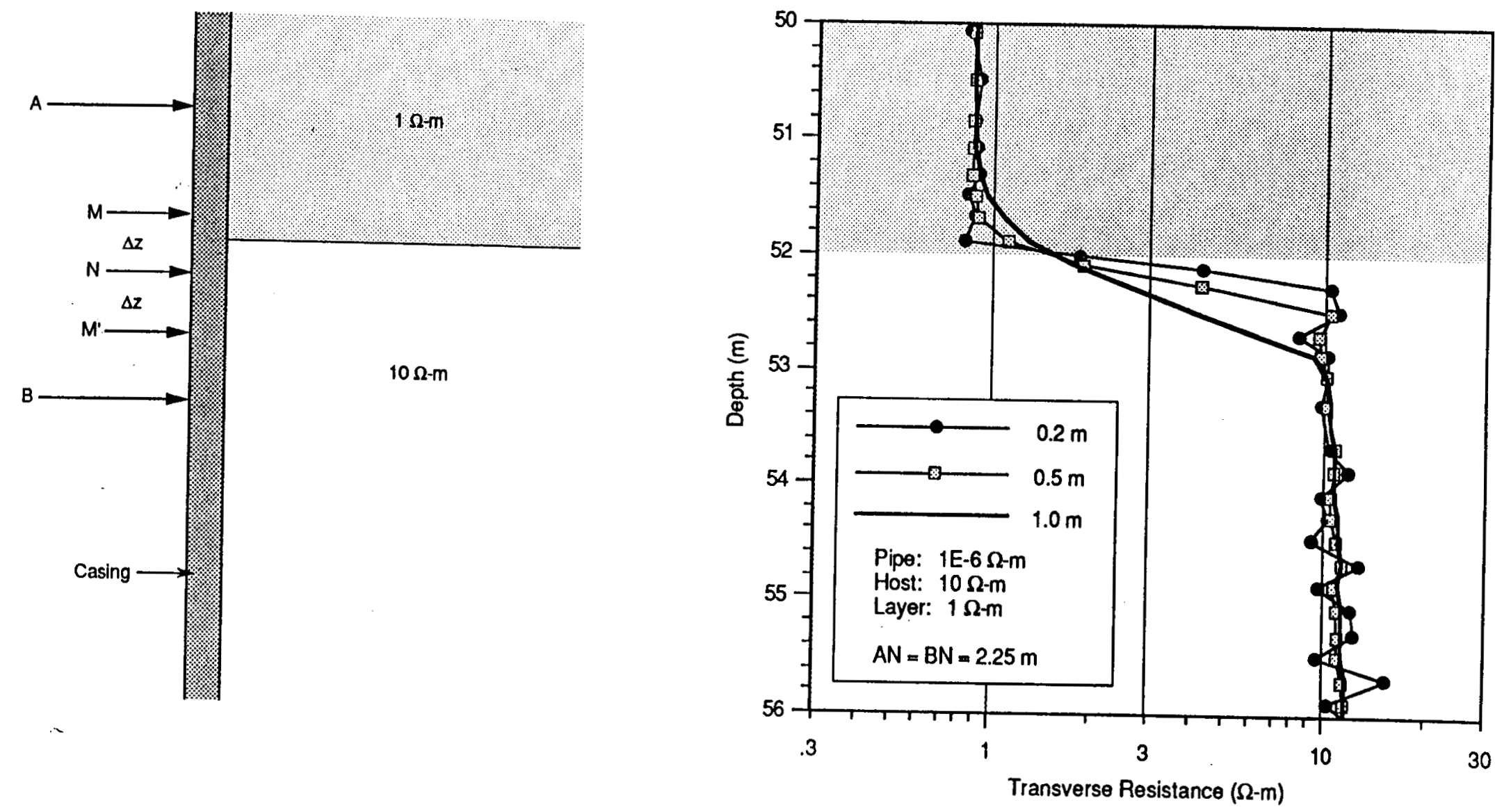

Figure 6-18: Model of the casing in a layered medium and electrode configuration (left) used to calculate the transverse resistance (right) for several electrode spacing. 


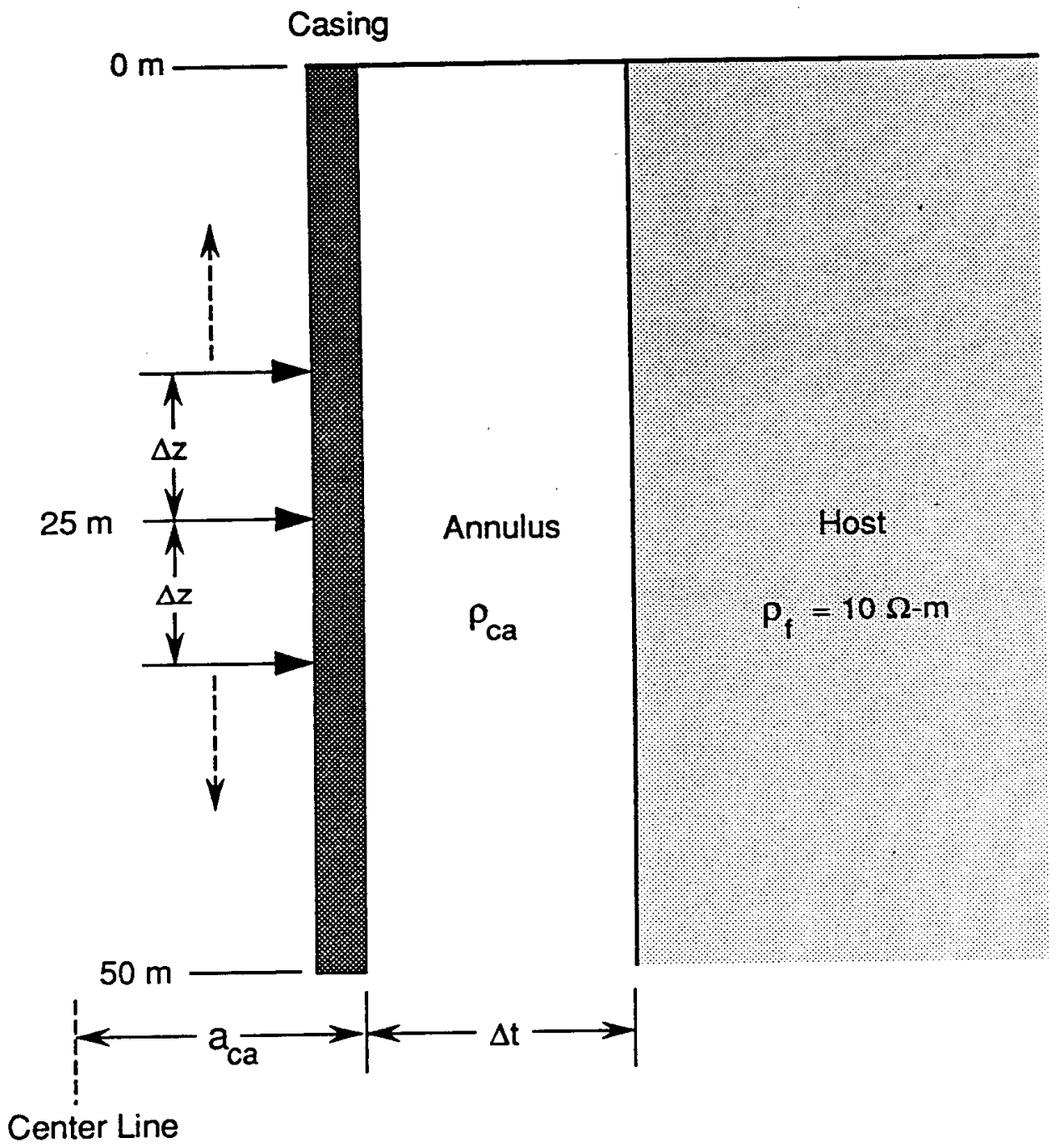

Figure 6-19: Model used to examine the relation of electrode spacing $\Delta z$ and center annulus thickness $\Delta t$. Center electrode is fixed and current electrodes are located $2 \mathrm{~m}$ from the outer potential electrodes. 

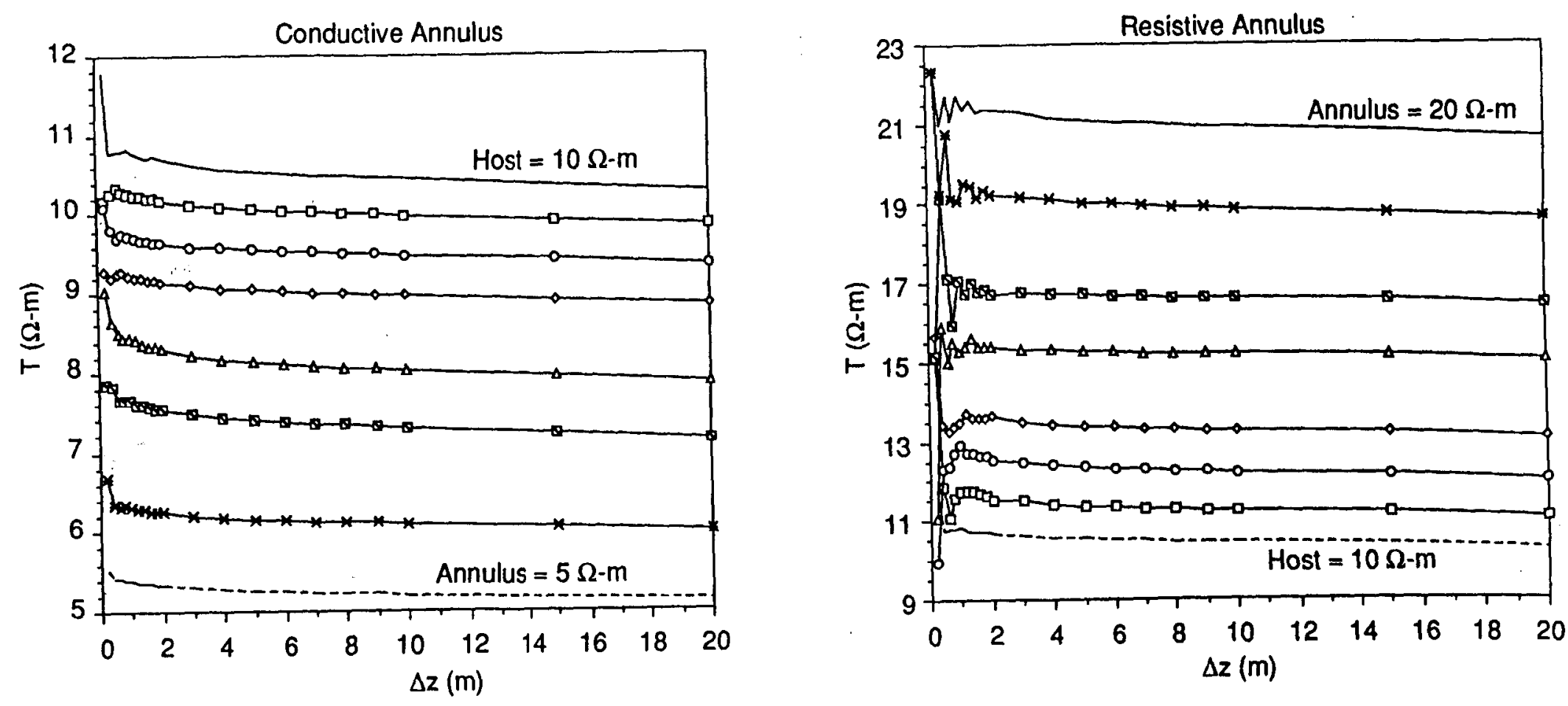

Annulus Thickness (m)

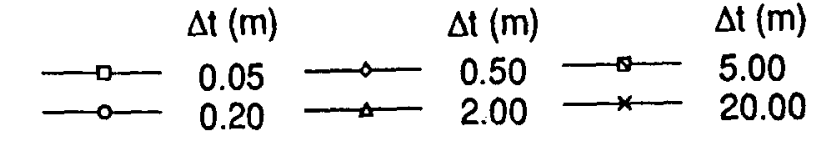

Figure 6-20: Transverse resistance calculated for several annular thickness $\Delta t$ as a function of electrode spacing $\Delta z$. The center electrode is fixed and the current electrodes are located $2 \mathrm{~m}$ from the outer potential electrodes. 

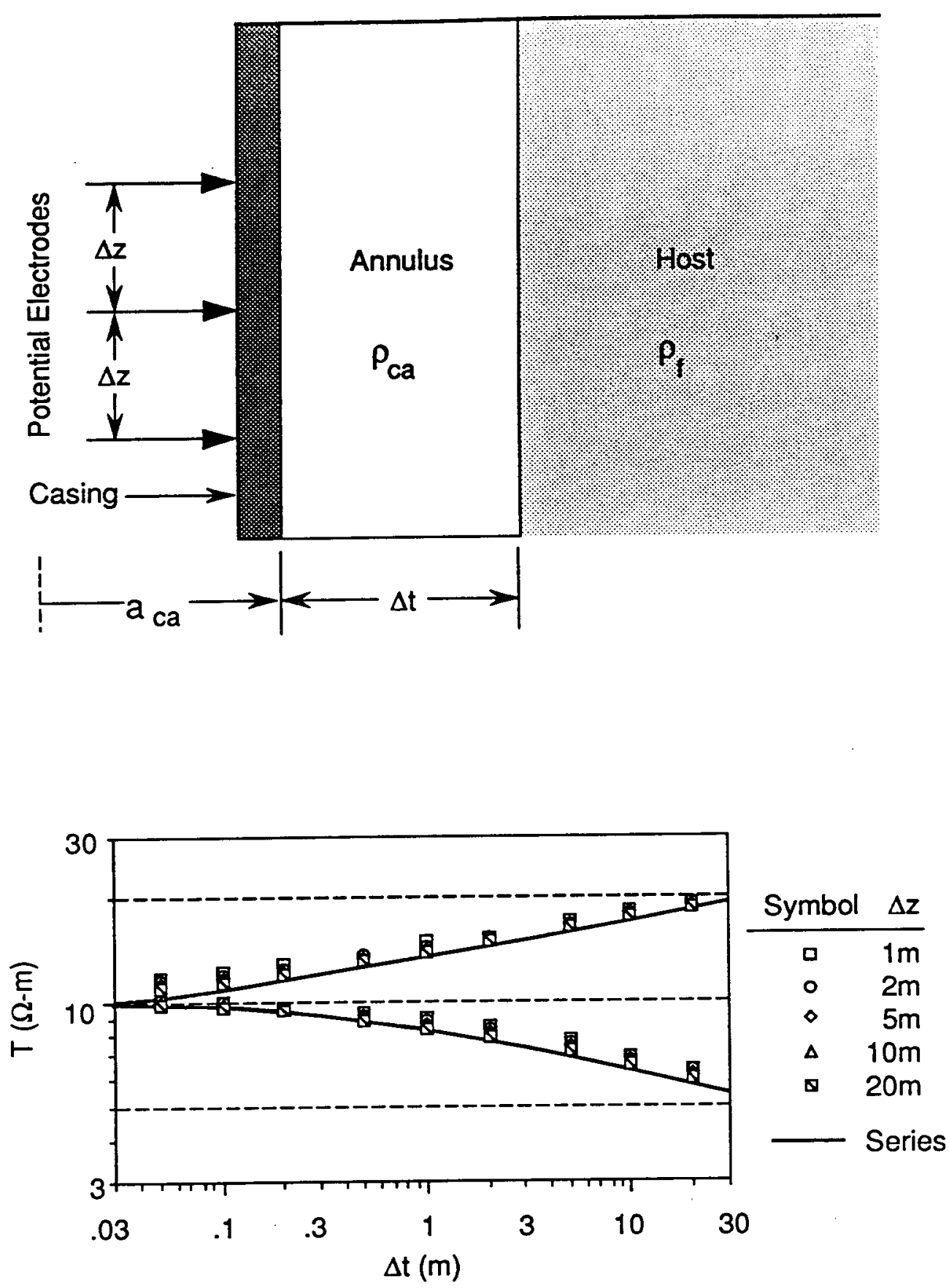

Figure 6-21: Model of $50 \mathrm{~m}$ casing in a $10 \Omega \cdot \mathrm{m}$ host (top) used to calculate the series resistance, equation (6-21), and transverse resistance, equation (6-11), for several annular thicknesses (bottom). The transverse resistances are calculated for several electrode spacings and the center electrode is fixed at $2 \mathrm{~m}$. The resistivities of the annulus are $5 \Omega \cdot \mathrm{m}$ and $10 \Omega \cdot \mathrm{m}$. 

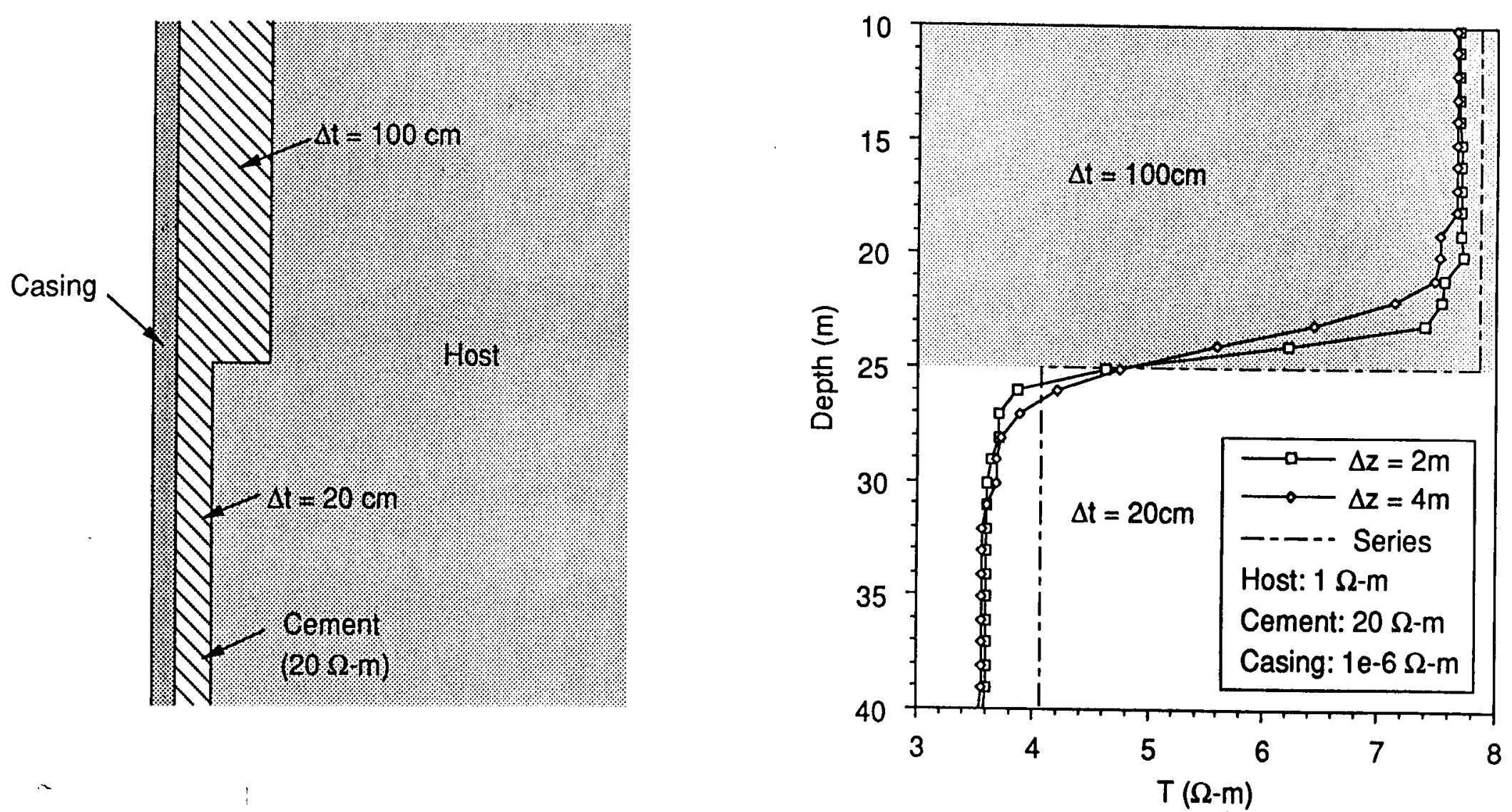

Figure 6-22: a) Model configuration of the cement annulus with variable thickness in a homogeneous medium (left). b) Series resistance (dashed line) and transverse resistance using two electrode spacings in a $1 \Omega$-m host. 

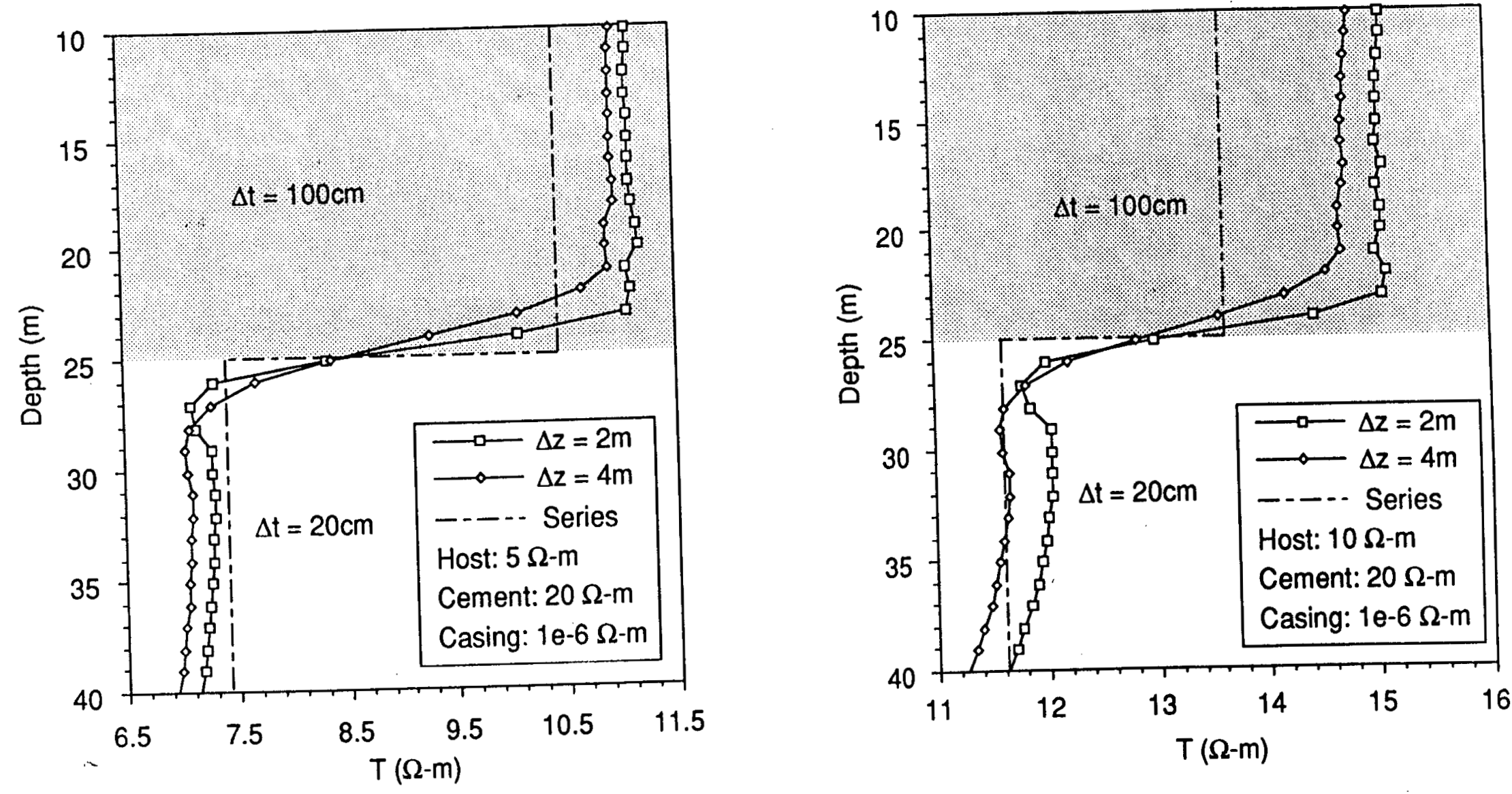

Figure 6-22: c) Series resistance (dashed line) and transverse resistance using two electrode spacings in a $10 \Omega-\mathrm{m}$ host. b) Series resistance (dashed line) and transverse resistance using two electrode spacings in a $100 \Omega$-m host. 

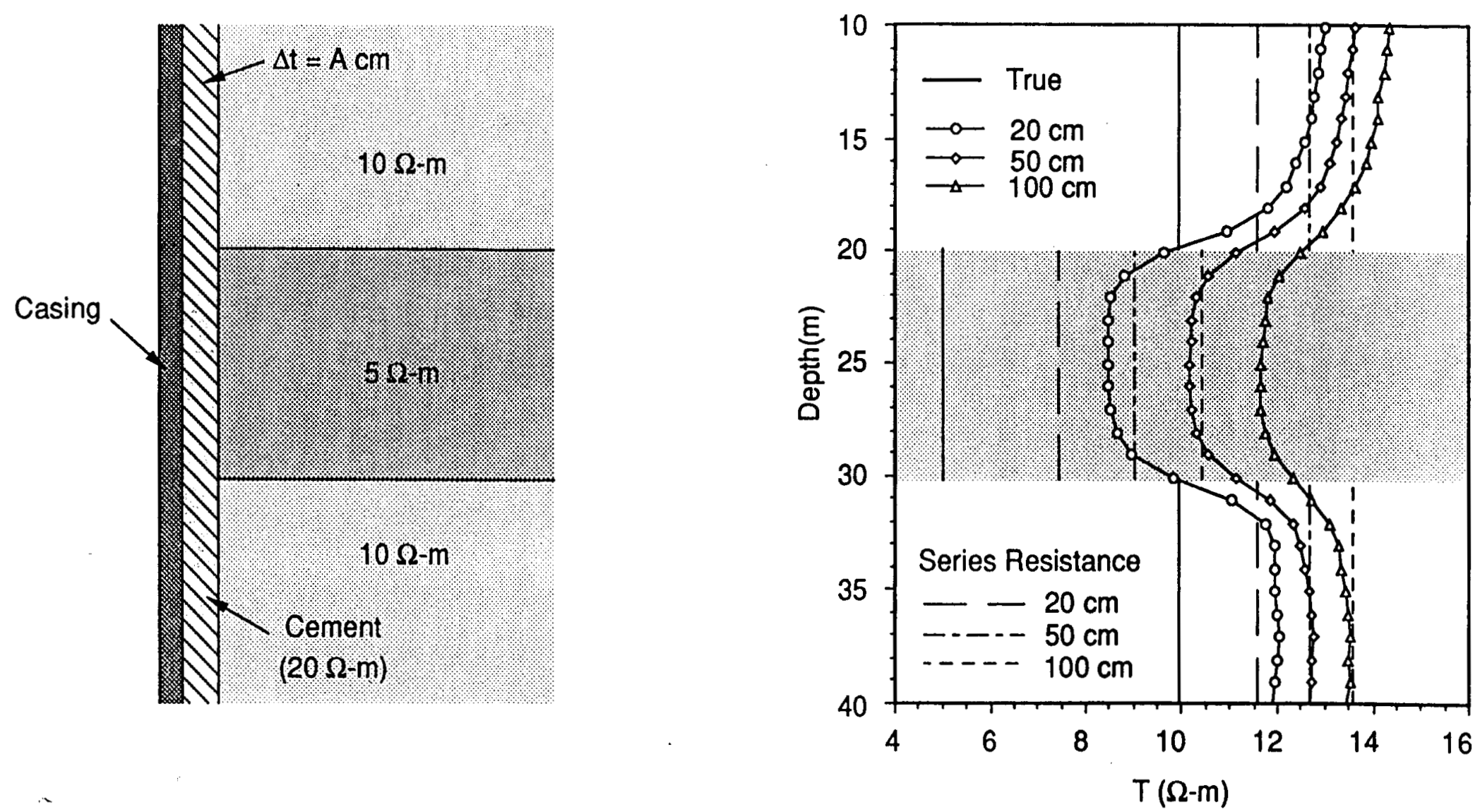

Figure 6-23: Model of the casing and uniform cement annulus in a layered medium (left). Series (lines only) and transverse (lines with symbols) resistances calculated using several annular thicknesses (right). 

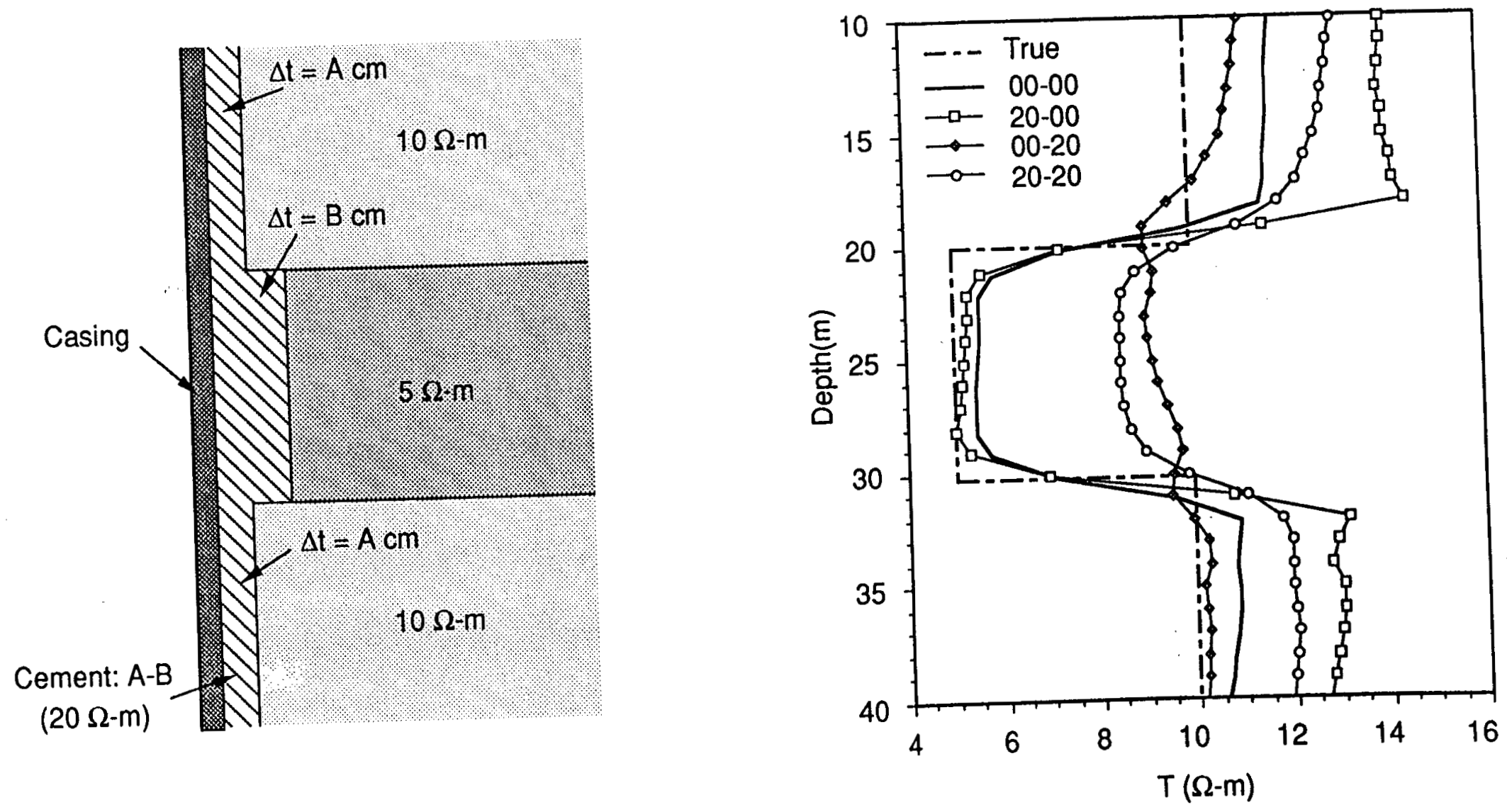

Figure 6-24: Model of the casing and varible cement annulus in a layered medium (left). Transverse resistances calculated using several annular thicknesses (right). 


\section{Chapter VII}

\section{FIELD EXAMPLE - Downhole to SuRface RESISTIVITY SuRVEY}

The contents of this chapter are based on a report ${ }^{1}$ submitted to the EPA on "The Feasibility Test of the Downhole-to-Surface Resistivity Method for Mapping the Areal Extent of Contaminants at Depth" by Schenkel and Morrison (1990b). The survey was conducted at DuPont's Hemby Branch test well 3.5 miles north of Waverly, ${ }^{2}$ Tennessee from the second to the sixth of October 1989. For the borehole-to-surface resistivity experiment, three radial surface arrays measured the voltages for a succession of current electrode depths in the well.

The test site is physiographically located in the westem part of the Western Highland Rim province which borders the Kentucky Lake/Tennessee River. The geology of the area is predominantly limestone formations with occasional dolomite and shale units. The limestone layers varied greatly in composition and in occurrence of fractures. Table (7-1) shows the stratigraphic units and their approximate depths obtained from core samples at the site. The topography near the well site can be described as rolling hills and ridges that are dissected by streams. The maximum relief in this area was approximately 150 feet. The drilling site was terraced into the side of a ridge. Figure (7-1) shows the topography and array configuration of the test area.

The first and major objective of this test was to determine the background noise levels for the voltage measurements and the practical values of electric current that can be achieved with the power supply of the $\mathrm{LBL}^{3}$ acquisition system in a resistive environment. In resistive limestone formations, we had no data on the effective contact resistance of the downhole elec-

1 US-British units were used in the report and are used in this chapter.

2 Waverly is located approximately 50-60 miles west of Nashville.

3 LBL is an abbreviation for Lawrence Berkeley Laboratory. Past experiments have been conducted in very conductive sediments and there has been no problem in injecting 20 to 30 Amps. 
trode. The second goal was to interpret the field data with simplified models using the surface IE method described in Chapters II and III.

\subsection{Field Experiment Design}

The principle of operation of this method is as follows. Electrical current is injected into the ground between a downhole electrode and a remote electrode on the surface. In a homogeneous medium, the current flows essentially radially away from the hole producing a predictable set of voltages at a radial array of measuring (potential) electrodes on the surface. When the current electrode is located in a zone of high conductivity, caused for example by the injection of a fluid with high tds, the current pattern is distorted by the zone. This distortion produces perturbations in the normal voltages seen on the measuring array. As injection continuous, a plume develops which can be monitored over time by the voltage changes. If the response of an arbitrary background conductivity distribution is known, then the changes due to the injection plume can be mapped.

The design of the experiment at the DuPont well is illustrated in Figure (7-2). At the time of the experiment, the well was drilled to a depth of approximately 2500 feet with steel casing down the first 450 feet. The acquisition system is shown in the schematics of Figure (7-3). This system consists of a timer/clock which controls the switching of the current transmitter and the sampling of the receiver or voltage measurement. The details of each are described below:

\section{Timer/Clock}

The timer is composed of a Hewlett-Packard (HP) function generator, which outputs an $80 \mathrm{~Hz}$ TTL square wave, and a divide-by circuit, which reduces the frequency to $0.05 \mathrm{~Hz}$. The low frequency square wave controls the transmitter waveform, and the high frequency triggers the receiver sampling rate. The setting of the frequency was chosen so as to reduce telluric noise and electromagnetic coupling. The high frequency sampling rate is dependent on the maximum speed of the scanner and voltmeter and the number of data channels desired. 
The transmitter was powered by 220 Volts three-phase power which was produced by a $5 \mathrm{kWatt}$ generator was supplied by DuPont. This AC is rectified and switched to produce a square wave voltage with a period of 20 seconds. This voltage waveform is controlled by a variable transformer at the input to the rectifier and can be varied from 0 to 200 Volts. The output of this switching rectifier is connected directly to the current electrodes which deliver the current to the ground. At the test site, 3-5 Amps was being supplied to the resistive ground. The current is monitored by a precision resistor serially comected in the output current path. The voltage measured across the resistor, calibrated at $10 \mu \mathrm{V} / \mathrm{Amp}$, is optically isolated to prevent coupling between the transmitter and receiver.

The remote current electrode consists of a 4 feet by 8 feet sheet of steel which is buried in a slit trench as shown in Figure (7-2). The slit trench is backfilled with soil and saline water. The remote electrode was located on a ridge approximately 1700 feet southwest from the well and elevated about 100 feet above the hole. The downhole current electrode is a 6 feet length steel pipe 4 inches in diameter. The electrode is lowered down the borehole by a steel cable insulated from the electrode by a length of polypropylene rope. The insulated current cable is attached directly to the electrode and is taped to the steel cable at 50 foot intervals. The current cable, connecting the remote and downhole current electrodes to the transmitter, is \#6 AWG copper with a tough $600 \mathrm{~V}$ neoprene insulation. The insulated cable attached to the remote electrode was placed along the power line access road.

\section{Receiver}

The voltages are measured between adjacent potential electrodes along three radial lines. The voltage measuring cables are multiconductor with 18 outlets spaced 15 meters apart, so that 17 dipoles are measured on each line. The potential electrodes were small 1 inch (diameter) by 6 inch copper-copper sulfate porous pots which were placed in 8-12 inch holes and covered during the experiment with wooden lids. The holes were filled with water to reduce the contact resistance.

The radial cables bring the voltages at each potential electrode back to the data acquisition van which is parked near the transmitter. The acquisition system is composed of antialias filters, an HP 3495A scanner, and an HP 3456A digital voltmeter. The input voltages 
from the 17 dipoles and the current monitor are filter by four-pole Butterworth filters with a cutoff frequency of $3 \mathrm{~Hz}$. The filtered signals are sequentially sampled by the scanner and measured by the voltmeter.

All the voltage differences on each radial line are sent from the voltmeter to an HP 330 computer where they are processed simultaneously. The measured signals are averaged over 10 to 20 periods of the transmitted current. The voltage-to-current (V/I) ratio averages and the standard deviations are calculated for every channel and stored into the computer. For each 10 second half-cycle of the current waveform, one channel would contain 20 sample points. Of the possible 20 samples, only 8 points, which showed no contamination from "filter ringing" and polarity switching, were used for the processing.

\subsection{Field Procedures}

The resistivity survey was conducted from October second to sixth. The first day was spent setting up the receiver and transmitter system. The last day was used for picking up the equipment, loading the system onto the truck, and transferring the data to floppies. The second day, October third, was spent checking the receiver system and background noise levels. The majority of the remaining time was used modifying software and hardware to correct for the problems caused by the resistive environment and repairing equipment damaged by outside sources. The total measurement time was less than 12 hours accumulated during the fourth and fifth of October. Many of the problems encountered during this experiment were not previously seen and were due to the highly resistive environment.

The main problem caused by the highly resistive earth was that the acquisition system was not configured to read large $V / I$ signals. In the past, experiments were done in fairly conductive sediments so that the acquisition system was configured to read $V / A$ signals in the range of tens to hundreds of $\mu \mathrm{V} / \mathrm{Amp}$. At the test site, some of the signals were over two orders of magnitude greater. Signals of this size exceeded the maximum of the voltmeter for the low signal setting and were clipped. Unfortunately, the computer program did not identify this situation but instead set all the values to zero which caused some problems. To handle these large signals, the voltmeter maximum setting was shifted up an order of magnitude and 
the software had to be modified to accommodate the change. After this modification, the voltages from the current monitor were below the resolution of the voltmeter. Since the dynamic range of the voltmeter could not be modified and no other shunt resistors were available to modify the current monitor voltage, an amplifier was used to increase the signal of the current monitor. Nearly the entire day of October fourth, was used to identify and correct these problems.

Other problems which occurred and caused a lost of time were: damage to one voltage measuring cable by the county roadside grass cutter, damage to the steel support cable and to the insulated current cable of the downhole electrode, and damage to the transformer of the transmitter caused by the large voltages applied on the transmitter.

\subsection{Field Results}

\section{Background Noise and Field Data}

Table (7-2) shows the noise level and the standard deviation at the test site calculated for a sampling size of 800 points per channel. The background and cultural noise at the site was extremely low. In most of the channels the noise level was below or near the resolution of the voltmeter. The standard deviation varied from channel to channel but was still quite small, less than $100 \mu \mathrm{V}$.

Originally, ten levels with 200 foot separation were planned. Due to unforeseen problems, the experiment concentrated on two depth levels. The two locations were the zone with brecciated limestone at about 1900-2300 feet and the area near the end of the casing, 450-500 feet. In the brecciated limestone zone, several repeat measurements were taken.

Table (7-3) is the tabulation of the field data for different current electrode depth levels. The range of the V/I signal amplitudes were from 170.0 to $0.5 \mathrm{mV} / \mathrm{A}$. The largest signals, which occurred for current electrodes near the casing, were thought to be due to the channeling of current up the conductive casing. The standard deviations were small for these signals which shows in the percent of the standard deviation-to-signal ratio. This ratio was one percent or less for nearly all cases. The cases where this ratio was larger oceurred when the signal was near zero. 
Repeat Measurements

Repeat measurement test were conducted at several (downhole current) electrode depth levels to check consistency of the measurements. Measurements were acquired for a depth separated in time ranging from one hour to one day. The percent differences for an electrode depth level are calculated from two temporally separated measurements and are listed in Table (7-4). The tests were conducted at shallow (500 ft) and deep $(\geq 1500 \mathrm{ft}$ ) electrodes for line 2 and only at the deep electrode depths for lines 2 and 3.

Most of the channels of lines 1 and 3 were repeatable to less than 2.5 percent. In general, the values for line 2 were 5 percent or less but in some cases exceeded 100 percent. For the shallow depths, the measurements of line 2 were acquired on October fourth and were repeatable to less than 1.6 percent. Most of the values at the shallow electrode depths were repeatable to less than 0.2 percent. The large percent differences appeared to occur for $\mathrm{V} / \mathrm{I}$ signals of less than $3.0 \mathrm{mV} / \mathrm{Amp}$ and $10.0 \mathrm{mV} / \mathrm{Amp}$ for line 2 and lines 1 and 3, respectively.

The poor repeat measurements may be atributed to two main factors. The first is the array configuration and the influence of the remote (current) electrode. The second is the variability of the current waveform. The geometry of the potential arrays and the current electrodes was an improper configuration to obtain optimum V/I signals. In many instances, the remote electrode was closer to the measuring potential electrodes than the downhole electrode. With this configuration, the remote electrode affected the fields which resulted in small (near zero) V/I signals for many of the channels. Even for changes that are in the range of the standard deviations of the noise $(\approx 0.1 \mathrm{mV} / \mathrm{Amp})$, large percent differences can occur if the signals are small. The variability of the current waveform at depth caused problems for repeatable measurements. The inconsistency of the waveform can be seen by the large standard deviations. The standard deviations for the greater depths were over an order of magnitude larger than that for the shallow depths. At depth, the formations were much more resistive than near the surface. The transmitter and the generator were exerted to their limit to inject enough current into the resistive formations to obtain a measurable signal. By running at the load limit, greater variations in the current waveform can occur which results in large standard deviations. 


\section{Noise-Signal Relation}

Figures (7-4) to (7-6) are scatter plots for each array line of the $V / I$ signal versus its standard deviation (noise). The upper plot of each figure is the data for electrode depths close to the casing ( $\leq 700 \mathrm{ft}$ ). The lower plot is the measurements for electrodes away from the pipe ( $\geq 1500 \mathrm{ft})$. The "100 level" line is an approximate maximum noise level, $100 \mu \mathrm{V} / \mathrm{Amp}$, that was determined from the standard deviation of the noise test. For data with little noise, the scatter pattern should be below this line. The "1\%" line represents the one percent noiseto-signal (N/S) ratio. Points lying below this line have N/S ratios of less that one percent. Note from the figures, that measurements can be above the $100 \mu \mathrm{V} / \mathrm{Amp}$ noise level and still have N/S ratios well below one percent. The "integer" line shows the smallest value possible for the survey. It is the result of the conversion of the data, $V / I$ signals and standard deviations, to an integer format. It also represents a N/S ratio of 0.01 percent.

The optimal scatter pattern would be one which is "flat" below the 100 level line. This type of response would represent a constant noise level for all signal sizes. Unfortunately, the data show some kind of linear correlation between the signal and the standard deviation. The measurements that have a large correlation and are above the $100 \mu \mathrm{V} / \mathrm{Amp}$ are: $700-4 \mathrm{~d}$ (all lines), 1500-5u (line 3), and 1700-5u (line 3). At this time, the source(s) for this noise correlation with the signal is unknown.

A pattern was observed with the noise levels and is well illustrated with the deep electrodes of line 3. The open symbols in this plot represent measurements taken as the electrode was lowered down the well. The solid symbols are the measurements acquired as the electrode was pulled upward which are taken in reverse order of the downward measurements. The " + " and " $x$ " are also data taken for the upward going electrode but have no equivalent downgoing measurements. As can be seen from the plot, the open symbols have the lowest noise levels. The solid symbols, 2300-1900 ft, have slightly higher noise levels with the 1900 foot level having the largest. The last two upward measurements, $1700-1500 \mathrm{ft}$, have very large noise levels. In fact, damage to the current and support cables were discovered after the last measurement. The high noise levels of the upward measurements may be due to the current leakage through the damaged current cable which had several gashés along its length. 


\subsection{Data Analysis}

Although most of the data had standard deviation of one percent or less, repeat measurements were inconsistent especially for the deeper electrode levels. The correlation of the noise and signal reduced the amount data could be analyzed. At the time of the analysis of the data, no well completion information was received and only the electric logs for the depths below the casing ( $>450 \mathrm{ft}$ ) were available to estimate formation depths and resistivities. Because of the suspect quality of the field data and the limited information available, the majority of the analysis was limited to models based on the data set of line 2.

\subsubsection{Apparent Resistivities}

The apparent resistivities of each channel for the three lines are plotted in Figures (7-7) to (7-9) and listed in Table (7-5). The apparent resistivities show several characteristics. First, most of the values follow similar trends and fluctuations along the array lines for various source locations. Next, the magnitude of the apparent resistivities greatly increases for the stations nearer the well. Last, negative apparent resistivities were observed on line 1 and line 2.

The trends and fluctuations are primarily due to topography and lateral inhomogeneities in the near surface. The anomalously large apparent resistivities near the well are due to inhomogeneities at or near the well distorting the fields near the well. This distortion of the fields can also be caused by the channeling of current through the conductive casing as discussed earlier in this thesis. Both of these effects were observed by Bevc et al. (1989) in a similar survey at Coalinga, California.

The negative values of apparent resistivities indicate that the electric fields or currents are in the opposite direction to those flowing at the same point in a homogeneous half-space. This is caused by several factors: array configuration, layering, and inhomogeneities. If the remote electrode is not placed far enough away to simulate an infinite electrode, it will affect the current paths. With eertain layered cases, negative apparent resistivities can be obtained with numerical models for the array configuration used for this experiment. The conductive pipe located above the current source also will greatly accentuate this phenomenon of negative 
apparent resistivities.

A layered earth model was used to simulate the apparent resistivities of Table (7-5). With a five-layer model, the negative apparent resistivities that were observed could be produced. But, the spatial fluctuation along the array and the large magnitudes near the well could not be obtained. The fluctuations observed were caused by topography and near-surface inhomogeneities. These effects could be minimized by applying a differencing scheme to the data. To use such a method, additional data in formations with different electrical responses must be collected. Hence, the analysis in this section will not account for the topographic and near-surface effects but will focus on the near-well effects. The large apparent resistivities near the well were assumed to be caused by the steel casing contaminating the signals. The program that can simulate the casing effects was available to model the data but required axial symmetric geometry. This symmetry was numerically created by superposing two data sets to produce a downhole current dipole source and eliminating the off-axis remote current source. For this analysis, one of the current electrodes was stationary, called "reference", and the other, called "roving", was moved in the well.

Table (7-6) gives the dipole-dipole apparent resistivities calculated for a reference electrode located one foot from the bottom of the casing at a depth of 451 feet. The roving electrode is placed at the other electrode depth levels. With the exception of channel 9 of line 1 , the negative values of apparent resistivities seen (Table 7-5) are eliminated. From Table (7-6), the axial symmetry of the apparent resistivities along the arrays is greatly improved. Previously, the values for a given radial distance from the well could vary several orders of magnitude. These variations could be produced by the array geometry, source locations, and a layered earth. After creating an axial symmetric dipole source, the apparent resistivities for a given channel showed some axial symmetry and differed only by small factors.

Since the geometry of this experiment is assumed to have axial symmetry, only one array line of the dipole-dipole measurements is needed for the analysis. The data of line 2 was used for two reasons. First, the topography effects on each channel measurement of line 2 should be nearly equal and small since the the line was placed on a gently sloping terrain. Second, this line had relatively low noise levels for nearly all the measurements. 
Figure (7-10) is a semi-log plot of the dipole-dipole apparent resistivities for line 2. From this plot, it appears that the deep roving electrode levels $(\geq 1500 \mathrm{ft}$ ) were in formations with the same resistivity. The near-casing roving electrode levels $(\leq 500 \mathrm{ft}$ ) were in formations which only differ slightly in resistivities. The 700 foot level values may represent measurements where the roving electrode was in a completely different formation from the nearcasing and deep levels. The near-well effects exist for approximately the first 3 to 5 channels. There may be near-surface effects along the array causing the small fluctuations and the steplike effects. Channels 206 through 213 of the shallow levels have an upward trend in the apparent resistivity. Whereas, the trends for the deep electrode levels of these channels are nearly constant and may be somewhat downward.

\subsubsection{Numerical Modeling}

The objective of the modeling is to come to a conceptual understanding of the field data and to determine feasible scenarios which can describe the data. These simplified models are based on general characteristics of the data and other information available. From the dipoledipole apparent resistivity data of line 2 , there are several characteristics that were used as guidelines for the models:

1) Large values of the apparent resistivities are observed near the well (first 3-5) channels.

2) For the deep roving electrode levels $(\geq 1500$ feet), the apparent resistivities are nearly all the same.

Excluding the near-well and near-surface effects;

3) The apparent resistivities tend to decrease for increasing roving electrode depth levels with the exception of the 483 foot level.

4) The apparent resistivities have trends along the array line. For the shallow roving electrode levels ( $\leq 700$ feet), the values tend to increase for increasing channel numbers. For deep roving electrode levels $(\geq 1500$ feet), the apparent resistivities are constant or may even decrease with larger channel numbers.

The other sources of information were the geologic-stratigraphic section based on core samples (Table 7-1) and the electric logs only for depths below the casing (see Table 7-7). 
Layered-Earth Models

With only the information obtained from the electric logs for formations below the casing (450 feet), the earth below the casing could be represented by a three-layer model. This model has a resistive half-space basement, $30,000 \Omega \cdot \mathrm{m}$, which begins at a depth of 1083 feet. This is overlain by a conductive layer, $200 \Omega \cdot \mathrm{m}$, with its top located at 482 feet. Above this layer is a resistive formation of unknown thickness and its resistivity constrainted to the values of 500 to $5000 \Omega \cdot \mathrm{m}$. With respect to the guidelines, this model produced poor results.

In order to follow the guidelines $2-4$, the addition of at least one layer is required in the upper 450 feet. An estimate of the depth of the interface between the first and second layer as 262 feet. It was obtained from the stratigraphic section which shows at this depth is the top of the Chattanooga Shale. Figure (7-11) is the model and the apparent resistivity for the fourlayer case. The resistivities of the first and second layer were chosen so that the model output matched the far-fields data (last few channels). In doing this, the resistivity of the second layer (5000 $\Omega \cdot \mathrm{m}$ ) which extends below the casing is about 1.5-2.5 times higher than what the electric logs showed. From the plot, the guidelines 2, 3, and the first part of 4 appear to be satisfied.

According to Table 7-1, the Chattanooga Shale is a relativity thick shale formation (3045 feet) sandwiched between (limestone?) units. From the electric logs, the shale units generally appears to be much less resistive than the limestone and dolomite units which dominate the area. Figure (7-12) is the model and calculated apparent resistivity that incorporated this shale unit. With this model, the resistivity of the bottom three layers were kept the same as the previous model. The resistivities of the first two layers, both contained within the first 300 feet, varied so that the far-field data and guidelines $2-4$ were satisfied. This five-layer model has a conductive second layer $(160 \Omega \cdot \mathrm{m})$ sandwiched between two resistive formations $(5000 \Omega \cdot \mathrm{m})$. With the addition of the extra layer, guideline 4 was satisfied much better. Like the four-layer model, the near-casing ( $<500 \mathrm{ft}$ ) apparent resistivity curves overlapped.

\section{Pipe-Layer Models}

With the exception of the near-well data, most of the apparent resistivities could be simulated with layer models. The large values seen for the near-well channels (guideline 1) 
are caused by inhomogeneities at or around the well and cannot be modeled with layers. The anomalously large values of the apparent resistivities were assumed to be caused by the steel casing and/or the borehole fluid channeling current up and distorting the fields near the well. The computer algorithm described in Chapter III can simulate this situation. The program is based on an integral equation approach of the potential fields and can model the casing, fluid, and several layers.

The new models were based on the five-layer model of the previous section. All depths and the resistivities of the bottom two layers were the same as the five-layer model. The resistivities of the upper three layers are allowed to vary so that the calculated values of the (outer) far-field channels matches the field data. No information was available to determine the properties of the casing and the borehole fluid. To illustrate the effects of the pipe and borehole fluid, the following values were used for the model. The borehole fluid column, that went down to the bottom of the well $(\approx 2500$ feet), had a resistivity of $10 \Omega \cdot \mathrm{m}$. The metal casing, which terminated at 450 feet, and was chosen to have a thickness of 0.5 inch. Two values of resistivities were used for the casing to simulate the quality of the contact between the casing and the formations.

The first model simulates a poor pipe-formation contact or possibly an insulated pipe. Figure (7-13) is the model and the calculated apparent resistivity for a casing resistivity of 0.1 $\Omega \cdot \mathrm{m}$. the resistivities of the top three layers slightly changed from the five-layer model. The resistivity of the first and third layers was half the value of the five-layer model $(2500 \Omega \cdot \mathrm{m})$. the conductive layer was reduced by a third from 160 to $50 \Omega \cdot \mathrm{m}$. The values used for the resistivity of the third layer are very close to the estimated values obtained from the electric logs.

The apparent resistivities of the pipe model follow the guidelines 1-4 of the field data. Excluding the near-surface effects, three main differences are seen. First, the calculated values are larger than the field data for the middle channels. Second, the effects of the casing is seen through the entire array for the curves representing roving electrode levels 700 feet and below. Third, the near-well channels have apparent resistivities much smaller than those of the field data. The first two discrepancies are probably due to the resistivity of the top 
layer being too large, thus extending the pipe effects throughout the array. The last difference indicates that the conductivity of the casing (area) is probably too small. This results in less distortion of the near-well fields and leads to smaller apparent resistivities.

The second model simulates the situation where the pipe-formation contact is good (the pipe acts as a good conductor). For this case, the resistivity of the pipe was $10^{-4} \Omega \cdot \mathrm{m}$ and produced the plot in Figure (7-14). The resistivity of the top layer is much more conductive (200 $\Omega \cdot \mathrm{m}$ ) than was previously used (2500 $\Omega \cdot \mathrm{m})$. For the second layer, the resistivity (50 $\Omega \cdot \mathrm{m}$ ) is half the value of the first pipe-layer model. Since the resistivity of the third layer was close to the well-log values, it remained the same $2500 \Omega \cdot \mathrm{m}$.

The calculated apparent resistivity curves follow the guidelines $1-4$ and in general match the field data well. Neglecting the near-surface effects, the apparent resistivities of Figure (714) and Figure (7-10) are very similar. The curves for the near-casing roving electrode levels ( $\leq 500$ feet) follow the general trends of the field data extremely well. In the deeper levels $\geq$ 700 feet) there are differences seen between the model results and the field data. The conductive pipe produced a larger apparent resistivity for the near-well channels which are slightly larger than the field data. These effects extended into the middle channels but is much less pronouned than the previous pipe-layer model. These discrepancies indicate that the conductance of the pipe and/or borehole fluid used for the model were too high. The far-field channels have values that are smaller than the field data but are still very close. This indicates that the top layers used for the model are too conductive.

In October 1990, the electric logs for the depth between 50 to 400 feet and a progress report, which contained conductivity results from borehole fluid samples (2100-3500 ft), were obtained. The resistivity from the upper $\log (50-400 \mathrm{ft}), \operatorname{lower} \log (450-2100 \mathrm{ft})$, and the fiuid test were estimated and listed in Table (7-7). The log shows that the relatively conductive $(\approx 200 \Omega \cdot \mathrm{m})$ Chattanooga Shale is located at a depth of 260 feet to 320 feet and is sandwiched in between resistive $(\approx 2000 \Omega \cdot \mathrm{m})$ formations. The upper log verifies the assumption used for the modeling that a conductive layer exist between resistive formations. Unfortunately, the electric $\log$ for the upper 50 feet was not received so that the electrical properties of the surface layers are unknown. 
The upper electric logs tend to agree with the resistive pipe modeling results for the formation resistivities. The conductive pipe model produced resistivity values that are too small. By reducing the pipe and/or fluid conductance (either by modifying the geometrical or electrical parameters), higher formation resistivities of the upper layers are needed in order to match the field data. This results in the desired modification of the model so that a better fit to the data can be obtained.

\subsection{Summary and Conclusion}

The background noise at the site was very low. For most of the channels, the noise was below or at the resolution of the voltmeter. The test showed noise levels of much less than $100 \mu \mathrm{V}$. In the resistive environment, approximately 3 to $5 \mathrm{Amps}$ could be injected into the ground with the power source and transmitter. The signal amplitudes ranged from 0.5 to 170 $\mu \mathrm{V} / \mathrm{Amp}$ with the largest signals occurring near the well. Most of the data had standard deviations of less than the one percent of the signal amplitudes and were below the noise level of $100 \mu \mathrm{V} / \mathrm{Amp}$.

The quality of repeat measurements varied depending on the depth and array line. For the shallow depths, the maximum deviation of the repeat measurement was 1.57 percent with most of the values being less than 0.2 percent. For the greater depths, the same array line could only repeat to values of 5 percent or less, but exceeded 100 percent for several channels. Poor repeat measurements were due to two factors: an inconsistent waveform caused by the highly resistive formations at depth and exerting the power source/transmitter to its load limit, and the close spatial proximity of the remote current electrode affecting the fields such that signals were not optimal. Several of the data sets showed high linear correlation between the signal and the noise. The source of this correlation is not known at this time but is thought to be caused by current leakage through the downhole cable.

The raw field data was influenced by several factors which caused trends, fluctuations, and jumps in the apparent resistivities as well as large near-well amplitudes and negative values. The trends, fluctuations, and jumps were most likely caused by topography and near-

surface lateral inhomogeneities. These effects can be minimized by applying a percent 
differencing technique (Asch and Morrison, 1989), but were not analyzed in this report. The negative apparent resistivities are mainly the result of the proximity of the remote current electrode. This effect can be simulated with a simple layered earth model and two current sources. This negative values can be eliminated by creating an axial symmetric source such as a downhole dipole. The anomalously large apparent resistivities may be due to near-well effects such as the casing as simulated by models.

The modeling analysis indicates that the field data can be simulated with a simplified model consisting of a conductive casing, borehole fluid, and four or five layers. The values of the outer channels can be produced by a layered earth model. The large anomalous apparent resistivities near the well are caused by casing effect. These responses may also be due to any near-well inhomogeneity that can produce equivalent effects. The pipe-layer models show an interaction between the casing and the near-surface layers strongly exists. The highly conductive pipe requires conductive near-surface layers to diffuse the anomalous currents quickly and thus restricting their effects near the well. A similar effect can be obtained with more resistive near-surface layers and pipe having a smaller conductance. Although it was not shown, additional model simulations suggested that borehole fluid in the uncased portion of the well is needed to act as a conduit for the currents of the downhole electrodes.

The numerical injection simulations in Chapter $\mathrm{V}$ indicate that in a resistive environment the monitoring of a conductive plume may be possible. Additionally, the metal casing or pipe may accentuate the ability to detect the changes that occur during the injection. Improved modeling algorithms where non-symmetric plumes are incorporated are needed to determine if directions of the plume can be determined from surface measurements.

The numerical results must be cautiously interpreted since they are based on idealized geometries and estimates of the depths and resistivities of the formations. Since the resistivity method is a nonunique problem, there exist numerous models that can produce results that corresponds to the data. But, all these models will have similar conductance characteristics. The results obtained in this analysis do give a conceptual understanding on the behavior of the casing and formation interactions. 
Several recommendations are suggested to help improve the acquisition and quality of the data for future experiments.

- Move the remote current electrode away from the well at least five times the maximum depth of the downhole electrode.

- Use a larger power source and/or transmitter to inject more current $(\approx 10 \mathrm{Amps})$ into the ground.

- Use a protected downhole current cable to guard against cuts, niches, and "balling-up" during movement of the electrode.

- Isolate and place all filters at the site of the potential electrodes and then line drive the signals to the acquisition van.

- Use longer periods and stack more waveforms to increase signal-to-noise ratio.

- Modify software to include additional in-field checks of the signal quality.

- Determine the electrical properties of the near-surface layers, casing, and borehole fluid to aid and improve interpretations.

- Use the steel casing as a current source.

- Place measurement dipoles orthogonal to the radial lines to reduce the effect of the casing. This experiment has given some insight to the problems that can be encountered in a resistive environment. To overcome these additional problems, new approaches and improvements of field procedures and acquisition system were identified. From this experiment, it appears that measurements can be acquired and interpreted in an environment with high resistivity. 


\begin{tabular}{|c|c|c|}
\hline Age & Formation & Depth to Top (ft) \\
\hline Mississippian & $\begin{array}{l}\text { Fort Payne Fm } \\
\text { New Providence Sh } \\
\text { Maury Sh }\end{array}$ & $\begin{array}{r}53 \\
? ? ? \\
? ? ?\end{array}$ \\
\hline Devonian & $\begin{array}{l}\text { Chattanooga Sh * } \\
\text { Camden-Harriman Fms } \\
\text { Ross Fm } \\
\text { Birdsong Sh } \\
\text { Rockhouse Ls }\end{array}$ & $\begin{array}{l}264 \\
308 \\
454 \\
483\end{array}$ \\
\hline Silurian & $\begin{array}{l}\text { Decatur Ls } \\
\text { Brownsport Group } \\
\text { Lobelville Ls } \\
\text { Bob Ls } \\
\text { Beech River Ls } \\
\text { Wayne Group } \\
\text { Dixon Ls } \\
\text { Lego Ls } \\
\text { Waldron Sh } \\
\text { Laurel Ls } \\
\text { Osgood Ls } \\
\text { Brassfield Ls }\end{array}$ & $\begin{array}{l}510 \\
557 \\
592 \\
610 \\
662 \\
703 \\
731 \\
734 \\
746 \\
787\end{array}$ \\
\hline Ordovician & 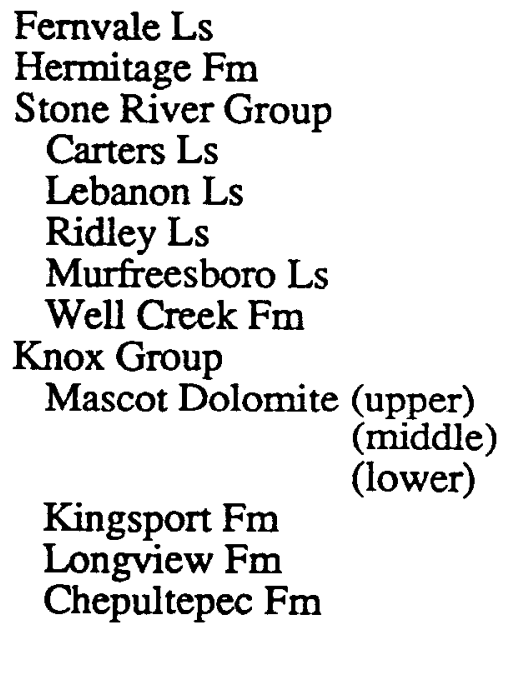 & $\begin{array}{r}845 \\
879 \\
\\
1096 \\
1186 \\
1305 \\
1456 \\
1734 \\
2018 \\
2498-\text { Oct } 1989 \\
2824 \\
3117 \\
3419 \\
3534 \quad \text { - Aug } 1990 \\
4200-\end{array}$ \\
\hline
\end{tabular}

Table 7-1: Geologic/stratigraphic section obtained from core samples in the test well.

* Tennessee division of Geology has adopted the age classification Mississippian and Devonian for the Chattanooga Shale (Wilson and Stearn, 1968). 


\begin{tabular}{|c||rr|rr|rr|}
\hline \multicolumn{7}{|c|}{ Background Noise Test } \\
\hline \hline \multicolumn{1}{|c||}{} & \multicolumn{2}{|c|}{ Line 1 } & \multicolumn{2}{c|}{ Line 2 } & \multicolumn{2}{c|}{ Line 3 } \\
\hline Dipole & Noise & STD & Noise & STD & Noise & STD \\
Channel & $(\mu \mathrm{V})$ & $(\mu \mathrm{V})$ & $(\mu \mathrm{V})$ & $(\mu \mathrm{V})$ & $(\mu \mathrm{V})$ & $(\mu \mathrm{V})$ \\
\hline 1 & 8.5 & 83.8 & -11.7 & 64.3 & 8.4 & 74.7 \\
2 & 3.5 & 8.5 & -1.5 & 13.7 & 0.8 & 8.0 \\
3 & 3.9 & 9.8 & 0.5 & 11.9 & -2.7 & 17.8 \\
4 & 0.0 & 0.0 & -1.9 & 9.3 & -2.7 & 16.9 \\
5 & 8.0 & 21.4 & -1.1 & 34.7 & -2.8 & 22.4 \\
6 & 5.1 & 12.0 & -1.1 & 8.1 & -0.7 & 10.6 \\
7 & 9.2 & 14.8 & 0.2 & 6.5 & 2.1 & 9.5 \\
8 & -51.6 & 45.8 & -2.1 & 10.2 & 1.4 & 9.9 \\
9 & 63.6 & 50.8 & 1.9 & 30.0 & -0.1 & 27.6 \\
10 & 3.7 & 15.7 & -3.9 & 14.6 & 1.0 & 13.6 \\
11 & 6.5 & 35.7 & -3.9 & 33.1 & 2.7 & 29.3 \\
12 & 6.9 & 29.1 & -3.0 & 32.5 & 4.4 & 25.8 \\
13 & 2.8 & 16.2 & -1.8 & 7.6 & -2.9 & 6.8 \\
14 & 0.9 & 6.3 & -2.1 & 5.8 & -0.8 & 7.3 \\
15 & 6.2 & 40.8 & -7.3 & 39.4 & 1.7 & 32.7 \\
16 & -1.8 & 13.1 & -4.3 & 7.5 & -3.3 & 8.5 \\
17 & 3.0 & 10.7 & -2.7 & 8.0 & 0.9 & 6.1 \\
\hline
\end{tabular}

Table 7-2: Background noise levels and standard deviations at the test site for a sample size of 800 points per channel. 


\begin{tabular}{|c||c|c|c|c|c|c|c|c|c|}
\hline \multicolumn{10}{|c|}{ V/ signals (mV/Amp) } \\
\hline $\begin{array}{c}\text { File } \\
\text { (feet-day) }\end{array}$ & 1 & 2 & 3 & 4 & 5 & 6 & 7 & 8 & 9 \\
\hline $0451-5 w$ & -124.87 & -71.36 & -39.74 & -26.58 & -63.93 & -47.39 & -65.00 & -77.87 & -9.21 \\
$0453-5 w$ & -119.78 & -68.20 & -38.01 & -25.57 & -61.69 & -45.60 & -62.54 & -76.41 & -7.09 \\
$0460-5 w$ & - & - & - & - & - & - & - & - & - \\
$0483-5 w$ & - & - & - & - & -- & - & - & - & - \\
$0500-4 d$ & -- & - & - & - & -- & - & - & - & - \\
$0500-4 d$ & - & - & - & - & - & - & -- & - & - \\
$0700-4 d$ & -43.64 & -25.45 & -13.45 & -8.80 & -28.79 & -18.88 & -24.84 & -22.58 & -9.69 \\
$1500-4 d$ & 0.0 & 0.0 & 0.0 & 0.0 & 0.0 & 0.0 & 0.0 & 0.0 & 0.0 \\
$1500-5 u$ & -44.75 & -32.62 & -20.97 & -10.19 & -29.80 & -20.31 & -27.30 & -25.05 & -10.80 \\
$1700-5 u$ & -40.11 & -28.13 & -17.14 & -8.93 & -28.94 & -19.22 & -25.35 & -23.28 & -9.65 \\
$1900-4 d$ & -36.46 & -21.53 & -10.84 & -6.65 & -27.85 & -17.26 & -22.21 & -20.67 & -8.14 \\
$1900-5 d$ & -38.73 & -22.12 & -10.47 & -6.47 & -28.40 & -17.67 & -22.41 & -21.36 & -8.30 \\
$1900-5 u$ & -38.15 & -22.13 & -11.24 & -6.80 & -28.08 & -17.80 & -22.92 & -21.10 & -8.32 \\
$2100-5 d$ & -38.77 & -23.05 & -11.83 & -7.34 & -28.94 & -18.19 & -23.49 & -21.84 & -8.66 \\
$2100-5 u$ & -39.92 & -23.39 & -12.06 & -7.37 & -28.99 & -18.60 & -24.09 & -22.05 & -8.81 \\
$2300-5 d$ & -37.64 & -22.23 & -11.33 & -6.80 & -28.25 & -17.88 & -23.04 & -21.35 & -8.38 \\
$2300-5 u$ & -38.62 & -22.56 & -11.47 & -6.92 & -28.50 & -18.09 & -23.34 & -21.57 & -8.53 \\
\hline
\end{tabular}

\begin{tabular}{|c||c|c|c|c|c|c|c|c|c|c|}
\hline \multicolumn{10}{|c|}{ V/I signals (mV/Amp) } \\
\hline $\begin{array}{c}\text { File } \\
\text { (feet-day) }\end{array}$ & 10 & 11 & 12 & 13 & 14 & 15 & 16 & 17 & I1 & I \\
\hline $0451-5 \mathrm{w}$ & -25.79 & -24.29 & -45.35 & -53.76 & -59.12 & -59.65 & -52.57 & -46.14 & 8.65 & 8.68 \\
$0453-5 \mathrm{w}$ & -24.62 & -23.30 & -44.07 & -52.60 & -58.14 & -58.47 & -51.73 & -45.40 & 8.11 & 8.13 \\
$0460-5 \mathrm{w}$ & -- & - & -- & - & -- & - & - & - & -- & -- \\
$0483-5 \mathrm{w}$ & -- & - & -- & - & -- & - & - & - & -- & -- \\
$0500-4 \mathrm{~d}$ & -- & - & -- & - & -- & - & -- & - & -- & - \\
$0500-4 \mathrm{~d}$ & -- & - & -- & - & -- & - & -- & - & -- & -- \\
$0700-4 \mathrm{~d}$ & -8.26 & -8.98 & -23.27 & -33.37 & -40.09 & -38.20 & -35.87 & -31.54 & 7.91 & 7.94 \\
$1500-4 \mathrm{~d}$ & 0.0 & 0.0 & 0.0 & 0.0 & 0.0 & 0.0 & 0.0 & 0.0 & 0.0 & 0.0 \\
$1500-5 \mathrm{u}$ & -9.18 & -9.96 & -25.87 & -37.40 & -45.08 & -42.79 & -40.10 & -35.33 & 7.76 & 7.74 \\
$1700-5 \mathrm{u}$ & -8.20 & -9.12 & -24.77 & -36.41 & -44.28 & -41.82 & -39.30 & -34.62 & 7.52 & 7.51 \\
$1900-4 \mathrm{~d}$ & -6.81 & -7.83 & -22.99 & -34.92 & -42.87 & -40.13 & -38.12 & -33.33 & 8.29 & 8.32 \\
$1900-5 \mathrm{~d}$ & -7.05 & -8.01 & -23.76 & -35.30 & -44.05 & -40.69 & -38.92 & -33.66 & 8.18 & 8.20 \\
$1900-5 \mathrm{u}$ & -6.98 & -8.04 & -23.35 & -35.40 & -43.45 & -40.68 & -38.51 & -33.82 & 8.14 & 8.15 \\
$2100-5 \mathrm{~d}$ & -7.32 & -8.29 & -24.03 & -35.40 & -44.01 & -40.82 & -38.99 & -33.73 & 6.85 & 6.86 \\
$2100-5 \mathrm{u}$ & -7.43 & -8.46 & -23.97 & -35.90 & -43.93 & -41.22 & -38.96 & -34.19 & 6.64 & 6.65 \\
$2300-5 \mathrm{~d}$ & -7.06 & -8.12 & -23.52 & -35.56 & -43.63 & -40.87 & -38.65 & -33.81 & 7.13 & 7.14 \\
$2300-5 \mathrm{u}$ & -7.20 & -8.23 & -23.66 & -35.72 & -43.78 & -41.03 & -38.80 & -33.98 & 8.11 & 8.12 \\
\hline
\end{tabular}

Table 7-3a: Line 1 field data for different current electrode depth levels. The top table has channels 1-9 and bottom table has channels 10-17 and currents 11 and 12 . The signals are in the form of voltage per unit current and the currents are peak-to-peak values. 


\begin{tabular}{|c|c|c|c|c|c|c|c|c|c|}
\hline \multicolumn{10}{|c|}{ V/I signals (mV/Amp) } \\
\hline \multirow{2}{*}{$\begin{array}{c}\text { File } \\
\text { (feet-day) }\end{array}$} & \multicolumn{9}{|c|}{ Channel Number } \\
\hline & 1 & 2 & 3 & 4 & 5 & 6 & 7 & 8 & 9 \\
\hline $0451-5 w$ & -148.76 & -80.30 & -45.68 & -42.95 & -39.23 & -22.03 & -18.18 & -14.50 & -14.06 \\
\hline $0453-5 w$ & -141.79 & -76.38 & -43.29 & -40.73 & -36.97 & -20.68 & -16.94 & -13.39 & -12.93 \\
\hline $0460-5 w$ & -124.98 & -67.09 & -37.25 & -35.18 & -31.39 & -17.32 & -13.91 & -10.71 & -10.19 \\
\hline $0483-5 w$ & -38.68 & -19.30 & -7.10 & -6.88 & -3.48 & -0.17 & 1.40 & 2.97 & 3.75 \\
\hline $0500-4 d$ & -22.02 & -10.43 & -2.06 & -2.18 & 0.76 & 225 & 3.41 & 4.59 & 5.36 \\
\hline $0500-4 d$ & -21.99 & -10.36 & -2.13 & -2.21 & 0.74 & 2.24 & 3.41 & 4.59 & 5.34 \\
\hline $0700-4 d$ & -48.16 & -24.63 & -11.66 & -10.89 & -7.75 & -2.82 & -1.01 & 0.80 & 1.70 \\
\hline $1500-4 d$ & -41.37 & -20.86 & -9.55 & -8.89 & -5.67 & -1.51 & 0.21 & 1.95 & 2.95 \\
\hline $1500-5 u$ & -55.79 & -31.44 & -8.28 & -10.29 & -7.12 & -2.39 & -0.52 & 1.39 & 2.44 \\
\hline $1700-5 u$ & -48.61 & -26.50 & -8.05 & -9.11 & -5.73 & -1.45 & 0.38 & 2.26 & 3.37 \\
\hline $1900-4 d$ & -38.15 & -18.80 & -7.87 & -7.37 & -3.73 & -0.08 & 1.70 & 3.53 & 4.71 \\
\hline $1900-5 d$ & -39.23 & -19.36 & -7.93 & -7.50 & -3.72 & -0.08 & 1.72 & 3.62 & 4.74 \\
\hline $1900-5 u$ & -39.90 & -19.59 & -8.27 & -7.75 & -4.03 & -0.28 & 1.53 & 3.38 & 4.57 \\
\hline $2100-5 d$ & -40.89 & -20.23 & -8.59 & -8.09 & -4.30 & -0.45 & 1.39 & 3.30 & 4.44 \\
\hline $2100-5 u$ & -42.26 & -20.90 & -9.12 & -8.55 & -4.82 & -0.76 & 1.10 & 3.00 & 4.18 \\
\hline $2300-5 d$ & -39.69 & -19.51 & -8.23 & -7.73 & -3.96 & -0.22 & 1.59 & 3.45 & 4.66 \\
\hline $2300-5 u$ & -40.47 & -19.91 & -8.51 & -7.99 & -4.24 & -0.39 & 1.44 & 3.31 & 4.50 \\
\hline
\end{tabular}

\begin{tabular}{|c|c|c|c|c|c|c|c|c|c|c|}
\hline \multicolumn{9}{|c|}{$\mathrm{V} / \mathrm{I}$ signals (mV/Amp) } & \multicolumn{2}{|c|}{ I-pp (Amp) } \\
\hline \multirow{2}{*}{$\begin{array}{c}\text { File } \\
\text { (feet-day) }\end{array}$} & \multicolumn{10}{|c|}{ Channel Number } \\
\hline & 10 & 11 & 12 & 13 & 14 & 15 & 16 & 17 & I1 & 12 \\
\hline $0451-5 w$ & -11.93 & -9.70 & -7.45 & -5.59 & -4.31 & -3.11 & -2.27 & -0.74 & 8.66 & 8.68 \\
\hline $0453-5 w$ & -10.94 & -8.82 & -6.70 & -4.95 & -3.64 & -2.43 & -1.67 & -0.25 & 8.11 & 8.13 \\
\hline $0460-5 w$ & -8.50 & -6.69 & -4.88 & -3.39 & -2.05 & -0.86 & -0.33 & 0.77 & 8.12 & 8.15 \\
\hline $0483-5 w$ & 4.02 & 4.20 & 4.53 & 4.64 & 6.23 & 7.38 & 6.82 & 6.30 & 8.11 & 8.13 \\
\hline $0500-4 d$ & 5.39 & 5.36 & 5.41 & 5.36 & 6.81 & 7.89 & 7.22 & 6.59 & 8.91 & 8.93 \\
\hline $0500-4 d$ & 5.39 & 5.35 & 5.41 & 5.35 & 6.81 & 7.89 & 7.22 & 6.59 & 8.91 & 8.94 \\
\hline $0700-4 d$ & 2.36 & 3.03 & 3.78 & 4.27 & 6.21 & 7.89 & 7.65 & 7.83 & 7.93 & 7.96 \\
\hline $1500-4 d$ & 3.56 & 4.19 & 4.90 & 5.33 & 7.48 & 9.40 & 9.13 & 9.38 & 2.10 & 2.10 \\
\hline $1500-5 \mathrm{u}$ & 3.19 & 3.93 & 4.78 & 5.31 & 7.60 & 9.65 & 9.41 & 9.73 & 7.56 & 754 \\
\hline $1700-5 u$ & 4.06 & 4.73 & 5.52 & 5.99 & 8.38 & 10.52 & 10.22 & 10.49 & 7.39 & 7.36 \\
\hline $1900-4 d$ & 5.31 & 5.89 & 6.62 & 6.99 & 9.57 & 11.79 & 11.49 & 11.67 & 8.29 & 8.32 \\
\hline $1900-5 d$ & 5.44 & 5.94 & 6.83 & 7.05 & 9.77 & 11.91 & 11.78 & 11.82 & 8.15 & 8.17 \\
\hline $1900-5 u$ & 5.17 & 5.78 & 6.51 & 6.90 & 9.43 & 11.69 & 11.31 & 11.55 & 8.27 & 8.28 \\
\hline $2100-5 d$ & 5.14 & 5.70 & 6.57 & 6.82 & 9.52 & 11.62 & 11.54 & 11.59 & 6.62 & 6.63 \\
\hline $2100-5 u$ & 4.82 & 5.47 & 6.24 & 6.67 & 9.19 & 11.44 & 11.09 & 11.36 & 6.42 & 6.43 \\
\hline $2300-5 d$ & 5.27 & 5.88 & 6.66 & 6.96 & 9.60 & 11.78 & 11.57 & 11.75 & 7.31 & 7.33 \\
\hline $2300-5 u$ & 5.12 & 5.74 & 6.50 & 6.89 & 9.45 & 11.72 & 11.36 & 11.60 & 8.00 & 8.01 \\
\hline
\end{tabular}

Table 7-3b: Line 2 field data for different current electrode depth levels. The top table has channels 1-9 and bottom table has channels 10-17 and currents I1 and 12 . The signals are in the form of voltage per unit current and the currents are peak-to-peak values. 


\begin{tabular}{|c||c|c|c|c|c|c|c|c|c|c|}
\hline \multicolumn{10}{|c|}{ V/I signals (mV/Amp) } \\
\hline File & \multicolumn{10}{c|}{ Channel Number } \\
(feet-day) & 1 & 2 & 3 & 4 & 5 & 6 & 7 & 8 & 9 \\
\hline $0451-5 \mathrm{w}$ & -160.59 & -30.93 & -121.66 & -124.54 & -99.23 & -127.41 & -117.90 & -99.81 & -107.58 \\
$0453-5 \mathrm{w}$ & -154.40 & -29.07 & -119.12 & -121.66 & -96.65 & -124.54 & -115.36 & -97.52 & -105.11 \\
$0460-5 \mathrm{w}$ & 0.0 & 0.0 & 0.0 & 0.0 & 0.0 & 0.0 & 0.0 & 0.0 & 0.0 \\
$0483-5 \mathrm{w}$ & 0.0 & 0.0 & 0.0 & 0.0 & 0.0 & 0.0 & 0.0 & 0.0 & 0.0 \\
$0500-4 \mathrm{~d}$ & -42.96 & 0.97 & -58.34 & -56.10 & -46.09 & -67.37 & -64.89 & -54.05 & -58.53 \\
$0500-4 \mathrm{~d}$ & 0.0 & 0.0 & 0.0 & 0.0 & 0.0 & 0.0 & 0.0 & 0.0 & 0.0 \\
$0700-4 \mathrm{~d}$ & -64.64 & -4.68 & -71.02 & -69.14 & -55.91 & -77.31 & -73.45 & -61.68 & -66.63 \\
$1500-4 \mathrm{~d}$ & -58.74 & -2.28 & -70.28 & -67.81 & -54.06 & -75.16 & -71.68 & -59.95 & -64.73 \\
$1500-5 \mathrm{u}$ & -71.28 & -6.81 & -78.51 & -76.62 & -61.76 & -89.28 & -84.32 & -70.01 & -75.51 \\
$1700-5 \mathrm{u}$ & -66.07 & -5.25 & -75.82 & -74.11 & -59.69 & -85.98 & -81.46 & -67.70 & -72.98 \\
$1900-4 \mathrm{~d}$ & -57.36 & -0.11 & -74.64 & -71.02 & -57.30 & -80.61 & -77.07 & -64.47 & -69.57 \\
$1900-5 \mathrm{~d}$ & -60.64 & -0.12 & -75.51 & -72.42 & -58.37 & -81.80 & -78.43 & -65.38 & -70.16 \\
$1900-5 \mathrm{u}$ & -61.69 & -3.01 & -74.89 & -72.96 & -58.70 & -82.28 & -78.65 & -65.63 & -70.77 \\
$2100-5 \mathrm{~d}$ & -62.63 & -1.60 & -75.63 & -73.41 & -59.02 & -82.30 & -78.94 & -65.77 & -70.60 \\
$2100-5 \mathrm{u}$ & -63.13 & -3.37 & -75.54 & -73.93 & -59.27 & -82.73 & -79.06 & -65.91 & -71.04 \\
$2300-5 \mathrm{~d}$ & -61.44 & -1.99 & -74.92 & -72.90 & -58.66 & -82.00 & -78.57 & -65.36 & -70.31 \\
$2300-5 \mathrm{u}$ & -62.05 & -2.53 & -74.69 & -73.01 & -58.83 & -82.18 & -78.73 & -65.55 & -70.60 \\
\hline
\end{tabular}

\begin{tabular}{|c||c|c|c|c|c|c|c|c|c|c|}
\hline \multicolumn{10}{|c|}{ V/I signals (mV/Amp) } \\
\hline $\begin{array}{c}\text { File } \\
\text { (feet-day) }\end{array}$ & 10 & 11 & 12 & 13 & 14 & 15 & 16 & 17 & I1 & I2 \\
\hline $0451-5 w$ & -104.17 & -92.36 & -84.35 & -69.37 & -68.16 & -71.17 & -56.98 & -70.65 & 8.67 & 8.69 \\
$0453-5 w$ & -101.75 & -90.52 & -82.62 & -68.15 & -67.10 & -70.10 & -56.23 & -69.82 & 8.12 & 8.15 \\
$0460-5 w$ & - & - & - & - & - & - & - & - & - & -- \\
$0483-5 w$ & -- & - & - & - & - & - & - & - & - & -- \\
$0500-4 d$ & -56.95 & -51.41 & -48.39 & -43.00 & -44.03 & -46.82 & -38.96 & -49.89 & 8.94 & 8.97 \\
$0500-4 d$ & - & - & -- & - & - & - & - & - & - & -- \\
$0700-4 d$ & -64.66 & -57.85 & -53.82 & -46.64 & -47.10 & -49.77 & -40.98 & -51.91 & 7.40 & 7.43 \\
$1500-4 d$ & -62.69 & -56.70 & -52.45 & -45.76 & -46.34 & -48.95 & -40.47 & -51.32 & 2.10 & 2.10 \\
$1500-5 u$ & -73.22 & -65.28 & -60.89 & -52.90 & -53.57 & -56.26 & -46.33 & -58.82 & 7.70 & 7.68 \\
$1700-5 \mathrm{u}$ & -71.03 & -63.26 & -59.08 & -51.64 & -52.45 & -55.15 & -45.48 & -57.91 & 7.39 & 7.38 \\
$1900-4 d$ & -67.28 & -61.14 & -56.53 & -49.50 & -50.35 & -53.18 & -44.09 & -56.09 & 8.31 & 8.34 \\
$1900-5 \mathrm{~d}$ & -68.00 & -61.67 & -57.21 & -49.80 & -51.11 & -53.71 & -44.48 & -56.74 & 8.15 & 8.17 \\
$1900-5 \mathrm{u}$ & -68.70 & -61.37 & -57.50 & -50.23 & -51.34 & -53.95 & -44.66 & -56.93 & 8.48 & 8.50 \\
$2100-5 \mathrm{~d}$ & -68.53 & -61.92 & -57.60 & -50.01 & -51.34 & -53.91 & -44.63 & -56.94 & 6.82 & 6.82 \\
$2100-5 \mathrm{u}$ & -68.97 & -61.73 & -57.88 & -50.28 & -51.50 & -54.13 & -44.76 & -57.05 & 6.59 & 6.60 \\
$2300-5 \mathrm{~d}$ & -68.17 & -61.49 & -57.40 & -49.81 & -51.25 & -53.86 & -44.48 & -56.80 & 7.16 & 7.17 \\
2300-5u & -68.48 & -61.56 & -57.61 & -49.95 & -51.36 & -53.97 & -44.55 & -56.90 & 7.97 & 7.97 \\
\hline
\end{tabular}

Table 7-3c: Line 3 field data for different current electrode depth levels. The top table has channels 1-9 and bottom table has channels 10-17 and currents I1 and 12 . The signals are in the form of voltage per unit current and the currents are peak-to-peak values. 


\begin{tabular}{|c||c|c|c|c|c|c|c|}
\hline \multicolumn{1}{|c||}{} & \multicolumn{6}{c|}{ Percent Difference: $2\left(V_{1}-V_{2}\right) /\left(V_{1}+V_{2}\right)^{*} 100 \%$} \\
\hline \hline File 1 & $500-4 \mathrm{~d} 1$ & $1500-4 \mathrm{~d}$ & $1900-4 \mathrm{~d}$ & $1900-4 \mathrm{~d}$ & $1900-5 \mathrm{~d}$ & $2100-5 \mathrm{~d}$ & $2300-5 \mathrm{~d}$ \\
File 2 & $500-4 \mathrm{~d} 2$ & $1500-5 \mathrm{u}$ & $1900-5 \mathrm{~d}$ & $1900-5 \mathrm{u}$ & $1900-5 \mathrm{u}$ & $2100-5 \mathrm{u}$ & $2300-5 \mathrm{u}$ \\
\hline Channel & & & & & & & \\
1 & -- & - & -6.03 & -4.51 & 1.52 & -2.92 & -2.58 \\
2 & -- & - & -2.69 & -2.74 & -0.05 & -1.48 & -1.48 \\
3 & -- & - & 3.45 & -3.64 & -7.08 & -1.85 & -1.26 \\
4 & -- & -- & 2.83 & -2.20 & -5.04 & -0.42 & -1.71 \\
5 & -- & - & -1.97 & -0.83 & 1.14 & -0.15 & -0.90 \\
6 & -- & - & -2.34 & -3.03 & -0.69 & -2.22 & -1.17 \\
7 & -- & - & -0.89 & -3.14 & -2.24 & -2.51 & -1.29 \\
8 & -- & - & -3.26 & -2.07 & 1.20 & -0.96 & -1.03 \\
9 & -- & - & -1.99 & -2.18 & -0.19 & -1.66 & -1.70 \\
10 & - & - & -3.38 & -2.47 & 0.91 & -1.49 & -1.92 \\
11 & - & - & -2.25 & -2.70 & -0.45 & -2.07 & -1.34 \\
12 & -- & - & -3.26 & -1.53 & 1.74 & 0.28 & -0.56 \\
13 & -- & - & -1.10 & -1.38 & -0.28 & -1.40 & -0.47 \\
14 & -- & -- & -2.72 & -1.36 & 1.37 & 0.18 & -0.33 \\
15 & -- & - & -1.40 & -1.37 & 0.03 & -0.97 & -0.41 \\
16 & -- & - & -2.07 & -1.01 & 1.06 & 0.06 & -0.37 \\
17 & -- & - & -0.96 & -1.45 & -0.49 & -1.34 & -0.49 \\
\hline
\end{tabular}

Table 7-4a: Percent difference of the apparent resistivities for line 1 at various current electrode depth levels. 


\begin{tabular}{|c||c|r|r|r|r|r|r|}
\hline \multicolumn{1}{|c||}{} & \multicolumn{7}{c|}{ Percent Difference: $2\left(V_{1}-V_{2}\right) /\left(V_{1}+V_{2}\right)^{*} 100 \%$} \\
\hline \hline File 1 & $500-4 \mathrm{~d} 1$ & $1500-4 \mathrm{~d}$ & $1900-4 \mathrm{~d}$ & $1900-4 \mathrm{~d}$ & $1900-5 \mathrm{~d}$ & $2100-5 \mathrm{~d}$ & $2300-5 \mathrm{~d}$ \\
File 2 & $500-4 \mathrm{~d} 2$ & $1500-5 \mathrm{u}$ & $1900-5 \mathrm{~d}$ & $1900-5 \mathrm{u}$ & $1900-5 \mathrm{u}$ & $2100-5 \mathrm{u}$ & $2300-5 \mathrm{u}$ \\
\hline Channel & & & & & & & \\
1 & 0.09 & -29.69 & -2.80 & -4.51 & -1.71 & -3.30 & -1.93 \\
2 & 0.33 & -40.47 & -2.90 & -4.09 & -1.19 & -3.28 & -2.02 \\
3 & -1.57 & 14.20 & -0.76 & -4.98 & -4.22 & -5.89 & -3.36 \\
4 & -0.58 & -14.61 & -1.76 & -5.05 & -3.29 & -5.51 & -3.26 \\
5 & -1.16 & -22.61 & 0.21 & -7.87 & -8.08 & -11.37 & -6.83 \\
6 & 0.81 & -45.33 & 5.71 & -111.11 & -115.00 & -51.33 & -54.22 \\
7 & 0.19 & -215.93 & -1.62 & 10.21 & 11.82 & 23.40 & 10.30 \\
8 & 0.13 & 33.36 & -2.60 & 4.26 & 6.85 & 9.47 & 4.22 \\
9 & 0.27 & 18.75 & -0.63 & 2.96 & 3.59 & 5.98 & 3.38 \\
10 & 0.08 & 11.11 & -2.41 & 2.72 & 5.13 & 6.36 & 2.79 \\
11 & 0.05 & 6.38 & -0.78 & 2.01 & 2.79 & 4.14 & 2.27 \\
12 & 0.02 & 2.56 & -3.17 & 1.58 & 4.76 & 5.11 & 2.45 \\
13 & 0.15 & 0.31 & -0.88 & 1.32 & 2.20 & 2.26 & 0.99 \\
14 & 0.06 & -1.55 & -2.09 & 1.43 & 3.52 & 3.58 & 1.58 \\
15 & 0.05 & -2.64 & -1.03 & 0.85 & 1.87 & 1.63 & 0.47 \\
16 & 0.06 & -2.96 & -2.47 & 1.55 & 4.02 & 3.91 & 1.83 \\
17 & 0.06 & -3.68 & -1.32 & 1.05 & 2.37 & 2.02 & 1.23 \\
\hline
\end{tabular}

Table 7-4b: Percent difference of the apparent resistivities for line 2 at various current electrode depth levels. 


\begin{tabular}{|c||c|c|r|r|r|r|r|}
\hline \multicolumn{1}{|c||}{} & \multicolumn{7}{c|}{ Percent Difference: $2\left(V_{1}-V_{2}\right) /\left(V_{1}+V_{2}\right)^{*} 100 \%$} \\
\hline \hline File 1 & $500-4 \mathrm{~d} 1$ & $1500-4 \mathrm{~d}$ & $1900-4 \mathrm{~d}$ & $1900-4 \mathrm{~d}$ & $1900-5 \mathrm{~d}$ & $2100-5 \mathrm{~d}$ & $2300-5 \mathrm{~d}$ \\
File 2 & $500-4 \mathrm{~d} 2$ & $1500-5 \mathrm{u}$ & $1900-5 \mathrm{~d}$ & $1900-5 \mathrm{u}$ & $1900-5 \mathrm{u}$ & $2100-5 \mathrm{u}$ & $2300-5 \mathrm{u}$ \\
\hline Channel & & & & & & & \\
1 & -- & -19.29 & -5.57 & -7.28 & -1.72 & -0.79 & -0.99 \\
2 & - & -99.82 & -7.69 & -185.84 & -184.75 & -71.11 & -23.72 \\
3 & -- & -11.08 & -1.15 & -0.33 & 0.82 & 0.12 & 0.31 \\
4 & -- & -12.21 & -1.96 & -2.70 & -0.75 & -0.71 & -0.15 \\
5 & -- & -13.31 & -1.85 & -2.40 & -0.55 & -0.43 & -0.28 \\
6 & - & -17.18 & -1.47 & -2.05 & -0.58 & -0.52 & -0.22 \\
7 & -- & -16.21 & -1.75 & -2.03 & -0.28 & -0.15 & -0.20 \\
8 & - & -15.49 & -1.40 & -1.79 & -0.39 & -0.20 & -0.29 \\
9 & -- & -15.38 & -0.85 & -1.72 & -0.86 & -0.62 & -0.41 \\
10 & -- & -15.49 & -1.06 & -2.10 & -1.04 & -0.64 & -0.45 \\
11 & -- & -14.07 & -0.86 & -0.38 & 0.49 & 0.32 & -0.11 \\
12 & -- & -14.89 & -1.21 & -1.71 & -0.50 & -0.48 & -0.37 \\
13 & -- & -14.48 & -0.60 & -1.46 & -0.86 & -0.53 & -0.27 \\
14 & -- & -14.50 & -1.50 & -1.96 & -0.45 & -0.30 & -0.22 \\
15 & -- & -13.92 & -0.99 & -1.45 & -0.45 & -0.41 & -0.21 \\
16 & -- & -13.51 & -0.90 & -1.30 & -0.40 & -0.30 & -0.15 \\
17 & -- & -13.62 & -1.16 & -1.49 & -0.33 & -0.19 & -0.18 \\
\hline
\end{tabular}

Table 7-4c: Percent difference of the apparent resistivities for line 3 at various current electrode depth levels. 


\begin{tabular}{|c||c|c|c|c|c|c|c|c|}
\hline \multicolumn{10}{|c|}{ Apparent Resistivity $(\Omega$-m) } \\
\hline $\begin{array}{c}\text { File } \\
\text { (feet-day) }\end{array}$ & 1 & 2 & 3 & 4 & \multicolumn{1}{|c|}{ Channel Number } & \multicolumn{1}{|c|}{6} & \multicolumn{1}{c|}{7} & \multicolumn{1}{|c|}{8} \\
\hline $0451-5 w$ & 6116 & 2287 & 1011 & 596 & 1352 & 989 & 1384 & 1730 \\
$0453-5 w$ & 5941 & 2213 & 978 & 579 & 1317 & 961 & 1343 & 1710 \\
$0460-5 w$ & - & - & - & - & - & - & - & - \\
$0483-5 w$ & - & - & - & - & - & - & - & -- \\
$0500-4 d$ & - & - & - & - & - & - & - & -- \\
$0500-4 d$ & - & - & - & - & - & - & - & - \\
$0700-4 d$ & 7208 & 2778 & 1130 & 619 & 1797 & 1088 & 1364 & 1211 \\
$1500-4 d$ & - & - & - & - & - & - & - & - \\
$1500-5 u$ & 37780 & 25174 & 15026 & 6874 & 19155 & 12579 & 16457 & 14835 \\
$1700-5 u$ & 39729 & 27933 & 17191 & 9115 & 30290 & 20788 & 28555 & 27525 \\
$1900-4 d$ & 40486 & 26044 & 14504 & 10022 & 48348 & 35519 & 56310 & 68342 \\
$1900-5 d$ & 43007 & 26757 & 14009 & 9751 & 49302 & 36363 & 56817 & 70623 \\
$1900-5 u$ & 42363 & 26770 & 15039 & 10248 & 48747 & 36630 & 58110 & 69763 \\
$2100-5 d$ & 46762 & 32541 & 20286 & 16135 & 89080 & 93563 & 361811 & -350579 \\
$2100-5 u$ & 48149 & 33021 & 20680 & 16201 & 89234 & 95672 & 371053 & -353950 \\
$2300-5 d$ & 48237 & 35428 & 24066 & 21803 & 185604 & -2688630 & -141245 & -67623 \\
2300-5u & 49493 & 35954 & 24364 & 22188 & 187247 & -2720208 & -143084 & -68319 \\
\hline
\end{tabular}

\begin{tabular}{|c||c|c|c|c|c|c|c|c|c|}
\hline \multicolumn{10}{|c|}{ Apparent Resistivity $(\Omega-m)$} \\
\hline $\begin{array}{c}\text { File } \\
\text { (feet-day) }\end{array}$ & 9 & 10 & 11 & 12 & 13 & 14 & 15 & 16 & 17 \\
\hline $0451-5 w$ & 158 & 634 & 613 & 1481 & 1730 & 1902 & 1964 & 1770 & 1537 \\
$0453-5 w$ & 122 & 608 & 591 & 1445 & 1698 & 1876 & 1931 & 1746 & 1516 \\
$0460-5 w$ & - & -- & - & - & -- & -- & - & - & -- \\
$0483-5 w$ & -- & -- & - & - & -- & -- & - & - & - \\
$0500-4 d$ & -- & -- & - & - & -- & -- & - & - & -- \\
$0500-4 d$ & -- & -- & - & - & -- & - & - & - & - \\
$0700-4 d$ & 320 & 362 & 380 & 1258 & 1584 & 1824 & 1793 & 1613 & 1357 \\
$1500-4 d$ & - & - & - & - & -- & - & - & - & - \\
$1500-5 u$ & 1094 & 1110 & 1056 & 3806 & 3481 & 3763 & 4000 & 3092 & 2465 \\
$1700-5 u$ & 1099 & 1103 & 1065 & 4099 & 3625 & 3928 & 4220 & 3209 & 2544 \\
$1900-4 d$ & 1009 & 989 & 980 & 4150 & 3646 & 3971 & 4280 & 3243 & 2542 \\
$1900-5 d$ & 1029 & 1024 & 1003 & 4289 & 3686 & 4081 & 4340 & 3311 & 2567 \\
$1900-5 u$ & 1031 & 1014 & 1007 & 4215 & 3696 & 4025 & 4339 & 3276 & 2580 \\
$2100-5 d$ & 1141 & 1124 & 1092 & 4626 & 3826 & 4207 & 4535 & 3418 & 2644 \\
$2100-5 u$ & 1161 & 1141 & 1114 & 4615 & 3880 & 4199 & 4580 & 3415 & 2680 \\
$2300-5 d$ & 1155 & 1130 & 1110 & 4752 & 3941 & 4267 & 4680 & 3464 & 2704 \\
2300-5u & 1176 & 1152 & 1125 & 4780 & 3959 & 4282 & 4699 & 3477 & 2718 \\
\hline
\end{tabular}

Table 7-5a: Apparent resistivities of line 1 for channels 1-8 (top) and channels 9-17 (bottom). 


\begin{tabular}{|c|c|c|c|c|c|c|c|c|}
\hline \multicolumn{9}{|c|}{ Apparent Resistivity ( $\Omega-\mathrm{m})$} \\
\hline \multirow{2}{*}{$\begin{array}{c}\text { File } \\
\text { (feet-day) }\end{array}$} & \multicolumn{8}{|c|}{ Channel Number } \\
\hline & 1 & 2 & 3 & 4 & 5 & 6 & 7 & 8 \\
\hline $0451-5 w$ & 12045 & 3385 & 1409 & 1123 & 946 & 517 & 431 & 357 \\
\hline $0453-5 w$ & 11721 & 3272 & 1355 & 1078 & 902 & 491 & 406 & 332 \\
\hline $0460-5 w$ & 11117 & 3041 & 1224 & 973 & 796 & 426 & 344 & 273 \\
\hline $0483-5 w$ & 4415 & 1053 & 274 & 220 & 100 & 5 & -37 & -82 \\
\hline $0500-4 d$ & 3058 & 653 & 89 & 77 & -23 & -67 & -100 & -137 \\
\hline $0500-4 d$ & 3054 & 649 & 93 & 78 & -22 & -66 & -100 & -137 \\
\hline $0700-4 d$ & -23938 & 14685 & 2336 & 1388 & 760 & 234 & 75 & -55 \\
\hline $1500-4 d$ & -6010 & -3371 & -1728 & -1820 & -1326 & -407 & 66 & 734 \\
\hline $1500-5 u$ & -8106 & -5081 & -1499 & -2107 & -1664 & -645 & -163 & 523 \\
\hline $1700-5 u$ & -6888 & -4091 & -1359 & -1691 & -1174 & -329 & 97 & 646 \\
\hline $1900-4 d$ & -5323 & -2824 & -1275 & -1292 & -709 & -16 & 384 & 873 \\
\hline $1900-5 d$ & -5474 & -2908 & -1285 & -1315 & -707 & -16 & 389 & 896 \\
\hline $1900-5 u$ & -5567 & -2942 & -1340 & -1358 & -766 & -57 & 346 & 836 \\
\hline $2100-5 d$ & -5649 & -2985 & -1356 & -1368 & -780 & -87 & 292 & 749 \\
\hline $2100-5 u$ & -5838 & -3084 & -1439 & -1446 & -874 & -147 & 231 & 681 \\
\hline $2300-5 d$ & -5447 & -2845 & -1276 & -1276 & -696 & -40 & 319 & 740 \\
\hline $2300-5 u$ & -5554 & -2903 & -1320 & -1319 & -746 & -72 & 289 & 710 \\
\hline
\end{tabular}

\begin{tabular}{|c||r|r|r|r|r|r|r|r|r|}
\hline \multicolumn{10}{|c|}{ Apparent Resistivity $(\Omega$-m) } \\
\hline File \\
(feet-day)
\end{tabular}

Table 7-5b: Apparent resistivities of line 2 for channels 1-8 (top) and channels 9-17 (bottom). 


\begin{tabular}{|c||c|c|c|c|c|c|c|c|}
\hline \multicolumn{10}{|c|}{ Apparent Resistivity $(\Omega$-m) } \\
\begin{tabular}{|c|c|c|c|c|c|c|}
\hline File \\
(feet-day)
\end{tabular} & 1 & 2 & 3 & 4 & 5 & 6 & 7 & 8 \\
\hline $0451-5 w$ & 5659 & 776 & 2500 & 2278 & 1707 & 2141 & 1986 & 1714 \\
$0453-5 w$ & 5490 & 737 & 2470 & 2244 & 1676 & 2108 & 1955 & 1684 \\
$0460-5 w$ & - & - & - & - & - & - & - & - \\
$0483-5 w$ & - & - & - & - & - & - & - & - \\
$0500-4 d$ & 1849 & -29 & 1485 & 1257 & 956 & 1342 & 1273 & 1063 \\
$0500-4 d$ & - & - & - & - & - & - & - & - \\
$0700-4 d$ & 4611 & 263 & 3342 & 2847 & 2082 & 2670 & 2399 & 1934 \\
$1500-4 d$ & 6422 & 229 & 6545 & 5868 & 4365 & 5683 & 5093 & 4016 \\
$1500-5 u$ & 7792 & 686 & 7312 & 6631 & 4987 & 6751 & 5991 & 4690 \\
$1700-5 u$ & 7364 & 544 & 7333 & 6705 & 5065 & 6859 & 6124 & 4806 \\
$1900-4 d$ & 6471 & 12 & 7398 & 6615 & 5024 & 6664 & 6017 & 4760 \\
$1900-5 d$ & 6842 & 13 & 7484 & 6745 & 5118 & 6762 & 6123 & 4828 \\
$1900-5 u$ & 6960 & 318 & 7423 & 6796 & 5147 & 6802 & 6140 & 4846 \\
$2100-5 d$ & 7123 & 171 & 7620 & 6973 & 5291 & 6972 & 6325 & 4991 \\
$2100-5 u$ & 7180 & 361 & 7611 & 7023 & 5314 & 7008 & 6335 & 5001 \\
$2300-5 d$ & 7027 & 215 & 7635 & 7020 & 5342 & 7067 & 6413 & 5057 \\
2300-5u & 7097 & 273 & 7611 & 7031 & 5357 & 7082 & 6426 & 5072 \\
\hline
\end{tabular}

\begin{tabular}{|c||c|c|c|c|c|c|c|c|c|}
\hline \multicolumn{10}{|c|}{ Apparent Resistivity $(\Omega$-m) } \\
\hline $\begin{array}{c}\text { File } \\
\text { (feet-day) }\end{array}$ & 9 & 10 & 11 & 12 & 13 & 14 & 15 & 16 & 17 \\
\hline $0451-5 w$ & 1904 & 1912 & 1764 & 1678 & 1436 & 1466 & 1587 & 1314 & 1683 \\
$0453-5 w$ & 1869 & 1876 & 1736 & 1649 & 1415 & 1447 & 1566 & 1299 & 1666 \\
$0460-5 w$ & - & - & - & - & - & - & - & - & - \\
$0483-5 w$ & - & - & - & - & - & - & - & - & - \\
$0500-4 d$ & 1168 & 1161 & 1077 & 1043 & 955 & 1007 & 1101 & 942 & 1238 \\
$0500-4 d$ & - & - & - & - & - & - & - & - & - \\
$0700-4 d$ & 2030 & 1932 & 1706 & 1575 & 1359 & 1370 & 1449 & 1197 & 1523 \\
$1500-4 d$ & 4100 & 3765 & 3239 & 2857 & 2383 & 2316 & 2354 & 1881 & 2315 \\
$1500-5 u$ & 4783 & 4398 & 3728 & 3316 & 2755 & 2677 & 2706 & 2153 & 2653 \\
$1700-5 u$ & 4903 & 4526 & 3832 & 3410 & 2847 & 2770 & 2798 & 2226 & 2746 \\
$1900-4 d$ & 4867 & 4466 & 3858 & 3398 & 2841 & 2766 & 2805 & 2241 & 2759 \\
$1900-5 d$ & 4908 & 4514 & 3892 & 3439 & 2858 & 2807 & 2832 & 2260 & 2791 \\
$1900-5 u$ & 4951 & 4560 & 3873 & 3456 & 2882 & 2820 & 2845 & 2270 & 2800 \\
2100-5d & 5080 & 4682 & 4022 & 3564 & 2954 & 2902 & 2924 & 2332 & 2878 \\
2100-5u & 5111 & 4712 & 4010 & 3582 & 2970 & 2911 & 2936 & 2338 & 2884 \\
$2300-5 d$ & 5162 & 4754 & 4079 & 3628 & 3005 & 2958 & 2983 & 2372 & 2930 \\
2300-5u & 5183 & 4776 & 4084 & 3641 & 3013 & 2964 & 2989 & 2376 & 2935 \\
\hline
\end{tabular}

Table 7-5c: Apparent resistivities of line 3 for channels 1-8 (top) and channels 9-17 (bottom). 


\begin{tabular}{|c||c|c|c|c|c|c|c|c|}
\hline \multicolumn{10}{|c|}{ Apparent Resistivity ( $\Omega$-m) $0451-5 w . d a t$} \\
\hline $\begin{array}{c}\text { File } \\
\text { (ft-day) }\end{array}$ & 1 & 2 & 3 & 4 & 5 & 6 & 7 & 8 \\
\hline $0453-5 w$ & 17467 & 7289 & 3328 & 1839 & 4193 & 3645 & 5621 & 3859 \\
$0460-5 w$ & - & - & - & - & -- & - & - & - \\
$0483-5 w$ & - & - & - & - & -- & - & - & - \\
$0500-4 d$ & - & - & - & - & - & - & - & - \\
$0700-4 d$ & 5653 & 2082 & 958 & 584 & 1123 & 933 & 1396 & 2098 \\
$1500-4 d$ & - & - & - & - & - & - & - & - \\
$1500-5 u$ & 4165 & 1295 & 494 & 379 & 746 & 585 & 832 & 1219 \\
$1700-5 u$ & 4367 & 1431 & 589 & 404 & 754 & 599 & 860 & 1236 \\
$1900-4 d$ & 4529 & 1640 & 749 & 453 & 772 & 635 & 918 & 1279 \\
$1900-5 d$ & 4413 & 1621 & 758 & 457 & 760 & 626 & 914 & 1264 \\
$1900-5 u$ & 4443 & 1620 & 738 & 450 & 767 & 624 & 903 & 1270 \\
$2100-5 d$ & 4395 & 1584 & 720 & 435 & 744 & 612 & 885 & 1243 \\
2100-5u & 4336 & 1573 & 714 & 435 & 743 & 603 & 872 & 1238 \\
2300-5d & 4442 & 1606 & 731 & 446 & 756 & 615 & 890 & 1247 \\
2300-5u & 4392 & 1596 & 727 & 443 & 751 & 611 & 884 & 1242 \\
\hline
\end{tabular}

\begin{tabular}{|c||c|c|c|c|c|c|c|c|c|}
\hline \multicolumn{10}{|c|}{ Apparent Resistivity $(\Omega$-m) $0451-5 w . d a t$} \\
\hline $\begin{array}{c}\text { File } \\
\text { (ft-day) }\end{array}$ & 9 & 10 & 11 & 12 & 13 & 14 & 15 & 16 & 17 \\
\hline $0453-5 w$ & 5586 & 5096 & 4987 & 8796 & 10018 & 9454 & 11734 & 10472 & 10371 \\
$0460-5 w w$ & - & - & -- & - & - & - & - & - & -- \\
$0483-5 w$ & - & - & -- & - & - & - & - & -- & -- \\
$0500-4 d$ & - & - & - & - & - & - & - & - & - \\
$0700-4 d$ & -17 & 979 & 957 & 1820 & 2038 & 2090 & 2366 & 2240 & 2156 \\
$1500-4 d$ & - & - & -- & - & - & - & - & - & -- \\
$1500-5 \mathrm{u}$ & -32 & 512 & 474 & 817 & 804 & 734 & 856 & 745 & 689 \\
$1700-5 \mathrm{u}$ & -8 & 528 & 488 & 836 & 825 & 748 & 871 & 760 & 702 \\
$1900-4 d$ & 21 & 561 & 520 & 891 & 876 & 800 & 929 & 805 & 757 \\
$1900-5 d$ & 18 & 554 & 514 & 860 & 858 & 742 & 902 & 760 & 738 \\
$1900-5 \mathrm{u}$ & 17 & 556 & 513 & 877 & 854 & 771 & 903 & 783 & 728 \\
$2100-5 \mathrm{~d}$ & 10 & 540 & 499 & 838 & 841 & 732 & 880 & 742 & 719 \\
$2100-5 \mathrm{u}$ & 8 & 537 & 494 & 840 & 818 & 736 & 862 & 743 & 692 \\
2300-5d & 16 & 543 & 500 & 849 & 825 & 742 & 867 & 750 & 704 \\
2300-5u & 13 & 539 & 496 & 844 & 818 & 735 & 860 & 742 & 694 \\
\hline
\end{tabular}

Table 7-6a: Apparent resistivities of line 1 for channels 1-8 (top) and channels 9-17 (bottom) using the dipole-dipole configuration where the reference electrode is 1 foot below the end of the casing and the roving electrode location varies. 


\begin{tabular}{|c|c|c|c|c|c|c|c|c|}
\hline \multicolumn{9}{|c|}{ Apparent Resistivity $(\Omega-m) 0451-5 w . d a t$} \\
\hline \multirow{2}{*}{$\begin{array}{c}\text { File } \\
\text { (ft-day) }\end{array}$} & \multicolumn{8}{|c|}{ Channel } \\
\hline & 1 & 2 & 3 & 4 & 5 & 6 & 7 & 8 \\
\hline $0453-5 w$ & 23922 & 9022 & 4608 & 4043 & 4234 & 2745 & 2836 & 2923 \\
\hline $0460-5 w$ & 26827 & 9964 & 5351 & 4649 & 4822 & 3134 & 3196 & 3262 \\
\hline $0483-5 w$ & 30834 & 11393 & 5982 & 5249 & 5286 & 3467 & 3460 & 3530 \\
\hline $0500-4 d$ & 24609 & 9018 & 4655 & 4062 & 4026 & 2608 & 2570 & 2585 \\
\hline $0700-4 d$ & 7004 & 2525 & 1240 & 1054 & 1006 & 628 & 597 & 580 \\
\hline $1500-4 d$ & 5583 & 1987 & 952 & 789 & 733 & 443 & 401 & 379 \\
\hline $1500-5 u$ & 4834 & 1633 & 985 & 757 & 702 & 424 & 389 & 366 \\
\hline $1700-5 u$ & 5160 & 1781 & 981 & 775 & 722 & 438 & 402 & 379 \\
\hline $1900-4 d$ & 5667 & 2024 & 980 & 809 & 759 & 462 & 426 & 403 \\
\hline $1900-5 d$ & 5612 & 2006 & 978 & 806 & 759 & 463 & 427 & 405 \\
\hline $1900-5 u$ & 5577 & 1998 & 965 & 800 & 753 & 458 & 423 & 400 \\
\hline $2100-5 d$ & 5506 & 19 & 957 & 789 & 743 & 452 & 417 & 395 \\
\hline 2100 & 5436 & 1947 & 943 & 778 & 732 & 445 & 411 & 388 \\
\hline 2300 & 5554 & 1988 & 96 & 794 & 748 & 455 & 419 & 396 \\
\hline $2300-5 u$ & 5514 & 1975 & 956 & 789 & 742 & 451 & 416 & 393 \\
\hline
\end{tabular}

\begin{tabular}{|c|c|c|c|c|c|c|c|c|c|}
\hline \multicolumn{10}{|c|}{ Apparent Resistivity $(\Omega$-m) $0451-5 \mathrm{w}$. dat } \\
\hline \multirow{2}{*}{$\begin{array}{c}\text { File } \\
\text { (ft-day) }\end{array}$} & \multicolumn{9}{|c|}{ Channel } \\
\hline & 9 & 10 & 11 & 12 & 13 & 14 & 15 & 16 & 17 \\
\hline $0453-5 w$ & 3500 & 3663 & 3874 & 393 & 4073 & 5042 & 6109 & 6367 & 6180 \\
\hline $0460-5 w$ & 3912 & 4131 & 4315 & 439. & 4532 & 5554 & 6597 & 6805 & 6288 \\
\hline $0483-5 w$ & 4186 & 4415 & $455^{\circ}$ & 466 & 4748 & 5815 & 6871 & 7042 & 6443 \\
\hline $0500-4 d$ & 3043 & 3184 & 3265 & 330 & 3337 & 4019 & 4709 & 4799 & 4367 \\
\hline $0700-4 d$ & 665 & 680 & 690 & 697 & 704 & 866 & 1046 & 1089 & 1085 \\
\hline $1500-4 d$ & 417 & 409 & 398 & 38 & 375 & 445 & 520 & 522 & 510 \\
\hline $1500-5 u$ & 405 & 399 & 391 & 38 & 374 & 449 & 530 & 534 & 528 \\
\hline $1700-5 u$ & 418 & 412 & 402 & $39 ?$ & 384 & 460 & 542 & 544 & 537 \\
\hline $1900-4 d$ & 444 & 437 & 427 & 418 & 407 & 490 & 575 & 581 & 573 \\
\hline $1900-5 d$ & 445 & 440 & 428 & 424 & 409 & 497 & 580 & 593 & 580 \\
\hline $1900-5 u$ & 441 & 433 & 423 & 415 & 404 & 485 & 571 & 573 & 567 \\
\hline $2100-5 d$ & 434 & 428 & 416 & 410 & 395 & 480 & 557 & 570 & 555 \\
\hline $2100-5 u$ & 428 & 420 & 410 & 401 & 390 & 468 & 550 & 551 & 545 \\
\hline $2300-5 d$ & 436 & 428 & 417 & 409 & 395 & 476 & 555 & 562 & 552 \\
\hline $2300-5 u$ & 432 & 424 & 414 & 404 & 393 & 471 & 553 & 553 & 546 \\
\hline
\end{tabular}

Table 7-6b: Apparent resistivities of line 2 for channels 1-8 (top) and channels 9-17 (bottom) using the dipole-dipole configuration where the reference electrode is 1 foot below the end of the casing and the roving electrode location varies. 


\begin{tabular}{|c||c|c|c|c|c|c|c|c|}
\hline \multicolumn{10}{|c|}{ Apparent Resistivity ( $\Omega$-m) $0451-5 w . d a t$} \\
\hline $\begin{array}{c}\text { File } \\
\text { (ft-day) }\end{array}$ & 1 & 2 & 3 & 4 & 5 & 6 & 7 & 8 \\
\hline $0453-5 w$ & 21264 & 4307 & 4906 & 5256 & 4836 & 5851 & 5824 & 6052 \\
$0460-5 w$ & - & - & - & - & - & - & - & - \\
$0483-5 w$ & - & - & - & - & - & - & - & - \\
$0500-4 d$ & 22841 & 4005 & 6759 & 6820 & 5351 & 6450 & 6310 & 6197 \\
$0700-4 d$ & 6681 & 1191 & 1846 & 1822 & 1385 & 1639 & 1545 & 1447 \\
$1500-4 d$ & 5295 & 958 & 1354 & 1315 & 987 & 1129 & 1020 & 920 \\
$1500-5 u$ & 4643 & 806 & 1137 & 1110 & 819 & 823 & 741 & 687 \\
$1700-5 u$ & 4870 & 850 & 1196 & 1155 & 853 & 881 & 790 & 727 \\
$1900-4 d$ & 5289 & 1014 & 1218 & 1217 & 897 & 987 & 876 & 790 \\
$1900-5 d$ & 5121 & 1014 & 1196 & 1185 & 874 & 961 & 847 & 770 \\
$1900-5 u$ & 5067 & 919 & 1212 & 1173 & 867 & 951 & 842 & 764 \\
$2100-5 d$ & 5001 & 961 & 1188 & 1158 & 856 & 945 & 830 & 755 \\
$2100-5 u$ & 4975 & 903 & 1190 & 1146 & 850 & 936 & 828 & 752 \\
$2300-5 d$ & 5049 & 946 & 1202 & 1165 & 860 & 947 & 834 & 760 \\
$2300-5 u$ & 5018 & 929 & 1208 & 1163 & 856 & 944 & 831 & 755 \\
\hline
\end{tabular}

\begin{tabular}{|c|c|c|c|c|c|c|c|c|c|}
\hline \multicolumn{10}{|c|}{ Apparent Resistivity $(\Omega-m) 0451-5 w . d a t$} \\
\hline \multirow{2}{*}{$\begin{array}{c}\text { File } \\
\text { (ft-day) }\end{array}$} & \multicolumn{9}{|c|}{ Channel } \\
\hline & 9 & 10 & 11 & 12 & 13 & 14 & 15 & 16 & 17 \\
\hline $0453-5 w$ & 7652 & 8914 & 8107 & 9120 & 7645 & 7974 & 9605 & 8003 & 10440 \\
\hline $0460-5 w$ & - & -. & -- & -- & - & - & -- & -- & -- \\
\hline $0483-5 w$ & - & - & .- & -- & -- & - & -- & -. & -- \\
\hline $0500-4 d$ & 7693 & 8679 & 8882 & 9241 & 8040 & 8723 & 10423 & 9113 & 12365 \\
\hline $0700-4 d$ & 1729 & 1881 & 1871 & 1897 & 1625 & 1736 & 2035 & 1756 & 2371 \\
\hline $1500-4 d$ & 1052 & 1096 & 1023 & 999 & 811 & 824 & 923 & 756 & 975 \\
\hline $1500-5 u$ & 787 & 818 & 777 & 735 & 565 & 550 & 619 & 487 & 597 \\
\hline $1700-5 u$ & 831 & 854 & 811 & 766 & 587 & 570 & 637 & 501 & 609 \\
\hline $1900-4 d$ & 900 & 936 & 855 & 827 & 643 & 629 & 694 & 544 & 672 \\
\hline $1900-5 d$ & 886 & 917 & 840 & 806 & 633 & 602 & 674 & 527 & 642 \\
\hline $1900-5 u$ & 871 & 899 & 848 & 798 & 619 & 594 & 665 & 520 & 633 \\
\hline $2100-5 d$ & 868 & 894 & 823 & 784 & 616 & 583 & 653 & 510 & 617 \\
\hline $2100-5 u$ & 857 & 883 & 828 & 776 & 608 & 578 & 645 & 504 & 612 \\
\hline $2300-5 d$ & 869 & 896 & 827 & 782 & 616 & 579 & 645 & 507 & 612 \\
\hline $2300-5 u$ & 862 & 889 & 826 & 776 & 611 & 575 & 641 & 505 & 608 \\
\hline
\end{tabular}

Table 7-6c: Apparent resistivities of line 3 for channels 1-8 (top) and channels 9-17 (bottom) using the dipole-dipole configuration where the reference electrode is 1 foot below the end of the casing and the roving electrode location varies. 


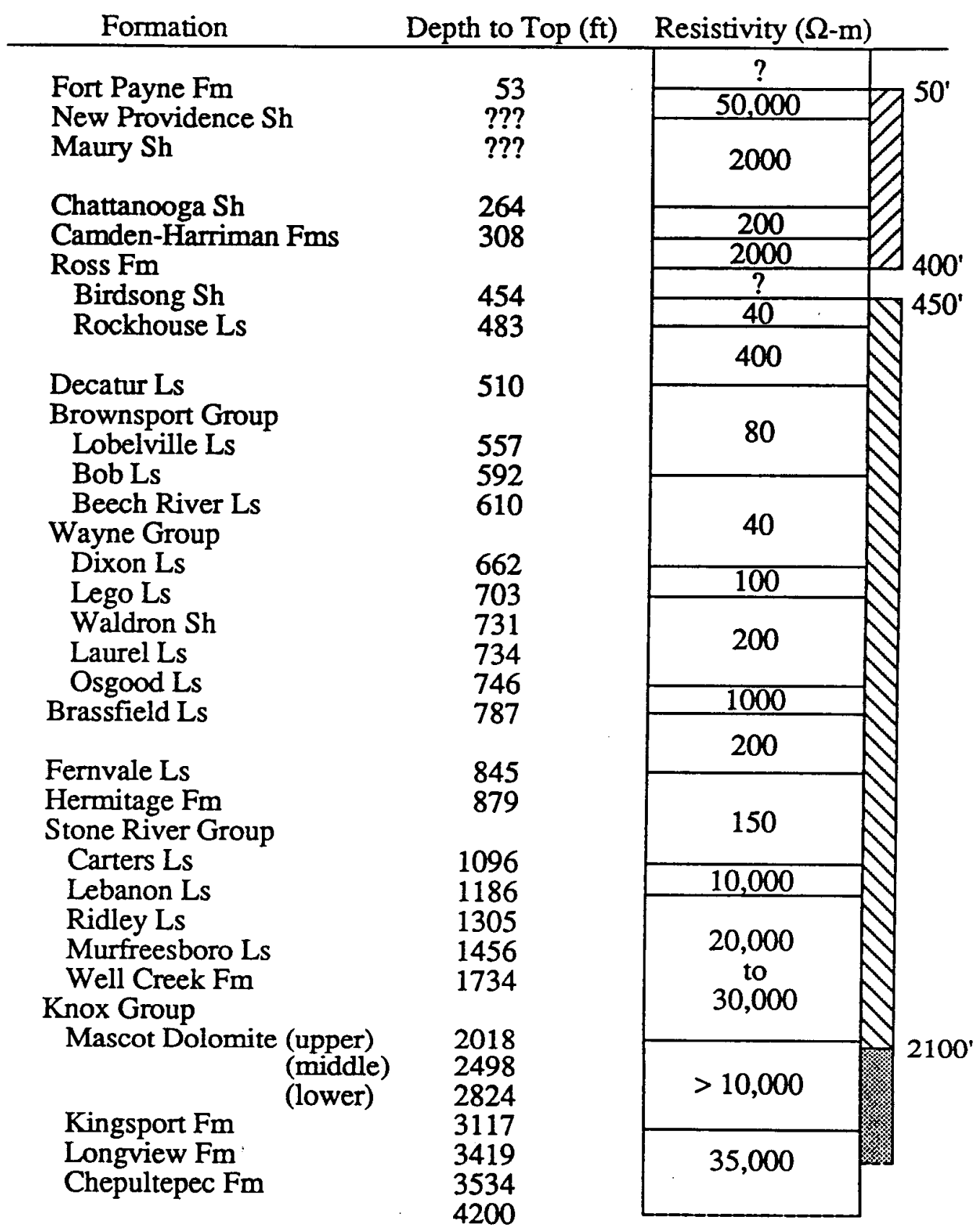

$\square$ Resistivity values estimated from electric logs (received October 1990)

$\triangle$ Resistivity values estimated from electric logs (received October 1989)

Resistivity values obtained from fluid samples (received October 1990)

Table 7-7: Formation resistivities obtained from electric logs and borehole fluid samples. 


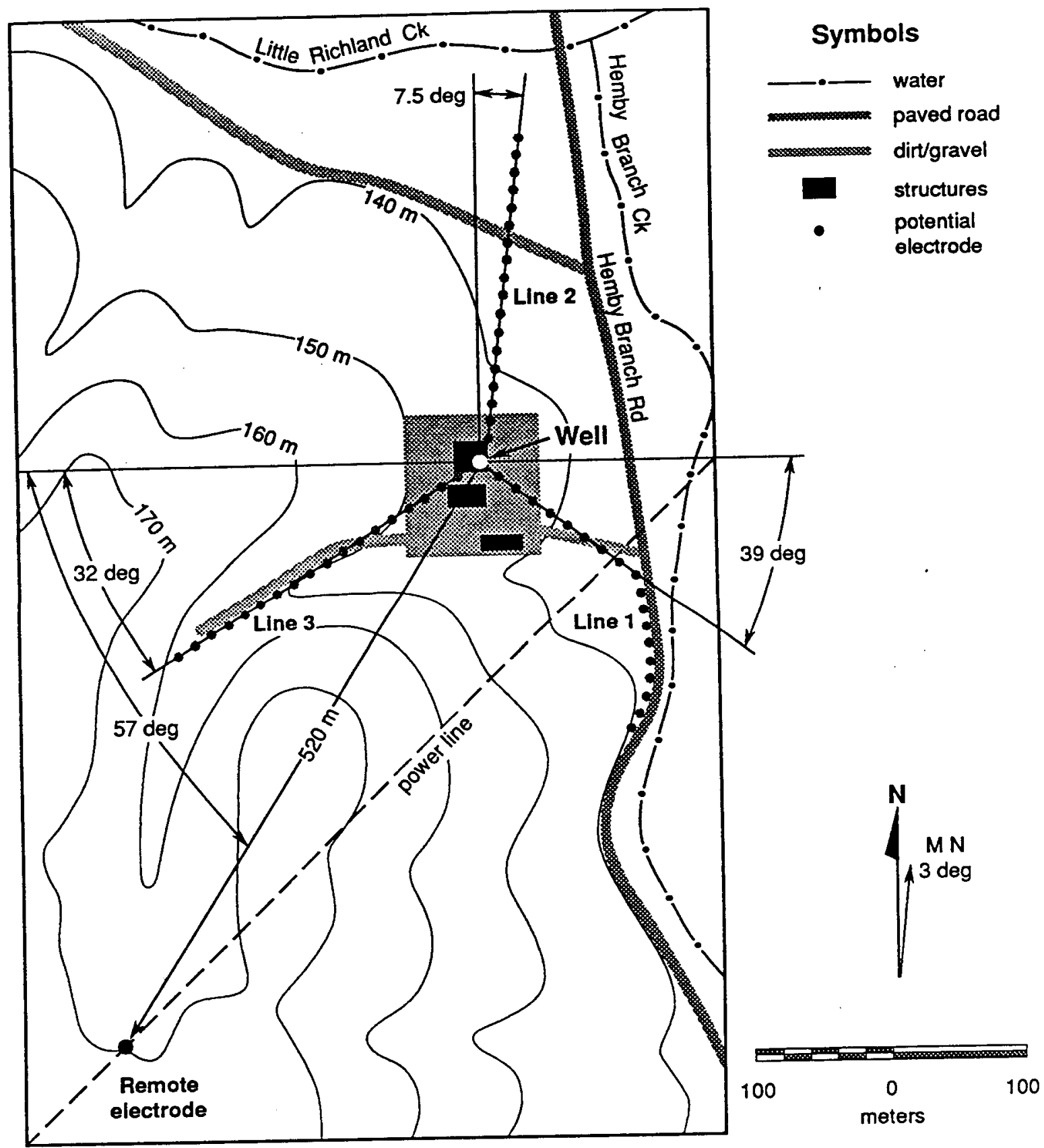

Figure 7-1: Map and array configuration at Dupont's Hemby Branch test well near Waverly, Tennessee. 


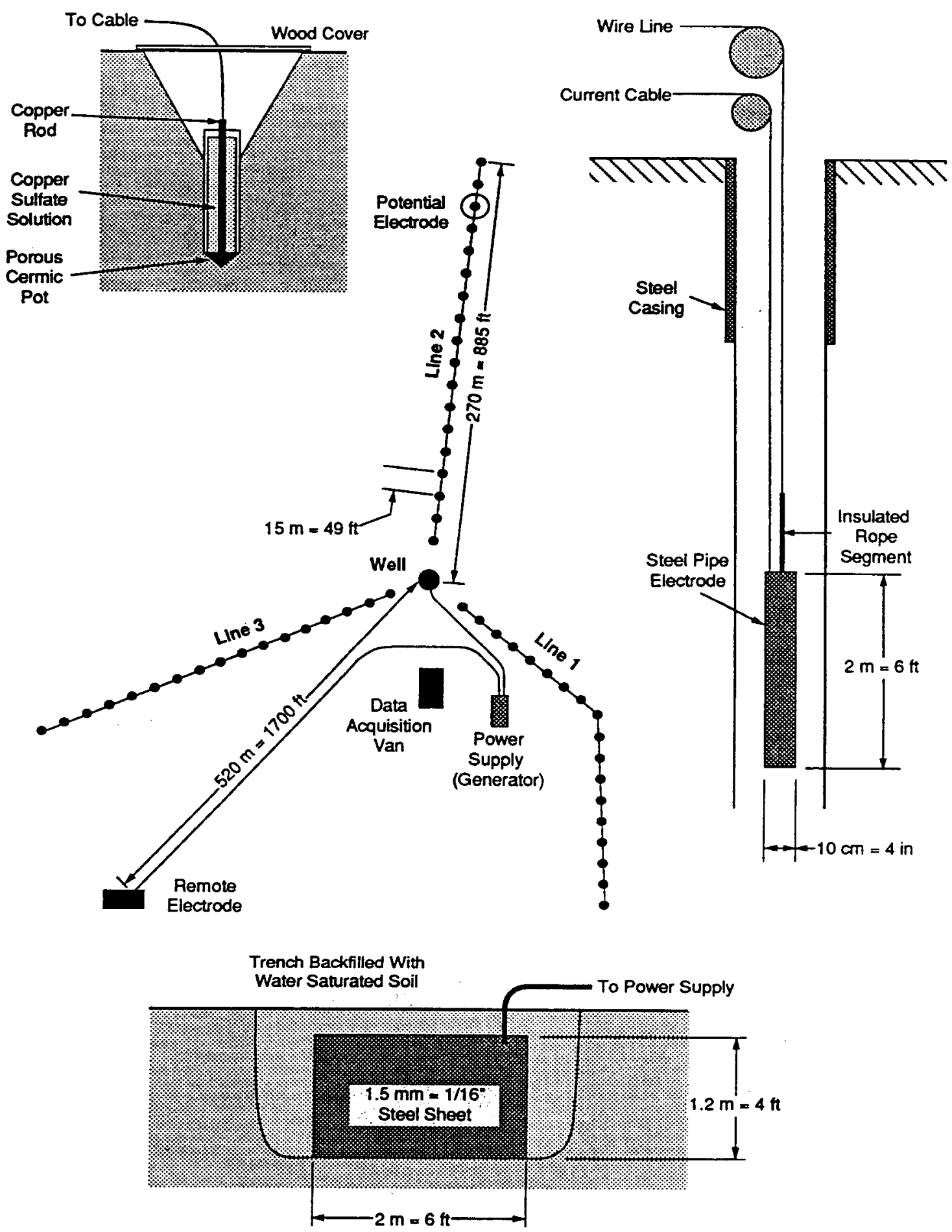

Figure 7-2: Illustrations of the field experiment design; potential electrode placed in the ground (top left), downhole current electrode (top right), potential array configuration (center), and remote current electrode (bottom). 


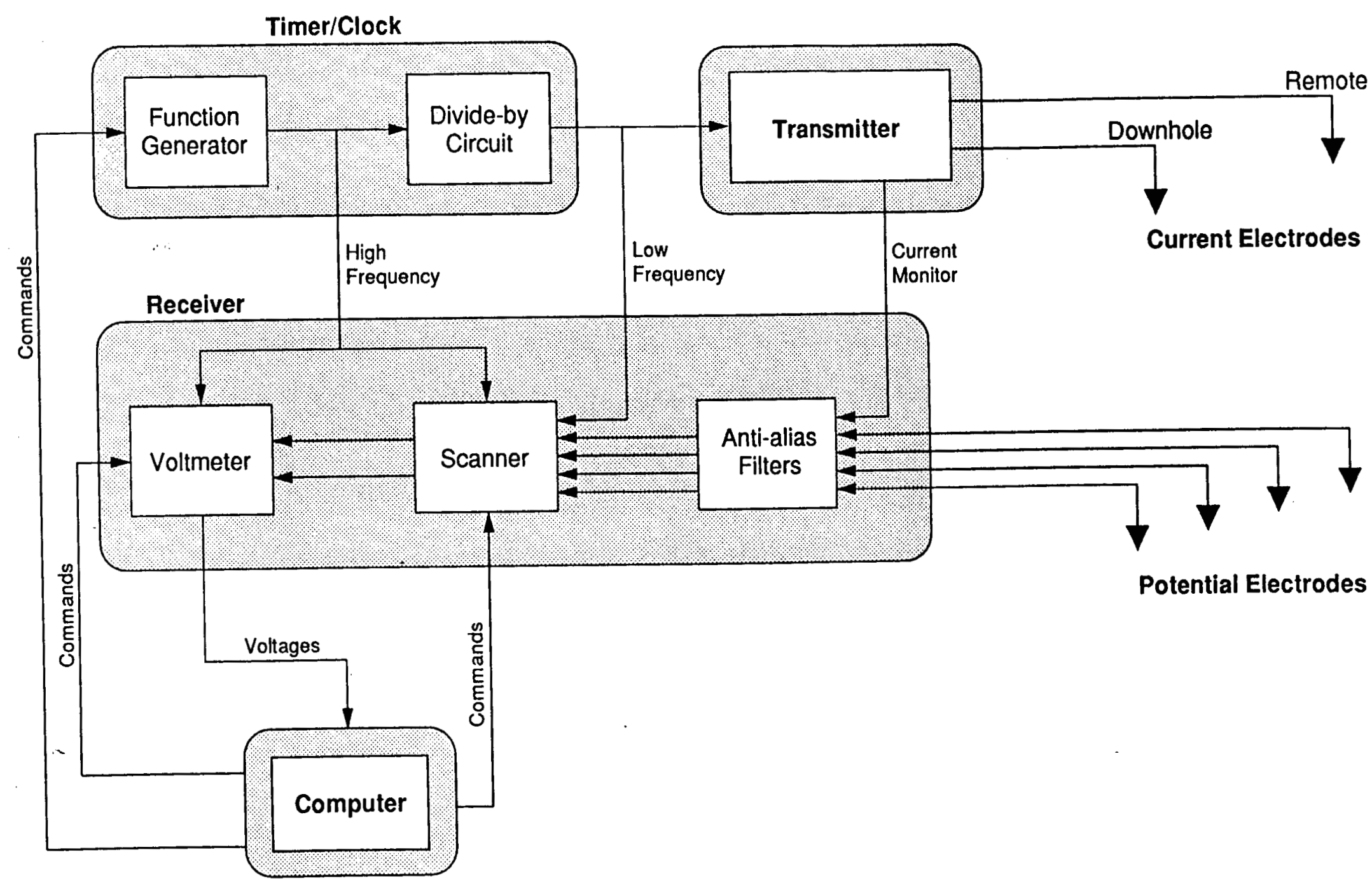

Figure 7-3: Schematic of the acquisition system used to collect and process the data 

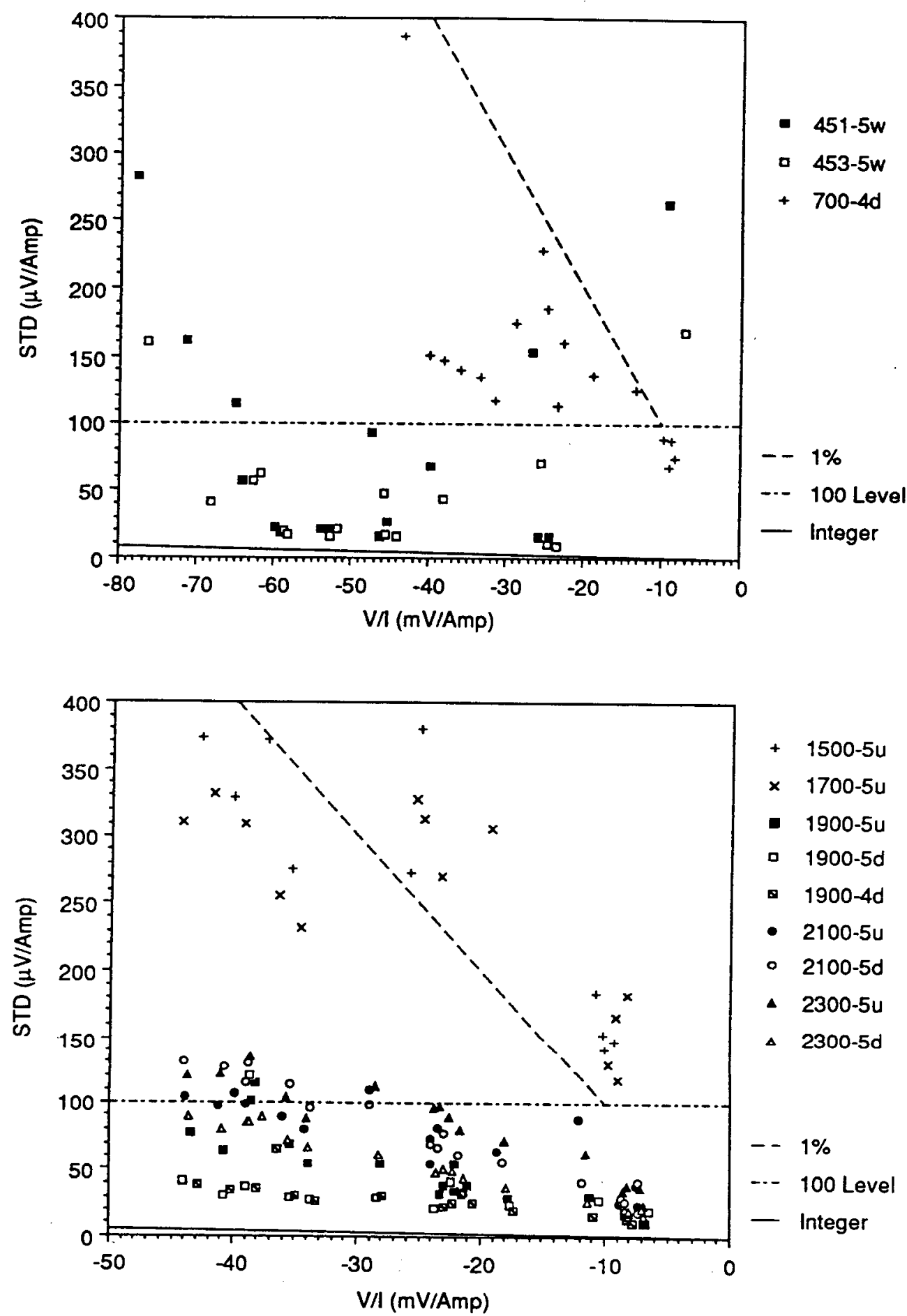

Figure 7-4: Scatter plots of the signal (V/I) vs. standard deviation (STD) of the field data for line 1; shallow (top) and deep (bottom). 

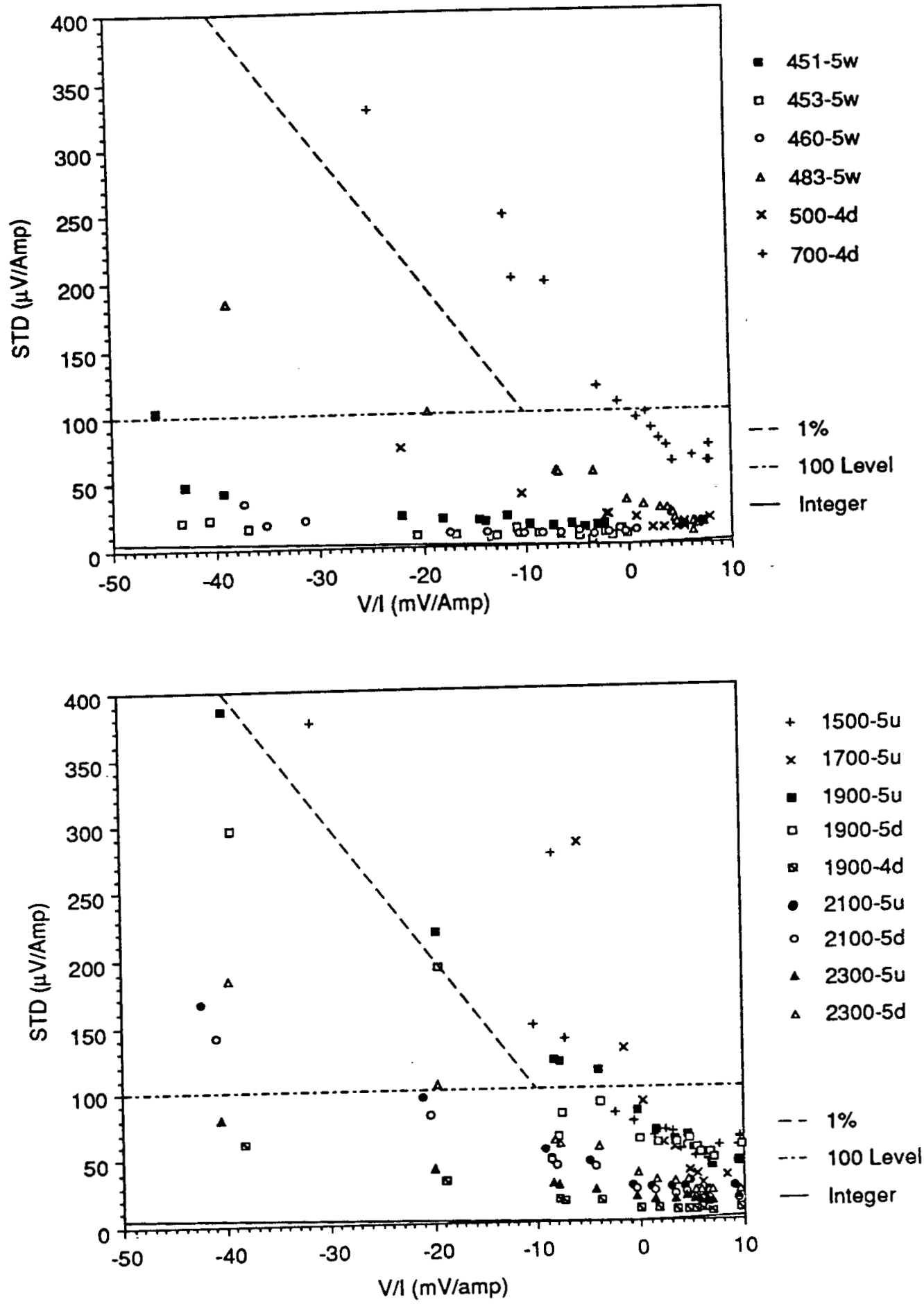

Figure 7-5: Scatter plots of the signal (V/I) vs. standard deviation (STD) of the field data for line 2; shallow (top) and deep (bottom). 

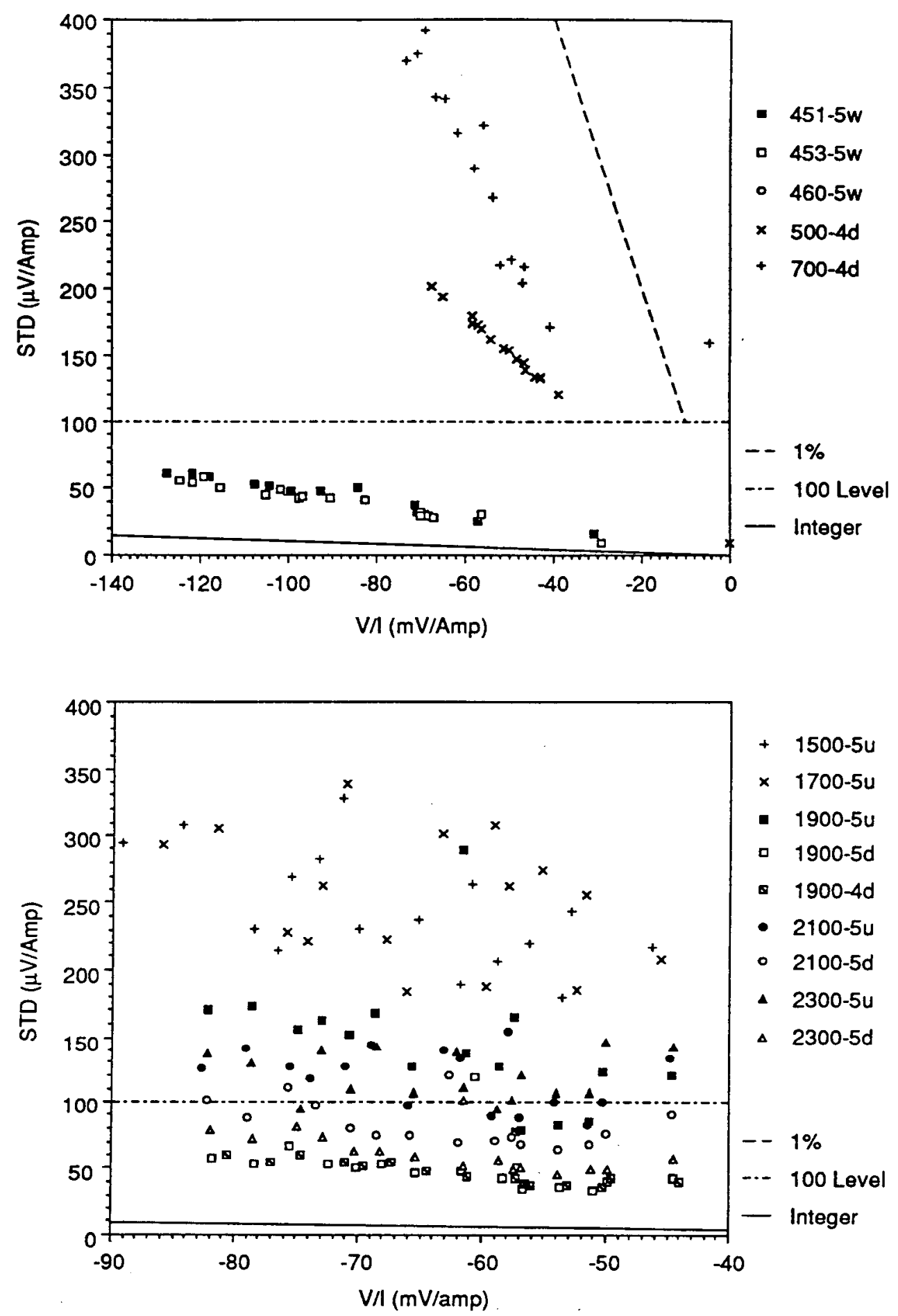

Figure 7-6: Scatter plots of the signal (V/I) vs. standard deviation (STD) of the field data for line 3; shallow (top) and deep (bottom). 

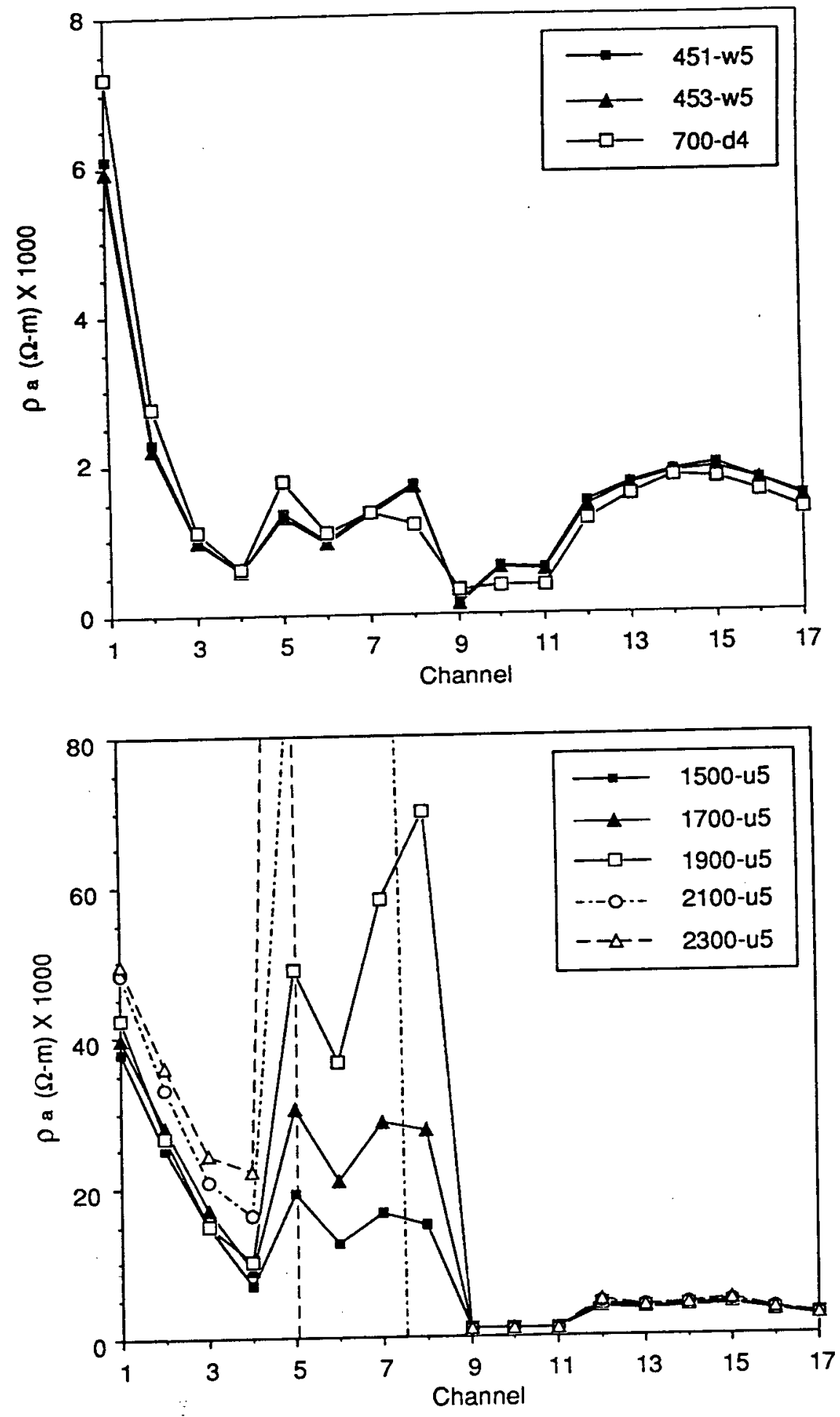

Figure 7-7: Apparent resistivities calculated from the field data for line 1 ; shallow (top) and deep (bottom). 

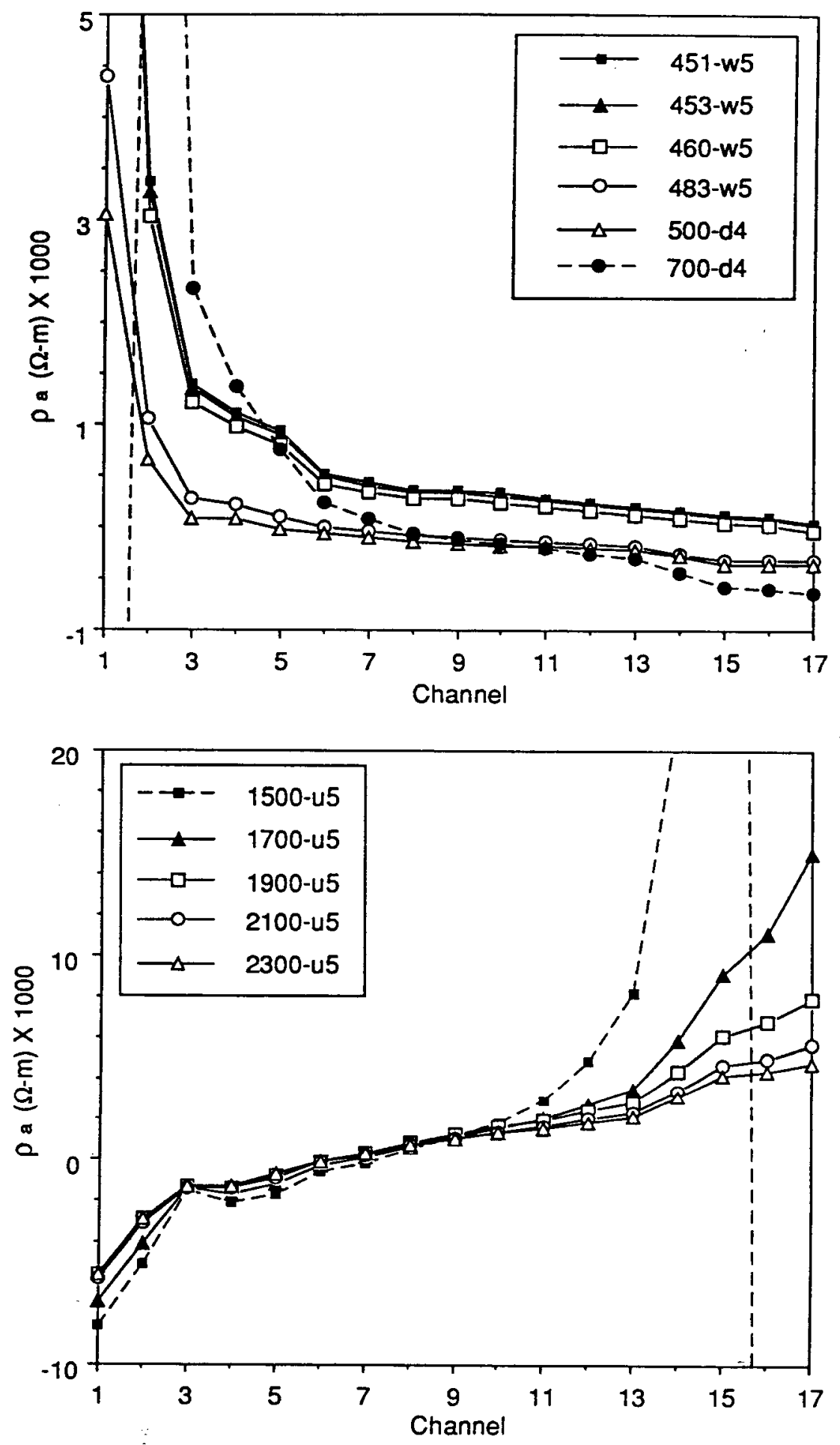

Figure 7-8: Apparent resistivities calculated from the field data for line 2; shallow (top) and deep (bottom). 

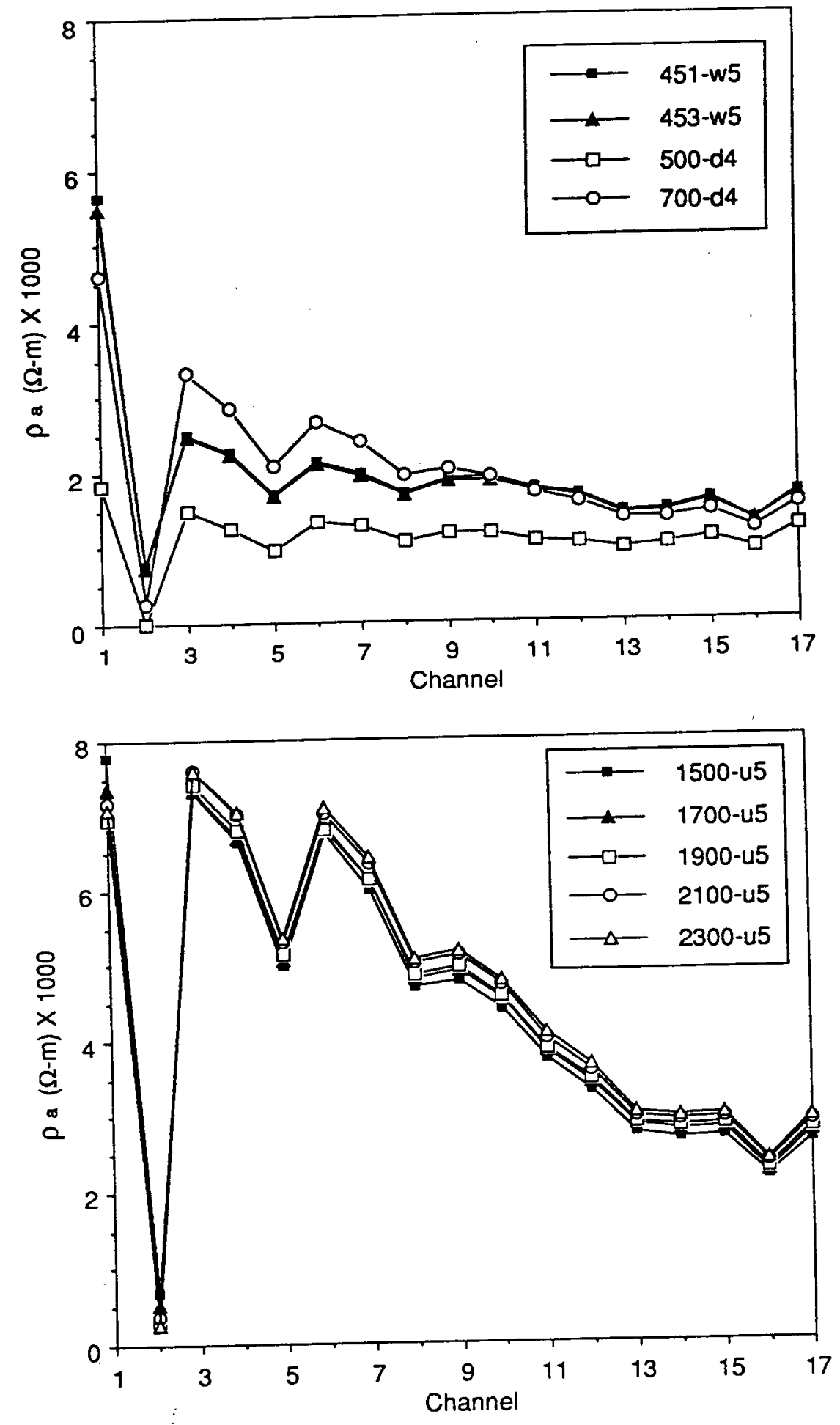

Figure 7-9: Apparent resistivities calculated from the field data for line 3; shallow (top) and deep (bottom). 

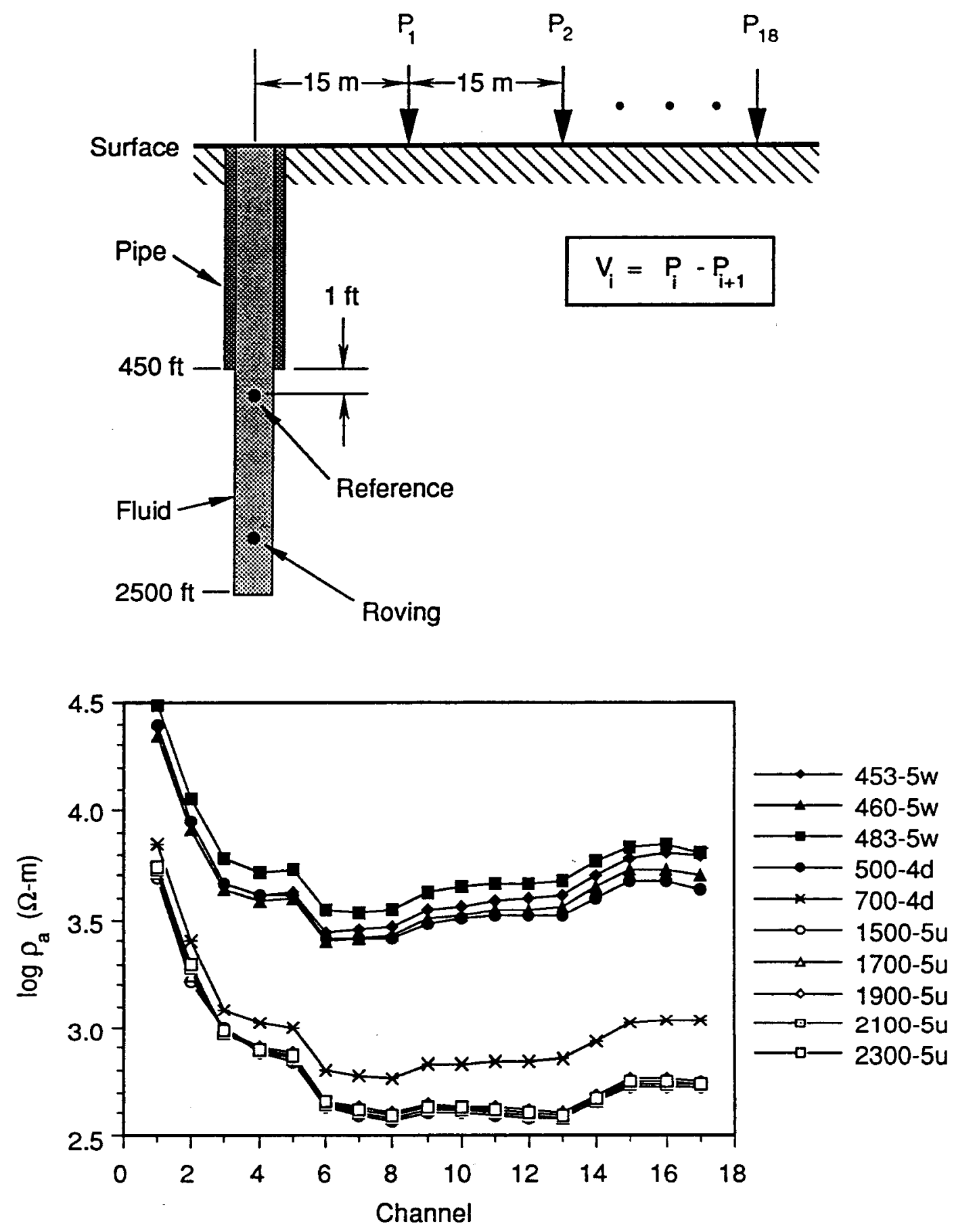

Figure 7-10: Illustration of the dipole-dipole configuration (top) used to calculate the apparent resistivities (bottom) for line 2 . 


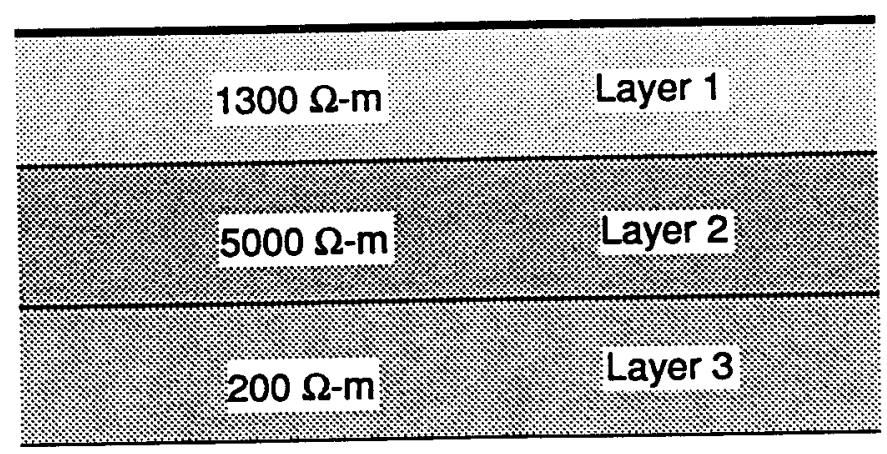

\section{Surface}

$262 \mathrm{ft}$

$482 \mathrm{ft}$

$1082 \mathrm{ft}$

$30,000 \Omega-m$

Basement

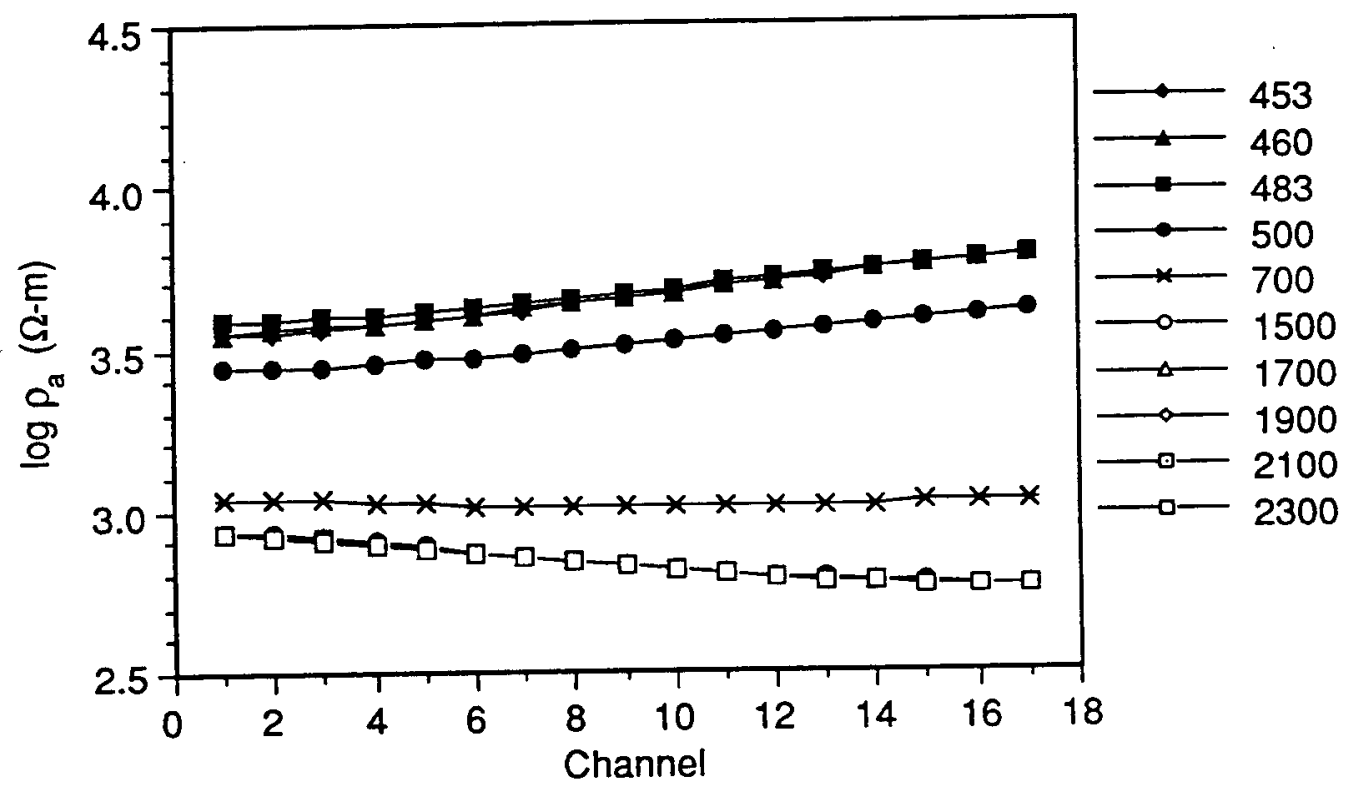

Figure 7-11: Four layer model (top) used to calculate the apparent resistivities (bottom). 

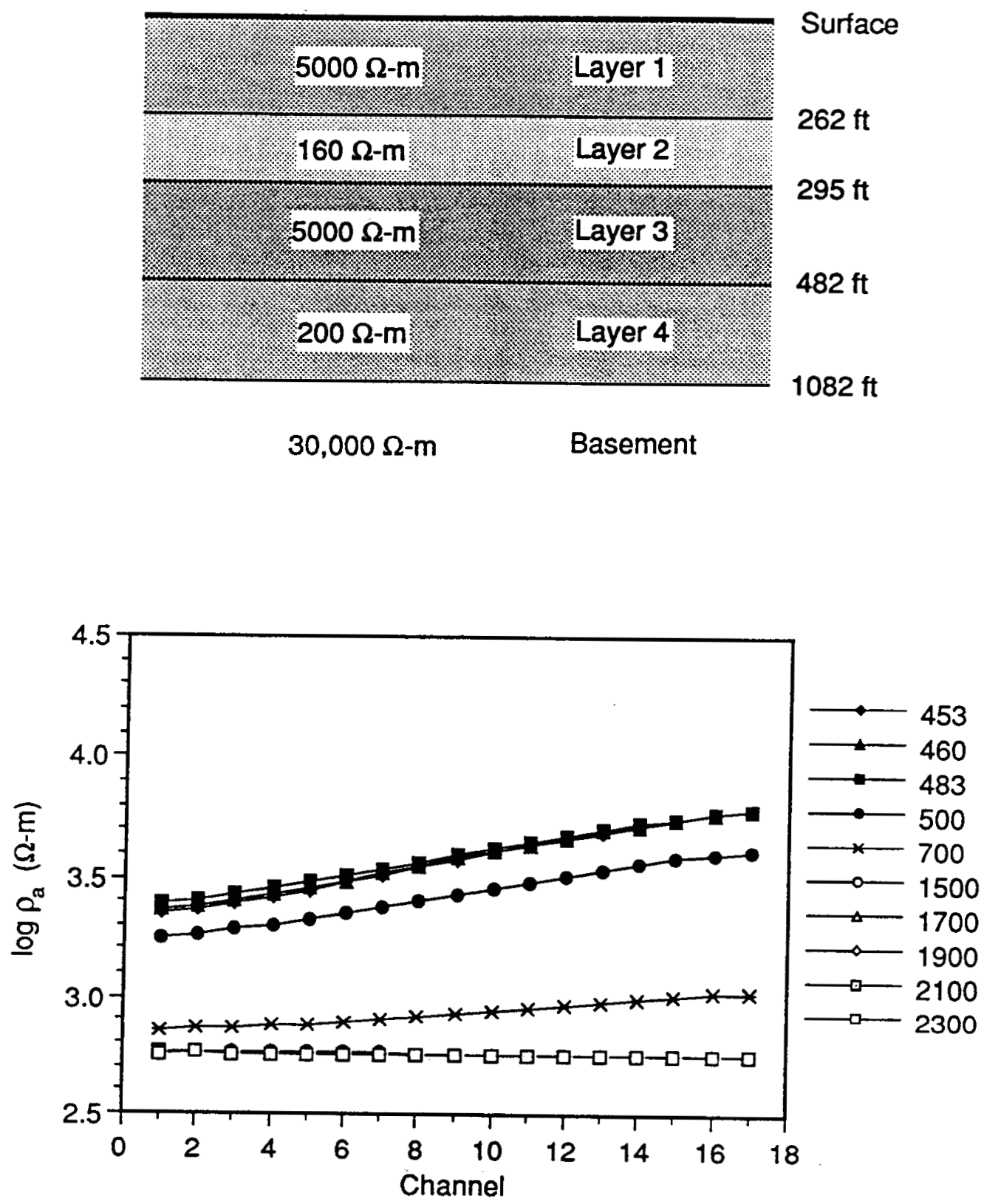

Figure 7-12: Five layer model (top) used to calculate the apparent resistivities (bottom). 

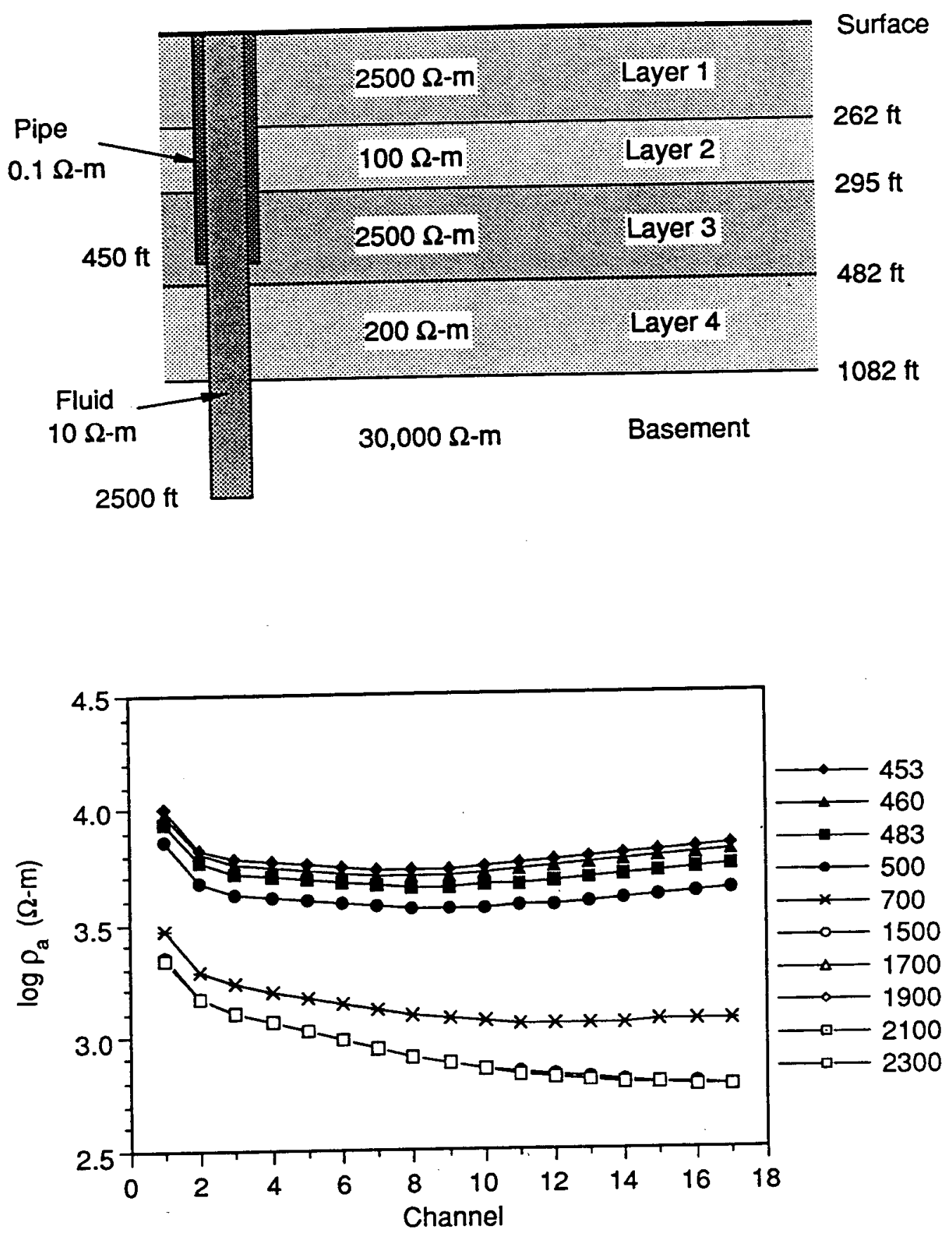

Figure 7-13: Pipe (resistive) and five layer model (top) used to calculate the apparent resistivities (bottom). 

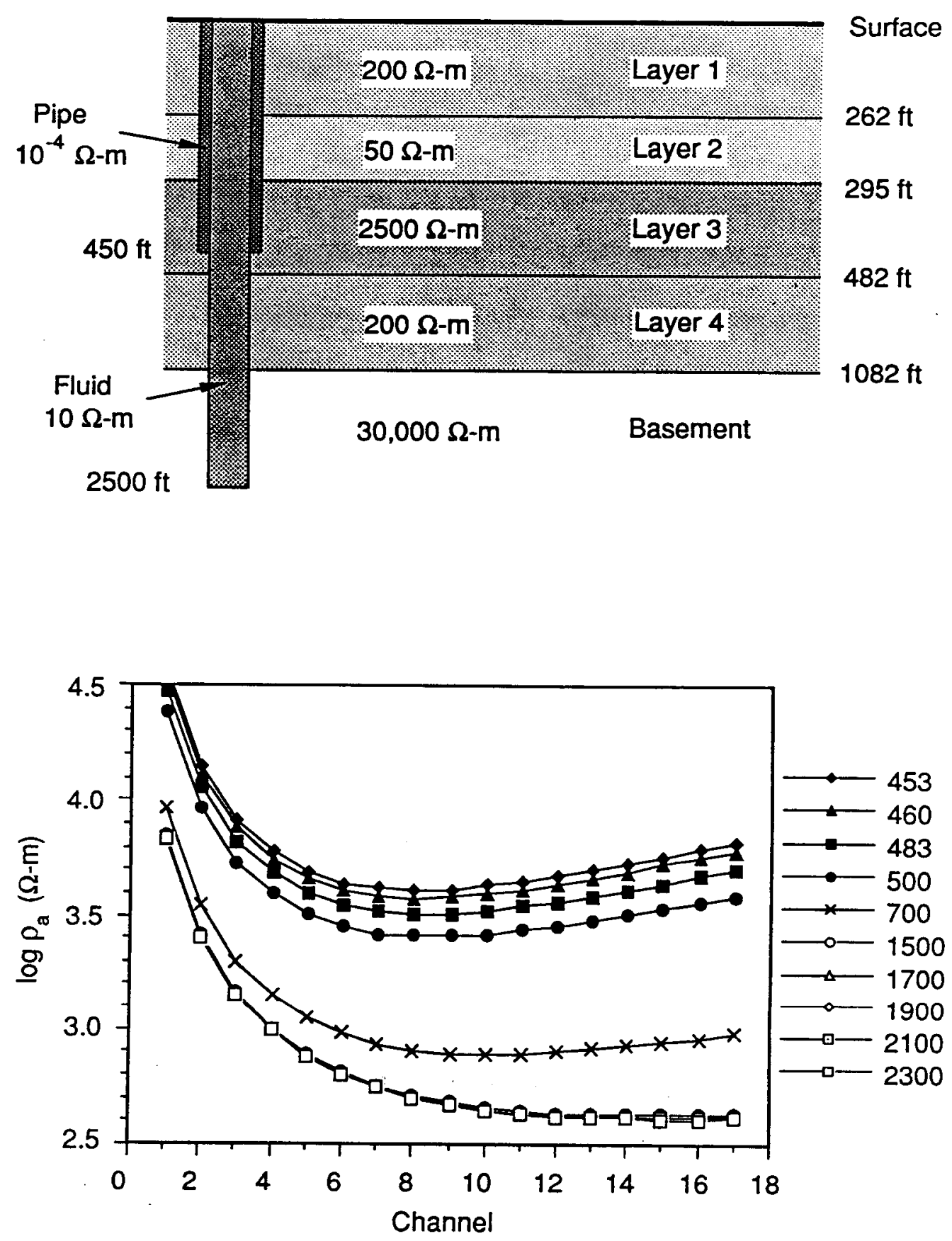

Figure 7-14: Pipe (conductive) and five layer model (top) used to calculate the apparent resistivities (bottom). 


\section{Chapter VIII}

\section{DIscussion}

The resistivity method in cased boreholes with downhole current sources has been analyzed using the integral equation (IE) technique. With the IE method, the casing and other bodies are represented as inhomogeneities in a background medium. For coaxial sources, there is axial symmetry and the casing can be characterized by ring segments. In this case, surface potential and E-field volume integral equation methods were used to solve the problem. A solution for the more complicated situation involving off-axis current sources and three-dimensional (3-D) bodies was formulated using the surface potential method. This solution which uses the 3-D Green's function was presented in both Cartesian and cylindrical coordinate systems.

Self-consistency and comparison checks showed that the theoretical formulation and numerical algorithms are valid and accurate. Numerical checks revealed that the current in the casing can be approximated by its vertical component except near the source and that the axial symmetry approximation for the casing is valid even for the 3-D problem.

During the analysis, it was found that the E-field volume IE method was an effective and efficient technique to simulate the response of the casing in a homogeneous medium. However, once other objects, such as layers, were included in the model, the surface potential IE approach was computationally better. Although more segments are needed to describe the casing for the surface potential IE approach, the number of unknowns to solve in the matrix equation is smaller than that for the E-field volume IE method. For the former, the surface of a body is discretized and the unknowns are scalar, whereas for the latter the volume is discretized and the unknowns are vectors quantities. 
Several configurations of the casing and current source were used to analyze the effects of the casing. In general, the field quantities are dependent on four characteristic factors. These are the conduction length, current source depth, casing depth, and casing length. The conduction length is the most important factor that influences the casing response in the medium. It relates the casing conductance to the conductivity of the host medium and is an indicator of the ability of the pipe to carry the current along its length.

When the source is located within the casing, the fields and spatial variables can be normalized by the casing length, reducing the characteristic parameters to three ratios: the conduction length to casing length (conduction ratio), the source position to casing length, and the casing depth to casing length. For a conduction ratio that is greater than two, the fields from the casing are similar to those produced by a line source. In a resistive medium, the line source approximation is valid for all field positions; the deviation is less than $5 \%$ at all points in the medium. In a conductive environment, surface measurements cannot be closer than one casing length but can be as close as $1 / 2$ casing length for cross-hole surveys.

When the source is located beneath the casing, the distortion of the fields also depends on the casing-source separation distance. For a current source near the casing $(\ll 100$ casing diameters), the casing greatly distorts the fields when compared to those produced by a pole source. When the source is greater than 100 casing diameters from the pipe, only the region near the casing is affected. For 5\% or less distortion, surface surveys must not be made closer than $1 / 2$ the casing length. Measurements can be made as close as $1 / 6$ pipe lengths for crosshole surveys.

The numerical results of Chapter VI show that resistivity measurements through metal casing are feasible provided one can distinguish small voltages. ${ }^{1}$ The discontinuity of the Efields corresponds to the layer boundary and the rate of change of the E-field is inversely proportional to the resistivity of the adjacent formation. ${ }^{2}$.

1 The E-fields, which are proportional to $I / S_{c}$, are in the order of 10 's $\mu \mathrm{V} / \mathrm{m}$ per Ampere.

2 For the formation resistivity range of 1 to $1000 \Omega \cdot m$, the rates of change of the E-field's are approximately 10.0 to $0.01 \mu \mathrm{V} / \mathrm{m}^{2}$ per Ampere. 
For a simple layer model, the formation resistivity can be estimated from the ratio of the potential and its second derivative provided that the casing conductance can be obtained. For an unknown casing conductance, two sets of measurements are needed to calculate the formation resistivity. One determines the casing conductance while the other measures the potentials used to estimate the second derivative. The resolution of the layer boundary is limited to the electrode spacing used to estimate the second derivative.

The borehole fluid resistivity has a negligible effect on the resistivity measurements in the casing. However, there are many other factors which can affect the measurements. Geometric factors, such as length and radius of the casing, as well as the array location within the casing affect the resistivity measurements. Variations of the casing conductance only affect the measurements when the discontinuity lies within the span of the array. The expression used by the patents cited in Chapter VI, equation (6-11), can only compensate for spatial variations of the casing that are longer than the array length. To compensate for smaller variations, an additional term is required (see equation 6-10).

Variation of the cement annulus parameters has a great effect on the estimate of the formation resistivity. At places where the contact resistance is low, the current tends to leak into the formation more readily and the formation resistivity will be underestimated. Since the radius of investigation is independent of the electrode spacing, no additional information about the cement conductance can be obtained by using different electrode spacing. Thus, estimating the true formation resistivity in the presence of a cement annulus may not be possible without prior knowledge of the thickness or resistivity of the cement.

The analysis of the downhole to surface field experiment showed that voltage measurements in a resistive environment are possible and that these voltages are quite large. Not only are casing effects large for measurements near the well, but they can also be seen at the far electrodes even for sources located far below the casing. Modeling indicates that strong interactions between the casing and near surface formations exist. The currents from the casing are channeled into the conductive layers and dissipate slowly into the resistive formations. 
Thus, the far-field electrodes are influenced by the casing and formation interaction.

\section{Further Studies}

The algorithms used here were limited to simulate problems involving downhole sources and axisymmetric bodies (logging, downhole to surface, and cross-hole surveys). A numerical code must be developed to combine the axisymmetric and 3-D solutions to simulate situations involving the casing and arbitrary 3-D bodies. The integral equation using the layered Green's function must be formulated to solve the problem involving the casing and 3-D targets in a layered earth. In this thesis, the layers were characterized as inhomogeneities. Here, formulating the integral equations with the layered Green's function may be computationally more efficient. Although employing this type of Green's function eliminates the use of analytic solution for the half-space Green's function, the matrix that must be inverted to solve for the unknowns will be much smaller, thus saving computational effort. ${ }^{3}$

The numerical simulations indicate that cross-hole and downhole to surface monitoring studies are not much affected by casing effects. In the practical example of using a dipole source at the end of the casing to monitor an injected conductive fluid (plume), the anomalies were enhanced by the presence of the casing. The enhanced response of a conductive zone was also observed when the casing was energized in another simulation. Thus, it may be advantageous to use the casing in experiments that monitor injection or extraction processes. A detailed analysis is needed to determine the configurations of the source, casing, and receivers that maximize the response and to verify whether a similar result is obtained with a resistive plume.

Iterative inversions or imaging of a target in the presence of the casing are numerically intractable if the forward model of the casing and target uses the IE approach. Most of the computation and memory : will be used to calculate the fields of the casing. If a set of line sources can be used to sufficiently approximate the casing response, iterative inversion and

3 Recall that the number of operations to invert a matrix of size $N \times N$ is approximately $N^{3}$. 
imaging techniques may become numerically feasible. One could use the IE algorithm to calculate the current distribution in the pipe for the homogeneous or layered medium problem. This current distribution can then be used as the "first guess" for the line sources. For nonlinear inversion techniques, the strengths of the line sources are adjusted at each iteration.

It appears that the transmission line formulation for the MTC method is valid. If this is true, then calculating the formation resistivity using the transmission line analogy presented by Kaufman (1990) will be orders of magnitude more efficient than using the IE method. However, this transmission line analogy (see Section 6.1) may also produce erroneous results. This formulation cannot account for current channeling effects in the formation since it assumes that the currents outside the casing are radial. To incorporate this effect, a transmission network with series and shunt resistors in the formations and annular zones is needed. This transmission network possibly may be used to explain the anomalous response caused by a cement annulus with variable thickness.

The analysis of the downhole to surface field data showed that the casing effects were large and radially symmetric. Hence, casing effects on field measurements can be minimized by placing the measuring dipoles orthogonal (azimuthally) to the radial lines. This configuration is insensitive to radial perturbations, such as casing effects and, unfortunately, plume responses. However, certain combinations of both azimuthal and radial dipoles may able to detect the anomalies produced by an injection plume, especially if there is a preferential flow direction of the fluid. A sensitivity analysis involving axisymmetric and nonaxisymmetric bodies in the presence of the casing is needed to determine optimal array configurations that will give a large plume response with minimal casing effects. 


\section{REFERENCES}

Asch, T.H. 1990. Mapping and monitoring of electrical resistivity with subsurface arrays. PhD Thesis, Department of Material Science and Mineral Engineering, University of Califomia, Berkeley.

Asch, T. and Morrison, H.F. 1989. Mapping and monitoring electrical resistivity with surface and subsurface electrode arrays. Geophysics, 54, 235-244.

Balanis, C.A. 1989. Advanced Engineering Electromagnetics. John Wiley and Sons, New York.

Baños, A. Jr. 1966. Dipole Radiation in the Presence of a Conducting Half-Space, Intemational series of monographs on electromagnetic waves, 9. Pergamon Press, Oxford.

Barnett, C.T. 1972. Theoretical Modelling of Induced Polarization Effects due to Arbitrarily Shaped Bodies. PhD thesis, Colorado School of Mines, Golden, Colorado.

Barnett, C.T. 1976. Theoretical modeling of the magnetic and gravitational fields of an arbitrarily shaped three-dimensional body. Geophysics, 41, 1353-1364.

Beasley, C.W. and Ward, S.H. 1986. Three-dimensional mise-à-la-masse modeling applied to mapping fracture zones. Geophysics, 51, 98-113.

Bevc, D. Lee, S., and Morrison, H.F. 1989. Mapping and monitoring an enhanced oil recovery processes with borehole-to-surface resistivity measurements: progress report and baseline data presentation. Engineering Geoscience Report, University of Califomia, Berkeley.

Bevc, D. and Morrison, H.F. 1990. Mapping and monitoring an enhanced oil recovery process with borehole-to-surface resistivity measurements. Engineering Geoscience Report, Department of Material Science and Mineral Engineering, University of California, Berkeley.

Brebbia, C.A., Telles, J.C.F., and Wrobel. L.C. 1984. Boundary Element Techniques. Springer-Verlag, Berlin.

Daily, W. and Yorkey, T.J. 1988. Evaluation of cross-borehole resistivity tomography. Expanded Abstracts, SEG 58th Annual International Meeting, 1, 201-203. 
Daniels, J.J. 1977. Three-dimensional resistivity and induced-polarization modeling using buried electrodes. Geophysics, 42, 1006-1019.

Daniels, J.J. and Dyck, A.V. 1984. Borehole resistivity and electromagnetic methods applied to mineral exploration. IEEE Transactions on Geoscience and Remote Sensing, GE-22, 80-87.

Dey, A. and Morrison, H.F. 1979. Resistivity modelling for arbitrary shaped threedimensional structures. Geophysics, 44, 753-780.

Dieter, K., Paterson, N.R. and Grant, F.S. 1969. IP and resistivity type curves for threedimensional bodies. Geophysics, 34, 615-632.

Dobecki, T.L. 1980. Borehole resistivity curves near spheroidal masses. Geophysics, 45, 1513-1522.

Eloranta, E. 1986. potential filed of a stationary electric current using Fredholm's integral equation of the second kind. Geophysical Prospecting, 22, 77-88.

Eskola, L. 1979. Calculation of galvanic effects by means of the method of sub-areas. Geophysical Prospecting, 27, 616-627.

Gard, M.F., Kingman, J.E.E., and Klein, J.D. 1989. Method and apparatus for measuring the electrical resistivity of geological formations through metal drill pipe or casing. U.S. patent $4,837,518$.

Gianzero, S. and Anderson, B. 1982. An integral transform solution to the fundamental problem in resistivity logging. Geophysics, 47, 946-956.

Gianzero, S. and Rau, R. 1977. The effect of sonde position in the hole on responses of resistivity logging tools. Geophysics, 42, 642-654.

Gravley, W. 1983. Review of downhole measurement-while-drilling systems. Journal of Petroleum Technology, p. 1439-1445.

Harrington, R.F. 1961. Time-Harmonic Electromagnetic Fields. McGraw-Hill Book Company, New York.

Harrington, R.F. 1968. Field Computation by Moment Methods. The Macmillan Co., New York.

Hohmann, G.W. 1971. Electromagnetic scattering by conductors in the earth near a line source of current. Geophysics, 36, 101-131. 
Hohmann, G.W. 1975. Three-dimensional induced polarization and electromagnetic modeling. Geophysics, 40, 309-324.

Hohmann, G.W. 1988. Numerical modeling for electromagnetic methods of geophysics. In Electromagnetic Methods in Applied Geophysics-Theory, 1, 313-363. Society of Exploration Geophysicists, Tulsa, Oklahoma.

Holbrook, P. 1985. The effect of mud filtrate invasion on the EWR ${ }^{\text {TM }} \log$ - a case history. SPWLA 26th Annual Logging Symposium, NNN 1-29.

Holladay J.S. and West G.F. 1984. Effect of well casing on surface electrical surveys. Geophysics, 49, 177-188.

Hvozdara, M. 1982. Potential field of a stationary electric current in a stratified medium with a three-dimensional perturbing body. Studia Geophysica et Geodaetica, 26, 160-172.

Hvozdara, M. 1983. Electric and magnetic field of a stationary current in a stratified medium with a three-dimensional conductivity inhomogeneity. Studia Geophysica et Geodaetica, 27, 59-84.

Jackson, P.D. 1981. Focussed electrical resistivity arrays: some theoretical and practical experiments. Geophysical Prospecting, 29, p. 601-626.

Johnson, R.H., Trofimenkoff, F.N. and Haslett, J.W. 1987. Resistivity response of a homogeneous earth with a finite-length contained vertical conductor. IEEE Transactions on Geoscience and Remote Sensing, GE-25, 414-421.

Kauahikaua, J., Mattice, M., and Jackson, D. 1980. Mise-a-la-masse mapping of the HGP-A geothermal reservoir, Hawaii. Geothermal Resource Council, Transactions, 4, 65-68.

Kaufman, A.A., 1989. Conductivity determination in a formation having a cased well. U.S. patent $4,796,186$.

Kaufman, A.A. 1990. The electric field in a borehole with a casing. Geophysics, 55, 29-38.

Kellogg, O.D. 1953. Foundations of Potential Theory. Dover Publications, Inc., New York.

LaBreque, D.J. and Ward, S.H. 1988. Effect of metal casing on a 3-D interpretation of borehole resistivity. Expanded Abstracts, SEG 58th Annual International Meeting, 1, 211-214.

Luke, Y.L. 1962. Integräls of Bessel Functions. McGraw-Hill Book Co., Inc., New York.

Lytle, R.J. 1982. Resistivity and induced-polarization probing in the vicinity of a spherical anomaly. IEEE Transactions on Geoscience and Remote Sensing, GE-20, 493-499. 
Merkel, R.H. and Alexander, S.S. 1971. Resistivity analysis for models of a sphere in a halfspace with buried current sources. Geophysical Prospecting, 19, 640-651.

Morse, P.M. and Feshbach, H. 1953. Methods of Theoretical Physics. McGraw-Hill Book Co., New York.

Okabe, M. 1976. Treatment of singularities in the integral equation approach. Geophysics, 44, 2004-2006.

Otto, D.V. 1968. A note on the mathematical representation of the source for tubular transmitting antennas. Radio Science, 862-864.

Panofsky, W.K.H. and Phillips, M. 1964. Classical Electricity and Magnetism. AddisonWesley Publishing Co., Reading, Massachusetts.

Parra, J.O., Owen, T.E., and Duff, B.M. 1986. A synthetically focused resistivity method for decting deep cavities. Expanded Abstracts, SEG 56th Annual International Meeting, 112-116.

Paul, M.K. 1974. The gravity effect of a homogeneous polyhedron for three-dimensional interpretation. Pure and Applied Geophysics, 112, 553-561.

Raiche, A.P. 1974. An integral equation approach to three-dimensional modelling. Geophysical Journal of the Royal Astronomical Society, 36, 363-376.

Robertson, R.C. 1987. The electromagnetic response of thin sheets buried in a uniform conducting half-space. Geophysics, 52, 108-117.

Rocroi, J.P. and Koulikov, A.V. 1985. The use of vertical line sources in electrical prospecting for hydrocarbons. Geophysical Prospecting, 33, 138-152.

SanFilipo, W.A. and Hohmann, G.W. 1985. Integral equation solution for the transient electromagnetic response of a three-dimensional body in a conductive half-space. Geophysics, 50, 798-809.

Sarkar, T.K. and Rao, S.M. 1982. An iterative method for solving electrostatic problems. IEEE Transactions on Antennas and Propagation, AP-30, 611-616.

Schenkel, C.J. and Morrison, H.F. 1990a. Effects of well casing of potential field measurements using downhole current sources. Geophysical Prospecting, 38, 663-686.

Schenkel, C.J. and Morrison, H.F. 1990b. Feasibility test on the borehole-to-surface resistivity method for mapping the areal extent of contaminants injected at depth. Report LBL-29088, Lawrence Berkeley Laboratory, Earth Science Division. 
Segesman, F. 1962. New SP correction charts. Geophysics, 27, 815-828.

Shima, H. and Saito, H. 1988. Application of resistivity tomography for detection of faults and evaluation of their hydraulic continuity: some numerical experiments. Expanded Abstracts, SEG 58th Annual International Meeting, 1, 204-207.

Sill, W.R. and Ward, S.H. 1978. Electrical energizing of well casings. Report 77-8, Contract EY-76-S-07-1601, University of Utah, Department of Geology and Geophysics.

Sill, W.R. 1983. Resistivity measurements before and after injection test 5 at Raft River KGRA, Idaho. Report DOE/ID/12079-104, University of Utah Research Institute, Earth Science Laboratory.

Smythe, W.R. 1968. Static and Dymamic Electricity, 3rd Edn., McGraw-Hill Book Co., New york.

Snyder, D.D. and Merkel, R.M. 1973. Analytic models for the interpretation of electrical surveys using buried current electrodes. Geophysics, 38, 513-529.

Snyder, D.D. 1976. A method for modeling the resistivity and IP response of twodimensional bodies. Geophysics, 41, 997-1015.

Sorensen, K. 1989. A method for measurement of the electrical formation resistivity while auger drilling. First Break, 7, 403-407.

Stinson, D.C. 1976. Intermediate Mathematics of Electromagnetics. Prentice-Hall, Inc., Englewood Cliffs, New Jersey.

Stratton, J.A. 1941. Electromagnetic Theory. McGraw-Hill Book Co., Inc., New York.

Sunde, E.D. 1968. Earth Conduction Effects in Transmission Systems. Dover Publications, Inc., New York.

Tai, Chen-To 1971. Dyadic Green's Functions in Electromagnetic Theory. International Textbook Co., Scranton.

Tagg, G.F. 1964. Earth Resistances. Pitman Publishing Corp., New York.

Ting, S.C. and Hohmann, G.W. 1981. Integral equation modeling of three-dimensional magnetotelluric response. Geophysics, 46, 182-197.

Vail, W.B., 1989a. Methods and apparatus for measurement of the resistivity of geological formations from within cased boreholes. U.S. patent 4,820,989. 
Vail, W.B., 1989b. Methods and apparatus for measurement of electronic properties of geological formations through borehole casing. U.S. patent 4,882,542.

Van Bladel, J. 1961. Some Remarks on Green's Dyadic for Infinite Space. IRE Transactions on Antennas and Propagation, 9, 563-566.

Van Bladel, J. 1964. Electromagnetic Fields. McGraw-Hill Book Co., Inc, New York.

Van Nostrand, R.G. and Cook, K.L. 1966. Interpretation of Resistivity Data, Geological Survey Professional Paper 499. U.S. Govemment Printing Office, Washington D.C.

Wait, J.R. 1952. The cylindrical orebody in the presence of a current carrying an oscillating current. Geophysics, 17, 378-386.

Wait, J.R. 1982. Geo-Electromagnetism. Academic Press, New York.

Wait, J.R. 1983. Resistivity response of homogeneous earth with a contained vertical condutor. IEEE Transactions on Geoscience and Remote Sensing, GE-21, 109-113.

Wait, J.R. and Williams, J.T., 1985. EM and IP response of a steel well casing for a fourelectrode surface array. Part I: theory. Geophysical Prospecting, 33, 723-735.

Ward, S.H. and Hohmann, G.W. 1988. Electromagnetic theory for geophysical applications; In Electromagnetic Methods in Applied Geophysics-Theory, 1, 131-311. Society of Exploration Geophysicists, Tulsa, Oklahoma.

Weidelt, P. 1975. Electromagnetic Induction in three-dimension structures. Journal of Geophysics, 41, 85-109.

Williams, J.T. and Wait, J.R. 1985. EM and IP response of a steel well casing for a fourelectrode surface array. Part II: numerical results. Geophysical Prospecting, 33, 736745.

Wilson, C.W., Jr. and Stearn, R.G. 1968. Geology of the Wells Creek structure, Tennessee. Tennessee Division of Geology, Bulletin 68, Nashville, TN.

Wilt, M.J. and Tsang, C.F. 1985. Monitoring of subsurface contaminants with borehole/surface resistivity measurements. In Proceedings from Surface and Borehole Geophysical Methods in Groundwater Investigation, National Water well Association Conference, February 12-14, Fort Worth, TX.

Yang, F.W. and Ward. S.H. 1985. Short Note; On sensitivity of surface-to-borehole resistivity measurements to the attitude and the depth to center of a three-dimensional spheroid. Geophysics, 50, 1173-1178. 


\section{General References}

Butler, C.M., and Wilton, D.R. 1980. General analysis of narrow strips and slots: IEEE Transactions on Antennas and Propagation, AP-28, 42-48.

Chang, D.C. 1967. On the electrically thick antenna. Radio Science, 2, 1043-1060.

Chang, D.C. 1973. Radiation of a buried dipole in the presence of a semi-infinte metallic tube. Radio Science, 8, 147-153.

Chang, W. and Otto, D.V. 1974. A simple approximate solution for the total current distribution on a thick short tubular antenna. IEEE Transactions on Antennas and Propagation, AP-22, 724-726.

Chang, W. and Otto, D.V. 1975. Current distribution on electrically thick tubular antennas of limited length. Internation Journal of Electronics, 39, 393-413.

Collin, R.E. 1986. The dyadic Green's function as an inverse operator. Radio Science, 21, 883-890.

Doherty, J. 1988. EM modelling useing surface integral equations. Geophysical Prospecting, 36, 644-668.

Drahos, D. 1984. Electrical modeling of the inhomogeneous invaded zone. Geophysics, 49, 1580-1585.

Eloranta, E. 1984. A method for calculation mise-a-la-masse anomalies in the case of high conductivity contrast by the integral equation technique. Geoexploration, 22, 77-88.

Eskola, L. 1984. Addendum to "The soultion of the stationary electric field strength and potential of a point current source in a 1/2-dimensional enviroment" by $L$. Eskola and $\mathrm{H}$. Hongisto. Geophysical Prospecting, 32, 510-511.

Eskola, L., Eloranta, E., and Puranen, R. 1984. A method for calculating IP anomalies for models with surface polarization. Geophysical Prospecting, 32, 79-87.

Eskola, L. and Hongisto, H. 1981. The solution of the stationary field strength and potential of a point current source in a $2 \frac{1}{2}$-dimensional enviroment. Geophysical Prospecting, 29, 260-273.

Graham, I.G. 1980. Some application areas for Fredholm integral equations of the second kind. In The Applications and Numerical Solutions of Integral Equations, Sijthoff and Nourdhoff, Alpen aan den Rijn, The Netherlands, 75-102.

Harrington, R.F. 1967. Matrix method for field problems. Proceedings of the IEEE, 55, 136-149. 
Harrison, C.W., Jr., Taylor, C.D., O'Donnell, E.E., and Aronson, E.A. 1967. On digital computer solutions of Fredholm integral equations of the first and second kind occurring in antenna theory. Radio Science, 2, 1067-1081.

Hill, S.C., Durney,C.H., and Christensen, D.A. 1983. Numerical calulations of low-frequency TE fields in arbitrarily shaped inhomogeneous lossy dielectric cylinders. Radio Science, 18, 328-226.

Jones, D.S. 1956. A critique of the variational method in scattering problems. IRE Transactions on Antennas and Propagation, AP-4, 297-301.

King, R.W. and Wu, T.T. 1967. The thick tubular transmitting antenna. Radio Science, 2, 1061-1065.

Lee, Hung-Mou 1983. Double series expansion of the Green's function for a perfectly conducting tubular cylinder of finite length Radio Science, 18, 48-56.

Lee, Hung-Mou 1987. Electromagnetic scattering of tubular cylindrical structure- double series formulation and some results. IEEE Transactions on Antennas and Propagation, AP-35, 384-390.

Lee, M.W. 1986. Low frequency radiation from point sources in a fluid-filled borehole. Geophysics, 51, 1801-1807.

Lee, T. 1975. An integral equation and its solution for some two- and three-dimensional problems in resistivity and induced polarization. Geophysical Joumal of the Royal Astronomical Society, 42, 81-95.

Le Masne, D. and Poirmeur, C. 1988. Three-dimensional model results for an electric holeto-surface method: Application to the interpretation of a field survey. Geophysics, 53, 85-103.

Lowry, T., Allen, M.B., and Shive, P.N. 1989. Singularity removal: a refinement of resistivity modeling techniques. Geophysics, 54, 766-774.

Newkirk, D.J. 1982. Downhole electrode resistivity interpretation with three-dimensional models. Technical Report DOE/ID/12079-47, Department of Geology and Geophysics, University of Utah.

Okabe, M. 1981. Boundary element method for the arbitrary inhomogeneities problem in electrical prospecting. Geophysical Prospecting, 29, 39-59.

Okabe, M. 1982. Reciprocal averaging techniques in the geoelectrical boundary element approach. Geophysical Prospecting, 30, 653-672. 
Otto, D.V. 1967. The admittance of cylindrical antennas driven from a coaxial line. Radio Science, 2, 1031-1042.

Otto, D.V. 1971. Singular integral equation solution for electrically small short cylindrical antenna. IEEE Transactions on Antennas and Propagation, AP-19, 532-533.

Pearson, C.E. 1957. On the finite strip problem. Quartary of Applied Mathematics, 15, 203211.

Pearson, L.W. 1983. On the spectral expansion of the electric and magnetic dyadic Green's functions in cylindrical harmonics. Radio Science, 18, 166-174.

Poirmeur, C. and Vasseur, G. 1988. Three-dimensional modeling of a hole-to-hole electrical method: Application to the interpretation of a field survey. Geophysics, 53, 402-414.

Pratt, D.A. 1972. The surface integral approach to the solution of the 3D resistivity problem. Australian Society of Exploration Geophysicists Bulletin, 3, 33-50.

Ross, H.P. and Ward, S.H. 1984. A review of the state-of-the-art and preliminary evaluation of the application to fracture mapping in geothermal systems. Report number DOE/SAN/12196-2, Earth Science Laboratory, University of Utah Research Institute.

Shirai, H. and Felsen, L.B. 1986. Modified GTD for generating complex resonances for flat strips and disks. IEEE Transactions an Antennas and Propagation, AP-34, 779-790.

Sloan, I.H. 1980. A review of numerical methods for Fredholm equations of the second kind. In The Applications and Numerical Solutions of Integral Equations, Sijthoff and Nourdhoff, Alpen aan den Rijn, The Netherlands, 51-74.

Tsang, L., Chan, A.K., and Gianzero, S. 1984. Solution of the fundamental problem in resistivity logging with a hybrid method. Geophysics, 49, 1596-1604.

Urankar, L.K. 1980. Vector potential and magnetic field of current-carrying finite arc segment in analytical form, part I: Filament approximation. IEEE Transaction on Magnetics, MAG-16, 1283-1288.

Urankar, L.K. 1982. Vector potential and magnetic field of current-carrying finite arc segment in analytical form, part II: Thin sheet approximation. IEEE Transaction on Magnetics, MAG-18, 911-917.

Urankar, L.K. 1982. Vector potential and magnetic field of current-carrying finite arc segment in analytical form, part III: Exact computation for rectangular cross section. IEEE Transaction on Magnetics, MAG-18, 1860-1867. 
Wait, J.R. 1978. Some earth resistivity problems involving buried cables. Quarterly Colorado School of Mines, 73, 1-21.

Wait, J.R. 1984. On modeling a well casing for resistivity and induced polarization. Geophysics, 49, 2061-2063.

Wait, J.R. 1986. Impedance conditions for a coated cylindrical conductor. Radio Science, 21, 623-626.

Wait, J.R. and Gruszka T.P. 1987. Resistivity an induced polarization response for a borehole model. IEEE Transaction on Geoscience and Remote Sensing, GE-25, 368-371.

Wait, J.R. and Umashankar, K.R. 1978/79. Analysis of the earth resistivity response of buried cables. Pageoph, 117, 711-742.

Wang, J.J.H. 1991. Generalized Moment Methods in Electromagnetics. John Wiley and Sons, Inc., New York.

Williams, J.T. and Dudley, D.G. 1987. Surface currents induced on a circular strip. Radio Science, 22, 216-226.

Yang, F.W. and Ward S.H. 1985. Single-borehole and cross-borehole resistivity anomalies of thin ellipsoids and spheroids. Geophysics, 50, 637-655.

Zemanian, A.H. and Anderson, B. 1987. Modeling of bolehole resistivity mesurements using infinite electrical grids. Geophysics, 52, 1525-1534. 


\section{APPENDIX A}

\section{GREEN'S FUNCTION}

For this study, the partial differential equation (PDE) that must be satisfied is Poisson's equation, either in scalar or vector form. Since the region of the homogeneous medium is considered unbounded, the Green's function method is a convenient technique to solve the inhomogeneous PDE. This technique represents the solution in terms of its source distribution. A solution to the inhomogeneous PDE is found for a unit driving source function, also known as an impulse or Dirac delta function. The general solution is written as a superposition of the impulse response solutions for the Dirac delta source at different locations. The strength of the general source at a particular point increases or decreases the effects of the unit source function at that point. The solution to a unit source function is called the Green's function.

\section{Scalar Green's Function}

Several forms of Green's functions exist depending on the governing PDE and boundary conditions. The various forms will not be given (see Morse and Feshbach, 1953 or Tai, 1971 for further discussion). This section will consider the Green's function related to the governing equation of $(2.1-11)$ which is:

$$
\nabla^{2} g\left(r, r^{*}\right)=-\delta\left(r-r^{*}\right)
$$

where $\mathbf{r}$ is the field (observation) point, $\mathbf{r}^{*}$ is the source point, $g$ is the Green's function for the unbounded homogeneous medium in this problem, and $\delta\left(\mathbf{r}-\mathbf{r}^{*}\right)$ is the Dirac delta function defined as: 


$$
\int_{v^{*}} \delta\left(\mathbf{r}-\mathbf{r}^{*}\right) d v^{*}= \begin{cases}1, & \mathbf{r} \text { in } V^{*} \\ 0, & \mathbf{r} \text { not in } V^{*}\end{cases}
$$

The solution which satisfies $(A-1)$ and the boundary condition, $\lim _{r \rightarrow} g\left(r, r^{*}\right)=0$ is:

$$
g\left(r, r^{*}\right)=\frac{1}{4 \pi} \frac{1}{\left|r-r^{*}\right|}
$$

Equation (A-3) is only given; see Morse and Feshbach (1953), Tai (1971), or Ward and Hohmann (1988) for the derivation of $g\left(r, r^{*}\right)$. From equation (A-3), the following relation can be shown:

$$
\nabla g\left(\mathbf{r}, \mathbf{r}^{*}\right)=-\nabla^{*} g\left(\mathbf{r}, \mathbf{r}^{*}\right)
$$

For many geophysical problems, the surface of the earth must be considered. Thus, a Green's function is needed for a half-space. For this situation, an additional boundary condition is required:

$$
\nabla_{g}\left(\mathbf{r}, \mathbf{r}^{*}\right) \cdot \mathbf{n}=0
$$

where $\mathbf{n}$ is the unit vector of the half-space (plane) surface.

Morse and Feshbach (1953) and Kellogg (1953) made use of the method of images to obtain the half-space Green's function which satisfies equations (A-1) and (A-5). The halfspace Green's function is:

$$
g\left(\mathbf{r}, \mathbf{r}^{*}\right)=\frac{1}{4 \pi}\left[\frac{1}{\left|\mathbf{r}-\mathbf{r}^{*}\right|}+\frac{1}{\left|\mathbf{r}-\mathbf{r}_{I}^{*}\right|}\right]
$$

where $\mathbf{r}_{i}^{*}$ is the image location of the source point $\mathbf{r}^{*}$, see Figure (A-1).

\section{Dyadic Green's Function}

Equation (A-4) is the scalar Green's function that can be used to solve the scalar inhomogeneous PDE. The vector form of the inhomogeneous PDE requires the use of the dyadic Green's function. The dyadic Green's function can be derived directly by solving the vector form of the PDE or by the steps used in Section (2.2). Before showing the dyadic Green's function, a brief review on dyads is presented. 
A dyad is defined by the juxtaposition ab where there is neither a scalar nor vector product of the vectors a and $\mathbf{b}$ (Balanis, 1989). In general, a dyad has nine components and in matrix form can be represented by:

$$
[\mathrm{ab}]=\left[\begin{array}{lll}
a_{1} b_{1} & a_{1} b_{2} & a_{1} b_{3} \\
a_{2} b_{1} & a_{2} b_{2} & a_{2} b_{3} \\
a_{3} b_{1} & a_{3} b_{2} & a_{3} b_{3}
\end{array}\right]
$$

The sum of the dyad is called a dyadic, $\mathbb{B}$ and for the case of equation (A-7) is:

$$
\begin{aligned}
\mathbb{B}=\sum_{n=1}^{3} \mathbf{a}_{n} \mathbf{b}_{n}= & \hat{a}_{1} a_{1} b_{1} \hat{\mathbf{b}}_{1}+\hat{a}_{2} a_{2} b_{1} \hat{\mathbf{b}}_{1}+\hat{\mathbf{a}}_{3} a_{3} b_{1} \hat{\mathbf{b}}_{1}+ \\
& \hat{\mathbf{a}}_{1} a_{1} b_{2} \hat{\mathbf{b}}_{2}+\hat{a}_{2} a_{2} b_{2} \hat{\mathbf{b}}_{2}+\hat{a}_{3} a_{3} b_{2} \hat{\mathbf{b}}_{2}+ \\
& \hat{\mathbf{a}}_{1} a_{1} b_{3} \hat{\mathbf{b}}_{3}+\hat{a}_{2} a_{2} b_{3} \hat{b}_{3}+\hat{a}_{3} a_{3} b_{3} \hat{\mathbf{b}}_{3}
\end{aligned}
$$

where $\hat{\mathbf{a}}_{i}$ and $\hat{\mathbf{b}}_{i}$ are unit vectors and $a_{i}$ and $b_{i}$ are components of $\mathbf{a}$ and $\mathbf{b}$. The dyadic $\hat{\mathbf{D}}$ satisfies the relation:

$$
(\mathbf{c} \cdot \mathbf{a}) \mathbf{b}=\mathbf{c} \cdot \mathbf{a b}=\mathbf{c} \cdot \mathbf{B}
$$

The quantities ab are operators such that the scalar product of the center expression in equation (A-8) is a vector pointed along the a base vectors with magnitude equal to the components of $\mathbf{c}$ along the $\mathbf{b}$ base vectors. In general, $\mathbf{c} \cdot \overrightarrow{\mathbf{D}} \neq \overrightarrow{\mathbf{D}}^{*} \cdot \mathbf{c}$ but $\mathbf{c} \cdot \overrightarrow{\mathbf{D}}=\overrightarrow{\mathbf{D}} \cdot \mathbf{c}$ where $\mathbb{\mathbf { D }}^{*}$ is the conjugate of $\mathbb{D}$.

The dyadic Green's function $\mathcal{G}\left(r, r^{*}\right)$ is defined as:

$$
\dot{G}\left(r, r^{*}\right)=\frac{1}{\sigma} \nabla \nabla g\left(r, r^{*}\right)
$$

where the operator $(\nabla \nabla)$ is called the double gradient dyad. By using the relation of equation (A-4), the following property exists:

$$
\mathrm{G}\left(\mathbf{r}, \mathbf{r}^{*}\right)=\frac{1}{\sigma} \nabla \nabla g\left(\mathbf{r}, \mathbf{r}^{*}\right)=-\frac{1}{\sigma} \nabla \nabla^{*} g\left(\mathbf{r}, \mathbf{r}^{*}\right)
$$

The tensor of the dyadic Green's function can be written as:

$$
\left[\dot{G}\left(\mathbf{r}, \mathbf{r}^{*}\right)\right]=\left[\begin{array}{ccc}
\mathbf{u}_{1} G_{11} \mathbf{u}_{1} & \mathbf{u}_{1} G_{12} \mathbf{u}_{2} & \mathbf{u}_{1} G_{13} \mathbf{u}_{3} \\
\mathbf{u}_{2} G_{21} \mathbf{u}_{1} & \mathbf{u}_{2} G_{22} \mathbf{u}_{2} & \mathbf{u}_{2} G_{23} \mathbf{u}_{3} \\
\mathbf{u}_{3} G_{31} \mathbf{u}_{1} & \mathbf{u}_{3} G_{32} \mathbf{u}_{2} & \mathbf{u}_{3} G_{33} \mathbf{u}_{3}
\end{array}\right]=\frac{1}{\sigma}\left[\begin{array}{ccc}
\mathbf{u}_{1} \frac{\partial^{2}}{\partial l_{1}^{2}} \mathbf{u}_{1} & \mathbf{u}_{1} \frac{\partial^{2}}{\partial l_{1} \partial l_{2}} \mathbf{u}_{2} & \mathbf{u}_{1} \frac{\partial^{2}}{\partial l_{1} \partial l_{3}} \mathbf{u}_{3} \\
\mathbf{u}_{2} \frac{\partial^{2}}{\partial l_{2} \partial l_{1}} \mathbf{u}_{1} & \mathbf{u}_{2} \frac{\partial^{2}}{\partial l_{2}^{2}} \mathbf{u}_{2} & \mathbf{u}_{2} \frac{\partial^{2}}{\partial l_{2} \partial l_{3}} \mathbf{u}_{3} \\
\mathbf{u}_{3} \frac{\partial^{2}}{\partial l_{3} \partial l_{1}} \mathbf{u}_{1} & \mathbf{u}_{3} \frac{\partial^{2}}{\partial l_{3} \partial l_{2}} \mathbf{u}_{2} & \mathbf{u}_{3} \frac{\partial^{2}}{\partial l_{3}^{2}} \mathbf{u}_{3}
\end{array}\right] g\left(\mathbf{r}, \mathbf{r}^{*}\right)(\mathrm{A}-12)
$$


where $l_{i}$ and $u_{i}$ for $(i=1,3)$ are the components and unit vectors The non-asterisk and asterisk superscripts represent the field and source coordinate system, respectively.

The dyadic Green's function components $G_{\alpha \beta}$ can be though of as relating the $\alpha$ component of the electric field at point $(r)$ to the $\beta$-component of the scattering current at point $\left(r^{*}\right)$. By applying the reciprocity theorem, the dyadic Green's function can take the form (Harrington, 1961):

$$
G_{\alpha \beta}\left(\mathbf{r}, \mathbf{r}^{*}\right)=G_{\beta \alpha}\left(\mathbf{r}, \mathbf{r}^{*}\right)
$$

which indicates that the dyadic Green's function is symmetric.

In addition to the matrix component representation of the dyadic Green's function, it can be represented by vectors in the form:

$$
\mathcal{G}\left(r, r^{*}\right)=u_{1} G_{1}+u_{2} G_{2}+u_{3} G_{3}
$$

where:

$$
\mathbf{G}_{k}=G_{k 1} \mathbf{u}_{1}+G_{k 2} \mathbf{u}_{2}+G_{k 3} \mathbf{u}_{3}
$$

The solution of the dyadic Green's function can be found by decomposing it into three vectors; one for each component of the unknown field to be solved. The solution for the vectors: $G_{1}, G_{2}$, and $G_{3}$, each satisfying the component form of the PDE and boundary conditions, are combined using equation (A-11) to form the dyadic Green's function which satisfies the PDE. 


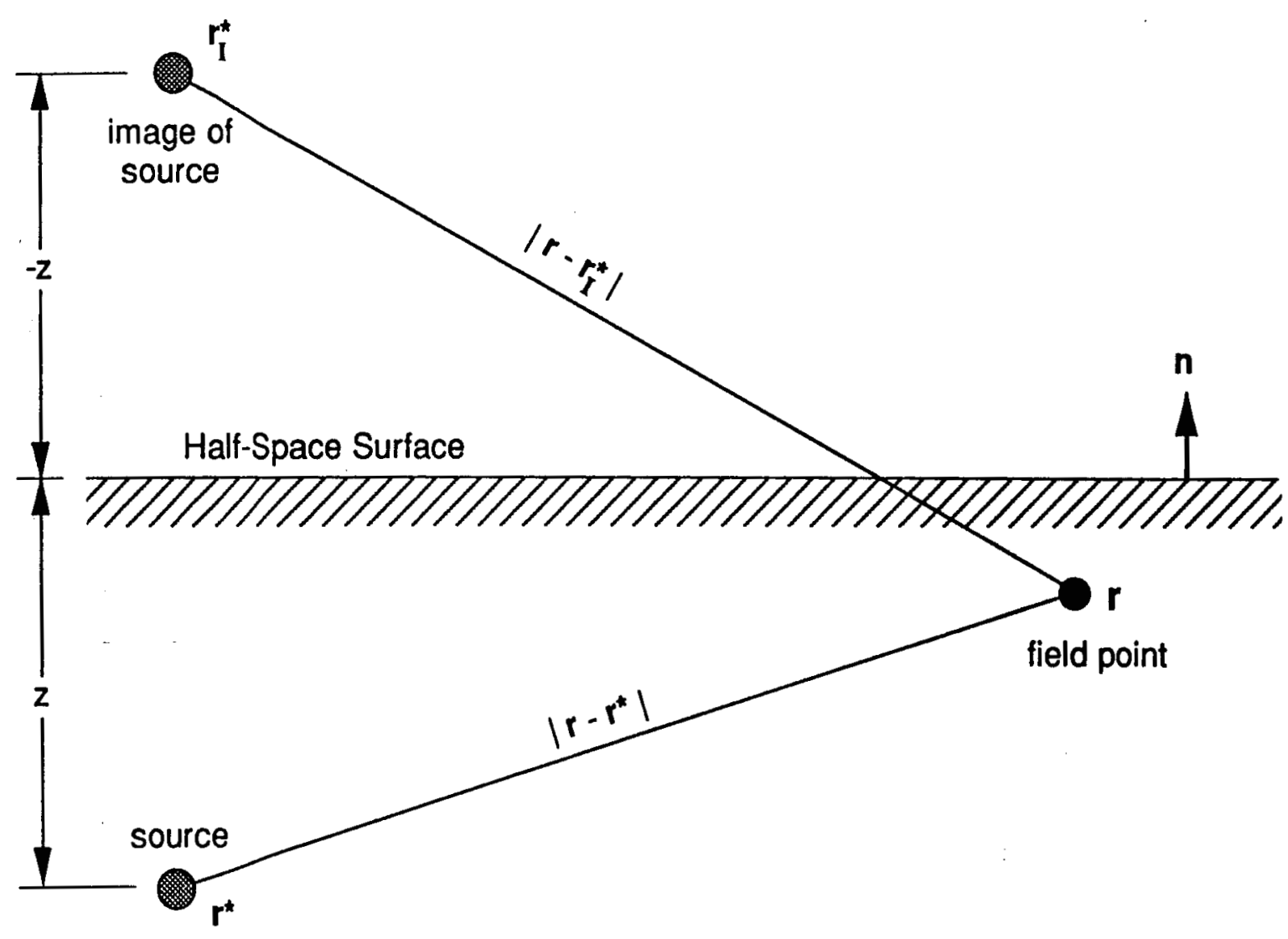

Figure A-1: The positions of the point source and its image with respect to the half-space plane. Both are used to calculate the half-space Green's function. 


\section{APPENDIX B}

\section{NUMERICAL APPROXIMATION: METHOD OF MOMENTS}

The integral equations derived in Chapter 2 are in the form of the Fredholm integral equation of the second kind. Very few of these types of equations, frequently encountered in geophysical electromagnetic (EM) problems, can be solved analytically in closed form. However, there are several approximation methods available that can obtain satisfactory solutions. One such technique is the method of moments, also known as the method of weighted residuals or point matching over subsectional basis. A solution to the field problem is obtained by reducing a functional equation into a matrix equation, and then solving the matrix equation by known techniques. Harrington (1968) is an excellent general reference on matrix methods to compute the solutions of field problems. Hohmann (1988) and Balanis (1989) are more specific and apply the method of moments to EM field problems.

This appendix will outline the method of moments described by Balanis as applied to the solving the IE. An IE can be represented as a linear inhomogeneous equation of the form:

$$
L(f)=g
$$

where $L$ is an integral operator, $g$ is the known source or excitation function, and $f$ is the unknown field or response function. Both $f$ and $g$ can be scalar or vector quantities. If the solution to equation (B-1) exist and is unique for all $g$, the inverse operator $L^{-1}$ exists such that:

$$
f=L^{-1}(g)
$$

Since $g$ is known, equation (B-2) represents the solution to the problem. To linearize the problem, the unknown field can be expanded into a finite series. ${ }^{1}$

1 An exact solution can be obtained if the series is an infinite summation. 


$$
f=\sum_{n=1}^{N} \alpha_{n} f_{n}
$$

where $\alpha_{n}$ are the constant coefficients and $f_{n}$ are the basis or expansion functions.

At this point, one would like to choose a basis function that can accurately represent the unknown function while minimizing the computational effort In practice, there are two classes of basis functions used. The first is the entire domain (ED) basis function where the bases exist over the entire region of interest. Although no segmentation of the domain is required, the ED basis functions usually have difficulty in modeling complicated or arbitrary unknown functions. One common ED basis set is that of sinusoidal functions. Other ED functions can be generated by polynomials, such as Tschebyscheff, Maclaurin, or Legendre.

The second class of basis function is the subdomain or subsectional bases. These bases exist only over subregions of the domain of interest. Unlike the ED basis functions, the subsectional bases may be used without a priori knowledge of the unknown function. Several kinds of subsectional basis functions exist: piecewise constant, piecewise linear, and piecewise sinusoid. The most common and easiest to use is the piecewise constant basis function which also is used for the method of methods. It is defined by:

$$
f_{n}=f_{n}(\mathbf{r})=\Pi\left(\mathbf{r}, \mathbf{r}_{n}\right)= \begin{cases}1, & \mathbf{r} \in \mathbf{r}_{n} \\ 0, & \mathbf{r} \notin \mathbf{r}_{n}\end{cases}
$$

This basis function, a rectangular function, is one only in the subregion where $r_{n}$ is defined and zero at all other locations.

Retuming to the problem of solving the linear inhomogeneous equation, by substituting equation (B-3) into equation (B-1) yields:

$$
L\left(\sum_{n} \alpha_{n} f_{n}\right)=\sum_{n} L\left(f_{n}\right)=g
$$

Since an approximation is used for the unknown field, there will be a residual $\varepsilon$ such that:

$$
\varepsilon=g-\sum_{n} \alpha_{n} L\left(f_{n}\right)
$$

The objective of the method of moments is not to force the residual to zero at every 
point $n$, but to produce a vanishing residual in an average sense over the entire domain. This is done by taking the inner product ${ }^{2}$ of the residual, equation (B-6), with a set of $N$ weighting or testing functions $\left(w_{m}\right)$ and setting the weighted residuals for all $m$ to zero:

$$
\left\langle w_{m}, \varepsilon\right\rangle=\left\langle w_{m}, g\right\rangle-\sum_{n=1}^{N} \alpha_{n}\left\langle w_{m}, L\left(f_{k}\right)\right\rangle=0
$$

This will force the residuals $\varepsilon$ to be orthogonal to the weighting functions which results in:

$$
<w_{m}, g>=\sum_{n=1}^{N} \alpha_{n}<w_{m}, L\left(f_{n}\right)>
$$

Equation (B-8) is a set of $N$ linear equations and can be written in matrix form as:

$$
\left[\mathbf{g}_{m}\right]=\left[\mathbf{L}_{m n}\right]\left[\alpha_{n}\right]
$$

If $\mathrm{L}_{m n}$ is nonsinguiar, then its inverse $\left(\mathrm{L}_{m n}{ }^{-1}\right)$ exist and the solution for the unknown coefficients $\alpha_{n}$ becomes:

$$
\left[\boldsymbol{\alpha}_{n}\right]=\left[\mathbf{L}_{m n}{ }^{-1}\right]\left[\mathbf{g}_{m}\right]
$$

The weighting function must be chosen such that the elements of $\mathbf{w}_{m}$ are linearly independent. Otherwise, the $N$ linear equations will not be independent and the matrix $\mathbf{L}_{m n}$ may be ill-conditioned. Another factor which affects the choice of the weighting function is the ease of evaluation for the matrix elements $\left[\mathbf{L}_{m n}\right]$.

For the method of moments, the weighting functions are replaced by the Dirac delta functions. This results in obtaining the solution at discrete points in the region of interest. In equation (B-8), replacing $\mathbf{w}_{m}$ with the Dirac delta function results in:

$$
\begin{aligned}
\left\langle\delta\left(r-r_{m}\right), g\right\rangle & =\sum_{n}\left\langle\delta\left(r-r_{m}\right), L\left(f_{n}\right)\right\rangle \\
\int_{\delta} \delta\left(r-r_{m}\right) \cdot g(r) d r & =\sum_{n} \alpha_{n} \delta\left(r-r_{m}\right) \cdot L\left(f_{n}(r)\right) d r \\
\left.\rightarrow \quad g(r)\right|_{r=r_{m}} & =\left.\sum_{n} \alpha_{n} L\left(f_{n}(r)\right)\right|_{r=r_{m}}
\end{aligned}
$$

2 The inner product between vectors $\mathrm{A}$ and $\mathrm{B}$ is:

$$
\langle\mathbf{A}, \mathbf{B}\rangle=\int\left[\mathbf{A}^{*} \cdot \mathbf{B}\right] d v
$$

where $\mathbf{A}^{*}$ is the complex conjugate of $\mathbf{A}$. 
The use of the Dirac delta function in equation (B-12) relaxes the condition that equation (B5) be valid for the entire domain and only enforces the condition at the $N$-points $\mathbf{r}_{m}$. Additionally, the Dirac delta weighting function does not increase the complexity of evaluating the linear operator $L\left(f_{n}\right)$.

The method of moments uses the piecewise constant function, equation (B-4), as the basis function for equation (B-12c). This results in set of the linear equations that is solved by equation (B-11). For the method of moments, the general form of the set of linear equations can be expressed as:

$$
g\left(\mathrm{r}_{m}\right)=\sum_{n} \alpha_{n} L\left(\Pi\left(\mathrm{r}_{m}, \mathrm{r}_{n}\right)\right)
$$




\section{APPENDIX C}

\section{AXISYMMETRIC GREEN'S FUNCTION}

In this appendix, the half-space axisymmetric (ring) Green's function uses the Dirac delta source function and its image and must satisfy the goveming field equation, Poisson's equation:

$$
\nabla^{2} g\left(\mathbf{r}, \mathbf{r}^{*}\right)=-\delta\left(\mathbf{r}-\mathbf{r}^{*}\right)
$$

In an axisymmetric cylindrical coordinate system, the Laplacian of the Green's function and the Dirac delta function can be written as:

$$
\left[\frac{\partial^{2}}{\partial \rho^{2}}+\frac{1}{\rho} \frac{\partial}{\partial \rho}+\frac{\partial^{2}}{\partial z^{2}}\right] g\left(\rho, z, \rho^{*}, z^{*}\right)=-\frac{1}{2 \pi \rho}\left[\delta\left(\rho-\rho^{*}\right) \delta\left(z-z^{*}\right)+\delta\left(\rho-\rho^{*}\right) \delta\left(z+z^{*}\right)\right]
$$

The representation of the Dirac delta function in the cylindrical coordinate system is found in Stinson (1976). The delta function term on the far left of equation (C-2) represents the primary source and the right term is its image. The image is offset in the negative $z^{*}$ position with respect to the primary so that the boundary condition at the surface is satisfied. Thus, equation (C-2) is the partial differential equation (PDE) that will be solved.

Taking the Hankel transform of zero order, equation (C-2) becomes:

$$
\left[\frac{\partial^{2}}{\partial z^{2}}-\lambda^{2}\right] g\left(\lambda, z, \rho^{*}, z^{*}\right)=-\frac{1}{2 \pi} J_{0}\left(\lambda \rho^{*}\right)\left[\delta\left(z-z^{*}\right)+\delta\left(z+z^{*}\right)\right]
$$

where $\mathrm{J}_{0}(x)$ is the Bessel function of the first kind of order zero.

Applying the Fourier transform, results in the following:

$$
-\left[\lambda^{2}+k^{2}\right] g\left(\lambda, k, \rho^{*}, z^{*}\right)=-\frac{1}{2 \pi} \mathrm{J}_{0}\left(\lambda \rho^{*}\right)\left[\mathrm{e}^{-i k z^{*}}+\mathrm{e}^{i k z^{*}}\right] \text {, }
$$

which leads to: 


$$
g\left(\lambda, k, \rho^{*}, z^{*}\right)=\frac{1}{2 \pi}\left[\lambda^{2}+k^{2}\right]^{-1} \mathrm{~J}_{0}\left(\lambda \rho^{*}\right)\left[\mathrm{e}^{-i k^{*}}+\mathrm{e}^{i k z^{*}}\right]
$$

The inverse Fourier transform of equation (C-5) is:

$$
\begin{aligned}
g\left(\lambda, z, \rho^{*}, z^{*}\right) & =\frac{1}{2 \pi} \int_{0}^{-} \frac{1}{2 \pi}\left[\lambda^{2}+k^{2}\right]^{-1} J_{0}\left(\lambda \rho^{*}\right)\left[e^{-i k^{*}}+e^{i k z^{*}}\right] \mathrm{e}^{i k z} d k \\
& =\frac{1}{4 \pi \lambda} \mathrm{J}_{0}\left(\lambda \rho^{*}\right)\left[\mathrm{e}^{-\lambda \mid z-z^{*} 1}+\mathrm{e}^{-\lambda\left(z+z^{*}\right)}\right]
\end{aligned}
$$

Inverse Hankel transforming yields:

$$
\begin{aligned}
g\left(\rho, z, \rho^{*}, z^{*}\right) & =\frac{1}{4 \pi} \int_{0}^{\infty} J_{0}\left(\lambda \rho^{*}\right)\left[\mathrm{e}^{-\lambda \mid z-z^{*} 1}+\mathrm{e}^{-\lambda\left(z+z^{*}\right)}\right] \mathrm{J}_{0}(\lambda \rho) d \lambda \\
& =\frac{1}{4 \pi} \int_{0}^{\infty} \mathrm{J}_{0}(\lambda \rho) \mathrm{J}_{0}\left(\lambda \rho^{*}\right)\left[\mathrm{e}^{-\lambda \mid z-z^{*}}+\mathrm{e}^{-\lambda\left(z+z^{*}\right)}\right] d \lambda
\end{aligned}
$$

Equation (C-7) is the half-space axisymmetric Green's function that satisfies Poisson's equation. It can be expressed in terms of elliptical integrals but will be left in this form for convenience.

The axisymmetric dyadic Green's function is found by applying the double gradient dyad, equation (A-10), to the axisymmetric scalar Green's function which can be expressed as:

$$
\begin{gathered}
\dot{G}\left(\mathbf{r}, \mathbf{r}^{*}\right)=\frac{1}{\sigma} \nabla \nabla g\left(r, r^{*}\right)=-\frac{1}{\sigma} \nabla \nabla^{*} g\left(r, r^{*}\right) \\
\rightarrow \quad G\left(\rho, z, \rho^{*}, z^{*}\right)=-\frac{1}{\sigma}\left[\begin{array}{cc}
\rho \frac{\partial^{2}}{\partial \rho \partial \rho^{*}} \rho^{*} z \frac{\partial^{2}}{\partial z \partial \rho^{*}} \rho^{*} \\
\rho \frac{\partial^{2}}{\partial \rho \partial z^{*}} z^{*} z \frac{\partial^{2}}{\partial z \partial z^{*}} z^{*}
\end{array}\right] g\left(\rho, z, \rho^{*}, z^{*}\right)
\end{gathered}
$$




\section{APPENDIX D}

\section{THE $\stackrel{P}{P}$ FUNCTIONS}

This appendix describes the derivation of the solutions for the axisymmetric Green's function volume integral $\vec{F}$-function. This $\vec{F}$-function can be expressed as:

$$
\vec{\Gamma}\left(\mathbf{r}, \mathbf{r}^{*}\right)=-\frac{2 \pi \Delta \sigma}{\sigma_{1}} \int_{z^{*}} \int_{p^{*}} \nabla \nabla^{*} g\left(\mathbf{r}, \mathbf{r}^{*}\right) \rho^{*} d \rho^{*} d z^{*}
$$

where $g\left(r, r^{*}\right)$ is the axisymmetric Green's function, equation (C-7) in Appendix C. The elements of the dyadic $\vec{P}$-function are:

$$
\left[\begin{array}{ll}
\Gamma_{p \rho^{*}} & \Gamma_{\rho z^{*}} \\
\Gamma_{z \rho^{*}} & \Gamma_{z z^{*}}
\end{array}\right]=-\frac{2 \pi \Delta \sigma}{\sigma_{1}} \int_{z^{*} \rho^{*}}\left[\begin{array}{ll}
\frac{\partial}{\partial \rho} \frac{\partial}{\partial \rho^{*}} & \frac{\partial}{\partial \rho} \frac{\partial}{\partial z^{*}} \\
\frac{\partial}{\partial z} \frac{\partial}{\partial \rho^{*}} & \frac{\partial}{\partial z} \frac{\partial}{\partial z^{*}}
\end{array}\right] g\left(r, r^{*}\right) \rho^{*} d \rho^{*} d z^{*}
$$

The remaining sections contain the derivation of the solution for each $\vec{F}$ component.

D1 The $\Gamma_{z^{*}}$-Function

Using the axisymmetric Green's function, the $\Gamma_{z z^{*}}$-function is

$$
\begin{aligned}
\Gamma_{z z^{*}} & =\frac{2 \pi \Delta \sigma}{\sigma_{1}} \int_{z^{*} \rho^{*}}\left[-\frac{\partial}{\partial z} \frac{\partial}{\partial z^{*}}\left[\frac{1}{4 \pi} \int_{0}^{\infty} J_{0}\left(\lambda \rho^{*}\right) J_{0}(\lambda \rho)\left(e^{-\lambda \mid z_{-} z^{*}}+e^{-\lambda\left(z+z^{*}\right)}\right) d \lambda\right] \rho^{*} d \rho^{*} d z^{*}\right. \\
& =\frac{\Delta \sigma}{2 \sigma_{1}} \int_{0}^{\infty}\left[\int_{\rho^{*}} J_{0}\left(\lambda \rho^{*}\right) \rho^{*} d \rho^{*}\right]\left[-\frac{\partial}{\partial z} \int_{z^{*}} \frac{\partial}{\partial z^{*}}\left(e^{-\lambda l_{z-z^{*} \mid}}+e^{-\lambda\left(z+z^{*}\right)}\right) d z^{*}\right] J_{0}(\lambda \rho) d \lambda
\end{aligned}
$$

The Bessel integral in equation (D1-1) is:

$$
\int_{\rho^{*}} J_{0}\left(\lambda \rho^{*}\right) \rho^{*} d \rho^{*}=\frac{1}{\lambda^{2}} \int_{b}^{c} J_{0}\left(\lambda \rho^{*}\right) \lambda \rho^{*} \lambda d \dot{\rho}^{*}=\frac{1}{\lambda}\left[b J_{1}(\lambda b)-c J_{1}(\lambda c)\right]
$$


where $b=a+\Delta a$ and $c=a-\Delta a$.

Equation (D1-2) is the solution of the integration, but can be approximated by the following procedure. Rearranging the bracketed term of equation (D1-2), one obtains:

$$
\lambda a\left[J_{1}(\lambda(a+\Delta a))-J_{1}(\lambda(a-\Delta a))\right]+\lambda \Delta a\left[J_{1}(\lambda(a+\Delta a))+J_{1}(\lambda(a-\Delta a))\right]
$$

The Taylor series expansion of the Bessel terms gives:

$$
J_{1}(\lambda(a+\Delta a))=J_{1}(\lambda a)+(\lambda \Delta a) J_{1}^{\prime}(\lambda a)+(\lambda \Delta a)^{2} J_{1}^{(2)}(\lambda a)+\cdots
$$

and

$$
J_{1}(\lambda(a-\Delta a))=J_{1}(\lambda a)-(\lambda \Delta a) J_{1}^{\prime}(\lambda a)+(\lambda \Delta a)^{2} J_{1}^{(2)}(\lambda a)-\cdots
$$

Substituting these expressions into equation (D1-3) yields:

$$
2 \lambda a\left[(\lambda \Delta a) J_{1}^{\prime}(\lambda a)+(\lambda \Delta a)^{3} J_{1}^{(3)}(\lambda a)+\cdots\right]+2 \lambda \Delta a\left[J_{1}(\lambda a)+(\lambda \Delta a)^{2} J_{1}^{(2)}(\lambda a)+\cdots\right]
$$

If one assumes that the pipe thickness $2 \Delta a$ is much less than the distance to the source and the pipe conductivity is much greater than the surrounding medium, then the "thin sheet" approximation can be used. For small thickness, the higher order terms of $\Delta a$ are negligible and equation (D1-5) can be approximated by the following:

$$
\begin{aligned}
2 \Delta a \lambda^{2} a J_{1}^{\prime}(\lambda a)+2 \Delta a \lambda J_{1}(\lambda a)=2 \Delta a \lambda\left[(\lambda a) J_{1}^{\prime}(\lambda a)+J_{1}(\lambda a)\right] & =2 \Delta a \lambda\left[\lambda a J_{0}(\lambda a)\right] \\
& =2 \Delta a \lambda^{2} a J_{0}(\lambda a)
\end{aligned}
$$

The $\lambda^{2}$ is cancelled by the $\lambda^{-2}$ term in equation (D1-2) and the Bessel integral becomes:

$$
\int_{\rho^{*}} J_{0}\left(\lambda, \rho^{*}\right) \rho^{*} d \rho^{*}=2 a \Delta a J_{0}(\lambda a)
$$

The evaluation of the exponential terms of equation (D1-1) is accomplished by the following procedure:

$$
\begin{aligned}
&-\frac{\partial}{\partial z} \int\left\{\frac{\partial}{\partial z^{*}}\left[e^{-\lambda\left|z-z^{*}\right|}+e^{-\lambda\left(z+z^{*}\right)}\right]\right\} d z^{*}=\frac{\partial}{\partial z} \frac{\partial}{\partial z} \int_{z^{*}}\left(e^{-\lambda\left|z-z^{*}\right|}+e^{-\lambda\left(z+z^{*}\right)}\right) d z^{*} \\
&=\frac{\partial}{\partial z} \frac{\partial}{\partial z} \xi(\lambda z)=\frac{\partial}{\partial z} \frac{\partial}{\partial z}\left[\int_{z_{0}^{-}}^{z_{0}^{+}} e^{-\lambda\left|z-z^{*}\right|} d z^{*}+\int_{z_{0}^{-}}^{z_{0}^{+}} e^{-\lambda\left(z+z^{*}\right)} d z^{*}\right]
\end{aligned}
$$


where $z_{0}^{+}=z_{0}+\Delta z, z_{0}^{-}=z_{0}-\Delta z$ and $\Delta z=h_{z} / 2$. Three cases occur for the evaluation of the first integral in equation (D1-8):

$$
\begin{aligned}
& \int_{z_{0}^{-}}^{z_{0}^{+}} e^{-\lambda z_{z}-z^{*}} d z^{*} \\
& = \begin{cases}\int_{z_{0}^{-}}^{z_{0}^{+}} e^{-\lambda\left(z^{*}-z\right)} d z^{*}=-\frac{1}{\lambda}\left[e^{-\lambda\left(z_{0}^{+}-z^{*}\right)}-e^{-\lambda\left(z_{0}^{-}-z\right)}\right], & z<z_{0}-\Delta z \\
z_{0}^{+} & z>z_{0}+\Delta z \\
z_{0}^{-} e^{-\lambda\left(z-z^{*}\right)} d z^{*}= & \frac{1}{\lambda}\left[e^{-\lambda\left(z-z_{0}^{+}\right)}-e^{-\lambda\left(z-z_{0}^{-}\right)}\right], \\
\int_{z_{0}^{-}}^{z} e^{-\lambda\left(z-z^{*}\right)} d z^{*}+\int_{z}^{z_{0}^{+}} e^{-\lambda\left(z^{*}-z\right)} d z^{*}=\frac{1}{\lambda}\left[2-e^{-\lambda\left(z_{0}^{+}-z\right)}-e^{-\lambda\left(z-z_{0}^{-}\right)}\right], & z_{0}-\Delta z \leq z \leq z_{0}+\Delta z\end{cases}
\end{aligned}
$$

For the second integral on the right side of equation (D1-8), the solution is:

$$
\int_{z_{0}^{-}}^{z_{0}^{+}} e^{-\lambda\left(z+z^{*}\right)} d z^{*}=-\frac{1}{\lambda} \int_{z_{0}^{-}}^{z_{0}^{+}} e^{-\lambda\left(z+z^{*}\right)} d z^{*}=-\frac{1}{\lambda}\left[e^{-\lambda\left(z+z_{0}^{+}\right)}-e^{-\lambda\left(z+z_{0}^{-}\right)}\right]
$$

Combining equations (D1-9) and (D1-10) yields,

$$
\xi(\lambda z)=\frac{1}{\lambda} \begin{cases}-e^{-\lambda\left(z_{0}^{+}-z\right)}+e^{-\lambda\left(z_{0}^{-}-z\right)}-e^{-\lambda\left(z+z_{0}^{+}\right)}+e^{-\lambda\left(z+z_{0}^{-}\right)} & z<z_{0}-\Delta z \\ +e^{-\lambda\left(z-z_{0}^{+}\right)}-e^{-\lambda\left(z-z_{0}^{-}\right)}-e^{-\lambda\left(z+z_{0}^{+}\right)}+e^{-\lambda\left(z+z_{0}^{-}\right)} & , z>z_{0}+\Delta z \\ -e^{-\lambda\left(z_{0}^{+}-z\right)}-e^{-\lambda\left(z-z_{0}^{-}\right)}-e^{-\lambda\left(z+z_{0}^{+}\right)}+e^{-\lambda\left(z+z_{0}^{-}\right)}+2, & z_{0}-\Delta z \leq z \leq z_{0}+\Delta z\end{cases}
$$

Taking the partial derivative with respect to $z$ of equation (D1-10) yields:

$$
\begin{aligned}
\xi_{z^{*}}(\lambda z)= & -\xi_{z}(\lambda z)=-\frac{\partial}{\partial z} \xi(\lambda z) \\
= & \begin{cases}+e^{-\lambda\left(z_{0}^{+}-z\right)}-e^{-\lambda\left(z_{0}^{-}-z\right)}+e^{-\lambda\left(z+z_{0}^{+}\right)}-e^{-\lambda\left(z+z_{0}^{-}\right)}, & z<z_{0}-\Delta z \\
+e^{-\lambda\left(z-z_{0}^{+}\right)}-e^{-\lambda\left(z-z_{0}^{-}\right)}+e^{-\lambda\left(z+z_{0}^{+}\right)}-e^{-\lambda\left(z+z_{0}^{-}\right)}, & z>z_{0}+\Delta z \\
+e^{-\lambda\left(z_{0}^{+}-z\right)}-e^{-\lambda\left(z-z_{0}^{-}\right)}+e^{-\lambda\left(z+z_{0}^{+}\right)}-e^{-\lambda\left(z+z_{0}^{-}\right)}, & z_{0}-\Delta z \leq z \leq z_{0}+\Delta z\end{cases}
\end{aligned}
$$

The second partial derivative with respect to $z$ gives:

$$
\lambda \xi_{z z^{*}}(\lambda z)=\lambda \begin{cases}-e^{-\lambda\left(z_{0}^{+}-z\right)}+e^{-\lambda\left(z_{0}^{-}-z\right)}+e^{-\lambda\left(z+z_{0}^{+}\right)}-e^{-\lambda\left(z+z_{0}^{-}\right)}, z<z_{0}-\Delta z \\ +e^{-\lambda\left(z-z_{0}^{+}\right)}-e^{-\lambda\left(z-z_{0}^{-}\right)}+e^{-\lambda\left(z+z_{0}^{+}\right)}-e^{-\lambda\left(z+z_{0}^{-}\right)}, \quad z>z_{0}^{\prime}+\Delta z \\ -e^{-\lambda\left(z_{0}^{+}-z\right)}-e^{-\lambda\left(z-z_{0}^{-}\right)}+e^{-\lambda\left(z+z_{0}^{+}\right)}-e^{-\lambda\left(z+z_{0}^{-}\right)}, \quad z_{0}-\Delta z \leq z \leq z_{0}+\Delta z\end{cases}
$$


The following form is used to simplify the expression in equation (D1-13).

$$
\lambda \xi_{z z^{*}}(\lambda z)=\frac{\partial}{\partial z} \xi_{z^{*}}(\lambda z)=-\frac{\partial}{\partial z} \xi_{z}(\lambda z)=\lambda \sum_{i=1}^{4}( \pm)_{i}^{z} e^{-\lambda \alpha_{i}}
$$

where: $\quad \alpha_{1}=\left|z-z_{0}^{+}\right|, \quad \alpha_{2}=\left|z-z_{0}^{-}\right|, \quad \alpha_{3}=\left(z+z_{0}^{+}\right), \quad \alpha_{4}=\left(z+z_{0}^{+}\right)$,

and the values of $( \pm)_{i}^{z}= \pm 1$ are dependent on $z, z_{0}$, and $\Delta z$ in equation (D1-13).

Combining the evaluations of the Bessel and exponential terms, equation (D1-1) is:

$$
\begin{aligned}
\Gamma_{z^{*}} & =\frac{\Delta \sigma}{2 \sigma_{1}} \int_{0}^{\infty}\left[\frac{1}{\lambda}\left[b_{1}(\lambda b)-\sigma_{1}(\lambda c)\right]\right]\left[\lambda \sum_{i=1}^{4}( \pm)_{i}^{2} e^{-\lambda \alpha_{i}}\right] J_{0}(\lambda \rho) d \lambda \\
& =\frac{\Delta \sigma}{2 \sigma_{1}} \sum_{i=1}^{4}( \pm)_{i}^{2}\left[b \int_{0}^{\infty} J_{1}(\lambda b) J_{0}(\lambda \rho) e^{-\lambda \alpha_{i}} d \lambda-c \int_{0}^{\infty} J_{1}(\lambda c) J_{0}(\lambda \rho) e^{-\lambda \alpha_{i}} d \lambda\right]
\end{aligned}
$$

The general solution for the integral in equation (D1-15) has the form of equation (3.1.1-9):

$$
\rho^{*} \int_{0}^{\infty} J_{1}\left(\lambda \rho^{*}\right) J_{0}(\lambda \rho) e^{-\lambda \alpha_{i}} d \lambda=F_{100}\left(\rho^{*}, \rho, \alpha_{i}\right)
$$

Thus, the form of the solution can be expressed as:

$$
\Gamma_{z z^{*}}=\frac{\Delta \sigma}{2 \sigma_{1}} \sum_{i=1}^{4}( \pm)_{i}^{z}\left[F_{100}\left(b, \rho, \alpha_{i}\right)-F_{100}\left(c, \rho, \alpha_{i}\right)\right]
$$

Using equation (D1-2), the approximate solution of the $\rho^{*}$-integration, equation (D1-1) is:

$$
\begin{aligned}
\Gamma_{z z^{*}} & =\frac{\Delta \sigma}{2 \sigma_{1}} \int_{0}^{\infty}\left[2 a \Delta a J_{0}(\lambda a)\right]\left[\lambda \sum_{i=1}^{4}( \pm)_{i}^{z} e^{-\lambda \alpha_{i}}\right] J_{0}(\lambda \rho) d \lambda \\
& =\frac{a \Delta a \Delta \sigma}{\sigma_{1}} \sum_{i=1}^{4}( \pm)_{i}^{z} \int_{0}^{\infty} J_{0}(\lambda a) J_{0}(\lambda \rho) e^{-\lambda \alpha_{i}} \lambda d \lambda
\end{aligned}
$$

The integral of the above equation (D1-18) can be found in Luke (1962) and is:

$$
\int_{0}^{\infty} J_{0}(\lambda a) J_{0}(\lambda \rho) e^{-\lambda \alpha_{i}} \lambda d \lambda=\frac{\alpha_{i}}{4 \pi(\rho a)^{3 / 2}} \frac{k_{i}^{3}}{\left(1-k_{i}\right)^{2}} \mathbf{E}\left(k_{i}\right)
$$

where:

$$
k_{i}=\left[\frac{4 \rho a}{\alpha_{i}^{2}+(\rho+a)^{2}}\right]^{1 / 2}
$$


and $\mathbf{E}(k)$ is the complete elliptic integral of the second kind.

Several tests were done to check the approximation solution equation (D1-2) with the exact solution equation (D1-17). These tests showed that the two solutions gave nearly the same results when using typical values of the pipe model. Since the exact solution required much more time to compute than the solution of the approximation, the approximate expression (D1-19) was used to solve the problem.

The approximation solution used for $\Gamma_{2 *}$ is:

$$
\Gamma_{z z^{*}}=\frac{a \Delta a \Delta \sigma}{\sigma_{1}} \sum_{i=1}^{4}( \pm)_{i}^{z}\left[\frac{\alpha_{i}}{4 \pi(\rho a)^{3 / 2}} \frac{k_{i}^{3}}{\left(1-k_{i}\right)^{2}} \mathbf{E}\left(k_{i}\right)\right]
$$

D2 The $\Gamma_{p z^{*}}$ Function

The integral form of $\Gamma_{\rho z^{*}}$ can be found by axisymmetric Green's function:

$$
\begin{aligned}
\Gamma_{\rho z^{*}} & =\frac{\Delta \sigma}{2 \sigma_{1}} \int_{z^{*}} \int_{\rho^{*}}\left[-\frac{\partial}{\partial \rho} \frac{\partial}{\partial z^{*}}\left[\int_{0}^{\infty} J_{0}\left(\lambda \rho^{*}\right) J_{0}(\lambda \rho)\left(e^{-\lambda\left|z-z^{*}\right|}+e^{-\lambda\left(z+z^{*}\right)}\right) d \lambda\right]\right] \rho^{*} d \rho^{*} d z^{*} \\
& =\frac{\Delta \sigma}{2 \sigma_{1}} \int_{0}\left[\int_{\rho^{*}} J_{0}\left(\lambda \rho^{*}\right) \rho^{*} d \rho^{*}\right]\left[-\int_{z^{*}} \frac{\partial}{\partial z^{*}}\left(e^{-\lambda \mid z-z^{*} 1}+e^{-\lambda\left(z+z^{*}\right)}\right) d z^{*}\right]\left[\frac{\partial}{\partial \rho} J_{0}(\lambda \rho)\right] d \lambda
\end{aligned}
$$

The Bessel integral evaluation is the same as that used for the $\Gamma_{2 z^{*}}$-function, equation (D1-7):

$$
\begin{aligned}
\int_{\rho^{*}} J_{0}\left(\lambda \rho^{*}\right) \rho^{*} d \rho^{*} & =\frac{1}{\lambda}\left[b J_{1}(\lambda b)-c J_{1}(\lambda c)\right] \\
& \approx 2 a \Delta a J_{0}(\lambda a)
\end{aligned}
$$

where $b=a+\Delta a$ and $c=a-\Delta a$.

The evaluation of the $z^{*}$-integration is also the same as equation (D1-11):

$$
\begin{aligned}
\xi_{z}(\lambda z) & =-\xi_{z^{*}}(\lambda z)=-\int_{z^{*}} \frac{\partial}{\partial z^{*}}\left[e^{-\lambda\left|z^{-z^{*}}\right|}-e^{-\lambda\left(z+z^{*}\right)}\right] d z^{*} \\
& =\left\{\begin{array}{ll}
-e^{-\lambda\left(z_{0}^{+}-z\right)}+e^{-\lambda\left(z_{0}^{-}-z\right)}-e^{-\lambda\left(z+z_{0}^{+}\right)}+e^{-\lambda\left(z+z_{0}^{-}\right)}, & z<z_{0}^{-} \\
-e^{-\lambda\left(z-z_{0}^{+}\right)}+e^{-\lambda\left(z-z_{0}^{-}\right)}-e^{-\lambda\left(z+z_{0}^{+}\right)}+e^{-\lambda\left(z+z_{0}^{-}\right)}, & z>z_{0}^{+} \\
-e^{-\lambda\left(z_{0}^{+}-z\right)}+e^{-\lambda\left(z-z_{0}^{-}\right)}-e^{-\lambda\left(z+z_{0}^{+}\right)}+e^{-\lambda\left(z+z_{0}^{-}\right)}, & z_{0}^{-} \leq z \leq z_{0}^{+}
\end{array}\right\}=\sum_{i=1}^{4}(-1)^{i} e^{-\lambda \alpha_{i}}
\end{aligned}
$$

where:

$$
\alpha_{1}=\left|z-z_{0}^{+}\right|, \quad \alpha_{2}=\left|z-z_{0}^{-}\right|, \quad \alpha_{3}=\left(z+z_{0}^{+}\right), \quad \alpha_{4}=\left(z^{\prime}+z_{0}^{-}\right),
$$




$$
z_{0}^{+}=z_{0}+\Delta z \quad \text { and } \quad z_{0}^{-}=z_{0}-\Delta z
$$

Evaluating the $\rho$-derivative of the zero order Bessel function and using equations (D2-2a) and (D2-3), the solution of equation (D2-1) is:

$$
\begin{aligned}
\Gamma_{\rho z^{*}} & =\frac{\Delta \sigma}{2 \sigma_{1}} \int_{0}^{\infty}\left[\frac{1}{\lambda}\left[b J_{1}(\lambda b)-c J_{1}(\lambda c)\right]\right]\left[\sum_{i=1}^{4}(-1)^{i} e^{-\lambda \alpha_{i}}\right]\left[-\lambda J_{1}(\lambda \rho)\right] d \lambda \\
& =\frac{\Delta \sigma}{2 \sigma_{1}} \sum_{i=1}^{4}(-1)^{i+1}\left[b \int_{0}^{-} J_{1}(\lambda b) J_{1}(\lambda \rho) e^{-\lambda \alpha_{i}} d \lambda-c \int_{0}^{-} J_{1}(\lambda c) J_{1}(\lambda \rho) e^{-\lambda \alpha_{i}} d \lambda\right]
\end{aligned}
$$

The general solution for the integral in equation (D2-4), found in Luke (1962), is:

$$
F_{110}\left(\rho^{*}, \rho, \alpha_{i}\right)=\rho^{*} \int_{0}^{\infty} J_{1}\left(\lambda \rho^{*}\right) J_{1}(\lambda \rho) e^{-\lambda \alpha_{i}} d \lambda=\frac{\rho^{*}}{\pi\left(\rho \rho^{*}\right)^{1 / 2} k_{i}}\left[\left(2-k_{i}^{2}\right) \mathbf{K}\left(k_{i}\right)-2 \mathbf{E}\left(k_{i}\right)\right]
$$

where:

$$
k_{i}=\left[\frac{4 \rho \rho^{*}}{\alpha_{i}^{2}+\left(\rho+\rho^{*}\right)^{2}}\right]^{1 / 2}
$$

$\mathbf{K}(k)$ and $\mathbf{E}(k)$ are the complete elliptic integrals of the first and second kind, respectively.

The form for the exact solution of equation (D2-1) is:

$$
\Gamma_{\rho z^{*}}=\frac{\Delta \sigma}{2 \sigma_{1}} \sum_{i=1}^{4}(-1)^{i+1}\left[F_{110}\left(b, \rho, \alpha_{i}\right)-F_{110}\left(c, \rho, \alpha_{i}\right)\right]
$$

Using the approximation for the $\rho^{*}$-integration (equation D1-7), equation (D2-1) can be written as:

$$
\begin{aligned}
\Gamma_{\rho z^{*}} & =\frac{\Delta \sigma}{2 \sigma_{1}} \int_{0}^{\infty}\left[2 a \Delta a J_{0}(\lambda a)\right]\left[\sum_{i=1}^{4}(-1)^{i} e^{-\lambda \alpha_{i}}\right]\left[-\lambda J_{1}(\lambda \rho)\right] d \lambda \\
& =\frac{a \Delta a \Delta \sigma}{\sigma_{1}} \sum_{i=1}^{4}(-1)^{i+1} \int_{0}^{\infty} J_{0}(\lambda a) J_{1}(\lambda \rho) e^{-\lambda \alpha_{i}} \lambda d \lambda
\end{aligned}
$$

The integral of the above equation (D2-9) is (Luke, 1962):

$$
\int_{0}^{\infty} J_{0}(\lambda a) J_{1}(\lambda \rho) e^{-\lambda \alpha_{i}} \lambda d \lambda=\frac{k_{i}}{2 \pi \rho(\rho a)^{1 / 2}}\left[\mathbf{K}\left(k_{i}\right)-\frac{\alpha_{i}^{2}+a^{2}-\rho^{2}}{\alpha_{i}^{2}+(a-\rho)^{2}} \mathbf{E}\left(k_{i}\right)\right]
$$

Again, the approximation solution, equation (D2-10), gave nearly the same result as the exact one for the model parameters used. Hence, the approximate expression for $\Gamma_{\rho z^{*}}$ can be used and is: 


$$
\Gamma_{\rho 2^{*}}=\frac{a \Delta a \Delta \sigma}{\sigma_{1}} \sum_{i=1}^{4}(-1)^{i+1} \frac{k_{i}}{2 \pi \rho(\rho a)^{1 / 2}}\left[\mathbf{K}\left(k_{i}\right)-\frac{\alpha_{i}^{2}+a^{2}-\rho^{2}}{\alpha_{i}^{2}+(a-\rho)^{2}} \mathbf{E}\left(k_{i}\right)\right]
$$

\section{D3 The $\Gamma_{z p}$-Function}

The derivation for $\Gamma_{z p^{*}}$-function is contained in this section. The $\Gamma_{z p^{*}}$-function can be expressed as:

$$
\begin{aligned}
\Gamma_{z \rho^{*}} & =\frac{\Delta \sigma}{2 \sigma_{1}} \int_{z^{*}} \int_{\rho^{*}}\left[-\frac{\partial}{\partial z} \frac{\partial}{\partial \rho^{*}}\left[\int_{0}^{\infty} J_{0}\left(\lambda \rho^{*}\right) J_{0}(\lambda \rho)\left(e^{-\lambda l z-z^{*} 1}+e^{-\lambda\left(z+z^{*}\right)}\right) d \lambda\right] \rho^{*} d \rho^{*} d z^{*}\right] \\
& =\frac{\Delta \sigma}{2 \sigma_{1}} \int_{0}^{\infty}\left[-\int_{\rho^{*}}\left[\frac{\partial}{\partial \rho^{*}} J_{0}\left(\lambda \rho^{*}\right)\right] \rho^{*} d \rho^{*}\right]\left[\int_{z^{*}} \frac{\partial}{\partial z}\left(e^{-\lambda l_{z-z^{*}}}+e^{-\lambda\left(z+z^{*}\right)}\right) d z^{*}\right] J_{0}(\lambda \rho) d \lambda(\mathrm{D} 3-1)
\end{aligned}
$$

Taking the $\rho^{*}$-derivative of the integrand gives:

$$
-\int_{\rho^{*}}\left[\frac{\partial}{\partial \rho^{*}} J_{0}\left(\lambda \rho^{*}\right)\right] \rho^{*} d \rho^{*}=-\int_{\rho^{*}}-\lambda J_{1}\left(\lambda \rho^{*}\right) \rho^{*} d \rho^{*}=\frac{1}{\lambda}\left[\int_{0}^{\lambda b} x J_{0}(x) d x-\int_{0}^{\lambda c} y J_{0}(y) d y\right]
$$

The solution of the $\rho^{*}$-integral of (D3-2) is much more complex than in the previous sections. The following steps are used to find $\Gamma_{z p^{*}}$. Both the integrals on the right side of (D3-2) have the same form and can be evaluated by integration by parts:

$$
\int_{0}^{w} \omega J_{1}(\omega) d \omega=-w J_{0}(w)+\int_{0}^{w} J_{0}(\omega) d \omega=\frac{\pi}{2} w\left[\mathbf{H}_{0}(w) J_{1}(w)-\mathbf{H}_{1}(w) J_{0}(w)\right]
$$

where $H_{0}(w)$ and $H_{1}(w)$ are Struve functions of the zero and first order, respectively. By letting $x=\lambda b, y=\lambda c$,

$$
\mathbf{F}(w)=\mathbf{H}_{0}(w) J_{1}(w),
$$

and

$$
\mathbf{G}(w)=\mathbf{H}_{1}(w) J_{0}(w),
$$

equation (D3-2) can be expressed as:

$$
\begin{aligned}
-\int_{\rho^{*}}\left[\frac{\partial}{\partial \rho^{*}} J_{1}\left(\lambda \rho^{*}\right)\right] \rho^{*} d \rho^{*} & =\frac{1}{\lambda}\left\{\frac{\pi}{2} x[\mathbf{F}(x)-\mathbf{G}(x)]-\frac{\pi}{2} y[\mathbf{F}(y)-\mathbf{G}(y)]\right\} \\
& =\frac{\pi}{2} b[\mathbf{F}(x)-\mathbf{G}(x)]-\frac{\pi}{2} c[\mathbf{F}(y)-\mathbf{G}(y)]
\end{aligned}
$$


Equations (D3-6) are the solution to the $\rho^{\bullet}$-integration. Unfortunately, the $\lambda$-integration of equation (D3-1) cannot be solved analytically using equation (D3-6). Therefore, an approximation of equation (D3-6) will be calculated. Rearranging the terms of equation (D3-6a) yields:

$$
\frac{\pi}{2} \frac{1}{\lambda}\{[x \mathbf{F}(x)-y \mathbf{F}(y)]-[x \mathbf{G}(x)-\mathbf{G}(y)]\}
$$

The Taylor series expansion of the $F(x)$ and $F(y)$ are:

$$
\mathbf{F}(x)=\mathbf{F}(\lambda(a+\Delta a))=\mathbf{F}(\lambda a)+(\lambda \Delta a) \mathbf{F}^{\prime}(\lambda a)+(\lambda \Delta a)^{2} \mathbf{F}^{(2)}(\lambda a)+\cdots
$$

and

$$
\mathbf{F}(y)=\mathbf{F}(\lambda(a-\Delta a))=\mathbf{F}(\lambda a)-(\lambda \Delta a) \mathbf{F}^{\prime}(\lambda a)+(\lambda \Delta a)^{2} \mathbf{F}^{(2)}(\lambda a)-\cdots
$$

The left bracketed term of equation (D3-7) can be written as:

$$
\begin{aligned}
{[x \mathbf{F}(x)-y \mathbf{F}(y)]=} & \lambda(a+\Delta a)\left[\mathbf{F}+(\lambda \Delta a) \mathbf{F}^{\prime}+(\lambda \Delta a)^{2} \mathbf{F}^{(2)}+\cdots\right] \\
& \quad-\lambda(a-\Delta a)\left[\mathbf{F}-(\lambda \Delta a) \mathbf{F}^{\prime}+(\lambda \Delta a)^{2} \mathbf{F}^{(2)}-\cdots\right] \\
= & 2\left\{\lambda a\left[(\lambda \Delta a) \mathbf{F}^{\prime}+(\lambda \Delta a)^{3} \mathbf{F}^{(3)}+\cdots\right]+\lambda \Delta a\left[\mathbf{F}+(\lambda \Delta a)^{2} \mathbf{F}^{(2)}+\cdots\right]\right\}
\end{aligned}
$$

where $\mathbf{F}=\mathbf{F}(\lambda a)$

If the thickness is much less than the distance to the field point and the conductivity of the pipe is much greater than the surrounding medium, then the higher order terms of $\Delta a$ are negligible and equation (D3-9) can be approximated:

$$
[x \mathrm{~F}(x)-y \mathrm{~F}(y)]=2 \lambda \Delta a\left[\mathrm{~F}(\lambda a)+(\lambda a) \mathrm{F}^{\prime}(\lambda a)\right]
$$

Likewise for the right bracketed term:

$$
[x \mathbf{G}(x)-y \mathbf{G}(y)] \approx 2 \lambda \Delta a\left[\mathbf{G}(\lambda a)+(\lambda a) \mathbf{G}^{\prime}(\lambda a)\right]
$$

The derivatives of $\mathbf{F}$ and $\mathbf{G}$ are:

$$
\begin{aligned}
\mathbf{F}^{\prime}(w) & =J_{1}^{\prime}(w) \mathbf{H}_{0}(w)+J_{1}(w) \mathbf{H}_{0}^{\prime}(w)=\left[J_{0}(w)-\frac{J_{1}(w)}{w}\right] \mathbf{H}_{0}(w)+J_{1}(w)\left[\frac{2}{\pi}-\mathbf{H}_{1}(w)\right] \\
& =J_{0}(w) \mathbf{H}_{0}(w)-\frac{J_{1}(w) \mathbf{H}_{0}(w)}{w}+\frac{2}{\pi} J_{1}(w)-J_{1}(w) \mathbf{H}_{1}(w)
\end{aligned}
$$

and 


$$
\begin{aligned}
\mathbf{G}^{\prime}(w) & =J_{0}^{\prime}(w) \mathbf{H}_{1}(w)+J_{0}(w) \mathbf{H}_{1}^{\prime}(w)=-J_{1}(w) \mathbf{H}_{1}(w)+J_{0}(w)\left[\mathbf{H}_{0}(w)-\frac{\mathbf{H}_{1}(w)}{w}\right] \\
& =J_{0}(w) \mathbf{H}_{0}(w)-J_{1}(w) \mathbf{H}_{1}(w)-\frac{J_{0} \mathbf{H}_{1}(w)}{w}
\end{aligned}
$$

Using the relation of equations (D3-4) and (D3-11) for $F, G, F^{\prime}$, and $\mathbf{G}^{\prime}$, the right side of equation (D3-10) becomes:

$$
\begin{aligned}
{\left[\mathbf{F}(\lambda a)+\lambda a \mathbf{F}^{\prime}(\lambda a)\right] } & =\mathbf{H}_{0}(\lambda a) J_{1}(\lambda a)+\lambda a\left[\mathbf{H}_{0}(\lambda a) J_{0}(\lambda a)-\frac{\mathbf{H}_{0}(\lambda a) J_{1}(\lambda a)}{\lambda a}+\frac{2}{\pi} J_{1}(\lambda a)-\mathbf{H}_{1}(\lambda a) J_{1}(\lambda a)\right] \\
& =\lambda a\left[J_{0}(\lambda a) \mathbf{H}_{0}(\lambda a)+\frac{2}{\pi} J_{1}(\lambda a)-J_{1}(\lambda a) \mathbf{H}_{1}(\lambda a)\right]
\end{aligned}
$$

and

$$
\begin{aligned}
{\left[\mathbf{G}(\lambda a)+\lambda a \mathbf{G}^{\prime}(\lambda a)\right] } & =\mathbf{H}_{1}(\lambda a) J_{0}(\lambda a)+\lambda a\left[\mathbf{H}_{0}(\lambda a) J_{0}(\lambda a)-\mathbf{H}_{1}(\lambda a) J_{1}(\lambda a)-\frac{J_{0}(\lambda a) \mathbf{H}_{1}(\lambda a)}{\lambda a}\right] \\
& =\lambda a\left[J_{0}(\lambda a) \mathbf{H}_{0}(\lambda a)-J_{1}(\lambda a) \mathbf{H}_{1}(\lambda a)\right]
\end{aligned}
$$

Using the relation between equations (D3-10) and (D3-12), equation (D3-7) becomes:

$$
\begin{aligned}
\frac{\pi}{2} \frac{1}{\lambda}\left\{2 \lambda ^ { 2 } a \Delta a \left[J_{0}(\lambda a) \mathbf{H}_{0}(\lambda a)\right.\right. & \left.\left.+\left[\frac{2}{\pi}-\mathbf{H}_{1}(\lambda a)\right] J_{1}(\lambda a)\right]\right\} \\
& -\frac{\pi}{2} \frac{1}{\lambda}\left\{2 \lambda^{2} a \Delta a\left[J_{0}(\lambda a) \mathbf{H}_{0}(\lambda a)-J_{1}(\lambda a) \mathbf{H}_{1}(\lambda a)\right]\right\}=2 a \Delta a \lambda J_{1}(\lambda a)
\end{aligned}
$$

The expression used to approximate the $\rho^{*}$-integral follows:

$$
-\int_{\rho^{*}}\left[\frac{\partial}{\partial \rho^{*}} J_{0}\left(\lambda \rho^{*}\right)\right] \rho^{*} d \rho^{*} \approx 2 a \Delta a \lambda J_{1}(\lambda a)
$$

The exponential term of equation (D3-1) is equivalent to the ones of the previous sections:

$$
\int_{z^{*}} \frac{\partial}{\partial z}\left[e^{-\lambda\left|z-z^{*}\right|}+e^{-\lambda\left(z+z^{*}\right)}\right] d z^{*}=\frac{\partial}{\partial z} \int_{z^{*}}\left[e^{-\lambda\left|z-z^{*}\right|}+e^{-\lambda\left(z+z^{*}\right)}\right] d z^{*}=\xi_{z}(\lambda z)=-\xi_{z z^{*}}(\lambda z)
$$

The solution to the exponential term is found in equation (zz-12) and can be written as:

$$
\xi_{z}(\lambda z)=\left\{\begin{array}{ll}
-e^{-\lambda\left(z_{0}^{+}-z\right)}+e^{-\lambda\left(z_{0}^{-}-z\right)}-e^{-\lambda\left(z+z_{0}^{+}\right)}+e^{-\lambda\left(z+z_{0}^{-}\right)}, \quad z<z_{0}^{-} \\
-e^{-\lambda\left(z-z_{0}^{+}\right)}+e^{-\lambda\left(z-z_{0}^{-}\right)}-e^{-\lambda\left(z+z_{0}^{+}\right)}+e^{-\lambda\left(z+z_{0}^{-}\right)}, & z>z_{0}^{+} \\
-e^{-\lambda\left(z_{0}^{+}-z\right)}+e^{-\lambda\left(z-z_{0}^{-}\right)}-e^{-\lambda\left(z+z_{0}^{+}\right)}+e^{-\lambda\left(z+z_{0}^{-}\right)}, & z_{0}^{-} \leq z \leq z_{0}^{+}
\end{array}\right\}=\sum_{i=1}^{4}(-1)^{i} e^{-\lambda \alpha_{i}}
$$


where: $\quad \alpha_{1}=\left|z-z_{0}^{+}\right|, \quad \alpha_{2}=\left|z-z_{0}^{-}\right|, \quad \alpha_{3}=\left(z+z_{0}^{+}\right), \quad \alpha_{4}=\left(z+z_{0}^{-}\right)$,

$$
z_{0}^{+}=z+\Delta z \text { and } z_{0}^{-}=z-\Delta z
$$

Using equations (D3-14) and (D3-17), the solution for equation (D3-1) can be expressed as:

$$
\begin{aligned}
\Gamma_{2 \rho^{*}} & =\frac{\Delta \sigma}{2 \sigma_{1}} \int_{0}^{\infty}\left[2 a \Delta a \lambda J_{1}(\lambda a)\right]\left[\sum_{i=1}^{4}(-1)^{i} e^{-\lambda \alpha_{i}}\right] J_{0}(\lambda \rho) d \lambda \\
& =\frac{a \Delta a \Delta \sigma}{\sigma_{1}} \sum_{i=1}^{4}(-1)^{i} \int_{0}^{-} J_{1}(\lambda a) J_{0}(\lambda \rho) e^{-\lambda \alpha_{i}} \lambda d \lambda
\end{aligned}
$$

The above integral has the same form of equation (2.3.2-10) except the $a$ and $\rho$ terms have reverse roles. Therefore, using the derivation from Section (2.3.2), the expression (D3-18) is:

$$
\int_{0}^{\infty} J_{1}(\lambda a) J_{0}(\lambda \rho) e^{-\lambda \alpha_{i}} \lambda d \lambda=\frac{k_{i}}{2 \pi a(\rho a)^{1 / 2}}\left[\mathbf{K}\left(k_{i}\right)-\frac{\alpha_{i}^{2}+\rho^{2}-a^{2}}{\alpha_{i}^{2}+(\rho-a)^{2}} \mathbf{E}\left(k_{i}\right)\right]
$$

and the expression for $\Gamma_{z \rho^{*}}$ becomes:

$$
\Gamma_{2 \mathrm{p}^{*}}=\frac{a \Delta a \Delta \sigma}{\sigma_{1}} \sum_{i=1}^{4}(-1)^{i}\left\{\frac{k_{i}}{2 \pi a(\rho a)^{1 / 2}}\left[\mathbf{K}\left(k_{i}\right)-\frac{\alpha_{i}^{2}+\rho^{2}-a^{2}}{\alpha_{i}^{2}+(\rho-a)^{2}} \mathbf{E}\left(k_{i}\right)\right]\right\}
$$

D4 The $\Gamma_{\rho \rho^{*}}-$ Function

The integral form of the $\Gamma_{p p^{*}}$-function can be expressed as:

$$
\begin{aligned}
\Gamma_{\rho \rho^{*}} & =\frac{\Delta \sigma}{2 \sigma_{1}} \int_{z^{*}} \int_{\rho^{*}}\left[-\frac{\partial}{\partial \rho^{*}} \frac{\partial}{\partial \rho}\left[\int_{0}^{\infty} J_{0}\left(\lambda \rho^{*}\right) J_{0}(\lambda \rho)\left(e^{-\lambda z_{z-z^{*} \mid}}+e^{-\lambda\left(z+z^{*}\right)}\right) d \lambda\right]\right] \rho^{*} d \rho^{*} d z^{*} \\
& =\frac{\Delta \sigma}{2 \sigma_{1}} \int_{0}^{\infty}\left[-\int_{\rho^{*}}\left[\frac{\partial}{\partial \rho^{*}} J_{0}\left(\lambda \rho^{*}\right)\right] \rho^{*} d \rho^{*}\right]\left[\int_{z^{*}}\left(e^{-\lambda\left|z-z^{*}\right|}+e^{-\lambda\left(z+z^{*}\right)}\right) d z^{*}\right]\left[\frac{\partial}{\partial \rho} J_{0}(\lambda \rho)\right] d \lambda
\end{aligned}
$$

The $\rho^{*}$-integration is the same one as equation (D3-14) which is approximated by the following:

$$
-\int_{\rho^{*}}\left[\frac{\partial}{\partial \rho^{*}} J_{0}\left(\lambda \rho^{*}\right)\right] \rho^{*} d \rho^{*}=2 a \Delta a \lambda J_{1}(\lambda a)
$$

The $z^{*}$-integration is also evaluated by equation (D1-11) and is:

$$
\int_{z^{*}}\left[e^{-\lambda\left|z-z^{*}\right|}+e^{-\lambda\left(z+z^{*}\right)}\right] d z^{*}=\xi(\lambda z)=-\frac{1}{\lambda} \xi_{\rho}(\lambda z)
$$

which can be written as: 


$$
\xi_{p}(\lambda z)= \begin{cases}+e^{-\lambda\left(z_{0}^{+}-z\right)}-e^{-\lambda\left(z_{0}^{-}-z\right)}-e^{-\lambda\left(z+z_{0}^{+}\right)}+e^{-\lambda\left(z+z_{0}^{-}\right)} & , z<z_{0}-\Delta z \\ -e^{-\lambda\left(z-z_{0}^{+}\right)}+e^{-\lambda\left(z-z_{0}^{-}\right)}-e^{-\lambda\left(z+z_{0}^{+}\right)}+e^{-\lambda\left(z+z_{0}^{-}\right)} & , z>z_{0}+\Delta z \\ +e^{-\lambda\left(z_{0}^{+}-z\right)}-e^{-\lambda\left(z-z_{0}^{-}\right)}-e^{-\lambda\left(z+z_{0}^{+}\right)}+e^{-\lambda\left(z+z_{0}^{-}\right)}-2, & z_{0}-\Delta z \leq z \leq z_{0}+\Delta z\end{cases}
$$

where: $\quad \alpha_{1}=\left|z-z_{0}^{+}\right|, \quad \alpha_{2}=\left|z-z_{0}^{-}\right|, \quad \alpha_{3}=\left(z+z_{0}^{+}\right), \quad \alpha_{4}=\left(z+z_{0}^{-}\right)$,

$$
z_{0}^{+}=z_{0}+\Delta z \text { and } z_{0}^{-}=z_{0}-\Delta z
$$

The following form is used to simplify the expression in equation (D4-4):

$$
\frac{1}{\lambda} \xi_{p}(\lambda z)=\frac{1}{\lambda}\left[\sum_{i=1}^{4}( \pm)_{i}^{p} e^{-\lambda \alpha_{i}}-\varepsilon_{z}\right]
$$

where $\varepsilon_{z}=2$ for $z_{0}^{-} \leq z \leq z_{0}^{+}$, and $\varepsilon_{z}=0$ for all other $z$ values.

Lastly, the $p$-derivative of equation (D4-1) is:

$$
\frac{\partial}{\partial \rho} J_{0}(\lambda \rho)=-\lambda J_{1}(\lambda \rho)
$$

Using equations (D4-3), (D4-5), and (D4-6), the solution of equation (D4-1) can be expressed as:

$$
\begin{aligned}
\Gamma_{p \rho^{*}} & =\frac{\Delta \sigma}{2 \sigma_{1}} \int_{0}^{\infty}\left[2 a \Delta a \lambda J_{1}(\lambda a)\right]\left[-\frac{1}{\lambda}\left[\sum_{i=1}^{4}( \pm)_{i}^{p} e^{-\lambda \alpha_{i}}-\varepsilon_{z}\right]\right]\left[-\lambda J_{1}(\lambda \rho)\right] d \lambda \\
& =\frac{a \Delta a \Delta \sigma}{\sigma_{1}} \sum_{i=1}^{4}\left[( \pm)_{i}^{\rho} \int_{0}^{\infty} J_{1}(\lambda a) J_{1}(\lambda \rho) e^{-\lambda \alpha_{i}} \lambda d \lambda-\varepsilon_{z} \int_{0}^{\infty} J_{1}(\lambda a) J_{1}(\lambda \rho) \lambda d \lambda\right]
\end{aligned}
$$

The solution of the left integral in equation (D4-7) is:

$$
\int_{0}^{\infty} J_{1}(\lambda a) J_{1}(\lambda \rho) e^{-\lambda \alpha_{i}} \lambda d \lambda=\frac{\alpha_{i} k_{i}}{4 \pi(\rho a)^{3 / 2}}\left[\frac{1+k^{\prime 2}}{k^{\prime 2}} \mathbf{E}\left(k_{i}\right)-2 \mathbf{K}\left(k_{i}\right)\right]
$$

where $k^{2}=1-k^{2}$.

The integral on the right of equation (D4-7) requires some manipulation. Let:

$$
\int_{0}^{\infty} J_{1}(\lambda a) J_{1}(\lambda \rho) \lambda d \lambda=-\frac{\partial}{\partial a} \int_{0}^{\infty} J_{0}(\lambda a) J_{1}(\lambda \rho) d \lambda
$$

The integral on the right side of equation (D4-9) is:

$$
\int_{0}^{\infty} J_{0}(\lambda a) J_{1}(\lambda \rho) d \lambda= \begin{cases}\rho^{-1} & , \rho>a \\ (2 a)^{-1} & , \rho=a \\ 0 & , \rho<a\end{cases}
$$


Taking the negative $a$-derivative yields:

$$
-\frac{\partial}{\partial a}\left\{\begin{array}{ll}
\rho^{-1} & , \rho>a \\
(2 a)^{-1} & , \rho=a \\
0 & , \rho<a
\end{array}\right\}=\frac{1}{2 a^{2}} \text { for }(\rho=a)
$$

Recall that $\varepsilon_{z}=2$ only for $z_{0}^{-}<z<z_{0}^{+}$, otherwise $\varepsilon=0$. So the integral on the right side of equation (D4-7) is:

$$
\varepsilon_{\rho}=\varepsilon_{z} \int_{0}^{\infty} J_{1}(\lambda a) J_{1}(\lambda \rho) \lambda d \lambda=\frac{1}{a^{2}} \quad \text { for }(\rho=a) \text { and }\left(z_{0}^{-}<z<z_{0}^{+}\right)
$$

Equation (D4-12) only occurs when evaluating the singular cell. Hence, the final form of the solution for $\Gamma_{\rho p^{*}}$ is:

$$
\Gamma_{\mathrm{p} \rho^{*}}=\frac{a \Delta a \Delta \sigma}{\sigma_{1}} \sum_{i=1}^{4}\left\{( \pm)_{i}^{\rho} \frac{\alpha_{i} k_{i}}{4 \pi(\rho a)^{3 / 2}}\left[\frac{1+k^{\prime 2}}{k^{\prime 2}} \mathrm{E}\left(k_{i}\right)-2 \mathrm{~K}\left(k_{i}\right)\right]-\varepsilon_{\rho}\right\}
$$

where:

$$
\varepsilon_{\rho}= \begin{cases}\frac{1}{a^{2}}, & (\rho=a) \text { and }\left(z_{0}^{-}<z<z_{0}^{+}\right) \\ 0, & \text { otherwise }\end{cases}
$$




\section{APPENDIX E}

\section{THE GRADIENT INTEGRAL}

This appendix solves the bracketed term in equation (3.1.2-10) which is:

$$
\begin{aligned}
\frac{\partial}{\partial z} \int_{V^{*}} g\left(\mathbf{r}, \mathbf{r}^{*}\right) d v^{*} & =2 \pi \frac{\partial}{\partial z} \int_{\rho^{*} z^{*}} \int\left\{\frac{1}{4 \pi} \int_{0}^{\infty} J_{0}\left(\lambda \rho^{*}\right) J_{0}(\lambda \rho)\left[e^{-\lambda\left|z-z^{*}\right|}-e^{-\lambda\left(z+z^{*}\right)}\right] d \lambda\right\} \rho^{*} d \rho^{*} d z^{*} \\
& =\frac{1}{2} \int_{0}^{\infty}\left[\int_{\rho^{*}} J_{0}\left(\lambda \rho^{*}\right) \rho^{*} d \rho^{*}\right]\left[\int_{z^{*}}\left[e^{-\lambda\left|z-z^{*}\right|}-e^{-\lambda\left(z+z^{*}\right)}\right) d z^{*}\right] J_{0}(\lambda \rho) d \lambda
\end{aligned}
$$

From appendix equation (D1-7), the $\rho^{*}$-integration is approximated by:

$$
\int_{\rho^{*}} J_{0}\left(\lambda \rho^{*}\right) \rho^{*} d \rho^{*}=2 a \Delta a J_{0}(\lambda a)
$$

The exponential term is found in appendix equation(D2-3) and is:

$$
\frac{\partial}{\partial z} \int_{z^{*}}\left[e^{-\lambda\left|z-z^{*}\right|}-e^{-\lambda\left(z+z^{*}\right)}\right] d z^{*}=\xi_{z}(\lambda z)=\sum_{i=1}^{4}(-1)^{i} e^{-\lambda \alpha_{i}}
$$

where: $\alpha_{1}=\left|z-\left(z_{0}+\Delta z\right)\right|, \alpha_{2}=\left|z-\left(z_{0}-\Delta z\right)\right|, \alpha_{3}=\left[z+\left(z_{0}+\Delta z\right)\right]$, and $\alpha_{4}=\left[z+\left(z_{0}-\Delta z\right)\right]$. Combining the results, equation (E1) is:

$$
\frac{\partial}{\partial z} \int_{v^{*}} g\left(\mathbf{r}, \mathbf{r}^{*}\right) d v^{*}=a \Delta a \int_{0}^{\infty} \sum_{i=1}^{4}(-1)^{i} e^{-\lambda \alpha_{i}} J_{0}(\lambda a) J_{0}(\lambda \rho) d \lambda=a \Delta a \sum_{i=1}^{4}(-1)^{i} \int_{0}^{\infty} J_{0}(\lambda a) J_{0}(\lambda \rho) e^{-\lambda \alpha_{i}} d \lambda
$$

The integral in equation (E4) is found in Luke (1962):

$$
\int_{0}^{\infty} J_{0}(\lambda a) J_{0}(\lambda \rho) e^{-\lambda \alpha_{i}} d \lambda=\frac{k_{i}}{\pi(p a)^{1 / 2}} \mathbf{K}\left(k_{i}\right)
$$

where:

$$
k_{i}=\left[\frac{4 \rho a}{\alpha_{i}{ }^{2}+(\rho+a)^{2}}\right]^{1 / 2}
$$

and $\mathbf{K}(k)$ is the complete elliptic integral of the first kind. The solution of equation $(E-1)$ is:

$$
\frac{\partial}{\partial z} \int_{v^{*}} g\left(\mathbf{r}, \mathbf{r}^{*}\right) d v^{*}=\sum_{i=1}^{4}(-1)^{i} \frac{k_{i}}{\pi(\rho a)^{1 / 2}} \mathbf{K}\left(k_{i}\right)
$$


Taking the negative $a$-derivative yields:

$$
-\frac{\partial}{\partial a}\left\{\begin{array}{ll}
\rho^{-1} & , \rho>a \\
(2 a)^{-1} & , \rho=a \\
0 & , \rho<a
\end{array}\right\}=\frac{1}{2 a^{2}} \text { for }(\rho=a)
$$

Recall that $\varepsilon_{z}=2$ only for $z_{0}^{-}<z<z_{0}^{+}$, otherwise $\varepsilon=0$. So the integral on the right side of equation (D4-7) is:

$$
\varepsilon_{p}=\varepsilon_{2} \int_{0}^{\infty} J_{1}(\lambda a) J_{1}(\lambda p) \lambda d \lambda=\frac{1}{a^{2}} \quad \text { for }(\rho=a) \text { and }\left(z_{0}^{-}<z<z_{0}^{+}\right)
$$

Equation (D4-12) only occurs when evaluating the singular cell. Hence, the final form of the solution for $\Gamma_{\rho \rho^{*}}$ is:

$$
\Gamma_{\mathrm{p} \mathrm{p}^{*}}=\frac{a \Delta a \Delta \sigma}{\sigma_{1}} \sum_{i=1}^{4}\left\{( \pm)_{i}^{\rho} \frac{\alpha_{i} k_{i}}{4 \pi(\rho a)^{3 / 2}}\left[\frac{1+k^{2}}{k^{\prime 2}} \mathrm{E}\left(k_{i}\right)-2 \mathrm{~K}\left(k_{i}\right)\right]-\varepsilon_{\mathrm{p}}\right\}
$$

where:

$$
\varepsilon_{\rho}= \begin{cases}\frac{1}{a^{2}}, & (\rho=a) \text { and }\left(z_{0}^{-}<z<z_{0}^{+}\right) \\ 0, & \text { otherwise }\end{cases}
$$




\section{APPENDIX E}

\section{THE GRADIENT INTEGRAL}

This appendix solves the bracketed term in equation (3.1.2-10) which is:

$$
\begin{aligned}
\frac{\partial}{\partial z} \int_{v^{*}} g\left(r, r^{*}\right) d v^{*} & =2 \pi \frac{\partial}{\partial z} \int_{\rho^{*} z^{*}}\left\{\frac{1}{4 \pi} \int_{0}^{\infty} J_{0}\left(\lambda \rho^{*}\right) J_{0}(\lambda \rho)\left[e^{-\lambda\left|z-z^{*}\right|}-e^{-\lambda\left(z+z^{*}\right)}\right] d \lambda\right\} \rho^{*} d \rho^{*} d z^{*} \\
& =\frac{1}{2} \int_{0}^{\infty}\left[\int_{\rho^{*}} J_{0}\left(\lambda \rho^{*}\right) \rho^{*} d \rho^{*}\right]\left[\int_{z^{*}}\left[e^{-\lambda\left|z-z^{*}\right|}-e^{-\lambda\left(z+z^{*}\right)}\right) d z^{*}\right] J_{0}(\lambda \rho) d \lambda
\end{aligned}
$$

From appendix equation (D1-7), the $\rho$ "-integration is approximated by:

$$
\int_{\rho^{*}} J_{0}\left(\lambda \rho^{*}\right) \rho^{*} d \rho^{*} \approx 2 a \Delta a J_{0}(\lambda a)
$$

The exponential term is found in appendix equation(D2-3) and is:

$$
\frac{\partial}{\partial z} \int_{z^{*}}\left[e^{-\lambda\left|z-z^{*}\right|}-e^{-\lambda\left(z+z^{*}\right)}\right] d z^{*}=\xi_{z}(\lambda z)=\sum_{i=1}^{4}(-1)^{i} e^{-\lambda \alpha_{i}}
$$

where: $\alpha_{1}=\left|z-\left(z_{0}+\Delta z\right)\right|, \alpha_{2}=\left|z-\left(z_{0}-\Delta z\right)\right|, \alpha_{3}=\left[z+\left(z_{0}+\Delta z\right)\right]$, and $\alpha_{4}=\left[z+\left(z_{0}-\Delta z\right)\right]$. Combining the results, equation (E1) is:

$\frac{\partial}{\partial z} \int_{v^{*}} g\left(\mathrm{r}, \mathrm{r}^{*}\right) d v^{*}=a \Delta a \int_{0}^{\infty} \sum_{i=1}^{4}(-1)^{i} e^{-\lambda \alpha_{i}} J_{0}(\lambda a) J_{0}(\lambda \rho) d \lambda=a \Delta a \sum_{i=1}^{4}(-1)^{i} \int_{0}^{\infty} J_{0}(\lambda a) J_{0}(\lambda \rho) e^{-\lambda \alpha_{i}} d \lambda$

The integral in equation (E4) is found in Luke (1962):

$$
\int_{0}^{\infty} J_{0}(\lambda a) J_{0}(\lambda \rho) e^{-\lambda \alpha_{i}} d \lambda=\frac{k_{i}}{\pi(p a)^{1 / 2}} \mathbf{K}\left(k_{i}\right)
$$

where:

$$
k_{i}=\left[\frac{4 \rho a}{\alpha_{i}^{2}+(\rho+a)^{2}}\right]^{1 / 2}
$$

and $\mathbf{K}(k)$ is the complete elliptic integral of the first kind. The solution of equation (E-1) is:

$$
\frac{\partial}{\partial z} \int_{v^{*}} g\left(\mathbf{r}, \mathbf{r}^{*}\right) d v^{*}=\sum_{i=1}^{4}(-1)^{i} \frac{k_{i}}{\pi(\rho a)^{1 / 2}} \mathbf{K}\left(k_{i}\right)
$$

